\title{
Identification and functional characterization of novel ionotropic glutamate receptor subunits at Drosophila neuromuscular synapse
}

\author{
Dissertation \\ zur Erlangung des Doktorgrades \\ der Mathematisch-Naturwissenschaftlichen Fakultäten \\ der Georg-August-Universitat zur Göttingen
}

Vorgelegt von

Gang Qin

aus Tianjin, V.R. China

Göttingen 2004 
D7

Referent: Prof. Dr. F. W. Schürmann

Korreferent: Prof. Dr. H. Jäckle

Tag der mündlichen Prüfung: 26 JAN 2005 

$\begin{array}{ll}\text { Summary } & 4\end{array}$

List of Figures

List of Tables

$\begin{array}{ll}\text { Acknowledgements } & 7\end{array}$

1 Introduction $\quad 8$

$\begin{array}{lr}\text { 1.1 Molecular basics of Synaptogenesis } & 8\end{array}$

1.1.1 The making of a synapse 8

1.1.2 Development of vertebrate NMJ 11

1.1.2.1 Initial AchR clustering 11

1.1.2.2 Agrin-MuSK signalling pathway 13

1.1.2.3 Developmental role of AchR per se 16

1.1.3 Development of mammalian CNS excitatory synapse 16

1.1.3.1 Cell adhesion molecules in the axon-dendrite contact 18

1.1.3.2 Assembly of synapse 21

1.1.3.2.1 Presynaptic assembly 21

1.1.3.2.2 Postsynaptic assembly 23

1.1.3.3 Time sequence of pre- and postsynaptic assembly 25

1.1.4 Drosophila NMJ as one model for synaptogenesis 25

1.1.4.1 Structural properties of Drosophila NMJ 25

1.1.4.2 Initiation and maintenance of neuromuscular connection 28

1.1.4.2.1 Initiation of neuromuscular contact---target recognition
(pathfinding)

1.1.4.2.2 Stabilization and maintenance of neuromuscular connection by cell adhesion molecules $\quad 29$

1.1.4.3 Synapse formation 31

1.2 Structure and functional relevance of ionotropic glutamate receptors 33

1.2.1 Modular design of ionotropic glutamate receptors
during evolution

1.2.2 Molecular mechanisms underlying selective assembly and transport of ionotropic glutamate receptors 34

1.2.3 Properties of ionotropic glutamate receptors expressed at Drosophila neuromuscular synapses $\quad 36$

1.3 The aim and strategy of this work 37 
2.1 Materials (Chemicals, enzymes and molecular kits)

2.2 Methods

2.2.1 Genechip analysis and quantitative real-time RT-PCR 39

2.2.1.1 Genechip analysis $\quad 39$

2.2.1.2 Real-time RT-PCR 45

2.2.2 Fly stocks and genetics 46

2.2.2.1 Fly stocks, crosses and rearing conditions 46

$\begin{array}{ll}\text { 2.2.2.2 Mutagenesis screening } & 47\end{array}$

2.2.3 Molecular constructs and transgenes 48

2.2.4 In situ hybridization $\quad 50$

2.2.5 Biochemistry (collaborated with Tobias Schwarz) 53

2.2.6 Immunohistochemistry $\quad 54$

2.2.6.1 Embryonic and Larval filet preparation $\quad 54$

2.2.6.2 Confocal epifluorescent microscopy 56

2.2.7 Ultrastructural analysis (collaborated with Carolin Wichmann) 56

2.2.8 Electrophysiology (collaborated with Robert Kittle) 57

3 Results $\quad 58$

3.1 Establishing the tissue specific transcriptome of Drosophila larval body wall muscle $\quad 58$

3.2 Identification of novel ionotropic glutamate receptor subunits expressed at the Drosophila neuromuscular synapse

3.2.1 Enrichment of various transcripts encoding novel ionotropic glutamate receptor subunits in larval body wall muscles $\quad 60$

3.2.2 Expression pattern of new ionotropic glutamate receptor genes 61

3.2.3 The GluR-IID and GluR-IIE represent a new type of muscle expressed glutamate receptor subunits

3.2.4 GluR-IIC, GluR-IID and GluR-IIE are specifically localized at postsynaptic densities of neuromuscular synapses 65

3.3 GluR-IID and GluR-IIE are essential for viability

3.4 Reciprocal dependence of all essential glutamate receptor subunits for glutamate receptor expression and function at synapse

3.4.1 Complete absence of all the other glutamate receptor subunits at synapse in knockout of any essential subunit genes

3.4.2 Genetically depriving the expression level of any single essential glutamate receptor subunits results into tight reduction of all of the other subunits at synapse

3.4.3 Depriving synaptic glutamate receptor subunits results into dramatic weakness of postsynaptic activity 
3.5 Structural role of ionotropic glutamate receptor during pre- and postsynaptic differentiation of neuromuscular synapse

3.5.1 Normal differentiation of presynaptic transmitter release mechinery at glutamate receptor deprived synapse

3.5.2 Defective assembly of PSD specialization at glutamate receptor deprived but not presynaptic neurotransmission deprived synapse

3.5.3 Ultrastructural evidence of abnormal synaptic differentiation at glutamate receptor deprived synapse

3.5.4 Defective compartmentation of the synaptic and perisynaptic area at glutamate receptor deprived but not neurotransmission deprived synapse

3.5.5 Similar defects in synaptic differentiation in the complete absence of glutamate receptors

4 Discussion

4.1 Genomic tools could speed up identifying essential genes involved in synapse formation and modulation

4.2 GluR-IID and GluR-IIE are novel glutamate receptor subunits crucial for glutamate receptor assembly and thus neurotransmission at neuromuscular synapse

4.3 Implications in the in vivo stoichiometry of Drosophila glutamate receptor based on genetic analysis

4.4 Structural role of ionotropic glutamate receptor per se in organizing synaptic assembly and time sequence of pre- and postsynaptic assembly

References

Appendix: Genes enriched in larval body wall preparations 


\section{Summary}

The molecular mechanisms triggering the formation of synapses in vivo are crucial for understanding the development as well as the activity dependent remodelling of synaptic circuits. The Drosophila neuromuscular junction (NMJ) provides an excellent platform for investigating fundamental aspects of how glutamatergic synapse form in vivo. The molecular mechanisms relevant to synaptogenesis and growth control of the Drosophila NMJ were addressed by combining genome-wide transcript analysis with functional genetics. This allowed the identification of two novel postsynaptic muscle expressed ionotropic glutamate receptor subunits, GluR-IID and GluR-IIE. Genetic elimination of either of the two novel subunits resulted in paralyzed lethal embryos, indicating that both new subunits are essential for forming the postsynaptic glutamate receptor complex. Further genetic, cell biological and electrophysiological studies then uncovered a tight interdependence of all NMJ glutamate receptor subunits for synaptic localization and function. These results imply that the NMJ glutamate receptor complex has strictly hetero-tetrameric stoichiometry. This so far was not described for mammalian ionotropic glutamate receptors which are usually considered to be dimers of dimers. In the second part of the thesis, glutamate receptor deprived synapses were closer inspected. Surprisingly, depleting glutamate receptors (but not depleting synaptic neurotransmission activity) provoked severe ultrastructural and molecular defects of postsynaptic membrane organization and compartment formation. Thus, the glutamate receptor complex per se but not its ligand-gated ion channel activity seemingly plays an instructive role for assembling mature postsynaptic specializations. Such a 'structural' role of glutamate receptor complexes in synaptic differentiation is novel. It might be relevant for the role 
ionotropic glutamate receptors play during synaptic plasticity of the mammalian brain, which is considered to be a cellular correlate of learning and memory processes. 


\section{List of Figures}

Fig.1-1 Basic structure of chemical synapse

Fig. 1-2 Representative molecular composition of CNS glutamatergic

Fig.1-3 Vertebrate neuromuscular junction

Fig. 1-4 Spiny dendrites from hippocampal pyramidal neuron

Fig. 1-5 Structure of Drosophila NMJ

Fig.1-6 Structure of iGluR subunit and model of iGluR assembly

Figure 3-1. GluR-IID and GluR-IIE: novel glutamate receptor subunits with muscle specific expression

Figure 3-2 Sequence analysis of GluR-IID and GluR-IIE

Fig.3-3 GluR-IID and GluR-IIE are expressed within postsynaptic Densities

Figure 3-4 Genetic analysis of GluR-IID and GluR-IIE

Figure 3-5 Interdependence between glutamate receptor subunits

Figure 3-6 A partial reduction of either GluR-IIE or GluR-IID provokes a significant reduction of all glutamate receptor subunits at the NMJ

Fig.3-7 Minimal mounts of GluR-IIA and no- IIB: expression of all glutamate receptor subunits and postsynaptic sensitivity are strongly reduced

Fig. 3-8 Normal organization of presynaptic neurotransmitter release machinery components at glutamate receptor deprived synapses

Fig.3-9 Defective PSD assembly at glutamate receptor deprived but not transmission deprived synapses

Fig. 3-10 Glutamate receptor per se but not presynaptic vesicle release are important for proper synapse formation at embryo NMJ

Fig.3-11 Electron microscopy: defective postsynaptic assembly at glutamate receptor deprived synapses

Fig.3-12 Defective synaptic compartments at glutamate receptor deprived but not neurotransmission deprived synapses 


\section{List of Tables}

Table 3-1 Described muscle / cuticle-specific transcripts displaying enrichment in gene chip analysis of body-wall-preparations

Table 3-2 GluR-IID and GluR-IIE are enriched in larval body wall mRNA 


\section{Acknowledgements}

First of all, I would like to express my gratitude to my advisors, Dr. Stephan Sigrist, Prof. Dr. F. W. Schürmann and Prof. Dr. H. Jäckle for their consistent support, helpful tips, kind advices and comments.

My very special thanks are to Dr. Stephan Sigrist, who provides me the opportunity to join his group and do exciting cutting-edge science. As one talented and serious scientist, Stephan taught me not only how to perform successful experiments but also how to ask the right question and address it in a logic way. During my whole thesis period, I was encouraged by our extensive and enthusiastic discussion, from which I really have learned a lot. Also, for me it is really nice to witness the rapid growth of our young group.

I would like to thank several collaborators, Robert Kittel for electrophysiology, Dr. Carolin Wichmann for Electron Microscopy and Tobias Schwarz for biochemistry. Without their excellent contributions, I could hardly reach current stage. The Gene Chip experiments were performed in Max-Planck Institute for Biophysical Chemistry, I am really indebted to Dr. Ronald Kühnlein and Dr. Sebastian Grönke for their warm and patient help.

I am also grateful to my colleagues Carlos Melino, Tobias Rasse, Andreas Schmid, Sara Mertel, Wernher, Laura Swan and Manuela Schmidt, with whom I can freely exchange ideas and reagents, which greatly facilitate my work. I want

to express my kindest gratefulness to Christine Quentin, Miriam Richter and Jasmin for their highly professional technical assistance.

Finally, my very special thanks are addressed to my family and my friends. 


\section{Introduction}

\subsection{Molecular basics of Synaptogenesis}

\subsubsection{The making of a synapse}

The human brain comprises a huge network of neurons (about $10^{11}$ ) which link with each other structurally and functionally through the special type of cell-cell junctions called synapses. Synapses transmit electrical signals between neurons, mediating neuronal signaling and computation, also they are able to undergo long-term changes (synaptic plasticity) that underlie aspects of learning and memory ( $\mathrm{Li}$ and Sheng, 2003). Although many of these synaptic contacts are highly specialized, all neurons make use of one of the two basic forms of synaptic transmission: electrical or chemical. Synaptic communication in the brain is mainly dependent on chemical mechanisms, that is, synaptic transmission is mediated via a chemical substance that is released by presynaptic terminal and diffuses through the synaptic cleft to activate relevant receptors on the postsynaptic membrane. Like other cell-cell junctions, synapses contain a wide variety of transmembrane proteins, cytoskeletal units and signaling components. However, they differ from most other junctions in being structurally asymmetric (Fig. 1-1): the presynaptic specialization, usually on the axon, contains a complex apparatus called active zone, which controls the transmitter-filled vesicles docking, fusing and the release of transmitter into the synaptic cleft. In contrast, the postsynaptic reception specialization, just juxtaposed to the active zone and recognized ultrastructurally by the presence of an electron-dense thickening called the postsynaptic density (PSD), is made of receptors, ion channels, signaling components and scaffold proteins clustered all together (Garner et al., 2002; Yamagata et al., 2003).

On average, each neuron forms about 1000 synaptic connections and receives even more, perhaps as many as 10,000 connections (Kandel ER et al. 2001). These synapses include excitatory, inhibitory and modulatory type. Most 
of them are excitatory and utilize glutamate as neurotransmitter. The inhibitory neurotransmission is mediated by glycine or GABA, whereas modulatory neurotransmission is mediated by 5-HT, dopamine, acetylcholine (Ach) and neuropeptides etc. Notably, although presynaptic terminals from any given neuron generally release one type of transmitter, neurons usually receive multiple forms of synaptic input, which can be excitatory, inhibitory or modulatory. Moreover, anatomical evidence has shown that the number of cellular contacts often exceeds the number of synaptic connections formed onto a single cell, suggesting that neurons are able to distinguish synaptic contact site and nonsynaptic site (White et al., 1976; Benson et al., 2001). Thus, for efficient and accurate neurotransmission, the site where synaptic contact forms is carefully selected, and, within an individual synapse, appropriate pre- and postsynaptic apparatus is assembled highly coordinately and precisely.
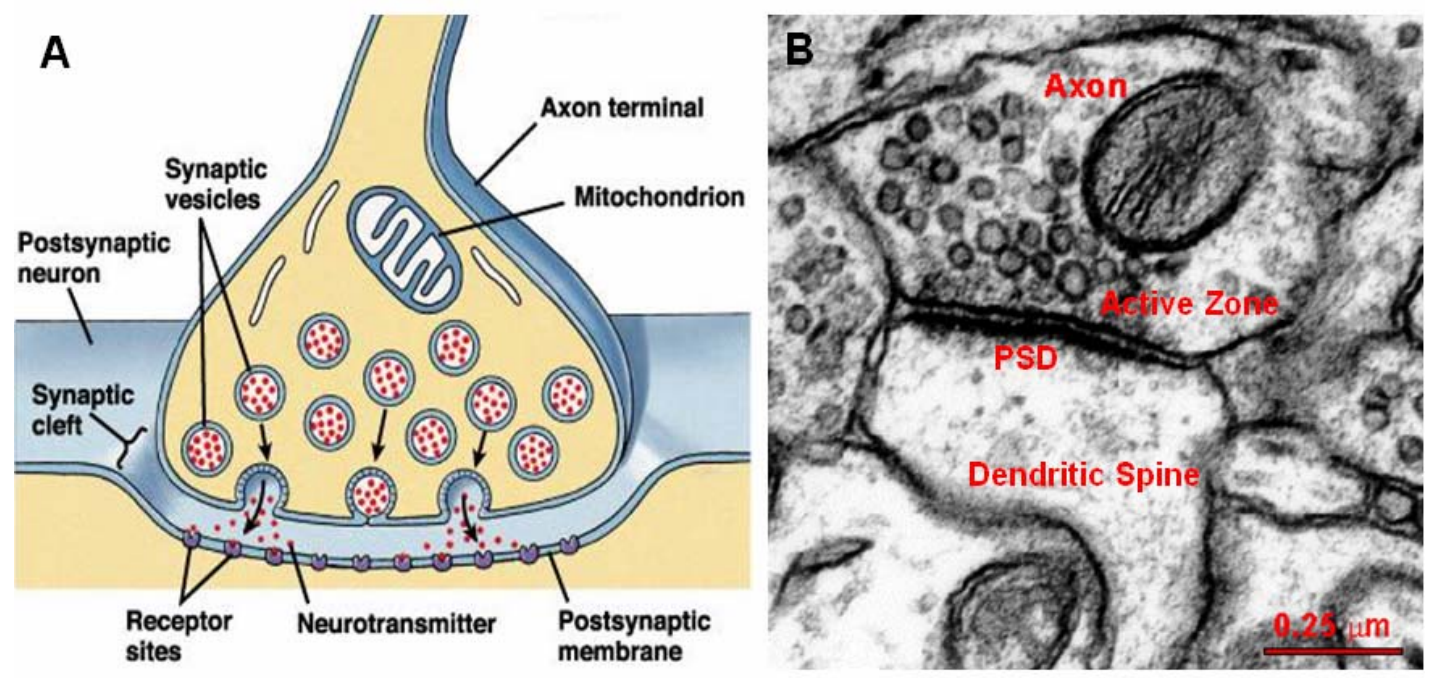

Fig.1-1 Basic structure of chemical synapse. A, diagram of a central nervous system chemical synapse. B, ultrastructure of a central chemical synapse (Adapted from www.synapses.mcg.edu/)

What is the molecular mechanisms controlling the site and type specific assembly of chemical synapses? To answer this fundamental question in neurobiology, extensive efforts have been invested to identify the molecular 
constituents of synapses and define their functional significance via the combination of molecular, biochemical and genetic approaches (Fernandez-Chacon and Sudhof, 1999; Scannevin and Huganir, 2000; Phillips et al., 2001; Sheng, 2001; Garner et al., 2002) (Fig. 1-2). Furthermore, recently the adoption of newly developed optical imaging technique has provided a way to track the dynamics of individual synaptic protein trafficking and targeting during synapse formation even in real-time resolution (Ahmari et al., 2000; Okabe et al., 2001; Prange and Murphy, 2001; Rosenberg et al., 2001; Zhai et al., 2001).
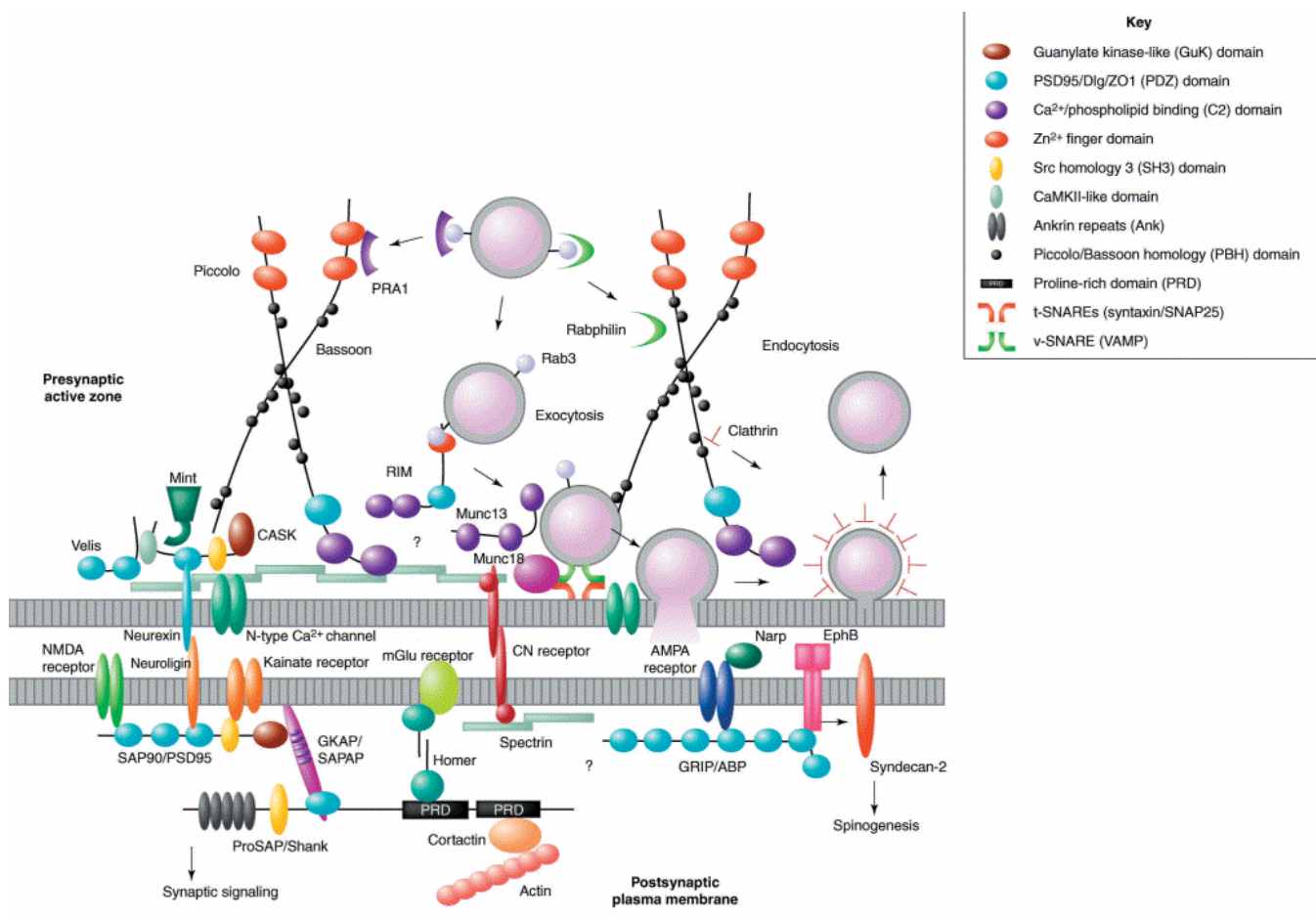

TRENOS in Neurosesiences

Fig. 1-2 Representative molecular composition of CNS glutamatergic synapse (Adapted from Garner et al., 2002).

Historically, much of what we know about the development of chemical synapses is based on studies of the vertebrate neuromuscular junction (NMJ) - a large peripheral synapse formed between a motor neuron and a skeletal muscle fibre. However, in recent years, with the incremental technical 
advances a picture of the synaptogenesis in the mammalian central nervous system has also begun to emerge.

\subsubsection{Vertebrate NMJ}

The vertebrate NMJ (Fig. 1-3) is ideal for studying chemical synaptogenesis because it is large, relatively simple and unparalleled accessible to experimentation. During development (starting at about E11 in mice) multinucleated skeletal muscle fibres form by fusion of precursor myoblasts, shortly after myotubes begin to form (at E12-13 in mice), motor neurons innervate the muscle at a specialized region of the muscle membrane called the end-plate. At the region where the motor axon approaches the muscle fibre, the axon loses its myelin sheath and splits into several fine branches. The ends of the fine branches form multiple expansions or varicosities, called synaptic boutons, from which the neurotransmitter (here is Ach) is released from the motor neuron. Each bouton is positioned over a postjunctional fold, a deep and narrow depression of muscle cell membrane that contains high densities of transmitter receptors (here is acetylcholine receptors, AchRs), and this precise apposition of postsynaptic specializations to the motor nerve terminal insures that transmitter comes into contact with its receptor within microseconds after release, thereby facilitating efficient synaptic transmission.

\subsubsection{Initial AchR clustering}

Once the myotube forms, the AchR is starting to be expressed and inserted into the muscle surface, but with low density (usually about 1000 per $\left.\mu \mathrm{m}^{2}\right)$; after synaptic maturation, by contrast, the AchRs are highly concentrated at the synaptic sites (to a density up to 10,000 per $\mu \mathrm{m}^{2}$ ) and depleted at the extrasynaptic membrane (10 per $\mu \mathrm{m}^{2}$ or less). One of the central questions is the contribution of muscle versus motor nerve in initiating 

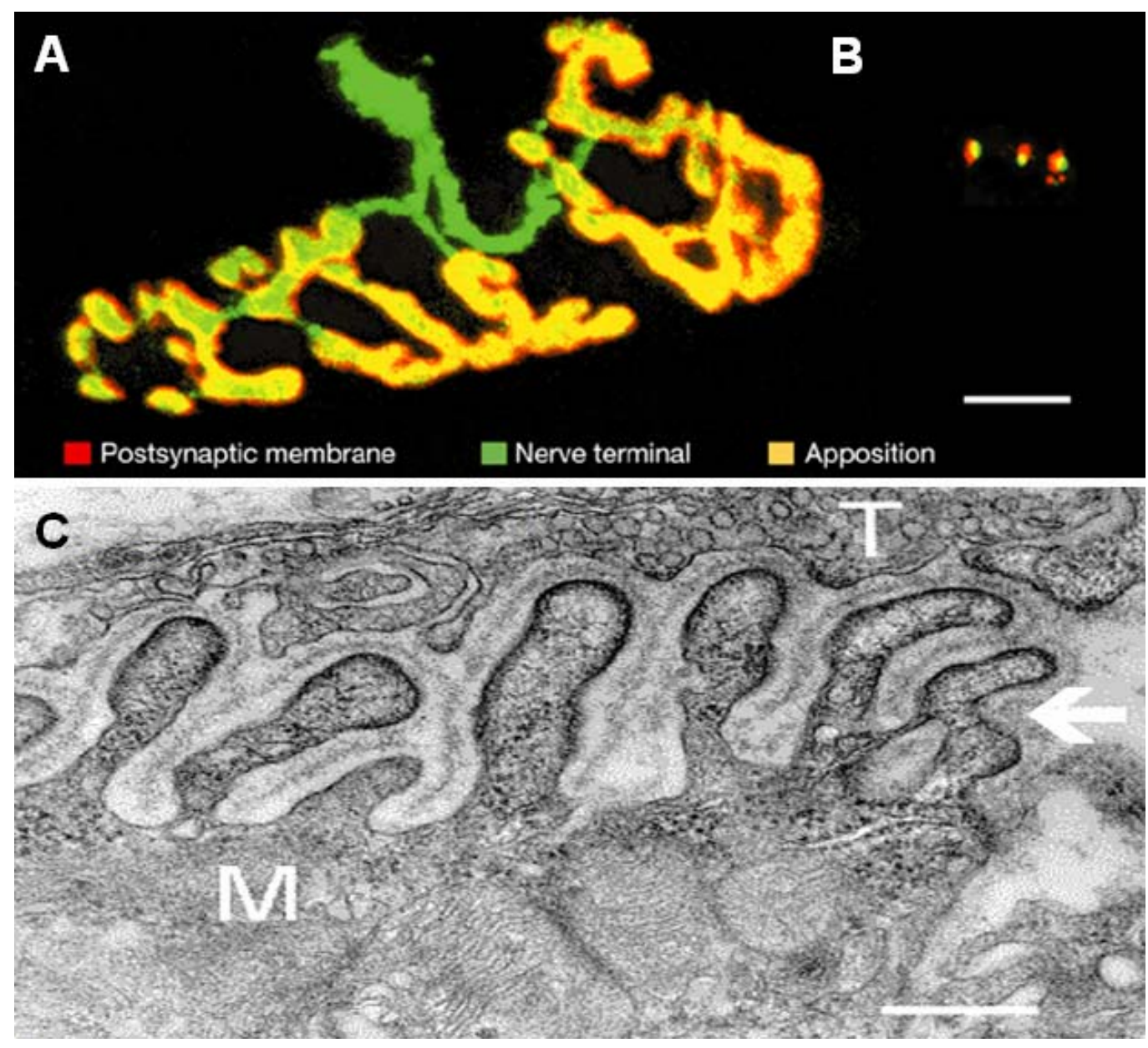

Fig.1-3 Vertebrate neuromuscular junction. A, The neuromuscular junction of an adult mouse. Scale $=5 \mu \mathrm{m}$. B, Three synapses on cultured mouse hippocampal neurons shown at the same scale(Adapted from Sanes J et al. 2001). C, Electron micrograph of motor end plate of abdominal muscle of frog. $\mathrm{T}$ - axon terminal, $\mathrm{M}$ - muscle fiber, arrow - foldings covered with basal lamina. Scale $=0.3 \mu \mathrm{m}$ (Adapted from www.synapses.mcg.edu/)

and maintaining postsynaptic differentiation. Recently, more and more evidences suggest that the muscle itself has the capacity to be prepatterned. Particularly pertinent observations are, during the brief period when synaptogenesis begins in mouse muscle, some AchR clusters are not apposed by nerves and some nerve endings are not opposed to AchR clusters; moreover, the AchR clusters are present in muscles even without innervation, most of these 
small clusters aggregate in the central end-plate band of the muscle, although this central band appears to be wider and more poorly defined than the one in innervated muscle (Lin et al., 2001; Yang et al., 2001).

\subsubsection{Arin-MuSK Signaling Pathway}

Upon innervation, AchR clusters are restricted to the central band of muscle. In the past three decades, numerous studies have established that motor nerve plays a major role in organizing this type of postsynaptic differentiation via two intramuscular mechanisms: clustering of AchRs at the synaptic contact sites; and selective transcription of AchR genes by myonuclei associated with synaptic sites (Burden, 1998; Sanes and Lichtman, 1999; Lin et al., 2001). Several nerve derived molecules have been identified and agrin is proved to be crucial in vivo. Agrin, isolated by McMahan and colleagues, is a large heparan sulphate proteoglycan that is synthesized by motor neurons, transported down motor axons, and released from nerve terminals, where it stably associates with the basal lamina of the synaptic cleft (Wallace, 1989; McMahan, 1990; Bowe and Fallon, 1995; Sanes and Lichtman, 2001). Agrin was identified from its ability to induce the aggregation AchRs on cultured myotubes, and was soon found to also aggregate many other components of the postsynaptic apparatus (Wallace, 1989). In Agrin-deficient mice, the number and density of AchR clusters, as well as synapse-specific transcription, are reduced markedly, which convincingly demonstrates that Agrin is crucial for AchR clustering in the muscle membrane opposite the presynaptic terminals (Gautam et al., 1996; Ruegg and Bixby, 1998).

MuSK, a transmembrane receptor tyrosine kinase, was discovered due to its selective abundance in synapse-rich Torpedo electric organ, whose principal cells are modified muscle cells that are innervated by cholinergic synapses (Jennings et al., 1993). Studies in mice reveal that MuSK is specifically expressed in skeletal muscles and is colocalized with AchRs in the postsynaptic membrane at the NMJ (Valenzuela et al., 1995). 
Unexpectedly, muscles in MuSK-null mutant mice show totally no detectable signs of postsynaptic differentiation, although the AchR genes are expressed at normal level (DeChiara et al., 1996; Lin et al., 2001; Yang et al., 2001). Several studies have established the linkage between Agrin and Musk signaling: application of Agrin to myotubes leads to rapid activation of MuSK; expression of a dominant negative form of MuSK inhibits Agrin-induced cluster formation in cultured myotubes (Glass et al., 1996); $\mathrm{MuSK}^{-/-}$ myotubes are completely unresponsive to agrin, but agrin sensitivity is restored by introducing wild-type MuSK (Zhou et al., 1999). Taken together, these results demonstrate that MuSK is essential for agrin-induced postsynaptic differentiation.

A crucial component downstream of MuSK that affects the postsynaptic differentiation is rapsyn, a $43-\mathrm{kDa}$ membrane associated cytoplasmic protein. Rapsyn is identified from its tight connection with AchR. It appears as soon as AchR starts clustering, perfectly colocalizes with AchR clusters, and in the electric organ it follows 1:1 stoichiometry (LaRochelle and Froehner, 1986; Noakes et al., 1993). Co-expression of AchR and rapsyn in all of the heterologous cells tested so far results into the formation of AchR- Rapsyn co-clusters, whereas AchRs are diffusely distributed when expressed along (Froehner et al., 1990; Phillips et al., 1991). Consistently, in rapsyn mutant mice, no AchR clusters form on the muscle surface (Gautam et al., 1995). Thus, rapsyn is necessary for all forms of AchR clusting. Intriguingly, rapsyn can cluster AchRs when they are co-expressed in heterologous non-muscle cells that lack agrin and MuSK (Apel et al., 1997). By contrast, not even spontaneous (agrin-independent) AchR clusters form in MuSK deficient myotubes (Zhou et al., 1999), and overexpression of rapsyn in wild-type myotubes induces virtually no further cluster formation (Yoshihara and Hall, 1993; Han et al., 1999). This raises the possibility that muscles contains intrinsic activity to prevent the MuSK signaling independent clustering of rapsyn, which might be an efficient way to avoid the AchR clustering at the 
wrong sites.

How does activation of Agrin-MuSK signaling lead to the clustering of AchRs? Previously it has been shown that cytoskeletal components are closely associated with AchR clusters, and disruption of cytoskeleton prevents the AchR cluster formation (Bloch and Pumplin, 1988). Thus, it is plausible that the activation of MuSK signaling upon agrin binding provide intrinsic cytoskeletal regulatory capacity required for remodelling and postsynaptic assembly. Consistent with this view, it has been found that agrin induces the polymerization of actin at the AchR clustering sites (Dai et al., 2000), moreover, it has been demonstrated that agrin-mediated clustering of AchRs depends on activation of $\mathrm{Rac} / \mathrm{Cdc} 42$, leading to the formation of AchR microclusters, followed by Rho activation, resulting in the consolidation of these microclusters into larger AcgR clusters (Weston et al., 2000; Weston et al., 2003).

Although there is little doubt that Agrin/ MuSK signaling is the major pathway that is crucial for NMJ synaptogenesis, the intracellular signaling mechanisms downstream of MuSK have only begun to be elucidated recently. In response to agrin binding, MuSK undergoes tyrosine phosphorylation, this might create docking sites for signaling molecules or promote proper folding of the kinase. As an example, the juxtamembrane tyrosine (Y553) is required for agrin-induced AchR clustering (Zhou et al., 1999; Herbst and Burden, 2000). Very recently, the Abl kinases were shown to be required for the agrin/ MuSK dependant synaptic assembly, Abl kinases and MuSK can effect reciprocal tyrosine phosphorylation and form a complex after agrin engagement (Finn et al., 2003). Dishevelled (Dvl), a signaling molecule important for planar cell polarity, has also been found to interact directly with MuSK and PAK, forming a signaling scaffold which is important for agrin/ MuSK mediated AchR clusting (Luo et al., 2002). Additionally, evidences have been provided that geranylgeranyltransferase I (GGT), a zinc 
metalloenzyme that tethers proteins to plasma membrane by prenylation (Zhang and Casey, 1996), serves as one important component in the agrin/ MuSK pathway, which reveals an important role of prenylation in regulating synapse formation and/ or maintenance (Luo et al., 2003).

\subsubsection{Developmental role of AchR per se}

During postsynaptic differentiation, do AchRs only occupy the slots within assembled scaffold and contribute nothing for the assembly? In tested non-muscle cells, this seems true, but in muscle cells it is obviously not the case: Rapsyn fails to cluster at synaptic sites in zebrafish mutants deficient in the AchRs, suggesting the requirement of AchRs for proper synaptic localization of postsynaptic components (Ono et al., 2001); consistently, in the variants of C2 myotubes virtually lacking AchRs, agrin also fails to aggregate rapsyn (Marangi et al., 2001); moreover, in mutant mice that lack an adult AchR subunit, the postsynaptic membrane undergoes a profound reorganization in which levels of several membrane and cytoskeletal components decline in parallel with AchR loss, and residual material forms abnormal small islands at the NMJ (Missias et al., 1997). Therefore, the AchR per se is not merely a passive ligand, but plays an active role in organizing synaptic assembly. Interestingly, this is reminiscent of the assembly of inhibitory CNS synapses, in which $\mathrm{GABA}_{\mathrm{A}}$ receptor subtypes are localized at synapses through their interaction with gephyrin (Kneussel et al., 1999). Gephyrin is not clustered at postsynaptic sites of mice lacking GABA $_{\mathrm{A}}$ receptor $\gamma$ subunits, suggesting that gephyrin requires the presence of $\mathrm{GABA}_{\mathrm{A}}$ receptors to efficiently form synaptic aggregates (Essrich et al., 1998). Thus, neurotransmitter receptors of both vertebrate NMJ and inhibitory CNS synapses are actively involved in postsynaptic assembly during synaptogenesis.

\subsubsection{Mammalian CNS excitatory synapses}

In the central nervous system, most excitatory synapses utilize glutamate 
as the neurotransmitter. Glutamatergic synapses contain excitatory, glutamate responsive ionotropic and metabotropic receptors (Hollmann and Heinemann, 1994; McGee and Bredt, 2003). Ionotropic glutamate receptors are supposed to be tetrameric complexes, and are categorized into three major classes, on the basis of pharmacology, electrophysiology and sequence homology: AMPA ( $\alpha$-amino-3-hydroxy-5-methyl-4-isoxazoleproprionic acid) receptors, NMDA (N-methyl-D-aspartic acid) receptors and kainate receptors.

Morphologically, most mature excitatory synapses are constructed on the characteristic tiny actin-rich structures---dendritic spines---the small lateral dendritic protrutions. Spines have variable size (up to a few $\mu \mathrm{m}$ in length and $0.8 \mu^{3}$ in volume) and shape (such as thin, stubby or mushroom-like) (Harris and Kater, 1994; Hering and Sheng, 2001; Li and Sheng, 2003) (Fig. 1-4). During brain development, there is morphological transition in spines. During the first two postnatal weeks, synapses are frequently observed on the dendritic shaft, on stubby spines and on filopodia (Fiala et al., 1998), with maturation of the brain, such synapses are gradually substituted by, or converted to, synapses on mushroom-like spines (Harris and Kater, 1994).

As described before, the pre- and postsynaptic membrane of vertebrate NMJ are separated by an extracellular basal membrane, the extracellular-matrix molecule agrin is secreted from the nerve terminal and induces the postsynaptic differentiation. However, in central synapses, the extracellular matrix is not well characterized and, so far, no secreted molecules has been convincingly shown to be crucial for the development of mammalian central synapses. Narp (neuronal activity-regulated pentraxin), a secreted molecule that accumulates at synaptic sites, can interact with and cluster AMPA receptors (O'Brien et al., 1999; O'Brien et al., 2002). However, just as its name implied, Narp is a neuronal activity-regulated molecule, it recruits AMPA receptors only when a synapse is undergoing activity-dependant changes. Narp is less likely to play a major role during synaptogenesis, since AMPA receptor clustering is not essential for initial 
synapse formation (see below for details).
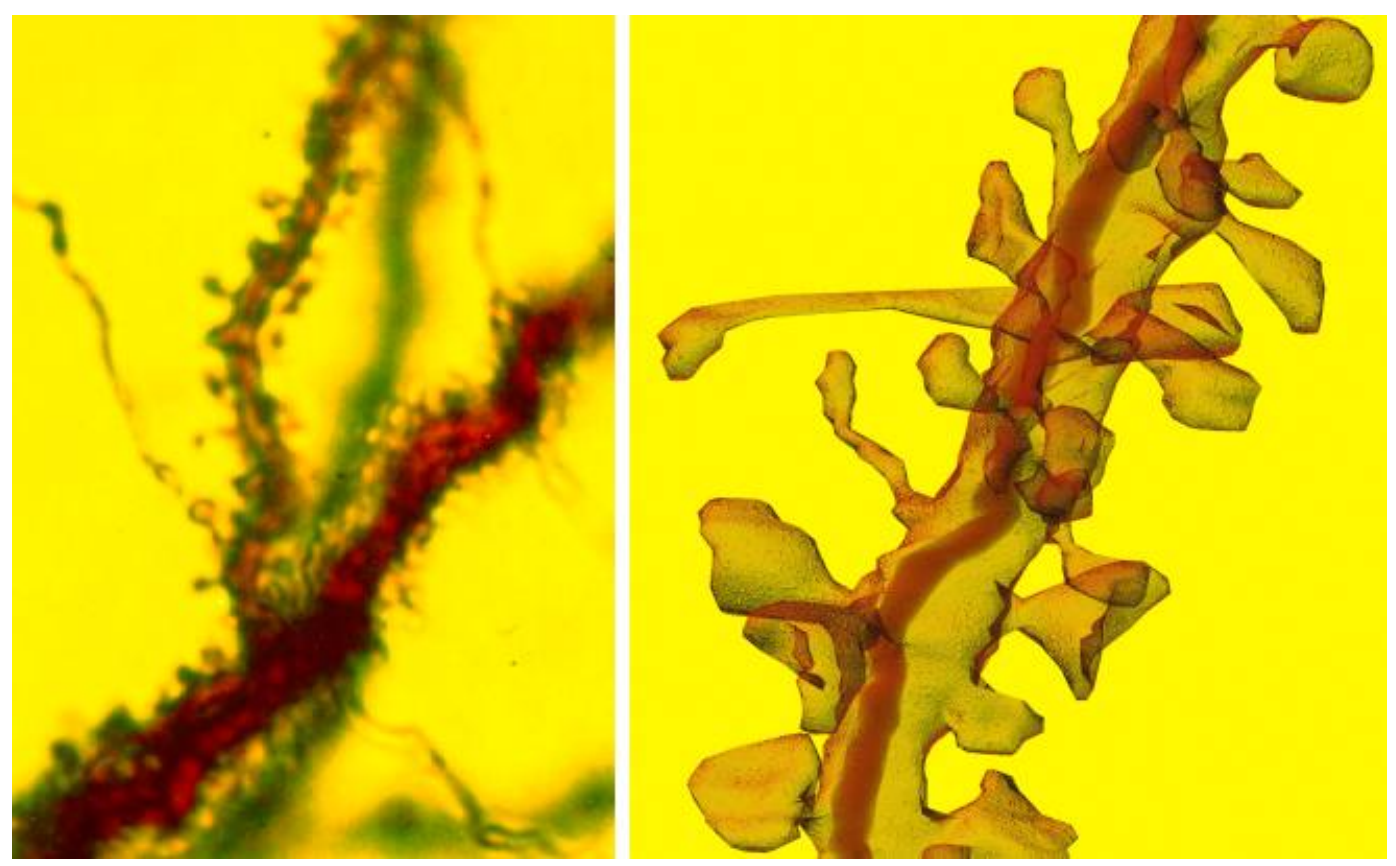

Fig. 1-4 Spiny dendrites from hippocampal pyramidal neuron.

Left: Light microscope image. Right: Reconstruction from serial electron microscopy (Adapted from www.synapses.mcg.edu/).

\subsubsection{Cell adhesion molecules in the axon-dendrite contact}

Synaptic assembly appears to be a multi-step process that is initiated from the axon-dendrite contact. Initial contact is followed by a stabilization step at the contact site and, subsequently, by further pre- and postsynaptic differentiation. Each process seems to require the interaction of various classes of cell adhesion molecules (CAMs). The Cadherin family of homophilic cell adhesion molecues is essential for the formation and stability of epithelian cell junctions. Cadherins are found to localize at both pre- and postsynaptic side, dominant-negative studies of the neuronal cadherins indicate that they are important for synapse integrity and morphological maturation of dendritic spines (Togashi et al., 2002). However, due to the large number of various Cadherin and Cadherin-related genes that are expressed in the central nervous system, it is still not possible to conclude 
whether cadherin plays an initiating role in synapse formation or a promoting role in subsequent synaptic growth.

Various types of integrins are expressed in the brain with different distribution patterns (Pinkstaff et al., 1999). Integrins function as cell-matrix or cell-cell adhesion molecules by binding, respectively, to extracellular matrix proteins or to immunoglobulin superfamily proteins (Aplin et al., 1998). Based on our current understanding, integrins have diverse functions in early stage of CNS development, including neuronal migration (Milner and Campbell, 2002), axon pathfinding and outgrowth (Hoang and Chiba, 1998; Pasterkamp and Kolodkin, 2003), they also appear to have a role in synapse maturation (Chavis and Westbrook, 2001).

Immunoglobulin superfamily molecules contain certain numbers of extracellular cyctein-looped domains first found in immunoglobulins. Synaptic cell adhesion molecule (SynCAM) belongs to such a superfamily and is found to be localized on the synaptic membranes. Importantly, expression of SynCAM in nonneuronal cells both induces neighboring neurons to form functional presynaptic terminals and, if gluamate receptors are coexpressed, endows the postsynaptic membranes with the capacity of responding to glutamate (Biederer et al., 2002). Thus, SynCAM seems an ideal candidate initiating central synapse formation, although its in vivo role has yet to be examined. Very recently, another two immunoglobulin superfamily members, SYG-1 and SYG-2 were isolated in genetic screen for C. elegans mutants defective in targeting specificity during synaptogenesis (Shen and Bargmann, 2003; Shen et al., 2004). SYG-1 is present at the presynaptic neuron HSNL and localizes to synaptic sites at the early stages of synaptic formation, while SYG-2 is expressed transiently in the guidepost epithelial cells during synapse formation. SYG-2 can interact with SYG-1 and thus instruct the accumulation of synaptic vesicles and the subsequent synaptic assembly at proper site. 
Presynaptic neurexins and postsynaptic neuroligins represent another group of synaptic CAMs proposed to function in synapse differentiation. The extracellular domain of presynaptic beta-neurexin interacts directly with the extracellular AchE-like domain of postsynaptic neuroligin (Nguyen and Sudhof, 1997). In a co-culture system, the overexpression of neuroligin in heterologous cells can induce the structural and functional presynaptic specialization in contacting axons (Scheiffele et al., 2000). More convincingly, in the context of intracellular interactions, the cytoplasmic tail of $\beta$-neurexin binds with PDZ protein CASK, a presynaptic scaffold protein; comparably, the intracellular part of neuroligin binds with PSD-95, which is an important PDZ domain scaffold protein of the postsynaptic density. Thus the direct 'talk' between $\beta$-neurexin and neuroligin across the synaptic cleft might bridge the adhesive and signaling components of both presynaptic transmitter release machinery and postsynaptic reception apparatus, allowing for a stepwise assembly of trans-synaptic complexes (Dean et al., 2003). Recent studies in knock-out mice have revealed that, in the absence of $\alpha$-neurexins the central synapse morphology is largely normal, but the presynaptic calcium channel activity and neurotransmitter release are severely impaired (Missler et al., 2003). Therefore, $\alpha$-neurexins probably have a role in organizing the molecular machinery at presynaptic side. Up to now, the in vivo role of $\beta$-neurexin in synaptic assembly remains to be answered.

Finally, Ephrin-Bs (EphB) are small transmembrane proteins identified as ligands for the EphB receptor tyrosine kinase. Through binding to EphB receptor, EphB can induce the clustering of NMDA receptors on the surface of fibroblasts and immature cultured hippocampal neurons (Dalva et al., 2000). The NMDA receptor clustering is mediated by a ligand-induced association of the extracellular domain of EphB receptor with the large extracellular domain of NR1 subunit, while the tyrosine kinase activity of EphB receptor are not necessary for this process. EphBs are unlikely to be the general inducers of postsynaptic differentiation, since in immature neurons it can only facilitate the 
clustering of NMDA receptors and its partner $\mathrm{Ca}^{2+}$-calmodulin-dependant protein kinase II (CaMKII) but not the other known PSD components (such as SAP90/ PSD95), moreover, although EpgB2 knock-out mice show deficits in activity-dependant synaptic plasticity, they have normal synapse density and structure, as well as largely intact synaptic function (Grunwald et al., 2001; Henderson et al., 2001). Syndecan2, a cell-surface heparan sulfate proteoglycan, is able to enhance the maturation of dendritic spines: when syndecan 2 is overexpressed in cultured immature hippocampal neurons, dendritic spines display morphology typical of mature spines (Ethell and Yamaguchi, 1999). Interestingly, EphB receptor is shown to be the upstream activators of syndecan2 (Ethell et al., 2001), thus EphB receptor pathway might not only function during the initial formation of synapses but also participate in their maturation process.

\subsubsection{Assembly of synapse}

The development of the time-lapse imaging techniques has allowed one to obtain information on the dynamic behavior of essential biological processes in living cells. Synaptic transmission can be detected within an hour of initial axon-dendrite contact, so the accumulation of certain pre- and postsynaptic proteins must occur even earlier. The time-lapse studies have shown that many synaptic components are assembled within tens of minutes after the initial contact of axons and dendrites, much faster than previous estimation (Friedman et al., 2000; Okabe et al., 2001; Washbourne et al., 2002).

\subsection{Presynaptic assembly}

Recently, evidence is growing to support the opinion that the presynaptic specialization is constructed from essentially two types of preassembled 'packets' of vesicles and/ or proteins (Hannah et al., 1999; Jahn and Sudhof, 1999; Sudhof, 2000). The biogenesis of these two precursor complexes occurs in the soma, apparently through the Golgi body. 
Using time-lapse imaging of green fluorescent protein (GFP) tagged VAMP (vesicle associated membrane protein, a synaptic vesicle protein), it has been found that even before the period of peak synaptogenesis in culture, VAMP-GFP is present in axons as mobile clusters (about 0.5-1.5 $\mu \mathrm{m}$ ) larger than single synaptic vesicles. These VAMP labelled puncta have varied vesicular and tubular membrane structures, as shown by EM, and they colocalize with presynaptic membrane proteins (such as the voltage-gated calcium channels) and other synaptic vesicle associated proteins (such as synaptic vesicle protein 2 and synapsin I). It is estimated that the amount of VAMP required in a single presynaptic bouton corresponds to 1-4 such precursor packets (Ahmari et al., 2000).

A second type of precursor packet, which is associated with dense-core vesicles that have a diameter of about $80 \mathrm{~nm}$, has been visualized and purified (Zhai et al., 2001). In contrast to synaptic vesicles, these dense-core vesicles contain many active zone components, including the presynaptic scaffold proteins Piccolo, Bassoon and RIM (Rab3-interacting molecule), the SNARE (soluble N-ethylmaleimide-sensitive-factor attachment protein receptor) proteins syntaxin and SNAP-25 (synaptosomal-associated protein 25), and the CAM N-Cadherin, suggesting that they constitute 'active zone presursor vesicles' that, upon fusing with the presynaptic plasma membrane, lead to rapid formation of active zones (Zhai et al., 2001). It was proposed that incorporation of only 2-3 of these transport vesicles supplies enough material for an active zone of average size (Shapira et al., 2003).

A common feature of these two types of precursor packets is that they are mobile along axons prior to synaptogenesis, but promptly immobilized at nascent synapses. During neuronal development, the Piccolo/ Bassoon dense-core vesicles are present earlier than the VAMP containing synaptic vesicle precursor packet, which might suggest that the formation of new neurotransmitter release sites is preceded by the recruitment and assembly of 
active zone components. The molecular mechanisms that target these precursor complexes to the nascent synaptic site still remain elusive, however, studies from immunoglobulin superfamily proteins (SYG-1 and SYG-2, see above) have already provided possible clue.

\subsection{Postsynaptic assembly}

Compared to the assembly of presynaptic specialization, the process of postsynaptic assembly is not well characterized yet, which is at least partially due to the fact that postsynaptic specialization is more complex and heterogeneous than presynaptic active zone. One of the best-studied complexes is associated with NMDA receptors. This complex comprises molecules such as SAP90/ PSD95, alfa-actinin, and CaMKII, neuroligin, the microtubule-binding protein Cript, the guanylate kinase domain-binding proteins GKAP/ SAPAPs, and other proteins such as ProSAP/ Shank, Homer and cortactin (which provide additional links to the actin cytoskeleton and other glutamate receptor complexes (Garner et al., 2002). The rapid recruitment of PSD-95 and the presence of non-synaptic clusters of PSD-95 (Rao et al., 1998; Marrs et al., 2001) are consistent with the idea that 'prefabricated' complexes are used to assemble the PSD---which is analogous to presynaptic differentiation. However, it is not known whether the non-synaptic clusters of PSD-95 represent real packets of PSD proteins in delivery. In sharp contrast to this view, however, independent studies suggest that although some synaptic PSD-95 clusters might be derived from the transport of pre-existing non-synaptic precursor packets, the de novo accumulation of PSD-95 clusters at nascent synapses seems to usually occur from diffuse 'cytoplasmic pools' (Bresler et al., 2001; Marrs et al., 2001). Notably, this model is supported by an updated report in which more PSD components have been examined, all the tagged PSD components tested are recruited to new synaptic sites gradually, with no discernable discrete transport packets involved (Bresler et al., 2004). Interestingly, in this new study, the non-synaptic PSD clusters are also observed, but they appear no more mobile than synaptic clusters, hence unlikely representing the transport intermediates. 
Ionotropic glutamate receptors are essential components of postsynaptic apparatus, in addition to converting the chemical signal released from presynaptic terminal to an electrical response in the postsynaptic neuron, they are critically involved in activity-dependant, long-term changes in synaptic strength, which may represent a central physiological correlate to learning and memory. Time-lapse imaging of GFP-tagged NR1 (one subunit of NMDA receptors) and GluR1 (one subunit of AMPA receptors) indicates that NMDA receptors and AMPA receptors are present in largely non-overlapping mobile clusters before synaptogenesis starts, NMDA receptors are recruited earlier than AMPA receptors to the axon-dendrite contact sites (Washbourne et al., 2002). It is still uncertain whether these glutamate receptor clusters represent prefabricated packets of synaptic components, or just reflect distinct classes of transport vesicles containing certain types of glutamate receptors. The fact that, the NMDA receptor scaffolding protein PSD-95 is transported to synaptic sites largely independently of NMDA receptors (Rao et al., 1998; Friedman et al., 2000; Rao et al., 2000; Washbourne et al., 2002), supports the latter interpretation. The non-overlapping of mobile AMPA and NMDA receptor clusters and the different dynamics of their recruitment to synaptic sites reflect the presence of distinct regulatory mechanisms controlling their synaptic traffic and localization. Consistently, the cytoplasmic parts of AMPA and NMDA receptor subunits bind to very different sets of structural and regulatory proteins (Scannevin and Huganir, 2000; Sheng, 2001; Sheng and Lee, 2001). The AMPA receptor binding protein, Stargazin, is crucial for the synaptic targeting of AMPA receptors, but not for the morphological development of synapses (Chen et al., 2000). Also combining with the discovery of 'silent synapses' present in rat hippocampus and somatosensory cortex, which only contain NMDA receptors but lack AMPA receptors (Liao et al., 1995; Isaac et al., 1997), it is clear that the AMPA receptor incorporation at the synaptic sites is not required for initial synaptic assembly. 


\subsubsection{Time sequence of pre- and postsynaptic assembly}

Although more and more pre- and postsynaptic proteins have been tagged with fluorescent probes and recorded in living cells, there is incomplete agreement on the time course of pre- and postsynaptic assembly. For example, DsRed tagged NMDA receptors have been reported to cluster at nascent synapses within a few minutes of axon-dendrite contact in young cortical culture (3-4 days in vitro) (Washbourne et al., 2002). However, another study in more mature hippocampal neurons (about 14 days in vitro) showed that the accumulation of NMDA receptors at nascent synapses takes 1-2 hours, and therefore lags behind the accumulation of presynaptic markers (Okabe et al., 2001). Thus, it is still not absolutely sure whether one synaptic side differentiates before the other, or whether the synaptic assembly undergoes simultaneously on both sides. Because the discrepancy raised here is at least partially due to the difference of experimental materials, it is also possible that divergent mechanisms for synaptic assembly are used by different neuron types, or even different stages of the same type. Further studies are apparently required to determine this basic issue in synaptogenesis, and it will be much more benificial to analyze, if possible, this process in vivo. Despite the uncertainty of the time course of pre- and postsynaptic assembly, as soon as a synapse is established, presynaptic active zone and PSD are exquisitely overlapped, indicating a close coordination of presynaptic and postsynaptic maintainence.

\subsubsection{Drosophila NMJ as a model for studying synaptogenesis}

\subsubsection{Structural properties of Drosophila NMJ}

The Drosophila neuromuscular junction (NMJ) is an excellent model system for addressing fundamental mechanisms governing synapse formation and growth. The advantageous features of Drosophila NMJ include its structural accessibility and amenability to powerful genetic, electrophysiological and microscopic techniques. 
In Drosophila late stage embryo and larva, body wall musculature shows a segmentally repeated pattern of 30 muscle cells (in each symmetric hemisegment) innervated by about 45 motor axons. Each of these muscles is easily identified by its distinct position and size, and is innervated by specific motorneurons. The NMJ morphology is highly sterotyped, the axons of motorneurons spread onto the muscle surface, forming a synaptic arbor composed of series of varicosities connected by thin axonal processes (Fig. 1-5 A). These varicosities, or called synaptic boutons, are categorized by three classes (Gramates and Budnik, 1999; Rheuben et al., 1999). The major class of boutons (type I) contain clear vesicles and are glutamatergic, and at larval stage, they are surrounded by the characteristic subsynaptic reticulum (SSR), an elaborately layered structure formed from the muscle membrane at the junctional region (Atwood et al., 1993; Jia et al., 1993)(Fig. 1-5 B). All 30 body wall muscles are innervated by type I glutamatergic boutons, some muscles are also innervated by type II and type III boutons, which contain octopamine or peptide neurotransmitters (Koh et al., 2000). The neuromuscular contact differentiates into distinct boutons containing functional synapses in late stage embryo, and the boutons continuously grow and sprout throughout the whole larval life. Extensive studies have been made on the third instar larval type I glutamatergic synapses, detailed analysis revealed that type I boutons can be further divided into type I small (Is) and type I big (Ib) by size, the extent of the folding of SSR, and electrophysiological properties. Due to the tiny size of embryo, it is not used as frequently as larva, however, it has been proved to be invaluable in understanding molecular events relevant to initial synapse formation. Unlike the vertebrate NMJ, the NMJ of Drosophila is not covered by glial cells, leaving $10-20 \mu \mathrm{m}$ of neuronal surface without insulation from the hemolymph. (Hall and Sanes, 1993; Auld et al., 1995).

Notably, Drosophila NMJ shares several important features with the excitatory central synapses in vertebrate brain: it is glutamatergic (unlike the cholinergic NMJ in vertebrate) and contains homologous ionotropic glutamate 

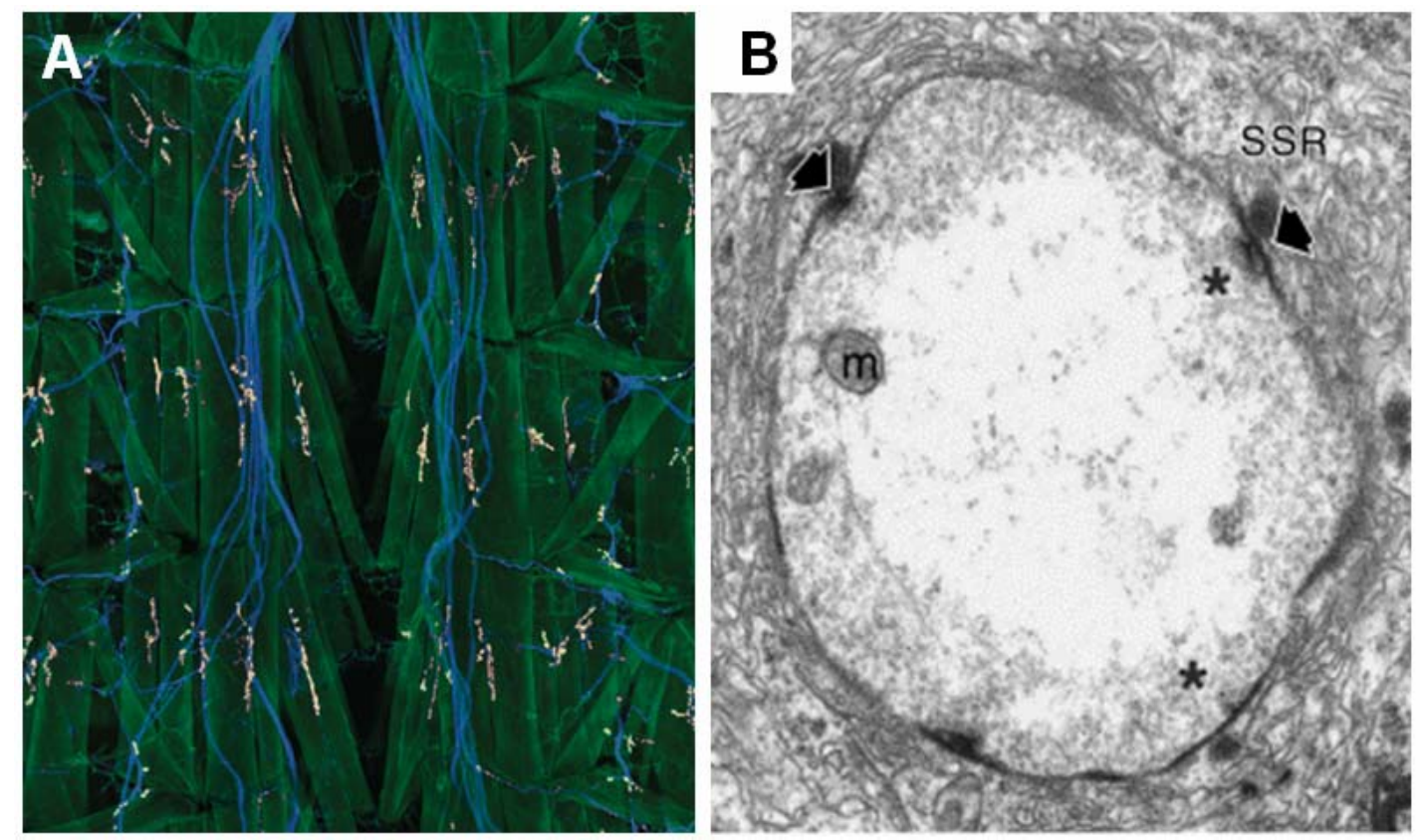

\section{Fig.1-5 Structure of Drosophila NMJ.}

A, Three segments of a dissected third instar Drosophila larva. Muscles are stained as green, neuromuscular junctions stained as red and nerves stained as blue (Adapted from (Daniels et al., 2004). B, Electron micrographs depicting cross-sections through a type-I bouton on muscle 6 in wild-type larvae. The subsynaptic reticulum (SSR), active zones (asterisks), T-bars (thick arrows), and mitochondria (m) are marked (Adapted from (Aberle et al., 2002).

receptors; there is no obvious basal lamina layer that separates pre- and postsynaptic membrane, so the synapse appears close membrane-membrane apposition ultrastructurally (Fig. 1-5 B); it is organized into a series of boutons that can be added or eliminated during development and plasticity (Petersen et al., 1997). During the period that larva grows from first instar to third instar, which normally takes several days, the muscles undergo a dramatic increase in size (more than 100 folds), therefore requiring a concomitant addition of more synaptic boutons and further elaboration of NMJ branches to efficiently depolarize the muscles.

Although the NMJ architecture changes dramatically during postembryonic 
development, the characteristics of individual neuromuscular synapses are rather similar between embryo and larva at ultrastructural level (Prokop, 1999): the pre- and postsynaptic membranes appear smoother and more electron dense compared to the extrasynaptic areas, indicating that the molecular composition of synaptic fields differs from extrasynaptic membranes. The intracellular face of presynaptic membrane harbors active zones, which contain characteristic T-shaped electron-dense structures (T-bars) surrounded by clusters of synaptic vesicles (Fig. 1-5 B). T-bars can still be seen in neuronal terminals that are depleted of synaptic vesicles and therefore appear to be independent molecular structures (Poodry and Edgar, 1979). The exact molecular composition and function of T-bars still remain unclear.

\subsubsection{Initiation and maintenance of neuromuscular connection 1.1.4.2.1 Initiation of neuromuscular contact---target recognition (pathfinding)}

So far, most studies on NMJ formation have been carried out on the ventral longitudinal muscles 6 and 7, which are innervated exclusively by glutamatergic type I boutons. It is believed that most NMJs, if not all, develop in a similar way and share the common architecture. At embryonic stage 15/ 16, muscles start sending out myopodia preferentially from the future innervation site (Ritzenthaler et al., 2000), and they are contacted by filopodia sent out from the motoneuronal growth cones in a random fashion. Those motoneuronal processes which are in contact with inappropriate muscles are soon withdrawn (Broadie and Bate, 1993; Yoshihara et al., 1997). This specific targeting recognition process is suggested to be mediated by both repulsion from inappropriate target muscle and contact-mediated attraction to the right target muscle (Bate and Broadie, 1995; Keshishian et al., 1996). Example of repellent molecule is the Toll, which is present on the surfaces of certain muscles and can prevent innervating by irrelevant motorneurons, removal of Toll leads to the ectopic 
innervation. Fasciclin III (FasIII), one homophilic cell adhesion molecule, appears to be mediate attractive cell-cell recognition, since misexpression of FasIII introduces innervation at the non-target cell. Up to now, many targeting cues have been identified, such as caspricious, semaphorins, connectin, neuromusculin and netrins (Bate and Broadie, 1995; Chiba and Rose, 1998), acting as either repellent or attractive molecules.

\subsection{Stabilization and maintenance of neuromuscular connection by cell adhesion molecules}

As soon as the initial neuromuscular contact is established, it has to be stabilized for further differentiation. This process, which should be also required for the new bouton growth during postembryonic period, is believed to be largely mediated by multiple cell adhesion molecules. Upon initial neuromuscular contact at embryonic stage 13, usually the junction consists of only short stretches of apposed membranes, often interrupted by stretches of non-connected cell surfaces (Schuster et al., 1996; Yoshihara et al., 1997; Prokop A et al., 1998). While at the mature NMJ of late stage 17 embryo such contact develops into an extended cell-cell junction, indicating that adhesive properties change during NMJ maturation. Consistent with this notion, FasIII, which contributes to target recognition and potentially also early phase of contact stabilization, disappears from NMJs of later stages (Broadie and Bate, 1993). In contrast, FasII, another homophilic CAM belonging to the immunoglobulin superfamily, is initially expressed strongly on the surfaces of all motor axons and at low levels in muscles during NMJ formation (Schuster et al., 1996). As synaptic connections are established, FasII is progressively

clustered at pre- and postsynaptic membranes. The late junctional maintenance of FasII is largely, but not all, mediated by the scaffold protein Discs large (Dlg), the Drosophila Membrane-Associated Guanylate Kinase (MAGUK) homologous to mammalian PSD-95/ SAP90 family proteins (Budnik et al., 1996; Thomas et al., 1997; Zito et al., 1997), which itself is not detectable at the NMJ until late stage 17 (Guan et al., 1996). Deleting FasII does not interfere with 
NMJ formation, but strongly affects postembryonic stabilization and maintenance of NMJs, suggesting its crucial role in controlling synaptic stability and growth (Schuster et al., 1996). Furthermore, it seems to be of developmental importance that FasII function is restricted to the late phase of NMJ differentiation: overexpression of FasII in muscles during initial NMJ formation seems to render muscle surfaces too 'sticky' and interferes with precise neuromuscular target recognition, as a result, ectopic motoneuronal branches (which normally occur only transiently) become trapped and form NMJs on inappropriate muscles (Davis et al., 1997). Apparently, there must exist other yet unidentified adhesion molecule(s) required for initial evens of synapse formation.

Another example relevant to switch in adhesive properties at NMJ comes from analysis of mef2 mutant embryos, where the initial neuromuscular contact can be genetically separated from the late phase of neuromuscular adhesion. In mef2 mutant embryos, muscle founder cells form, and motoneurons establish initial contact with proper target cells (Prokop et al., 1996). However, the muscle founder cells remain immature and fail to acquire properties of general muscle differentiation. In late-stage 17 mef2 mutant embryos, proper cell junctions between motoneurons and improperly differentiated muscle fibers are never observed, but instead the abnormal muscle surfaces are covered by basement membrane. This phenotype could be explained by lack of mef2-dependent late synaptic CAMs, which directly maintain the established neuromuscular connection. Alternatively, mef2 might be required to exclude basement membrane receptors from the NMJ, thus preventing dissociation of the pre- and postsynaptic membranes from competitive invasion of basement membrane material. No matter which explanation is true, it is clear that appropriate adhesive strength is the prerequisite for proper NMJ maturation and further maintenance. 


\subsubsection{Synapse formation}

Synapse formation involves the coordinated assembly of pre- and postsynaptic apparatus. Positioning cues are required to direct the juxtaposition of synaptic components at the pre- and postsynaptic membranes. In Drosophila NMJ, candidate that might serve as positioning cue is the special type of cleft-spanning adhesion molecules, as supposed by the function of Neuroligin/ Neuexin at vertebrate central synapses and immunoglobulin superfamily members SYG-1/ SYG-2 in C. elegans (see above), but this still remains to be proved.

In contrast to the essential role that nerve-secreted factor agrin plays in vertebrate NMJ differentiation, no agrin orthologue seems to be present in the Drosophila genome. However, recent evidence indicates that other sets of secreted molecules, Wnts and members of the TGF-beta family might take similar responsiblility in the development of glutamatergic synapses at Drosophila NMJ. Wingless (Wg), a member of the Wnt family of ligands, is secreted from motorneuron, loss of $\mathrm{Wg}$ leads to dramatic impairment of synapse formation, with boutons lacking active zones and postsynaptic structures (Packard et al., 2002). In consistence with this, a mammalian Wg homologue Wnt7a has been implicated in presynaptic differentiation in the cerebellum (Hall et al., 2000). Wishful thinking (wit) encodes a BMP (bone-morphogenetic-protein) type II receptor, which is present in a subsets of neurons, including motorneurons; its ligand, the BMP ortholog Glass Bottom Boat (Gbb), mainly fuctions in muscles. Mutations in both wit and Gbb result in much smaller NMJs, defective synaptic transmission and presynaptic abmormalities (such as synaptic membrane detatchment at active zone region, presence of floating T-bodies) (Aberle et al., 2002; McCabe et al., 2003). Taken together, these studies indicate that $\mathrm{Wg}$ and $\mathrm{Gbb}$ function in opposite directions at Drosophila NMJ to assure proper differentiation of pre- and postsynaptic structures. 
Before motorneuron innervation, low amounts of glutamate receptors are already expressed in muscles and diffusely distributed over the muscle surfaces (Broadie and Bate, 1993; Currie et al., 1995; Saitoe et al., 1997). Upon the initial neuromuscular contact, glutamate receptors begin clustering rapidly at the innervation sites, and within a few hours, new glutamate receptors are synthesized and inserted into newly formed synapses. Compared to the innervation-independent spontaneous AchR clustering at vertebrate NMJ, the clustering of glutamate receptors is innervation-dependent, the aneural muscles neither cluster existing receptors nor synthesize additional receptors. Intriguingly, disruption or complete elimination of neurotransmission does not seem to inhibit glutamate receptor field formation (Broadie et al., 1995; Featherstone and Broadie, 2000), however, the glutamate receptors will not cluster if the action potential of the neuron is blocked genetically or with tetrodotoxin (Broadie and Bate, 1993). Thus, glutamate receptor clustering appears to require an electrical activity dependant signal derived from motorneuron, and this signal is not related to neurotransmission. The latter notion is completely consistent with findings in mammalian central synapses: in Munc-13 or Munc-18 null mutant mice, in which neurotransmitter release is completely absent, both brain anatomy and synapses appear normal (Verhage et al., 2000; Varoqueaux et al., 2002). Thus, evidences from both Drosophila and vertebrates highly suggest that, at least in glutamatergic synapses, synaptic activity is not necessary for initiating synaptogenesis.

Analysis on mutants deficient in muscle development indicates that the presynaptic apparatus can be assembled independently of the postsynaptic cell (Prokop et al., 1996), which again contrasts with vertebrate NMJ differentiation. In the latter case, muscular secretion of s-laminin into synaptic basement membrane is essential for the assembly of morphologically normal active zones (Noakes et al., 1995). Anyhow, although the assembly of active zones at Drosophila NMJ can be independent of muscle cells, the precise apposition of 
pre- and postsynaptic apparatus must rely on the close communication between motorneuron and target muscle.

\subsection{Structure and functional relevance of ionotropic glutamate receptors}

The ionotropic glutamate receptors (iGluRs), including AMPA, NMDA and Kainate types, mediate excitatory synaptic transmission at most mammalian central synapses. In addition to converting transmitter released from presynaptic terminal to electrical response in the postsynaptic neuron, these receptors are thought to contribute to the activity-dependant, long-term changes in synaptic strength which is proposed to underlie learning and memory (Contractor and Heinemann, 2002). Besides the physiological importance of iGluRs, their dysfunction is implicated in various neuropathologies, including epilepsy, stroke damage and the perception of pain (Dingledine et al., 1999).

\subsubsection{Modular design of ionotropic glutamate receptors during evolution}

Ligand-gated ion channels are generally formed as homo- or hetero-oligomeric assemblies of integral membrane protein subunits. One important feature of iGluR structure is its modular construction. As illustrated in Fig.1-6 A, one typical iGluR subunit consists of an amino-terminal domain (NTD), a ligand-binding domain (S1S2), three transmembrane domains, a re-entrant pore loop, and a carboxy-terminal domain (Hollmann and Heinemann, 1994; Madden, 2002). The NTD shows homology to the leucine/ isoleucine/ valine-binding protein LIVBP, one of the bacterial periplasmic binding proteins (PBPs). In NR2 subunits, it forms or contributes to the binding site of many NMDA receptor modulators and affects receptor desensitization. For the non-NMDA receptors, it is supposed to be involved in receptor assembly. The $\mathrm{S} 1 \mathrm{~S} 2$ ligand-binding domain is formed by two sequences ( the S1 region which is the N-terminal of transmembrane domain 1, and the S2 region which is C-terminal of transmembrane domain 2) sharing sequence and structural 
homology with the glutamine-binding protein QBP. The re-entrant pore loop is homologous to the P-loop sequences of other channels, the P-loop sequence lines the channel and determines many of its electrophysiological properties. In addition, the cytoplasmic carboxy-terminal contains various interaction domains and phosphorylation sites, which likely controls the transport and localization of these receptors. The modular feature of iGluR indicates that it might have been assembled from discrete components during evolution, consistent with the 'genes-in-pieces' hypothesis (Gilbert, 1978). Supporting this notion, a prokaryotic glutamate receptor ion channel (GluR0) was found, which lacks NTD and has only two transmembrane domains but is indeed glutamate-gated and selective for potassium ions (Chen et al., 1999).
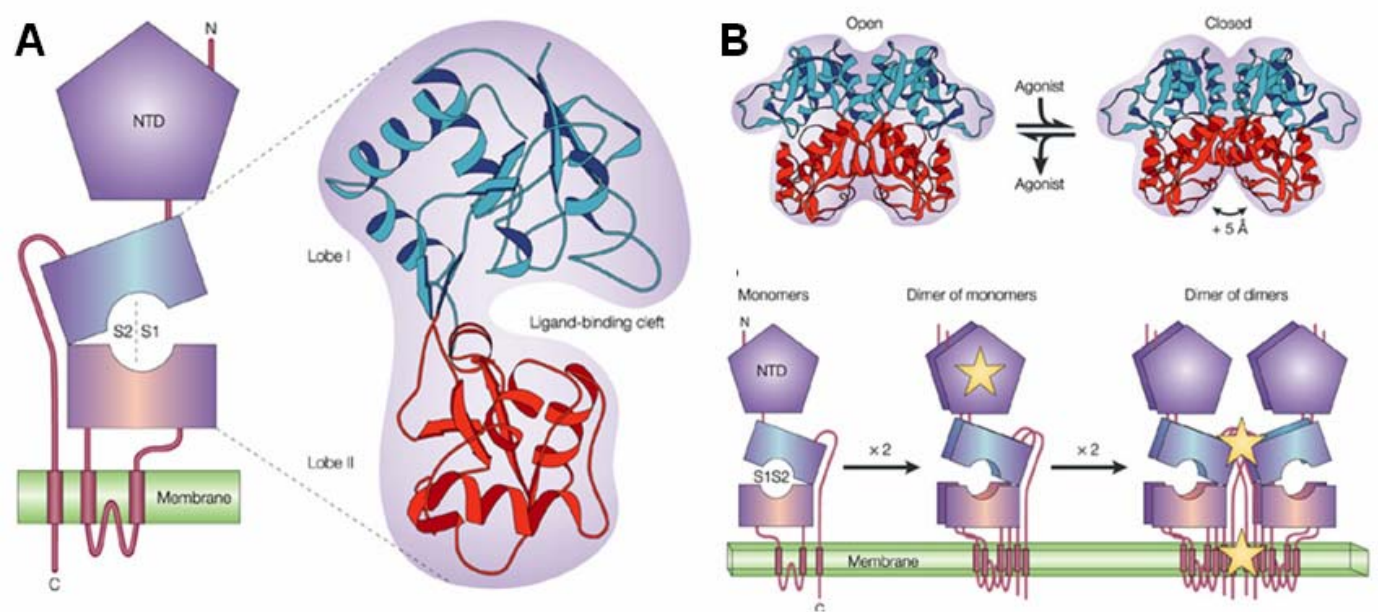

Fig.1-6 Structure of iGluR subunit and model of iGluR assembly. A, The modular structure of iGluR subunit. B, 'Dimer of dimers' model of iGluR assembly (Adapted from Madden, 2002).

\subsubsection{Molecular mechanisms underlying selective assembly and transport of ionotropic glutamate receptors}

Despite the schematic understanding of the sequence of building blocks that constitutes glutamate receptor subunits, still little is known about the spatial disposition of these domains within individual subunit and assembled functional 
ion channel. Recently, dissecting the molecular details underlying the functional assembly and cell surface trafficking of various types of iGluRs has become one of the central focuses in molecular neuroscience.

On the basis of electrophysiological, biochemical and hydrodynamic analysis, iGluRs are proposed to be tetramers (Laube et al., 1998; Mano and Teichberg, 1998; Rosenmund et al., 1998; Kuusinen et al., 1999; Safferling et al., 2001). AMPA receptors are homo- or hetero-tetramers composed of GluR1-4 (Hollmann and Heinemann, 1994; Dingledine et al., 1999). Kainate receptors are homo- or hetero-tetramers of the subunits GluR5-7, KA1 and KA2. NMDA receptors require both NR1 and at least one type of the four NR2 subunits (A-D) to form functional channels, in some cases NR3 subunits are also included (Meguro et al., 1992; Monyer et al., 1992; Perez-Otano et al., 2001; Matsuda et al., 2003). Recently, using a series of epitope-tagged receptor chimeras (Ayalon and Stern-Bach, 2001), Ayalon and Stern-Bach provided evidence of sequential assembly of tetrameric iGluRs as dimmer of dimmers (Fig.1-7 B). In this model, initial subunit dimerization is mediated primarily by interactions between compatible NTDs. Then, assembly of functional receptors requires that these dimmers undergo a second dimerization, this time requiring compatibility between the S2 and transmembrane domains of the subunits. This secondary dimerization is not observed in the absence of NTD compatibility, indicating that it might be weaker than the primary dimerization. This dimmer-of-dimmers model of iGluR assembly is consistent with images of GluR2 homomers determined by electron microscopy (Safferling et al., 2001).

In non-neuronal cells, the step of exit from the endoplasmic reticulum (ER) is often under the most stringent control during transport of membrane proteins to the cell surface (Ellgaard and Helenius, 2003). Like other multimeric cell membrane proteins, glutamate receptors are synthesized, folded, assembled in the ER; similarly, traffic of glutamate receptors through ER is also tightly regulated, which decides the type and number of glutamate receptor at synapse 
(Vandenberghe and Bredt, 2004). ER contains rigorous quality control system to ensure that only correctly folded and assembled proteins exit to the Golgi body, which in the case of glutamate receptors is evidenced by the presence of various ER retention and export signals in NMDA, AMPA and Kainate receptor subunits. For NMDA and Kainate receptors, both ER retention and export signals localize within the intracellular tails (Standley and Baudry, 2000; Scott et al., 2001; Xia et al., 2001; Ren et al., 2003; Hawkins et al., 2004; Jaskolski et al., 2004). Intriguingly, AMPA receptor subunit GluR2 contains a unique ER retention motif which just localizes in the re-entrant membrane loop (Greger et al., 2002), this single arginine (R607) is generated by mRNA editing and critically controls the ion permeability (Burnashev et al., 1992). Unlike other known ER export signal, the ER export signal of AMPA receptor subunit Glu1 appears within the extra-cellular NTD (Xia et al., 2002).

\subsubsection{Properties of ionotropic glutamate receptors expressed at Drosophila neuromuscular synapses}

In contrast to mammalian acetylcholinergic NMJ, the Drosophila type I junctional bouton is glutamatergic. So far, three ionotropic glutamate receptor subunits have been identified to be specifically expressed at the postsynaptic muscle cell (Schuster et al., 1991; Marrus et al., 2004). These glutamate receptor subunits are structurally and functionally similar to mammalian AMPA-/ Kainate-type receptors. GluR-IIA and GluR-IIB are genomic neighbors and their encoding proteins share $44 \%$ amino acid identity, animals with double knock-out of both genes die at late embryonic stage, while the lethality can be fully rescued by transgenic expression of either gene; moreover, modulation of subunit composition via genetically changing the copy number of GluR-IIA and GluR-IIB gene results into distinct single channel kinetic properties and is able to trigger long term changes of synaptic strength (Petersen et al., 1997; DiAntonio et al., 1999; Sigrist et al., 2002). GluRIII (also called GluR-IIC) is crucial for animal vitality and required for synaptic localization of GluR-IIA and GluR-IIB; while GluR-IIA and GluR-IIB do not precisely colocalize at 
many synapses, they both perfectly colocalize with GluR-III, implicating that GluR-III acts as a obligate subunit for functional receptor assembly (Marrus et al., 2004).

\subsection{The aim and strategy of this work}

The purpose of this study is to improve our understanding of the molecular mechanisms relevant to the synaptogenesis and growth control/ regulation of Drosophila NMJ. To this end, newly developed DNA microarray technique was applied to sort potential genes relevant to the NMJ formation and functioning through the whole genome. Via RNA profiling of postsynaptic cell (here is the somatic body wall muscle), a pool of genes with specific or enriched expression pattern were obtained, which would greatly facilitate systematic characterization of certain interesting functional groups. Data mining from these obtained RNA profiles and online genomic resources resulted into the identification of several muscle expressed but uncharacterized ionotropic glutamate receptor subunit genes. The expression pattern of these subunits were further verified at both RNA and protein level, via quantitative RT-PCR, in situ hybridization and immunohistochemistry. Ionotropic glutamate receptors play the major roles in mediating the signal transmission at excitatory glutamatergic synapses in the central nervous system, moreover, these receptors are also thought to contribute to the activity-dependant, long-term plasticity of neuronal circuits which is proposed to underlie learning and memory (Contractor A et al., 2002). The Drosophila neuromuscular synapse provides an excellent platform for investigating the behavior of glutamate receptors, due to its structural and functional similarity to excitatory central synapse and its rather simple organization. Reasonably, characterizing the functional significance of these newly identified glutamate receptor subunits at Drosophila NMJ in principle would help better understand their orthologs in the central synapses and thus became the main body of this thesis work. 
To study the in vivo roles of these novel glutamate receptor subunits, knock-out animals were generated via standard transposon-based mutagenesis screening. The null mutant animals of either subunit die at late embryonic stage, indicating that they are all essential genes. Further genetic, cell biological and electrophysiological studies were performed and uncovered a tight interdependence of all essential glutamate receptor subunits for synaptic localization and functioning, implying a novel "strictly hetero-tetrameric stoichiometry" model which is so far not reported in mammalian ionotropic glutamate receptors.

Closer inspection on glutamate receptor deprived synapses was also performed, with the combination of immunohistochemistry and electron microscopy based ultrastructural analysis. Surprisingly, it was found that with deprivation of glutamate receptors, the typical postsynaptic differentiation and synaptic compartmentation displayed severe defects, further analysis of a series of neurotransmission deficient mutant backgrounds excluded the involvement of neurotransmission activity in instructing differentiation of synaptic specializations. Thus, the glutamate receptor per se, but not its ligand-gated ion channel activity, is essential for normal synaptic construction. This important 'structural' role of glutamate receptor in organizing synaptic differentiation is firstly reported, raising highly interesting questions such as, which part of glutamate receptor is responsible for facilitating synaptic assembly? Do the ionotropic glutamate receptors in mammalian central synapses play similar roles during synaptogenesis? 


\section{Materials and methods}

\subsection{Materials (Chemicals, enzymes and molecular biology kits)}

All of the chemicals were purchased from Roth and Sigma unless stated elsewhere. For molecular cloning, all of the enzymes, including various restriction endonuclease enzymes, T4 DNA ligases, Alkaline Phosphotase were purchased from Roche (Mannheim, Germany). The Vent polymerase used for PCR cloning was from NEB (USA). All of the oligonucleotides were synthesized from MWG (Germany). The E. Coli strains used for transformation were either self-made DH5 $\alpha$ chemical competent cell or SURE electroporation competent cell from Stratagene (USA). Unless described elsewhere all of the molecular biology kits were from Qiagen (Germany).

\subsection{Methods}

\subsubsection{Genechip analysis and real-time RT-PCR}

\subsubsection{Genechip analysis}

Part I Protocol for the Affymatrix GeneChip Target Preparation

Material and Reagents:

Qiagen RNeasy RNA purification kit

3M Sodium Acetate, pH 5.2 Sigma

$80 \%$ ethanol kept cold

Absolute ethanol kept cold

Glycogen 5mg/ml, Roche

Ethedium bromide

7.5M Ammonium Acetate, Sigma

1). Synthesis of Double-Stranded cDNA from Total RNA

Materials and Reagents:

T7- (dT)24 Primer, (MWG Corp) HPLC purified DNA

Phase lock Gel, Eppendorf 
Phenol/ Chloroform/isoamyl alcohol, Roth

Superscript Choice System, Invitrogen

Ice bath

Heat/cool block for $70^{\circ} \mathrm{C}, 42^{\circ} \mathrm{C}, 16^{\circ} \mathrm{C}$

PE 9700 PCR machine

\subsection{First strand cDNA synthesis}

Prepare an Rnase- free $0.5 \mathrm{ml}$ reaction tube containing the following reagents.

They are to be added in the order listed. The total reaction volume is $20 \mu l$.

1. In a $0.5 \mathrm{ml}$ Rnase-free tube add total volume of $10 \mu \mathrm{l}$ of total RNA sample $(2.5 \mu \mathrm{g})$.

2. Add $1 \mu 1$ of T7- (dT) 24 primer (100 pmol/ $\mu \mathrm{l})$.

3. Incubate at $70^{\circ} \mathrm{C}$ for $10 \mathrm{Min}$, quick spin and place on ice.

4. Add $4 \mu 1$ of $5 X$ First strand cDNA buffer.

5. Add $2 \mu 1$ of 0.1 M DTT.

6. Then add $1 \mu 1$ of $10 \mathrm{mM}$ dNTP mix.

7. Add this mixture to the RNA sample then Incubate at $42^{\circ} \mathrm{C}$ for $2 \mathrm{~min}$.

8. While in the bath Add $2 \mu 1 \mathrm{SSII}$

9. Incubate for 1 hour at $42^{\circ} \mathrm{C}$.

10. Place reaction on ice and proceed onto second strand synthesis.

11. Equilibrate a $16^{\circ} \mathrm{C}$ bath.

\subsection{Second Strand Synthesis}

12. Briefly centrifuge first strand reaction and return to ice.

13. Add the following reagents in the order listed to the first strand reaction.

14. Make sure to add the reagents while the reaction is on ice.

$91 \mu \mathrm{l}$ of DEPC-treated $\mathrm{dH}_{2} \mathrm{O}$

$30 \mu \mathrm{l}$ of $5 \mathrm{X}$ Second Strand Reaction Buffer

$3 \mu \mathrm{l}$ of $10 \mathrm{mM}$ dATP, dCTP, dGTP, DTTP

$1 \mu \mathrm{l}$ of DNA ligase $(10 \mathrm{U} / \mu \mathrm{l})$

$4 \mu 1$ of DNA polymerase I $(10 \mathrm{U} / \mu \mathrm{l})$ 
$1 \mu \mathrm{l}$ of Rnase $\mathrm{H}(2 \mathrm{U} / \mu \mathrm{l})$

To yield a final reaction volume of $150 \mu 1$.

15. Gently tap tube to mix. Briefly spin to remove condensation.

16. Incubate the reaction at $16^{\circ} \mathrm{C}$ for 2 hours in a cooling water bath

17. While in the bath add $2 \mu 1$ of T4 DNA Polymerase.

18. Incubate for an additional 5 minutes at $16^{\circ} \mathrm{C}$.

19. Add $10 \mu \mathrm{l}$ of $0.5 \mathrm{M} \mathrm{Na}_{2}$ EDTA

The reaction product can be stored at $-20^{\circ} \mathrm{C}$ for later use.

\subsection{Clean Up of Double-Stranded cDNA}

STEP 1-Phase Lock Gels Phenol/Chloroform Extraction

1. Pellet the Phase lock gel in a micro-centrifuge tube by spinning it at full speed (1,3200rpm) for 20 to 30 seconds.

2. Add $162 \mu 1$ of Phenol: Chloroform: isoamyl alcohol (saturated with $10 \mathrm{mM}$ Tris- $\mathrm{HCl} \mathrm{pH} 8.0 / 1 \mathrm{mM}$ EDTA) to the cDNA reaction sample, mix by vortexing for one minute.

3. Transfer the entire cDNA Phenol Chloroform mixture into the PLG tube. Spin the PLG tube at full speed for 2 minutes

4. Avoiding contact with the gel transfer the aqueous upper phase to a fresh 1.5 $\mathrm{ml}$ tube (Tilt the tube for better recovery).

Proceed to step 2.

STEP 2 - Ethanol precipitation

1. Add $1 \mu 1$ of glycogen to the sample.

2. Add 0.5 volumes of $7.5 \mathrm{M}$ NH4Ac plus 2.5 volumes of absolute ethanol (stored at $-20^{\circ} \mathrm{C}$ ) to the sample and vortex.

3. Immediately spin at full speed in a micro-centrifuge at room temp for $20 \mathrm{~min}$, discard supernatant.

4. Wash pellet with $500 \mu \mathrm{l}$ of $80 \%$ ethanol (stored at $-20^{\circ} \mathrm{C}$ )

5. Spin at full speed for 5minutes at room temp.

6. Remove the ethanol very carefully so as not to disturb the pellet and repeat the wash. (This is an optional stopping point. The pellet may be left in the wash 
at $-20^{\circ} \mathrm{C}$ overnight.)

7. Air dry the pellet at room temp completely (this may require over an hour).

8 . Resuspend the pellet in $12 \mu 1$ of Rnase-free water.

9. Proceed onto Synthesis of biotin-labeled cRNA.

To reduce overdrying leave the pellet in a very small volume of the last wash.

2). Synthesis of biotin-labeled cRNA

Materials and Reagents:

Enzo RNA transcript labeling Kit 9 (BioArray High Yeald RNA Transcript

Labeling Kit)

Rneasy Mini Kit, Qiagen

DEPC-treated water

10X TBE, BioWhittaker

Reagent preparation

5X Fragmentation Buffer:

In an Rnase-free vessel combine

$4.0 \mathrm{ml}$ of $1 \mathrm{M}$ Tris-acetate $\mathrm{pH} 8.1$ (adjusted with glacial acetic acid).

$0.64 \mathrm{~g} \mathrm{MgOAc}$.

$0.98 \mathrm{~g} \mathrm{KOAc.}$

DEPC-treated water to make a final volume of $20 \mathrm{~mL}$.

Mix thoroughly then filter through a $0.2 \mu \mathrm{m}$ vacuum filter unit. This reagent should be aliquoted and stored.

2.1 protocol for the IVT reaction

1. Equilibrate a $37^{\circ} \mathrm{C}$ bath / block

2. Thaw the Enzo reagents on ice.

3. When thawed check for precipitate, vortex if necessary.

4. The volume of cDNA to be used in the IVT reaction should corresponding to the total RNA range of 8.0 and $16.0 \mu \mathrm{g}$.

5. From the Enzo BioArray RNA labeling kit add the following reagents in the 
order listed.

Reagent Volume

Template DNA Variable to give $1 \mu \mathrm{g}$ of cDNA $(10 \mu \mathrm{l})$

Deionized water To make final volume equal to $40 \mu \mathrm{l}(\sim 12 \mu \mathrm{l})$

10X HY Buffer $4 \mu 1$

10X Biotin-labeled nucleotides $4 \mu 1$

10X DTT $4 \mu \mathrm{l}$

10X RNase inhibitor $4 \mu 1$

20X T7 RNA polymerase $2 \mu \mathrm{l}$

Total volume $40 \mu 1$

6. Carefully mix the reagents in the tube then spin the sample briefly

7. Immediatly place the tube in a water bath at $37^{\circ} \mathrm{C}$ incubate for 5.5 hours gently mixing every 30 to 45 minutes

8. Remove $1 \mu 1$ aliquot for gel analysis

9. At the end of the incubation the reaction may be stored at $-20.0^{\circ} \mathrm{C}$ for later use.

2.2 Purification and Quantification of in vitro Transcription STEP 1 - Column purification of RNA

1. Pre-heat a small working stock of Rnase-free water at $60-65^{\circ} \mathrm{C}$.

2. Pre- -heat elution buffer at $60-65^{\circ} \mathrm{C}$.

3. Aliquot one half $(\sim 20 \mu \mathrm{l})$ of the cRNA reaction into a $1.5 \mathrm{ml}$ tube.

4. Adjust volume to $100 \mu \mathrm{l}$ with Rnase-free water.

5. Add $350 \mu 1$ of buffer RLT (lysis buffer) to the sample. Mix thoroughly.

6. Add $250 \mu 1$ of ethanol (96-100\%) to the lysate and mix well by pipetting up and down.

7. Apply sample $(700 \mu 1)$ to Rneasy mini-spin column sitting in a 2 ml-collection tube.

8. Centrifuge for $30 \mathrm{sec}$ at 10,000 rpm.

9. Reapply the flow-through onto the column and spin again.

10. Transfer the column into a new $2 \mathrm{ml}$-collection tube.

11. Add $500 \mu 1$ of buffer RPE (ensure ethanol has been added to the buffer) and 
let stand for $1 \mathrm{~min}$.

12. Spin at max for 30 s and discard flow-through.

13. Add another $500 \mu \mathrm{l}$ of buffer RPE onto the column and let it stand for $1 \mathrm{~min}$.

14. Centrifuge and discard flow-through.

15. Spin at full speed for an additional $3 \mathrm{~min}$.

16. Transfer the column to a new $1.5 \mathrm{ml}$ tube and pipet $33 \mu \mathrm{l}$ of the pre-heated Rnase free water directly onto the membrane. Let stand for $1 \mathrm{~min}$.

17. Spin at full speed for $1 \mathrm{~min}$.

18. Repeat elution using the flow-through from the first elution.

STEP 2 - Ethanol precipitation: if the cRNA yield is poor

19. Add 0.5 volume of $7.5 \mathrm{M}$ Ammonium Acetate and 2.5 times volume of absolute ethanol

20. Incubate at $-20^{\circ} \mathrm{C}$ for 60 min then spin at full speed for 30 minutes.

21 . Decant the supernatant then wash the pellet by adding $500 \mu 1$ of $80 \%$ Ethanol and spin at full speed for 5 minutes.

22. Decant the supernatant and repeat the wash step

23. Decant the final wash and dry the pellet at room temperature.

STEP 3 - Electrophoresis

24. Prepare sample in a RNA gel-loading buffer.

25. Heat sample $65^{\circ} \mathrm{C}, 5 \mathrm{~min}$.

26. Run one lane of unpurified labeled cRNA $(0.5 \mu 1)$ along side a lane of the purified labeled probe $(1.5 \mu \mathrm{l})$ on standard 1\% agarose gel.

Fragmentation of cRNA for target preparation

27. Add $2 \mu 1$ of $5 \mathrm{X}$ Fragmentation buffer to every $8 \mu 1$ of RNA plus water. The final RNA concentration in the reaction can range between $0.8 \mu \mathrm{g} / \mu \mathrm{l}$ and 2 $\mu \mathrm{g} / \mu 1$.

28. The following are guidelines that should be followed:

$20 \mu \mathrm{g}$ cRNA 1 to $16 \mu \mathrm{l}$

$5 \mathrm{X}$ Fragmentation buffer $4 \mu 1$

Rnase-free water to $20 \mu \mathrm{L}$ 
Component Volume

$15 \mu \mathrm{g}$ cRNA (extreme case) 1 to $12 \mu \mathrm{l}$

$5 X$ Fragmentation buffer $3 \mu 1$

Rnase-free water $15 \mu 1$

29. Incubate the reaction at $94^{\circ} \mathrm{C}$ for $35 \mathrm{~min}$, quick spin and place on ice immediately following the incubation.

30. Run $1 \mu \mathrm{g}$ of the fragmented RNA on a $1 \%$ agarose gel to check the completion of fragmentation.

31. The fragmented RNA is ready for hybridization.

Hybridization was performed in Affymetrix hybridization oven at $50^{\circ} \mathrm{C}$ for 16 hours. The hybridized Gene chips were then stained and washed in Affymetrix fluidics station following the standard program. Data analysis was performed via Microarray Suite 4.0 and exported via Excel.

\subsubsection{Real-time RT-PCR}

1. Extract total RNAs using QIAGEN's RNeasy kit (as described in 2.2.1.1), check the concentration of each sample.

2. Perform reverse transcription using QIAGEN's Omniscript Reverse Transcriptase:

Master mix:

10X Buffer RT

$2.0 \mu 1$

dNTP Mix (5mM each dNTP) $\quad 2.0 \mu \mathrm{l}$

Random hexamer $\quad 2.0 \mu \mathrm{l}$

Rnase inhibitor (40 units $/ \mu 1$ ) $\quad 0.25 \mu 1$

Ominiscript Reverse Transcriptase $\quad 1.0 \mu l$

Rnase-free $\mathrm{H}_{2} \mathrm{O} \quad \mathrm{X} \mu \mathrm{l}$ 
Template RNA

Incubate the mixture for 1 hour at $37^{\circ} \mathrm{C}$, then inactivate the Reverse Transcriptase by heating the reaction mixture to $93^{\circ} \mathrm{C}$ for $5 \mathrm{~min}$ followed by rapid cooling on ice.

3. Perform the real-time PCR in 96 well plate :

Master mix $(50 \mu 1)$ :

2X PCR mixture

$25 \mu 1$

$\mathrm{H}_{2} \mathrm{O}$

$22 \mu 1$

Primer (forward)

$1.0 \mu 1$

Primer (reverse)

$1.0 \mu 1$

cDNA template

$1.0 \mu 1$

4. Quantitate the relative transcript level by analyzing $\mathrm{Ct}$ value of each sample.

5. Check the amplified products by agrose gel electrophoresis.

\subsubsection{Fly stocks and genetics}

\subsubsection{Fly stocks, crosses and rearing conditions}

Fly stocks used are: All of the wild type control strain used is $w^{1}(B)$ except stated elsewhere. $\mathrm{w}^{-} ; \mathrm{Df}(2 \mathrm{~L})^{\mathrm{SP22}}\left(\right.$ Gglur-IIA $^{-/}$, Gglur-IIB $\left.^{-/}\right) /$Cyo, Kr::GFP (Petersen et al., 1997). w; $\mathrm{wf}^{-2}(2 \mathrm{~L}) \mathrm{cl}^{\mathrm{h}}{ }^{4} /$ Cyo, Kr::GFP. w;OK319-Gal4. $\mathrm{w}^{-}$;UAS-TNTe (Sweeney et al., 1995). Syx ${ }^{229}$ is a null mutation allele of syx-1A that completely eliminates mRNA and protein expression (Schulze et al., 1995). $S h i^{\text {ts } 1}$ mutants were shifted from a permissive $\left(25^{\circ} \mathrm{C}\right)$ to a non-permissive temperature $\left(32^{\circ} \mathrm{C}\right)$ after $13 \mathrm{~h}$ of embryogenesis (Featherstone D et al., 2002). $\mathrm{III}^{1} / \mathrm{Gla}$ Bc (Marrus et al., 2004).

Double mutants in GluR-IIA and GluR-IIB were described previously (Petersen et al., 1997; DiAntonio et al., 1999). In short, GluR-IIA and GluR-IIB 
double mutant embryos were recovered by crossing $D f(2 L) G l u R-I I A \& B^{S P 22}$ to $D f(2 L) C^{h 4}$, mutant embryos were selected using GFP marked balancer chromosomes.

To generate GluRIIA hypo, GluR-IIB ${ }^{\text {null }}$ condition having an extremely reduced amount of GluR-IIA and no GluR-IIB expression, genomic fragment of GluR-IIA encompassing promotor region and the whole open reading frame while missing most part of the 3'-UTR was used. This transgene still produces full length GluR-IIA while in dramatically reduced amount due a loss of message stability (see 3.4.2 for details). This construct was expressed from pUAST vector (using the GluR-IIA endogenous promotor). A single transgene copy rescues embryos null for both GluR-IIA and GluR-IIB (Df(2L)GluRIIA\& $B^{S P 22} / D f(2 L) C l^{\text {h4 }}$ ) giving GluRIIA $A^{\text {hypo }}$, GluR-IIB ${ }^{\text {null }}$ larvae (see results).

Unless stated elsewhere, all of the crosses were established at $25^{\circ} \mathrm{C}$ with $60 \%$ humidity.

\subsubsection{Mutagenesis screening}

The GluR-IID and GluR-IIE loci are situated at 92F4. In a recently released pBac transposon insertion collection (Thibault et al., 2004), a piggyBac transposon ( $\mathrm{pBac}\{\mathrm{RB}\} \mathrm{e} 01443, \# 17952$,) was found to be inserted into intron 6 at amino acid position 427 of the GluR-IID open reading frame. This allele GluR-IIDe $e^{01443}$ is embryonic lethal both homozygous or over $D f(3 R) H-B 79$ (Bloomington Drosophila stock center) which deletes a large genomic region including the GluR-IID and GluR-IIE locus, and over deficiency GluRIID\&-IIE $E^{E 3}$ (see below). For GluR-IIE, imprecise excision screening was performed using the $\mathrm{P}$ element line GE28753 (commercially available with Genexcel), which is inserted $\sim 150 \mathrm{bp}$ downstream of the end of the GluR-IIE transcript. In brief, P-element GE28753 was remobilized by crossing to the $\Delta 2-3$ transposase source, white eye progenies were selected and mated individually, 
then single fly genomic PCR reactions were performed to map deletions flanking the P-element insertion site. Nearly 1,000 eye color revertants were checked, one line (GluR-IIE $E^{E 1}$ ) was found to delete $1.2 \mathrm{~kb}$ flanking region in direction of the GluR-IIE gene, removing the C-term and transmembrane domain 4 of GluR-IIE. GluR-IIE ${ }^{E 1}$ is embryonic lethal homozygously, over $d f(3 R) H-B 79$ and over GluR-IID\&-IIE $E^{E 3}$. GluR-IID\&-IIE $E^{E 3}$ is a larger deletion also recovered from exision mutagenesis of GE28753, which removes both GluR-IIE and GluRIID and thus was used in combination with either GluR-IID ${ }^{e 01443}$ or GluR-IIE $E^{E 1}$ as deficiency.

\subsubsection{Molecular Constructs and Transgenes}

A genomic fragment covering the DGluR-IIA gene (containg $1.3 \mathrm{~kb}$ sequence 5' of the ATG) was firstly subcloned into pSL1180 as EcoRI/ XhoI fragment $(5.6 \mathrm{~Kb})$ from a BAC clone RPCI-98-35L07. The wild type rescue construct was made by directly inserting the EcoRI/ XhoI fragment into pUAST (Brand and Perrimon, 1993). For the 3' UTR deleted version, the subclone was cut with NcoI and XhoI, end-blunted and religated, then the EcoRI/ Asp718 fragment $(4.7 \mathrm{~Kb})$ was inserted into pUAST. All constructs were confirmed by double-strand sequencing, transgenic flies were produced in w1 background via standard procedures and crossed into the $\operatorname{Df}(2 \mathrm{~L}) \mathrm{cl}^{\mathrm{h} 4} / \mathrm{Gla}$, Bc background.

Genomic fragments covering the GluR-IID gene and GluR-IIE gene were generated by PCR using for IID

5'GGTCTAGAGCGGCCGCGGCCACGAACTGACCCACGGTTTC3' and 5'GCGGCCCTCGAGCGACGTCAAGGATGTGCCCAC3'

and for IIE

5'GGTCTAGAGCGGCCGCACCTCCCCAAGCTGTCAACTTC3' and 5'GCGGCCCTCGAGACTGCTCAAAGCTGCTGCCCTG3'. The products were double strand sequenced and cloned into $p U A S T$. Several independent lines of transgenic animals were generated. For overexpression studies, 
UAS-GluRIID and UAS-GluRIIE were generated by introducing the full length cDNA into the transformation vector $p U A S T$. Full-length cDNAs of GluR-IID (RE24732) and GluR-IIE (RE07945) were obtained from Berkeley Drosophila Genome Project cDNA libraries.

The construct for inducible RNA interference (RNAi-GluR-IIE) was made based on the pUASTi plasmid (contains an intron between insertion sites for sense and antisense frgaments; generous gift by Amin Ghabria, Krasnow lab). Selected cDNA fragments coving part of 5'-UTR and coding region were PCR-amplified by using the following primer pairs:

5'GCGCGCCTCGAGCTGTTCGGGAAACTCAAGAAT3' and 5'GGTCTAGAGCGGCCGCCGTGGTTAGCTCGTTCAAAATG3' and 5'GCTGGTACCTGTTCGGGAAACTCAAGAAT3' and 5'GCGTCTAGATCGTGGTTAGCTCGTTCAAAATG3'

The two fragments were inserted into pUASTi plasmid sequentially and verified by sequencing. Several independent lines of transgenic animals carrying UAS-GluR-IIE were generated.

To generate GluR-IIE::EGFP fusion. The EGFP ORF was inserted into the C-terminal of GluR-IIE coding sequence (the insertion site and the linker sequences are as described in (Sheridan et al., 2002) via 'blant-end ligation' strategy. Briefly, a HindIII fragment covering the C-terminal region of GluRIIE was cut from full-length cDNA (RE07945) and subcloned into pSL1180; PCR reaction was performed to generate the whole length linear fragment using the following primers which are standing at the EGFP insertion site but opposite in direction:

GGCAGATGTGTATAAGAGACAGTCGCCAGTCCTCGATGTCAGTAGCTT and

AGATGTGTATAAGAGACAGGACTGGCGACTGCGCGAGTAGATGG, at the same time EGFP coding sequence was amplified from EGFP vector (Clontech) using the following primers with 5' phosphate modification: 
pGGCGCGCCGAGCAAGGGCGAGGAGCTGTTCACCGG

and

pCGGGCGCGCCGCCCTTGTACAGCTCGTCCATGCCGAGA, the two PCR products were put together for blant-end ligation and the positive clones with right orientation of EGFP were verified by PCR and sequencing; then the HindIII fragment (now containing EGFP insert) was put back into the full-length cDNA backbone and the reading frame was verified by PCR and sequencing; finally the full-length DGluRIIE cDNA tagged with EGFP was PCR cloned into the NotI/ XbaI site of pUAST and pFasBac vector and verified by sequencing. Several independent lines of transgenic animals were generated from injecting the pUAST construct. The pFastBac construct was used for driving expression in Sf9 cells (for details see 2.2.5)

\subsubsection{In Situ Hybridization}

I. Preparation of embryos

1. Collect embryos every 3 hours for 24 hours and wash $3 \mathrm{X}$ with $0.02 \%$ Triton X (Tx)

( $15 \mathrm{ml}$ for up to $2 \mathrm{ml}$ embryos in a $50 \mathrm{ml}$ falcon tube).

2. Dechorionate $3 \mathrm{~min}$. in $50 \%$ bleach.

3. Wash $3 \mathrm{X}$ with $0.02 \% \mathrm{Tx}$.

4. Fill tube with equal parts heptane and $4 \%$ formaldehyde/PBS (filtered).

5. Incubate $25 \mathrm{~min}$. with frequent shaking.

6. Remove LOWER aqueous phase and replace with equal volume methanol.

7. Shake for $1 \mathrm{~min}$. allowing embryos to settle. Do not vortex.

8. Remove UPPER phase along with embryos and vitelline membranes and remaining at the interphase.

9. Rinse settled embryos $3 \mathrm{X}$ with methanol (Embryos can be stored at -20 degrees $\mathrm{C}$ at this point).

10. Rehydrate in 3:1 (MeOH: 4\% formaldehyde/PBS) for 2 min., then 1:3 (MeOH: 4\% formaldehyde/PBS) for $5 \mathrm{~min}$. 
11. Fix $10 \mathrm{~min}$. in $4 \%$ formaldehyde/PBS.

12. Rinse $6 \mathrm{X}$ with $\mathrm{PBS}+0.1 \%$ Tween 20 (PBT).

II. Preparation of Probe

1. Perform restriction digestion of the vectors containing the sequences of interest. For preparing antisense RNA probes of, GluR-IID and GluR-IIE, RE24732 (GluR-IID) and RE07945 (GluR-IIE) plasmids were cut with NotI, respectively.

2. Run the digest on agrose gel and extract the DNA (elute the DNA in $10 \mu 1$ of $\mathrm{H} 2 \mathrm{O}$ )

3. Run $1 \mu 1$ of DNA on a test gel.

4. In-vitro transcription:

Set the following reaction mixture:

DNA $\quad 9 \mu 1$

RNAse free water $\quad 3 \mu \mathrm{l}$

10xTrancription Buffer $2 \mu 1$

RNAse inhibitor $\quad 2 \mu 1$

DIG-labelling mix $\quad 2 \mu 1$

T3 RNA polymerase $\quad 2 \mu \mathrm{l}$

Incubate at $37^{\circ} \mathrm{C}$ for 2 hours.

5. Then add $2 \mu \mathrm{l}$ RNAse free DNAse and incubate at $37^{\circ} \mathrm{C}$ for 15 minutes.

6. Extract the RNAs by using RNAeasy Kit.

7. Fragmentation of RNA

RNA sample in Rnase free water $25 \mu 1$

2xcarbonate buffer $\quad 25 \mu 1$

8. Divide the above mixture into two parts. Incubate $25 \mu 1$ at $60^{\circ} \mathrm{C}$ for $5 \mathrm{~min}$ and incubate other $25 \mu \mathrm{l}$ at $60^{\circ} \mathrm{C}$ for $15 \mathrm{~min}$.

9. Add Rnase free water to the fragmented RNA sample to get a final volume of $100 \mu 1$, add $10 \mu 1$ of NaAc buffer (PH 5.2) and $1 \mu 1$ of LPA, and $250 \mu l$ of 
absolute ethanol. Mix and centrifuge for $5 \mathrm{~min}$ at full speed, remove supernatant and wash the pellet with $75 \%$ ethanol.

10. Dissolve the RNAs sample with $25 \mu$ of probe resuspension buffer.

III. Hybridisation of embryos (fillets)

1. Wash the prepared embryos (fillets) in 1ml Pre-hybridisation Buffer(-DS)

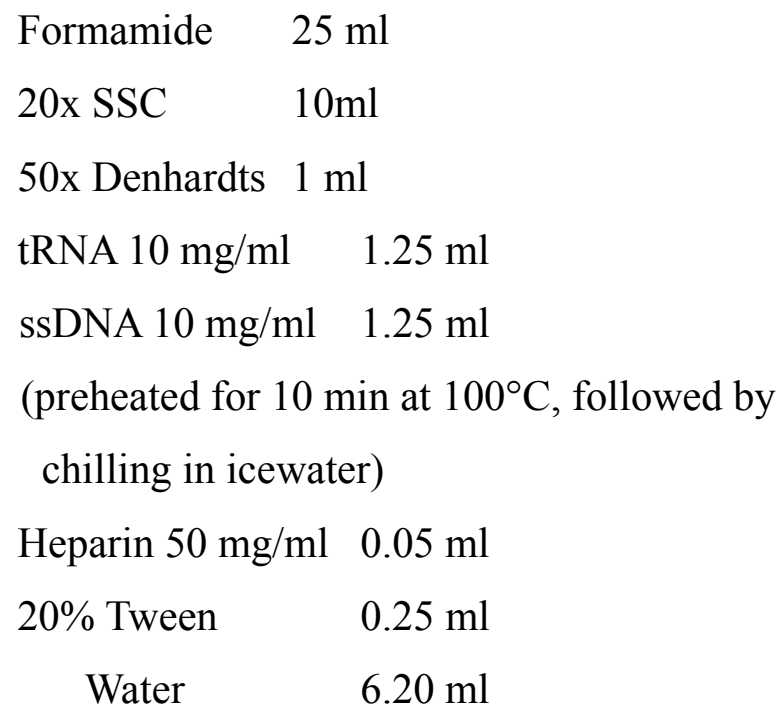

Combine $9 \mathrm{ml}$ with $1 \mathrm{ml}$ water to get the Pre-hybridisation Buffer (-DS) 2. Incubate the samples in $1 \mathrm{ml}$ of Pre-hybridisation Buffer(-DS) for $1 \mathrm{hr}$ at room temperature.

3. Add $1 \mu \mathrm{l}$ of probe to $0.5 \mathrm{ml}$ hybridisation Buffer (+DS) (9:1). Incubate overnight at $55^{\circ} \mathrm{C}$.

4. 2x wash with $0.5 \mathrm{ml}$ Washbuffer prewarmed at $55^{\circ} \mathrm{C}$.

Wash buffer:

Formamide $250 \mathrm{ml}$

20x SSC $\quad 50 \mathrm{ml}$

Water $\quad 200 \mathrm{ml}$

$20 \%$ Tween $202.5 \mathrm{ml}$

$5.7 \times 0.5 \mathrm{ml}$ warmed wash buffer $1 \mathrm{hr}$ at $55^{\circ} \mathrm{C}$.

6. Incubate in $0.5 \mathrm{ml}$ warmed wash buffer overnight at $55^{\circ} \mathrm{C}$.

7. 2x wash with PBTw. 
8. Incubate for $30 \mathrm{~min}$ in PBTw at RT.

9. Incubate in $0.5 \mathrm{ml} \mathrm{PBTw}+5 \% \mathrm{NGS}+$ Antikörper (1:2000) (anti-DIG-AP

FAB-Frag.) for $2 \mathrm{hrs}$. at RT.

10. 2x wash with PBTw.

11. 9x 10 min. PBTw at RT.

12. $2 \mathrm{x}$ wash with Alkaline Phosphatase Buffer $+0.1 \%$ Tween-20

$$
\begin{aligned}
& 100 \mathrm{mM} \mathrm{NaCl}^{2} \quad(=20 \mathrm{ml} 5 \mathrm{M} \mathrm{NaCl}) \\
& 5 \mathrm{mM} \mathrm{MgCl}_{2} \quad\left(=5 \mathrm{ml} \mathrm{MgCl}_{2}\right) \\
& 100 \mathrm{mM} \text { Tris/HCl pH } 9.5 \quad(=100 \mathrm{ml} 1 \mathrm{M} \text { Tris }+875 \\
& \text { ml water }) \\
& 1 / 100 \mathrm{~V} 10 \% \text { Tween-20 }
\end{aligned}
$$

13. 5 min of incubation in AP buffer at RT.

14. Transfer the samples into 24 well plates, wash one time with $0.2 \mathrm{ml}$ of BM Purple AP substrate, then add $0.2 \mathrm{ml}$ of BM Purple AP substrate.

15. At proper stages, stop staining by 3 times of wash with PBTw and add $80 \%$ Glycerin/ H2O. Store the plate at 4 degrees.

IIII. Mounting and light microscopy

\subsubsection{Biochemistry (collaborated with Tobias Schwarz)}

GluR-IID and GluR-IIE-EGFP were inserted into pFastbac1 vector (Invitrogen) and transformed into DH10Bac cells. Then the recombinant bacmid DNA was used for transfecting Sf9 cells with the aid of the Bac-to-Bac Baculovirus expression system (Invitrogen). For immunoblot experiments Sf9 cells were infected with the recombinant GluR-IID and GluR-IIE-EGFP baculovirus at a MOI (multiplicity of infection) of 1. Sf9 cells were harvested 40 hours post-infection washed twice with PBS, denatured in Laemmli buffer and heated at $100^{\circ} \mathrm{C}$ for 10 minutes.

Drosophila wild-type embryos were collected and dechorionated 18h AEL, froze in liquid nitrogen and stored at $-80^{\circ} \mathrm{C}$. Embryo lysates were prepared as described (Gillespie and Wasserman, 1994). The embryonic debris was removed 
by centrifugation at $13000 \mathrm{rpm}$ for 15 minutes and the supernatant was transferred to a new tube and centrifuged again for further purification. A portion of both pellet and supernatant fraction were mixed with an equal volume of $2 \mathrm{x}$ Laemmli buffer and heated at $100^{\circ} \mathrm{C}$ for 10 minutes.

Standard western blot was performed as described before. Anti-IID (1:1000), anti-IIC (1:1000) and rabbit anti-GFP antibodies (1:2000, Abcam) were incubated for 2 hours with the blot membranes at $4^{\circ} \mathrm{C}$. For the visualization of the bands horseradish peroxidase conjugated secondary antibodies were used with the ECL Western blot system (Amersham).

\subsubsection{Immunohistochemistry}

\subsubsection{Embryonic and Larval preparation}

Solutions used for dissection:

Haemolymph-like (HL-3) saline without Ca

$14 \mathrm{ml} 5 \mathrm{M} \mathrm{NaCl}$

$5 \mathrm{ml} 1 \mathrm{M} \mathrm{KCl}$

$20 \mathrm{ml} 1 \mathrm{M} \mathrm{MgCl}_{2}$

$5 \mathrm{ml}$ Trehalose

$115 \mathrm{ml} 1 \mathrm{M}$ Sucrose

Add distilled and deionized water to $1000 \mathrm{ml}$, adjust $\mathrm{PH}$ to 7.2

10X PBS (Phosphate-buffered saline)

$80 \mathrm{~g} \mathrm{NaCl}$

$2 \mathrm{~g} \mathrm{KCl}$

$2 \mathrm{~g} \mathrm{KH}_{2} \mathrm{PO}_{4}$

11.5g Na $2 \mathrm{HPO}_{4} \cdot 2 \mathrm{H}_{2} \mathrm{O}$

Add distilled and deionized water to $1000 \mathrm{ml}$, adjust $\mathrm{PH}$ to 7.4 
PBT

$1 \mathrm{X}$ PBS

$0.05 \%$ Tween-20

4\% PFA (Fixation solution)

$4 \%$ paraformaldehyde

$1 \mathrm{X}$ PBS

Bouwin Fixation solution

Antibodies

Rabbit anti-GluR-IIC/III antibodies were generated against a c-terminal peptide (PRRSLDKSLDRTPKS). Rabbit anti-GluR-IID antibodies were generated against a c-terminal peptide of GluR-IID (ESLKTDSEENMPVED). Both sera were affinity purified and used at 1:500 dilution. Other primary antibodies were used at the following concentrations: mouse monoclonal anti-GluR-IIA antibody (8B4D2, DSHB), 1:100; Goat anti-HRP-Cy5, 1:250; mouse anti-FasII (1D4, DSHB), 1:40; mouse anti-Dlg (DSHB), 1:500; Nc82 (generous gift of Erich Buchner, Würzburg), 1:100. Except for samples stained with 8B4D2, which were fixed for 5 min with cold methanol, all of the other stainings were fixed for 10 min with $4 \%$ paraformaldehyd.

Dissection and staining

Mid stage 3rd instar larvae were put on a dissection plate, both ends were fixed by fine pins and the specimen was covered by a drop of ice cold HL-3 solution (see below). Dissection scissors were used to make a small hole at the dorsal midline of the larva (near to the posterior end) which was then completely opened along the dorsal midline from the hole to the anterior end. The epidermis was stretched flat and pinned down, then the internal organs and central nervous system were removed carefully with forceps. Late stage embryos (20- $22 \mathrm{hrs}$ after egg laying) were dissected on sylgard plates, fixed 
with fine clips and opened using a pair of sharp tungsten needles. The dissected samples were fixed and then incubated with primary antibodies overnight, followed by fluorescence-labelled secondary antibodies (Dianova) and mounted in VectaShield mounting media (Vector Laboratories).

\subsubsection{Confocal and epifluorescent microscopy}

Imaging of embryonic and larval body wall preparations was performed on a Leica DM IRE2 microscope equipped with a Leica TCS SP2 AOBS scan head, using a Leica HCX PL Apo CS 63x 1.32 NA OIL UV objective. Epifluoresence images were taken on a Zeiss Axioscope with Axiocam camera, using a 100x oil objective of NA 1.4. Image processing was performed using ImageJ and Photoshop software.

\subsubsection{Ultrastructural analysis (collaborated with Carolin Wichmann)}

Dissected preparations were primary fixed in a mixture of $4 \%$ paraformaldehyde and $0.5 \%$ glutaraldehyde in $0.1 \mathrm{M}$ PBS (pH7.2) for $10 \mathrm{~min}$ and fixed additionally $60 \mathrm{~min}$ with secondary fixative comprising of $2 \%$ glutaraldehyde in $0.1 \mathrm{M}$ sodium cacodylate buffer ( $\mathrm{pH} 7.2$ ), washed three times for $5 \mathrm{~min}$ in sodium cacodylate buffer, and postfixed on ice for $1 \mathrm{hr}$ with $1 \%$ osmium tetroxide, followed by an $1 \mathrm{hr}$ washing step in sodium cacodylate buffer and three brief washing steps in destilled water. The samples were stained en bloc with $1 \%$ uranyl acetate in destilled water for $1 \mathrm{hr}$ on ice. After a brief wash with destilled water, the samples were dehydrated in increasing ethanol concentrations $(30 \%, 50 \%, 70 \%, 95 \%, 100 \%)$, infiltrated in Epon resin $(100 \%$ EtOH/Epon 1:1, 30 min and $90 \mathrm{~min} ; 100 \%$ Epon, over night) and embedded for $24 \mathrm{hr}$ at $85^{\circ} \mathrm{C}$.

The samples were trimmed, and series of $80 \mathrm{~nm}$ ultrathin sections were cut from muscle 6/7, abdominal segment 2 and 3 with a diamond knife on a Reichert Ultracut Ultramicrotome and mounted on Formvar-coated grids. 
The sections were stained in uranyl acetate (4\%) and photographed with a Philips (EM 301) transmission electron microscope.

\subsubsection{Eletrophysiology (collaborated with Robert Kittel)}

Intracellular recordings were made at $22^{\circ} \mathrm{C}$ from muscle fiber 6 of abdominal segments 2 and 3, of late third instar larvae. The larvae were dissected in ice-cold, calcium-free haemolymph-like saline (HL-3) according to Stewart et al. (1994). Larval filets were rinsed with $2 \mathrm{ml}$ of HL-3 saline containing $1 \mathrm{mM} \mathrm{Ca}^{2+}$, before being transferred to the recording chamber where two-electrode voltage clamp (TEVC) recordings were performed in $1 \mathrm{mM}$ extra cellular $\mathrm{Ca}^{2+}$. The larval NMJ was visualized with a fixed-stage upright microscope (Olympus, 40x water immersion lens). Whole muscle recordings of both miniature and evoked postsynaptic currents were recorded in TEVC mode (AxoClamp 2B, Axon Instruments) using sharp microelectrodes (borosilicate glass with filament, $1,5 \mathrm{~mm}$ outer diameter) with resistances of 15-35 $\mathrm{M} \Omega$ and filled with $3 \mathrm{M} \mathrm{KCL}$. All cells selected for analysis had resting potentials between -55 and $-70 \mathrm{mV}$. For stimulation, the cut end of the segmental nerve was pulled into a fire-polished suction electrode and brief $(300 \mu \mathrm{s})$ depolarizing pulses were passed at $0.2 \mathrm{~Hz}$ (npi stimulus generator and isolation unit). To ensure the stable recruitment of both innervating motoneurons, the amplitude of the pulse was determined by increasing the stimulation strength to 1.5 times the amplitude needed to reach the threshold of double motoneuron recruitment. The clamp was tuned such that it responded to a voltage step from -60 to $-70 \mathrm{mV}$ with settling times of $1 \mathrm{~ms}$ for mEJCs and $500-750 \mu$ s for eEJCs, this gave voltage errors of maximally $4 \mathrm{mV}$ for eEJCs of approx. 100nA. Both eEJCs (voltage clamp at $-60 \mathrm{mV}$ ) and $\mathrm{mEJCs}$ (voltage clamp at $-80 \mathrm{mV}$ ) were low-pass filtered at $1 \mathrm{kHz}$. For each cell, 20 eEJCs and 90s of mEJCs recordings were used for subsequent analysis (pClamp9, Axon Instruments). For the paired-pulse stimulation, responses to 20 consecutive paired stimuli (separated by $4 \mathrm{~s}$ rest) with $20 \mathrm{~ms}$ interpulse interval were averaged. 


\section{Results}

\subsection{Establishing the tissue specific transcriptome of Drosophila larval body wall muscles}

The total number of genes in Drosophila genome is predicted to be approximately 14,000 . The high-density DNA microarray technique provides a fast and high throughput way to identify genes expressed at specific tissues or involved in certain processes (Stathopoulos and Levine, 2002; Butler et al., 2003; Li and White, 2003). Here, initial goal was to obtain a whole genome scaled map of molecular components involved in the construction and regulation of the postsynaptic muscle cells. Thus, using a Drosophila wild type strain (CS10), the transcripts of $3^{\text {rd }}$ instar larval epidermis preparations, mainly consisting of body wall somatic muscles (with cuticles) but not central nervous systems, were prepared, amplified, labeled and hybridized to the Affymetrix Drosophila Genome arrays, generating the RNA expression profiles. RNA expression profiles of total larvae of Drosophila CS10 strain were also produced and set as baseline to compare with those from epidermis samples. Since equal amount of RNAs were utilized for different samples, transcripts displaying higher expression level in epidermis samples might represent the muscle specific or enriched genes and were selected.

The transcripts were ranked by average fold increase. Transcripts showing 2 fold or greater enrichment in epidermis samples were collected and shown in the appendix. One direct way to check the specificity of the data obtained is to check those transcripts with known tissue distribution pattern. As expected, among the pool of nearly 700 transcripts showing increasing abundance in epidermis samples, about one tenth are already known to be specifically (or abundantly) present in muscles or cuticles, thus directly proving the reliability of the data. These genes are categorized into various groups, including RNA-binding proteins, transcription factors, cell adhesion molecules, enzymes, 
motor proteins, receptors and ion channels, and structural proteins, etc.(representatively shown in Table 3-1).

Table 3-1 Described muscle / cuticle-specific transcripts displaying enrichment in gene chip analysis of body-wall-preparations

\begin{tabular}{|c|c|c|}
\hline Gene & Fold change & Proposed Function \\
\hline \multicolumn{3}{|l|}{ RNA binding proteins } \\
\hline How (held out wings) & 4.5 & RNA binding \\
\hline \multicolumn{3}{|l|}{ Transcription factors } \\
\hline Mef2 & 4.4 & RNA polymerase II transcription factor \\
\hline Muscle LIM protein at $84 \mathrm{~B}$ & 4.4 & transcription factor \\
\hline \multicolumn{2}{|l|}{ Cell adhesion molecules } & cell adhesion \\
\hline \multicolumn{3}{|l|}{ Enzymes } \\
\hline Mlc-k & 12.2 & myosin light chain kinse \\
\hline myosin light chain Kinase & 5 & myosin light chain kinase \\
\hline CamKII & 3.8 & Calcium/ Calmodulin-dependent protein kinase II \\
\hline dlg1 (discs large 1) & 2.9 & Synaptic and muscular scaffolding \\
\hline \multicolumn{3}{|l|}{ Motor proteins } \\
\hline Tropomysin 1 & 2.6 & Molecular motor \\
\hline Tropomysin 2 & 3.6 & Molecular motor \\
\hline upheld (troponin T) & 3.6 & tropomyosin binding \\
\hline sanpodo (tropomodulin) & 5.8 & tropomyosin binding \\
\hline Mhc (myosin II heavy chain) & 2.5 & muscle motor protein \\
\hline Mlc2 (myosin light chain 2) & 2.1 & muscle motor protein \\
\hline \multicolumn{3}{|l|}{ Receptors and ion channels } \\
\hline GluR-IIA & 4.3 & glutamate receptor \\
\hline GluR-IIB & 2.6 & glutamate receptor \\
\hline GluR-III/ IIC & 3.5 & glutamate receptor \\
\hline \multicolumn{3}{|l|}{ Structural proteins } \\
\hline Lcp65Ag1 & 3.3 & structural constituent of larval cuticle \\
\hline Lcp1 & 2.9 & structural constituent of larval cuticle \\
\hline Lcp2 & 2.8 & structural constituent of larval cuticle \\
\hline Lcp65Ag2 & 2.1 & structural constituent of larval cuticle \\
\hline Lcp4 & 2.1 & structural constituent of larval cuticle \\
\hline Lcp3 & 2 & structural constituent of larval cuticle \\
\hline \multicolumn{3}{|l|}{ Others } \\
\hline Mp20 (muscle protein 20) & 2.9 & calcium-binding \\
\hline TpnC73F (Troponin C) & 4.3 & calcium-binding \\
\hline TpnC47D & 2.7 & calcium-binding \\
\hline
\end{tabular}




\subsection{Identification of novel ionotropic glutamate receptor subunits expressed at the Drosophila neuromuscular synapse}

\subsubsection{Enrichment of various transcripts encoding novel ionotropic glutamate receptor subunits in larval body wall muscles}

The Drosophila genome encodes about 30 potential ionotropic glutamate receptor subunits (Littleton, 2000; Littleton and Ganetzky, 2000; Sprengel et al., 2001). Using the Affymetrix Drosophila gene chip result, the expression level of each subunit gene was compared between larval body wall and whole larvae RNA pools. As expected, GluR-IIA and GluR-IIB mRNA (Schuster et al., 1991; Petersen et al., 1997) were found to be enriched in body wall preparations. Another subunit annotated as CG4226, first referred to as GluR-IIC (Saitoe et al., 2001) and later as GluR-III (Marrus et al., 2004) was found to be enriched in body walls as well. A recent publication has shown that GluR-III null mutants die at late embryonic stages most likely due to a defect of glutamatergic transmission. In addition to these already described subunits with muscle expression, another glutamate receptor (annotated as CG18039) was found to be also enriched in body wall preparations.

Since the probe design of the Drosophila gene chip used here is based on an earlier version of the Drosophila genome annotation database, some genes might be omitted. To check if there are any newly anotated glutamate receptor genes which were not represented by the chip used, each of these selected glutamate receptor genes was blasted against the updated Drosophila genome annotation database (www.Flybase.com). Indeed, when blasting CG18039 coding sequence one gene annotated as CG31201 was found, which is strikingly similar to CG18039 (see below, Fig3-2). Interestingly, these two genes are located as direct neighbors within the genome, which is reminiscent of the organization of GluR-IIA and GluR-IIB (Petersen et al., 1997). Recently, it has been found that genes with similar expression pattern often cluster at certain regions of genome (Boutanaev et al., 2002; Roy et al., 2002), likely facilitating 
transcriptional control of to be co-expressed genes. Intuitively, CG31201 might thus be expressed in a similar pattern with its neighbor CG18039.

Next, real time quantitative RT-PCR was applied to independently quantify the expression level of these candidate glutamate receptor genes. As shown in table3-2, real time quantitative PCR data were consistent with the observation from gene chip analysis. Moreover, CG31201 was shown to be enriched within body wall mRNA pool as well. From now on we will refer to the locus encoding subunit CG18039 as GluR-IID and the locus encoding CG31201 as GluR-IIE. These names are meant to reflect their muscle expression (see below) along with Glu-IIA, -IIB and -IIC/III.

\begin{tabular}{lccccc}
\hline & $\begin{array}{c}\mathrm{C}_{\mathrm{t}} \\
\text { (body wall) }\end{array}$ & \% control & $\begin{array}{c}\mathrm{C}_{\mathrm{t}} \\
\text { (total larva) }\end{array}$ & \% control & $\begin{array}{c}\text { Body wall } \\
\text { /total larva }\end{array}$ \\
\hline $\begin{array}{l}\text { GluR-IIA } \\
\text { (CG6992) }\end{array}$ & 26,7 & 15,4 & 28,3 & 4,4 & 3,5 \\
$\begin{array}{l}\text { GluR-IIB } \\
\text { (CG7234) }\end{array}$ & 28,2 & 5,6 & 29,5 & 1,9 & 3,0 \\
$\begin{array}{l}\text { GluR-IIC/III } \\
\text { (CG4226) }\end{array}$ & 26,4 & 18,7 & 28,9 & 3,1 & 6,3 \\
$\begin{array}{l}\text { GluR-IID } \\
\text { (CG18039) }\end{array}$ & 25,5 & 35,5 & 27,0 & 10,5 & 3,4 \\
$\begin{array}{l}\text { GluR-IIE } \\
\text { (CG31201) }\end{array}$ & 24,5 & 73,7 & 26,3 & 17,5 & 4,2 \\
$\begin{array}{l}\text { tbp-1 } \\
\text { (internal } \\
\text { control) }\end{array}$ & 23,8 & 100,0 & 23,8 & 100,0 & 1,0 \\
\hline
\end{tabular}

Table 3-2 GluR-IID and GluR-IIE are enriched in larval body wall mRNA.

Abundances of glutamate receptor subunit transcripts estimated using real time PCR. The control transcript (proteasome subunit tbp-1) had the same abundance in body wall preps and whole larvae. The glutamate receptor subunits shown were enriched in body wall RNA when compared to whole larva RNA. The abundances of mRNAs are expressed as $C_{t}$ values indicating the cycle number with which amplification exceeds detection threshold ( $\mathrm{Ct}$ difference of one indicates a two-fold difference in abundance). All data are averages of three independent experiments where each sample was run two times in parallel.

\subsubsection{Expression pattern of new ionotropic glutamate receptor genes}


To further confirm the muscle expression of these new subunit genes, in situ hybridization on Drosophila embryos and larvae was performed. In fact, the mRNAs of GluR-IID (Fig.3-1A-D) and GluR-IIE (Fig.3-1E, F) are specifically expressed in somatic muscles of both Drosophila embryo (Fig.3-1A-C, E, F) and larva (Fig.3-1D). Expression of GluR-IID (Fig.3-1C-D) and GluR-IIE (Fig.3-1E) starts in somatic muscles of late stage 12 embryos (Fig.3-1A, E) and extends throughout embryonic and larval development (Fig.3-1D, F), a pattern very similar to that of GluR-IIA, -IIB and -IIC/III (data not shown; Petersen et al., 1997; Marrus et al., 2004). Intriguingly, in addition to the somatic muscle expression GluR-IID is also abundantly present in cardiac precursor cells (Fig.3-1A, C), which was not found for the other muscle subunit genes.

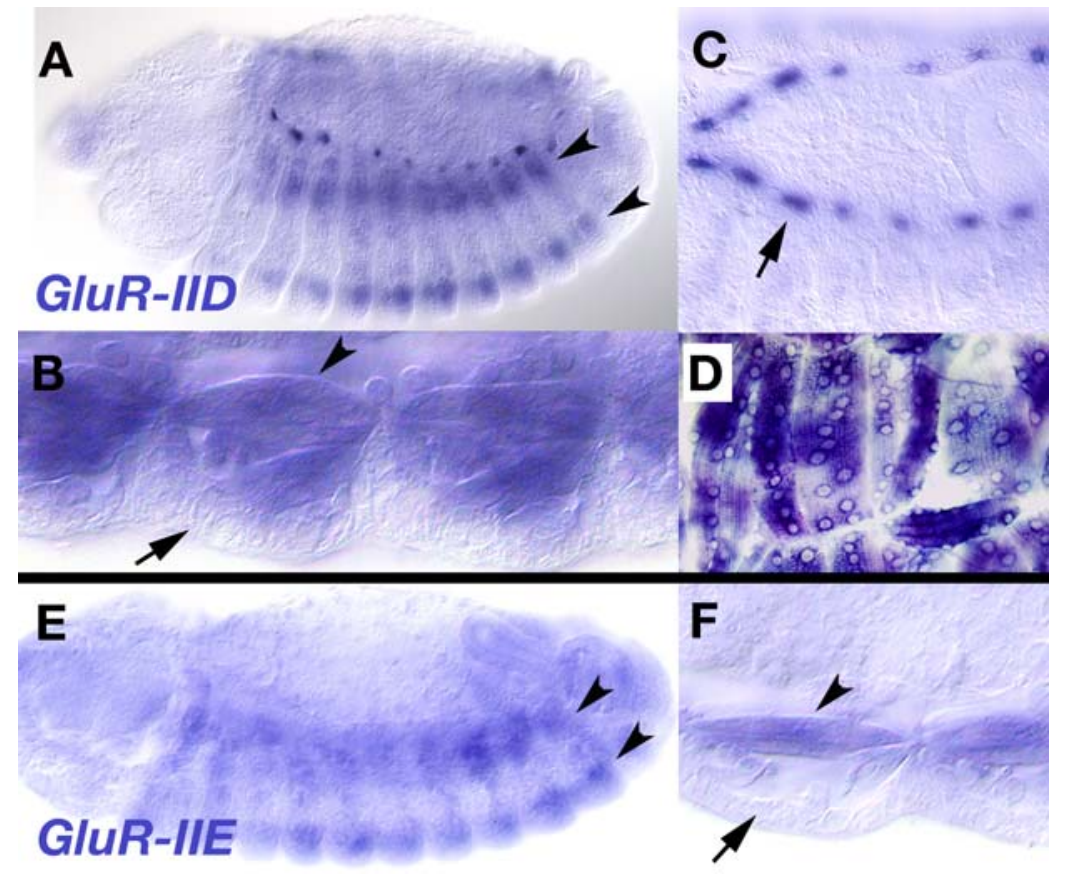

Figure 3-1. GluR-IID and GluR-IIE: novel glutamate receptor subunits with muscle specific expression

In situ hybridizations on Drosophila embryos (A-C, E,F) and larvae (D) for GluRIID (A-D) and GluRIIE mRNA (E,F). Both subunits are expressed specifically in presumptive somatic muscle cells (A, B, E, F, arrowheads) but are for example not found in the adjacent epidermis (A, B, F, arrows). Expression of GluR-IID and GluR-IIE transcript in the presumptive somatic muscles starts in late stage 12 and peaks at around stage 14 (A, E), to then persist during later embryogenesis (B: stage 16; E: stage 17) and larval development (D). GluR-IID is also expressed within heart precursor cells (A and B, arrows). 


\subsubsection{GluR-IID and GluR-IIE represent a new type of muscle-expressed glutamate receptor subunit}

In a 'simple' synaptic model system like the Drosophila NMJ the coexistence of so many distinct glutamate receptor subunits is somewhat unexpected. To get a closer view of the relationship among them, full-length cDNA clones of GluR-IIC, GluR-IID and GluR-IIE were obtained, the reading frames were verified by double strand sequencingand the deduced amino acid sequences compared. Clearly, these proteins encode all structural features typical in glutamate receptor subunits. Importantly, all putative transmembrane domains were found at similar positions (see Figure 3-2A). As previously noted (Marrus et al., 2004), the GluR-IIC/III sequence is closely related to GluR-IIA and IIB. GluR-IID and GluR-IIE are also highly related with each other (Fig. 3-2A, B). However, GluR-IID and GluR-IIE are distant from GluR-IIA, IIB and IIC/III group (Fig.3-2B). The fact is, GluR-IID and GluR-IIE are even slightly closer to human kainate receptor GluR6 than they are to the GluR-IIA, -IIB and -IIC/III group (see dendrogram in Fig.3-2B). Thus, in contrast to the GluR-IIA, -IIB, -IIC/III type, GluR-IID and GluR-IIE represent a new type of glutamate receptor subunits which is expressed in somatic Drosophila muscle. In a previous alignment entailing all identified ionotropic glutamate receptor subunits of the Drosophila genome, several other non-NMDA type subunits group in between GluRIID, -IIE and GluR-IIA, -IIB, -IIC/III groups (Littleton and Ganetzky, 2000). Notably, all of the muscle glutamate receptor subunits (from GluR-IIA to -IIE) have direct orthologs in Drosophila Pseudoobscura (data not shown), suggesting that the differentiation of the insect muscle-expressed glutamate receptor subunits into two structurally different groups might be conserved. 


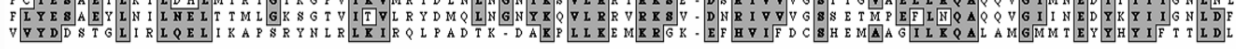

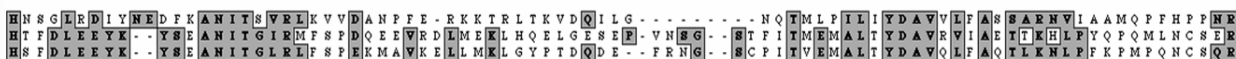

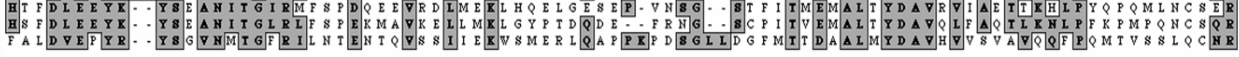

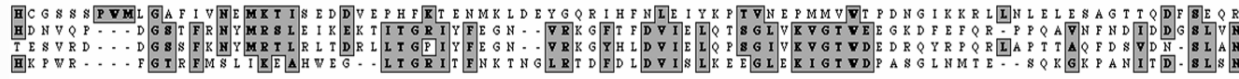

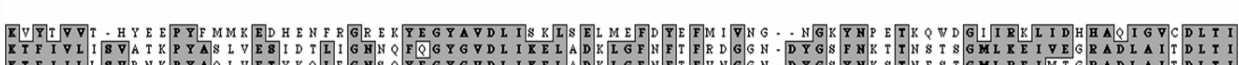

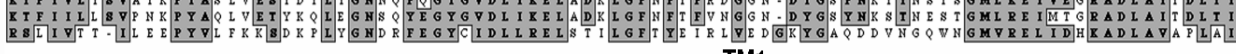

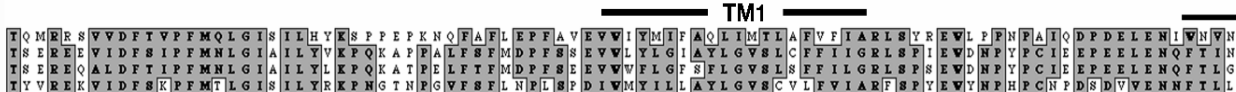

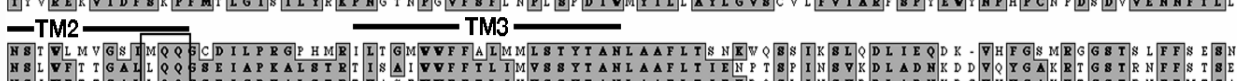

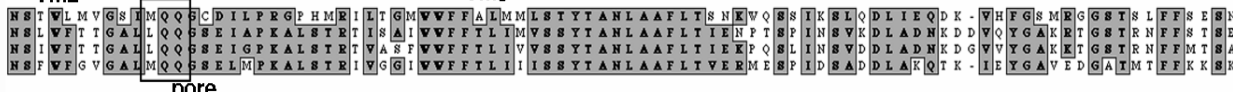

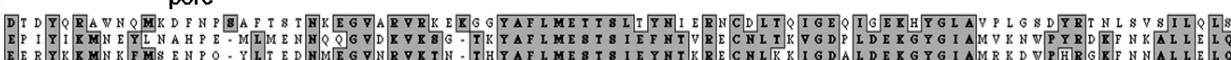

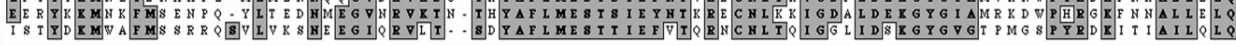

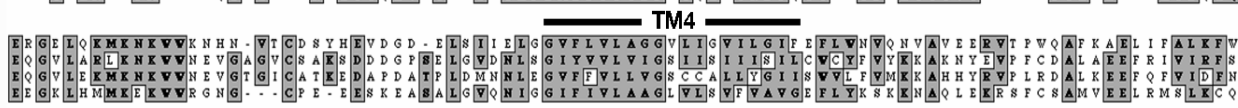

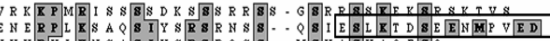

B

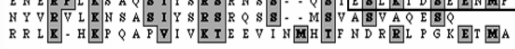

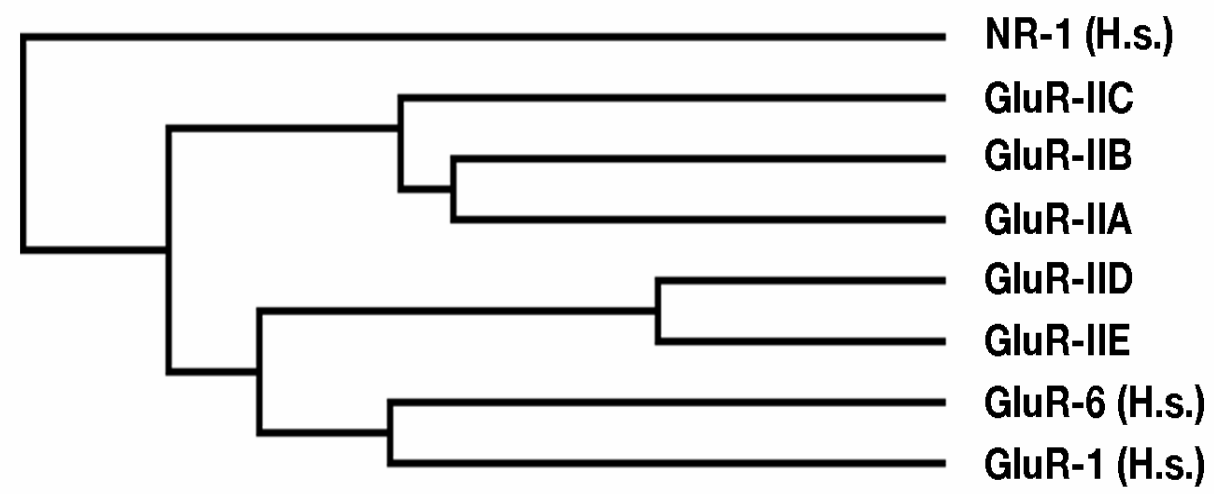

Figure 3-2 Sequence analysis of GluR-IID and GluR-IIE

A, sequence alignment of predicted amino acid sequences of (from top to bottom) GluRIIC/III, GluR-IID, GluR-IIE and human kainate receptor subunit GluR-6, similar amino acids are indicated by shaded boxes. Putative trans-membrane domains (TM1-4), the channel pore region and the c-terminal peptide of GluR-IID which was used for immunization are indicated as well.

$\mathrm{B}$, dendrogram analysis comparing muscle expressed glutamate receptor subunits of Drosophila, together with AMPA receptor subunit GluR1, kainate receptor subunit GluR-6 and NMDA receptor subunit NR-1 (all Homo sapiens). Dendrogram was generated using MacVector software. 


\subsubsection{GluR-IIC, GluR-IID and GluR-IIE are specifically localized at postsynaptic densities of neuromuscular synapses}

To directly test the subcellular distribution of these novel glutamate receptor subunits, polyclonal antibodies were produced. In brief, rabbits were immunized with GluR-IIC/III C-terminus peptide (PRRSLDKSLDRTPKS) and GluR-IID C-terminus peptide (ESLKTDSEENMPVED) respectively. Collected sera were affinity-purified via their corresponding peptides. In western blotting assay, the antibodies against GluR-IIC/III and GluR-IID recognize a single band of about predicted $109 \mathrm{kD}$ and $102 \mathrm{KD}$ in wild type Drosophila embryo extracts respectively (Fig.3-3A, right lane). Cross reactivity of our sera between receptor subunits is not observed, since the antiserum against DGluR-IID can specifically recognize SF9-cells infected with recombinant virus containing GluR-IID cDNA but not the SF9-cells with expressing GluR-IIE (Fig.3-3A, left lanes).

For immuno-fluorescence microscopy studies, previously a monoclonal antibody (8B4D2) against GluR-IIA has been characterized. In both late stage embryos and larvae 8B4D2 stains the postsynaptic membrane along the NMJ with a discrete dotty manner (Fethetherston et al., 2001; Packard et al., 2002). This antibody thus allows direct visualization of PSDs opposite the corresponding active zones. In the wild type larvae and embryos stained with our antibodies against GluR-IIC and GluR-IID, similar findings were obtained: localization of both GluR-IIC and GluR-IID is confined to typical punctae (Fig.3-3B, C, arrow heads) corresponding to individual postsynaptic densities (PSDs), which are surrounded by the HRP labels known to have perisynaptic expression (Sone et al., 2000). Moreover, these punctae are found directly opposite to the presynaptic Nc82 label. The Nc82 monoclonal antibody specifically recognizes the presynaptic active zone (Heimbeck et al., 1999; Wucherpfennig et al., 2003). Thus, it can be concluded that both GluR-IIC and GluR-IID are expressed within the PSD region of individual neuromuscular 
synapses.
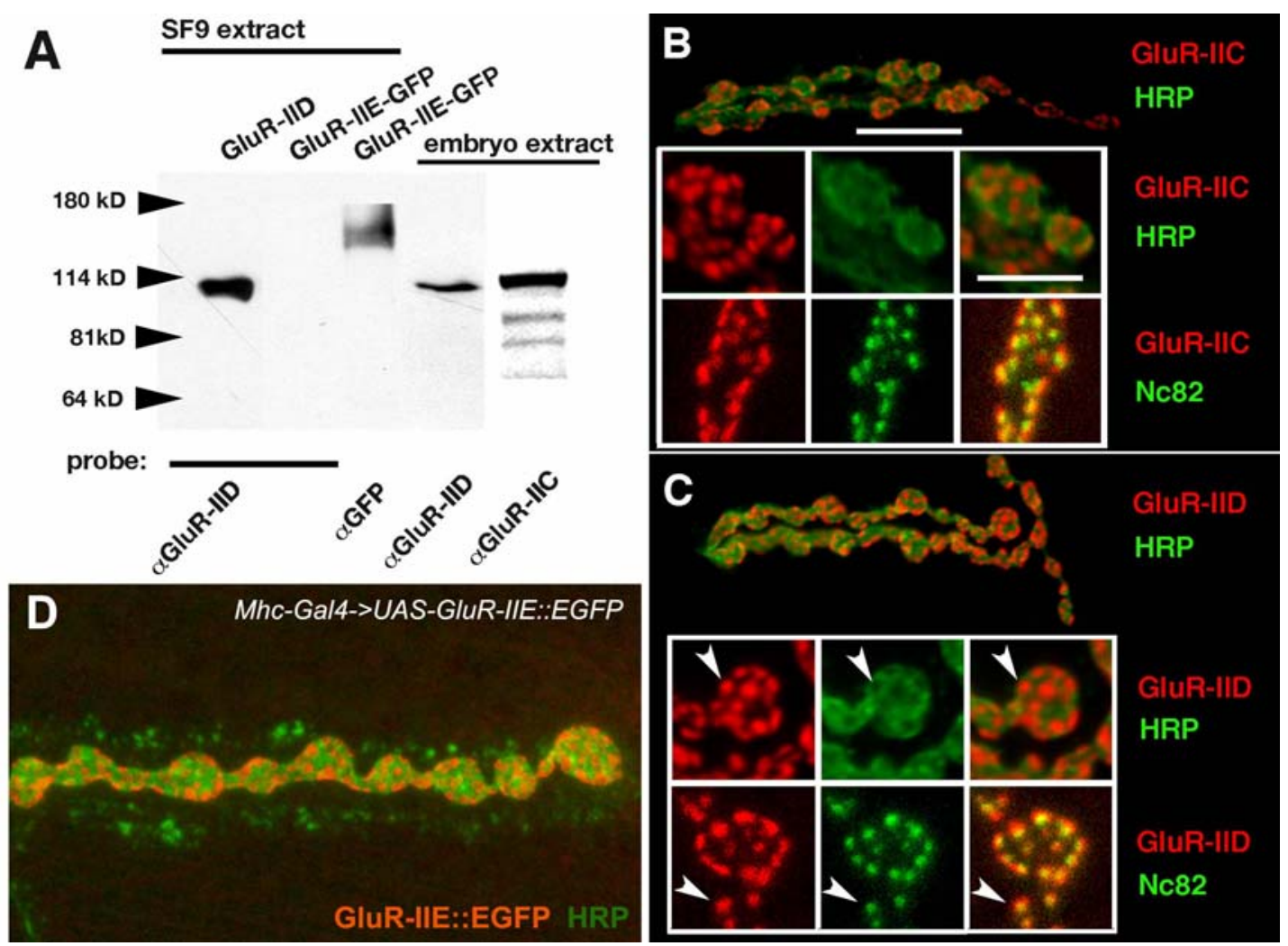

Fig.3-3 GluR-IID and GluR-IIE are expressed within postsynaptic densities A: western blot analysis: the anti-IID peptide antibody recognizes SF9-cell expressed GluR-IID and endogenous GluR-IID from Drosophila embryo extract. It does not cross react with the related GluR-IIE protein, which is SF9-cell expressed as a GFP fusion and is recognized using anti-GFP antibody with the predicted size of about 145 $\mathrm{kD}$. GluR-IIC/III is recognized with our peptide antibody in Drosophila embryo extract with predicted size as well.

B-C, shown are epifluorescence pictures (upper two panels in B and C) and confocal pictures (lower panels in $\mathrm{B}$ and $\mathrm{C}$ ) of receptor subunits GluR-IIC/III (B, red) and GluR-IID (C, red) together with the perisynaptic marker HRP (upper panels in B and C, green) or active zone marker Nc82 (lower panels in B and C, green), scale bars: 8 and $4 \mu \mathrm{m}$.

D, confocal picture of GluR-IIE::EGFP (red) together with perisynaptic marker HRP (green).

Several GluR-IIE-specific peptides were used to immunize rabbits as well. Unfortunately, specific antibodies could not be obtained, mainly due to the fact that the GluR-IIE amino acid sequence is closely related to GluR-IID. In fact, it is hard to find a GluR-IIE specific peptide sequence which would be proper for 
immunization. To overcome this difficulty, the enhanced green fluoerscent protein (EGFP) reading frame was inserted into the near C-terminus GluR-IIE to generate an autofluorescent fusion protein. Fusion of EGFP into the same position of mammalian GluR2 has been previously shown not to interfere with glutamate receptor assembly and function (Sheridan et al., 2002). Indeed, upon overexpressing this GluR-IIE::EGFP in larval muscle (driven by MHC-Gal4), an antibody directed against EGFP strongly labeled the postsynaptic density region (Fig3-3D). This result makes it very likely that the endogenous GluR-IIE protein localizes to the PSD region as well.

\subsection{GluR-IID and GluR-IIE are essential for viability}

To genetically investigate what roles GluR-IID and GluR-IIE play in NMJ glutamatergic transmission, null mutants for each of the two genes were required. For Drosophila molecular genetics, transposon mediated mutagenesis often is an efficient way of knocking out a candidate gene function. In the presence of transposase activity, integrated transposons can be remobilized and randomly jump into other chromosmal locations. Upon remobilization, apart from the transposon seqeunce as well often genomic sequences flanking the insertion site of the mobilized transposon will be eliminated as well. Thus, transposon mapping in the near of candidate loci can be used to produce gene-specific deletions. Here, this strategy was used to obtain mutants of GluR-IID and GluR-IIE.

From a collection of piggyBac transposon lines which covers $53 \%$ of all loci annotated for Drosophila (Thibault et al., 2004), one line (pBac $\{\mathrm{RB}\} \mathrm{e} 01443)$ was described to have an insertion in the GluR-IID locus. This line was obtained and genomic PCR was performed using one primer corresponding to the flanking region the proposed insertion site and another primer corresponding to the end of the piggyBac transposon. The resulting PCR 


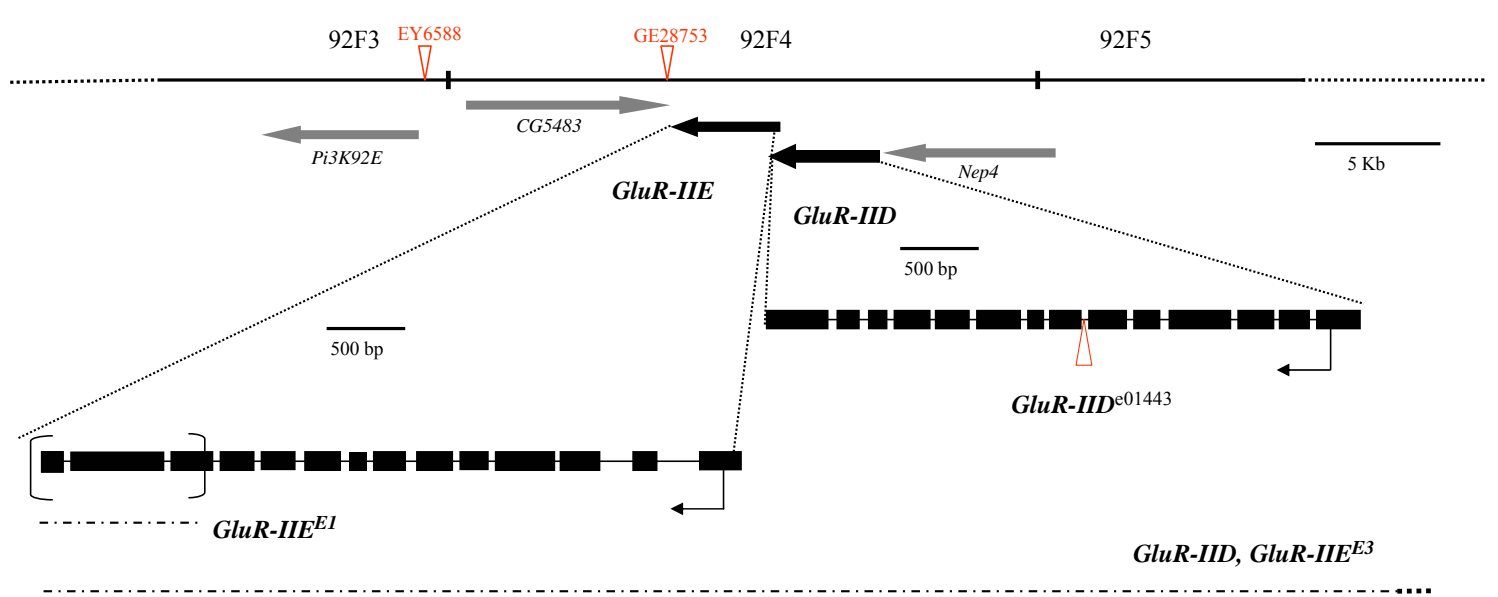

GluR-IIE genomic rescue

Figure 3-4 Genetic analysis of GluR-IID and GluR-IIE

GluR-IID and GluR-IIE map to position 92F on chromosome III. Exon-intron structure of both loci is shown, exons are boxed. The null allele GluR-IIDe01443 is based on a piggyBac transposon insertion within the open reading frame of the GluR-IID locus. Null allele GluR-IIEE1 lacks c-terminal sequence of the protein including the last transmembrane domain of the receptor subunit. GluR-IIEE3 deletes at least GluR-IIE and GluR-IID alleles and thus is used as deficiency allele. The genomic stretches used for genomic rescue constructs are shown below. 
product was purified and subjected to sequencing. It was found that in fact in this line the transposon has integrated directly into intron 6 of DGluR-IID (Fig.3-4). This could well interfere with normal transcript splicing and hence the generation of GluRIID protein. Interestingly, this line is embryonic lethal in homozygous condition. We from now on refer to this allele as GluR-IID ${ }^{\mathrm{e} 01443}$.

For GluR-IIE, imprecise excision mutagenesis screening was performed using transposon line EY6588 (http://flypush.imgen.bcm.tmc.edu/pscreen/) and later GE28753 (http://www.genexel.com) whose insertion sites are located 14Kb and $150 \mathrm{bp}$ downstream of the 3' end of GluR-IIE transcript, respectively (Fig.3-4). From the screening, about 30 imprecise excision events (Fig.3-4) were recovered, genomic PCR with a series of primer pairs corresponding to the flanking region of GE28753 insertion position were utilized to recover the deleted region in each line. Among them, a line called GluR-IIE ${ }^{E 1}$ was found to have the genomic sequence encoding the C-terminal part of GluR-IIE including the last transmembrane domain deleted. Importantly however, the GluR-IIE $E^{E 1}$ deletion does not extend into neighboring loci. Similar to GluR-IID ${ }^{\mathrm{e} 01443}$, GluR-IIE $E^{E 1}$ is also embryonic lethal in homozygous condition. In addition, no GluR-IID protein could be observed at the neuromuscular synapses of either GluR-IID ${ }^{\mathrm{e} 01443}$ or GluR-IIE ${ }^{E 1}$ homozygous embryos (see 3.4.1 for details). GluR-IID $D^{\mathrm{e} 01443}$ and GluR-IIE $E^{E 1}$ are also embryonic lethal over another independent excision allele GluR-IID\&IIE E3 (Fig.3-4), a larger deficiency which deletes both GluR-IID and GluR-IIE genomic sequences. However, GluR-IID $D^{\mathrm{e} 01443}$ is fully viable when crossed over GluR-IIE ${ }^{E 1}$. Both GluR-IID $D^{\mathrm{e} 01443}$ and GluR-IIE $E^{E 1}$ are fully rescued by transgenic addition of one copy of genomic GluR-IID or GluR-IIE, respectively; however, neither the GluR-IID mutant nor the GluR-IIE mutant can be rescued by the respective other transgene. Thus, several lines of evidence (here and 3.4.1) suggest that GluR-IID $D^{\mathrm{e} 01443}$ and GluR-IIE E1 represent specific null mutant alleles for GluR-IID and GluR-IIE, respectively. While the neighboring loci GluR-IID and GluR-IIE encode very similar proteins with largely overlapping expression patern, both of them are essential for embryonic viability. 


\subsection{Reciprocal dependence of all essential glutamate receptor subunits for synaptic expression and function}

3.4.1 Complete synaptic absence of all other glutamate receptor subunits after knockout of any essential subunit genes

Homozygous GluR-IID ${ }^{\mathrm{e} 01443}$ and GluR-IIE ${ }^{E 1}$ embryos were subjected to further inspection. These embryos, while apparently developing normally, show no coordinated movements and do not hatch. The same phenotype is also observed previously within GluR-IIC/III single or GluR-IIA\&IIB double null mutant embryos. This phenotype was interpreted as failure of neuromuscular neurotransmission due to the absence of functional postsynaptic glutamate receptors (Petersen et al., 1997; Marrus et al., 2004). Consistent with a role in synaptic transmission, GluR-IID and GluR-IIE mRNA expression starts within embryonic muscles well before the onset of neurotransmission (Fig.3-1), just like the cases of GluR-IIA, -IIB and -IIC (Petersen et al., 1997; Marrus et al., 2004).

In mammals, ionotropic glutamate receptors appear to be composed of rather closely related subunits (Wenthold et al., 1996; Mulle et al., 2000). Hence, whether the rather similar GluR-IID and GluR-IIE subunits directly form glutamate receptors was investigated via immuno-fluorescence microscopy. In rpinciple, if the formation of a certain type of glutamate receptor fails, the synaptic localization of its consituent receptor subunits must be affected. In wild type late stage embryos, GluR-IID proteins stably localize at the PSD region of all NMJ synapses (Fig.3-5B, wild type). In GluR-IIE E1 embryos, neuromuscular contacts still form, as shown by staining against anti-HRP and Nc82 antibodies (Fig.3-5A, blue and green channel). However, GluR-IID is completely absent from GluR-IIE ${ }^{E 1}$ embryonic NMJs (Fig.3-5B). This finding suggests that GluR-IIE and GluR-IID might join into a common glutamate receptor which in turn is essential for synaptic neurotransmission at the embryonic NMJ. 


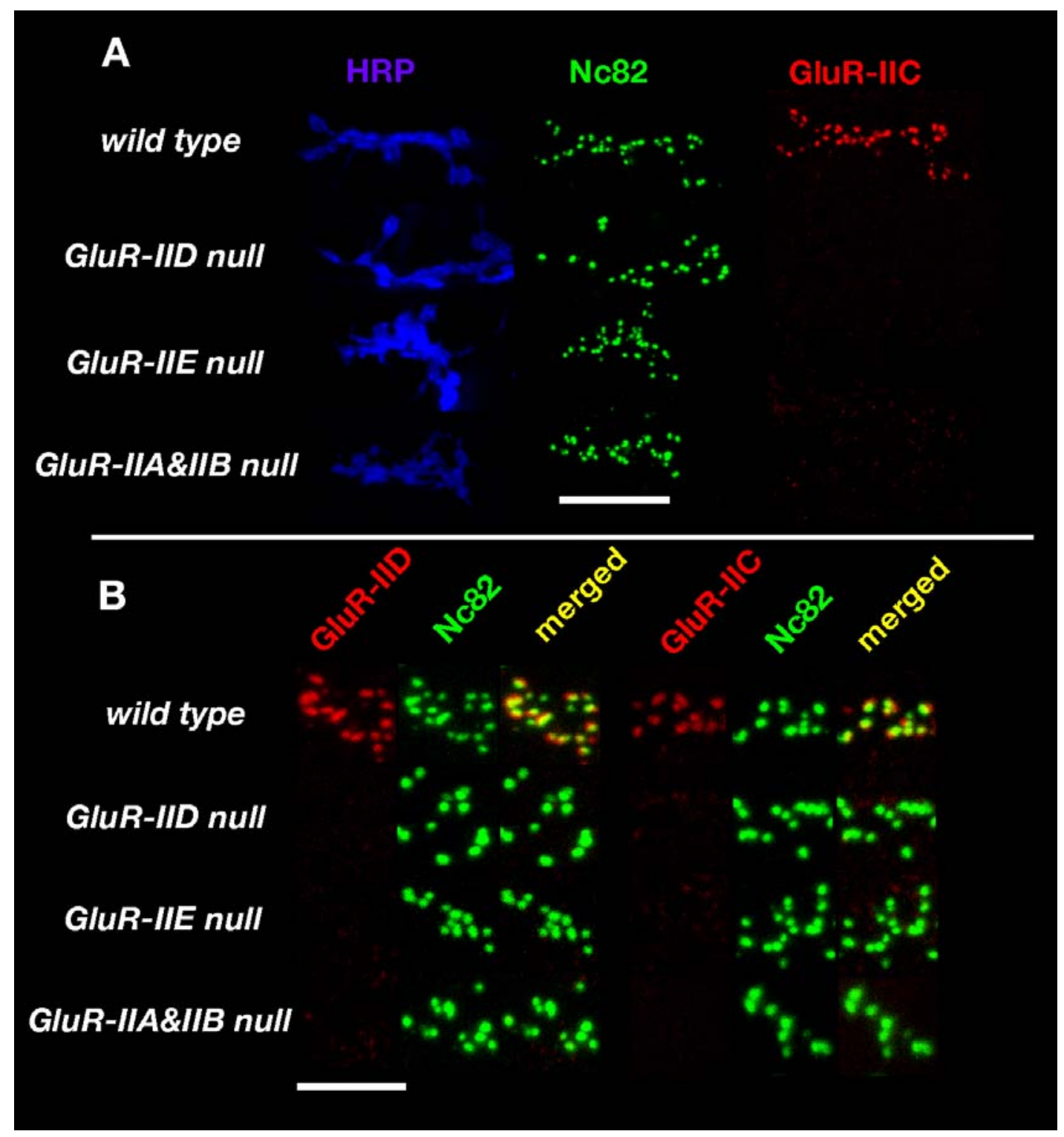

Figure 3-5 Interdependence between glutamate receptor subunits for NMJ expression

A, Confocal images of NMJs in wild-type, GluR-IID null, GluR-IIE null or GluR-IIA\&IIB double null mutant embryos (20-22 hrs old) stained with antibodies against GluR-IIC/III (red), Nc82 (green) and HRP (blue). Nc82 staining indicates differentiation of presynaptic release sites at the mutant NMJs. Synaptic expression of all glutamate receptor subunits fails at the mutant NMJs. Scale bar: $10 \mu \mathrm{m}$ $\mathrm{B}$, Higher magnifications of confocal stainings similar as in A. Stainings of either GluR-IID (left panels) or GluR-IIC/III (right panels) are shown together with Nc82. Scale bar: $5 \mu \mathrm{m}$.

Based on the analysis of GluR-IIC/III and GluRII-A hypomorphic larvae, it 
has been suggested that GluR-IIC/III is an obligatory subunit, which associates with either GluR-IIA or GluR-IIB to form functionally distinct glutamate receptors (Marrus et al., 2004). This idea was tested again but directly by immunostaining GluRII-A\&IIB double mutant embryos. Consistent with Marrus et al., GluR-IIC/III label is completely absent from NMJs of Glur-IIA\&IIB null mutant embryos (Fig.3-5A and B). Thus GluR-IIA together with GluR-IIB can be considered a "synthetic essential subunit". In principle, the two "groups" of subunits (GluR-IID, -IIE versus -IIA, -IIB, -IIC/III) could be independently essential for embryonic neurotransmission. Alternatively, synaptic localization and thus receptor function could be interdependent between both groups. To distinguish these two possibilities, it was first checked whether the synaptic localization of the GluRIIC/III glutamate receptor subunit is dependent on the presence of GluR-IID and GluR-IIE. Clearly, no synaptic localization of GluR-IIC/III can be found in either GluR-IID or GluR-IIE null mutant embryos (Fig.3-5A and B). Vice versa, the synaptic expression of GluR-IID was absent from GluR-IIA\&-IIB double null mutant embryos (Fig. 5B). Thus, at the embryonic NMJ, the synaptic expression of all essential glutamate receptor subunits is absolutely interdependent. The easiest explanation for these data is that at the Drosophila NMJ the glutamate receptor is formed by four different essential glutamate receptor subunits: GluR-IIC, GluR-IID, and GluR-IIE together with either GluR-IIA or GluR-IIB.

\subsubsection{Genetically depriving the expression level of any single essential glutamate receptor subunit results into corresponding reduction of all other subunits}

If the glutamate receptor is indeed assembled from four different subunits, partially suppressing the level of any single such subunit should interfere with the synaptic expression of the other essential subunits and the overall glutamate receptor formation. To test this, several independent experiments have been performed. 
First, the expression of GluR-IIE was knocked down in vivo via transgenic RNA interference technique (Kennerdell and Carthew, 2000). When RNA interference against GluR-IIE was conducted in a muscle-specific manner, the GluR-IIE transcript level was knocked down to about $20 \%$ of wild type level while the transcription level of all other muscle subunit genes remained unchanged (not shown), indicating the highly selective RNA degradation mediated by RNA interference. In contrast to the GluR-IIE null mutants, these animals do not die at embryonic stage but instead develop into mature larvae and adult flys. So the effects on the distribution of other subunits in $3^{\text {rd }}$ larvae were examined. Significantly, all of the other known subunits (GluR-IIA, GluR-IIB, GluR-IIC/III and GluR-IID) were greatly reduced at NMJ when the RNA interference was driven in muscles (using G14-Gal4 or MHC-Gal4), while

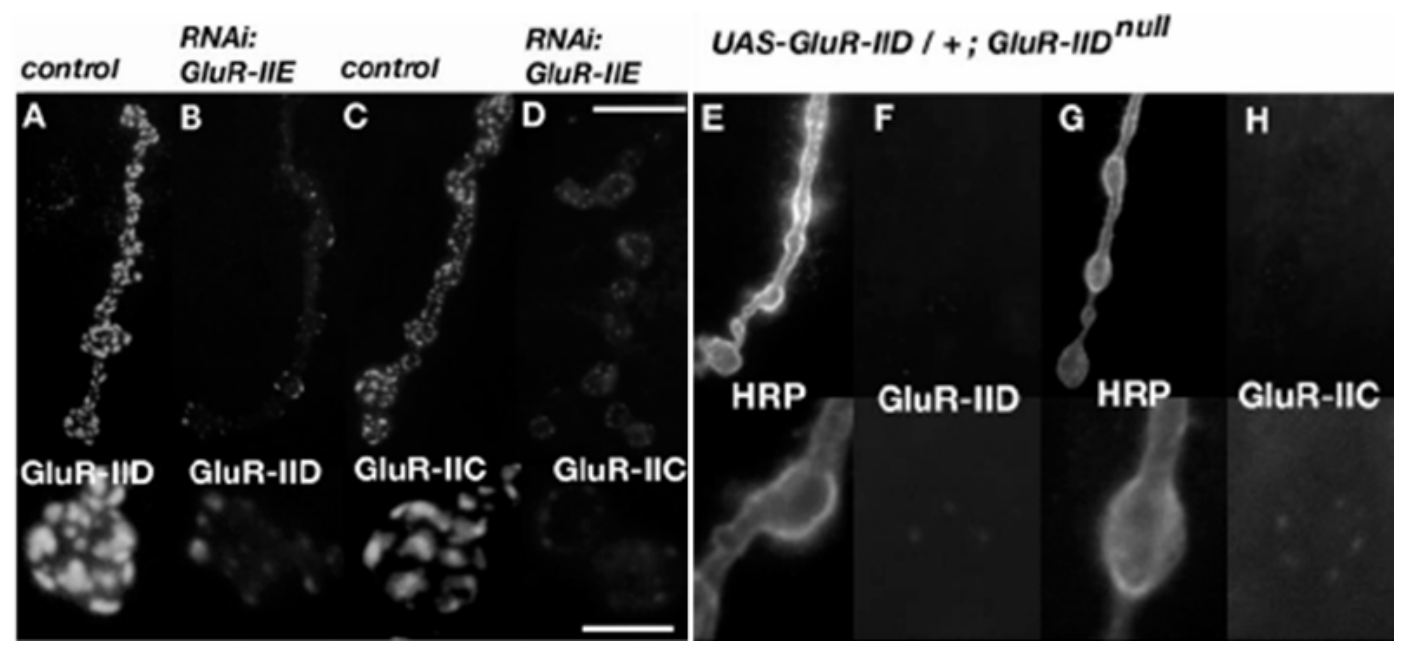

Figure 3-6 A partial reduction of either GluR-IIE or GluR-IID provokes a significant reduction of all glutamate receptor subunits at the NMJ

(A-D) Larval NMJs (muscle 4, abdominal segment 2) stained for GluR-IID (A, B) or GluR-IIC/III (C, D) in control larvae (G14-gal4/ +, A and C) or larvae experiencing muscle specific RNA interference against GluR-IIE (G14-gal/+; UAS-GluR-IIE-RNAi, $\mathrm{B}$ and D). Suppression of GluR-IIE leads also to a reduction in the NMJ expression of GluR-IIC/III and GluR-IID. Lower pictures represent higher magnifications.

(E-H) Shown are NMJs (muscle 4, abdominal segment 2) of GluR-IID null mutant larvae rescued with a single copy of UAS-GluR-IID (UAS-GluR-IID/+; GluR-IID ${ }^{e 01443} /$ GluR-IID\&IIE E3. Only trace amounts of GluR-IID (F) and GluR-IIC (H) are expressed at the NMJ, HRP stainings $(E, G)$ are added to visualize the NMJ. Non-linear contrasting had to be used to make the trace amounts of GluR-IID (F) and GluR-IIC (H) visible. Scale bar in upper panel 7,5 $\mu \mathrm{m}$, in lower panel showing higher magnification $2,5 \mu \mathrm{m}$. 
there is no obvious effects when driving the expression of RNA interference construct in the central nervous system (using ELAV-Gal4) (Fig.3-6B, D; data not shown), which further verify that GluR-IIE functions specifically in somatic muscles and intrinsically associates with other subunits.

For a second experiment, it was found that minimal amounts of GluR-IID (produced by the "leaky" expression from pure UAS-cDNA transgenes) can already rescue GluR-IID null mutant embryos into mature larvae (Fig.3-6E-H). In these rescued larvae, both GluR-IID and all other glutamate receptor subunits were strongly reduced at the NMJs (Fig.3-6H). Re-expressing GluR-IID to normal level by using the muscle specific Gal4-driver line Mhc-Gal4 at $18^{\circ} \mathrm{C}$ restored the synaptic localization of GluR-IID and all other glutamate receptor subunits (not shown; overexpression of GluR-IID cDNA in both wild type and GluR-IID null background at $25^{\circ} \mathrm{C}$ resulted into significant reduction of synaptic glutamate receptor level with abnormal accumulation of GluR-IID proteins within the ER). Thus, the levels of GluR-IID and GluR-IIE within the muscles directly control the overall amount of glutamate receptors that localize at the NMJ.

The next question addressed is, whether vice versa the amounts of muscle GluR-IIA, -IIB and -IIC/III can control synaptic level of GluR-IID and -IIE. To this end, a genetic situation in which GluRIIB was fully absent and simultaneously GluR-IIA was suppressed to dramatically low level (GluRIIA ${ }^{\text {hypo, }}$, $G l u R-I I B^{\text {null }}$, see 2.2.2.1) was used. Previously it has been proposed that local postsynaptical translation plays important roles in controlling the growth and transmission efficacy of synapses at Drosophila NMJ. GluR-IIA appears might be a substrate under subsynaptic translational control (Sigrist et al., 2000). Since translational control elements often localize within the 3'UTR region of a gene, transgenic flys expressing GluR-IIA lacking most 3'UTR were produced and tested for their ability to rescue the GluR-IIA\&IIB double null mutants, which is 
embryonic lethal (Diantonio et al., 1999). Among several independent transgenic lines tested, only one of them could rescue the embryonic lethality with low rate and the rescued larvae were clearly paralyzed. Real-time quantitative PCR assay showed that the GluR-IIA message level was below 5\% of endogenous level. In accordance with the low message level, the GluR-IIA protein was hardly detectable at the larval NMJ (not shown). Notably, the level of both GluR-IIC/III and GluR-IID were drastically low (Fig.3-7B, D). Collectively, all the above data suggest all essential glutamate receptor subunits together controls receptor formation and proper synaptic localization. Furthermore, this rule seems true for both initial formation and further development of NMJs, where many new synapses get added continuously (Schuster et al., 1996; Gramates and Budnik, 1999).

Fig.3-7 Minimal mounts of GluR-IIA and no- IIB: expression of all glutamate receptor subunits and postsynaptic sensitivity are strongly reduced

(A-D) Shown are 3rd instar larval NMJs (muscle 4, abdominal segment 2) stained for GluR-IID (A, B) or GluR-IIC/III (C, D) in wild type controls (A, C) or in animals having only about 5\% GluR-IIA and no GluR-IIB (GluR-IIAhypo, GluR-IIBnull, see Material and Methods). NMJ morphology in HRP labeling is shown in insets to allow NMJ visualization independent of receptor label. In GluR-IIAhypo, GluR-IIBnull larvae, the synaptic localization of both GluR-IID and GluR-IIC/III is strongly reduced. Apparent differences in between residual GluR-IID and GluR-IIC/III are likely due to slightly different sensitivity of our antibodies.

E-G, Postsynaptic sensitivity is dramatically reduced at larval NMJs of GluRIIAhypo,GluRIIBnull larvae. E, F, Two-electrode voltage-clamp recordings of eEJCs and mEJCs from muscle 6 of abdominal segment A2 and A3. Representative traces of mEJC recordings (E) and average traces of 10 consecutively recorded eEJCs (F) of the indicated genotypes are shown. The mean amplitudes of mEJCs (white bars) are indistinguishable between wild type and GluRIIA\&IIB double mutant larvae rescued with a complete genomic GluRIIA transgene (exact genotype: $\operatorname{df}(2 \mathrm{~L}) \mathrm{clh} 4$, $\mathrm{P}$ [GluRIIA 43 'UTR] / GluRIIA\&BSP22). In all muscle cells of GluRIIAhypo,GluRIIBnull larvae, no signs of mEJCs could be recorded despite extensive and sensitive recording. Evoked responses (F) are also dramatically reduced in GluRIIAhypo,GluRIIBnull larvae compared to both control groups. The increase in evoked response in animals rescued with wild type GluRIIA (and thus without expressing of GluRIIB) when compared to wild type has been described before (Petersen et al., 1997; DiAntonio et al., 1999). G, Paired pulses (stimulation interval ms) provoke slight facilitation in the controls but depression in GluRIIAhypo,GluRIIB-/larvae. Data are derived from the indicated number of cells (number in the column) and represent means \pm SEM. 

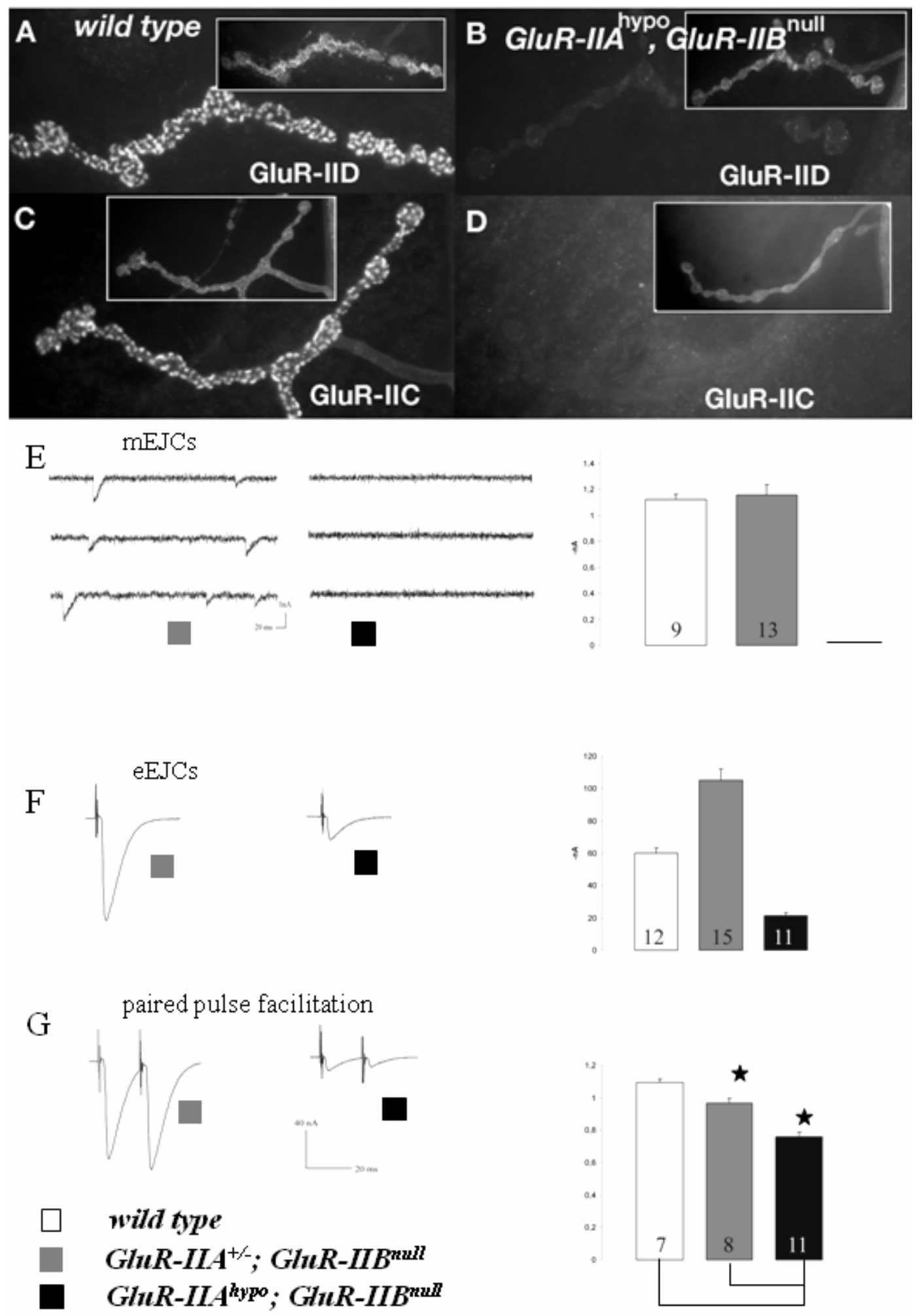


\subsubsection{Depriving synaptic glutamate receptor subunits results into dramatic weakness of postsynaptic activity}

To test whether the physiological function of glutamate receptor correlates well with its presence at synapse reflected from the above morphological data, electrophysiological analysis is highly necessary. Among the different genetic situations which partially deprive certain glutamate receptor subunits in embryos and larvae, the GluRIIA ${ }^{\text {hypo }}$, GluR-IIB ${ }^{\text {null }}$ larvae maintain the lowest overall level of glutamate receptors (Fig.3-7 B, D) and display clear signs of paralysis. Thus GluRIIA hypo, GluR-IIB ${ }^{\text {null }}$ larvae were subjected to two-electrode-voltage-clamp recordings in collaboration with Robert Kittle (Fig.3-7 E, F). As expected, compared to wild type conditions, spontaneous miniature junctional currents (mEJCs) in these animals were below the detection limit indicating an extreme drop of postsynaptic glutamate receptor function. Accordingly, evoked currents (eEJCs) were also significantly low (wild type: 61 $\mathrm{nA}, \mathrm{n}=12$, GluRIIAhypo, GluR-IIBnull: $21 \mathrm{nA}, \mathrm{n}=11 ; \mathrm{p}<0.00005$, Mann-Whitney, two-sided non-parametric test).

In conclusion, strongly reducing the expression of a single 'essential' glutamate receptor subunit is sufficient in severely downregulating the overall level of functional glutamate receptors at the NMJ, which in turn results into weakening of postsynaptic activity in response to neurotransmitter release.

\subsection{Structural role of ionotropic glutamate receptor during pre- and postsynaptic differentiation of neuromuscular synapse}

\subsubsection{Normal differentiation of presynaptic transmitter release machinery at glutamate receptor deprived synapse}

As shown in chapter 3.4, GluRIIA ${ }^{\text {hypo }}$, GluR-IIB ${ }^{\text {null }}$ larval junctions produce evoked currents of about one third of that of wild type junctions. Even more drastically, the spontaneous currents are hardly detectable. We considered the fact that evoked responses appeared less affected than spontaneous responses in 
GluRIIA $^{\text {hypo }}$, GluR-IIB ${ }^{\text {null }}$ larvae as indication of normal or even increased presynaptic transmitter vesicle release as compensation of severe postsynaptic defects. Consistent with the concomitant increase in presynaptic vesicle release, paired pulse stimulation of GluRIIA ${ }^{\text {hypo }}$, GluR-IIB ${ }^{\text {null }}$ NMJ leads to depression of evoked currents while as for wild type controls the currents remain slightly increased in the same condition. The organization of presynaptic release machinery was further characterized morphologically. The active zone is the region of the presynaptic plasma membrane where synaptic vesicles dock, fuse, and release the neurotransmitters (Shapira et al., 2003). Monoclonal antibody NC82 was shown to specifically label the Active zones of synapses of Drosophila NMJ (Wucherpfennig et al., 2003) and central synapses (Heimbeck et al., 1999). In GluRIIA ${ }^{\text {hypo }}$, GluR-IIB ${ }^{\text {null }}$ larvae, nc82 labels are clearly present at NMJ with both density and individual size comparable to wild type controls (Fig.3-8 C, D; Fig.3-9 D-E). $\alpha$-Adaptin is essential for presynaptic endocytosis/vesicle retrieval (Gonzalez-Gaitan and Jackle, 1997) and is restricted to the presynaptic membrane, forming a network-like structure that surrounds the active zones (Gonzalez-Gaitan and Jackle, 1997) (Fig. 3-8 A-C). In GluRIIA $^{\text {hypo }}$, GluR-IIB ${ }^{\text {null }}$ larvae, subcellular distribution of $\alpha$-Adaptin is not apparently distinct with that of wild type controls (Fig.3-8 A, B), suggesting that the differentiation of presynaptic release apparatus is not affected by depriving postsynaptic glutamate receptor.

Fig. 3-8 Normal organization of presynaptic neurotransmitter release machinery components at glutamate receptor deprived synapses

NMJs double-labeled with the antibodies against presynaptic component $\alpha$-Adaptin (Gonzalez-Gaintan M et al., 1997; A-B) and Nc82 antibody recognizing an active zone epitope (Wucherpfennig et al., 2003, Heimbeck et al., 1999; C-D). In wild type animals, $\alpha$-Adaptin is restricted to the presynaptic membrane, forming a network-like structure that surrounds the active zones represented by $\mathrm{Nc} 82$ spots (A, C, E). In GluRIIA $^{\text {hypo }}$, GluRIIB $^{-/-}$larvae, both Nc82 and $\alpha$-Adaptin spots appear no different from wild type control (B, D, F). 

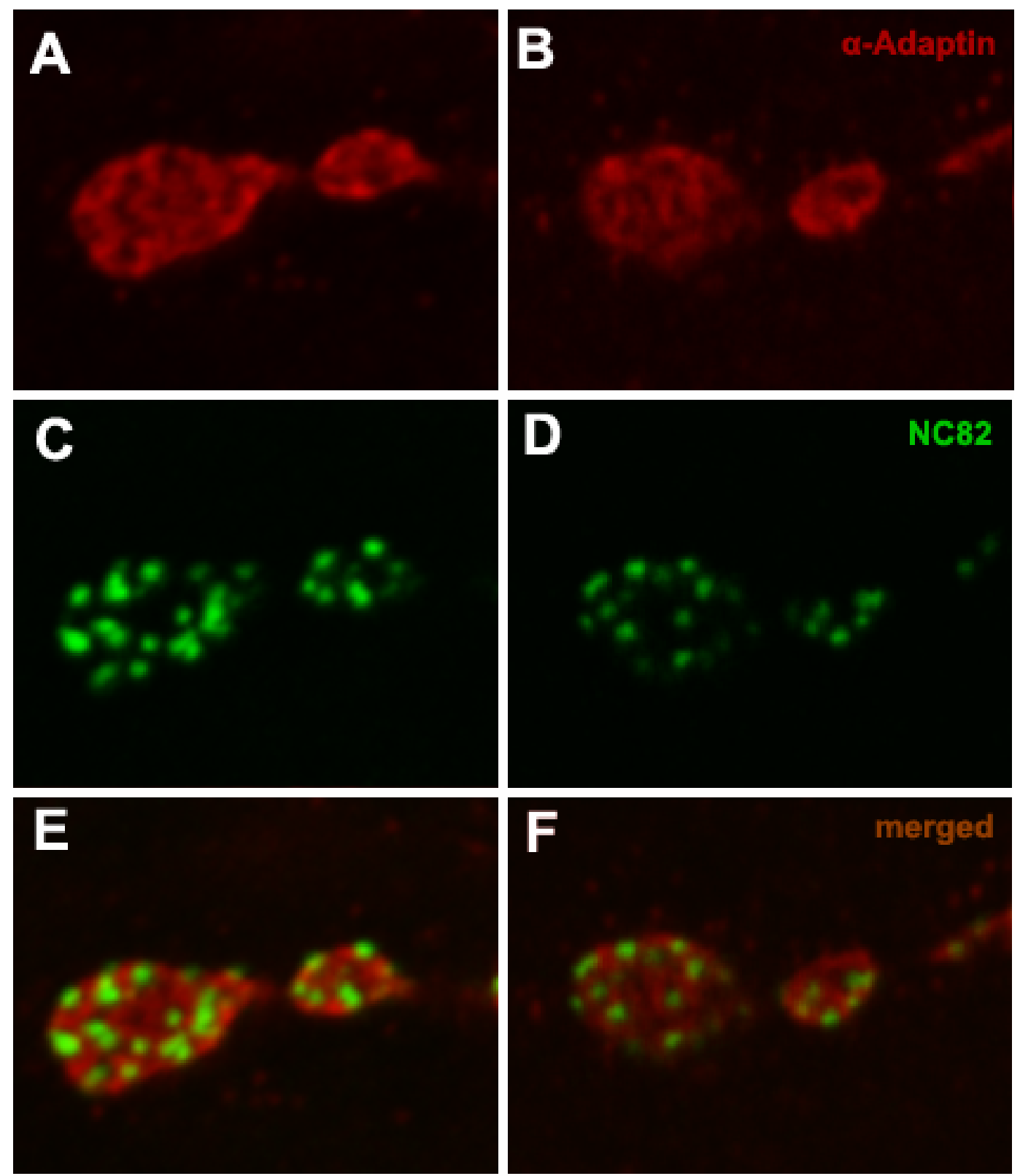

Wild type

GluR-IIAhypo, GluR-IIBnuII

3.5.2 Defective assembly of PSD specialization at glutamate receptor deprived but not presynaptic neurotransmission activity deprived synapse

The differentiation of postsynaptic apparatus was also analyzed in GluRIIA $^{\text {hypo }}$, GluR-IIB ${ }^{\text {null }}$ larvae. So far, only a few PSD components have been identified and characterized at Drosophila NMJ (Sone et al., 2000; Wan et al., 
2000; Parnas et al., 2001). PAKs are a family of serine/threonine kinases that serve as targets for the small GTP-binding proteins Cdc42 and Rac, they are implicated in modulating the cytoskeleton organization (Bagrodia and Cerione, 1999). In Drosophila, dPak has been shown to specifically localize at the PSD of neuromuscular synapse at both immunofluorescence microscopic and immuno-EM level (Sone et al., 2000; Wan et al., 2000). In dpak mutants, the levels of DLG and GluRIIA at the synapse are reduced and the SSR folding is also disrupted (Parnas et al., 2001; Albin and Davis, 2004) In wild type larvae, dPAK antibodies labeled the well defined patches with approximately even distribution over the surface of bouton, (Fig. 3-9), and as expected NC82 perfectly colocalized with dPAK staining (Fig.3-9 ). In GluRIIA ${ }^{\text {hypo }}$, GluR-IIB ${ }^{\text {null }}$ bouton, however, the dPAK staining displayed apparently lower density, with the size of individual spots much more diverse---from huge irregular aggregates to tiny dots, and often, at some bouton areas the dPAK even did not aggregate at all but just 'wrap' the bouton like cloud, (Fig.3-9, arrow).

The above data suggest that, when glutamate receptors are deprived from synapse other constitutive components of postsynaptic specialization can not be assembled efficiently. In principle, these defects could be either due to the absence of glutamate receptor per se or lack of glutamate mediated transmission activity. To distinguish these possibilities, several genetic situations in which the NMJ transmission was either strongly reduced (survival to larval stage) or

Fig.3-9 Defective PSD assembly at glutamate receptor deprived but not transmission deprived synapses

NMJs triple-labeled with the Nc82 antibody recognizing an active zone epitope (Wucherpfennig et al., 2003, Heimbeck et al., 1999; D-F), antibodies against PSD marker DPAK (Sone et al., 2000; G-I)) and HRP (A-C). In wild type animals, Nc82 spots localize at presynaptically juxtaposed to postsynaptic DPAK spots (J). In GluRIIA $^{\text {hypo }}$,GluRIIB ${ }^{-/-}$larvae, Nc82 spots localize normally (E), however, DPAK spots display significantly lower density and more variable individual size $(\mathrm{H})$. In OK319-Gal4->UAS-TNT larvae, both Nc82 and DPAK spots appear no different from wild type control (F, I, L). 

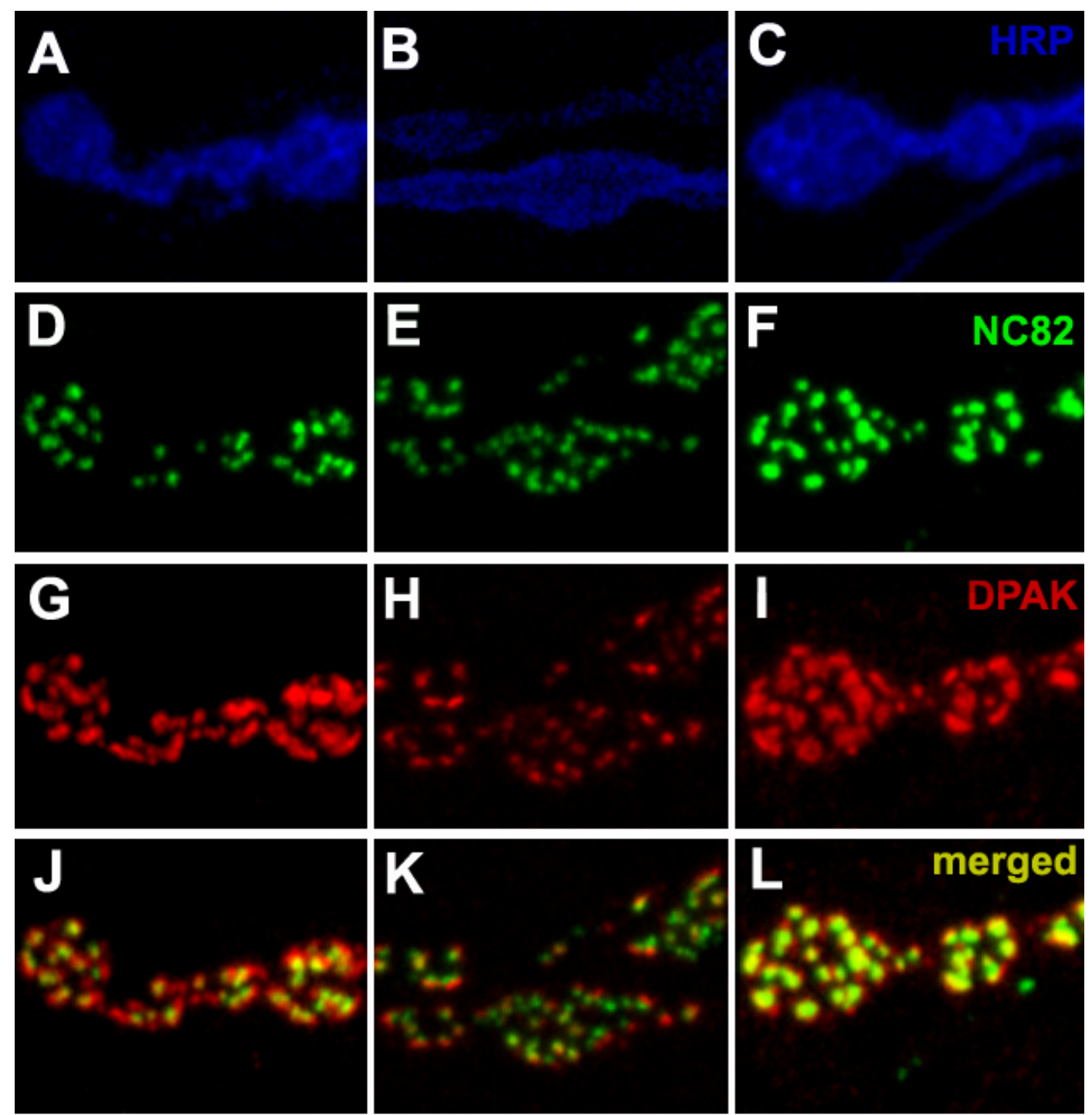

Wild type

GluR-IIAhypo, GluR-IIBnull Ok319-Gal4->UAS-TNT

completely suppressed (lethal at late embryonic stage) were analyzed for postsynaptic assembly. Targeted expression of tetanus toxin light chain (TNT) in Drosophila neurons completely eliminates evoked, but not spontaneous synaptic vesicle release (Sweeney et al., 1995). When using the motor neuron driver ok319-Gal4 to drive TNT expression, low number of animals can survive to $3^{\text {rd }}$ instar larval stage (this survival is likely due to the mosaic property of the driver, Cahir O'Kane, personal communication). Surviving larvae were very strongly paralyzed, much stronger than GluRIIA ${ }^{\text {hypo }}$, GluR-IIB ${ }^{\text {null }}$ larvae. However, the distribution of neither postsynaptic components $\mathrm{dPAK}$ nor glutamate receptor 
show any discernible defects in all the NMJs checked, in contrast they both colocalize well with active zone shown by NC82 staining (Fig.3-9 F, I).

Since the expression of tetanus toxin does not fully eliminate spontaneous synaptic vesicle release (Sweeney et al., 1995), it might be argued that in the above ok319-Gal4 driven situation remaining activity (especially spontaneous activity) could sufficiently induce the assembly of postsynaptic specializations at normal level. Indeed, recently a tight link between spontaneous vesicle exocytosis and glutamate receptor clustering during Drosophila embryonic development has been reported (Saitoe et al., 2001). It should be noted, however, that this report is clearly controversial to some other reports (Broadie et al., 1995; Featherstone et al., 2001). It has been proved previously that, in either null mutants of syntaxin or temperature sensitive allele of dynamin $\left(\mathrm{shi}^{\mathrm{ts} 1}\right)$ reared at nonpermissive temperature, both spontaneous and evoked vesicle exocytosis and hence synaptic transmission are completely eliminated (Broadie et al., 1995). Using these 'cleaner' conditions, the immuno-fluoresence microscopic analysis was performed with our antibodies against GluR-IIC and GluR-IID. In contrast to the findings of Saitoe $M$ et al but consistent with those of Featherstone et al, the glutamate receptor localization within individual PSDs is essentially unaffected as seen in GluR-IIC and GluR-IID staining (Fig.3-10). Moreover, in these animals DPAK proteins also cluster at normal size at the PSD region as evidenced by colocalization with NC82 labels (Fig3-10). Therefore, it is the glutamate receptor per se but not synaptic transmission activity essential for the postsynaptic specialization (including glutamate receptor field) assembly.

Besides the main finding described above, another interesting phenomena found is found. At the NMJs of glutamate receptor deprived larvae (GluRIIA ${ }^{\text {hypo }}$, GluR-IIB ${ }^{\text {null }}$ ), often postsynaptic dPAK label was not opposed by presynptic active zone marker (identified via NC82 staining) (Fig.3-9). One explanation is that these free NC82 labels might represent new synapse growth sites. However, 
this is quite unlikely since in wild type NMJs stained with same antibodies, the free NC82 labels without aligned dPAK patches were very rarely found (Fig.3-9). Intriguingly, free dPAK labels could be stably detected in wide type NMJs, and in most cases such free dPAK patches are small and localize at the tips of junctions or the linkage regions between boutons, which likely represent the potential growth sites of new synapses. Thus, these free dPAK labels might reflect the precursors of new synapses, which implicates that, in contrast to the finding in synapses of cultured hippocampal neurons, at Drosophila NMJ the postsynaptic assembly might precede presynptic assembly. In GluRIIA ${ }^{\text {hypo, }}$ GluR-IIB $^{\text {null }}$ NMJs, the precise coordination of pre- and postsynaptic differentiation is strongly affected. However, it is unlikely that this coordination defect is merely due to absence of glutamate receptor per se, since in embryos with complete loss of presynptic transmitter release, although individual PSDs are assembled rather normally, there also exist free active zones (Fig.3-10 F). Because the glutamate receptor null mutants lack both glutamate receptor and the concomitant transmission activity, it remains to be further addressed whether the precise coordination of pre- and postsynaptic differentiation is activity-dependant.

Fig. 3-10 Glutamate receptor per se but not presynaptic vesicle release are important for proper synapse formation at embryo NMJ

Confocal microscopy on embryonic NMJs (muscle 6 and 7), stained with the synaptic markers in wild type (A-D), in GluR-IIA\&IIB ${ }^{-/-}$double mutant embryos (B, E) and in shibire temperature sensitive mutant $\left(s h i^{\text {ts1 }}\right)$ embryos reared at nonpermissive temperature $(\mathrm{C}, \mathrm{F})$.

A-C, Embryonic NMJs double labeled with active zone recognizing antibody Nc82, glutamate receptor subunits GluR-IIC/ III. In situation with defective presynaptic release (C), postsynaptic glutamate receptor fields appear identical as in wild type control. As expected, staining for GluR-IIC, which depends on the presence of either GluR-IIA or GluR-IIB expression, is absent from synapses of dglurIIA\&IIB-/-(B).

D-F, Embryonic NMJs double labeled with the active zone recognizing antibody Nc82 and antibodies against PSD marker DPAK. . Wild type embryos show typical DPAK patches juxtaposed to presynaptic Nc82 labels (D). In GluR-IIA\&IIB ${ }^{-/-}$and shibire shits embryos reared at nonpermissive temperature, $\mathrm{Nc} 82$ often is not associated with postsynaptic DPAK spots (E, F), DPAK spots are irregular in size and shape and show lower density in GluR-IIA\&IIB ${ }^{-/}$embryos (E). 

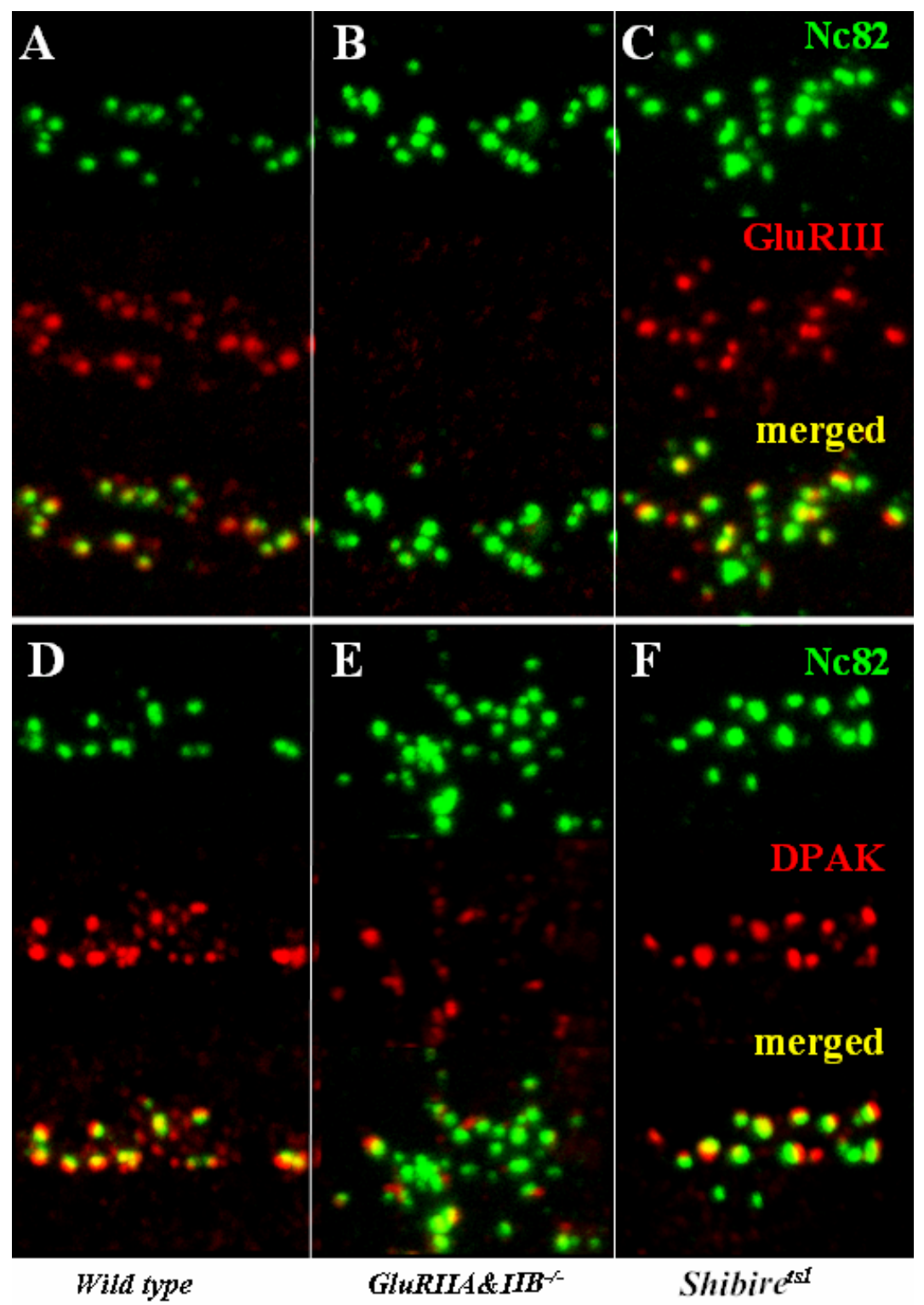

\subsubsection{Ultrastructural evidence of abnormal synaptic differentiation at glutamate receptor deprived synapse}

Next, to check whether such assembly defects of postsynaptic components at glutamate receptor deprived synapse could be directly visualized at the ultrastructural level, neuromuscular junctions 6 and 7 of $3^{\text {rd }}$ instar GluR-IIA ${ }^{\text {hypo }}$, GluR-IIB ${ }^{\text {null }}$ larvae were subjected to transmission electron microscopy (collaborated with Carolin Wichman). At normal Drosophila neuromuscular synapses, active zones are characterized by the presence of 

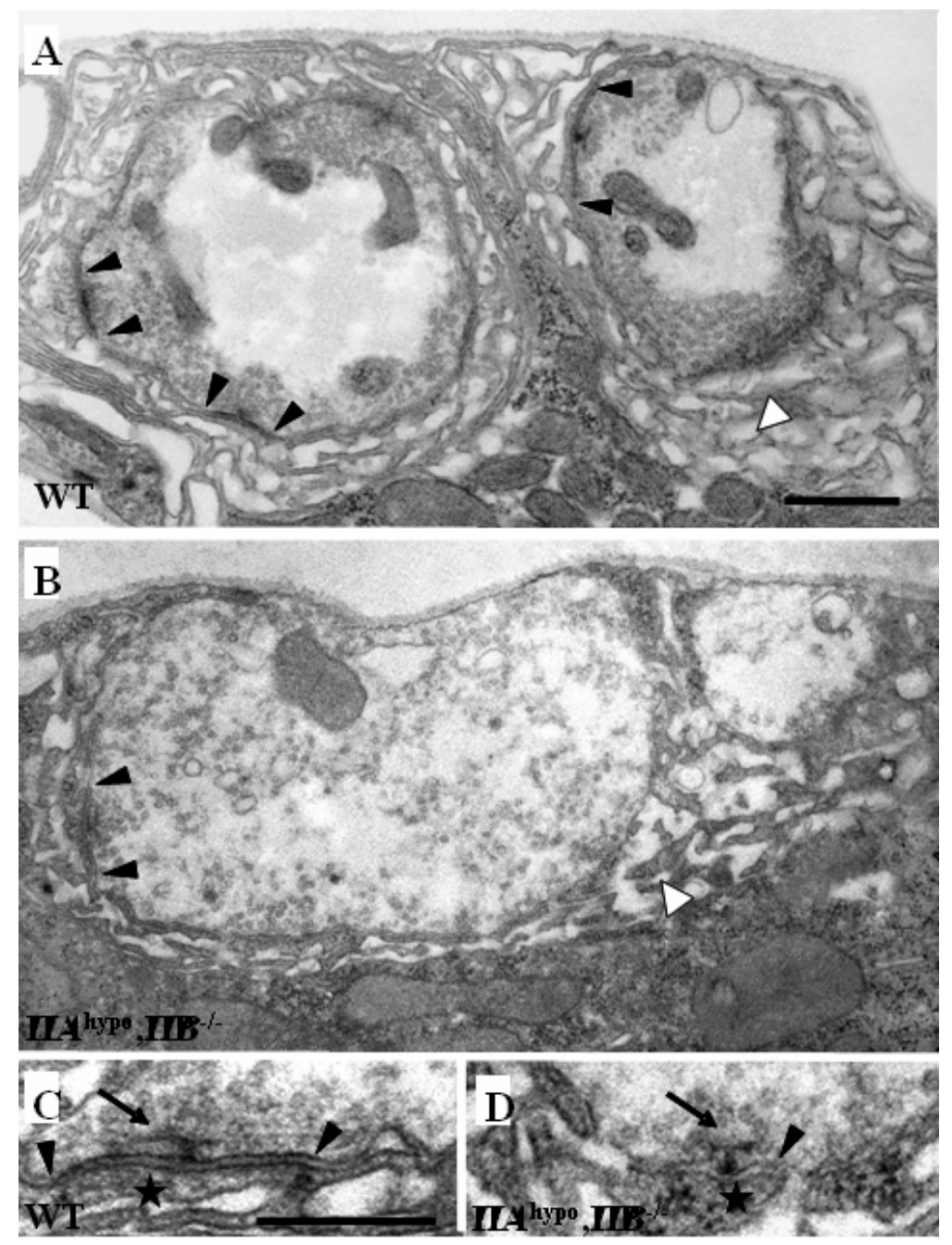

Fig.3-11 Electron microscopy: defective postsynaptic assembly at glutamate receptor deprived synapses

(A) Electron micrograph of type I nerve terminals of NMJ 6/7 in a wild type situation showing several clearly visible electron dense synapses (black arrowheads) and the SSR (white arrowhead). The synaptic vesicles are arranged along the presynaptic membrane. (B) GluRIIA ${ }^{\text {hypo }}$, GluRIIB ${ }^{-/ 2}$ type 1 b bouton of NMJ 6/7, respectively. The black arrowheads indicate a mutant, clearly electron translucend synapses decorated with a T-bar. The SSR (white arrowhead) is significantly reduced. (C, D) Synaptic sites in wild type (C) and GluRIIA ${ }^{\text {hypo }}$, GluRIIB ${ }^{-/}$(D) larvae. The electron dense T-bars are indicated by arrows. The PSD, in wild type detectable as electron dense material $(C$, asterisk) is in GluRIIA ${ }^{\text {hypo }}$, GluRIIB $^{-/-}$synapses absent (D, asterisk) or in less frequent weaker cases dramatically reduced (not shown). In GluRIIA ${ }^{\text {hypo }}$, GluRIIB $^{-/-}$synapses the lack of PSD organization also affects the close alignment of the pre- and postsynaptic membranes, which usually extends over several $100 \mathrm{~nm}$ (C, arrowheads). In GluRIIA $^{\text {hypo }}$, GluRIIB $^{-/-}$larvae this alignment is severely defective and the SSR formation already begins in direct vicinity to the T-bar (D, arrowhead) or extends asymmetrically. 
electron-dense T-shaped structures called 'T-bars' at the presynaptic side (Wojtowicz et al., 1994; Cooper et al., 1995; Cooper et al., 1996). PSDs are opposite to the T-bars and easily recognized by electron dense linear membrane-membrane appositions (Fig.3-11), a characteristic structure also found in mammalian CNS glutamatergic synapses. Within the PSD region, preand postsynaptic membrane associate closely with each other and are visualized as linear apposition in cross section (Fig.3-11). At most synaptic sites, the PSD diameter clearly exceeds the diameter of the presynaptic T-bar. In region between individual PSDs (so called "perisynaptic region"), pre- and postsynaptic membrane (motorneuron and muscle membrane) are organized into various in-foldings and associate only at restricted contact points. This structure is continuous with the subsynaptic reticulum (SSR).

In GluR-IIA ${ }^{\text {hypo }}$,GluR-IIB ${ }^{\text {null }}$ larvae, apparently normal presynaptic T-bars were observed in boutons. However, PSD organization is severely defective (Fig.3-11) in these animals: the muscle membrane domains juxtaposed to the presynaptic T-bars, where typically PSDs should be formed, show very low amount or even complete absence of electron dense material (Fig. 3-11 D). Moreover, the typical extended and close alignment between pre- and postsynaptic membranes could not be found from GluRIIA $^{\text {hypo }}$, GluRIIB ${ }^{\text {null }}$ larvae, in which with only significantly narrower and "wrinkled" apposition of membranes observed (Fig3-11 D). Thus, the ultrastructural analysis directly shows that in the absence of glutamate receptors, the PSD can not be efficiently assembled. In contrast, in the dpak mutants, despite the dramatic reduction of SSR foldings, the ultrastructure of individual synapse appeared rather normal, with smooth electron dense thickening at PSD region (Parnas et al., 2001). Rather normal ultrastructural active zone and PSD assembly was also found in embryos with neuronal TNT expression or lack of Syntaxin (Broadie et al., 1995). In combination of all these data, it is the glutamate receptor per se, obviously independent of transmission activity, controls the normal PSD assembly. 


\subsubsection{Defective compartmentation of the synaptic and perisynaptic zones at glutamate receptor deprived but not neurotransmission deprived synapse}

The ultrastructural analysis showed that the close apposition in between pre- and postsynaptic membrane is largely abolished after reducing glutamate receptor level. It is thus interesting to know, whether the strong defects in ultrastructural PSD organization at glutamate receptor deprived synapses might also reflect defects in the molecular organization of synaptic compartments. Therefore, to address this issue, the GluRIIA ${ }^{\text {hypo }}$, GluRIIB $^{\text {null }}$ larval NMJs were further investigated by antibody staining against compartment specific marker molecules. At normal Drosophila NMJ boutons, individual synapses are separated and surrounded by so called "perisynaptic" or "periactive zone" compartment (Sone et al., 2000; Sigrist et al., 2002), characterized by the presence of various molecular markers such as Fasciclin II (FasII) and Disc Large (Dlg). FasII is one NCAM-related cell adhesion molecule involved in synaptic growth, stabilization and structural plasticity (Shuster et al., 1996). DLG is the Drosophila PSD95 homologue, it belongs to the membrane-associated guanylate kinase homologs (MAGUKs) family and is generally involved in synaptic clustering of various molecules such as potassium channel Shaker and FasII through the PDZ domains mediated interactions (Tejedor et al., 1997; Thomas et al., 1997; Zito et al., 1997). As reported previously, in wild type controls both FasII and DLG label the boutons in a characterized network pattern, which is clearly complementary with dPAK staining (Fig.3-12 A, D). Since normally presynaptic active zone is always precisely aligned with DPAK as evidenced by NC82 and DPAK double staining (see Fig.3-9), the perisynaptic components such as FasII and DLG clearly decorate the synaptic region.

At GluRIIA ${ }^{\text {hypo }}$, GluRIIB $^{\text {null }}$ boutons, both FasII and DLG staining appear reduced and lose the typical network pattern, in contrast, they are distributed almost evenly throughout boutons (Fig.3-12 B, E). Notably, this homogeneous distribution pattern of Fas II and DLG indicates the intrusion of perisynaptic 

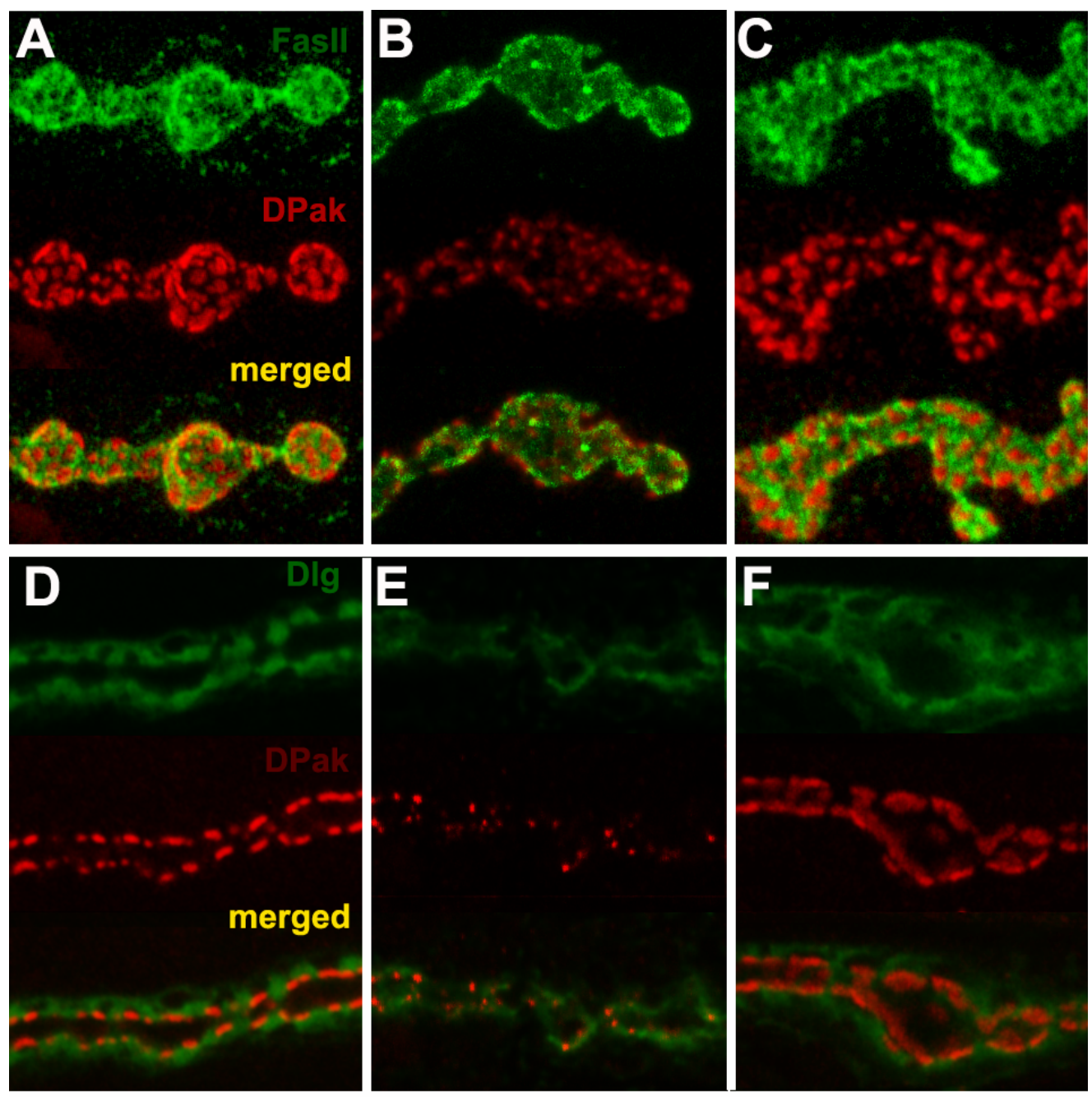

Wild type

GluR-IIAhypo, GluR-IIBnull

OK319-Gal4->UAS-TNT

Fig.3-12 Defective synaptic compartments at glutamate receptor deprived but not neurotransmission deprived synapses

Confocal microscopy on NMJs of mid 3rd instar larvae at muscle 4, stained with compartment specific antibodies in wild type (A, D), GluRIIA ${ }^{\text {hypo }}$, GluRIIB $^{-/}$larvae (B, E) and animals with presynaptic expression of Tetanus-toxin light chain (OK319-Gal4->UAS-TNT, Sweeney, et al., 1995) (C, F).

A-C, NMJs double labeled with FASII together with DPAK. In wild type animals, FasII shows typical perisynaptic distribution surrounding individual PSDs which are marked by DPAK expression (A). In GluRIIA ${ }^{\text {hypo }}$, GluRIIB ${ }^{-/-}$larvae, FasII show rather even distribution over the whole bouton (B), DPAK spots display significantly lower density and more variable individual size (B). In OK319-Gal4->UAS-TNT larvae, both FasII and DPAK show normal distribution (C).

D-F, single cross section image of larval NMJs double labeled with DLG and DPAK. In wild type animals, DLG shows typical perisynaptic distribution surrounding individual PSDs which are marked by DPAK expression (D). In GluRIIA ${ }^{\text {hypo }}$, GluRIIB $^{-1-}$ larvae, DLG show rather even distribution over the whole bouton surface thus invade the synaptic area (E), DPAK spots display significantly lower density and more variable individual size and overlay with DLG labels (E). In OK319-Gal4->UAS-TNT larvae, both DLG and DPAK show normal distribution $(\mathrm{F})$. 
components into the synaptic/ active zone region, thus destroying the compartmentation of synaptic and perisynaptic area in boutons. Concerning the still rather normal localization of active zones within these boutons, clearly the major driving force to prevent perisynaptic components entering synaptic area comes from the efficiently assembled PSDs, glutamate receptors play either direct or indirect roles in this process.

\subsubsection{Similar defects in synaptic differentiation in the complete absence of glutamate receptors}

The above studies using glutamate receptor largely deprived larvae strongly support the structural role of glutamate receptor in organizing synaptic differentiation. To further confirm this finding, analysis on synapses with complete loss of all glutamate receptors is needed. To this end, the GluR-IIA\&IIB double null mutant background was used. At late stage embryos of this mutant, none of any known muscle glutamate receptor subunits can be detected at NMJs (Fig.3-5 B; Fig.3-10). In consistent with the result obtained

from GluRIIA ${ }^{\text {hypo }}$, GluRIIB $^{\text {null }}$ larvae, although the active zone localize normally at the presynaptic terminal, much fewer amount of assemble dPAK aggregates are present at junctions compared to control conditions (Fig.3-10 E). 


\section{Discussion}

\subsection{Genomic tools could speed up identifying essential genes involved in synapse formation and modulation}

Drosophila has long been an excellent model organism due to the availability of well established, classical genetic tools. With the recent release of the whole Drosophila genome sequence information and development of systematic functional genomic research tools, reverse genetics has further strengthened the power of this model organism. Recently genomic scale microarray screening has been stably applied to a wide range of studies to obtain molecular insights or identify novel relevant components of complicated regulatory processes (Furlong et al., 2001; Stathopoulos and Levine, 2002; Butler et al., 2003; Li and White, 2003).

Here, to screen genes relevant to synapse formation and growth control at whole genome scale, the microarray technique was applied to the Drosophila model synapse system, the NMJ. Thus, the Drosophila body wall filets which mainly consist of somatic muscles were sampled and the RNAs were extracted. Tissue specific RNA profiling was performed and pools of genes (about 700) with specific or enriched expression in postsynaptic muscles were obtained. These genes represent wide variety of functional groups, including enzymes, signal transduction components, receptors and ion channels, cell adhesion molecules, transcriptional factors, RNA-binding proteins, structural proteins and so on. Among them, some are previously already shown to be specifically or abundantly expressed within muscle cells, indicating that our strategy is absolutely feasible and the gene chip methodology is highly reliable. Notably, a large body of genes within the pools are not characterized yet, thus the challenge will be to study their functional significance in high-throughput way. To this end, the application of RNA interference (RNAi) will be the best choice since recently Drosophila whole genomic RNAi has been set up by various labs and proved to be very efficient assay (Kiger et al., 2003; Kim et al., 2004). 
Undoubtedly, the microarray oriented reverse genetics platform can well complement the classical genetic screening based strategy and their application into neuronal system will greatly enhance our precise understanding of molecular details related to synapse construction and modulation. The main part of this thesis work is studying the molecular behaviors of glutamate receptor subunits, which is originated from the microarray screening and thus provide direct proof of its value.

\subsection{GluR-IID and GluR-IIE are novel glutamate receptor subunits crucial for glutamate receptor assembly and thus neurotransmission at neuromuscular synapse}

The glutamatergic neuromuscular synapses of Drosophila, rather similar to the excitatory CNS synapses of vertebrate brain in terms of ultrastructure and molecular composition, have been intensely utilized to investigate synaptic function in vivo capitalizing on the efficient genetics and superb experimental accessibility of this model system. At neuromuscular synapses three non-NMDA type glutamate receptor subunits (GluR-IIA, -IIB, -IIC/III) have been described previously. While only GluR-IIA\&IIB double null mutants but not either single null mutants completely lack neurotransmission (Petersen et al., 1997; DiAntonio et al., 1999), lack of GluRIIC/ III alone is fatal with a phenotype consistent with the absence of neurotransmission at neuromuscular synapses (Marrus et al., 2004). The full picture of subunit composition of synaptic glutamate receptors at the NMJ still remains elusive.

Here via the genomic scale microarray screening, I report the identification of two additional non-NMDA glutamate receptor subunits, GluR-IID and GluR-IIE, which are proved to be specifically localized at the postsynaptic site of all neuromuscular synapses. Interestingly, while GluR-IID and GluR-IIE are genomic neighbors and very similar to each other, they are not particularly close to GluR-IIA, -IIB and -IIC/III. Specific null mutants for both GluR-IID and GluR-IIE have been obtained to further functional characterization. While both 
GluR-IID and GluR-IIE null embryos still fully develop, they do not hatch and are incapable of performing any coordinated movement. Consistent with my finding, via embryonic electrophysiological recording, Featherstone et al. have recently demonstrated that no postsynaptic sensitivity to glutamate was detected at the embryonic muscles of one independent GluR-IID null mutant allele (personal communication). Thus, GluR-IID and GluR-IIE appear critically important for mediating glutamate-gated ionic currents at the postsynaptic site of neuromuscular synapses. Since in the case of GluR-IIA\&IIB double as well as the GluR-IIC/III single mutant background the same paralysis phenotype was found, it is therefore most likely, that they also lack any glutamate gated ionic current.

Taken together, lack of either GluR-IIC/III, -IID, -IIE or both -IIA and -IIB leads to total loss of glutamate receptor function at the embryonic NMJ. The following evidences support that this is directly due to the loss of the respective glutamate receptor subunits hence all the functional glutamate receptors within the embryonic muscles. Firstly, despite the fact that GluR-IID is also present in cardio-precursor cells, the expression of GluR-IIA, -IIB, -IIC/III and -IIE is most likely restricted to the somatic muscles (Petersen et al., 1997; DiAntonio et al., 1999; Fig.3-1). Secondly, the paralysis phenotype of all these null mutants could always be fully rescued by a muscle-specific re-expression of the corresponding cDNAs (DiAntonio et al., 1999; Marrus et al., 2004; 3.4). Thirdly, muscle-specific down-regulation of GluR-IIE via RNA interference could effectively down-regulate synaptic expression of GluR-IID, -IIA, -IIB and -IIC/III as well. Last, in each of these null mutants, all of the other subunits are also completely absent at NMJ. Thus clearly, lack of any essential glutamate receptor subunits within the postsynaptic muscle cells will result into a complete loss of all glutamate receptors, which is the direct cause of embryonic paralysis. 


\subsection{Implications in the in vivo stoichiometry of Drosophila glutamate receptor based on genetic analysis}

Several recent studies have suggested that the mammalian ionotropic glutamate receptors form as a tetramer of subunits (Kuusinen A et al., 1999; Rosenmund C et al., 1998; Laube B et al., 1998; Mano I et al., 1998; Safferling $\mathrm{M}$ et al., 2001). At the Drosophila Neuromuscular synapses, the synaptic expression of all glutamate receptor subunits is completely abolished after eliminating either GluR-IID or IIE alone or GluR-IIA and IIB together (Fig. 3-5), clearly indicating that essential glutamate receptor subunits (GluR-IIC/III, -IID and -IIE) are obligatory part of all postsynaptic glutamate receptors. In addition, the presence of either GluRIIA or GluR-IIB seems obligate for receptor formation as well. One likely scenario therefore is that the glutamate receptor population at the Drosophila NMJ is a mix of (IIA)(IIC/III)(IID)(IIE) receptors with (IIB)(IIC/III)(IID)(IIE) receptors. However, since the current tetrameric structure model of ionotropic glutamate receptors was suggested solely on the basis of either electrophysiological and biochemical studies in heterologous system or crystal structure analysis of ligand-binding domains, an unequivocal determination of the stoichiometry of functional glutamate receptor thus still awaits efficient method that reliably resolute the structure of the endogeneous receptor itself. In theory, as was proposed in the past (Premkumar et al., 1997), pentameric (or higher) stoichiometry still cannot be totally excluded in the moment. Therefore, stoichiometry as (A)2(C)(D)(E), (B)2(C)(D)(E) or $(A)(B)(C)(D)(E)$ still need to be considered.

Previous studies have established that GluR-IIA- and GluR-IIB-containing receptors differ strongly in their biophysical properties, with GluR-IIA-containing receptors showing slow desensitization kinetics and GluR-IIB-containing receptors showing fast desensitization kinetics (DiAntonio et al., 1999). Thus, GluR-IIA and GluR-IIB containing complexes are likely to play different roles in synaptic function and development. Acordingly, it has 
been recently found that, increased postsynaptic GluR-IIA level or reduced GluR-IIB gene dosage results into an increased strength of NMJ transmission and an addition of boutons harboring increased numbers of synapses; this phenotypes were suppressed by overexpression of GluR-IIB (Sigrist et al., 2002). Thus, GluR-IIC/III, IID and IIE might establish a "receptor assembly platform" where the incorporation of either the GluR-IIA or the GluR-IIB subunit could determine the specific functions of the respective glutamate receptor.

As discussed above, the interdependency between all essential subunits observed for synaptic localization is easiest explained by assuming the existence of common glutamate receptor. Another possibility might be that eliminating a certain glutamate receptor subunit could provoke an early defect during the developmental set up of neuromuscular synapses, which in turn might interfere with the localization of other glutamate receptor complexes. This way, the genetic elimination of a certain subunit could also cause the loss of synaptic expression (and thus also synaptic function) of another subunit even if these two subunits would not be coassembled in the same glutamate receptor complex. At this juncture, such a scenario appears unlikely because of the following reasons. First, partial suppression of any essential glutamate receptor subunit always provokes a corresponding down-regulation of other essential glutamate receptor subunits at larval neuromuscular synapses (Fig.3-6 and3-7; Marrus et al., 2004). This suggests that all essential subunits obey a tight stoichiometric relationship, where the availability of the least abundant subunit defines the overall amount of glutamate receptors, and that this relation exists throughout development. Secondly, Featherstone et al. have convincingly demonstrated a complete loss of glutamate mediated currents on embryonic muscle surfaces of one independent GluR-IID null mutant allele (Featherstone D et al., personal communication). In contrast, extrasynaptic glutamate receptor mediated currents have been stably detected on wild type embryonic muscle membranes using the same experimental settings (Broadie and Bate, 1993; Featherstone et al., personal 
communication). Thus, elimination of GluR-IID abolishes not only synaptic but also extrasynaptic populations of glutamate receptors on embryonic muscles. Therefore, if interaction between independently forming glutamate receptors is involved in their proper targeting, it would have to be absolutely essential already during the intracellular transport and insertion of glutamate receptors. Since all known muscle receptor subunits from GluR-IIA to GluR-IID (GluR-IIE also quite likely the case as evidenced by EGFP fusion protein distribution) are clearly expressed in substantial amounts within postsynaptic densities, the existence of subunits evolved only to mediate the intracellular transport or assembly of glutamate receptor complexes appears rather unlikely unless it was omitted from the Gene chip analysis.

Collectively, the in vivo data presented here clearly favor the notion that, at Drosophila neuromuscular synapses the functional glutamate receptors can be composed of four different subunits. To our knowledge, a "strictly hetero-tetrameric stoichiometry" has so far not been described for other types of ionotropic glutamate receptors in all model synapses. This finding thus potentially reveal that, apart from our current understanding on glutamate receptor behavior mainly based on heterologous studies, the in vivo glutamate receptors might have a rather fixed stochiometry, which is reminiscent of that of other types of ligand-gated ion channels such as the nicotinic acetylcholine receptor (Colquhoun and Sivilotti, 2004).

\subsection{Structural role of ionotropic glutamate receptor per se in organizing synaptic assembly}

In the brain, huge amount of neurons are precisely connected via synapses, which display respectively characteristic structural properties at both pre and postsynaptic sides. Most excitatory synapses are glutamatergic, and their initial formation, stabilization, and elimination are under strict control all the time. Moreover, the local changes in synapse strength are proposed to be relevant to 
the process of learning and memory. Apparently, it is of great importance to understand how the original synaptic contact is initiated at the proper site and how exquisite coordination of presynaptic and postsynaptic development is accomplished. Although up to now great efforts have been devoted in these directions with more and more synaptic components identified and characterized, still the molecular details driving synaptic assembly remain elusive.

At central excitatory synapses, synaptic transmission is mediated by various ionotropic glutamate receptors including AMPA, kainite and NMDA subfamilies. Recent work has revealed that certain classes of AMPA receptors recycle rapidly at the synaptic sites, suggesting that placeholders or 'slots' might be brought to, or assembled at the postsynaptic membrane with de novo insertion of AMPA receptors. The molecular identity of the slots is unknown, but is likely to involve one or more receptor-binding or scaffolding proteins (Barry and Ziff, 2002).

Taking the advantages of the powerful Drosophila genetic tools and the rather simpler architecture of $\mathrm{NMJ}$, the consequences of losing synaptic glutamate receptor partially or completely in vivo were investigated. Despite the rather normal differentiation of transmitter release machinery at the presynaptic membrane, which is consistent with previous findings that the presynaptic differentiation can undergo at the nerve terminal even without contact with the postsynaptic cell (Prokop et al., 1996), the postsynaptic assembly is apparently deficient, as evidenced by not only inefficient clustering of other PSD component ( $\mathrm{dPAK})$ but also the intrusion of perisynaptic components (FASII and DLG) into synaptic (active zone) territory. PAK is known to regulate cytoskeleton dynamics, the cytoskeleton organization is crucial for the clustering of synaptic components and /or maintaining the synaptic structure at diverse types of synapses (Kirsch and Betz, 1995; Allison et al., 1998; Dai et al., 2000; Hirai, 2000). Recently it has been proposed that dPAK is important in 
clustering glutamate receptors at Drosophila NMJ since in dpak hypomorphic mutant animals, glutamate receptor subunit GluR-IIA is significantly reduced but still localize normally at synapses (Parnas et al., 2001). Interestingly, the data presented here indicate that proper aggregation and synaptic distribution of dPAK is definitely dependant on the availability of glutamate receptors; with glutamate receptors depleted the $\mathrm{APAK}$ is no longer able to cluster and localize properly. At mammalian neuromuscular synapse it has been shown that agrin-mediated clustering of AchR is mediated by the corporation of various signaling pathways: first the activation of Rac/ $\mathrm{Cdc} 42$, leading to formation of AchR microclusters, followed by Rho activation, resulting into combining these microclusters into larger clusters (Weston C et al., 2003). DPAK does not necessarily need to interact directly with glutamate receptors, but via various direct and indirect interactions glutamate receptors might be able to recruit various PSD components (including dPAK, other signaling molecules and scaffold proteins) during synaptogenesis, as soon as these components are recruited together, the relevant signaling cascades are efficiently activated and thus trigger the membrane and cytoskeleton remodeling which finally results into the stable development of mature synaptic structure; in depletion of glutamate receptors, either the dPAK signaling cascade is not efficiently activated to reorganize actin cytoskeleton essential for proper clustering, or activating dPAK cascade along is not sufficient for effective PSD components clustering and maintaining. Currently, it remains elusive whether other signaling pathways are involved in PSD formation, but based on the clear defects in synaptic compartmentation that perisynaptic components intrude into synaptic region, the cytoskeleton organization must have been improperly regulated. This notion is also supported from ultrastructural analysis, the electron dense material typical present at the PSD region is essentially absent from glutamate receptor deprived synapses, implicating that the components of postsynaptic scaffold are either not recruited together at all or no longer stable without the integration of glutamate receptors. Interestingly, at the neuromuscular synapses of dapk hypomorphic mutant, despite much fewer SSR, the ultrastructure of 
both PSD and active zone appears quite normal, further supporting the relatively minor role of $\mathrm{dPAK}$ in postsynaptic differentiation. Notably, the role of glutamate receptor in synaptic development was shown not to be relevant to its ion channel activity, since in all the backgrounds tested which deprive presynaptic neurotransmitter release (partially or completely), the glutamate receptor can be clustered as effectively as wild type control. Correspondingly, the postsynaptic reception apparatus in these transmission depleted conditions appear functional since responses can be stably detected when applying glutamate at junctions (Broadie, 1996).

Another interesting phenomena at glutamate receptor deprived synapse is that the number of presynaptic specializations exceeds postsynaptic ones, thus resulting into free active zones without postsynaptic partners, which is hardly found in normal conditions. In principle, this can be due to either lack of glutamate receptor per se, or that of the transmission activity. The latter idea appears more likely since in complete transmitter release deficient situations, free active zones were also observed although certain levels of PSDs (glutamate receptor fields) were assembled normally.

In conclusion, apart from functioning as ligand-gated ion channel, the glutamate receptor at Drosophila NMJ also plays crucial role in organizing and /or maintaining the synaptic architecture, which is reminiscent of AchR at vertebrate NMJ (Ono F et al., 2001; Marangi PA et al., 2001; Missias AC et al., 1997) and glycin and GABA receptor at mammalian central synapses (Essrich et al., 1998). In mammalian CNS synapses similar roles of ionotropic glutamate receptors have not been reported, mainly due to the fact that there exist highly divergent classes of glutamate receptors at central synapses and each type of receptors play distinct roles, therefore it is not easy to obtain a condition with all types of glutamate receptors removed together. 


\section{Reference}

Aberle H, Haghighi AP, Fetter RD, McCabe BD, Magalhaes TR, Goodman CS (2002) wishful thinking encodes a BMP type II receptor that regulates synaptic growth in Drosophila. Neuron 33:545-558.

Ahmari SE, Buchanan J, Smith SJ (2000) Assembly of presynaptic active zones from cytoplasmic transport packets. Nat Neurosci 3:445-451.

Albin SD, Davis GW (2004) Coordinating structural and functional synapse development: postsynaptic p21-activated kinase independently specifies glutamate receptor abundance and postsynaptic morphology. J Neurosci 24:6871-6879.

Allison DW, Gelfand VI, Spector I, Craig AM (1998) Role of actin in anchoring postsynaptic receptors in cultured hippocampal neurons: differential attachment of NMDA versus AMPA receptors. $J$ Neurosci 18:2423-2436.

Apel ED, Glass DJ, Moscoso LM, Yancopoulos GD, Sanes JR (1997) Rapsyn is required for MuSK signaling and recruits synaptic components to a MuSK-containing scaffold. Neuron 18:623-635.

Aplin AE, Howe A, Alahari SK, Juliano RL (1998) Signal transduction and signal modulation by cell adhesion receptors: the role of integrins, cadherins, immunoglobulin-cell adhesion molecules, and selectins. Pharmacol Rev 50:197-263.

Atwood HL, Govind CK, Wu CF (1993) Differential ultrastructure of synaptic terminals on ventral longitudinal abdominal muscles in Drosophila larvae. J Neurobiol 24:1008-1024.

Auld VJ, Fetter RD, Broadie K, Goodman CS (1995) Gliotactin, a novel transmembrane protein on peripheral glia, is required to form the blood-nerve barrier in Drosophila. Cell 81:757-767.

Ayalon G, Stern-Bach Y (2001) Functional assembly of AMPA and kainate receptors is mediated by several discrete protein-protein interactions. Neuron 31:103-113.

Bagrodia S, Cerione RA (1999) Pak to the future. Trends Cell Biol 9:350-355.

Barry MF, Ziff EB (2002) Receptor trafficking and the plasticity of excitatory synapses. Curr Opin Neurobiol 12:279-286.

Bate M, Broadie K (1995) Wiring by fly: the neuromuscular system of the Drosophila embryo. Neuron 15:513-525.

Benson DL, Colman DR, Huntley GW (2001) Molecules, maps and synapse specificity. Nat Rev Neurosci 2:899-909.

Biederer T, Sara Y, Mozhayeva M, Atasoy D, Liu X, Kavalali ET, Sudhof TC (2002) SynCAM, a synaptic adhesion molecule that drives synapse assembly. Science 297:1525-1531.

Bloch RJ, Pumplin DW (1988) Molecular events in synaptogenesis: nerve-muscle adhesion and postsynaptic differentiation. Am J Physiol 254:C345-364.

Boutanaev AM, Kalmykova AI, Shevelyov YY, Nurminsky DI (2002) Large clusters of co-expressed genes in the Drosophila genome. Nature 420:666-669.

Bowe MA, Fallon JR (1995) The role of agrin in synapse formation. Annu Rev Neurosci 18:443-462.

Brand AH, Perrimon N (1993) Targeted gene expression as a means of altering cell fates and generating dominant phenotypes. Development 118:401-415.

Bresler T, Ramati Y, Zamorano PL, Zhai R, Garner CC, Ziv NE (2001) The dynamics of SAP90/PSD-95 recruitment to new synaptic junctions. Mol Cell Neurosci 18:149-167.

Bresler T, Shapira M, Boeckers T, Dresbach T, Futter M, Garner CC, Rosenblum K, Gundelfinger ED, Ziv NE (2004) Postsynaptic density assembly is fundamentally different from presynaptic active zone assembly. J Neurosci 24:1507-1520.

Broadie K, Bate M (1993) Activity-dependent development of the neuromuscular synapse during Drosophila embryogenesis. Neuron 11:607-619.

Broadie K, Bate M (1993) Innervation directs receptor synthesis and localization in Drosophila embryo synaptogenesis. Nature 361:350-353.

Broadie K, Prokop A, Bellen HJ, O'Kane CJ, Schulze KL, Sweeney ST (1995) Syntaxin and synaptobrevin function downstream of vesicle docking in Drosophila. Neuron 15:663-673.

Broadie KS (1996) Regulation of the synaptic vesicle cycle in Drosophila. Biochem Soc Trans 24:639-645.

Budnik V, Koh YH, Guan B, Hartmann B, Hough C, Woods D, Gorczyca M (1996) Regulation of 
synapse structure and function by the Drosophila tumor suppressor gene dlg. Neuron 17:627-640.

Burden SJ (1998) The formation of neuromuscular synapses. Genes Dev 12:133-148.

Burnashev N, Khodorova A, Jonas P, Helm PJ, Wisden W, Monyer H, Seeburg PH, Sakmann B (1992) Calcium-permeable AMPA-kainate receptors in fusiform cerebellar glial cells. Science 256:1566-1570.

Butler MJ, Jacobsen TL, Cain DM, Jarman MG, Hubank M, Whittle JR, Phillips R, Simcox A (2003) Discovery of genes with highly restricted expression patterns in the Drosophila wing disc using DNA oligonucleotide microarrays. Development 130:659-670.

Chavis P, Westbrook G (2001) Integrins mediate functional pre- and postsynaptic maturation at a hippocampal synapse. Nature 411:317-321.

Chen GQ, Cui C, Mayer ML, Gouaux E (1999) Functional characterization of a potassium-selective prokaryotic glutamate receptor. Nature 402:817-821.

Chen L, Chetkovich DM, Petralia RS, Sweeney NT, Kawasaki Y, Wenthold RJ, Bredt DS, Nicoll RA (2000) Stargazin regulates synaptic targeting of AMPA receptors by two distinct mechanisms. Nature 408:936-943.

Chiba A, Rose D (1998) "Painting" the target: how local molecular cues define synaptic relationships. Bioessays 20:941-948.

Colquhoun D, Sivilotti LG (2004) Function and structure in glycine receptors and some of their relatives. Trends Neurosci 27:337-344.

Contractor A, Heinemann SF (2002) Glutamate receptor trafficking in synaptic plasticity. Sci STKE 2002:RE14.

Cooper RL, Marin L, Atwood HL (1995) Synaptic differentiation of a single motor neuron: conjoint definition of transmitter release, presynaptic calcium signals, and ultrastructure. J Neurosci 15:4209-4222.

Cooper RL, Harrington CC, Marin L, Atwood HL (1996) Quantal release at visualized terminals of a crayfish motor axon: intraterminal and regional differences. J Comp Neurol 375:583-600.

Currie DA, Truman JW, Burden SJ (1995) Drosophila glutamate receptor RNA expression in embryonic and larval muscle fibers. Dev Dyn 203:311-316.

Dai Z, Luo X, Xie H, Peng HB (2000) The actin-driven movement and formation of acetylcholine receptor clusters. J Cell Biol 150:1321-1334.

Dalva MB, Takasu MA, Lin MZ, Shamah SM, Hu L, Gale NW, Greenberg ME (2000) EphB receptors interact with NMDA receptors and regulate excitatory synapse formation. Cell 103:945-956.

Daniels RW, Collins CA, Gelfand MV, Dant J, Brooks ES, Krantz DE, Diantonio A (2004) Increased Expression of the Drosophila Vesicular Glutamate Transporter Leads to Excess Glutamate Release and a Compensatory Decrease in Quantal Content. J Neurosci 24:10466-10474.

Davis GW, Schuster CM, Goodman CS (1997) Genetic analysis of the mechanisms controlling target selection: target-derived Fasciclin II regulates the pattern of synapse formation. Neuron 19:561-573.

Dean C, Scholl FG, Choih J, DeMaria S, Berger J, Isacoff E, Scheiffele P (2003) Neurexin mediates the assembly of presynaptic terminals. Nat Neurosci 6:708-716.

DeChiara TM, Bowen DC, Valenzuela DM, Simmons MV, Poueymirou WT, Thomas S, Kinetz E, Compton DL, Rojas E, Park JS, Smith C, DiStefano PS, Glass DJ, Burden SJ, Yancopoulos GD (1996) The receptor tyrosine kinase MuSK is required for neuromuscular junction formation in vivo. Cell 85:501-512.

DiAntonio A, Petersen SA, Heckmann M, Goodman CS (1999) Glutamate receptor expression regulates quantal size and quantal content at the Drosophila neuromuscular junction. J Neurosci 19:3023-3032.

Dingledine R, Borges K, Bowie D, Traynelis SF (1999) The glutamate receptor ion channels. Pharmacol Rev 51:7-61.

Dingledine R, Borges K, Bowie D, Traynelis SF (1999) The glutamate receptor ion channels. Pharmacol Rev 51:7-61.

Ellgaard L, Helenius A (2003) Quality control in the endoplasmic reticulum. Nat Rev Mol Cell Biol 4:181-191.

Essrich C, Lorez M, Benson JA, Fritschy JM, Luscher B (1998) Postsynaptic clustering of major GABAA receptor subtypes requires the gamma 2 subunit and gephyrin. Nat Neurosci 1:563-571.

Ethell IM, Yamaguchi Y (1999) Cell surface heparan sulfate proteoglycan syndecan-2 induces the maturation of dendritic spines in rat hippocampal neurons. J Cell Biol 144:575-586.

Ethell IM, Irie F, Kalo MS, Couchman JR, Pasquale EB, Yamaguchi Y (2001) EphB/syndecan-2 
signaling in dendritic spine morphogenesis. Neuron 31:1001-1013.

Featherstone DE, Broadie K (2000) Surprises from Drosophila: genetic mechanisms of synaptic development and plasticity. Brain Res Bull 53:501-511.

Fernandez-Chacon R, Sudhof TC (1999) Genetics of synaptic vesicle function: toward the complete functional anatomy of an organelle. Annu Rev Physiol 61:753-776.

Fiala JC, Feinberg M, Popov V, Harris KM (1998) Synaptogenesis via dendritic filopodia in developing hippocampal area CA1. J Neurosci 18:8900-8911.

Finn AJ, Feng G, Pendergast AM (2003) Postsynaptic requirement for Abl kinases in assembly of the neuromuscular junction. Nat Neurosci 6:717-723.

Friedman HV, Bresler T, Garner CC, Ziv NE (2000) Assembly of new individual excitatory synapses: time course and temporal order of synaptic molecule recruitment. Neuron 27:57-69.

Froehner SC, Luetje CW, Scotland PB, Patrick J (1990) The postsynaptic 43K protein clusters muscle nicotinic acetylcholine receptors in Xenopus oocytes. Neuron 5:403-410.

Furlong EE, Andersen EC, Null B, White KP, Scott MP (2001) Patterns of gene expression during Drosophila mesoderm development. Science 293:1629-1633.

Garner CC, Zhai RG, Gundelfinger ED, Ziv NE (2002) Molecular mechanisms of CNS synaptogenesis. Trends Neurosci 25:243-251.

Gautam M, Noakes PG, Mudd J, Nichol M, Chu GC, Sanes JR, Merlie JP (1995) Failure of postsynaptic specialization to develop at neuromuscular junctions of rapsyn-deficient mice. Nature 377:232-236.

Gautam M, Noakes PG, Moscoso L, Rupp F, Scheller RH, Merlie JP, Sanes JR (1996) Defective neuromuscular synaptogenesis in agrin-deficient mutant mice. Cell 85:525-535.

Gilbert W (1978) Why genes in pieces? Nature 271:501.

Gillespie SK, Wasserman SA (1994) Dorsal, a Drosophila Rel-like protein, is phosphorylated upon activation of the transmembrane protein Toll. Mol Cell Biol 14:3559-3568.

Glass DJ, Bowen DC, Stitt TN, Radziejewski C, Bruno J, Ryan TE, Gies DR, Shah S, Mattsson K, Burden SJ, DiStefano PS, Valenzuela DM, DeChiara TM, Yancopoulos GD (1996) Agrin acts via a MuSK receptor complex. Cell 85:513-523.

Gonzalez-Gaitan M, Jackle H (1997) Role of Drosophila alpha-adaptin in presynaptic vesicle recycling. Cell 88:767-776.

Gramates LS, Budnik V (1999) Assembly and maturation of the Drosophila larval neuromuscular junction. Int Rev Neurobiol 43:93-117.

Greger IH, Khatri L, Ziff EB (2002) RNA editing at arg607 controls AMPA receptor exit from the endoplasmic reticulum. Neuron 34:759-772.

Grunwald IC, Korte M, Wolfer D, Wilkinson GA, Unsicker K, Lipp HP, Bonhoeffer T, Klein R (2001) Kinase-independent requirement of EphB2 receptors in hippocampal synaptic plasticity. Neuron 32:1027-1040.

Guan B, Hartmann B, Kho YH, Gorczyca M, Budnik V (1996) The Drosophila tumor suppressor gene, $\mathrm{dlg}$, is involved in structural plasticity at a glutamatergic synapse. Curr Biol 6:695-706.

Hall AC, Lucas FR, Salinas PC (2000) Axonal remodeling and synaptic differentiation in the cerebellum is regulated by WNT-7a signaling. Cell 100:525-535.

Hall ZW, Sanes JR (1993) Synaptic structure and development: the neuromuscular junction. Cell 72 Suppl:99-121.

Han H, Noakes PG, Phillips WD (1999) Overexpression of rapsyn inhibits agrin-induced acetylcholine receptor clustering in muscle cells. J Neurocytol 28:763-775.

Hannah MJ, Schmidt AA, Huttner WB (1999) Synaptic vesicle biogenesis. Annu Rev Cell Dev Biol 15:733-798

Harris KM, Kater SB (1994) Dendritic spines: cellular specializations imparting both stability and flexibility to synaptic function. Annu Rev Neurosci 17:341-371.

Hawkins LM, Prybylowski K, Chang K, Moussan C, Stephenson FA, Wenthold RJ (2004) Export from the endoplasmic reticulum of assembled N-methyl-d-aspartic acid receptors is controlled by a motif in the $\mathrm{c}$ terminus of the NR2 subunit. J Biol Chem 279:28903-28910.

Heimbeck G, Bugnon V, Gendre N, Haberlin C, Stocker RF (1999) Smell and taste perception in Drosophila melanogaster larva: toxin expression studies in chemosensory neurons. J Neurosci 19:6599-6609.

Henderson JT, Georgiou J, Jia Z, Robertson J, Elowe S, Roder JC, Pawson T (2001) The receptor tyrosine kinase EphB2 regulates NMDA-dependent synaptic function. Neuron 32:1041-1056.

Herbst R, Burden SJ (2000) The juxtamembrane region of MuSK has a critical role in agrin-mediated signaling. Embo J 19:67-77.

Hering H, Sheng M (2001) Dendritic spines: structure, dynamics and regulation. Nat Rev Neurosci 
2:880-888.

Hirai H (2000) Clustering of delta glutamate receptors is regulated by the actin cytoskeleton in the dendritic spines of cultured rat Purkinje cells. Eur J Neurosci 12:563-570.

Hoang B, Chiba A (1998) Genetic analysis on the role of integrin during axon guidance in Drosophila. J Neurosci 18:7847-7855.

Hollmann M, Heinemann S (1994) Cloned glutamate receptors. Annu Rev Neurosci 17:31-108.

Isaac JT, Crair MC, Nicoll RA, Malenka RC (1997) Silent synapses during development of thalamocortical inputs. Neuron 18:269-280.

Jahn R, Sudhof TC (1999) Membrane fusion and exocytosis. Annu Rev Biochem 68:863-911.

Jaskolski F, Coussen F, Nagarajan N, Normand E, Rosenmund C, Mulle C (2004) Subunit composition and alternative splicing regulate membrane delivery of kainate receptors. J Neurosci 24:2506-2515.

Jennings CG, Dyer SM, Burden SJ (1993) Muscle-specific trk-related receptor with a kringle domain defines a distinct class of receptor tyrosine kinases. Proc Natl Acad Sci U S A 90:2895-2899.

Jia XX, Gorczyca M, Budnik V (1993) Ultrastructure of neuromuscular junctions in Drosophila: comparison of wild type and mutants with increased excitability. J Neurobiol 24:1025-1044.

Kennerdell JR, Carthew RW (2000) Heritable gene silencing in Drosophila using double-stranded RNA. Nat Biotechnol 18:896-898.

Keshishian H, Broadie K, Chiba A, Bate M (1996) The Drosophila neuromuscular junction: a model system for studying synaptic development and function. Annu Rev Neurosci 19:545-575.

Kiger AA, Baum B, Jones S, Jones MR, Coulson A, Echeverri C, Perrimon N (2003) A functional genomic analysis of cell morphology using RNA interference. J Biol 2:27.

Kim YO, Park SJ, Balaban RS, Nirenberg M, Kim Y (2004) A functional genomic screen for cardiogenic genes using RNA interference in developing Drosophila embryos. Proc Natl Acad Sci U S A 101:159-164.

Kirsch J, Betz H (1995) The postsynaptic localization of the glycine receptor-associated protein gephyrin is regulated by the cytoskeleton. J Neurosci 15:4148-4156.

Kneussel M, Brandstatter JH, Laube B, Stahl S, Muller U, Betz H (1999) Loss of postsynaptic GABA(A) receptor clustering in gephyrin-deficient mice. J Neurosci 19:9289-9297.

Koh YH, Gramates LS, Budnik V (2000) Drosophila larval neuromuscular junction: molecular components and mechanisms underlying synaptic plasticity. Microsc Res Tech 49:14-25.

Kuusinen A, Abele R, Madden DR, Keinanen K (1999) Oligomerization and ligand-binding properties of the ectodomain of the alpha-amino-3-hydroxy-5-methyl-4-isoxazole propionic acid receptor subunit GluRD. J Biol Chem 274:28937-28943.

LaRochelle WJ, Froehner SC (1986) Determination of the tissue distributions and relative concentrations of the postsynaptic $43-\mathrm{kDa}$ protein and the acetylcholine receptor in Torpedo. $\mathrm{J}$ Biol Chem 261:5270-5274.

Laube B, Kuhse J, Betz H (1998) Evidence for a tetrameric structure of recombinant NMDA receptors. J Neurosci 18:2954-2961.

Li TR, White KP (2003) Tissue-specific gene expression and ecdysone-regulated genomic networks in Drosophila. Dev Cell 5:59-72.

Li Z, Sheng M (2003) Some assembly required: the development of neuronal synapses. Nat Rev Mol Cell Biol 4:833-841.

Liao D, Hessler NA, Malinow R (1995) Activation of postsynaptically silent synapses during pairing-induced LTP in CA1 region of hippocampal slice. Nature 375:400-404.

Lin W, Burgess RW, Dominguez B, Pfaff SL, Sanes JR, Lee KF (2001) Distinct roles of nerve and muscle in postsynaptic differentiation of the neuromuscular synapse. Nature 410:1057-1064.

Littleton JT (2000) A genomic analysis of membrane trafficking and neurotransmitter release in Drosophila. J Cell Biol 150:F77-82.

Littleton JT, Ganetzky B (2000) Ion channels and synaptic organization: analysis of the Drosophila genome. Neuron 26:35-43.

Luo ZG, Je HS, Wang Q, Yang F, Dobbins GC, Yang ZH, Xiong WC, Lu B, Mei L (2003) Implication of geranylgeranyltransferase I in synapse formation. Neuron 40:703-717.

Luo ZG, Wang Q, Zhou JZ, Wang J, Luo Z, Liu M, He X, Wynshaw-Boris A, Xiong WC, Lu B, Mei L (2002) Regulation of AChR clustering by Dishevelled interacting with MuSK and PAK1. Neuron 35:489-505.

Madden DR (2002) The structure and function of glutamate receptor ion channels. Nat Rev Neurosci 3:91-101.

Mano I, Teichberg VI (1998) A tetrameric subunit stoichiometry for a glutamate receptor-channel complex. Neuroreport 9:327-331. 
Marangi PA, Forsayeth JR, Mittaud P, Erb-Vogtli S, Blake DJ, Moransard M, Sander A, Fuhrer C (2001) Acetylcholine receptors are required for agrin-induced clustering of postsynaptic proteins. Embo J 20:7060-7073.

Marrs GS, Green SH, Dailey ME (2001) Rapid formation and remodeling of postsynaptic densities in developing dendrites. Nat Neurosci 4:1006-1013.

Marrus SB, Portman SL, Allen MJ, Moffat KG, DiAntonio A (2004) Differential localization of glutamate receptor subunits at the Drosophila neuromuscular junction. J Neurosci 24:1406-1415.

Matsuda K, Fletcher M, Kamiya Y, Yuzaki M (2003) Specific assembly with the NMDA receptor 3B subunit controls surface expression and calcium permeability of NMDA receptors. J Neurosci 23:10064-10073.

McCabe BD, Marques G, Haghighi AP, Fetter RD, Crotty ML, Haerry TE, Goodman CS, O'Connor MB (2003) The BMP homolog Gbb provides a retrograde signal that regulates synaptic growth at the Drosophila neuromuscular junction. Neuron 39:241-254.

McGee AW, Bredt DS (2003) Assembly and plasticity of the glutamatergic postsynaptic specialization. Curr Opin Neurobiol 13:111-118.

McMahan UJ (1990) The agrin hypothesis. Cold Spring Harb Symp Quant Biol 55:407-418.

Meguro H, Mori H, Araki K, Kushiya E, Kutsuwada T, Yamazaki M, Kumanishi T, Arakawa M, Sakimura K, Mishina M (1992) Functional characterization of a heteromeric NMDA receptor channel expressed from cloned cDNAs. Nature 357:70-74

Milner R, Campbell IL (2002) The integrin family of cell adhesion molecules has multiple functions within the CNS. J Neurosci Res 69:286-291.

Missias AC, Mudd J, Cunningham JM, Steinbach JH, Merlie JP, Sanes JR (1997) Deficient development and maintenance of postsynaptic specializations in mutant mice lacking an 'adult' acetylcholine receptor subunit. Development 124:5075-5086.

Missler M, Zhang W, Rohlmann A, Kattenstroth G, Hammer RE, Gottmann K, Sudhof TC (2003) Alpha-neurexins couple Ca2+ channels to synaptic vesicle exocytosis. Nature 423:939-948.

Monyer H, Sprengel R, Schoepfer R, Herb A, Higuchi M, Lomeli H, Burnashev N, Sakmann B, Seeburg PH (1992) Heteromeric NMDA receptors: molecular and functional distinction of subtypes. Science 256:1217-1221.

Mulle C, Sailer A, Swanson GT, Brana C, O'Gorman S, Bettler B, Heinemann SF (2000) Subunit composition of kainate receptors in hippocampal interneurons. Neuron 28:475-484.

Nguyen T, Sudhof TC (1997) Binding properties of neuroligin 1 and neurexin 1beta reveal function as heterophilic cell adhesion molecules. J Biol Chem 272:26032-26039.

Noakes PG, Phillips WD, Hanley TA, Sanes JR, Merlie JP (1993) 43K protein and acetylcholine receptors colocalize during the initial stages of neuromuscular synapse formation in vivo. Dev Biol 155:275-280.

Noakes PG, Gautam M, Mudd J, Sanes JR, Merlie JP (1995) Aberrant differentiation of neuromuscular junctions in mice lacking s-laminin/laminin beta 2. Nature 374:258-262.

O'Brien R, Xu D, Mi R, Tang X, Hopf C, Worley P (2002) Synaptically targeted narp plays an essential role in the aggregation of AMPA receptors at excitatory synapses in cultured spinal neurons. $\mathrm{J}$ Neurosci 22:4487-4498.

O'Brien RJ, Xu D, Petralia RS, Steward O, Huganir RL, Worley P (1999) Synaptic clustering of AMPA receptors by the extracellular immediate-early gene product Narp. Neuron 23:309-323.

Okabe S, Miwa A, Okado H (2001) Spine formation and correlated assembly of presynaptic and postsynaptic molecules. J Neurosci 21:6105-6114.

Okabe S, Urushido T, Konno D, Okado H, Sobue K (2001) Rapid redistribution of the postsynaptic density protein PSD-Zip45 (Homer 1c) and its differential regulation by NMDA receptors and calcium channels. J Neurosci 21:9561-9571.

Ono F, Higashijima S, Shcherbatko A, Fetcho JR, Brehm P (2001) Paralytic zebrafish lacking acetylcholine receptors fail to localize rapsyn clusters to the synapse. J Neurosci 21:5439-5448.

Packard M, Koo ES, Gorczyca M, Sharpe J, Cumberledge S, Budnik V (2002) The Drosophila Wnt, wingless, provides an essential signal for pre- and postsynaptic differentiation. Cell 111:319-330.

Parnas D, Haghighi AP, Fetter RD, Kim SW, Goodman CS (2001) Regulation of postsynaptic structure and protein localization by the Rho-type guanine nucleotide exchange factor dPix. Neuron 32:415-424.

Pasterkamp RJ, Kolodkin AL (2003) Semaphorin junction: making tracks toward neural connectivity. Curr Opin Neurobiol 13:79-89.

Perez-Otano I, Schulteis CT, Contractor A, Lipton SA, Trimmer JS, Sucher NJ, Heinemann SF (2001) 
Assembly with the NR1 subunit is required for surface expression of NR3A-containing NMDA receptors. J Neurosci 21:1228-1237.

Petersen SA, Fetter RD, Noordermeer JN, Goodman CS, DiAntonio A (1997) Genetic analysis of glutamate receptors in Drosophila reveals a retrograde signal regulating presynaptic transmitter release. Neuron 19:1237-1248.

Phillips GR, Huang JK, Wang Y, Tanaka H, Shapiro L, Zhang W, Shan WS, Arndt K, Frank M, Gordon RE, Gawinowicz MA, Zhao Y, Colman DR (2001) The presynaptic particle web: ultrastructure, composition, dissolution, and reconstitution. Neuron 32:63-77.

Phillips WD, Maimone MM, Merlie JP (1991) Mutagenesis of the 43-kD postsynaptic protein defines domains involved in plasma membrane targeting and AChR clustering. J Cell Biol 115:1713-1723.

Pinkstaff JK, Detterich J, Lynch G, Gall C (1999) Integrin subunit gene expression is regionally differentiated in adult brain. J Neurosci 19:1541-1556.

Poodry CA, Edgar L (1979) Reversible alteration in the neuromuscular junctions of Drosophila melanogaster bearing a temperature-sensitive mutation, shibire. J Cell Biol 81:520-527.

Prange O, Murphy TH (2001) Modular transport of postsynaptic density-95 clusters and association with stable spine precursors during early development of cortical neurons. J Neurosci 21:9325-9333.

Prokop A (1999) Integrating bits and pieces: synapse structure and formation in Drosophila embryos. Cell Tissue Res 297:169-186.

Prokop A, Landgraf M, Rushton E, Broadie K, Bate M (1996) Presynaptic development at the Drosophila neuromuscular junction: assembly and localization of presynaptic active zones. Neuron 17:617-626.

Prokop A, Martin-Bermudo MD, Bate M, Brown N (1998) Absence of PS integrins or laminin A affects extracellular adhesion, but not intracellular assembly, of hemiadherens and neuromuscular junctions in Drosophila embryos. Dev Biol 196:58-76.

Rao A, Cha EM, Craig AM (2000) Mismatched appositions of presynaptic and postsynaptic components in isolated hippocampal neurons. J Neurosci 20:8344-8353.

Rao A, Kim E, Sheng M, Craig AM (1998) Heterogeneity in the molecular composition of excitatory postsynaptic sites during development of hippocampal neurons in culture. J Neurosci 18:1217-1229.

Ren Z, Riley NJ, Needleman LA, Sanders JM, Swanson GT, Marshall J (2003) Cell surface expression of GluR5 kainate receptors is regulated by an endoplasmic reticulum retention signal. J Biol Chem 278:52700-52709.

Rheuben MB, Yoshihara M, Kidokoro Y (1999) Ultrastructural correlates of neuromuscular junction development. Int Rev Neurobiol 43:69-92.

Ritzenthaler S, Suzuki E, Chiba A (2000) Postsynaptic filopodia in muscle cells interact with innervating motoneuron axons. Nat Neurosci 3:1012-1017.

Rosenberg M, Meier J, Triller A, Vannier C (2001) Dynamics of glycine receptor insertion in the neuronal plasma membrane. J Neurosci 21:5036-5044.

Rosenmund C, Stern-Bach Y, Stevens CF (1998) The tetrameric structure of a glutamate receptor channel. Science 280:1596-1599.

Roy PJ, Stuart JM, Lund J, Kim SK (2002) Chromosomal clustering of muscle-expressed genes in Caenorhabditis elegans. Nature 418:975-979.

Ruegg MA, Bixby JL (1998) Agrin orchestrates synaptic differentiation at the vertebrate neuromuscular junction. Trends Neurosci 21:22-27.

Safferling M, Tichelaar W, Kummerle G, Jouppila A, Kuusinen A, Keinanen K, Madden DR (2001) First images of a glutamate receptor ion channel: oligomeric state and molecular dimensions of GluRB homomers. Biochemistry 40:13948-13953.

Saitoe M, Tanaka S, Takata K, Kidokoro Y (1997) Neural activity affects distribution of glutamate receptors during neuromuscular junction formation in Drosophila embryos. Dev Biol 184:48-60.

Saitoe M, Schwarz TL, Umbach JA, Gundersen CB, Kidokoro Y (2001) Absence of junctional glutamate receptor clusters in Drosophila mutants lacking spontaneous transmitter release. Science 293:514-517.

Sanes JR, Lichtman JW (1999) Development of the vertebrate neuromuscular junction. Annu Rev Neurosci 22:389-442.

Sanes JR, Lichtman JW (2001) Induction, assembly, maturation and maintenance of a postsynaptic apparatus. Nat Rev Neurosci 2:791-805.

Scannevin RH, Huganir RL (2000) Postsynaptic organization and regulation of excitatory synapses. Nat 
Rev Neurosci 1:133-141.

Scheiffele P, Fan J, Choih J, Fetter R, Serafini T (2000) Neuroligin expressed in nonneuronal cells triggers presynaptic development in contacting axons. Cell 101:657-669.

Schulze KL, Broadie K, Perin MS, Bellen HJ (1995) Genetic and electrophysiological studies of Drosophila syntaxin-1A demonstrate its role in nonneuronal secretion and neurotransmission. Cell 80:311-320.

Schuster CM, Davis GW, Fetter RD, Goodman CS (1996) Genetic dissection of structural and functional components of synaptic plasticity. I. Fasciclin II controls synaptic stabilization and growth. Neuron 17:641-654.

Schuster CM, Ultsch A, Schloss P, Cox JA, Schmitt B, Betz H (1991) Molecular cloning of an invertebrate glutamate receptor subunit expressed in Drosophila muscle. Science 254:112-114.

Scott DB, Blanpied TA, Swanson GT, Zhang C, Ehlers MD (2001) An NMDA receptor ER retention signal regulated by phosphorylation and alternative splicing. J Neurosci 21:3063-3072.

Shapira M, Zhai RG, Dresbach T, Bresler T, Torres VI, Gundelfinger ED, Ziv NE, Garner CC (2003) Unitary assembly of presynaptic active zones from Piccolo-Bassoon transport vesicles. Neuron 38:237-252.

Shen K, Bargmann CI (2003) The immunoglobulin superfamily protein SYG-1 determines the location of specific synapses in C. elegans. Cell 112:619-630.

Shen K, Fetter RD, Bargmann CI (2004) Synaptic specificity is generated by the synaptic guidepost protein SYG-2 and its receptor, SYG-1. Cell 116:869-881.

Sheng M (2001) Molecular organization of the postsynaptic specialization. Proc Natl Acad Sci U S A 98:7058-7061.

Sheng M, Lee SH (2001) AMPA receptor trafficking and the control of synaptic transmission. Cell 105:825-828.

Sheridan DL, Berlot CH, Robert A, Inglis FM, Jakobsdottir KB, Howe JR, Hughes TE (2002) A new way to rapidly create functional, fluorescent fusion proteins: random insertion of GFP with an in vitro transposition reaction. BMC Neurosci 3:7.

Sigrist SJ, Thiel PR, Reiff DF, Schuster CM (2002) The postsynaptic glutamate receptor subunit DGluR-IIA mediates long-term plasticity in Drosophila. J Neurosci 22:7362-7372.

Sigrist SJ, Thiel PR, Reiff DF, Lachance PE, Lasko P, Schuster CM (2000) Postsynaptic translation affects the efficacy and morphology of neuromuscular junctions. Nature 405:1062-1065.

Sone M, Suzuki E, Hoshino M, Hou D, Kuromi H, Fukata M, Kuroda S, Kaibuchi K, Nabeshima Y, Hama C (2000) Synaptic development is controlled in the periactive zones of Drosophila synapses. Development 127:4157-4168.

Sprengel R, Aronoff R, Volkner M, Schmitt B, Mosbach R, Kuner T (2001) Glutamate receptor channel signatures. Trends Pharmacol Sci 22:7-10.

Standley S, Baudry M (2000) The role of glycosylation in ionotropic glutamate receptor ligand binding, function, and trafficking. Cell Mol Life Sci 57:1508-1516.

Stathopoulos A, Levine M (2002) Whole-genome expression profiles identify gene batteries in Drosophila. Dev Cell 3:464-465.

Sudhof TC (2000) The synaptic vesicle cycle revisited. Neuron 28:317-320.

Sweeney ST, Broadie K, Keane J, Niemann H, O'Kane CJ (1995) Targeted expression of tetanus toxin light chain in Drosophila specifically eliminates synaptic transmission and causes behavioral defects. Neuron 14:341-351.

Tejedor FJ, Bokhari A, Rogero O, Gorczyca M, Zhang J, Kim E, Sheng M, Budnik V (1997) Essential role for dlg in synaptic clustering of Shaker K+ channels in vivo. J Neurosci 17:152-159.

Thibault ST, Singer MA, Miyazaki WY, Milash B, Dompe NA, Singh CM, Buchholz R, Demsky M, Fawcett R, Francis-Lang HL, Ryner L, Cheung LM, Chong A, Erickson C, Fisher WW, Greer K, Hartouni SR, Howie E, Jakkula L, Joo D, Killpack K, Laufer A, Mazzotta J, Smith RD, Stevens LM, Stuber C, Tan LR, Ventura R, Woo A, Zakrajsek I, Zhao L, Chen F, Swimmer C, Kopczynski C, Duyk G, Winberg ML, Margolis J (2004) A complementary transposon tool kit for Drosophila melanogaster using P and piggyBac. Nat Genet 36:283-287.

Thomas U, Kim E, Kuhlendahl S, Koh YH, Gundelfinger ED, Sheng M, Garner CC, Budnik V (1997) Synaptic clustering of the cell adhesion molecule fasciclin II by discs- large and its role in the regulation of presynaptic structure. Neuron 19:787-799.

Togashi H, Abe K, Mizoguchi A, Takaoka K, Chisaka O, Takeichi M (2002) Cadherin regulates dendritic spine morphogenesis. Neuron 35:77-89.

Valenzuela DM, Stitt TN, DiStefano PS, Rojas E, Mattsson K, Compton DL, Nunez L, Park JS, Stark JL, Gies DR, et al. (1995) Receptor tyrosine kinase specific for the skeletal muscle lineage: expression in embryonic muscle, at the neuromuscular junction, and after injury. Neuron 
15:573-584

Vandenberghe W, Bredt DS (2004) Early events in glutamate receptor trafficking. Curr Opin Cell Biol 16:134-139.

Varoqueaux F, Sigler A, Rhee JS, Brose N, Enk C, Reim K, Rosenmund C (2002) Total arrest of spontaneous and evoked synaptic transmission but normal synaptogenesis in the absence of Munc13-mediated vesicle priming. Proc Natl Acad Sci U S A 99:9037-9042.

Verhage M, Maia AS, Plomp JJ, Brussaard AB, Heeroma JH, Vermeer H, Toonen RF, Hammer RE, van den Berg TK, Missler M, Geuze HJ, Sudhof TC (2000) Synaptic assembly of the brain in the absence of neurotransmitter secretion. Science 287:864-869.

Wallace BG (1989) Agrin-induced specializations contain cytoplasmic, membrane, and extracellular matrix-associated components of the postsynaptic apparatus. J Neurosci 9:1294-1302.

Wan HI, DiAntonio A, Fetter RD, Bergstrom K, Strauss R, Goodman CS (2000) Highwire regulates synaptic growth in Drosophila. Neuron 26:313-329.

Washbourne P, Bennett JE, McAllister AK (2002) Rapid recruitment of NMDA receptor transport packets to nascent synapses. Nat Neurosci 5:751-759.

Wenthold RJ, Petralia RS, Blahos J, II, Niedzielski AS (1996) Evidence for multiple AMPA receptor complexes in hippocampal CA1/CA2 neurons. J Neurosci 16:1982-1989.

Weston C, Yee B, Hod E, Prives J (2000) Agrin-induced acetylcholine receptor clustering is mediated by the small guanosine triphosphatases Rac and Cdc42. J Cell Biol 150:205-212.

Weston C, Gordon C, Teressa G, Hod E, Ren XD, Prives J (2003) Cooperative regulation by Rac and Rho of agrin-induced acetylcholine receptor clustering in muscle cells. J Biol Chem 278:6450-6455.

White JG, Southgate E, Thomson JN, Brenner S (1976) The structure of the ventral nerve cord of Caenorhabditis elegans. Philos Trans R Soc Lond B Biol Sci 275:327-348.

Wojtowicz JM, Marin L, Atwood HL (1994) Activity-induced changes in synaptic release sites at the crayfish neuromuscular junction. J Neurosci 14:3688-3703.

Wucherpfennig T, Wilsch-Brauninger M, Gonzalez-Gaitan M (2003) Role of Drosophila Rab5 during endosomal trafficking at the synapse and evoked neurotransmitter release. J Cell Biol 161:609-624.

Xia H, Hornby ZD, Malenka RC (2001) An ER retention signal explains differences in surface expression of NMDA and AMPA receptor subunits. Neuropharmacology 41:714-723.

Xia H, von Zastrow M, Malenka RC (2002) A novel anterograde trafficking signal present in the $\mathrm{N}$-terminal extracellular domain of ionotropic glutamate receptors. J Biol Chem 277:47765-47769.

Yamagata M, Sanes JR, Weiner JA (2003) Synaptic adhesion molecules. Curr Opin Cell Biol 15:621-632.

Yang X, Arber S, William C, Li L, Tanabe Y, Jessell TM, Birchmeier C, Burden SJ (2001) Patterning of muscle acetylcholine receptor gene expression in the absence of motor innervation. Neuron 30:399-410.

Yoshihara CM, Hall ZW (1993) Increased expression of the 43-kD protein disrupts acetylcholine receptor clustering in myotubes. J Cell Biol 122:169-179.

Yoshihara M, Rheuben MB, Kidokoro Y (1997) Transition from growth cone to functional motor nerve terminal in Drosophila embryos. J Neurosci 17:8408-8426.

Zhai RG, Vardinon-Friedman H, Cases-Langhoff C, Becker B, Gundelfinger ED, Ziv NE, Garner CC (2001) Assembling the presynaptic active zone: a characterization of an active one precursor vesicle. Neuron 29:131-143.

Zhang FL, Casey PJ (1996) Protein prenylation: molecular mechanisms and functional consequences. Annu Rev Biochem 65:241-269.

Zhou H, Glass DJ, Yancopoulos GD, Sanes JR (1999) Distinct domains of MuSK mediate its abilities to induce and to associate with postsynaptic specializations. J Cell Biol 146:1133-1146.

Zito K, Fetter RD, Goodman CS, Isacoff EY (1997) Synaptic clustering of Fascilin II and Shaker: essential targeting sequences and role of Dlg. Neuron 19:1007-1016. 


\section{Appendix}

\section{Genes enriched in larval body wall preparations}

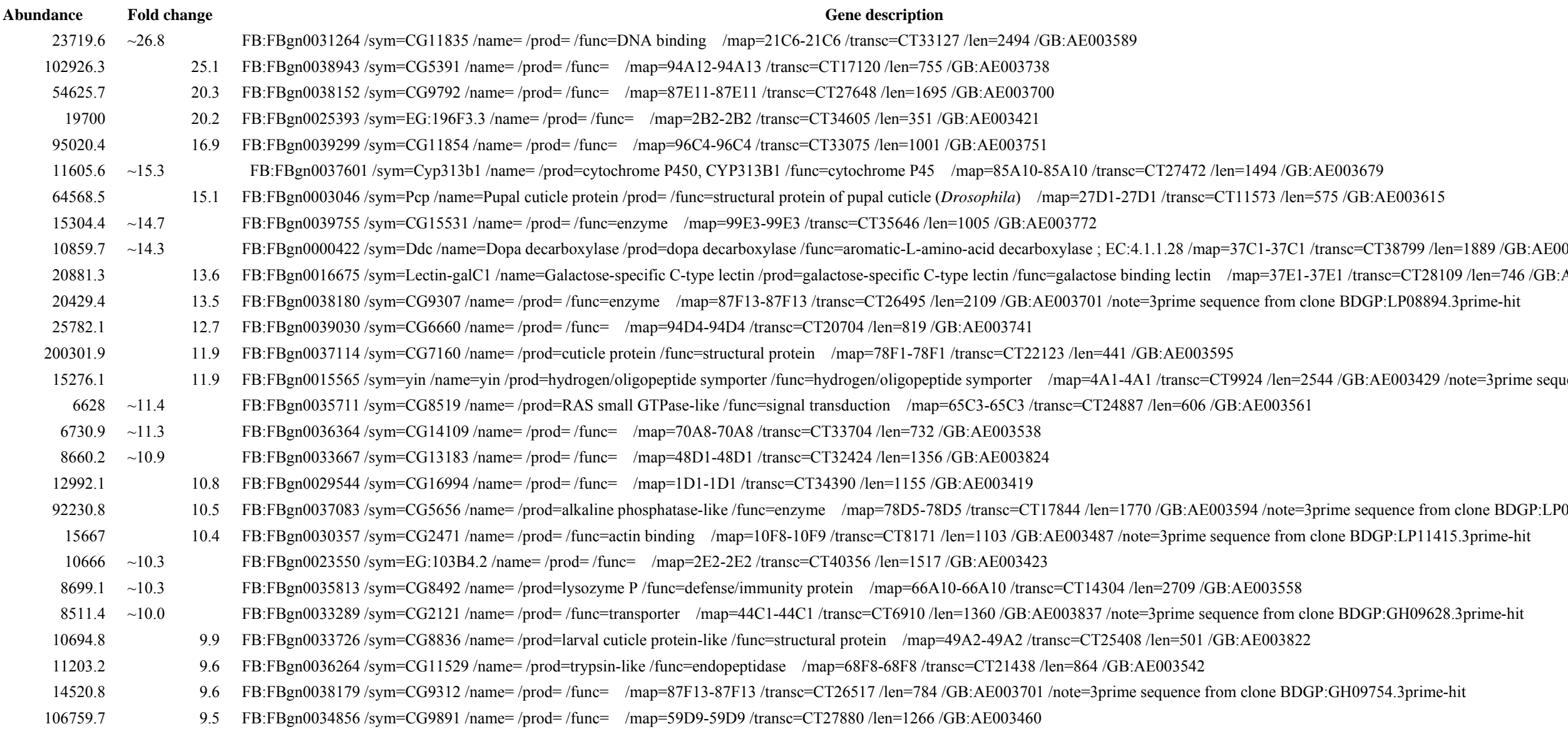

Gene description

FB:FBgn0031264 $/$ sym $=$ CG11835 $/$ name $=/$ prod $=/$ func=DNA binding $/$ map=21C6-21C6 $/$ transc $=$ CT33127 /len=2494 /GB:AE003589

25.1 FB:FBgn0038943 $/$ sym $=$ CG5391 $/$ name $=/$ prod $=/$ func $=/$ map $=94$ A12-94A13 $/$ transc $=\mathrm{CT} 17120 /$ len $=755 /$ GB:AE003738

20.3 FB:FBgn0038152/sym $=$ CG9792 $/$ name $=/$ prod $=/$ func $=/$ map $=87$ E $11-87$ E $11 /$ transc $=$ CT27648 $/$ len $=1695 /$ GB:AE003700

20.2 FB:FBgn0025393 /sym=EG:196F3.3 /name $=/$ prod= $/$ func $=/$ map=2B2-2B2 $/$ transc $=$ CT34605 /len=351 /GB:AE003421

16.9 FB:FBgn0039299/sym=CG1 $1854 /$ name $=/$ prod $=/$ func $=/$ map=96C4-96C4 $/$ transc=CT33075/len=1001 /GB:AE003751

FB:FBgn0037601 /sym=Cyp313b1 /name $=/$ prod=cytochrome P450, CYP313B1 /func=cytochrome P45 /map=85A10-85A10/transc=CT27472 /len=1494 /GB:AE003679

15.1 FB:FBgn0003046/sym=Pcp /name=Pupal cuticle protein /prod=/func=structural protein of pupal cuticle (Drosophila) $/$ map=27D1-27D1/transc=CT11573/len=575/GB:AE003615 FB:FBgn0039755 /sym=CG15531 /name=/prod= /func=enzyme $/$ map=99E3-99E3 $/$ transc=CT35646/len=1005 /GB:AE003772

4.3

$13.6 \mathrm{FB} \cdot \mathrm{FBgn} 0016675 / \mathrm{sym}=$ Lectin-galC1/name $=$ Galactose-specific $\mathrm{C}$-type lectin $/ \mathrm{prod}=$ galactose-specific C-type lectin $/ \mathrm{func}=\mathrm{galactose}$ binding lectin $/ \mathrm{map}=37 \mathrm{E} 1-37 \mathrm{E} 1 /$ transc $=\mathrm{CT} 28109 / \mathrm{len}=746 / \mathrm{GB}$ -

13.5 FB:FBgn0038180/sym=CG9307 /name=/prod= /func=enzyme /map=87F13-87F13/transc=CT26495/len=2109/GB:AE003701 /note=3prime sequence from clone BDGP:LP08894.3prime-hit

12.7 FB:FBgn0039030 $/$ sym $=$ CG6660 $/$ name $=/$ prod $=/$ func $=/$ map=94D4-94D4 $/$ transc $=$ CT20704 $/$ len $=819 /$ GB:AE003741

11.9 FB:FBgn0037114 $/$ sym=CG7160 $/$ name $=/$ prod=cuticle protein $/$ func=structural protein $/$ map=78F1-78F1 $/$ transc=CT22123/len=441 /GB:AE003595

11.9 FB:FBgn0015565/sym=yin /name=yin /prod=hydrogen/oligopeptide symporter /func=hydrogen/oligopeptide symporter $/$ map=4A1-4A1/transc=CT9924/len=2544 /GB:AE003429/note=3prime sequ FB:FBgn0035711 /sym=CG8519/name=/prod=RAS small GTPase-like /func=signal transduction /map=65C3-65C3/transc=CT24887/len=606/GB:AE003561

FB:FBgn0036364 $/$ sym $=$ CG14109 $/$ name $=/$ prod $=/$ func $=/$ map=70A8-70A $8 /$ transc $=$ CT33704 $/$ len $=732 /$ GB:AE003538

FB:FBgn0033667/sym $=$ CG13183 $/$ name $=/$ prod $=/$ func $=/$ map $=48 D 1-48 D 1 /$ transc $=$ CT32424 $/$ len $=1356 /$ GB:AE003824

10.8 FB:FBgn0029544 $/$ sym $=$ CG16994 $/$ name $=/$ prod $=/$ func $=/$ map=1D1-1D1 $/$ transc $=$ CT34390 $/$ len=1155 $/$ GB:AE003419

10.5 FB:FBgn0037083/sym=CG5656/name $=/$ prod=alkaline phosphatase-like $/$ func=enzyme $/$ map=78D5-78D5 /transc=CT17844 /len=1770 /GB:AE003594 /note=3prime sequence from clone BDGP:LP0

10.4 FB:FBgn0030357/sym=CG2471/name=/prod=/func=actin binding $/$ map=10F8-10F9/transc=CT8171/len=1103 /GB:AE003487/note=3prime sequence from clone BDGP:LP11415.3prime-hit FB:FBgn0023550 $/$ sym=EG:103B4.2 $/$ name $=/$ prod $=/$ func $=/$ map $=2 \mathrm{E} 2-2 \mathrm{E} 2 /$ transc $=\mathrm{CT} 40356 / \mathrm{len}=1517 / \mathrm{GB}: \mathrm{AE} 003423$

FB:FBgn0035813 /sym=CG8492/name $=/$ prod=lysozyme $\mathrm{P} /$ func=defense/immunity protein $/ \mathrm{map}=66 \mathrm{~A} 10-66 \mathrm{~A} 10 /$ transc $=\mathrm{CT} 14304 / \mathrm{len}=2709 / \mathrm{GB}: \mathrm{AE} 003558$

FB:FBgn0033289/sym=CG2121 /name=/prod=/func=transporter $/$ map=44C1-44C1 /transc=CT6910/len=1360 /GB:AE003837/note=3prime sequence from clone BDGP:GH09628.3prime-hit

9.9 FB:FBgn0033726/sym=CG8836/name=/prod=larval cuticle protein-like /func=structural protein $/$ map=49A2-49A2/transc=CT25408/len=501 /GB:AE003822

9.6 FB:FBgn0036264/sym=CG11529/name $=/$ prod=trypsin-like $/$ func $=$ endopeptidase $/ \mathrm{map}=68 \mathrm{~F} 8-68 \mathrm{~F} 8 /$ transc $=\mathrm{CT} 21438 /$ len $=864 / \mathrm{GB}: \mathrm{AE} 003542$

9.6 FB:FBgn0038179/sym $=$ CG9312 $/$ name $=/$ prod $=/$ func $=/$ map $=87 F 13-87 F 13 /$ transc $=$ CT26517 /len=784 /GB:AE003701 /note=3prime sequence from clone BDGP:GH09754.3prime-hit

9.5 FB:FBgn0034856 $/$ sym $=$ CG9891 $/$ name $=/$ prod $=/$ func $=/$ map $=59 \mathrm{D} 9-59 D 9 /$ transc $=\mathrm{CT} 27880 /$ len $=1266 / \mathrm{GB}: \mathrm{AE} 003460$ 


$\begin{aligned} 8081 & \sim 9.1 \\ 7297.3 & \sim 9.1 \\ 6026.4 & \sim 8.9 \\ 10038.8 & \\ 17201.8 & \\ 30755.5 & \\ 22531.1 & \\ 10634.5 & \\ 11276.3 & \\ 19312.7 & \\ 7383.3 & \sim 7.7 \\ 6329 & \sim 7.4 \\ 19301.8 & \\ 7576.3 & \sim 7.3 \\ 22097.3 & \\ 63005.9 & \\ 5453.9 & \sim 7.2 \\ 101119.9 & \\ 6255.2 & \sim 7.1 \\ 5606.4 & \sim 7.0 \\ 7498.3 & \\ 5278.4 & \sim 7.0 \\ 5163.4 & \sim 6.8 \\ 69802.4 & \\ 11072.1 & \\ 19916.4 & \\ 7115.8 & \\ 2204.1 & \sim 6.6 \\ 4567.9 & \sim 6.6 \\ 5343.8 & \sim 6.6 \\ 78514.5 & \\ 80923 & \\ & \\ & \\ & \\ & \\ & \\ & \\ & \\ & \\ & \\ & \\ & \\ & \\ & \\ & \\ & \end{aligned}$

80923
FB:FBgn0032349 $/$ sym $=$ CG4779 $/$ name $=/$ prod $=/$ func $=/$ map $=32 \mathrm{D} 4-32 \mathrm{D} 4 /$ transc $=\mathrm{CT} 15337 /$ len $=1272 / \mathrm{GB}: \mathrm{AE} 003631$ FB:FBgn0033858 $/$ sym $=$ CG13335 $/$ name $=/$ prod $=/$ func $=/$ map $=50 \mathrm{~B} 7-50 \mathrm{~B} 7 /$ transc $=\mathrm{CT} 32654 / \mathrm{len}=408 / \mathrm{GB}: \mathrm{AE} 003818$ FB:FBgn0040868 $/ \mathrm{sym}=\mathrm{CG} 15762 /$ name $=/$ prod $=/$ func $=/ \mathrm{map}=12 \mathrm{~B} 10-12 \mathrm{~B} 10 /$ transc $=\mathrm{CT} 36016 / \mathrm{len}=240 / \mathrm{GB}: \mathrm{AE} 003493$

8.9 FB:FBgn0037319/sym=CG2666/name $=/$ prod=chitin synthase-like $/$ func=enzyme $/$ map=83A5-83A5 /transc=CT9021 /len=3897/GB:AE003603

8.6 FB:FBgn0034443/sym=CG10460 /name $=/$ prod $=/$ func=endopeptidase $/ \mathrm{map}=56 \mathrm{D} 4-56 \mathrm{D} 4 /$ transc=CT29364 /len=485 /GB:AE003796

8.5 FB:FBgn0035964/sym=CG4665/name=dihydropteridine reductase $/$ prod=dihydropteridine reductase-like $/$ func $=$ dihydropteridine reductase $/ \mathrm{map}=66 \mathrm{~F} 6-66 \mathrm{~F} 6 /$ transc $=\mathrm{CT} 15055 /$ len $=708 / \mathrm{GB}: \mathrm{AE} 0035$

8.3 FB:FBgn0035427 /sym $=\mathrm{CG} 14959 /$ name $=/$ prod $=/$ func $=/ \mathrm{map}=63 \mathrm{C} 2-63 \mathrm{C} 2 /$ transc $=\mathrm{CT} 34804 / \mathrm{len}=450 / \mathrm{GB}: \mathrm{AE} 003477$

8.2 FB:FBgn0029821/sym=CG4020/name $=/$ prod $=/$ func $=/ \mathrm{map}=5 \mathrm{C} 6-5 \mathrm{C} 6 /$ transc $=\mathrm{CT} 13322 /$ len $=1485 / \mathrm{GB}: \mathrm{AE} 003435$

7.8 FB:FBgn0035300 $/$ sym $=\mathrm{CG} 1139 /$ name $=/$ prod $=$ amino-acid permease-like $/$ func=transporter $/ \mathrm{map}=62 \mathrm{~B} 11-62 \mathrm{~B} 11 / \mathrm{transc}=\mathrm{CT} 1131 / \mathrm{len}=1511 / \mathrm{GB}: \mathrm{AE} 003473$

7.7 FB:FBgn0038348 $/$ sym $=$ CG18519 $/$ name $=/$ prod $=/$ func $=/$ map $=88 \mathrm{~F} 8-88 \mathrm{~F} 8 /$ transc $=\mathrm{CT} 42266 / \mathrm{len}=3671 / \mathrm{GB}: \mathrm{AE} 003709$

FB:FBgn0034253 /sym=CG10936/name $=/$ prod $=/$ func $=$ endopeptidase $/$ map=54D3-54D4 /transc=CT30629 /len=4407 /GB:AE003802

FB:FBgn0038629 $/$ sym $=$ CG14304 $/$ name $=/$ prod $=/$ func $=/$ map=91B4-91B4 $/$ transc $=$ CT33934 /len=3585 $/$ GB:AE003722

7.3 FB:FBgn0031791 $/$ sym $=\mathrm{CG} 9486 /$ name $=/$ prod $=/$ func $=$ enzyme $/ \mathrm{map}=26 \mathrm{C} 1-26 \mathrm{C} 1 /$ transc $=\mathrm{CT} 26862 / \mathrm{len}=651 / \mathrm{GB}: \mathrm{AE} 003613$

FB:FBgn0037503 $/$ sym $=$ CG14598 $/$ name $=/$ prod $=/$ func $=/$ map $=84 \mathrm{D} 3-84 \mathrm{D} 3 /$ transc $=\mathrm{CT} 34346 /$ len $=690 /$ GB:AE003671

7.3 FB:FBgn0037761 $/$ sym $=$ CG8534 $/$ name $=/$ prod $=/$ func $=/$ map $=85 \mathrm{E} 10-85 \mathrm{E} 10 /$ transc $=\mathrm{CT} 24919 / \mathrm{len}=818 / \mathrm{GB}: \mathrm{AE} 003684$

7.2 FB:FBgn0030326 $/ \mathrm{sym}=\mathrm{CG} 2444 / \mathrm{name}=/ \mathrm{prod}=/$ func $=/ \mathrm{map}=10 \mathrm{D} 4-10 \mathrm{D} 4 /$ transc $=\mathrm{CT} 8084 / \mathrm{len}=635 / \mathrm{GB}: \mathrm{AE} 003487$

FB:FBgn0000594 /sym=Est-P /name=Esterase P $/$ prod=carboxylesterase $/$ func=carboxyesterase $;$ EC:3.1.1.1 /map=68F8-68F8 /transc=CT38084 /len=1635 /GB:AE003542

7.1 FB:FBgn0034162 $/ \mathrm{sym}=\mathrm{CG} 6426 /$ name $=/$ prod=calcium binding protein-like $/$ func=ligand binding or carrier $/ \mathrm{map}=53 \mathrm{D} 15-53 \mathrm{D} 15 /$ transc $=\mathrm{CT} 19886 / \mathrm{len}=426 / \mathrm{GB}: \mathrm{AE} 003805$ FB:FBgn0033136 $/$ sym $=\mathrm{CG} 12838 /$ name $=/$ prod $=/$ func $=/$ map $=42 \mathrm{E} 2-42 \mathrm{E} 2 /$ transc $=\mathrm{CT} 31970 / \mathrm{len}=633 / \mathrm{GB}: \mathrm{AE} 003842$

FB:FBgn0026190/sym=prolyl-4-hydroxylase-alpha /name=prolyl 4-hydroxylase alpha /prod=procollagen-proline,2-oxoglutarate-4-dioxygenase, alpha subunit /func=procollagen-proline,2-oxoglutarate

7 FB:FBgn0037905 $/$ sym $=$ CG14703 $/$ name $=/$ prod $=/$ func $=/$ map=86E6-86E $6 /$ transc $=$ CT34493 $/$ len $=1397 /$ GB:AE003691

FB:FBgn0033099 $/$ sym $=$ CG $9435 /$ name $=/$ prod $=/$ func $=/$ map $=42 \mathrm{C} 3-42 \mathrm{C} 3 /$ transc $=$ CT26754 $/$ len $=594 /$ GB:AE003789

FB:FBgn0031564 /sym=CG2816/name =/prod=chymotrypsin inhibitor-like /func=enzyme inhibitor $/$ map=24A5-24A5/transc=CT9603/len=292/GB:AE003579

6.7 FB:FBgn0033595 $/$ sym $=\mathrm{CG} 18337 /$ name $=/$ prod $=/$ func $=/$ map $=47 \mathrm{E} 1-47 \mathrm{E} 1 /$ transc $=\mathrm{CT} 41641 / \mathrm{len}=540 / \mathrm{GB}: \mathrm{AE} 003826$

6.7 FB:FBgn0022702 /sym=Cht2 /name=Chitinase $2 /$ prod=chitinase /func=chitinase ; EC:3.2.1.14/map=62B1-62B1/transc=CT6562/len=1597/GB:AE003472 /note=3prime sequence from clone BDGP:L

6.6 FB:FBgn0022770 /sym=Peritrophin-A /name=Peritrophin A /prod=peritrophin A /func=chitin binding /map=19C1-19C1 /transc=CT35567/len=1232/GB:AE003572 /note=3prime sequence from clon FB:FBgn0010222/sym=Nmdmc /name=NAD-dependent methylenetetrahydrofolate dehydrogenase $/$ prod=NAD-dependent methylenetetrahydrofolate dehydrogenase $/$ func $=$ methenyltetrahydrofolate cy 6.6 /GB:AE003

FB:FBgn0039098 $/$ sym $=$ CG13822 $/$ name $=/$ prod $=/$ func $=/$ map=95B1-95B1 $/$ transc $=$ CT33322 $/$ len $=651 /$ GB:AE003744

FB:FBgn0035553/sym=CG13722/name=/prod=prolin-rich protein-like /func=structural protein $/$ map=64B12-64B12/transc=CT33188/len=2124/GB:AE003481

FB:FBgn0027570/sym=BcDNA:GH07643 /name=/prod=membrane-anchored zinc metalloendopeptidase-like $(\mathrm{M} 13$ peptidase $) /$ func $=$ endopeptidase $/$ map $=82 \mathrm{D} 6-82 \mathrm{D} 7 /$ transc $=\mathrm{CT} 27583 /$ len $=2739 / \mathrm{C}$ 6.5 FB:FBgn0039482 $/$ sym $=$ CG14258 $/$ name $=/$ prod $=/$ func $=/$ map $=97$ E6-97E6 $/$ transc $=$ CT33878 $/$ len $=777 /$ GB $:$ AE003759

6.5 FB:FBgn0034517/sym=CG18066/name $=/$ prod $=/$ func $=/$ map=57A8-57A8 $/$ transc=CT40491/len=625 /GB:AE003791 /note $=3$ prime sequence from clone BDGP:GH09112.3prime-hit 
6.5 FB:FBgn0030976 $/$ sym $=\mathrm{CG} 7378 /$ name $=/$ prod $=$ protein phosphatase-like $/$ func $=$ protein phosphatase $/$ map $=17 \mathrm{E} 8-17 \mathrm{E} 8 /$ transc $=\mathrm{CT} 22699 / \mathrm{len}=738 / \mathrm{GB}: \mathrm{AE} 003510$

6.5 FB:FBgn0033022 $/ \mathrm{sym}=\mathrm{CG} 10398 /$ name $=/$ prod $=/$ func $=/ \mathrm{map}=41 \mathrm{C} 1-41 \mathrm{C} 1 /$ transc $=\mathrm{CT} 29180 / \mathrm{len}=501 / \mathrm{GB}: \mathrm{AE} 003786$

6.4 FB:FBgn0038053 $/$ sym $=C$ CG18549 $/$ name $=/$ prod $=/$ func $=/$ map $=87 \mathrm{C} 1-87 \mathrm{C} 1 /$ transc $=\mathrm{CT} 17408 / \mathrm{len}=1634 / \mathrm{GB}: \mathrm{AE} 003696$

6.4 FB:FBgn0031542 $/$ sym $=\mathrm{CG} 15414 /$ name $=/$ prod $=/$ func $=/$ map $=23 F 3-23 F 3 /$ transc $=\mathrm{CT} 35473 /$ len $=963 /$ GB:AE003579

FB:FBgn0035755 $/ \mathrm{sym}=\mathrm{CG} 14830 /$ name $=/$ prod $=/$ func $=/ \mathrm{map}=65 \mathrm{E} 10-65 \mathrm{E} 10 /$ transc $=\mathrm{CT} 34646 / \mathrm{len}=972 / \mathrm{GB}: \mathrm{AE} 003559$

6.3 FB:FBgn0035684/sym=CG10461 /name $=/$ prod=cuticle protein-like $/$ func=structural protein $/$ map=65A5-65A5 /transc=CT29366/len=321 /GB:AE003563

6.3 FB:FBgn0034860 $/$ sym $=$ CG9812 $/$ name $=/$ prod $=/$ func $=/$ map=59D10-59D $11 /$ transc $=C T 42639 /$ len=611 $/$ GB:AE003460

6.3 FB:FBgn0030816/sym $=$ CG16700/name $=/$ prod=amino-acid permease-like $/$ func $=\mathrm{G}$ protein linked receptor $/ \mathrm{map}=15 \mathrm{D} 2-15 \mathrm{D} 3 /$ transc $=\mathrm{CT} 37167 / \mathrm{len}=1673 / \mathrm{GB}: \mathrm{AE} 003504$ FB:FBgn0038449 $/$ sym $=$ CG17562 $/$ name $=/$ prod $=/$ func=enzyme $/$ map=89D5-89D5 /transc=CT34716 /len=1416 /GB:AE003714

6.2 FB:FBgn0030558 /sym=CG1461 /name $=/$ prod=tyrosine aminotransferase $/$ func=enzyme $/$ map=12E1-12E1 /transc=CT3554/len=1844 /GB:AE003495

FB:FBgn0039111/sym=CG10371/name=/prod $=/$ func $=$ protein phosphatase $/$ map=95B7-95B7/transc=CT29124/len=585/GB:AE003744

6.1 FB:FBgn0039895/sym=CG11288 /name $=/$ prod=fibrillin-like $/$ func $=$ cell adhesion $/ \mathrm{map}=102 \mathrm{~A} 3-102 \mathrm{~A} 3 /$ transc $=\mathrm{CT} 31491 / \mathrm{len}=914 / \mathrm{GB}: \mathrm{AE003844}$

6.1 FB:FBgn0031737/sym=CG11142/name=/prod=peritrophin-like $/$ func=structural protein $/ \mathrm{map}=26 \mathrm{~A} 1-26 \mathrm{~A} 1 /$ transc $=\mathrm{CT} 31147 / \mathrm{len}=1457 / \mathrm{GB}: \mathrm{AE} 003611$

6 FB:FBgn0038366 $/$ sym $=$ CG4576 $/$ name $=/$ prod $=/$ func $=/$ map $=89$ A $8-89$ A $8 /$ transc $=$ CT14818 $/$ len $=2581 /$ GB:AE003710

FB:FBgn0023540 $/$ sym=EG:152A3.3 $/$ name $=/$ prod $=/$ func $=/$ map=2E1-2E1 $/$ transc $=$ CT12189/len=1918 /GB:AE003423 /note=3prime sequence from clone BDGP:HL01173.3prime-hit

5.9 FB:FBgn0035936 $/$ sym $=$ CG4999 $/$ name $=/$ prod $=/$ func $=/$ map $=66 \mathrm{E} 3-66 \mathrm{E} 3 /$ transc $=\mathrm{CT} 16000 /$ len $=1131 /$ GB:AE003553

5.9 FB:FBgn0020439 $/$ sym=fau $/$ name $=/$ prod=anoxia upregulated protein $/$ func $=/$ map $=86 C 4-86 C 4 /$ transc $=$ CT41978 $/$ len=2121 /GB:AE003688

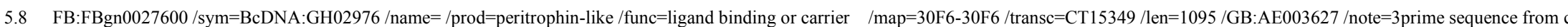
FB:FBgn0037186/sym=CG11241 /name $=/$ prod $=$ pyridoxal-phosphate-dependent aminotransferase class-III $/$ func $=$ enzyme $/$ map $=79 F 2-79 F 2 /$ transc $=\mathrm{CT} 31379 /$ len $=1856 / \mathrm{GB}: \mathrm{AE} 003598 /$ note $=3 \mathrm{prime}$ FB:FBgn0030595 $/$ sym $=$ CG14406 $/$ name $=/$ prod $=/$ func $=/$ map $=13$ A1-13A $1 /$ transc $=$ CT34057 $/$ len $=312 /$ GB:AE003497

BDGP:GH188.3prime-hit $/$ ESTpos=maps in FB:FBgn0036365 $/$ sym $=$ CG10732 $/$ name $=/$ prod $=/$ func $=$ signal transduction $/$ map $=7$ A8-7A8 $/$ transc $=\mathrm{CT} 369 /$ len $=535$

5.8 FB:FBgn0010019/sym=Cyp4g1/name=Cytochrome P45-4g1/prod=cytochrome P450, CYP4G1/func=cytochrome P45 ; EC:1.14.14.1/map=1B3-1B3 /transc=CT13187/len=2267 /GB:AE003417/nc 5.7 FB:FBgn0033725/sym=CG8502/name $=/$ prod $=$ cuticle protein /func=structural protein $/$ map=49A2-49A2/transc=CT24855/len=1413 /GB:AE003822 /note=3prime sequence from clone BDGP:LP07

5.7 FB:FBgn0029838 $/$ sym $=$ CG4666 $/$ name $=/$ prod $=/$ func $=/$ map $=5 \mathrm{D} 2-5 \mathrm{D} 2 /$ transc $=\mathrm{CT} 15013 / \mathrm{len}=729 / \mathrm{GB}: \mathrm{AE} 003436$

FB:FBgn0035304/sym=CG1282 $/$ name $=/$ prod=titin/twitchin-like $/$ func=cell adhesion $/ \mathrm{map}=62 \mathrm{C} 3-62 \mathrm{C} 3 /$ transc $=\mathrm{CT} 2687 /$ len=2392 $/ \mathrm{GB}: \mathrm{AE} 003473$

FB:FBgn0028922 /sym=BG:DS00929.8/name $=/$ prod $=/$ func $=/$ map=35C1-35C1 $/$ transc $=$ CT12287 /len $=1508 /$ GB:AE003646

5.6 FB:FBgn0030394 /sym=CG2560/name $=/$ prod $=$ cuticle protein-like $/$ func $=$ structural protein $/ \mathrm{map}=11 \mathrm{~B} 7-11 \mathrm{~B} 7 /$ transc $=\mathrm{CT} 8657 / \mathrm{len}=893 / \mathrm{GB}: \mathrm{AE} 003489$

FB:FBgn0036185 $/$ sym $=$ CG7346 $/$ name $=/$ prod=ATP-binding cassette transporter $/$ func=enzyme $/$ map=68C10-68C11 /transc=CT22647/len=1794 $/$ GB:AE003544

FB:FBgn0033290 $/$ sym $=$ CG8698 $/$ name $=/$ prod $=/$ func $=/ \mathrm{map}=44 \mathrm{C} 1-44 \mathrm{C} 1 /$ transc $=\mathrm{CT} 8775 / \mathrm{len}=504 / \mathrm{GB}: \mathrm{AE} 003837$

FB:FBgn0035289 $/ \mathrm{sym}=\mathrm{CG} 12026 /$ name $=/$ prod $=/$ func $=/$ map $=62 \mathrm{~B} 9-62 \mathrm{~B} 9 /$ transc $=\mathrm{CT} 1797 / \mathrm{len}=1455 / \mathrm{GB}: \mathrm{AE} 003473 /$ note $=3$ prime sequence from clone BDGP $:$ LP10272.3prime-hit

5.5 FB:FBgn0031872/sym=CG9211/name=/prod=/func=cell adhesion $/$ map=27C5-27C6 /transc=CT26314 /len=3247 /GB:AE003615 /note=3prime sequence from clone BDGP:GH03927.3prime-hit

5.5 FB:FBgn0032497 $/$ sym $=$ CG6043 $/$ name $=/$ prod $=/$ func $=/$ map $=34$ A11 $-34 \mathrm{~B} 1 /$ transc $=\mathrm{CT} 18935 /$ len $=3263 /$ GB:AE003639 
FB:FBgn0035457 $/$ sym $=\mathrm{CG} 12604 /$ name $=/$ prod $=/$ func $=/$ map $=63 \mathrm{E} 8-63 \mathrm{E} 8 / \mathrm{transc}=\mathrm{CT} 34822 / \mathrm{len}=936 / \mathrm{GB}: \mathrm{AE} 003479$

5.4 FB:FBgn0037534/sym=CG2781/name $=/$ prod $=/$ func=1,3-beta-glucan synthase $/$ map=84E5-84E5 /transc $=\mathrm{CT} 9465 /$ len $=831 / \mathrm{GB}: \mathrm{AE} 003677$

5.3 FB:FBgn0034920 $/ \mathrm{sym}=\mathrm{CG} 5597 /$ name $=/$ prod $=/$ func $=/ \mathrm{map}=60 \mathrm{~A} 8-60 \mathrm{~A} 8 /$ transc $=\mathrm{CT} 17698 / \mathrm{len}=897 /$ GB:AE003462 $/$ note $=3$ prime sequence from clone BDGP $:$ GH04238.3prime-hit

5.3 FB:FBgn0035985/sym=CG3672/name=/prod=/func=structural protein $/$ map=67B2-67B2/transc=CT12317/len=997 /GB:AE003552/note=3prime sequence from clone BDGP:GH12638.3prime-hit FB:FBgn0030065/sym=CG12075/name=/prod=/func=transcription factor $/ \mathrm{map}=8 \mathrm{~B} 4-8 \mathrm{~B} 4 /$ transc=CT5152/len=3406 /GB:AE003445 /note=3prime sequence from clone BDGP:LD23217.3prime-hit

5.3 FB:FBgn0032230 $/$ sym $=$ CG13139 $/$ name $=/$ prod $=/$ func $=/$ map $=31 \mathrm{D} 10-31 \mathrm{D} 10 /$ transc $=\mathrm{CT} 32379 / \mathrm{len}=807 / \mathrm{GB}: \mathrm{AE} 003628$

FB:FBgn0037805 $/$ sym $=$ CG1 $1871 /$ name $=/$ prod $=/$ func $=/$ map $=86$ A1-86A1 $/$ transc $=$ CT37022 $/$ len $=3575 /$ GB:AE003686

5.3 FB:FBgn0035705 /sym=CG8352/name $=/$ prod $=/$ func $=/$ map=65B4-65B5 /transc=CT24625/len=1728 /GB:AE003562 /note=3prime sequence from clone BDGP:LD26402.3prime-hit

5.3 FB:FBgn0031971 /sym $=$ CG7224 $/$ name $=/$ prod $=/$ func $=/$ map $=28 \mathrm{D} 11-28 \mathrm{D} 11 /$ transc $=\mathrm{CT} 22279 / \mathrm{len}=755 /$ GB:AE003619 $/$ note $=3$ prime sequence from clone BDGP $:$ GH18422.3prime-hit

5.2 FB:FBgn0033509/sym=CG12908/name=/prod=nidogen-like $/$ func=cell adhesion $/$ map=47A1-47A1 /transc=CT32053 /len=4591 /GB:AE003830 /note=3prime sequence from clone BDGP:LD35637

5.2 FB:FBgn0036985/sym=CG5847/name $=/$ prod $=/$ func $=$ structural protein $/$ map=77B9-77B9 $/$ transc=CT18331/len=6855/GB:AE003591

5.2 FB:FBgn0022701 /sym=Cht3 /name=Chitinase $3 /$ prod=chitinase $/$ func=chitinase ; EC:3.2.1.14 / map=40D5-40D5/transc=CT40797/len=3899/GB:AE002743

5.2 FB:FBgn0039690 /sym=CG1969 /name =/prod $=/$ func=glucosamine-phosphate N-acetyltransferase $;$ EC:2.3.1.4 /map=99C1-99C1/transc=CT4002 /len=1206 /GB:AE003771 /note=3prime sequence fro

5.2 FB:FBgn0029898/sym=CG14439/name=/prod=permease-like $/$ func=transporter $/ \mathrm{map}=6 \mathrm{C} 11-6 \mathrm{C} 11 /$ transc $=\mathrm{CT} 34101 / \mathrm{len}=1608 / \mathrm{GB}: \mathrm{AE} 003438$

5.2 FB:FBgn0032993 /sym=CG18117/name=/prod=chitinase-like /func=enzyme $/ \mathrm{map}=40 \mathrm{E} 1-40 \mathrm{E} 1 /$ transc=CT40795 /len=1393 /GB:AE002743 /note=3prime sequence from clone BDGP:LP05745.3prin

5.2 FB:FBgn0032601 /sym=yellow-b $/$ name=yellow-b $/$ prod $=/$ func $=/$ map=36A8-36A8 $/$ transc=CT39906 /len=1625 /GB:AE003652 /note=3prime sequence from clone BDGP:LD43175.3prime-hit

5.1 FB:FBgn0035398 /sym=CG1869 /name $=/$ prod=chitinase $/$ func=enzyme $/$ map=63B8-63B $/$ transc=CT5720/len=2346 /GB:AE003477

FB:FBgn0039658 /sym=CG11956/name=/prod=/func=/map=99A5-99A5/transc=CT39158/len=2937/GB:AE003768 /note=3prime sequence from clone BDGP:LD35296.3prime-hit

5.1 FB:FBgn0010388/sym=Dro $/$ name=Drosocin $/$ prod=drosocin $/$ func=antibacterial response protein $/ \mathrm{map}=51 \mathrm{C} 2-51 \mathrm{C} 2 /$ transc=CT30318/len=195/GB:AE003813

5 FB:FBgn0031908/sym=CG5177/name =/prod=/func=enzyme /map=27F6-27F6/transc=CT16575/len=980 /GB:AE003617 /note=3prime sequence from clone BDGP:LD18740.3prime-hit FB:FBgn0000152/sym=Axs /name=Abnormal X segregation $/$ prod $=/$ func $=/$ map=15A1-15A1/transc=CT27428/len=2396 /GB:AE003503

5 FB:FBgn0032281 $/$ sym $=$ CG17107 $/$ name $=/$ prod $=/$ func $=/$ map $=32 \mathrm{~A} 1-32 \mathrm{~A} 1 /$ transc $=$ CT33651 $/$ len $=291 /$ GB:AE003629

5 FB:FBgn0002440 /sym=1(3)mbn /name=lethal (3) malignant blood neoplasm $/$ prod $=/$ func $=/$ map $=65$ A4-65A4 $/$ transc $=\mathrm{CT} 29378 /$ len=1957 $/$ GB:AE003563

5 FB:FBgn0035103 $/$ sym $=$ CG7047 $/$ name $=/$ prod $=$ arrestin-like $/$ func $=/$ map $=61$ A5-61A5 $/$ transc $=$ CT42521 $/$ len $=1187 /$ GB:AE003467

5 FB:FBgn0039894/sym=CG10323/name=/prod=fibulin/fibrillin-like /func=cell adhesion $/ \mathrm{map}=102 \mathrm{~A} 1-102 \mathrm{~A} 3 /$ transc $=\mathrm{CT} 7856 / \mathrm{len}=1684 / \mathrm{GB}: \mathrm{AE} 003844$

5 FB:FBgn0036271 /sym=CG10335/name $=/$ prod=porphobilinogen synthase-like $/$ func $=$ enzyme $/$ map $=69$ A3 $-69 A 3 /$ transc $=$ CT29022 $/$ len $=984 / \mathrm{GB}: \mathrm{AE} 003542$

5 FB:FBgn0034420/sym=CG10737/name =/prod=protein kinase-like /func=protein kinase /map=56B6-56C1/transc=CT21097/len=3105/GB:AE003797 /note=3prime sequence from clone BDGP:GN FB:FBgn0013718 $/ \mathrm{sym}=$ nuf $/$ name $=$ nuclear fallout $/$ prod $=/$ func $=$ cytoskeletal structural protein $/$ map=70D2-70D2 $/$ transc $=$ CT23449 $/$ len $=2261 / \mathrm{GB}:$ AE003535

4.9 FB:FBgn0033130/sym=CG12843/name=Tetraspanin 42Ei /prod=tetraspanin /func $=/ \mathrm{map}=42 \mathrm{E} 1-42 \mathrm{E} 1 /$ transc $=\mathrm{CT} 31975 / \mathrm{len}=967 / \mathrm{GB}: \mathrm{AE} 003842$

4.9 FB:FBgn0040631/sym=CG13249 $/$ name $=/$ prod $=/$ func $=/$ map=77C2-77C2 $/$ transc=CT32502 /len=432 /GB:AE003591 /note=3prime sequence from clone BDGP $:$ LD43096.3prime-hit FB:FBgn0032382/sym=CG14935/name=/prod=maltase 2-like /func=enzyme $/$ map=33B2-33B2/transc=CT34763/len=1790/GB:AE003634

4.9 FB:FBgn0038774 /sym=CG5023/name $=/$ prod=calponin-like /func=ligand binding or carrier $/$ map=92D2-92D2/transc=CT16114 /len=697 /GB:AE003729 $/$ note $=3$ prime sequence from clone BDGP: 
4.9 FB:FBgn0032774 /sym=CG17549 /name $=/$ prod $=/$ func $=/$ map=37E1-37E1 /transc=CT38763/len=1051 /GB:AE003662 /note=3prime sequence from clone BDGP:GH19142.3prime-hit FB:FBgn0035155/sym=CG12015/name $=/$ prod $=/$ func $=$ transporter $/$ map=61C $9-61 \mathrm{C} 9 /$ transc $=\mathrm{CT} 1655 / \mathrm{len}=728 / \mathrm{GB}: \mathrm{AE} 003469$

FB:FBgn0036561 /sym=CG5891/name $=/$ prod=ankyrin-like $/$ func $=$ cytoskeletal structural protein $/$ map=72D1-72D3 $/$ transc=CT18415 /len=3517 /GB:AE003528

4.8 FB:FBgn0038250/sym=CG3505/name $=/$ prod $=/$ func $=$ endopeptidase $/ \mathrm{map}=88 \mathrm{C} 11-88 \mathrm{C} 11 /$ transc=CT11805/len=1161 /GB:AE003705 /note=3prime sequence from clone BDGP:LP10895.3prime-hit 4.7 FB:FBgn0033724/sym $=$ CG8501 $/$ name $=/$ prod $=/$ func $=/$ map=49A2-49A2 /transc=CT24861 /len=902 /GB:AE003822 /note=3prime sequence from clone BDGP:HL07915.3prime-hit

4.7 FB:FBgn0027617/sym=Rbp9/name=/prod $=/$ func=RNA binding $/$ map=23C1-23C2 /transc=CT38165/len=3320/GB:AE003581 /note $=3$ prime sequence from clone BDGP:GH07919.3prime-hit

4.7 FB:FBgn0036454/sym=CG17839 $/$ name $=/$ prod $=/$ func $=$ cell adhesion $/$ map=71A1-71A1 /transc=CT39634/len=3570 /GB:AE003533

4.7 FB:FBgn0034603/sym=CG9480/name=/prod=glycogenin glucosyltransferase-like /func=enzyme $/$ map=57C7-57C7/transc=CT26834/len=950/GB:AE003453

FB:FBgn0003888 /sym=betaTub60D /name=betaTubulin6D /prod=beta-tubulin /func=cytoskeletal structural protein $/ \mathrm{map}=60 \mathrm{C} 5-60 \mathrm{C} 5 /$ transc $=\mathrm{CT} 11425 /$ len=1542 $/ \mathrm{GB}: \mathrm{AE} 003463$

4.7 FB:FBgn0038151/sym=CG17044 $/$ name $=/$ prod $=/$ func $=/$ map $=87$ E11-87E $11 /$ transc $=$ CT37841 /len $=1281 /$ GB:AE003700

4.6 FB:FBgn0029662 $/ \mathrm{sym}=\mathrm{CG} 12206 /$ name $=/$ prod $=/$ func $=/ \mathrm{map}=3 \mathrm{D} 6-3 \mathrm{D} 6 /$ transc $=\mathrm{CT} 10991 / \mathrm{len}=1446 / \mathrm{GB}: \mathrm{AE} 003427$

FB:FBgn0034112 $/$ sym $=$ CG3896 $/$ name $=/$ prod $=/$ func=ligand binding or carrier $/$ map=53A3-53A4/transc=CT12657/len=3264 $/$ GB:AE003807

4.6 FB:FBgn0037067/sym $=\mathrm{CG} 11310 /$ name $=/$ prod $=/$ func $=$ structural protein $/ \mathrm{map}=78 \mathrm{C} 8-78 \mathrm{C} 8 /$ transc $=\mathrm{CT} 23453 /$ len=384 $/ \mathrm{GB}: \mathrm{AE} 003594$

4.6 FB:FBgn0033679/sym=CG8888 /name=/prod=/func=enzyme /map=48E4-48E6 /transc=CT25512 /len=1694 /GB:AE003823 /note=3prime sequence from clone BDGP:GH26015.3prime-hit

4.6 FB:FBgn0033630 $/ \mathrm{sym}=\mathrm{CG} 13200 /$ name $=/$ prod $=/$ func $=/ \mathrm{map}=47 \mathrm{~F} 6-47 \mathrm{~F} 6 /$ transc $=\mathrm{CT} 32444 / \mathrm{len}=309 / \mathrm{GB}: \mathrm{AE} 003826$

4.6 FB:FBgn0034522 $/$ sym $=$ CG13432 $/$ name $=/$ prod $=/$ func $=/$ map $=57$ A9-57A10 $/$ transc $=$ CT32789 $/$ len $=1355 /$ GB:AE003791

4.6 FB:FBgn0040992 $/ \mathrm{sym}=\mathrm{CG} 10570 /$ name $=/$ prod $=/$ func $=/$ map $=37 \mathrm{~B} 1-37 \mathrm{~B} 1 /$ transc $=\mathrm{CT} 29654 / \mathrm{len}=802 / \mathrm{GB}: \mathrm{AE} 003660 /$ note $=3$ prime sequence from clone BDGP:GH23934.3prime-hit

4.6 FB:FBgn0030798 $/ \mathrm{sym}=\mathrm{CG} 13003 / \mathrm{name}=/ \mathrm{prod}=/$ func $=/ \mathrm{map}=15 \mathrm{~A} 10-15 \mathrm{~A} 10 /$ transc $=\mathrm{CT} 32210 / \mathrm{len}=3477 / \mathrm{GB}: \mathrm{AE} 003503$

4.6 FB:FBgn0029843 /sym=CG5894/name=/prod=endothelin-converting enzyme-like zinc metalloendopeptidase $(\mathrm{M} 13$ peptidase $)$-like /func=endopeptidase $/ \mathrm{map}=5 \mathrm{D} 2-5 \mathrm{D} 2 /$ transc $=\mathrm{CT} 18487 /$ len=2314 FB:FBgn0038896 $/$ sym $=$ CG6328 $/$ name $=/$ prod $=/$ func $=/$ map $=93 F 4-93 F 4 /$ transc $=$ CT19790 $/$ len $=1317 /$ GB:AE003736

FB:FBgn0038977 $/$ sym $=$ CG5376 $/$ name $=/$ prod $=/$ func $=$ motor $/$ map=94B4-94B $/$ transc $=$ CT17018 $/$ len $=763 /$ GB:AE003739

FB:FBgn0039433/sym=CG5467/name=/prod=/func= /map=97B9-97B9 /transc=CT17334/len=2934 /GB:AE003757 /note=3prime sequence from clone BDGP:GH07007.3prime-hit

4.5 FB:FBgn0037697/sym=CG9363/name $=/$ prod $=/$ func=enzyme $/$ map=85D18-85D18 /transc=CT26609/len=1037 /GB:AE003682 /note=3prime sequence from clone BDGP:GH17960.3prime-hit

4.5 FB:FBgn0037721 /sym=CG9427 /name $=/$ prod $=/$ func $=/$ map=85E1-85E2 $/$ transc=CT26734/len=836/GB:AE003683 /note=3prime sequence from clone BDGP:GH19763.3prime-hit

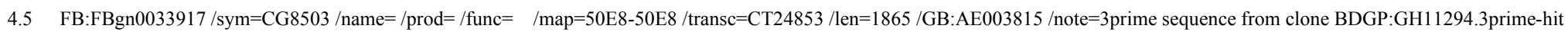
FB:FBgn0031193 $/$ sym $=$ CG17602 $/$ name $=/$ prod $=/$ func $=/$ map $=20 A 1-20 A 1 /$ transc $=$ CT34368 $/$ len $=1152 /$ GB:AE003574

4.4 FB:FBgn0038088 $/$ sym $=$ CG10126/name $=/$ prod $=$ calcium binding protein-like $/$ func $=$ ligand binding or carrier $/$ map $=87 \mathrm{D} 3-87 \mathrm{D} 3 /$ transc $=\mathrm{CT} 28495 /$ len $=879 / \mathrm{GB}: \mathrm{AE} 003697 /$ note $=3$ prime sequence fro

4.4 FB:FBgn0015801 /sym=Reg-5/name=Rhythmically expressed gene $5 /$ prod $=/$ func $=/ \mathrm{map}=60 \mathrm{D} 15-60 \mathrm{D} 16 /$ transc $=\mathrm{CT} 9939 /$ len $=1650 /$ GB:AE003465 $/$ note $=3$ prime sequence from clone BDGP:HL047

4.4 FB:FBgn0036547/sym $=$ CG17032 $/$ name $=/$ prod $=/$ func $=/$ map $=72 \mathrm{C} 1-72 \mathrm{C} 1 /$ transc $=\mathrm{CT} 32296 / \mathrm{len}=1007 / \mathrm{GB}: \mathrm{AE} 003529$

4.4 FB:FBgn0033109/sym $=\mathrm{CG} 9446 /$ name $=/$ prod $=/$ func $=$ chaperone $/ \mathrm{map}=42 \mathrm{C} 8-42 \mathrm{C} 8 /$ transc $=\mathrm{CT} 11617 / \mathrm{len}=1880 / \mathrm{GB}: \mathrm{AE} 003790$

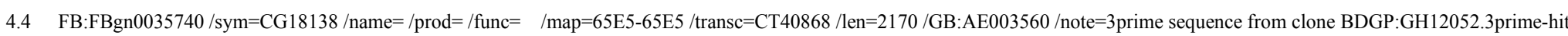

4.4 FB:FBgn0035917/sym=CG6416/name $=/$ prod $=/$ func $=$ enzyme $/$ map=66D9-66D10/transc=CT20013 /len=1175 /GB:AE003554/note=3prime sequence from clone BDGP:GH19182.3prime-hit 
$3588.9 \sim 4.4$

$2988.3 \sim 4.4$

110661.9

34249.9

25547.3

11289.1

82296.8

37898.4

11320.1

3702.5

4432.4

6566.3

1895.4

16413.7

26327.7

$3681.9 \sim 4.2$

20932.2

$2247.7 \sim 4.2$

$3098.4 \sim 4.2$

$3047.3 \sim 4.2$

11736.9

23343.7

175868.2

99849.6

180907.8

58068.8

10733.4
FB:FBgn0036101 /sym=CG6449 /name $=/$ prod=ninjurin $/$ func $=$ cell adhesion $\quad /$ map=67E5-67E $5 /$ transc $=$ CT20102 $/$ len $=591 /$ GB:AE003547

BDGP:SD1276.3prime-hit /ESTpos=maps 3prime of FB:FBgn0003638/sym=su(w[a]) /name=suppressor of white-apricot $/$ prod=/func=RNA binding $/$ map=1E1-1E1/transc=CT1162 /len=584

4.3 FB:FBgn0034044 /sym=CG12969 /name $=/$ prod $=/$ func $=$ enzyme $/$ map=52C7-52C $/$ transc=CT32161 /len=1203 /GB:AE003809

4.3 FB:FBgn0032685/sym=CG10211/name=/prod=peroxidase-like /func=enzyme $/$ map=37A1-37A1/transc=CT28633 /len=3691/GB:AE003659 /note=3prime sequence from clone BDGP:LD42267.3p

4.3 FB:FBgn0033033 $/ \mathrm{sym}=\mathrm{CG} 11066 /$ name $=/$ prod $=/$ func $=$ endopeptidase $/ \mathrm{map}=41 \mathrm{E} 3-41 \mathrm{E} 4 /$ transc $=\mathrm{CT} 30959 / \mathrm{len}=1377 / \mathrm{GB}: \mathrm{AE} 003785$

4.3 FB:FBgn0030355/sym $=\mathrm{CG} 2467 /$ name $=/$ prod $=/$ func $=/$ map=10F7-10F $/$ transc $=$ CT8145 $/$ len $=3418 /$ GB:AE003487 $/$ note $=3$ prime sequence from clone BDGP:GH09980.3prime-hit

4.3 FB:FBgn0031037/sym=CG14207/name $=/$ prod=heat shock protein HSP20-like /func=chaperone $/$ map=18D8-18D8 /transc $=\mathrm{CT} 33820 /$ len=1175 /GB:AE003512 /note $=3$ prime sequence from clone B FB:FBgn0000275/sym=Pka-R1 /name $=\mathrm{cAMP}-$ dependent protein kinase R1 /prod $=\mathrm{cAMP}-$ dependent protein kinase, regulatory subunit /func $=\mathrm{cAMP}-$ dependent protein kinase regulator $/$ map $=77 \mathrm{~F} 1-7$ 4.3 clon

4.3 BDGP:GH4896.3prime-hit /ESTpos=maps in FB:FBgn0033462/sym=CG12138/name=/prod=/func=DNA binding $/ \mathrm{map}=46 \mathrm{C} 4-46 \mathrm{C} 4 /$ transc $=\mathrm{CT} 7814 / \mathrm{len}=700$

FB:FBgn0011656/sym=Mef2 /name=Myocyte enhancing factor $2 /$ prod= /func=RNA polymerase II transcription factor $/$ map=46C6-46C7 /transc=CT3413/len=3211 /GB:AE003831 /note=3prime sec

4.3 FB:FBgn0031938 $/$ sym $=$ CG18590 $/$ name $=/$ prod $=/$ func $=/$ map $=28 \mathrm{C} 2-28 \mathrm{C} 2 /$ transc $=\mathrm{CT} 42499 / \mathrm{len}=176 / \mathrm{GB}: \mathrm{AE} 003618$

4.3 FB:FBgn0036673 /sym=CG11915/name=/prod=/func=ligand binding or carrier $/$ map=73D3-73D3 /transc=CT37088 /len=2999 /GB:AE003525 /note=3prime sequence from clone BDGP:GH17145.3 FB:FBgn0033936 $/$ sym $=$ CG17386 $/$ name $=/$ prod= $/$ func=RNA binding $/$ map=51A1-51A1/transc=CT33484/len=2609 $/$ GB:AE003814

4.3 FB:FBgn0032456/sym=CG6214 /name=/prod=ATP-binding cassette transporter; multidrug resistance protein-like $/$ func $=$ ion channel $/ \mathrm{map}=33 \mathrm{~F} 2-33 \mathrm{~F} 3 /$ transc $=\mathrm{CT} 19412 /$ len $=6461 / \mathrm{GB}: \mathrm{AE} 003637 / \mathrm{n}$

4.2 FB:FBgn0033133 $/$ sym $=$ CG12841 $/$ name $=/$ prod $=/$ func $=/$ map $=42 \mathrm{E} 1-42 \mathrm{E} 1 /$ transc $=\mathrm{CT} 31973 / \mathrm{len}=648 / \mathrm{GB}: \mathrm{AE} 003842$

FB:FBgn0004620/sym=Glu-RIIA /name=Glutamate receptor IIA /prod=glutamate receptor/func=glutamate receptor /map=25F1-25F1/transc=CT21587/len=3022 /GB:AE003610

4.2 FB:FBgn0029517 $/$ sym $=$ CG13377 $/$ name $=/$ prod $=/$ func $=/$ map $=1$ A5-1 $55 /$ transc $=$ CT32709 $/$ len $=993 /$ GB:AE003417

FB:FBgn0038987 $/$ sym $=$ CG6926 $/$ name $=/$ prod $=/$ func $=/$ map $=94 B 8-94 B 8 /$ transc $=C T 21454 /$ len $=789 /$ GB:AE003739

FB:FBgn0032616 $/$ sym $=$ CG15131 $/$ name $=/$ prod $=/$ func $=/$ map $=36$ A10-36A10 $/$ transc $=$ CT35026 $/$ len $=862 /$ GB:AE003652

FB:FBgn0022703 /sym=Cht1 /name=Chitinase $1 /$ prod=chitinase $/$ func=chitinase ; EC:3.2.1.14/map=40D5-40E1/transc=CT39094/len=1527/GB:AE002743

4.2 FB:FBgn0038720 $/$ sym $=$ CG6231 $/$ name $=/$ prod $=$ organic cation transporter $/$ func=transporter $/$ map $=92 \mathrm{~A} 13-92 \mathrm{~A} 13 /$ transc $=\mathrm{CT} 19536 / \mathrm{len}=1743 / \mathrm{GB}: \mathrm{AE} 003727$

4.2 FB:FBgn0030876 $/$ sym $=\mathrm{CG} 6762 /$ name $=/$ prod $=/$ func $=/ \mathrm{map}=16 \mathrm{D} 7-16 \mathrm{D} 7 /$ transc $=\mathrm{CT} 20293 / \mathrm{len}=580 / \mathrm{GB}: \mathrm{AE} 003507$

4.2 FB:FBgn0033729 $/ \mathrm{sym}=\mathrm{CG} 8510 /$ name $=/$ prod=cuticle protein-like $/$ func $=$ structural protein $/ \mathrm{map}=49 \mathrm{~A} 3-49 \mathrm{~A} 3 / \mathrm{transc}=\mathrm{CT} 24877 / \mathrm{len}=381 / \mathrm{GB}: \mathrm{AE} 003822$

4.2 FB:FBgn0001258 /sym=ImpL3 /name=Ecdysone-inducible gene L3 /prod=L-lactate dehydrogenase $/$ func=L-lactate dehydrogenase $; \mathrm{EC}: 1.1 .1 .27 / \mathrm{map}=65 \mathrm{~A} 8-65 \mathrm{~A} 8 /$ transc $=\mathrm{CT} 28577 /$ len=999 $/ \mathrm{GB}: \mathrm{AE} 0$

4.1 FB:FBgn0032777 $/$ sym $=$ CG18576 $/$ name $=/$ prod $=/$ func $=/$ map $=37 \mathrm{E} 1-37 \mathrm{E} 1 /$ transc $=\mathrm{CT} 42454 / \mathrm{len}=1593 / \mathrm{GB}: \mathrm{AE} 003662$

4.1 FB:FBgn0037069/sym=CG7658 /name $=/$ prod=cuticle protein-like $/$ func $=$ structural protein $/ \mathrm{map}=78 \mathrm{C} 8-78 \mathrm{C} 8 /$ transc $=\mathrm{CT} 23381 / \mathrm{len}=511 / \mathrm{GB}: \mathrm{AE} 003594$

4.1 FB:FBgn0017397/sym=how $/$ name=held out wings $/$ prod= $/$ func=RNA binding $/$ map=94A1-94A2/transc=CT28865 /len=1922 /GB:AE003737

FB:FBgn0032851 $/$ sym $=$ CG13970 $/$ name $=/$ prod $=/$ func $=/$ map $=38 \mathrm{C} 1-38 \mathrm{C} 1 /$ transc $=\mathrm{CT} 33523 / \mathrm{len}=618 / \mathrm{GB}: \mathrm{AE} 003665$

FB:FBgn0004852 /sym=Ac76E /name=Adenylyl cyclase 76E /prod=adenylate cyclase $/$ func=adenylate cyclase $;$ EC:4.6.1.1 /map=76D8-76E1/transc=CT23940 $/$ len=5189 $/$ GB:AE003515 /note=3prime

4.1 FB:FBgn0030759 $/ \mathrm{sym}=\mathrm{CG} 13014 /$ name $=/$ prod $=/$ func $=/ \mathrm{map}=14 \mathrm{~F} 2-14 \mathrm{~F} 2 /$ transc $=\mathrm{CT} 32227 / \mathrm{len}=699 / \mathrm{GB}: \mathrm{AE} 003502$

4.1 FB:FBgn0032025/sym $=\mathrm{CG} 7778 /$ name $=/$ prod $=/$ func $=/ \mathrm{map}=29 \mathrm{~B} 1-29 \mathrm{~B} 2 /$ transc $=\mathrm{CT} 23662 / \mathrm{len}=1222 / \mathrm{GB}: \mathrm{AE} 003620 /$ note $=3$ prime sequence from clone BDGP:GH17475.3prime-hit

4.1 FB:FBgn0014863 /sym=Mlp84B /name=Muscle LIM protein at 84B /prod=muscle LIM protein at 84B /func=transcription factor $/ \mathrm{map}=84 \mathrm{C} 3-84 \mathrm{C} 3 /$ transc $=\mathrm{CT} 1058 /$ len=1863 $/ \mathrm{GB}: \mathrm{AE} 003672$ 
4.1 FB:FBgn0038842 $/$ sym $=$ CG5630 $/$ name $=/$ prod $=/$ func $=/$ map $=93$ A4-93A4 $/$ transc $=C T 17794 /$ len $=1360 /$ GB:AE003732

4.1 FB:FBgn0037213 $/$ sym=CG12581 $/$ name $=/$ prod $=/$ func $=/$ map=82A1-82A1 /transc=CT34405/len=2382 /GB:AE003607 /note=3prime sequence from clone BDGP:GM02933.3prime-hit FB:FBgn0031700 $/$ sym $=$ CG14022 $/$ name $=/$ prod $=/$ func $=$ enzyme $/$ map=25D5-25D $/$ transc $=$ CT33581 /len=334 /GB:AE003609

4.1 FB:FBgn0034067/sym=CG8399 $/$ name $=/$ prod $=/$ func $=/$ map=52E1-52E3 $/$ transc=CT18617/len=2268 /GB:AE003808 /note=3prime sequence from clone BDGP:LD47639.3prime-hit FB:FBgn0037275/sym=CG14655/name=/prod=/func=nucleic acid binding $/$ map=82E1-82E1/transc=CT34432/len=2167/GB:AE003605/note=3prime sequence from clone BDGP:GH23506.3prim

4 FB:FBgn0034575/sym=CG15652/name $=/$ prod $=/$ func $=/$ map $=57$ B16-57B16 $/$ transc $=$ CT35836/len=1311 /GB:AE003452 /note=3prime sequence from clone BDGP:GH14953.3prime-hit

4 FB:FBgn0033532/sym $=$ CG18380 $/$ name $=/$ prod $=/$ func $=/$ map $=47$ A11-47A11 $/$ transc $=$ CT41783 /len $=657 /$ GB:AE003829

FB:FBgn0008649 /sym=dei /name $=$ delilah /prod $=/$ func $=$ specific RNA polymerase II transcription factor $/$ map=97B2-97B2 /transc=CT17256/len=1862 /GB:AE003756 /note $=3$ prime sequence from c

4 FB:FBgn0022800/sym=HD-14 /name $=/$ prod=fibroblast growth factor receptor-like /func=protein tyrosine kinase ; EC:2.7.1.11 inferred from sequence similarity $/ \mathrm{map}=96 \mathrm{C} 2-96 \mathrm{C} 2 /$ transc $=\mathrm{CT} 28777 / 1$

4 FB:FBgn0039667/sym=CG2010 /name=/prod= /func= /map=99B3-99B3 /transc=CT6431/len=1752 /GB:AE003769 /note=3prime sequence from clone BDGP:GH26184.3prime-hit

4 FB:FBgn0004028/sym=wupA /name=wings up A /prod=troponin I /func=cytoskeletal structural protein /map=16F7-17A1/transc=CT40012/len=389/GB:AE003507 FB:FBgn0033345 / $/$ ym $=$ CG13750 $/$ name $=/$ prod $=/$ func $=/$ map $=44 F 9-44 F 9 /$ transc $=$ CT33227 $/$ len $=228 /$ GB:AE003835

FB:FBgn0030758 /sym=CG9819/name=/prod=/func=protein phosphatase $/$ map=14F1-14F1/transc=CT27722/len=1724 /GB:AE003502

4 FB:FBgn0032284 $/$ sym $=$ CG7294 $/$ name $=/$ prod $=/$ func $=/$ map $=32 \mathrm{~A} 1-32 \mathrm{~A} 1 /$ transc $=\mathrm{CT} 22525 / \mathrm{len}=527 / \mathrm{GB}: \mathrm{AE} 003629$

4 FB:FBgn0031097/sym=CG17052/name=/prod=peritrophin-like /func=structural protein $/ \mathrm{map}=19 \mathrm{C} 1-19 \mathrm{C} 1 /$ transc $=\mathrm{CT} 37860 / \mathrm{len}=1383 / \mathrm{GB}: \mathrm{AE} 003572$

4 FB:FBgn0032279 $/ \mathrm{sym}=\mathrm{CG17104} /$ name $=/$ prod $=/$ func $=/ \mathrm{map}=32 \mathrm{~A} 1-32 \mathrm{~A} 1 /$ transc=CT38006 $/$ len=1746 $/$ GB:AE003629 $/$ note $=3$ prime sequence from clone BDGP:LD16579.3prime-hit

4 FB:FBgn0039742/sym=CG15528/name=/prod=protein phosphatase-like /func=protein phosphatase /map=99D5-99D5/transc=CT35642/len=639/GB:AE003772

4 FB:FBgn0036674 /sym=CG11916/name $=/$ prod= $/$ func=transcription factor $/$ map=73D4-73D4/transc=CT37092/len=707 /GB:AE003525

4 FB:FBgn0022341 /sym $=\mathrm{CG} 17467 /$ name $=/$ prod $=/$ func $=/$ map $=102 \mathrm{E} 3-102 \mathrm{E} 3 /$ transc $=\mathrm{CT} 38621 / \mathrm{len}=1002 / \mathrm{GB}: \mathrm{AE} 003846$

4 FB:FBgn0034701 $/$ sym $=$ CG13505 $/$ name $=/$ prod $=/$ func $=/$ map $=58 \mathrm{C} 5-58 \mathrm{C} 5 /$ transc $=\mathrm{CT} 32873 / \mathrm{len}=1011 / \mathrm{GB}: \mathrm{AE} 003456$

3.9 FB:FBgn0036675/sym=CG9959/name $=/$ prod $=/$ func=transcription factor $/$ map=73D4-73D5 /transc=CT28055/len=883 /GB:AE003525

FB:FBgn0032178 $/$ sym=CG4804 /name $=/$ prod=serpin $/$ func=serpin $/$ map=31A1-31A1 /transc $=C$ T15443 /len=1259 /GB:AE003627

3.9 FB:FBgn0028855/sym=BG:DS07721.3 $/$ name $=/$ prod $=/$ func $=/$ map=35B4-35B4 $/$ transc $=$ CT35230 $/$ len=465 $/$ GB:AE003644

3.9 FB:FBgn0038674 /sym=CG14285/name $=/$ prod $=/$ func=transcription factor binding $/$ map=91E4-91E4 $/$ transc $=\mathrm{CT} 33914 /$ len $=894 / \mathrm{GB}: \mathrm{AE} 003724$

3.9 FB:FBgn0036572/sym=CG5165/name=/prod=phosphoglucomutase-like /func=enzyme /map=72D7-72D7/transc=CT16539/len=1766 /GB:AE003528 /note=3prime sequence from clone BDGP:LD

3.9 FB:FBgn0035710/sym $=$ SP1173 $/$ name $=/$ prod $=/$ func $=/$ map $=65 \mathrm{C} 1-65 \mathrm{C} 1 /$ transc $=\mathrm{CT} 28481 / \mathrm{len}=2494 / \mathrm{GB}: \mathrm{AE} 003561 /$ note $=3$ prime sequence from clone BDGP:GH14073.3prime-hit

3.9 FB:FBgn0004507 /sym=GlyP /name=Glycogen phosphorylase /prod=glycogen phosphorylase /func=phosphorylase ; EC:2.4.1.1 inferred from direct assay $/ \mathrm{map}=22 \mathrm{C} 3-22 \mathrm{C} 3 /$ transc=CT22383 /len=287

3.9 FB:FBgn0032474 /sym $=\mathrm{CG} 9828 /$ name $=/$ prod $=/$ func $=$ chaperone $/ \mathrm{map}=34 \mathrm{~A} 8-34 \mathrm{~A} 8 /$ transc $=\mathrm{CT} 27734 / \mathrm{len}=1253 / \mathrm{GB}: \mathrm{AE} 003639$

FB:FBgn0037972 $/$ sym $=$ CG10005 $/$ name $=/$ prod $=/$ func $=/$ map $=87$ A8 -87 A $8 /$ transc $=$ CT28191 $/$ len $=684 /$ GB:AE003693

3.9 FB:FBgn0004577/sym=Pxd /name=Peroxidase $/$ prod=peroxidase $/$ func=peroxidase $; \mathrm{EC}: 1.11 .1 .7 / \mathrm{map}=89 \mathrm{E} 11-89 \mathrm{E} 11 /$ transc $=\mathrm{CT} 11695 / \mathrm{len}=2071 / \mathrm{GB}: \mathrm{AE} 003716$

3.8 FB:FBgn0035494/sym=CG14993 /name=/prod=fumarylacetoacetase-like /func=enzyme $/$ map=64A5-64A5 /transc=CT34846/len=1050/GB:AE003480 FB:FBgn0040587 $/$ sym $=$ CG17618 $/$ name $=/$ prod $=/$ func $=/$ map $=94$ C4 $-94 \mathrm{C} 4 /$ transc $=$ CT38876 $/$ len $=576 /$ GB:AE003740 
3.8 FB:FBgn0032332 $/ \mathrm{sym}=\mathrm{CG} 14917 / \mathrm{name}=/ \mathrm{prod}=/ \mathrm{func}=/ \mathrm{map}=32 \mathrm{D} 1-32 \mathrm{D} 1 / \operatorname{transc}=\mathrm{CT} 34744 / \mathrm{len}=1863 / \mathrm{GB}: \mathrm{AE} 003630$ FB:FBgn0035385/sym=CG2114/name $=/$ prod=G-protein coupled receptor-like $/$ func $=\mathrm{G}$ protein linked receptor $/ \mathrm{map}=63 \mathrm{~B} 2-63 \mathrm{~B} 2 /$ transc $=\mathrm{CT} 2366 /$ len $=1650 / \mathrm{GB}: \mathrm{AE} 003476$

3.8 BDGP:GH1742.3prime-hit /ESTpos=maps in FB:FBgn0033441 / $/ \mathrm{sym}=\mathrm{CG} 1776 /$ name $=/$ prod=myosin light chain kinase $/$ func $=$ protein kinase $/ \mathrm{map}=46 \mathrm{~A} 3-46 \mathrm{~A} 4 / \mathrm{transc}=\mathrm{CT} 5322 / \mathrm{len}=488$

3.8 FB:FBgn0037167/sym=CG11425/name=/prod=phosphatidate phosphohydrolase type 2-like /func=enzyme /map=79E4-79E4/transc=CT21215/len=956 /GB:AE003597

3.8 FB:FBgn0032785 /sym=CG10026/name = /prod=alpha-tocopherol transfer relative protein /func=transcription factor $/ \mathrm{map}=37 \mathrm{E} 3-37 \mathrm{E} 3 / \mathrm{transc}=\mathrm{CT} 28189 / \mathrm{len}=1143 / \mathrm{GB}: \mathrm{AE} 003663 /$ note $=3 \mathrm{prime}$ sequ

3.8 FB:FBgn0014141/sym=cher /name=cheerio /prod=filamin /func=actin binding /map=89F1-89F $1 /$ transc=CT12961 /len=7617 /GB:AE003716 /note=3prime sequence from clone BDGP:SD05640.3pri FB:FBgn0010015/sym=CanA1 /name=Calcineurin A1 /prod=protein serine/threonine phosphatase /func=protein serine/threonine phosphatase ; EC:3.1.3.16 | inferred from sequence similarity $/$ map=10

3.8 FB:FBgn0032280 $/$ sym $=\mathrm{CG} 17105 /$ name $=/$ prod $=/$ func $=/$ map $=32 \mathrm{~A} 1-32 \mathrm{~A} 1 /$ transc $=\mathrm{CT} 33652 / \mathrm{len}=312 / \mathrm{GB}: \mathrm{AE} 003629$

FB:FBgn0030586/sym=CG12539/name=/prod=glucose dehydrogenase (acceptor) /func=enzyme $/$ map=13A1-13A1 /transc=CT34058/len=1503/GB:AE003497

3.8 FB:FBgn0032283 $/$ sym $=$ CG7296 $/$ name $=/$ prod $=/$ func $=/$ map $=32 \mathrm{~A} 1-32 \mathrm{~A} 1 /$ transc $=\mathrm{CT} 22519 / \mathrm{len}=575 / \mathrm{GB}: \mathrm{AE} 003629$

3.8 FB:FBgn0036550/sym $=\mathrm{CG} 17026 /$ name $=/$ prod $=/$ func $=/ \mathrm{map}=72 \mathrm{C} 1-72 \mathrm{C} 1 /$ transc $=\mathrm{CT} 37805 / \mathrm{len}=855 / \mathrm{GB}: \mathrm{AE} 003529$ FB:FBgn0033092 $/$ sym $=$ CG9422 $/$ name $=/$ prod $=/$ func $=/$ map $=42 \mathrm{C} 1-42 \mathrm{C} 1 /$ transc $=\mathrm{CT} 26724 / \mathrm{len}=621 / \mathrm{GB}: \mathrm{AE} 003789$ FB:FBgn0038451/sym=CG14893 /name=/prod=/func=enzyme $/$ map=89D6-89D6 /transc=CT34717/len=1491 /GB:AE003714

3.8 FB:FBgn0031198 $/$ sym $=\mathrm{CG} 13238 /$ name $=/$ prod $=/$ func $=/$ map $=20 \mathrm{~A} 1-20 \mathrm{~A} 1 /$ transc $=\mathrm{CT} 32487 / \mathrm{len}=405 / \mathrm{GB}: \mathrm{AE} 003574$

3.8 FB:FBgn0011296/sym=1(2)efl /name=lethal (2) essential for life /prod=/func=chaperone /map=59F4-59F4/transc=CT14702/len=717/GB:AE003461 /note=3prime sequence from clone BDGP:GH0

3.8 FB:FBgn0035303/sym=CG5699/name=/prod=/func=cell adhesion $/ \mathrm{map}=62 \mathrm{C} 3-62 \mathrm{C} 3 /$ transc=CT2689 /len=1990 /GB:AE003473 /note=3prime sequence from clone BDGP:GH09541.3prime-hit

3.8 FB:FBgn0031534/sym=CG2774 /name=/prod=sorting nexin 1-like /func=transporter $/$ map=23F2-23F2/transc=CT9125/len=1450/GB:AE003579

3.8 FB:FBgn0031421 /sym $=$ CG9867 $/$ name $=/$ prod $=/$ func $=/$ map=22E1-22E2 $/$ transc $=$ CT27848 $/$ len=1826 $/$ GB:AE003583 $/$ note $=3$ prime sequence from clone BDGP:GH05422.3prime-hit FB:FBgn0039927/sym=CG11155/name=/prod=glutamate receptor-like /func=ion channel $/$ map=102E7-102F1/transc=CT30863/len=2062/GB:AE003846

FB:FBgn0031080 $/$ sym $=$ CG12655 $/$ name $=/$ prod $=/$ func $=/$ map $=19 A 3-19$ A3 $/$ transc $=$ CT35310 $/$ len $=496 /$ GB:AE00261 1

3.7 FB:FBgn0033034 $/ \mathrm{sym}=\mathrm{CG} 15900 /$ name $=/$ prod $=/$ func $=/ \mathrm{map}=41 \mathrm{E} 4-41 \mathrm{E} 4 /$ transc $=\mathrm{CT} 34160 / \mathrm{len}=519 / \mathrm{GB}: \mathrm{AE} 003785$

3.7 FB:FBgn0032422 $/ \mathrm{sym}=\mathrm{CG} 6579 /$ name $=/$ prod $=/$ func $=/ \mathrm{map}=33 \mathrm{C} 3-33 \mathrm{C} 4 /$ transc $=\mathrm{CT} 20347 / \mathrm{len}=834 / \mathrm{GB}: \mathrm{AE} 003635$

3.7 FB:FBgn0023023 /sym=CRMP $/$ name $=$ Collapsin Response Mediator Protein $/$ prod $=/$ func $=$ enzyme $/$ map $=83 B 3-83 B 4 /$ transc $=\mathrm{CT} 3290 /$ len $=2554 / \mathrm{GB}: \mathrm{AE} 003602 /$ note $=3$ prime sequence from clone B

3.7 FB:FBgn0038096 $/$ sym $=$ CG7340 $/$ name $=/$ prod $=/$ func $=/$ map=87D7-87D7 $/$ transc $=$ CT22347 /len=1953 /GB:AE003698 $/$ note $=3$ prime sequence from clone BDGP $:$ LD13869.3prime-hit

3.7 FB:FBgn0038009/sym $=$ CG17738 $/$ name $=/$ prod $=/$ func $=/$ map $=87 \mathrm{~B} 8-87 \mathrm{~B} 8 /$ transc $=$ CT34537 $/$ len $=333 /$ GB:AE003695 FB:FBgn0037813 $/$ sym $=\mathrm{CG} 17100 /$ name $=/$ prod $=/$ func $=/ \mathrm{map}=86 \mathrm{~A} 5-86 \mathrm{~A} 5 /$ transc $=\mathrm{CT} 34464 / \mathrm{len}=2030 / \mathrm{GB}: \mathrm{AE} 003687$

3.7 FB:FBgn0034797/sym=CG12781/name=/prod=/func=protein kinase $/$ map=59B4-59B4/transc=CT36793/len=4470/GB:AE003459/note=3prime sequence from clone BDGP:GH04942.3prime-hit

3.7 BDGP:LD43683.3prime-hit /ESTpos=maps in FB:FBgn0031097/sym=CG17052/name=/prod=peritrophin-like /func=structural protein $/ \mathrm{map}=19 \mathrm{C} 1-19 \mathrm{C} 1 /$ transc $=\mathrm{CT} 3786 /$ len $=595$

3.7 FB:FBgn0035550/sym=CG11349 $/$ name $=/$ prod $=/$ func $=$ structural protein $/ \mathrm{map}=64 \mathrm{~B} 12-64 \mathrm{~B} 12 / \operatorname{transc}=\mathrm{CT} 31656 /$ len $=1880 / \mathrm{GB}: \mathrm{AE} 003481$

3.7 FB:FBgn0015276/sym=Pcmt/name=Protein-L-isoaspartate (D-aspartate) O-methyltransferase /prod=protein-L-isoaspartate (D-aspartate) O-methyltransferase /func=protein-L-isoaspartate (D-aspartate)

3.7 FB:FBgn0033309/sym=CG8735/name $=/$ prod $=/$ func=electron transfer $/$ map=44D1-44D2/transc=CT8725/len=1421 /GB:AE003836/note=3prime sequence from clone BDGP:GH24644.3prime-hit FB:FBgn0040874 $/$ sym $=\mathrm{CG} 15600 /$ name $=/$ prod $=/$ func $=/$ map $=13 \mathrm{E} 12-13 \mathrm{E} 12 /$ transc $=\mathrm{CT} 35723 / \mathrm{len}=153 / \mathrm{GB}: \mathrm{AE} 003499$ 
3.7 FB:FBgn0036902 $/$ sym $=$ CG8748 $/$ name $=/$ prod $=/$ func $=/$ map $=76 \mathrm{C} 4-76 \mathrm{C} 4 /$ transc $=\mathrm{CT} 25246 /$ len $=1263 / \mathrm{GB}: \mathrm{AE} 003516$

3.7 FB:FBgn0036901 /sym=CG8756/name $=/$ prod=low-density lipoprotein-receptor-like $/$ func=receptor $/$ map=76C3-76C3 /transc $=\mathrm{CT} 25252 /$ len=2157 /GB:AE003516 $/$ note $=3$ prime sequence from clone FB:FBgn0032469 $/$ sym $=$ CG9932 $/$ name $=/$ prod $=/$ func $=$ nucleic acid binding $/$ map=34A5-34A5 $/$ transc=CT27960/len=6516 $/$ GB:AE003638 FB:FBgn0040981 /sym=CG15268 $/$ name $=/$ prod $=/$ func $=/$ map $=35$ B9-35B $/$ transc $=$ CT35215 $/$ len $=195 /$ GB:AE003645

3.7 FB:FBgn0034042 /sym $=$ CG8242 $/$ name $=/$ prod $=/$ func $=$ transcription factor $/ \mathrm{map}=52 \mathrm{C} 7-52 \mathrm{C} 7 /$ transc $=\mathrm{CT} 24455 /$ len $=4875 / \mathrm{GB}: \mathrm{AE} 003809$

3.6 FB:FBgn0033502 $/ \mathrm{sym}=\mathrm{CG} 12910 /$ name $=/$ prod $=/$ func $=/ \mathrm{map}=46 \mathrm{~F} 7-46 \mathrm{~F} 7 /$ transc $=\mathrm{CT} 32055 /$ len $=1690 / \mathrm{GB}: \mathrm{AE} 003830 /$ note $=3$ prime sequence from clone BDGP $:$ GH17442.3prime-hit

3.6 FB:FBgn0031064 /sym=CG12531/name $=/$ prod=amino-acid permease $/$ func=transporter $/ \mathrm{map}=18 \mathrm{E} 3-18 \mathrm{E} 5 /$ transc $=\mathrm{CT} 33850 / \mathrm{len}=2415 / \mathrm{GB}: \mathrm{AE} 003513$

3.6 FB:FBgn0035612/sym=CG10625/name=/prod=/func= /map=64E2-64E2 /transc=CT29764/len=3883 /GB:AE003565 /note=3prime sequence from clone BDGP:LD39545.3prime-hit FB:FBgn0034194 /sym=CG15611/name $=/$ prod=guanyl-nucleotide exchange factor $/$ func=signal transduction $/ \mathrm{map}=53 \mathrm{~F} 12-53 \mathrm{~F} 13 /$ transc $=\mathrm{CT} 35747 /$ len=1742 $/ \mathrm{GB}: \mathrm{AE} 003804 /$ note $=3$ prime sequence

3.6 FB:FBgn0000565 /sym=Eip71CD /name=Ecdysone-induced protein 28/29kD /prod=protein-methionine-S-oxide reductase-like /func=protein-methionine-S-oxide reductase ; EC:1.8.4.6 | inferred from s FB:FBgn0004879/sym=plx /name=pollux $/$ prod $=/$ func $=/$ map $=83$ B8-83B9 $/$ transc $=$ CT1567 /len $=5472 /$ GB:AE003602

3.6 FB:FBgn0034075/sym=CG18658/name=/prod=peptide-aspartate beta-dioxygenase-like /func=enzyme $/ \mathrm{map}=52 \mathrm{~F} 1-52 \mathrm{~F} 1 /$ transc $=\mathrm{CT} 18815 /$ len=1662 $/$ GB:AE003808 FB:FBgn0033942 $/$ sym $=\mathrm{CG} 10112 /$ name $=/$ prod $=/$ func=structural protein $/ \mathrm{map}=51 \mathrm{~A} 6-51 \mathrm{~A} 6 /$ transc $=\mathrm{CT} 28465 / \mathrm{len}=435 / \mathrm{GB}: \mathrm{AE} 003814$

3.6 FB:FBgn0037911 /sym=CG10898 /name $=/$ prod=7,8-dihydro-8-oxoguanine-triphosphatase $/$ func=DNA repair protein $/$ map $=86 \mathrm{E} 13-86 \mathrm{E} 13 /$ transc $=\mathrm{CT} 30521 / \mathrm{len}=1936 / \mathrm{GB}: \mathrm{AE} 003692 /$ note $=3$ prime 3.6 FB:FBgn0040993 $/$ sym $=$ CG17325 $/$ name $=/$ prod $=/$ func $=/$ map $=37$ B1 -37 B $2 /$ transc $=$ CT35069 $/$ len $=765 /$ GB:AE003660

3.6 FB:FBgn0028915/sym=BG:DS01068.5/name=/prod=serine endopeptidase $/$ func=endopeptidase $/ \mathrm{map}=35 \mathrm{~A} 1-35 \mathrm{~A} 1 /$ transc=CT23067 /len=530/GB:AE003643

3.6 FB:FBgn0036659/sym=CG9701/name=/prod=beta-glucosidase-like /func=ion channel $/$ map=73B5-73B5 /transc=CT27420/len=1936/GB:AE003526 /note=3prime sequence from clone BDGP:LP0

3.6 FB:FBgn0004117/sym=Tm2 $/$ name=Tropomyosin $2 /$ prod=tropomyosin $/$ func=motor $/$ map=88E11-88E $12 /$ transc=CT15467 /len=914 /GB:AE003708

3.6 FB:FBgn0036875/sym=CG9449/name=/prod=acid phosphatase-like /func=enzyme /map=76B6-76B6/transc=CT26772/len=1179/GB:AE003516

3.6 FB:FBgn0033968 $/ \mathrm{sym}=\mathrm{CG} 10200 /$ name $=/$ prod $=/$ func $=/ \mathrm{map}=51 \mathrm{C} 5-51 \mathrm{C} 5 /$ transc $=\mathrm{CT} 28697 /$ len $=659 /$ GB:AE003813 $/$ note $=3$ prime sequence from clone BDGP $:$ LP02570.3prime-hit

3.5 FB:FBgn0033919/sym $=\mathrm{CG} 8547 /$ name $=/$ prod $=/$ func $=$ DNA binding $/ \mathrm{map}=50 \mathrm{~F} 1-50 \mathrm{~F} 1 /$ transc $=\mathrm{CT} 24943 / \mathrm{len}=1313 / \mathrm{GB}: \mathrm{AE} 003815$ FB:FBgn0035590 $/$ sym $=\mathrm{CG} 10673 /$ name $=/$ prod $=/$ func=protein kinase $/$ map $=64 \mathrm{C} 12-64 \mathrm{C} 12 /$ transc $=\mathrm{CT} 29894 /$ len $=675 / \mathrm{GB}: \mathrm{AE} 003567$

3.5 FB:FBgn0030745/sym=CG4239/name $=/$ prod $=/$ func $=/ \mathrm{map}=14 \mathrm{C} 3-14 \mathrm{C} 4 /$ transc=CT13936/len=1639 /GB:AE003502 /note=3prime sequence from clone BDGP:GH25683.3prime-hit FB:FBgn0000719 $/$ sym=fog $/$ name $=$ folded gastrulation $/$ prod $=/$ func $=/$ map=20A4-20A5 $/$ transc $=$ CT16970 $/$ len=3059 $/$ GB:AE003573 $/$ note $=3$ prime sequence from clone BDGP:SD02223.3prime-hit

3.5 FB:FBgn0031293/sym=CG4226/name=/prod=AMPA/kainate-selective ionotrophic glutamate receptor-like /func=ion channel $/ \mathrm{map}=21 \mathrm{D} 4-21 \mathrm{D} 4 /$ transc $=\mathrm{CT} 13021 / \mathrm{len}=2979 / \mathrm{GB}: \mathrm{AE} 003588 / \mathrm{note}=3$

3.5 FB:FBgn0026077/sym=Gasp /name=/prod=peritrophin-like /func=chitin binding $/$ map=83D4-83D4/transc=CT28895/len=1460/GB:AE003600/note=3prime sequence from clone BDGP:LD05259.

3.5 FB:FBgn0036051/sym $=$ CG8154 $/$ name $=/$ prod $=/$ func $=/$ map $=67 \mathrm{C} 2-67 \mathrm{C} 2 /$ transc $=\mathrm{CT} 24352 / \mathrm{len}=1726 / \mathrm{GB}: \mathrm{AE} 003550$

3.5 BDGP:LD31422.3prime-hit /ESTpos=maps in FB:FBgn0004624/sym=CaMKII /name=Calcium/calmodulin-dependent protein kinase II /prod=calcium/calmodulin-dependent protein kinase II /func=cal

3.5 FB:FBgn0038181/sym=CG9297/name $=/$ prod $=/$ func $=$ enzyme $/$ map $=87 F 13-87 \mathrm{~F} 13 /$ transc $=$ CT26475 /len=2985 $/$ GB:AE003701

3.5 FB:FBgn0031199 $/ \mathrm{sym}=\mathrm{CG} 9557 /$ name $=/$ prod $=/$ func $=/ \mathrm{map}=20 \mathrm{~A} 1-20 \mathrm{~A} 1 /$ transc $=\mathrm{CT} 17180 /$ len $=420 / \mathrm{GB}: \mathrm{AE} 003574$

3.5 FB:FBgn0038294 $/ \mathrm{sym}=\mathrm{CG} 6803 /$ name $=/$ prod $=/$ func $=/ \mathrm{map}=88 \mathrm{E} 6-88 \mathrm{E} 6 /$ transc $=\mathrm{CT} 21060 / \mathrm{len}=1488 / \mathrm{GB}: \mathrm{AE} 003707 /$ note $=3$ prime sequence from clone BDGP $:$ GH14252.3prime-hit FB:FBgn0033996 /sym=CG11807/name=/prod=/func=receptor $/$ map=51E7-51E7/transc=CT14564/len=1924 /GB:AE003811 /note=3prime sequence from clone BDGP:SD03973.3prime-hit 
3.4 FB:FBgn0032897 /sym $=$ CG9336 $/$ name $=/$ prod $=/$ func $=/$ map $=38 \mathrm{~F} 1-38 \mathrm{~F} 1 /$ transc $=\mathrm{CT} 5238 / \mathrm{len}=660 / \mathrm{GB}: \mathrm{AE} 003668$

3.4 FB:FBgn0034632 $/$ sym $=$ CG15668 $/$ name $=/$ prod $=/$ func $=/$ map $=57$ E8 -57 E9 $/$ transc $=$ CT35852 $/$ len=1976 $/$ GB:AE003454 $/$ note $=3$ prime sequence from clone BDGP $:$ GH02495.3prime-hit

3.4 FB:FBgn0035844 $/ \mathrm{sym}=\mathrm{CG} 13676 /$ name $=/ \mathrm{prod}=/$ func $=/ \mathrm{map}=66 \mathrm{~B} 10-66 \mathrm{~B} 10 /$ transc $=\mathrm{CT} 33112 / \mathrm{len}=2625 / \mathrm{GB}: \mathrm{AE} 003556$

3.4 FB:FBgn0036903/sym=CG8747/name=/prod=low-density lipoprotein-like /func=receptor $/ \mathrm{map}=76 \mathrm{C} 4-76 \mathrm{C} 4 /$ transc=CT25242 /len=651 /GB:AE003516

3.4 FB:FBgn0034819 $/$ sym $=\mathrm{CG} 9877 /$ name $=/$ prod $=/$ func $=/$ map $=59$ C4 $-59 \mathrm{C} 4 /$ transc $=\mathrm{CT} 27864 /$ len $=267 / \mathrm{GB}: \mathrm{AE} 003459$

FB:FBgn0003995/sym=vvl/name=ventral veins lacking /prod=/func=RNA polymerase II transcription factor /map=65C5-65D1/transc=CT28091/len=2556/GB:AE003561

3.4 FB:FBgn0001123/sym=G-salpha60A /name $=\mathrm{G}$ protein salpha $6 \mathrm{~A} /$ prod $=\mathrm{G}$ protein alphas-subunit $/$ func=heterotrimeric G protein $/ \mathrm{map}=60 \mathrm{~A} 12-60 \mathrm{~A} 12 / \mathrm{transc}=\mathrm{CT} 40310 /$ len $=1626 / \mathrm{GB}: \mathrm{AE} 003462 / \mathrm{no}$

3.4 FB:FBgn0033872/sym=CG6329/name $=/$ prod $=/$ func $=/$ map=50C12-50C12 /transc=CT19784 /len=1312 /GB:AE003817 /note $=3$ prime sequence from clone BDGP:HL02087.3prime-hit

3.4 FB:FBgn0003162 /sym=Pu /name=Punch /prod=GTP cyclohydrolase I /func=GTP cyclohydrolase I ; EC:3.5.4.16/map=57C5-57C6/transc=CT26766 /len=1623 /GB:AE003453 /note=3prime sequence

3.4 FB:FBgn0038610/sym=CG7675/name=/prod=/func=enzyme /map=91A2-91A2/transc=CT30419/len=1100/GB:AE003721 /note=3prime sequence from clone BDGP:GH26851.3prime-hit

3.4 FB:FBgn0028694/sym $=$ Rpn $11 /$ name $=/$ prod $=19 \mathrm{~S}$ proteasome regulatory particle, non-ATPase protein, subunit S13 $/$ func $=/ \mathrm{map}=25 \mathrm{C} 3-25 \mathrm{C} 3 /$ transc $=\mathrm{CT} 41032 / \mathrm{len}=1165 / \mathrm{GB}: \mathrm{AE} 003608$ FB:FBgn0036028 $/$ sym $=$ CG16717 $/$ name $=/$ prod $=/$ func $=/$ map $=67$ B11-67B $11 /$ transc $=$ CT37199 $/$ len $=903 /$ GB:AE00355

3.4 FB:FBgn0031200 $/ \mathrm{sym}=\mathrm{CG} 9558 /$ name $=/$ prod $=/$ func $=/ \mathrm{map}=20 \mathrm{~A} 1-20 \mathrm{~A} 1 /$ transc $=\mathrm{CT} 17174 / \mathrm{len}=261 / \mathrm{GB}: \mathrm{AE} 003574$

3.4 FB:FBgn0039897/sym=CG1674 $/$ name $=/$ prod $=/$ func $=$ motor $/$ map=102A3-102A $4 /$ transc $=$ CT4686 /len=2388 $/$ GB:AE003844

FB:FBgn0011327/sym=Uch-L3 /name=Ubiquitin C-terminal hydrolase /prod=ubiquitinyl hydrolase L3 /func=ubiquitinyl hydrolase 1 /map=67B5-67B5/transc=CT11565 /len=1137/GB:AE003552 /1

3.3 FB:FBgn0030309/sym $=\mathrm{CG} 1572 / \mathrm{name}=/ \mathrm{prod}=/$ func $=/ \mathrm{map}=10 \mathrm{C} 4-10 \mathrm{C} 4 /$ transc $=\mathrm{CT} 4080 / \mathrm{len}=955 /$ GB:AE003486 $/$ note $=3$ prime sequence from clone BDGP $:$ LD04844.3prime-hit

3.3 FB:FBgn0016756/sym=Ubp64E /name=Ubiquitin-specific protease 64E /prod=ubiquitin-specific protease /func=ubiquitin-specific protease $/ \mathrm{map}=64 \mathrm{E} 13-64 \mathrm{~F} 1 /$ transc $=\mathrm{CT} 17382 / \mathrm{len}=4202 / \mathrm{GB}: \mathrm{AE} 00$

3.3 FB:FBgn0033446/sym $=\mathrm{CG} 1648 /$ name $=/$ prod $=/$ func $=/ \mathrm{map}=46 \mathrm{~B} 10-46 \mathrm{~B} 10 /$ transc $=\mathrm{CT} 4516 / \mathrm{len}=960 / \mathrm{GB}: \mathrm{AE} 003832 /$ note $=3$ prime sequence from clone BDGP $:$ GH20817.3prime-hit

FB:FBgn0039335/sym=CG5127/name=/prod=vacuolar protein sorting-like /func=transporter $/$ map=96E1-96E1 /transc=CT16445/len=2021 /GB:AE003752/note=3prime sequence from clone BDG

3.3 FB:FBgn0033596/sym $=\mathrm{CG} 7738 /$ name $=/$ prod $=/$ func $=/$ map $=47 \mathrm{E} 1-47 \mathrm{E} 1 /$ transc $=\mathrm{CT} 23557 / \mathrm{len}=405 / \mathrm{GB}: \mathrm{AE} 003826$

3.3 FB:FBgn0032002/sym=CG8353 /name=/prod=cytidine deaminase-like $/$ func=enzyme $/$ map=28F4-28F4 /transc=CT24599 /len=513/GB:AE003620

FB:FBgn0037542/sym=CG2723 /name = /prod= /func= /map=84E6-84E6/transc=CT9257/len=1314 /GB:AE003677 /note=3prime sequence from clone BDGP:LD11958.3prime-hit

3.3 FB:FBgn0019972/sym=Ice $/$ name=Ice $/$ prod=caspase /func=caspase ; EC:3.4.22.- $/ \mathrm{map}=99 \mathrm{C} 4-99 \mathrm{C} 4 /$ transc=CT4706 /len=1932/GB:AE003771 /note=3prime sequence from clone BDGP:GH24292.3pr

3.3 FB:FBgn0023517/sym=EG:63B12.4 /name $=/$ prod= $/$ func $=/$ map=2B13-2B13 /transc=CT34629/len=1066 /GB:AE003422 /note=3prime sequence from clone BDGP:GH02880.3prime-hit

FB:FBgn0036843 $/$ sym $=$ CG6812 $/$ name $=/$ prod $=/$ func $=/$ map $=75 F 4-75 F 5 /$ transc $=$ CT2 $1117 /$ len $=1585 /$ GB:AE003518

3.3 FB:FBgn0039719/sym=CG15515/name $=/$ prod=cuticle protein-like $/$ func $=$ structural protein $/$ map=99D1-99D1 $/$ transc=CT35628 /len=327 /GB:AE003771

3.3 FB:FBgn0031821 /sym $=$ CG9542 $/$ name $=/$ prod $=/$ func $=/$ map=26D7-26D7 $/$ transc $=$ CT26992 $/$ len $=903 /$ GB:AE003613

3.3 FB:FBgn0039208 $/$ sym $=$ CG6643 $/$ name $=/$ prod $=/$ func $=$ enzyme $/ \mathrm{map}=96$ A7-96A7 $/$ transc $=$ CT20638 $/$ len=2769 $/$ GB:AE003748 $/$ note $=3$ prime sequence from clone BDGP:GH21511.3prime-hit FB:FBgn0031502/sym=CG3524/name=/prod=/func=enzyme /map=23C5-23D1/transc=CT11835/len=7590/GB:AE003581/note=3prime sequence from clone BDGP:GH02912.3prime-hit FB:FBgn0035054 $/$ sym $=$ CG9189 $/$ name $=/$ prod $=/$ func $=/$ map $=60 \mathrm{D} 14-60 \mathrm{D} 14 /$ transc $=\mathrm{CT} 26264 / \mathrm{len}=1121 / \mathrm{GB}: \mathrm{AE} 003465$

3.3 FB:FBgn0039529 $/$ sym $=$ CG5612 $/$ name $=/$ prod $=/$ func $=/$ map=98A4-98A4 $/$ transc $=$ CT17752 $/$ len $=1352 /$ GB:AE003761

FB:FBgn0029542 $/$ sym $=\mathrm{CG} 3708 /$ name $=/$ prod $=/$ func $=/$ map $=1 \mathrm{C} 5-1 \mathrm{C} 5 /$ transc $=\mathrm{CT} 12445 /$ len $=1188 / \mathrm{GB}: \mathrm{AE} 003419 /$ note $=3$ prime sequence from clone BDGP $: \mathrm{GH} 17085.3$ prime-hit 
FB:FBgn0040542 $/$ sym $=C G 12815 /$ name $=/$ prod $=/$ func $=/$ map $=85$ F15-85F15 $/$ transc $=$ CT31943 $/$ len $=213 /$ GB:AE003686

3.3 FB:FBgn0031695/sym=Cyp4ac3 /name=/prod=cytochrome P450, CYP4AC3 /func=cytochrome P45 /map=25D2-25D2/transc=CT33590/len=939/GB:AE003609 /note=3prime sequence from clon FB:FBgn0031153/sym=CG15448/name=/prod=/func=transmembrane receptor $/$ map=19E7-19E7/transc=CT35512/len=669 /GB:AE003569 FB:FBgn0031211/sym=CG2776/name=/prod=/func=ligand binding or carrier $/$ map=21A4-21A4/transc=CT9453/len=615 /GB:AE003590

3.2 FB:FBgn0027540 /sym=BcDNA:GH12504/name=/prod=/func=transmembrane receptor $/$ map=85B3-85B4/transc=CT27712/len=3771 /GB:AE003680/note=3prime sequence from clone BDGP:GH

3.2 BDGP:GH1453.3prime-hit /ESTpos=maps in FB:FBgn0031737/sym=CG11142/name=/prod=peritrophin-like /func=structural protein $/ \mathrm{map}=26 \mathrm{~A} 1-26 \mathrm{~A} 1 /$ transc $=\mathrm{CT} 31147 /$ len=899 FB:FBgn0031251 /sym $=$ CG4213 $/$ name $=/$ prod $=/$ func $=$ motor $/$ map $=21 \mathrm{C} 2-21 \mathrm{C} 2 /$ transc $=\mathrm{CT} 13886 / \mathrm{len}=3696 / \mathrm{GB}: \mathrm{AE} 003589$

3.2 FB:FBgn0038407/sym=CG6126/name=/prod=sugar transporter /func=transporter $/$ map=89B13-89B13/transc=CT19169/len=2108 /GB:AE003712 /note=3prime sequence from clone BDGP:GH092

3.2 FB:FBgn0034525/sym=CG13435/name=/prod=/func=ligand binding or carrier $/ \mathrm{map}=57 \mathrm{~B} 1-57 \mathrm{~B} 1 /$ transc=CT32792 /len=1983 /GB:AE003791

3.2 FB:FBgn0003499 /sym=sr /name=stripe /prod=/func=RNA polymerase II transcription factor $/$ map=90E1-90E2 $/$ transc=CT23724 /len=4829/GB:AE003720 FB:FBgn0035772 $/$ sym $=$ CG8582 $/$ name $=/$ prod $=/$ func $=/$ map $=65 F 4-65 F 4 /$ transc $=$ CT14420 $/$ len $=1739 /$ GB:AE003559

FB:FBgn0036562 $/$ sym $=$ CG5600 $/$ name $=/$ prod $=/$ func $=/$ map=72D3-72D4 /transc $=C$ T17536 $/$ len $=1858 /$ GB:AE003528 $/$ note $=3$ prime sequence from clone BDGP:GH16214.3prime-hit

3.2 FB:FBgn0039606 /sym $=$ CG1448 /name $=/$ prod $=/$ func $=/$ map=98E6-98E6 $/$ transc $=C$ T3509 /len=1544 /GB:AE003767 /note=3prime sequence from clone BDGP:LD34202.3prime-hit

$3.2 \quad \mathrm{FB}$

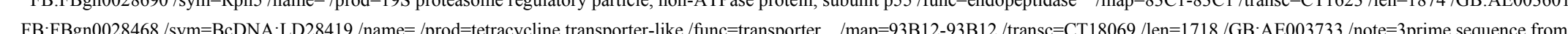
FB:FBgn0039870 $/$ sym=CG1896/name $=/$ prod $=/$ func $=/$ map=100E2-100E2 $/$ transc=CT5870 /len=806 /GB:AE003779 /note=3prime sequence from clone BDGP:LD39576.3prime-hit

3.2 FB:FBgn0010424 /sym=TpnC73F /name=Troponin $\mathrm{C}$ at $73 \mathrm{~F} /$ prod=troponin $\mathrm{C} /$ func=calcium binding $/ \mathrm{map}=73 \mathrm{E} 4-73 \mathrm{E} 5 /$ transc $=\mathrm{CT} 23822 /$ len=935 $/ \mathrm{GB}: \mathrm{AE} 003525$

FB:FBgn0034029 $/$ sym $=$ CG8190 $/$ name $=/$ prod=translation initiation factor 2B-gamma-like $/$ func=translation factor $/$ map $=52 \mathrm{~A} 10-52 \mathrm{~A} 10 /$ transc $=\mathrm{CT} 20341 /$ len $=1503 / \mathrm{GB}: \mathrm{AE} 003810 /$ note $=3$ prime sec 3.2 FB:FBgn0030028 $/ \mathrm{sym}=\mathrm{CG} 10965 / \mathrm{name}=/ \mathrm{prod}=/ \mathrm{func}=/ \mathrm{map}=7 \mathrm{D} 21-7 \mathrm{D} 21 /$ transc $=\mathrm{CT} 7616 / \mathrm{len}=1052 / \mathrm{GB}: \mathrm{AE} 003443 /$ note $=3$ prime sequence from clone BDGP $:$ GH26991.3prime-hit

3.2 FB:FBgn0038149 $/$ sym $=$ CG9796 $/$ name $=/$ prod $=/$ func $=/$ map=87E10-87E10 /transc=CT27692/len=1109 /GB:AE003700 /note $=3$ prime sequence from clone BDGP $:$ LD47508.3prime-hit FB:FBgn0034045/sym=CG8249/name=/prod=glucose transporter-like /func=transporter $/$ map=52D2-52D2 /transc=CT21797/len=1807/GB:AE003809 FB:FBgn0034579 $/$ sym $=$ CG9353 $/$ name $=/$ prod $=/$ func $=/$ map $=57$ B20-57B20 $/$ transc $=$ CT26571 $/$ len=647 $/$ GB:AE003452 $/$ note $=3$ prime sequence from clone BDGP $:$ SD09147.3prime-hit

3.2 FB:FBgn0011716/sym=spdo /name=sanpodo /prod=tropomodulin /func=tropomyosin binding /map=100A1-100A1/transc=CT3919/len=5374/GB:AE003774 /note=3prime sequence from clone BD 3.2 FB:FBgn0032899 $/$ sym $=$ CG9338 $/$ name $=/$ prod $=/$ func $=/$ map $=38 \mathrm{~F} 1-38 \mathrm{~F} 1 /$ transc $=$ CT5240 $/$ len $=660 /$ GB:AE003668 $/$ note $=3$ prime sequence from clone BDGP $:$ GH07967.3prime-hit

3.2 FB:FBgn0029958/sym=CG12151/name=/prod=pyruvate dehydrogenase phosphatase /func=protein phosphatase $/ \mathrm{map}=7 \mathrm{~B} 8-7 \mathrm{~B} 8 /$ transc $=\mathrm{CT} 8439 / \mathrm{len}=1910 / \mathrm{GB}: \mathrm{AE} 003441 /$ note $=3$ prime sequence fr

3.2 FB:FBgn0032596/sym $=\mathrm{CG} 17331 /$ name $=/$ prod=20S proteasome, beta2 subunit $/$ func $=$ endopeptidase $/ \mathrm{map}=36 \mathrm{~A} 7-36 \mathrm{~A} 7 /$ transc $=\mathrm{CT} 32562 / \mathrm{len}=760 / \mathrm{GB}: \mathrm{AE} 003652$

3.2 FB:FBgn0038817 /sym $=$ CG18039 $/$ name $=/$ prod $=/$ func $=/$ map $=92 F 1-92 F 1 /$ transc $=C T 13538 /$ len $=961 /$ GB:AE003731

3.2 FB:FBgn0027501/sym=BcDNA:LD24639/name=/prod=UDP-N-acetylglucosamine pyrophosphorylase-like $/$ func=enzyme $/ \mathrm{map}=26 \mathrm{D} 5-26 \mathrm{D} 5 /$ transc $=\mathrm{CT} 26974 / \mathrm{len}=2033 / \mathrm{GB}: \mathrm{AE} 003613 / \mathrm{note}=3 \mathrm{pri}$

3.2 FB:FBgn0039898/sym=CG1748 /name=/prod=RHO GTPase activator-like /func=signal transduction /map=102A4-102A4/transc=CT4742/len=717/GB:AE003844

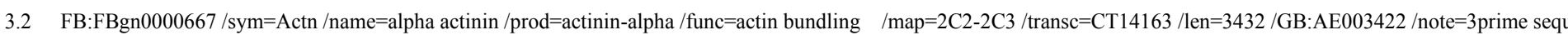
FB:FBgn0028428 $/$ sym $=\mathrm{Ih} /$ name $=/$ prod=voltage-gated ion channel protein $/$ func=voltage-gated ion channel $/ \mathrm{map}=50 \mathrm{~F} 1-50 \mathrm{~F} 1 /$ transc $=\mathrm{CT} 24973 /$ len $=3924 / \mathrm{GB}: \mathrm{AE} 003815$ FB:FBgn0038866 $/$ sym $=$ CG5810 $/$ name $=/$ prod $=/$ func $=$ cell adhesion $/$ map=93C3-93C $3 /$ transc $=\mathrm{CT} 18214 /$ len=1287 $/$ GB:AE003733 
FB:FBgn0037762 $/$ sym $=$ CG16905 $/$ name $=/$ prod $=/$ func $=/$ map $=85 \mathrm{E} 10-85 \mathrm{E} 10 /$ transc $=\mathrm{CT} 37510 / \mathrm{len}=781 / \mathrm{GB}: \mathrm{AE} 003684$

3.1 FB:FBgn0004586 /sym=grh /name=grainy head /prod=/func=specific RNA polymerase II transcription factor $/ \mathrm{map}=54 \mathrm{~F} 1-54 \mathrm{~F} 4 /$ transc $=\mathrm{CT} 42182 / \mathrm{len}=4748 / \mathrm{GB}: \mathrm{AE} 003801 /$ note $=3$ prime sequence fre FB:FBgn0034366 /sym=CG5489 /name=/prod=/func=enzyme $/$ map=55E4-55E $4 /$ transc $=$ CT42577 /len=1577 /GB:AE003799

FB:FBgn0028895/sym=BG:DS02740.8/name=/prod=/func=nucleic acid binding /map=35F8-35F8/transc=CT32539/len=1158 /GB:AE003650

FB:FBgn0039807/sym=CG15546/name $=/$ prod $=/$ func $=/$ map=100B1-100B1 $/$ transc $=$ CT35662 $/$ len $=1233 /$ GB:AE003775

FB:FBgn0037817/sym=Cyp12e1 /name=/prod=cytochrome P450, CYP12E1/func=cytochrome P45 /map=86A7-86A7/transc=CT34465/len=1518/GB:AE003687

3.1 FB:FBgn0023548 $/$ sym $=$ msta $/$ name $=/$ prod $=/$ func $=/$ map $=2 \mathrm{E} 2-2 \mathrm{E} 2 /$ transc $=\mathrm{CT} 40358 / \mathrm{len}=3150 / \mathrm{GB}: \mathrm{AE} 003423$

FB:FBgn0032490 $/$ sym $=$ CG16813 $/$ name $=/$ prod $=/$ func $=/$ map $=34$ A10-34A10 $/$ transc $=$ CT35578 $/$ len $=555 /$ GB:AE003639

FB:FBgn0031457/sym=CG3077/name=/prod=/func=ligand binding or carrier /map=23B1-23B1/transc=CT10334/len=1317 /GB:AE003582/note=3prime sequence from clone BDGP:LD12265.3p

3.1 FB:FBgn0024989/sym=EG:125H10.1/name=/prod=/func=motor $/$ map=1A8-1A8/transc=CT12604/len=2479/GB:AE003417

FB:FBgn0031933 $/ \mathrm{sym}=\mathrm{CG} 7068 /$ name $=/$ prod $=/$ func $=/ \mathrm{map}=28 \mathrm{C} 1-28 \mathrm{C} 1 /$ transc $=\mathrm{CT} 21827 /$ len=4745 $/$ GB:AE003618 $/$ note $=3$ prime sequence from clone BDGP:GH05679.3prime-hit

3.1 FB:FBgn0033807/sym=CG12251/name=aquaporin /prod=aquaporin /func=water transporter $/$ map=49F11-49F11/transc=CT14932/len=1180/GB:AE003819

3.1 FB:FBgn0023175 /sym=Prosalpha7 /name=Proteasome alpha7 subunit $/$ prod=20S proteasome, alpha7 subunit /func=multicatalytic endopeptidase ; EC:3.4.99.46 /map=46B13-46B13 /transc $=\mathrm{CT} 3927 / /$

3.1 FB:FBgn0030917 $/$ sym $=$ CG6267 $/$ name $=/$ prod $=/$ func $=/$ map=17A11 -17 A $11 /$ transc $=$ CT19600 $/$ len $=678 /$ GB:AE003508

FB:FBgn0031027/sym=CG14201/name=/prod=/func=transcription factor $/$ map=18D3-18D3/transc=CT33814/len=702 /GB:AE003512

FB:FBgn0032330 $/$ sym $=$ CG12291 $/$ name $=/$ prod $=/$ func $=/$ map $=32$ D1 $-32 \mathrm{D} 1 /$ transc $=$ CT19324 $/$ len $=382 /$ GB:AE003630

3.1 FB:FBgn0034142 /sym=CG8306 /name $=/$ prod $=/$ func $=$ enzyme $/$ map $=53 \mathrm{C} 7-53 \mathrm{C} 8 /$ transc $=\mathrm{CT} 24503 / \mathrm{len}=1937 / \mathrm{GB}: \mathrm{AE} 003806$

3.1 FB:FBgn0001250/sym=if /name=inflated /prod=integrin, alpha-subunit $/$ func $=$ cell adhesion receptor $/ \mathrm{map}=15 \mathrm{~A} 3-15 \mathrm{~A} 5 /$ transc $=\mathrm{CT} 27194 /$ len $=5726 / \mathrm{GB}: \mathrm{AE} 003503$

3.1 FB:FBgn0036370 $/$ sym $=$ CG14108 $/$ name $=/$ prod $=/$ func $=/$ map $=70 B 1-70 B 1 /$ transc $=\mathrm{CT} 33703 /$ len $=435 /$ GB:AE003537

3.1 FB:FBgn0003060 $/$ sym $=$ CG9757 $/$ name $=/$ prod $=/$ func $=/ \mathrm{map}=87 \mathrm{~F} 5-87 \mathrm{~F} 5 /$ transc $=\mathrm{CT} 27573 / \mathrm{len}=566 / \mathrm{GB}: \mathrm{AE} 003701$

3.1 FB:FBgn0000014/sym=abd-A /name=abdominal A /prod=/func=specific RNA polymerase II transcription factor $/$ map=89E4-89E4/transc=CT29034 /len=1931/GB:AE003715

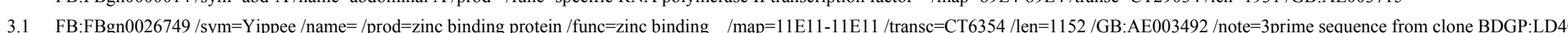

3.1 FB:FBgn0038293/sym=CG6904/name=/prod=/func=enzyme /map=88E5-88E5/transc=CT21366/len=2705/GB:AE003707/note=3prime sequence from clone BDGP:LD46952.3prime-hit

3.1 FB:FBgn0001112/sym=Gld /name=Glucose dehydrogenase $/$ prod=glucose dehydrogenase (acceptor) $==\mathrm{EC} 1.1 .99 .10 /$ func=glucose dehydrogenase $($ acceptor $)$; EC:1.1.99.10 $/$ map $=84 \mathrm{C} 7-84 \mathrm{C} 8 /$ transc=

3 FB:FBgn0003206 $/$ sym=Ras64B $/$ name $=$ Ras oncogene at 64B $/$ prod $=/$ func $=$ RAS small GTPase $/ \mathrm{map}=64 \mathrm{~A} 10-64 \mathrm{~A} 10 /$ transc $=\mathrm{CT} 1405 / \mathrm{len}=588 / \mathrm{GB}: \mathrm{AE} 003480$

3 FB:FBgn0033600/sym=CG9077 /name $=/$ prod $=$ cuticle protein $/$ func $=$ structural protein $/$ map=47E1-47E1 /transc $=\mathrm{CT} 26058 /$ len=396 /GB:AE003826 FB:FBgn0029585/sym=CG11511/name=/prod=/func=transcription factor $/ \mathrm{map}=2 \mathrm{~B} 6-2 \mathrm{~B} 6 /$ transc=CT34608 /len=1000/GB:AE003421

FB:FBgn0003511/sym=Sry-beta $/$ name=Serendipity beta $/$ rod $=/$ func=RNA polymerase II transcription factor $/$ map=99D5-99D5/transc=CT5812/len=1247/GB:AE003772/note=3prime sequence fi

3 FB:FBgn0003071 /sym=Pfk /name=Phosphofructokinase $/$ prod=6-phosphofructokinase $/$ func $=6$-phosphofructokinase $; \mathrm{EC}: 2.7 .1 .11 / \mathrm{map}=46 \mathrm{E} 4-46 \mathrm{E} 4 /$ transc $=\mathrm{CT} 13302 / \mathrm{len}=3176 / \mathrm{GB}: \mathrm{AE} 003830 /$ note $=$

3 FB:FBgn0040559 $/ \mathrm{sym}=\mathrm{CG} 14359 / \mathrm{name}=/ \mathrm{prod}=/$ func $=/ \mathrm{map}=88 \mathrm{~B} 1-88 \mathrm{~B} 1 / \mathrm{transc}=\mathrm{CT} 33994 / \mathrm{len}=237 / \mathrm{GB}: \mathrm{AE} 003703$

3 FB:FBgn0028693/sym=Rpn12/name=/prod=19S proteasome regulatory particle, non-ATPase protein, subunit S14/func=endopeptidase $/ \mathrm{map}=73 \mathrm{~A} 8-73 \mathrm{~A} 8 /$ transc $=\mathrm{CT} 13736 /$ len $=989 / \mathrm{GB}: \mathrm{AE} 00352$ FB:FBgn0038536 $/$ sym $=\mathrm{CG} 7655 /$ name $=/$ prod $=$ multipass nuclear envelope protein-like $/$ func $=$ transmembrane receptor $/$ map $=90 \mathrm{C} 1-90 \mathrm{C} 1 /$ transc $=\mathrm{CT} 23407 /$ len $=965 / \mathrm{GB}: \mathrm{AE} 003718 / \mathrm{note}=3 \mathrm{prime}$ seq 
44484.6

3552.4

38368.3

41645.2

14310.2

104072.4

30341.6

4080.2

4064.4

16524

86366.2

32359.3

2061.3

29445.5

38242.4

29174.8

6818

10857

67758.7

13006.7

1649.5

34712.3

25527.5

1435.5

32146.3

2417.1

6095.4

22931.3

$1923 \sim 2.9$

$2417.5 \sim 2.9$

143693.8
3 FB:FBgn0029533 $/$ sym $=$ CG5254 $/$ name $=/$ prod $=/$ func $=/$ map $=1 \mathrm{C} 1-1 \mathrm{C} 1 /$ transc $=\mathrm{CT} 16777 /$ len $=993 / \mathrm{GB}: \mathrm{AE} 003418$

3 FB:FBgn0032128 $/$ sym $=$ CG13115 $/$ name $=/$ prod $=/$ func $=/$ map=30B11-30B $11 /$ transc $=$ CT32352 $/$ len=956 $/$ GB:AE003625 $/$ note $=3$ prime sequence from clone BDGP:GH05993.3prime-hit

3 FB:FBgn0035030/sym $=\mathrm{CG} 3541 / \mathrm{name}=/ \mathrm{prod}=/ \mathrm{func}=/ \mathrm{map}=60 \mathrm{D} 7-60 \mathrm{D} 8 /$ transc $=\mathrm{CT} 11882 / \mathrm{len}=2417 /$ GB:AE003464 $/$ note $=3$ prime sequence from clone BDGP:GH12163.3prime-hit

3 FB:FBgn0022359/sym=Sodh-2 /name=Sorbitol dehydrogenase-2 /prod=L-iditol 2-dehydrogenase /func=L-iditol 2-dehydrogenase ; EC:1.1.1.14 /map=86C7-86C7 /transc=CT14906 /len=1345/GB:AEC

3 FB:FBgn0028685 $/$ sym=Rpt4 /name=/prod=19S proteasome regulatory particle, triple-A protein, subunit S10b $/$ func=proteasome ATPase ; EC:3.6.4.8 $/$ map $=5 \mathrm{E} 1-5 \mathrm{E} 1 /$ transc $=\mathrm{CT} 11623 / \mathrm{len}=1453 / \mathrm{GB}$

3 BDGP:HL664.3prime-hit /ESTpos=maps 3prime of FB:FBgn0038181/sym=CG9297/name $=/$ prod $=/$ func $=$ enzyme $/$ map $=87 \mathrm{~F} 13-87 \mathrm{~F} 13 /$ transc $=\mathrm{CT} 26475 /$ len $=608$

3 FB:FBgn0028691 /sym=Rpn4 /name=/prod=19S proteasome regulatory particle, non-ATPase protein, subunit S13/func=endopeptidase /map=95B5-95B5/transc=CT28751/len=1330/GB:AE00374

3 FB:FBgn0005771/sym=noc /name=no ocelli /prod=/func=RNA polymerase II transcription factor $/$ map=35A4-35A4 /transc=CT14619/len=2668/GB:AE003644 /note=3prime sequence from clone $\mathrm{B}$

3. FB:FBgn0031637/sym=CG2950/name =/prod=/func=RNA binding $/ \mathrm{map}=25 \mathrm{~B} 3-25 \mathrm{~B} 3 /$ transc=CT40278 /len=2649 /GB:AE003575/note=3prime sequence from clone BDGP:GH06479.3prime-hit

3 FB:FBgn0040520 $/$ sym $=$ CG12447 $/$ name $=/$ prod $=/$ func $=/$ map=20A1-20A $1 /$ transc $=$ CT32486 $/$ len $=132 /$ GB:AE003574

FB:FBgn0003944/sym=Ubx /name=Ultrabithorax /prod=/func=specific RNA polymerase II transcription factor /map=89D8-89E2/transc=CT29154/len=2204 /GB:AE003714

3 FB:FBgn0034497/sym=CG9090/name = /prod=phosphate transporter /func=carrier type transporter $/$ map=56F16-56F16/transc=CT25968 /len=1371 /GB:AE003792

2.9 FB:FBgn0029876 /sym=CG3960/name=/prod=/func=actin binding /map=6B3-6C1 /transc=CT13158/len=2129 /GB:AE003438 /note=3prime sequence from clone BDGP:GH02414.3prime-hi

FB:FBgn0027550/sym=BcDNA:GH10711/name=/prod=/func=receptor $/$ map=32A4-32A5/transc=CT20185 /len=2550/GB:AE003629/note=3prime sequence from clone BDGP:GH10711.3prime-

2.9 FB:FBgn0034399 $/ \mathrm{sym}=\mathrm{CG} 15083 / \mathrm{name}=/ \mathrm{prod}=/ \mathrm{func}=/ \mathrm{map}=55 \mathrm{~F} 3-55 \mathrm{~F} 3 /$ transc $=\mathrm{CT} 34958 / \mathrm{len}=453 / \mathrm{GB}: \mathrm{AE} 003798$

2.9 FB:FBgn0033553/sym $=$ CG12323 $/$ name $=/$ prod $=/$ func $=/$ map $=47 \mathrm{C} 1-47 \mathrm{C} 1 /$ transc $=\mathrm{CT} 22275 /$ len=1114 $/$ GB:AE003828 $/$ note $=3$ prime sequence from clone BDGP:LD08717.3prime-hit

2.9 FB:FBgn0031307/sym=CG4726/name $=/$ prod=sodium/phosphate cotransporter /func=transporter $/$ map=21E4-21E4 /transc=CT15243/len=2003 /GB:AE003587

2.9 FB:FBgn0032129 $/$ sym $=$ CG4405 $/$ name $=/$ prod $=/$ func $=$ RNA binding $/ \mathrm{map}=30 \mathrm{~B} 11-30 \mathrm{~B} 12 /$ transc $=\mathrm{CT} 14344 / \mathrm{len}=3801 / \mathrm{GB}: \mathrm{AE} 003625$

2.9 FB:FBgn0035927/sym=CG5775 /name $=/$ prod $=/$ func $=/$ map=66E1-66E1 $/$ transc $=$ CT18132 $/$ len=837 /GB:AE003554 /note=3prime sequence from clone BDGP:SD08909.3prime-hit

2.9 FB:FBgn0034470/sym $=\mathrm{CG} 11218 / \mathrm{name}=/$ prod $=$ antennal binding protein X-like $/$ func $=$ ligand binding or carrier $/ \mathrm{map}=56 \mathrm{E} 4-56 \mathrm{E} 4 / \mathrm{transc}=\mathrm{CT} 31326 / \mathrm{len}=574 / \mathrm{GB}: \mathrm{AE} 003795$

2.9 FB:FBgn0036891/sym=CG9372/name=/prod=serine protease-like /func=endopeptidase $/$ map=76B11-76B11/transc=CT26619/len=1569/GB:AE003516

FB:FBgn0039706 $/$ sym $=$ CG18040 $/$ name $=/$ prod $=/$ func $=/$ map $=99$ C5-99C $6 /$ transc $=$ CT40392 $/$ len $=786 /$ GB:AE003771

2.9 FB:FBgn0032427 /sym=CG5453 /name $=/$ prod $=/$ func $=/$ map=33D4-33D4/transc=CT17294 /len=819 /GB:AE003636/note=3prime sequence from clone BDGP:GH02216.3prime-hit

2.9 FB:FBgn0010397/sym=L

FB:FBgn0001624/sym=dlg1 /name=discs large $1 /$ prod=guanylate kinase $/$ func=guanylate kinase $; \mathrm{EC}: 2.7 .4 .8 / \mathrm{map}=10 \mathrm{~B} 11-10 \mathrm{~B} 12 / \mathrm{transc}=\mathrm{CT} 41310 / \mathrm{len}=631 / \mathrm{GB}: \mathrm{AE} 003486 / \mathrm{note}=3 \mathrm{prime}$ sequence fr

2.9 FB:FBgn0035517 $/ \mathrm{sym}=\mathrm{CG} 1265 / \mathrm{name}=/ \mathrm{prod}=/ \mathrm{func}=/ \mathrm{map}=64 \mathrm{~B} 4-64 \mathrm{~B} 4 /$ transc $=\mathrm{CT} 2591 / \mathrm{len}=549 / \mathrm{GB}: \mathrm{AE} 003480$

FB:FBgn0032013 $/$ sym $=$ CG7851 $/$ name $=/$ prod $=/$ func $=/$ map $=29 A 4-29 \mathrm{~A} 4 /$ transc $=C T 23800 /$ len $=1391 /$ GB:AE003620

2.9 FB:FBgn0030494 /sym=CG15757 /name $=/$ prod $=$ cuticle protein-like $/$ func $=$ structural protein $/ \mathrm{map}=12 \mathrm{~A} 1-12 \mathrm{~A} 1 /$ transc=CT36009 $/$ len=522 $/ \mathrm{GB}: \mathrm{AE} 003492$

2.9 FB:FBgn0040575 $/$ sym $=$ CG15922 $/$ name $=/$ prod $=/$ func $=/$ map=92E10-92E $10 /$ transc $=$ CT35885 $/$ len $=159 /$ GB:AE003731

FB:FBgn0035199/sym=CG9134/name=/prod=C-type lectin-like /func=ligand binding or carrier $/$ map=61F4-61F4 /transc=CT10115/len=1161 /GB:AE003471 /note=3prime sequence from clone BD FB:FBgn0004957 /sym=por $/$ name=porcupine $/$ prod $=/$ func $=/$ map=17A10-17A10 $/$ transc $=$ CT19254 $/$ len=2331 $/$ GB:AE003508

2.9 FB:FBgn0003075/sym=Pgk /name=Phosphoglycerate kinase $/$ prod=phosphoglycerate kinase $/$ func=phosphoglycerate kinase $;$ EC:2.7.2.3 /map=23A7-23A7/transc=CT10484 /len=1361 /GB:AE003582 
2.9 FB:FBgn0035542 $/$ sym $=$ CG1 $1347 /$ name $=/$ prod $=/$ func $=\quad /$ map $=64 \mathrm{~B} 11-64 \mathrm{~B} 11 /$ transc $=\mathrm{CT} 31652 /$ len=2045 $/$ GB:AE003481 $/$ note $=3$ prime sequence from clone BDGP:GH28550.3prime-hit

2.8 FB:FBgn0031914 /sym=CG5973 /name=/prod=/func=ligand binding or carrier $/$ map=27F7-28A1/transc=CT18751 /len=1519/GB:AE003617 /note=3prime sequence from clone BDGP:HL01515.3p 2.8 FB:FBgn0028688 /sym=Rpn7 /name $=/$ prod=19S proteasome regulatory particle, non-ATPase protein, subunit S10a /func=endopeptidase $/ \mathrm{map}=94 \mathrm{~B} 3-94 \mathrm{~B} 3 / \mathrm{transc}=\mathrm{CT} 17076 / \mathrm{len}=1374 / \mathrm{GB}: \mathrm{AE} 0037$ 2.8 FB:FBgn0035062 $/ \mathrm{sym}=\mathrm{CG} 16914 / \mathrm{name}=/$ prod=larval cuticle protein-like $/$ func $=$ structural protein $/ \mathrm{map}=60 \mathrm{D} 15-60 \mathrm{D} 15 /$ transc $=\mathrm{CT} 37520 / \mathrm{len}=279 / \mathrm{GB}: \mathrm{AE} 003465$

2.8 FB:FBgn0034064 /sym=CG8392 /name $=/$ prod $=20$ S proteasome, beta1 subunit $/$ func $=$ endopeptidase $/ \mathrm{map}=52 \mathrm{E} 1-52 \mathrm{E} 1 /$ transc $=\mathrm{CT} 18263 /$ len $=829 / \mathrm{GB}: \mathrm{AE} 003808 /$ note $=3$ prime sequence from clone 2.8 FB:FBgn0040941 $/$ sym $=$ CG15308 $/$ name $=/$ prod $=/$ func $=/$ map=9B6-9B6 $/$ transc $=$ CT35285 $/$ len $=249 /$ GB:AE003449

2.8 FB:FBgn0031629/sym=CG3244 /name=/prod=selectin-like /func=ligand binding or carrier $/$ map=25B1-25B1/transc=CT10874 /len=1267/GB:AE003575

2.8 FB:FBgn0032727 $/$ sym $=$ CG10623 $/$ name $=/$ prod $=/$ func $=/$ map=37B8-37B8 $/$ transc $=$ CT29758 $/$ len $=1114 /$ GB:AE00366

2.8 FB:FBgn0020369/sym=Pros $45 /$ name=Saccharomyces cerevisiae UAS construct a of Cheng /prod=19S proteasome regulatory particle, triple-A protein, subunit S8 /func=proteasome ATPase ; EC 3.6 .

2.8 FB:FBgn0034975 /sym=CG11290/name $=/$ prod=histone acetyltransferase-like $/$ func=enzyme $/$ map=60B5-60B5 /transc=CT31509/len=6956 /GB:AE003463 /note=3prime sequence from clone BDGP FB:FBgn0024887 /sym=kin $17 /$ name $=/$ prod= $/$ func $=$ DNA binding $/$ map=77B4-77B4 $/$ transc $=$ CT17834 /len=1241 /GB:AE003591

2.8 BDGP:GH13437.3prime-hit /ESTpos=maps 3prime of FB:FBgn0039493 $/$ sym=CG5889 $/$ name $=/$ prod $=/$ func $=/$ map=97E11-97F1 $/$ transc=CT18483 $/$ len=374

2.8 FB:FBgn0028544/sym=BG:DS00180.3/name=/prod=/func= /map=34E1-34E1/transc=CT25718/len=1397 /GB:AE003641/note=3prime sequence from clone BDGP:HL02234.3prime-hit

2.8 FB:FBgn0031067/sym $=$ CG12533 $/$ name $=/$ prod $=/$ func $=$ actin binding $/ \mathrm{map}=18 \mathrm{~F} 1-18 \mathrm{~F} 1 /$ transc $=\mathrm{CT} 33853 / \mathrm{len}=2079 / \mathrm{GB}: \mathrm{AE} 003513$

2.8 FB:FBgn0034140 $/ \mathrm{sym}=\mathrm{CG} 8317 /$ name $=/$ prod $=/$ func $=/ \mathrm{map}=53 \mathrm{C} 7-53 \mathrm{C} 7 /$ transc $=\mathrm{CT} 24573 / \mathrm{len}=693 / \mathrm{GB}: \mathrm{AE} 003806$

2.8 FB:FBgn0014869/sym=Pglym78 /name=Phosphoglyceromutase /prod=phosphoglycerate mutase /func=phosphoglycerate mutase ; EC:5.4.2.1 /map=99A1-99A1 /transc=CT4904 /len=1125 /GB:AE003

2.8 FB:FBgn0004066 /sym=Pros28.1 /name=Proteasome $28 \mathrm{kD}$ subunit $1 /$ prod=20S proteasome, alpha4 subunit /func=multicatalytic endopeptidase $; \mathrm{EC}: 3.4 .99 .46 / \mathrm{map}=14 \mathrm{~B} 11-14 \mathrm{~B} 11 /$ transc $=\mathrm{CT} 11501 / 1$

2.8 FB:FBgn0000084 /sym=AnnX /name=Annexin X/prod=annexin X /func=calcium-dependent phospholipid binding /map=19C1-19C1/transc=CT17636/len=1216/GB:AE002611 /note=3prime seque

2.8 FB:FBgn0035334 /sym=CG8993 /name=/prod=mitochondrial thioredoxin-like /func=chaperone $/ \mathrm{map}=62 \mathrm{E} 1-62 \mathrm{E} 1 / \mathrm{transc}=\mathrm{CT} 25846 / \mathrm{len}=484 / \mathrm{GB}: \mathrm{AE} 003474$

2.8 FB:FBgn0032538 $/$ sym $=$ CG16885 $/$ name $=/$ prod $=/$ func $=/$ map $=34 \mathrm{E} 1-34 \mathrm{E} 1 /$ transc $=\mathrm{CT} 35249 / \mathrm{len}=780 / \mathrm{GB}: \mathrm{AE} 003641$

2.8 FB:FBgn0033959 $/$ sym $=\mathrm{CG} 18372 /$ name $=/$ prod $=/$ func $=/$ map $=51 \mathrm{C} 2-51 \mathrm{C} 2 /$ transc $=\mathrm{CT} 41763 / \mathrm{len}=674 / \mathrm{GB}: \mathrm{AE} 003813$

2.8 FB:FBgn0031322 $/ \mathrm{sym}=\mathrm{CG} 5001 / \mathrm{name}=/ \mathrm{prod}=/$ func $=$ chaperone $/ \mathrm{map}=21 \mathrm{~F} 1-21 \mathrm{~F} 1 /$ transc $=\mathrm{CT} 15884 / \mathrm{len}=1356 / \mathrm{GB}: \mathrm{AE} 003587$

2.8 FB:FBgn0026380 $/$ sym $=$ Prosbeta3 $/$ name $=/$ prod $=20 \mathrm{~S}$ proteasome, beta3 subunit $/$ func $=$ multicatalytic endopeptidase $; \mathrm{EC}: 3.4 .99 .46 / \mathrm{map}=85 \mathrm{C} 3-85 \mathrm{C} 3 / \mathrm{transc}=\mathrm{CT} 32122 / \mathrm{len}=915 / \mathrm{GB}: \mathrm{AE} 003681 / \mathrm{note}=$ 2.8 BDGP:LD8622.3prime-hit /ESTpos=maps 3prime of FB:FBgn0013718 $/$ sym=nuf $/$ name=nuclear fallout $/$ prod $=/$ func=cytoskeletal structural protein $/ \mathrm{map}=7 \mathrm{D} 2-7 \mathrm{D} 2 /$ transc $=\mathrm{CT} 23449 /$ len=291

2.8 FB:FBgn0035788 $/ \mathrm{sym}=\mathrm{CG} 8541 /$ name $=/$ prod $=$ cuticle protein $/$ func=ligand binding or carrier $/ \mathrm{map}=66 \mathrm{~A} 1-66 \mathrm{~A} 1 / \mathrm{transc}=\mathrm{CT} 15726 / \mathrm{len}=828 / \mathrm{GB}: \mathrm{AE} 003559$

FB:FBgn0038063 $/$ sym $=\mathrm{CG} 6989 /$ name $=/$ prod=beta adrenergic receptor-like $/$ func $=\mathrm{G}$ protein linked receptor $/ \mathrm{map}=87 \mathrm{C} 2-87 \mathrm{C} 2 /$ transc $=\mathrm{CT} 21650 / \mathrm{len}=1224 / \mathrm{GB}: \mathrm{AE} 003696$

FB:FBgn0000462 /sym=dl /name=dorsal /prod=/func=specific RNA polymerase II transcription factor $/$ map=36C2-36C2 /transc=CT42418/len=4565 /GB:AE003655

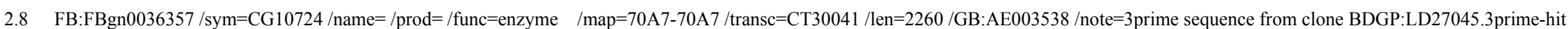
FB:FBgn0039484/sym=CG6124 /name $=/$ prod $=/$ func=cell adhesion $/$ map=97E6-97E6 $/$ transc $=$ CT19227/len=2673 /GB:AE003759

2.8 FB:FBgn0037744/sym=CG8417/name=/prod=mannose-6-phosphate isomerase $/$ func=enzyme $/$ map=85E8-85E8 /transc=CT24707 /len=1398 $/$ GB:AE003684 $/$ note $=3$ prime sequence from clone BDC 2.8 FB:FBgn0020304/sym=drongo $/$ name=drongo $/$ prod= /func=defense/immunity protein $/$ map=21D2-21D2/transc=CT42210 /len=2383/GB:AE003588 $/$ note=3prime sequence from clone BDGP:GH1 2.8 FB:FBgn0035390 $/$ sym $=\mathrm{CG} 1893 /$ name $=/$ prod $=/$ func $=/$ map $=63$ B5 $-63 \mathrm{~B} 5 /$ transc $=\mathrm{CT} 5862 / \mathrm{len}=834 / \mathrm{GB}: \mathrm{AE} 003476$ 
2.8 FB:FBgn0005666 /sym=bt /name=bent /prod=projectin /func=cell adhesion $/$ map=102D6-102E1 /transc=CT3598 /len=23764 /GB:AE003843 /note=3prime sequence from clone BDGP:GH07636.3pri FB:FBgn0037651/sym $=$ CG1 $1978 /$ name $=/$ prod $=/$ func $=/$ map $=85 \mathrm{C} 3-85 \mathrm{C} 3 /$ transc $=\mathrm{CT} 32124 / \mathrm{len}=456 / \mathrm{GB}: \mathrm{AE} 003681$

FB:FBgn0033526 $/$ sym=CG12892 $/$ name $=/$ prod $=/$ func $=$ enzyme $/$ map=47A9-47A9 $/$ transc $=$ CT32037 /len=2244 $/$ GB:AE003829

2.8 FB:FBgn0016762 $/$ sym=angel $/$ name $=$ angel $/$ prod $=/$ func $=$ enzyme $/$ map=59F4-59F4 $/$ transc $=$ CT17360 $/$ len=1184 $/$ GB:AE003461 /note=3prime sequence from clone BDGP:GH06351.3prime-hit

2.8 FB:FBgn0030526 $/ \mathrm{sym}=\mathrm{CG} 11102 / \mathrm{name}=/$ prod $=/$ func $=/ \mathrm{map}=12 \mathrm{~B} 9-12 \mathrm{~B} 9 /$ transc $=\mathrm{CT} 31063 / \mathrm{len}=1671 / \mathrm{GB}: \mathrm{AE} 003493$

2.8 FB:FBgn0003738/sym=Tpi /name=Triose phosphate isomerase /prod=triosephosphate isomerase /func=triosephosphate isomerase ; EC:5.3.1.1/map=99E1-99E1/transc=CT6334/len=1186/GB:AE003 2.8 BDGP:LD1876.3prime-hit $/$ ESTpos=maps 3prime of FB:FBgn0038501 $/ \mathrm{sym}=\mathrm{CG} 5319 / \mathrm{name}=/ \mathrm{prod}=/$ func $=/ \mathrm{map}=9 \mathrm{~A} 6-9 \mathrm{~A} 6 / \mathrm{transc}=\mathrm{CT} 1693 / \mathrm{len}=567$

2.8 FB:FBgn0002789 /sym=Mp20/name=Muscle protein $2 /$ prod=calcium-binding protein /func=calcium binding $/$ map=49F15-49F15/transc=CT15161 /len=852 /GB:AE003819

2.7 FB:FBgn0030753/sym=CG4420 /name=/prod=/func=DNA binding /map=14D1-14D1/transc=CT14402/len=1880 /GB:AE003502/note=3prime sequence from clone BDGP:GM04721.3prime-hit

2.7 FB:FBgn0039914/sym=CG1901/name=/prod=transforming growth factor beta-like $/$ func=signal transduction $/$ map=102D1-102D1/transc=CT5854/len=2528 $/$ GB:AE003843

2.7 FB:FBgn0032282 $/$ sym $=$ CG7299 $/$ name $=/$ prod $=/$ func $=/$ map $=32 \mathrm{~A} 1-32 \mathrm{~A} 1 /$ transc $=\mathrm{CT} 22515 / \mathrm{len}=534 / \mathrm{GB}: \mathrm{AE} 003629$

2.7 FB:FBgn0037138/sym=CG7145/name=/prod=1-pyrroline-5-carboxylate dehydrogenase-like /func=enzyme /map=79A5-79A5/transc=CT22083/len=1890/GB:AE003595 FB:FBgn0036656/sym $=$ CG13026 $/$ name $=/$ prod $=/$ func $=/$ map $=73$ B5-73B $5 /$ transc $=$ CT32244 $/$ len $=405 /$ GB:AE003526

2.7 FB:FBgn0033593 $/$ sym $=$ CG9080 $/$ name $=/$ prod $=/$ func $=/$ map $=47 \mathrm{E} 1-47 \mathrm{E} 1 /$ transc $=\mathrm{CT} 26066 / \mathrm{len}=435 / \mathrm{GB}: \mathrm{AE} 003826$

2.7 FB:FBgn0000486 /sym=Dox-A2 /name=Diphenol oxidase A2 /prod=proteasome, regulatory subunit S3 /func=multicatalytic endopeptidase ; EC:3.4.99.46 | inferred from sequence similarity /map=37B 2.7 FB:FBgn0035232 $/$ sym $=\mathrm{CG} 12099 /$ name $=/$ prod $=/$ func $=/$ map $=62 \mathrm{~A} 7-62 \mathrm{~A} 7 /$ transc $=\mathrm{CT} 6023 / \mathrm{len}=2445 / \mathrm{GB}: \mathrm{AE} 003472$

2.7 FB:FBgn0010423 /sym=TpnC47D /name=Troponin C at 47D /prod=troponin C /func=calcium binding /map=47E1-47E1 /transc=CT26048/len=586/GB:AE003826

2.7 FB:FBgn0004169/sym=up /name=upheld /prod=troponin T /func=tropomyosin binding /map=12A2-12A4/transc=CT41714/len=1424/GB:AE003493

BDGP:GH27479.3prime-hit /ESTpos=maps in FB:FBgn0031850/sym=CG11326/name=/prod=thrombospondin-3 like $/$ func=cell adhesion $/ \mathrm{map}=26 \mathrm{~F} 6-27 \mathrm{~A} 1 /$ transc $=\mathrm{CT} 31613 /$ len $=523$

2.7 FB:FBgn0000568 /sym=Eip75B /name=Ecdysone-induced protein 75B /prod=nuclear receptor NR1D3 /func=ligand-dependent nuclear receptor $/ \mathrm{map}=75 \mathrm{~A} 10-75 \mathrm{~B} 6 /$ transc=CT24290 /len=4527 /GB:A FB:FBgn0035345 $/$ sym $=C$ CG16764 $/$ name $=/$ prod $=/$ func $=/$ map $=62$ E $5-62$ E $6 /$ transc $=$ CT37287 $/$ len $=577 /$ GB:AE003475

2.7 FB:FBgn0037821 /sym=CG14682 $/$ name $=/$ prod $=/$ func=nucleic acid binding $/ \mathrm{map}=86 \mathrm{C} 2-86 \mathrm{C} 2 /$ transc=CT34468 $/$ len=3926 $/$ GB:AE003688 $/$ note $=3$ prime sequence from clone BDGP:GH12580.3prin

2.7 FB:FBgn0039136/sym $=$ CG5902 $/$ name $=/$ prod $=/$ func $=/$ map=95D1-95D $1 /$ transc $=$ CT18529 $/$ len=1603 $/$ GB:AE003745 $/$ note $=3$ prime sequence from clone BDGP:SD10002.3prime-hit

2.7 FB:FBgn0037747/sym=CG8481/name=/prod=N-acetyltransferase /func=enzyme /map=85E8-85E8/transc=CT24815/len=1390/GB:AE003684 /note=3prime sequence from clone BDGP:GH04732

2.7 FB:FBgn0034412 /sym $=$ CG15105 /name $=/$ prod $=/$ func $=$ transcription factor $/ \mathrm{map}=56 \mathrm{~A} 1-56 \mathrm{~A} 2 /$ transc $=\mathrm{CT} 34980 /$ len $=3668 /$ GB:AE003797 $/$ note $=3$ prime sequence from clone BDGP:GH06739.3prim

2.7 FB:FBgn0038130 $/$ sym $=$ CG8630 $/$ name $=/$ prod $=/$ func $=$ enzyme $/$ map $=87$ E5-87E5 $/$ transc $=$ CT25031 /len $=1227 /$ GB:AE003699

2.7 FB:FBgn0002772/sym=Mlc1 /name=Myosin alkali light chain $1 /$ prod=myosin muscle class II essential light chain /func=muscle motor protein $/$ map=98A6-98A6/transc=CT17694 /len=722 /GB:AEC 2.7 FB:FBgn0039268 $/$ sym=CG11819 $/$ name $=/$ prod $=/$ func=protein kinase $/ \mathrm{map}=96 \mathrm{~B} 15-96 \mathrm{~B} 16 /$ transc $=\mathrm{CT} 36931 / \mathrm{len}=2933 / \mathrm{GB}: \mathrm{AE} 003750$

FB:FBgn0023180/sym=Orc6 $/$ name=Origin recognition complex subunit $6 /$ prod=origin recognition complex, subunit $6 /$ func $=$ DNA replication factor $/ \mathrm{map}=46 \mathrm{~B} 13-46 \mathrm{~B} 13 /$ transc $=\mathrm{CT} 4175 /$ len $=774$ 2.7 FB:FBgn0032218 $/ \mathrm{sym}=\mathrm{CG} 5381 / \mathrm{name}=/ \mathrm{prod}=/$ func $=$ transcription factor $/ \mathrm{map}=31 \mathrm{D} 8-31 \mathrm{D} 8 /$ transc $=\mathrm{CT} 17078 / \mathrm{len}=1884 / \mathrm{GB}: \mathrm{AE} 003628$

2.7 FB:FBgn0033631/sym=CG9027/name=/prod=superoxide dismutase-like /func=enzyme /map=47F6-47F7/transc=CT25938/len=477/GB:AE003826

2.7 FB:FBgn0004646 /sym=ogre /name =optic ganglion reduced /prod=innexin /func=ion channel $/$ map=6E4-6E4 /transc=CT9674/len=2219/GB:AE003439 /note=3prime sequence from clone BDGP:HL 
FB:FBgn0038572 $/$ sym $=$ CG7901 $/$ name $=/$ prod $=/$ func $=/$ map $=90 \mathrm{E} 4-90 \mathrm{E} 4 /$ transc $=\mathrm{CT} 42545 /$ len $=937 /$ GB:AE003721

2.7 FB:FBgn0028695/sym=Rpn $/$ name $=/$ prod=19S proteasome regulatory particle, non-ATPase protein, subunit S2 $/$ func=endopeptidase $/$ map=76D7-76D7/transc=CT23606 /len=2967 /GB:AE003515 FB:FBgn0037806 $/$ sym $=$ CG1 $1872 /$ name $=/$ prod $=/$ func $=/$ map $=86$ A $1-86$ A $2 /$ transc $=$ CT37024 $/$ len $=4311 /$ GB:AE003686

2.7 FB:FBgn0034969/sym=CG10485/name $=/$ prod=ribosomal protein L12-like $/$ func=structural protein of ribosome $/ \mathrm{map}=60 \mathrm{~B} 2-60 \mathrm{~B} 2 / \mathrm{transc}=\mathrm{CT} 29426 / \mathrm{len}=675 / \mathrm{GB}: \mathrm{AE} 003462$

2.7 FB:FBgn0039909 /sym=CG1970/name $=/$ prod=NADH-ubiquinone oxidoreductase $/$ func $=$ enzyme $/ \mathrm{map}=102 \mathrm{C} 5-102 \mathrm{C} 5 /$ transc $=\mathrm{CT} 6146 /$ len $=1482 / \mathrm{GB}: \mathrm{AE} 003843$

2.7 FB:FBgn0003149/sym=Prm /name=Paramyosin /prod=paramyosin /func=structural protein of muscle /map=66D14-66D14/transc=CT18619/len=2721 /GB:AE003554/note=3prime sequence from c

2.6 FB:FBgn0003178 /sym=PyK /name=Pyruvate kinase /prod=pyruvate kinase $/$ func=pyruvate kinase $;$ EC:2.7.1.40 /map=94A15-94A15/transc=CT21861/len=2091 /GB:AE003738/note=3prime sequenc FB:FBgn0016697/sym=ProsMA5 $/$ name=Proteasome alpha subunit $/$ prod=20S proteasome, alpha5 subunit $/$ func $=$ multicatalytic endopeptidase $; \mathrm{EC}: 3.4 .99 .46 / \mathrm{map}=54 \mathrm{C} 1-54 \mathrm{C} 1 /$ transc $=\mathrm{CT} 30641 /$ len= 2.6 BDGP:LD33318.3prime-hi

FB:FBgn0038591/sym=CG7150/name=/prod=transcriptional adaptor-like /func=transcription factor binding /map=90F4-90F4/transc=CT22097/len=1876 /GB:AE003721

2.6 FB:FBgn0032148 /sym=CG13122 /name $=/$ prod= /func= /map=30D1-30D1/transc=CT32359/len=1567 /GB:AE003626/note=3prime sequence from clone BDGP:HL02309.3prime-hit

2.6 FB:FBgn0003074 /sym=Pgi /name=Phosphoglucose isomerase /prod=phosphogluconate dehydrogenase (decarboxylating)/func=phosphogluconate dehydrogenase (decarboxylating); EC:1.1.1.44/map=

2.6 FB:FBgn0031187/sym=CG14619/name =/prod=ubiquitin-specific protease $/$ func=ubiquitin-specific protease $/ \mathrm{map}=19 \mathrm{~F} 5-19 \mathrm{~F} 6 /$ transc $=\mathrm{CT} 34376 /$ len=2865 $/ \mathrm{GB}: \mathrm{AE} 003574 /$ note $=3$ prime sequence fro

2.6 FB:FBgn0033126 $/$ sym $=C$ CG10106 $/$ name $=/$ prod $=/$ func $=/$ map $=42$ E $1-42 \mathrm{E} 1 /$ transc $=$ CT7946 $/$ len $=1113 /$ GB:AE003842

FB:FBgn0037543 $/$ sym $=$ CG10903 $/$ name $=/$ prod $=/$ func $=/$ map $=84$ E7-84E7 $/$ transc $=C T 10979 /$ len $=831 /$ GB:AE003677

FB:FBgn0040733 $/$ sym $=$ CG15068 $/$ name $=/$ prod $=/$ func $=/$ map $=55$ C $9-55$ C $9 /$ transc $=$ CT34939 $/$ len $=189 /$ GB:AE003799

2.6 FB:FBgn0034952 $/ \mathrm{sym}=\mathrm{CG} 18021 /$ name $=/$ prod $=/$ func $=/ \mathrm{map}=60 \mathrm{~A} 15-60 \mathrm{~A} 15 /$ transc $=\mathrm{CT} 40326 / \mathrm{len}=2017 /$ GB:AE003462 $/$ note $=3$ prime sequence from clone BDGP $:$ GH20492.3prime-hit

2.6 FB:FBgn0036108 $/ \mathrm{sym}=\mathrm{CG} 7941 / \mathrm{name}=/$ prod $=$ cuticle protein-like $/$ func $=$ structural protein $/ \mathrm{map}=67 \mathrm{~F} 4-67 \mathrm{~F} 4 /$ transc $=\mathrm{CT} 23954 / \mathrm{len}=405 / \mathrm{GB}: \mathrm{AE} 003546$

2.6 FB:FBgn0035464 $/ \mathrm{sym}=\mathrm{CG} 12006 /$ name $=/$ prod $=/$ func $=/ \mathrm{map}=63 \mathrm{~F} 1-63 \mathrm{~F} 1 /$ transc $=\mathrm{CT} 1387 / \mathrm{len}=1873 / \mathrm{GB}: \mathrm{AE} 003479 /$ note $=3$ prime sequence from clone BDGP:LD47795.3prime-hit

2.6 BDGP:LD23884.3prime-hit $/$ ESTpos=maps in FB:FBgn0030430/sym=CG4410/name=/prod=chaperone-like protein /func=chaperone $/ \mathrm{map}=11 \mathrm{C} 4-11 \mathrm{C} 4 /$ transc $=\mathrm{CT} 4259 / \mathrm{len}=500$

2.6 FB:FBgn0023215 /sym=EG:114E2.2/name=/prod=/func=transcription factor binding /map=3F2-3F2 /transc=CT9776/len=1830/GB:AE003428 /note=3prime sequence from clone BDGP:GH28809.

2.6 FB:FBgn0037252/sym $=\mathrm{CG} 14650 /$ name $=/$ prod $=/$ func $=$ chaperone $/ \mathrm{map}=82 \mathrm{C} 1-82 \mathrm{C} 1 /$ transc $=\mathrm{CT} 34422 / \mathrm{len}=3606 /$ GB:AE003606 $/$ note $=3$ prime sequence from clone BDGP:GH27269.3prime-hit

2.6 FB:FBgn0033179/sym $=$ CG11139 $/$ name $=/$ prod $=/$ func $=/ \mathrm{map}=43 \mathrm{C} 4-43 \mathrm{C} 5 /$ transc $=\mathrm{CT} 31143 /$ len=1419 $/$ GB:AE003841 /note $=3$ prime sequence from clone BDGP:GH01724.3prime-hit

2.6 FB:FBgn0037314 $/$ sym $=$ CG12000 $/$ name $=/$ prod $=20 \mathrm{~S}$ proteasome, beta4 subunit-like $/$ func $=$ endopeptidase $/ \mathrm{map}=83 \mathrm{~A} 4-83 \mathrm{~A} 4 /$ transc $=\mathrm{CT} 1070 /$ len $=1216 / \mathrm{GB}: \mathrm{AE} 003603 / \mathrm{note}=3$ prime sequence from

2.6 FB:FBgn0028689/sym=Rpn6 /name=Proteasome p44.5 subunit/prod=19S proteasome regulatory particle, non-ATPase protein, subunit S9 /func=multicatalytic endopeptidase ; EC:3.4.99.46 inferred

2.6 FB:FBgn0012042/sym=AttA /name=Attacin-A /prod=attacin $/$ func=antibacterial response protein $/$ map $=51 \mathrm{C} 2-51 \mathrm{C} 2 /$ transc $=\mathrm{CT} 28545 / \mathrm{len}=895 / \mathrm{GB}: \mathrm{AE} 003813 / \mathrm{note}=3$ prime sequence from clone $\mathrm{BD}$

2.6 FB:FBgn0022986 /sym=qkr58E-1 /name=quaking related 58E-1 /prod=/func=RNA binding /map=58D8-58D8 /transc=CT12115/len=1618/GB:AE003457 /note=3prime sequence from clone BDGP:

2.6 FB:FBgn0032538 $/$ sym $=$ CG16885 $/$ name $=/$ prod $=/$ func $=/$ map $=34 \mathrm{E} 1-34 \mathrm{E} 1 /$ transc $=\mathrm{CT} 35249 / \mathrm{len}=780 / \mathrm{GB}: \mathrm{AE} 003641$

2.6 FB:FBgn0039110/sym=CG10225/name=/prod=/func=structural protein $/ \mathrm{map}=95 \mathrm{~B} 7-95 \mathrm{~B} 7 /$ transc=CT28739/len=1726 /GB:AE003744 /note=3prime sequence from clone BDGP:LD02979.3prime-1

2.6 FB:FBgn0002787/sym=Mov34/name=Mov34/prod=19S proteasome regulatory particle, non-ATPase protein,subunit S12 /func=multicatalytic endopeptidase ; EC:3.4.99.46/map=60D1-60D1/transc

2.6 FB:FBgn0030479/sym=CG1987/name=/prod=RNA binding protein-like /func=RNA binding /map=11E11-11E11 /transc=CT6330/len=393/GB:AE003492

2.6 FB:FBgn0000480/sym=Doa $/$ name=Darkener of apricot $/$ prod=protein serine/threonine kinase $/$ func=protein kinase $/$ map $=98 \mathrm{~F} 1-98 \mathrm{~F} 2 /$ transc $=\mathrm{CT} 4592 / \mathrm{len}=2232 / \mathrm{GB}: \mathrm{AE} 003767 /$ note $=3 \mathrm{prime}$ sequen FB:FBgn0039688/sym=CG1964 /name=/prod=ADAM10 family metalloendopeptidase/disintegrin-like /func=endopeptidase $/$ map=99C1-99C1/transc=CT3146/len=4614/GB:AE003770 
2.6 FB:FBgn0002641 /sym=mal /name=maroon-like $/$ prod=molybdopterin cofactor sulfurase-like $/$ func=molybdopterin cofactor sulfurase $/ \mathrm{map}=19 \mathrm{D} 2-19 \mathrm{D} 3 / \mathrm{transc}=\mathrm{CT} 4746 /$ len=2717 $/ \mathrm{GB}: \mathrm{AE} 003571 / \mathrm{nc}$

2.6 FB:FBgn0039423 $/ \mathrm{sym}=\mathrm{CG} 6166 /$ name $=/$ prod $=/$ func $=/ \mathrm{map}=97 \mathrm{~A} 9-97 \mathrm{~A} 9 /$ transc $=\mathrm{CT} 19364 / \mathrm{len}=2031 / \mathrm{GB}: \mathrm{AE} 003756 /$ note $=3$ prime sequence from clone BDGP:GH14066.3prime-hit FB:FBgn0038041 /sym $=\mathrm{CG} 6525 /$ name $=/$ prod $=/$ func $=$ ligand binding or carrier $/$ map $=87 \mathrm{~B} 15-87 \mathrm{~B} 15 /$ transc $=\mathrm{CT} 20279 / \mathrm{len}=6051 / \mathrm{GB}: \mathrm{AE} 003695$

2.5 FB:FBgn0034688 $/ \mathrm{sym}=\mathrm{CG} 11474 /$ name $=/$ prod $=/$ func $=/ \mathrm{map}=58 \mathrm{C} 1-58 \mathrm{C} 1 /$ transc=CT36283/len=1521 /GB:AE003456 /note=3prime sequence from clone BDGP:LD03212.3prime-hit

2.5 FB:FBgn0029851/sym $=\mathrm{CG} 14445 /$ name $=/$ prod $=/$ func $=/ \mathrm{map}=5 \mathrm{D} 6-5 \mathrm{D} 6 / \mathrm{transc}=\mathrm{CT} 34116 / \mathrm{len}=1143 / \mathrm{GB}: \mathrm{AE} 003437$

FB:FBgn0034242 $/$ sym $=$ CG14480 $/$ name $=/$ prod $=/$ func $=/$ map $=54 \mathrm{D} 1-54 \mathrm{D} 1 /$ transc $=\mathrm{CT} 34191 / \mathrm{len}=750 /$ GB:AE003802

2.5 FB:FBgn0028687/sym=Rpt1 /name=/prod=19S proteasome regulatory particle, triple-A protein, subunit S7 /func=proteasome ATPase ; EC:3.6.4.8 /map=43E6-43E6 /transc=CT3016/len=1397 /GB: FB:FBgn0035081 /sym=CG2858 /name $=/$ prod $=/$ func $=$ enzyme $/$ map=60E5-60E5 $/$ transc $=$ CT $9732 /$ len $=1449 /$ GB:AE003465

FB:FBgn0038688/sym=CG3768/name $=/$ prod=calcium binding protein-like /func=ligand binding or carrier $/$ map=91F8-91F8 /transc=CT12576/len=1030 /GB:AE003725 /note=3prime sequence fron 2.5 FB:FBgn0029715/sym=CG11444 /name $=/$ prod $=/$ func $=/$ map $=4$ C4 $-4 \mathrm{C} 4 /$ transc $=$ CT9463 /len=1230 /GB:AE003431 /note=3prime sequence from clone BDGP:GM14292.3prime-hit

2.5 FB:FBgn0024988 /sym=EG:131F2.2 /name $=/$ prod= /func=enzyme $/ \mathrm{map}=2 \mathrm{~B} 12-2 \mathrm{~B} 12 /$ transc=CT34614 /len=2176 /GB:AE003422 /note=3prime sequence from clone BDGP:SD04906.3prime-hit

2.5 FB:FBgn0037346/sym=CG2922 $/$ name $=/$ prod $=/$ func $=/$ map=83B4-83B4 $/$ transc=CT7240/len=2082 $/$ GB:AE003602 $/$ note $=3$ prime sequence from clone BDGP $:$ LD21309.3prime-hit

2.5 FB:FBgn0036109 $/$ sym $=$ CG18349 $/$ name $=/$ prod $=/$ func $=/$ map $=67 F 4-67 F 4 /$ transc $=$ CT41690 $/$ len $=536 /$ GB:AE003546

2.5 Drosophila gene for Gapdh2 (_5,_M,_3 represent transcript regions 5 prime, Middle, and 3 prime respectively)

2.5 FB:FBgn0001092 /sym=Gapdh2 /name=Glyceraldehyde 3 phosphate dehydrogenase $2 /$ prod=glyceraldehyde 3-phosphate dehydrogenase (phosphorylating) 2 /func=glyceraldehyde 3-phosphate dehydro 2.5 FB:FBgn0038420 $/$ sym $=$ CG10311 $/$ name $=/$ prod $=/$ func $=/$ map $=89 \mathrm{~B} 22-89 \mathrm{~B} 22 /$ transc $=\mathrm{CT} 28967 / \mathrm{len}=719 / \mathrm{GB}: \mathrm{AE} 003713$

FB:FBgn0033745/sym=CG8824/name $=/$ prod=beta-N-acetylhexosaminidase-like $/$ func $=$ enzyme $/$ map $=49 A 9-49 \mathrm{~A} 9 /$ transc $=\mathrm{CT} 25388 /$ len $=2555 / \mathrm{GB}: \mathrm{AE} 003822 /$ note $=3$ prime sequence from clone BI 2.5 FB:FBgn0038878 $/$ sym=CG3301 $/$ name $=/$ prod $=/$ func $=$ enzyme $/$ map=93D4-93D4 /transc=CT11093 /len=913 /GB:AE003734 /note=3prime sequence from clone BDGP:GH01837.3prime-hit FB:FBgn0031206 /sym $=$ CG12466 $/$ name $=/$ prod $=/$ func=enzyme $/ \mathrm{map}=20 \mathrm{~B} 1-20 \mathrm{~B} 1 /$ transc=CT32678 /len=1204 /GB:AE003573 /note=3prime sequence from clone BDGP:GH12380.3prime-hit

2.5 FB:FBgn0030672/sym=CG9281/name=/prod=ATP-binding cassette transporter /func=enzyme $/$ map=13E14-13E14/transc $=\mathrm{CT} 26402 /$ len $=2568 / \mathrm{GB}: \mathrm{AE} 003500 /$ note $=3$ prime sequence from clone B

2.5 FB:FBgn0026781/sym=Prosalpha1 $/$ name=Proteasome alpha1 subunit $/$ prod $=20 \mathrm{~S}$ proteasome alpha1 subunit $/$ func $=$ multicatalytic endopeptidase $; \mathrm{EC}: 3.4 .99 .46 / \mathrm{map}=43 \mathrm{~F} 1-43 \mathrm{~F} 1 / \mathrm{transc}=\mathrm{CT} 42048 / \mathrm{len}=$

2.5 FB:FBgn0036811 $/$ sym $=$ CG6884 $/$ name $=/$ prod $=/$ func $=/$ map $=75 \mathrm{D} 4-75 \mathrm{D} 4 /$ transc $=\mathrm{CT} 21320 /$ len $=610 /$ GB:AE003519

$2.5 \quad$ FB:FBgn0032574/sym $=\mathrm{CG} 18629 / \mathrm{name}=/ \mathrm{prod}=/$ func $=/ \mathrm{map}=35 \mathrm{E} 1-35 \mathrm{E} 1 / \mathrm{transc}=\mathrm{CT} 41822 / \mathrm{len}=588 / \mathrm{GB}: \mathrm{AE} 003649$

2.5 FB:FBgn0034094/sym=CG3666/name=/prod=transferrin-like /func=ligand binding or carrier $/$ map=52F10-52F10/transc=CT12185/len=2352/GB:AE003807/note=3prime sequence from clone BD 2.5 FB:FBgn0038154/sym $=$ CG18290 $/$ name $=/$ prod $=/$ func $=/$ map $=87$ E $11-87$ E $11 /$ transc $=$ CT41497 /len $=1195 /$ GB:AE003700

2.5 FB:FBgn0000250/sym=cact $/$ name $=$ cactus $/$ prod $=/$ func=transcription factor, cytoplasmic sequestering $/ \mathrm{map}=35 \mathrm{~F} 8-35 \mathrm{~F} 9 /$ transc=CT18347 /len=1918 $/ \mathrm{GB}: \mathrm{AE} 003650 /$ note $=3$ prime sequence from clo 2.5 FB:FBgn0040754 $/$ sym $=$ CG17059 $/$ name $=/$ prod $=/$ func $=/$ map $=49 \mathrm{~F} 13-49 \mathrm{~F} 13 /$ transc $=\mathrm{CT} 37898 / \mathrm{len}=358 / \mathrm{GB}: \mathrm{AE} 003819$

2.5 FB:FBgn0000045/sym=Act79B /name=Actin 79B /prod=actin /func=muscle motor protein $/ \mathrm{map}=79 \mathrm{~B} 3-79 \mathrm{~B} 3 / \mathrm{transc}=\mathrm{CT} 22987 / \mathrm{len}=1172 / \mathrm{GB}: \mathrm{AE} 003596$

2.5 FB:FBgn0023174 /sym=Prosbeta2 $/$ name=Proteasome beta2 subunit $/$ prod=20S proteasome, beta2 subunit $/$ func=multicatalytic endopeptidase $; \mathrm{EC}: 3.4 .99 .46 / \mathrm{map}=71 \mathrm{~A} 3-71 \mathrm{~A} 3 / \mathrm{transc}=\mathrm{CT} 10524 / \mathrm{len}=1$ 2.5 FB:FBgn0001301 /sym=kel /name=kelch /prod=/func=transcription factor $/ \mathrm{map}=36 \mathrm{E} 3-36 \mathrm{E} 5 /$ transc=CT22235/len=5604 /GB:AE003657 /note=3prime sequence from clone BDGP:LD29455.3prime-

$2.4 \mathrm{FB}: \mathrm{FBg} 0032202 / \mathrm{syn}=\mathrm{CG} 18619 / \mathrm{name}=/ \mathrm{p}$ 
396588.5

2.4 FB:FBgn0002531/sym=Lcp $1 /$ name=Larval cuticle protein $1 /$ prod=larval cuticle protein $1 /$ func=structural protein of larval cuticle $($ Drosophila $) \quad / \mathrm{map}=44 \mathrm{C} 1-44 \mathrm{C} 1 /$ transc $=\mathrm{CT} 36649 /$ len=543 $/ \mathrm{GB}: \mathrm{AE}$

2.4 FB:FBgn0033129 $/$ sym $=$ CG12844 $/$ name $=/$ prod $=/$ func $=/$ map $=42 \mathrm{E} 1-42 \mathrm{E} 1 /$ transc $=\mathrm{CT} 31976 / \mathrm{len}=673 / \mathrm{GB}: \mathrm{AE} 003842$

FB:FBgn0035649 $/$ sym $=$ CG10483 $/$ name $=/$ prod $=/$ func $=/$ map $=64 F 5-64 F 5 /$ transc $=$ CT29432 /len=2221 /GB:AE003564 /note=3prime sequence from clone BDGP:GH26628.3prime-hit BDGP:LD4689.3prime-hit $/$ ESTpos=maps in FB:FBgn0037937/sym=CG6913 $/$ name $=/$ prod $=/$ func $=$ DNA binding $/ \mathrm{map}=86 \mathrm{~F} 1-86 \mathrm{~F} 1 /$ transc $=\mathrm{CT} 21412 / \mathrm{len}=536$

2.4 BDGP:LD1981.3prime-hit /ESTpos=maps 3prime of FB:FBgn0027356/sym=amphiphysin /name=amphiphysin /prod=amphiphysin /func=protein kinase $/ \mathrm{map}=49 \mathrm{~B} 3-49 \mathrm{~B} 3 / \mathrm{transc}=\mathrm{CT} 259 / \mathrm{len}=527$

2.4 FB:FBgn0015282 /sym=Pros26.4 /name=Proteasome $26 \mathrm{~S}$ subunit subunit 4 ATPase /prod=19S proteasome regulatory particle, triple-A protein, subunit S4 /func=proteasome ATPase ; EC:3.6.4.8 /map FB:FBgn0030941 $/$ sym $=$ CG6531 $/$ name $=/$ prod $=/$ func $=/$ map $=17$ C3-17C3 $/$ transc $=C T 20295 /$ len $=1260 /$ GB:AE003509

2.4 FB:FBgn0029534 $/$ sym $=$ CG5273 $/$ name $=/$ prod $=/$ func $=/$ map $=1 \mathrm{C} 1-1 \mathrm{C} 1 /$ transc $=\mathrm{CT} 16821 /$ len $=2072 /$ GB:AE003418

2.4 FB:FBgn0015283 /sym=Pros54 /name=Proteasome 54kD subunit /prod=19S proteasome regulatory particle, non-ATPase protein, subunit S5a /func=multicatalytic endopeptidase ; EC:3.4.99.46 /map= FB:FBgn0033026 /sym=CG12183/name $=/$ prod $=/$ func=actin binding $/$ map=41C4-41C4 /transc=CT9399/len=1982 /GB:AE003786

2.4 FB:FBgn0039238 $/$ sym $=$ CG7016 $/$ name $=/$ prod $=/$ func $=/$ map $=96 \mathrm{~B} 4-96 \mathrm{~B} 4 /$ transc $=\mathrm{CT} 21718 / \mathrm{len}=1089 / \mathrm{GB}: \mathrm{AE} 003749$

FB:FBgn0036359/sym $=$ CG14105 $/$ name $=/$ prod $=/$ func $=/$ map $=70$ A $8-70 A 8 /$ transc $=$ CT33698 $/$ len $=558 /$ GB:AE003538

2.4 FB:FBgn0035089/sym=CG9358 $/$ name $=/$ prod $=/$ func=ligand binding or carrier $/ \mathrm{map}=60 \mathrm{E} 7-60 \mathrm{E} 7 /$ transc $=\mathrm{CT} 26583 / \mathrm{len}=366 / \mathrm{GB}: \mathrm{AE} 003465$

2.4 FB:FBgn0038260/sym=CG14855/name=/prod=organic cation transporter-like /func=transporter $/$ map=88D5-88D5/transc=CT34672 /len=1671 /GB:AE003706

2.4 FB:FBgn0029639 $/ \mathrm{sym}=\mathrm{CG} 14419 /$ name $=/ \mathrm{prod}=/ \mathrm{func}=/ \mathrm{map}=3 \mathrm{C} 3-3 \mathrm{C} 3 /$ transc $=\mathrm{CT} 34076 / \mathrm{len}=588 / \mathrm{GB}: \mathrm{AE} 003425$

2.4 FB:FBgn0001091 /sym=Gapdh1 /name=Glyceraldehyde 3 phosphate dehydrogenase 1 /prod=glyceraldehyde 3-phosphate dehydrogenase (phosphorylating) 1 /func=glyceraldehyde 3-phosphate dehydro FB:FBgn0030991 $/$ sym $=C$ CG7453 $/$ name $=/$ prod $=/$ func $=/$ map $=18$ B4 -18 B $4 /$ transc $=C T 22925 /$ len $=1285 /$ GB:AE00351

2.4 FB:FBgn0035978 /sym=CG4347 /name=/prod=/func=UTP--glucose-1-phosphate uridylyltransferase $/$ map=67A9-67B1/transc=CT14147/len=2009/GB:AE003552 /note=3prime sequence from clon FB:FBgn0024993 /sym=EG:100G10.6 /name = /prod=/func=transcription factor $/ \mathrm{map}=3 \mathrm{~B} 4-3 \mathrm{~B} 5 /$ transc=CT9011 /len=1402 /GB:AE003425

2.4 FB:FBgn0036111/sym=CG6391/name=/prod=diphosphoinositol polyphosphate phosphohydrolase $/$ func=enzyme $/$ map=67F4-67F4/transc=CT19950/len=1704 /GB:AE003546

2.4 FB:FBgn0032392 $/$ sym $=$ CG17749 $/$ name $=/$ prod $=/$ func $=/$ map $=33$ B9-33B $/$ transc $=$ CT39339 $/$ len $=476 /$ GB:AE003634

2.4 FB:FBgn0036580 $/$ sym $=$ CG13072 $/$ name $=/$ prod $=/$ func $=/$ map $=72 \mathrm{D} 12-72 \mathrm{D} 12 /$ transc $=\mathrm{CT} 32291 / \mathrm{len}=402 / \mathrm{GB}: \mathrm{AE} 003528$

2.4 FB:FBgn0033683 $/ \mathrm{sym}=\mathrm{CG} 18343 / \mathrm{name}=/ \mathrm{prod}=/ \mathrm{func}=/ \mathrm{map}=48 \mathrm{E} 10-48 \mathrm{E} 10 /$ transc $=\mathrm{CT} 41671 / \mathrm{len}=463 / \mathrm{GB}: \mathrm{AE} 003823$

2.4 FB:FBgn0039358 $/ \mathrm{sym}=\mathrm{CG} 5028 / \mathrm{name}=/ \mathrm{prod}=/$ func $=$ enzyme $/ \mathrm{map}=96 \mathrm{E} 10-96 \mathrm{E} 10 /$ transc $=\mathrm{CT} 16155 / \mathrm{len}=1312 / \mathrm{GB}: \mathrm{AE} 003753$

FB:FBgn0015569/sym=alpha-Est10 /name=alpha-Esterase-1 /prod=esterase, unknown substrate /func=esterase, unknown substrate ; EC:3.1.1.- /map=84D5-84D5 /transc=CT1811 /len=1650 /GB:AE0 2.4 FB:FBgn0024754 /sym=Flo /name=flotillin /prod=flotillin /func=ligand binding or carrier $/$ map=52A13-52A13/transc=CT37018 /len=1779/GB:AE003810 /note=3prime sequence from clone BDGP: 2.4 FB:FBgn0030151/sym=CG1354/name=/prod=GTP-binding protein /func=ligand binding or carrier $/ \mathrm{map}=8 \mathrm{~F} 10-8 \mathrm{~F} 10 /$ transc $=\mathrm{CT} 3048 /$ len $=1200 / \mathrm{GB}: \mathrm{AE} 003448$

2.4 FB:FBgn0028686 $/ \mathrm{sym}=\mathrm{Rpt} 3 / \mathrm{name}=/$ prod=19S proteasome regulatory particle, triple-A protein, subunit S6b $/$ func=proteasome ATPase $; \mathrm{EC}: 3.6 .4 .8 / \mathrm{map}=10 \mathrm{~A} 6-10 \mathrm{~A} 6 /$ transc $=\mathrm{CT} 35131 / \mathrm{len}=1242 / \mathrm{G}$ 2.4 FB:FBgn0000064 /sym=Ald /name=Aldolase /prod=fructose-bisphosphate aldolase /func=fructose-bisphosphate aldolase ; EC:4.1.2.13 inferred from direct assay /map=97A6-97A6/transc=CT41919/1 2.4 FB:FBgn0037007 $/ \mathrm{sym}=\mathrm{CG} 5059 / \mathrm{name}=/ \mathrm{prod}=/ \mathrm{func}=/ \mathrm{map}=77 \mathrm{C} 4-77 \mathrm{C} 4 /$ transc $=\mathrm{CT} 16233 / \mathrm{len}=939 / \mathrm{GB}: \mathrm{AE} 003591$

2.4 FB:FBgn0004551/sym=Ca-P60A /name=Calcium ATPase at 6A /prod=calcium-transporting ATPase, sarco/endoplasmic reticulum type /func=calcium-transporting ATPase ; EC:3.6.1.38 /map=60A11 2.4 FB:FBgn0027591/sym=BcDNA:GH04245/name=/prod=/func=/map=41A1-41A2 /transc=CT9123 /len=3519/GB:AE003787 /note=3prime sequence from clone BDGP:LD16758.3prime-hit 
FB:FBgn0030563 $/$ sym $=\mathrm{CG} 18157 /$ name $=/$ prod $=/$ func $=/$ map $=12 \mathrm{E} 2-12 \mathrm{E} 2 /$ transc $=\mathrm{CT} 40954 / \mathrm{len}=594 / \mathrm{GB}: \mathrm{AE} 003495$

2.4 FB:FBgn0040954 $/$ sym $=\mathrm{CG} 13779 /$ name $=/$ prod $=/$ func $=/$ map $=27 \mathrm{D} 5-27 \mathrm{D} 5 /$ transc $=\mathrm{CT} 33267 / \mathrm{len}=240 / \mathrm{GB}: \mathrm{AE} 003616$

2.4 FB:FBgn0035981/sym=CG4452/name=/prod=/func=endopeptidase /map=67B1-67B1/transc=CT14468 /len=1633/GB:AE003552/note=3prime sequence from clone BDGP:LD21662.3prime-hit

2.4 FB:FBgn0040764 $/$ sym $=$ CG13230 $/$ name $=/$ prod $=/$ func $=/$ map=47D4-47D4 $/$ transc $=$ CT32474 /len=219 $/$ GB:AE003827

2.4 Drosophila gene for Gapdh2 (_5,_M, 3 represent transcript regions 5 prime, Middle, and 3 prime respectively)

2.4 FB:FBgn0026777 /sym=Rad23 /name=/prod=/func=DNA repair protein $/$ map=102A8-102A8 /transc=CT5572/len=1477 /GB:AE003844 /note=3prime sequence from clone BDGP:LD10153.complet FB:FBgn0036074 $/$ sym $=$ CG1 $1965 /$ name $=/$ prod $=/$ func $=/$ map $=67 D 2-67 D 2 /$ transc $=$ CT37129 $/$ len $=1464 /$ GB:AE003549

2.4 FB:FBgn0015614 /sym=CanB2 /name=Calcineurin B2 /prod=calcineurin, calcium-binding, regulatory (B)-subunit $2 /$ func=calcium binding $/$ map=43E16-43E16 /transc=CT31322 /len=718 /GB:AE0

2.3 FB:FBgn0024753/sym=Flo-2 /name=flotillin $2 /$ prod=flotillin $2 /$ func= /map=13A3-13A4/transc=CT34056/len=1791 /GB:AE003497/note=3prime sequence from clone BDGP:LP11503.3prime-hit

2.3 FB:FBgn0027493/sym=BcDNA:LD32788/name=/prod=adenylosuccinate synthase /func=adenylosuccinate synthase ; EC:6.3.4.4/map=92F13-92F13/transc=CT35906/len=1986/GB:AE003732 /not

2.3 FB:FBgn0039213 /sym=CG6668 /name $=/$ prod $=/$ func $=/$ map=96A14-96A15/transc=CT20689/len=2852 /GB:AE003748 /note=3prime sequence from clone BDGP:GH09383.3prime-hit

FB:FBgn0034946/sym=CG3065/name $=/$ prod $=/$ func $=$ nucleic acid binding $/$ map=60A14-60A14 /transc $=$ CT10306 /len=1916 /GB:AE003462 /note $=3$ prime sequence from clone BDGP:GM01315.3pr 2.3 FB:FBgn0033886 $/ \mathrm{sym}=\mathrm{CG} 13349 /$ name $=/ \mathrm{prod}=/$ func $=/ \mathrm{map}=50 \mathrm{C} 20-50 \mathrm{C} 20 /$ transc $=\mathrm{CT} 32670 / \mathrm{len}=1170 / \mathrm{GB}: \mathrm{AE} 003816$

2.3 FB:FBgn0002284 $/ \mathrm{sym}=$ Pros $26 /$ name $=$ Proteasome $26 \mathrm{kD}$ subunit $/ \mathrm{prod}=20 \mathrm{~S}$ proteasome, beta6 subunit $/$ func $=$ multicatalytic endopeptidase $; \mathrm{EC}: 3.4 .99 .46 / \mathrm{map}=73 \mathrm{~A} 10-73 \mathrm{~A} 10 / \mathrm{transc}=\mathrm{CT} 13566 /$ len $=9$

2.3 FB:FBgn0017565 $/ \mathrm{sym}=$ Nacalpha $/$ name $=$ Nascent polypeptide associated complex protein alpha subunit $/$ prod=nascent polypeptide associated complex protein alpha subunit $/ \mathrm{func}=/ \mathrm{map}=49 \mathrm{C} 2-49 \mathrm{C} 2$

2.3 FB:FBgn0030310/sym $=$ CG11709 $/$ name $=/$ prod=peptidoglycan recognition protein-like $/$ func $=$ defense/immunity protein $/$ map $=10 \mathrm{C} 4-10 \mathrm{C} 4 /$ transc $=\mathrm{CT} 35112 /$ len $=612 / \mathrm{GB}: \mathrm{AE} 003486$

2.3 FB:FBgn0030724 /sym=CG9212/name=/prod=/func=4-nitrophenylphosphatase $/ \mathrm{map}=14 \mathrm{A6}-14 \mathrm{~A} 6 /$ transc=CT26318 /len=1359/GB:AE003501 /note=3prime sequence from clone BDGP:LD01807.3 FB:FBgn0029970 $/$ sym $=$ CG17256 $/$ name $=/$ prod $=$ protein serine/threonine kinase-like $/$ func $=$ protein kinase $/$ map=7C6-7C6 $/$ transc $=\mathrm{CT} 38234 /$ len $=2435 / \mathrm{GB}: \mathrm{AE} 003442 /$ note $=3$ prime sequence from cle

2.3 FB:FBgn0036630/sym $=\mathrm{CG} 4561 /$ name $=/$ prod $=/$ func $=$ tyrosine--tRNA ligase $/ \mathrm{map}=73 \mathrm{~A} 1-73 \mathrm{~A} 1 /$ transc $=\mathrm{CT} 14730 /$ len $=1717 / \mathrm{GB}: \mathrm{AE} 003527$

BDGP:GH06247.3prime-hit /maps to FB:FBgn0036239 (/sym=CG5684/name=/prod=/func=transcription factor $)$ and FB:FBgn0036238 (/sym=CG5688 $/$ name $=/$ prod=/func=motor $)$

2.3 FB:FBgn0003151/sym=Pros35/name=Proteasome 35kD subunit /prod=20S proteasome, alpha6 subunit /func=multicatalytic endopeptidase $; \mathrm{EC}: 3.4 .99 .46 / \mathrm{map}=31 \mathrm{~B} 3-31 \mathrm{~B} 4 / \mathrm{transc}=\mathrm{CT} 15762 /$ len=94

2.3 FB:FBgn0035206 $/$ sym=CG9186 $/$ name $=/$ prod $=/$ func $=/$ map=61F6-61F6 $/$ transc $=$ CT8283 /len=1400 $/$ GB:AE003471 /note $=3$ prime sequence from clone BDGP:LP01162.3prime-hit

2.3 FB:FBgn0010406/sym=RNaseX25 /name=Ribonuclease X25/prod=ribonuclease-like /func=ribonuclease /map=66A22-66A22/transc=CT24383/len=1658 /GB:AE003557/note=3prime sequence fro FB:FBgn0029798 $/$ sym=CG4078 $/$ name $=/$ prod= $/$ func=DNA repair protein $/ \mathrm{map}=5 \mathrm{~B} 3-5 \mathrm{~B} 3 /$ transc=CT13546 $/$ len=3246 $/$ GB:AE003435

2.3 FB:FBgn0005649/sym=Rox8 /name=Rox8 /prod=nucleolysin-like /func=poly(A) binding /map=95D5-95D5/transc=CT17178 /len=3165/GB:AE003746

FB:FBgn0039901 $/$ sym $=$ CG10322 $/$ name $=/$ prod $=/$ func $=/$ map $=102 \mathrm{~A} 7-102 \mathrm{~A} 8 /$ transc $=\mathrm{CT} 7685 /$ len $=630 /$ GB:AE003844

2.3 FB:FBgn0033352/sym=CG8232/name=/prod=PAB-dependent poly(A)-specific ribonuclease subunit /func=enzyme $/ \mathrm{map}=44 \mathrm{~F} 9-44 \mathrm{~F} 11 /$ transc=CT8229 $/$ len=3925 $/ \mathrm{GB}: \mathrm{AE} 003835 /$ note=3prime sequ

2.3 FB:FBgn0039265 $/ \mathrm{sym}=\mathrm{CG1} 1790 / \mathrm{name}=/ \mathrm{prod}=/ \mathrm{func}=$ chaperone $/ \mathrm{map}=96 \mathrm{~B} 15-96 \mathrm{~B} 15 / \mathrm{transc}=\mathrm{CT} 33049 / \mathrm{len}=794 / \mathrm{GB}: \mathrm{AE} 003750$

2.3 FB:FBgn0034618 /sym=CG9485 /name $=/$ prod=glycogen debranching enzyme $/$ func $=$ enzyme $/$ map $=57$ D4-57D5 /transc $=$ CT26790 $/$ len=4780 $/$ GB:AE003453 $/$ note $=3$ prime sequence from clone BDGI

2.3 FB:FBgn0038922 /sym=CG6439/name $=/$ prod=isocitrate dehydrogenase $[\mathrm{NAD}]$ subunit $/$ func=enzyme $/ \mathrm{map}=93 \mathrm{~F} 14-93 \mathrm{~F} 14 /$ transc $=\mathrm{CT} 20062 /$ len=1598 $/ \mathrm{GB}: \mathrm{AE} 003737 /$ note $=3 \mathrm{prime}$ sequence from FB:FBgn0016122/sym=Acer /name=Angiotensin-converting enzyme-related $/$ prod=angiotensin I-converting enzyme /func=peptidyl-dipeptidase A ; EC:3.4.15.1/map=29D1-29D2/transc=CT29700 /le 2.2 BDGP:LD28328.3prim

2.2 Drosophila gene for Gapdh2 (_5,_M,_3 represent transcript regions 5 prime, Middle, and 3 prime respectively) 
FB:FBgn0025186/sym=ari2 $/$ name $=$ ariadne $2 /$ prod $=/$ func $=/$ map=58C7-58D $1 /$ transc $=$ CT17832 $/$ len $=3002 /$ GB:AE003456

FB:FBgn0004237 /sym $=$ Hrb87F $/$ name $=$ Heterogeneous nuclear ribonucleoprotein at $87 \mathrm{~F} /$ prod=heterogeneous nuclear ribonucleoprotein A1 /func $=$ ribonucleoprotein $\quad / \mathrm{map}=87 \mathrm{~F} 7-87 \mathrm{~F} 7 /$ transc $=\mathrm{CT} 2725$ 2.2 BDGP:GH05625.3

2.2 FB:FBgn0003150/sym=Pros $29 /$ name=Proteasome $29 \mathrm{kD}$ subunit $/$ prod=20S proteasome, alpha3 subunit $/$ func=multicatalytic endopeptidase $; \mathrm{EC}: 3.4 .99 .46 / \mathrm{map}=57 \mathrm{~B} 15-57 \mathrm{~B} 15 /$ transc $=\mathrm{CT} 26525 /$ len= 2.2 FB:FBgn0032537/sym=CG18634 $/$ name $=/$ prod $=/$ func $=/$ map $=34 \mathrm{E} 1-34 \mathrm{E} 1 /$ transc $=\mathrm{CT} 42158 / \mathrm{len}=552 / \mathrm{GB}: \mathrm{AE} 003641$

2.2 FB:FBgn0035631/sym=CG5495/name=Thioredoxin-like /prod=thioredoxin-like /func=thioredoxin $/ \mathrm{map}=64 \mathrm{~F} 1-64 \mathrm{~F} 1 /$ transc $=\mathrm{CT} 17420 /$ len=957 $/ \mathrm{GB}: \mathrm{AE} 003565 /$ note $=3$ prime sequence from clone B

2.2 FB:FBgn0027910/sym=BcDNA:GM14618 $/$ name $=/$ prod $=/$ func $=/$ map $=25 \mathrm{C} 2-25 \mathrm{C} 3 /$ transc $=\mathrm{CT} 26154 / \mathrm{len}=1161 / \mathrm{GB}:$ AE003608

2.2 FB:FBgn0033356/sym $=$ CG8229 $/$ name $=/$ prod $=/$ func $=/$ map=44F12-45A1 / transc=CT8591 /len=1918 /GB:AE003835 /note=3prime sequence from clone BDGP:LD27667.3prime-hit

2.2 FB:FBgn0000579/sym=Eno $/$ name=Enolase $/$ prod=phosphopyruvate hydratase $/$ func=phosphopyruvate hydratase $;$ EC:4.2.1.11 /map=22B1-22B1/transc=CT32526 /len=1931/GB:AE003585

2.2 FB:FBgn0040985 $/ \mathrm{sym}=\mathrm{CG} 6115 / \mathrm{name}=/ \mathrm{prod}=/$ func $=/ \mathrm{map}=36 \mathrm{~A} 11-36 \mathrm{~A} 11 /$ transc $=\mathrm{CT} 19203 / \mathrm{len}=566 / \mathrm{GB}: \mathrm{AE} 003653$

2.2 FB:FBgn0022959/sym=yps /name=ypsilon schachtel $/$ prod $=/$ func $=/ \mathrm{map}=68 \mathrm{~F} 4-68 \mathrm{~F} 4 /$ transc $=\mathrm{CT} 17850 /$ len=2254 $/$ GB:AE003542 $/$ note $=3$ prime sequence from clone BDGP:LD37574.3prime-hit

2.2 FB:FBgn0032776 $/$ sym $=$ CG18061 $/$ name $=/$ prod $=/$ func $=/$ map=37E1-37E1 $/$ transc $=$ CT40481 /len=2027 /GB:AE003662 $/$ note $=3$ prime sequence from clone BDGP:SD04793.3prime-hit

FB:FBgn0011754/sym=PhKgamma /name=Phosphorylase kinase gamma /prod=phosphorylase kinase, catalytic gamma subunit /func=phosphorylase kinase catalyst $/$ map=10D2-10D4 /transc=CT55 2.2 BDGP:GH28523.3pri

2.2 FB:FBgn0032203 $/ \mathrm{sym}=\mathrm{CG} 4946 / \mathrm{name}=/ \mathrm{prod}=/ \mathrm{func}=/ \mathrm{map}=31 \mathrm{C} 6-31 \mathrm{C} 6 /$ transc $=\mathrm{CT} 15842 / \mathrm{len}=834 / \mathrm{GB}: \mathrm{AE} 003628$

2.2 FB:FBgn0032941 /sym=CG8669 /name $=/$ prod $=/$ func $=/$ map=39D2-39D2 /transc $=$ CT5302 /len=1793 /GB:AE003670 /note=3prime sequence from clone BDGP:GH1 1210.3 prime-hit FB:FBgn0029913/sym=CG3044 /name=/prod=chitinase $/$ func=enzyme $/$ map=6D3-6D3/transc=CT10053 /len=1299 /GB:AE003439

FB:FBgn0004170/sym=sc /name=scute /prod=/func=specific RNA polymerase II transcription factor $/$ map=1B2-1B2/transc=CT12777/len=1038/GB:AE003417

2.2 FB:FBgn0037377 $/$ sym $=\mathrm{CG} 1218 /$ name $=/$ prod $=/$ func $=/ \mathrm{map}=83 \mathrm{C} 1-83 \mathrm{C} 1 /$ transc $=\mathrm{CT} 2292 / \mathrm{len}=1479 / \mathrm{GB}: \mathrm{AE} 003601 /$ note $=3$ prime sequence from clone BDGP $:$ LD28626.3prime-hit FB:FBgn0040737/sym $=$ CG14503 $/$ name $=/$ prod $=/$ func $=/$ map $=55$ C5-55C $5 /$ transc $=$ CT34218 $/$ len $=177 /$ GB:AE003800

2.2 FB:FBgn0028490/sym=BcDNA:GH07269/name=/prod=/func=DNA binding /map=33A2-33A2/transc=CT20317 /len=3403 /GB:AE003632 /note=3prime sequence from clone BDGP:GH07269.3p FB:FBgn0028854/sym=BG:DS07721.6 $/$ name $=/$ prod $=/$ func $=/$ map=35B5-35B $/$ transc $=$ CT35229 $/$ len $=3129 /$ GB:AE003644

2.2 FB:FBgn0031024 $/ \mathrm{sym}=\mathrm{CG} 12233 / \mathrm{name}=/ \mathrm{prod}=/$ func $=$ enzyme $/ \mathrm{map}=18 \mathrm{D} 3-18 \mathrm{D} 3 /$ transc $=\mathrm{CT} 13154 / \mathrm{len}=1375 / \mathrm{GB}: \mathrm{AE} 003512$

2.2 FB:FBgn0034110/sym $=\mathrm{CG} 3615 /$ name $=/$ prod $=/$ func $=/$ map=53A2-53A2 $/$ transc $=$ CT12045/len=2831 /GB:AE003807 /note=3prime sequence from clone BDGP:SD01812.3prime-hit

2.2 FB:FBgn0037537/sym=CG2767/name=/prod=alcohol dehydrogenase /func=enzyme /map=84E5-84E6/transc=CT9417/len=1293/GB:AE003677 /note=3prime sequence from clone BDGP:LD2467

2.2 FB:FBgn0015239/sym=Hr78 /name=Hormone-receptor-like in $78 /$ prod=nuclear receptor NR2D1 /func=ligand-dependent nuclear receptor $/ \mathrm{map}=78 \mathrm{D} 7-78 \mathrm{D} 7 /$ transc=CT22217 /len=2246 $/ \mathrm{GB}: \mathrm{AE} 003$

2.2 FB:FBgn0033128 /sym=CG12142 /name=Tetraspanin 42Eg /prod=tetraspanin /func $=/$ map=42E1-42E1 /transc $=$ CT7934 /len=1170 /GB:AE003842 /note $=3$ prime sequence from clone BDGP:GM0696

2.2 FB:FBgn0036661 $/ \mathrm{sym}=\mathrm{CG} 9705 /$ name $=/$ prod $=$ calcium-regulated heat stable protein-like $/$ func $=/$ map $=73 \mathrm{C} 1-73 \mathrm{C} 1 /$ transc $=\mathrm{CT} 27440 /$ len $=1386 / \mathrm{GB}: \mathrm{AE} 003526 /$ note $=3$ prime sequence from clone $\mathrm{BD}$

2.2 FB:FBgn0027108/sym=inx2 /name=/prod=innexin $2 /$ func=neurotransmitter transporter $/$ map=6E4-6E5 /transc=CT14874 /len=1819/GB:AE003439

2.2 FB:FBgn0035904 $/ \mathrm{sym}=\mathrm{CG} 6776 /$ name $=/$ prod $=$ glutathione transferase $/$ func $=$ glutathione transferase $/ \mathrm{map}=66 \mathrm{D} 4-66 \mathrm{D} 4 /$ transc $=\mathrm{CT} 21027 / \mathrm{len}=776 / \mathrm{GB}: \mathrm{AE} 003555$

2.2 FB:FBgn0004432 /sym=Cyp1 /name=Cyclophilin $1 /$ prod=cyclophilin /func=cyclophilin $/$ map=14B15-14B15/transc=CT27926/len=910/GB:AE003501 /note=3prime sequence from clone BDGP:SD FB:FBgn0037190/sym=CG7651 /name=/prod=P-type ATPase /func=transporter $/$ map=79F3-79F3 /transc=CT22775/len=3566 /GB:AE003598

2.2 FB:FBgn0036299 $/ \mathrm{sym}=\mathrm{CG} 10620 /$ name $=/$ prod=transferrin-like $/$ func=transporter $/ \mathrm{map}=69 \mathrm{C} 4-69 \mathrm{C} 4 /$ transc $=\mathrm{CT} 29752 /$ len=2860 $/$ GB:AE003541 /note=3prime sequence from clone BDGP:LD22449. 2.2 FB:FBgn0037245 $/$ sym $=$ CG14648 $/$ name $=/$ prod $=/$ func $=$ enzyme $/$ map=82B3-82B3 $/$ transc $=$ CT34420 $/$ len $=1773 /$ GB:AE003606 
2.2 FB:FBgn0033385 $/$ sym $=$ CG8055 $/$ name $=/$ prod $=/$ func $=\quad /$ map=45B1-45B1/transc=CT8052/len=1251 /GB:AE003834 /note=3prime sequence from clone BDGP:GH13992.3prime-hit FB:FBgn0034578 $/$ sym $=$ CG15653 $/$ name $=/$ prod $=/$ func $=/$ map $=57 \mathrm{~B} 20-57 \mathrm{~B} 20 /$ transc $=\mathrm{CT} 35837 / \mathrm{len}=513 / \mathrm{GB}: \mathrm{AE} 003452$

FB:FBgn0025802/sym=Sbf $/$ name $=$ SET domain binding factor $/$ prod $=/$ func=ligand binding or carrier $/$ map=86F9-86F9 $/$ transc $=\mathrm{CT} 21495 /$ len $=6619 / \mathrm{GB}: \mathrm{AE} 003693 /$ note $=3$ prime sequence from clon 2.2 FB:FBgn0031454/sym=CG9960 $/$ name $=/$ prod $=/$ func $=/$ map=23B1-23B1 $/$ transc=CT28077 /len=799 /GB:AE003582 /note=3prime sequence from clone BDGP:GH13185.3prime-hit FB:FBgn0039443 $/$ sym $=$ CG14242 $/$ name $=/$ prod $=/$ func $=/$ map $=97 \mathrm{C} 1-97 \mathrm{C} 1 /$ transc $=\mathrm{CT} 33862 /$ len $=687 / \mathrm{GB}: \mathrm{AE} 003757$

2.1 FB:FBgn0037465 $/$ sym $=$ CG1 $105 /$ name $=/$ prod $=/$ func $=/$ map $=84 \mathrm{C} 1-84 \mathrm{C} 1 /$ transc $=\mathrm{CT} 1653 /$ len $=1336 / \mathrm{GB}: \mathrm{AE} 003673$

FB:FBgn0034494 /sym=CG10444 /name=/prod=sodium-dependent multivitamin transporter-like $/$ func=transporter $/ \mathrm{map}=56 \mathrm{~F} 15-56 \mathrm{~F} 16 /$ transc $=\mathrm{CT} 29322 / \mathrm{len}=2772 / \mathrm{GB}: \mathrm{AE} 003792 /$ note $=3$ prime seq

2.1 FB:FBgn0028684 /sym=Rpt5 /name=Tat-binding protein-1 /prod=19S proteasome regulatory particle, triple-A protein, subunit S6a /func=proteasome ATPase ; EC:3.6.4.8 /map=95B7-95B7/transc=C

2.1 FB:FBgn0034583/sym=CG10527 /name $=/$ prod $=/$ func $=/$ map=57B20-57B20 /transc=CT29543/len=1133 /GB:AE003452/note=3prime sequence from clone BDGP:LD46156.3prime-hit

2.1 FB:FBgn0016700/sym=Rab1 /name=Rab-protein $1 /$ prod=/func=RHO small GTPase $/$ map=93D4-93D4 /transc=CT11153/len=1791/GB:AE003734

2.1 FB:FBgn0038298/sym=CG18525/name $=/$ prod $=/$ func $=/$ map=88E7-88E7 /transc=CT42292/len=1494 /GB:AE003708 /note=3prime sequence from clone BDGP:GH14439.3prime-hit

2.1 FB:FBgn0037686/sym=CG9354/name=/prod=ribosomal protein L34-like/func=structural protein of ribosome /map=85D15-85D15/transc=CT25494/len=771 /GB:AE003682 FB:FBgn0036722 $/$ sym $=$ CG13729 $/$ name $=/$ prod $=/$ func $=/$ map $=74$ C1-74C1 $/$ transc $=$ CT33196 $/$ len $=360 /$ GB:AE003524

2.1 FB:FBgn0002626/sym=RpL32 /name=Ribosomal protein L32 /prod=ribosomal protein L32/func=large-subunit cytosol ribosomal protein /map=99D5-99D5 /transc=CT6405/len=505 /GB:AE003772

2.1 FB:FBgn0030087/sym=CG7766 $/$ name $=/$ prod $=/$ func=protein kinase $/ \mathrm{map}=8 \mathrm{C} 13-8 \mathrm{C} 14 /$ transc $=\mathrm{CT} 23171 / \mathrm{len}=3994 / \mathrm{GB}: \mathrm{AE} 003446$

2.1 FB:FBgn0023211 /sym=Elongin-C $/$ name=Elongin $\mathrm{C} /$ prod=elongin $\mathrm{C} /$ func=transcription factor $/ \mathrm{map}=56 \mathrm{D} 7-56 \mathrm{D} 7 / \operatorname{transc}=\mathrm{CT} 26431 / \mathrm{len}=557 / \mathrm{GB}: \mathrm{AE} 003796$

FB:FBgn0040974 $/$ sym $=$ CG9260 $/$ name $=/$ prod $=/$ func $=/$ map $=34 \mathrm{C} 1-34 \mathrm{C} 1 /$ transc $=\mathrm{CT} 26204 / \mathrm{len}=460 /$ GB:AE003640

2.1 FB:FBgn0002533/sym=Lcp2 /name=Larval cuticle protein $2 /$ prod=larval cuticle protein $2 /$ func $=$ structural protein of larval cuticle $($ Drosophila $) \quad /$ map $=44 \mathrm{C} 1-44 \mathrm{C} 1 /$ transc $=\mathrm{CT} 3631 /$ len $=526 / \mathrm{GB}: \mathrm{AEO}$

2.1 FB:FBgn0026088 $/$ sym=EG:63B12.12 $/$ name $=/$ prod $=/$ func $=/$ map=2B14-2B $14 /$ transc $=$ CT34631 /len $=460 /$ GB:AE003422

FB:FBgn0039353/sym=CG5046/name=/prod= /func=cell adhesion $/$ map=96E10-96E10/transc=CT16193/len=840 /GB:AE003753

2.1 FB:FBgn0011768 /sym=Fdh /name=Formaldehyde dehydrogenase /prod=formaldehyde dehydrogenase (glutathione) /func=formaldehyde dehydrogenase (glutathione) ; EC:1.2.1.1 | inferred from direct

2.1 FB:FBgn0032725/sym=CG10679/name $=/$ prod= $/$ func=structural protein of ribosome $/$ map=37B8-37B8 /transc=CT29904 /len=255/GB:AE003661

2.1 FB:FBgn0032134 /sym=CG3864 /name $=/$ prod $=/$ func $=/$ map=30C1-30C2 $/$ transc=CT12877/len=966 /GB:AE003625 /note=3prime sequence from clone BDGP:SD03042.3prime-hit

2.1 FB:FBgn0033635/sym $=\mathrm{CG} 7777 /$ name $=/$ prod=water transporter $/$ func $=$ transporter $/$ map $=47 F 13-47 F 13 /$ transc $=\mathrm{CT} 23658 /$ len $=1371 / \mathrm{GB}: \mathrm{AE} 003826 /$ note $=3$ prime sequence from clone

2.1 FB:FBgn0002535/sym=Lcp $4 /$ name=Larval cuticle protein $4 /$ prod=larval cuticle protein $4 /$ func $=$ structural protein of larval cuticle $($ Drosophila $) \quad / \mathrm{map}=44 \mathrm{C} 1-44 \mathrm{C} 1 /$ transc $=\mathrm{CT} 6607 /$ len $=682 / \mathrm{GB}: \mathrm{AEO}$

2.1 FB:FBgn0002741 /sym=Mhc /name=Myosin heavy chain $/$ prod=myosin II heavy chain $/$ func=muscle motor protein $/$ map=36A8-36A9/transc=CT39920/len=5794 /GB:AE003652

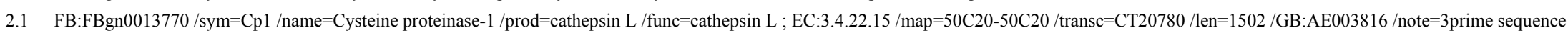
FB:FBgn0033024/sym=CG10416/name=/prod=/func=RNA-directed DNA polymerase, group II intron encoded $/$ map=41C2-41C2/transc=CT29248 /len=1195/GB:AE003786

2.1 FB:FBgn0030847/sym $=$ CG12991 $/$ name $=/$ prod $=/$ func $=/$ map=16B8-16B8 $/$ transc $=$ CT32195 $/$ len $=1380 /$ GB:AE003506

FB:FBgn0035026/sym=CG12252/name $=/$ prod=RNA polymerase CTD phosphatase $/$ func=protein phosphatase $/$ map=60D5-60D5/transc $=\mathrm{CT} 15027 /$ len=3150 $/$ GB:AE003464 $/$ note $=3$ prime sequenc 2.1 FB:FBgn0037752/sym=CG8495/name=/prod=ribosomal protein S29-like /func=structural protein of ribosome $/$ map=85E9-85E9/transc=CT24837/len=979/GB:AE003684

2.1 FB:FBgn0030350 $/$ sym $=\mathrm{CG} 1844 /$ name $=/$ prod $=/$ func $=/$ map=10F4-10F4 $/$ transc $=$ CT5645 /len=828 /GB:AE003487 /note=3prime sequence from clone BDGP $:$ GH03581.3prime-hit 
2.1 FB:FBgn0035549 $/$ sym $=$ CG1 $1346 /$ name $=/$ prod $=/$ func $=$ structural protein $/ \mathrm{map}=64 \mathrm{~B} 12-64 \mathrm{~B} 12 / \operatorname{transc}=\mathrm{CT} 31648 /$ len $=564 / \mathrm{GB}: \mathrm{AE} 003481$ BDGP:LD32469.3prime-hit /maps to FB:FBgn0032432 (/sym=CG5442 /name=/prod=/func=) and FB:FBgn0032431 (/sym=CG5435/name $=/$ prod=/func= $)$

2.1 FB:FBgn0038570 $/ \mathrm{sym}=\mathrm{CG} 7217 /$ name $=/$ prod $=/$ func $=/ \mathrm{map}=90 \mathrm{E} 4-90 \mathrm{E} 4 /$ transc $=\mathrm{CT} 22257 / \mathrm{len}=728 / \mathrm{GB}: \mathrm{AE} 003721 /$ note $=3$ prime sequence from clone BDGP $:$ LD45324.3prime-hit

2.1 FB:FBgn0023081/sym=gek /name=genghis khan /prod=protein serine/threonine kinase $/$ func=protein serine/threonine kinase $;$ EC:2.7.1.37 /map=60B5-60B5/transc=CT13314 /len=5090 /GB:AE00346 2.1 FB:FBgn0037874 $/$ sym $=\mathrm{CG} 4800 /$ name $=/$ prod $=/$ func $=/$ map $=86 \mathrm{D} 7-86 \mathrm{D} 7 /$ transc $=\mathrm{CT} 15437 / \mathrm{len}=782 / \mathrm{GB}: \mathrm{AE} 003690$

2.1 FB:FBgn0020249/sym=stck /name=steamer duck /prod= /func=transcription factor $/$ map=84F15-84F16/transc=CT23960 /len=1335 /GB:AE003678 /note=3prime sequence from clone BDGP:LD393 FB:FBgn0039333 $/$ sym $=$ CG1 $1917 /$ name $=/$ prod $=/$ func $=/$ map $=96$ D2-96D $2 /$ transc $=C T 37090 /$ len $=718 /$ GB:AE003751

2.1 FB:FBgn0024923 /sym=TER94/name=Saccharomyces cerevisiae UAS construct a of McKearin /prod=transitional endoplasmic reticulum adenosinetriphosphatase /func=adenosinetriphosphatase ; EC:3

2.1 FB:FBgn0027844 /sym=CAH1 /name=Carbonic anhydrase 1 /prod=carbonate dehydratase /func=carbonate dehydratase ; EC:4.2.1.1 /map=34D1-34D1 /transc=CT23642/len=1439 /GB:AE003641 /note FB:FBgn0033900/sym=CG8257/name=/prod=cysteine--tRNA ligase-like /func=enzyme /map=50E2-50E2 /transc=CT24479/len=1770 /GB:AE003815 /note=3prime sequence from clone BDGP:GH

2.1 FB:FBgn0000116/sym=Argk /name=Arginine kinase $/$ prod=arginine kinase $/$ func=arginine kinase $; \mathrm{EC}: 2.7 .3 .3 / \mathrm{map}=66 \mathrm{~F} 2-66 \mathrm{~F} 2 /$ transc $=\mathrm{CT} 16565 /$ len=2106 $/ \mathrm{GB}: \mathrm{AE} 003553 / \mathrm{note}=3 \mathrm{prime}$ sequence fro

2 FB:FBgn0037253 $/$ sym $=$ CG9798 $/$ name $=/$ prod $=/$ func $=/$ map $=82 \mathrm{C} 1-82 \mathrm{C} 2 /$ transc $=\mathrm{CT} 27682 / \mathrm{len}=1914 / \mathrm{GB}: \mathrm{AE} 003606$

2 FB:FBgn0027066/sym=BcDNA:LD08743/name $=/$ prod $=/$ func $=$ cytoskeletal structural protein $/$ map $=42 \mathrm{C} 3-42 \mathrm{C} 4 /$ transc $=\mathrm{CT} 10989 / \mathrm{len}=1860 / \mathrm{GB}: \mathrm{AE} 003789$

2 FB:FBgn0030541/sym=CG1 1584/name=/prod=/func=ligand binding or carrier $/$ map=12D3-12D3/transc=CT36540/len=3048 /GB:AE003495

2 BDGP:GH18222.3prime-hit /ESTpos=maps in FB:FBgn0033295/sym=CG8689/name=/prod=maltase L-like /func=enzyme /map=44C3-44C3/transc=CT6492/len=521

2 FB:FBgn0011726/sym=tsr /name=twinstar $/$ prod=cofilin $/$ func=actin binding $/$ map=60B2-60B2 /transc=CT13858 /len=733 /GB:AE003462

2 FB:FBgn0033134 $/$ sym $=$ CG12840 $/$ name $=$ Tetraspanin 42El $/$ prod=tetraspanin $/$ func $=/$ map=42E2-42E2 $/$ transc $=$ CT31972 $/$ len=1034 $/$ GB:AE003842 $/$ note $=3$ prime sequence from clone BDGP:GH1495

2 FB:FBgn0026630 $/$ sym $=$ nes $/$ name $=$ nessy $/$ prod $=/$ func $=/$ map $=76$ A3-76A3 $/$ transc $=$ CT27290 $/$ len $=2247 /$ GB:AE003517

2 FB:FBgn0039595/sym=CG10001/name=/prod=allatostatin receptor-like /func=allatostatin receptor $/$ map=98E2-98E2/transc=CT28187/len=990/GB:AE003766

2 FB:FBgn0032518/sym=CG9282/name=/prod=ribosomal protein L24/func=structural protein of ribosome /map=34B6-34B6/transc=CT26439/len=774 /GB:AE003640

2 J04423 E coli bioD gene dethiobiotin synthetase ( -5 and -3 represent transcript regions 5 prime and 3 prime respectively)

2 FB:FBgn0010808 $/$ sym=l(3)03670 $/$ name $=/$ prod $=/$ func $=/$ map=100B4-100B4 $/$ transc $=$ CT4906 /len=906 $/$ GB:AE003776

2 FB:FBgn0035272/sym $=\mathrm{CG} 13922 /$ name $=/$ prod $=/$ func $=$ structural protein of ribosome $/$ map $=62 \mathrm{~B} 4-62 \mathrm{~B} 4 /$ transc $=\mathrm{CT} 33461 /$ len $=830 / \mathrm{GB}: \mathrm{AE} 003472$

2 FB:FBgn0035201 $/$ sym $=$ CG9146 $/$ name $=/$ prod $=/$ func $=/$ map $=61 \mathrm{~F} 5-61 \mathrm{~F} 5 /$ transc $=\mathrm{CT} 26188 / \mathrm{len}=4092 / \mathrm{GB}: \mathrm{AE} 003471$

2 FB:FBgn0027525/sym=BcDNA:LD21529/name $=/$ prod $=/$ func $=/$ map=47C3-47C3 /transc=CT23459/len=1643 /GB:AE003828 /note=3prime sequence from clone BDGP:LD21529.3prime-hit

2 FB:FBgn0025366 /sym=Ip259/name=Intronic Protein $259 /$ prod= /func= /map=31E1-31E1/transc=CT16819/len=838 /GB:AE003628 /note=3prime sequence from clone BDGP:GM13959.3prime-hit

2 FB:FBgn0033188 /sym=CG1600 /name = /prod=/func=enzyme /map=43D3-43D3 /transc=CT37723/len=1747 /GB:AE003840/note=3prime sequence from clone BDGP:GH18014.3prime-hit

2 BDGP:LP1 1629.3prime-hit /ESTpos=maps 3prime of FB:FBgn0033505/sym=CG3451 /name $=/$ prod $=/$ func $=$ cell cycle regulator $/ \mathrm{map}=46 \mathrm{F9}-46 \mathrm{F9} / \mathrm{transc}=\mathrm{CT} 7898 / \mathrm{len}=250$

2 FB:FBgn0000261 /sym=Cat /name=Catalase /prod=catalase $/$ func=catalase $;$ EC:1.11.1.6 $/$ inferred from direct assay $/$ map=75D7-75D8 /transc=CT21282 /len=1977 /GB:AE003519

2 FB:FBgn0031213/sym=CG11372/name $=/$ prod $=/$ func=ligand binding or carrier $/ \mathrm{map}=21 \mathrm{~A} 5-21 \mathrm{~A} 5 /$ transc $=\mathrm{CT} 31742 / \mathrm{len}=1517 / \mathrm{GB}: \mathrm{AE} 003590$

2 FB:FBgn0025828 /sym=EG:EG0003.7/name=/prod=cytosolic translation release factor-like /func=cytosolic translation release factor /map=53D14-53D15/transc=CT19804/len=790/GB:AE003805 2 FB:FBgn0025582/sym=Int6 $/$ name $=$ Int6 homologue $/$ prod=translation initiation factor 3 , subunit $6 /$ func $=$ cytosolic translation initiation factor $/ \mathrm{map}=73 \mathrm{C} 1-73 \mathrm{C} 1 /$ transc $=\mathrm{CT} 27364 / \mathrm{len}=1560 / \mathrm{GB}: \mathrm{AE}$ 
2 FB:FBgn0030531/sym=CG11058/name=/prod=/func=enzyme /map=12B9-12B10/transc=CT30929/len=2254 /GB:AE003493 /note=3prime sequence from clone BDGP:GH10492.3prime-hit

2 FB:FBgn0015268 /sym=Nap1 /name=Nucleosome assembly protein $1 /$ prod=nucleosome assembly protein $1 /$ func=nucleosome assembly chaperone $/ \mathrm{map}=60 \mathrm{~A} 9-60 \mathrm{~A} 9 /$ transc $=\mathrm{CT} 16956 /$ len $=1381 / \mathrm{G}$

2 FB:FBgn0025637/sym=skpA /name=/prod=/func=cell cycle regulator /map=1B10-1B11/transc=CT32694/len=1059/GB:AE003418/note=3prime sequence from clone BDGP:HL01263.3prime-hit

2 FB:FBgn0020236/sym=ATPCL /name=ATP citrate lyase /prod=ATP-citrate (pro-S)-lyase /func=ATP-citrate (pro-S)-lyase ; EC:4.1.3.8 /map=52E1-52E1 /transc=CT18257 /len=3745/GB:AE003808

2 FB:FBgn0038446 $/$ sym $=$ CG14903 $/$ name $=/$ prod $=/$ func $=/$ map $=89 \mathrm{D} 3-89 \mathrm{D} 3 /$ transc $=\mathrm{CT} 34727 / \mathrm{len}=360 / \mathrm{GB}: \mathrm{AE} 003714$

2 FB:FBgn0035471 $/$ sym $=$ CG10849 $/$ name $=/$ prod $=/$ func $=/$ map=63F6-63F6 $/$ transc $=$ CT30379 $/$ len=1056 $/$ GB:AE003479 $/$ note $=3$ prime sequence from clone BDGP:SD09294.3prime-hit

2 FB:FBgn0038826/sym=CG17838/name=/prod=RNA binding protein /func=RNA binding /map=92F10-92F10/transc=CT39628 /len=1802 /GB:AE003732 /note=3prime sequence from clone BDGP 2 FB:FBgn0003498/sym=sqd /name=squid /prod=/func=ribonucleoprotein $/ \mathrm{map}=87 \mathrm{~F} 7-87 \mathrm{~F} 7 /$ transc=CT39414/len=1137/GB:AE003701

2 FB:FBgn0001297/sym=kay /name=kayak/prod=/func=DNA binding /map=99C2-99C4/transc=CT35622/len=2642 /GB:AE003771 /note=3prime sequence from clone BDGP:SD04477.3prime-hit 


\section{Lebenslauf}

Ich bin am 13.12.1975 in Tianjin, Volkrepublik China, geboren und habe die chinesische Staatsangehörigkeit. 1994 schloss ich die No.1 High School in Tianjin ab und begann im selben Jahr mein Studium an der Nankai Universität in Tianjin. 1998 erhielt ich den Bachelor of Science im Hauptfach Molekularbiologie. 2001erhielt ich den Master of Science (First class honours degree) der Nankai Universität in Tianjin.

Am 15. August 2001 begann ich in der Abteilung von Dr. Stephan J. Sigrist am European Neuroscience Institute des Bereichs Humanmedizin der Georg-August-Universität Göttingen unter der Anleitung von Prof. Dr. Friedrich-Wilhelm Schürmann die Arbeit an der Dissertation mit dem Thema „Identification and functional characterization of novel ionotropic glutamate receptor subunits at Drosophila neuromuscular synapse“. 
$\begin{array}{ll}\text { Summary } & 4\end{array}$

List of Figures

List of Tables

$\begin{array}{ll}\text { Acknowledgements } & 7\end{array}$

1 Introduction $\quad 8$

$\begin{array}{lr}\text { 1.1 Molecular basics of Synaptogenesis } & 8\end{array}$

1.1.1 The making of a synapse 8

1.1.2 Development of vertebrate NMJ 11

1.1.2.1 Initial AchR clustering 11

1.1.2.2 Agrin-MuSK signalling pathway 13

1.1.2.3 Developmental role of AchR per se 16

1.1.3 Development of mammalian CNS excitatory synapse 16

1.1.3.1 Cell adhesion molecules in the axon-dendrite contact 18

1.1.3.2 Assembly of synapse 21

1.1.3.2.1 Presynaptic assembly 21

1.1.3.2.2 Postsynaptic assembly 23

1.1.3.3 Time sequence of pre- and postsynaptic assembly 25

1.1.4 Drosophila NMJ as one model for synaptogenesis 25

1.1.4.1 Structural properties of Drosophila NMJ 25

1.1.4.2 Initiation and maintenance of neuromuscular connection 28

1.1.4.2.1 Initiation of neuromuscular contact---target recognition
(pathfinding)

1.1.4.2.2 Stabilization and maintenance of neuromuscular connection by cell adhesion molecules $\quad 29$

1.1.4.3 Synapse formation 31

1.2 Structure and functional relevance of ionotropic glutamate receptors 33

1.2.1 Modular design of ionotropic glutamate receptors
during evolution

1.2.2 Molecular mechanisms underlying selective assembly and transport of ionotropic glutamate receptors 34

1.2.3 Properties of ionotropic glutamate receptors expressed at Drosophila neuromuscular synapses $\quad 36$

1.3 The aim and strategy of this work 37 
2.1 Materials (Chemicals, enzymes and molecular kits)

2.2 Methods

2.2.1 Genechip analysis and quantitative real-time RT-PCR 39

2.2.1.1 Genechip analysis $\quad 39$

2.2.1.2 Real-time RT-PCR 45

2.2.2 Fly stocks and genetics 46

2.2.2.1 Fly stocks, crosses and rearing conditions 46

$\begin{array}{ll}\text { 2.2.2.2 Mutagenesis screening } & 47\end{array}$

2.2.3 Molecular constructs and transgenes 48

2.2.4 In situ hybridization $\quad 50$

2.2.5 Biochemistry (collaborated with Tobias Schwarz) 53

2.2.6 Immunohistochemistry $\quad 54$

2.2.6.1 Embryonic and Larval filet preparation $\quad 54$

2.2.6.2 Confocal epifluorescent microscopy 56

2.2.7 Ultrastructural analysis (collaborated with Carolin Wichmann) 56

2.2.8 Electrophysiology (collaborated with Robert Kittle) 57

3 Results $\quad 58$

3.1 Establishing the tissue specific transcriptome of Drosophila larval body wall muscle $\quad 58$

3.2 Identification of novel ionotropic glutamate receptor subunits expressed at the Drosophila neuromuscular synapse

3.2.1 Enrichment of various transcripts encoding novel ionotropic glutamate receptor subunits in larval body wall muscles $\quad 60$

3.2.2 Expression pattern of new ionotropic glutamate receptor genes 61

3.2.3 The GluR-IID and GluR-IIE represent a new type of muscle expressed glutamate receptor subunits

3.2.4 GluR-IIC, GluR-IID and GluR-IIE are specifically localized at postsynaptic densities of neuromuscular synapses 65

3.3 GluR-IID and GluR-IIE are essential for viability

3.4 Reciprocal dependence of all essential glutamate receptor subunits for glutamate receptor expression and function at synapse

3.4.1 Complete absence of all the other glutamate receptor subunits at synapse in knockout of any essential subunit genes

3.4.2 Genetically depriving the expression level of any single essential glutamate receptor subunits results into tight reduction of all of the other subunits at synapse

3.4.3 Depriving synaptic glutamate receptor subunits results into dramatic weakness of postsynaptic activity 
3.5 Structural role of ionotropic glutamate receptor during pre- and postsynaptic differentiation of neuromuscular synapse

3.5.1 Normal differentiation of presynaptic transmitter release mechinery at glutamate receptor deprived synapse

3.5.2 Defective assembly of PSD specialization at glutamate receptor deprived but not presynaptic neurotransmission deprived synapse

3.5.3 Ultrastructural evidence of abnormal synaptic differentiation at glutamate receptor deprived synapse

3.5.4 Defective compartmentation of the synaptic and perisynaptic area at glutamate receptor deprived but not neurotransmission deprived synapse

3.5.5 Similar defects in synaptic differentiation in the complete absence of glutamate receptors

4 Discussion

4.1 Genomic tools could speed up identifying essential genes involved in synapse formation and modulation

4.2 GluR-IID and GluR-IIE are novel glutamate receptor subunits crucial for glutamate receptor assembly and thus neurotransmission at neuromuscular synapse

4.3 Implications in the in vivo stoichiometry of Drosophila glutamate receptor based on genetic analysis

4.4 Structural role of ionotropic glutamate receptor per se in organizing synaptic assembly and time sequence of pre- and postsynaptic assembly

References

Appendix: Genes enriched in larval body wall preparations 


\section{Summary}

The molecular mechanisms triggering the formation of synapses in vivo are crucial for understanding the development as well as the activity dependent remodelling of synaptic circuits. The Drosophila neuromuscular junction (NMJ) provides an excellent platform for investigating fundamental aspects of how glutamatergic synapse form in vivo. The molecular mechanisms relevant to synaptogenesis and growth control of the Drosophila NMJ were addressed by combining genome-wide transcript analysis with functional genetics. This allowed the identification of two novel postsynaptic muscle expressed ionotropic glutamate receptor subunits, GluR-IID and GluR-IIE. Genetic elimination of either of the two novel subunits resulted in paralyzed lethal embryos, indicating that both new subunits are essential for forming the postsynaptic glutamate receptor complex. Further genetic, cell biological and electrophysiological studies then uncovered a tight interdependence of all NMJ glutamate receptor subunits for synaptic localization and function. These results imply that the NMJ glutamate receptor complex has strictly hetero-tetrameric stoichiometry. This so far was not described for mammalian ionotropic glutamate receptors which are usually considered to be dimers of dimers. In the second part of the thesis, glutamate receptor deprived synapses were closer inspected. Surprisingly, depleting glutamate receptors (but not depleting synaptic neurotransmission activity) provoked severe ultrastructural and molecular defects of postsynaptic membrane organization and compartment formation. Thus, the glutamate receptor complex per se but not its ligand-gated ion channel activity seemingly plays an instructive role for assembling mature postsynaptic specializations. Such a 'structural' role of glutamate receptor complexes in synaptic differentiation is novel. It might be relevant for the role 
ionotropic glutamate receptors play during synaptic plasticity of the mammalian brain, which is considered to be a cellular correlate of learning and memory processes. 


\section{List of Figures}

Fig.1-1 Basic structure of chemical synapse

Fig. 1-2 Representative molecular composition of CNS glutamatergic

Fig.1-3 Vertebrate neuromuscular junction

Fig. 1-4 Spiny dendrites from hippocampal pyramidal neuron

Fig. 1-5 Structure of Drosophila NMJ

Fig.1-6 Structure of iGluR subunit and model of iGluR assembly

Figure 3-1. GluR-IID and GluR-IIE: novel glutamate receptor subunits with muscle specific expression

Figure 3-2 Sequence analysis of GluR-IID and GluR-IIE

Fig.3-3 GluR-IID and GluR-IIE are expressed within postsynaptic Densities

Figure 3-4 Genetic analysis of GluR-IID and GluR-IIE

Figure 3-5 Interdependence between glutamate receptor subunits

Figure 3-6 A partial reduction of either GluR-IIE or GluR-IID provokes a significant reduction of all glutamate receptor subunits at the NMJ

Fig.3-7 Minimal mounts of GluR-IIA and no- IIB: expression of all glutamate receptor subunits and postsynaptic sensitivity are strongly reduced

Fig. 3-8 Normal organization of presynaptic neurotransmitter release machinery components at glutamate receptor deprived synapses

Fig.3-9 Defective PSD assembly at glutamate receptor deprived but not transmission deprived synapses

Fig. 3-10 Glutamate receptor per se but not presynaptic vesicle release are important for proper synapse formation at embryo NMJ

Fig.3-11 Electron microscopy: defective postsynaptic assembly at glutamate receptor deprived synapses

Fig.3-12 Defective synaptic compartments at glutamate receptor deprived but not neurotransmission deprived synapses 


\section{List of Tables}

Table 3-1 Described muscle / cuticle-specific transcripts displaying enrichment in gene chip analysis of body-wall-preparations

Table 3-2 GluR-IID and GluR-IIE are enriched in larval body wall mRNA 


\section{Acknowledgements}

First of all, I would like to express my gratitude to my advisors, Dr. Stephan Sigrist, Prof. Dr. F. W. Schürmann and Prof. Dr. H. Jäckle for their consistent support, helpful tips, kind advices and comments.

My very special thanks are to Dr. Stephan Sigrist, who provides me the opportunity to join his group and do exciting cutting-edge science. As one talented and serious scientist, Stephan taught me not only how to perform successful experiments but also how to ask the right question and address it in a logic way. During my whole thesis period, I was encouraged by our extensive and enthusiastic discussion, from which I really have learned a lot. Also, for me it is really nice to witness the rapid growth of our young group.

I would like to thank several collaborators, Robert Kittel for electrophysiology, Dr. Carolin Wichmann for Electron Microscopy and Tobias Schwarz for biochemistry. Without their excellent contributions, I could hardly reach current stage. The Gene Chip experiments were performed in Max-Planck Institute for Biophysical Chemistry, I am really indebted to Dr. Ronald Kühnlein and Dr. Sebastian Grönke for their warm and patient help.

I am also grateful to my colleagues Carlos Melino, Tobias Rasse, Andreas Schmid, Sara Mertel, Wernher, Laura Swan and Manuela Schmidt, with whom I can freely exchange ideas and reagents, which greatly facilitate my work. I want

to express my kindest gratefulness to Christine Quentin, Miriam Richter and Jasmin for their highly professional technical assistance.

Finally, my very special thanks are addressed to my family and my friends. 


\section{Introduction}

\subsection{Molecular basics of Synaptogenesis}

\subsubsection{The making of a synapse}

The human brain comprises a huge network of neurons (about $10^{11}$ ) which link with each other structurally and functionally through the special type of cell-cell junctions called synapses. Synapses transmit electrical signals between neurons, mediating neuronal signaling and computation, also they are able to undergo long-term changes (synaptic plasticity) that underlie aspects of learning and memory ( $\mathrm{Li}$ and Sheng, 2003). Although many of these synaptic contacts are highly specialized, all neurons make use of one of the two basic forms of synaptic transmission: electrical or chemical. Synaptic communication in the brain is mainly dependent on chemical mechanisms, that is, synaptic transmission is mediated via a chemical substance that is released by presynaptic terminal and diffuses through the synaptic cleft to activate relevant receptors on the postsynaptic membrane. Like other cell-cell junctions, synapses contain a wide variety of transmembrane proteins, cytoskeletal units and signaling components. However, they differ from most other junctions in being structurally asymmetric (Fig. 1-1): the presynaptic specialization, usually on the axon, contains a complex apparatus called active zone, which controls the transmitter-filled vesicles docking, fusing and the release of transmitter into the synaptic cleft. In contrast, the postsynaptic reception specialization, just juxtaposed to the active zone and recognized ultrastructurally by the presence of an electron-dense thickening called the postsynaptic density (PSD), is made of receptors, ion channels, signaling components and scaffold proteins clustered all together (Garner et al., 2002; Yamagata et al., 2003).

On average, each neuron forms about 1000 synaptic connections and receives even more, perhaps as many as 10,000 connections (Kandel ER et al. 2001). These synapses include excitatory, inhibitory and modulatory type. Most 
of them are excitatory and utilize glutamate as neurotransmitter. The inhibitory neurotransmission is mediated by glycine or GABA, whereas modulatory neurotransmission is mediated by 5-HT, dopamine, acetylcholine (Ach) and neuropeptides etc. Notably, although presynaptic terminals from any given neuron generally release one type of transmitter, neurons usually receive multiple forms of synaptic input, which can be excitatory, inhibitory or modulatory. Moreover, anatomical evidence has shown that the number of cellular contacts often exceeds the number of synaptic connections formed onto a single cell, suggesting that neurons are able to distinguish synaptic contact site and nonsynaptic site (White et al., 1976; Benson et al., 2001). Thus, for efficient and accurate neurotransmission, the site where synaptic contact forms is carefully selected, and, within an individual synapse, appropriate pre- and postsynaptic apparatus is assembled highly coordinately and precisely.
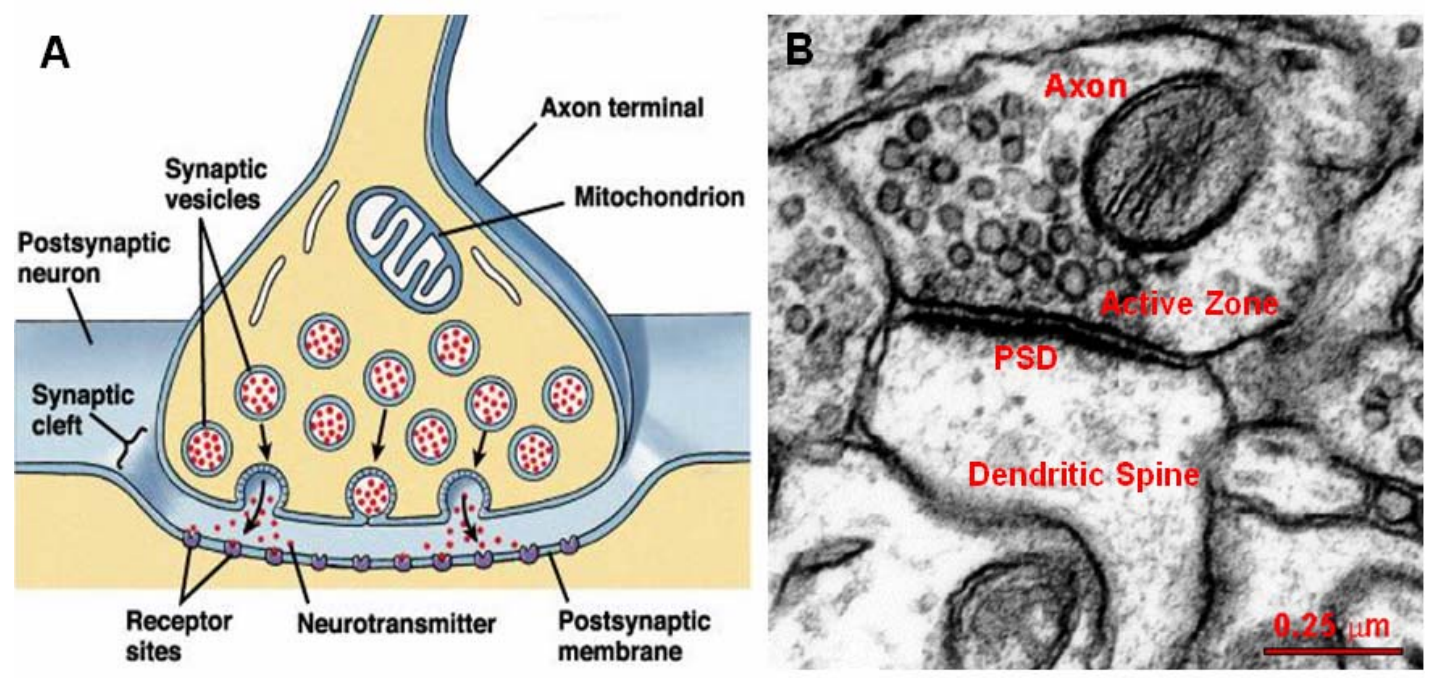

Fig.1-1 Basic structure of chemical synapse. A, diagram of a central nervous system chemical synapse. B, ultrastructure of a central chemical synapse (Adapted from www.synapses.mcg.edu/)

What is the molecular mechanisms controlling the site and type specific assembly of chemical synapses? To answer this fundamental question in neurobiology, extensive efforts have been invested to identify the molecular 
constituents of synapses and define their functional significance via the combination of molecular, biochemical and genetic approaches (Fernandez-Chacon and Sudhof, 1999; Scannevin and Huganir, 2000; Phillips et al., 2001; Sheng, 2001; Garner et al., 2002) (Fig. 1-2). Furthermore, recently the adoption of newly developed optical imaging technique has provided a way to track the dynamics of individual synaptic protein trafficking and targeting during synapse formation even in real-time resolution (Ahmari et al., 2000; Okabe et al., 2001; Prange and Murphy, 2001; Rosenberg et al., 2001; Zhai et al., 2001).
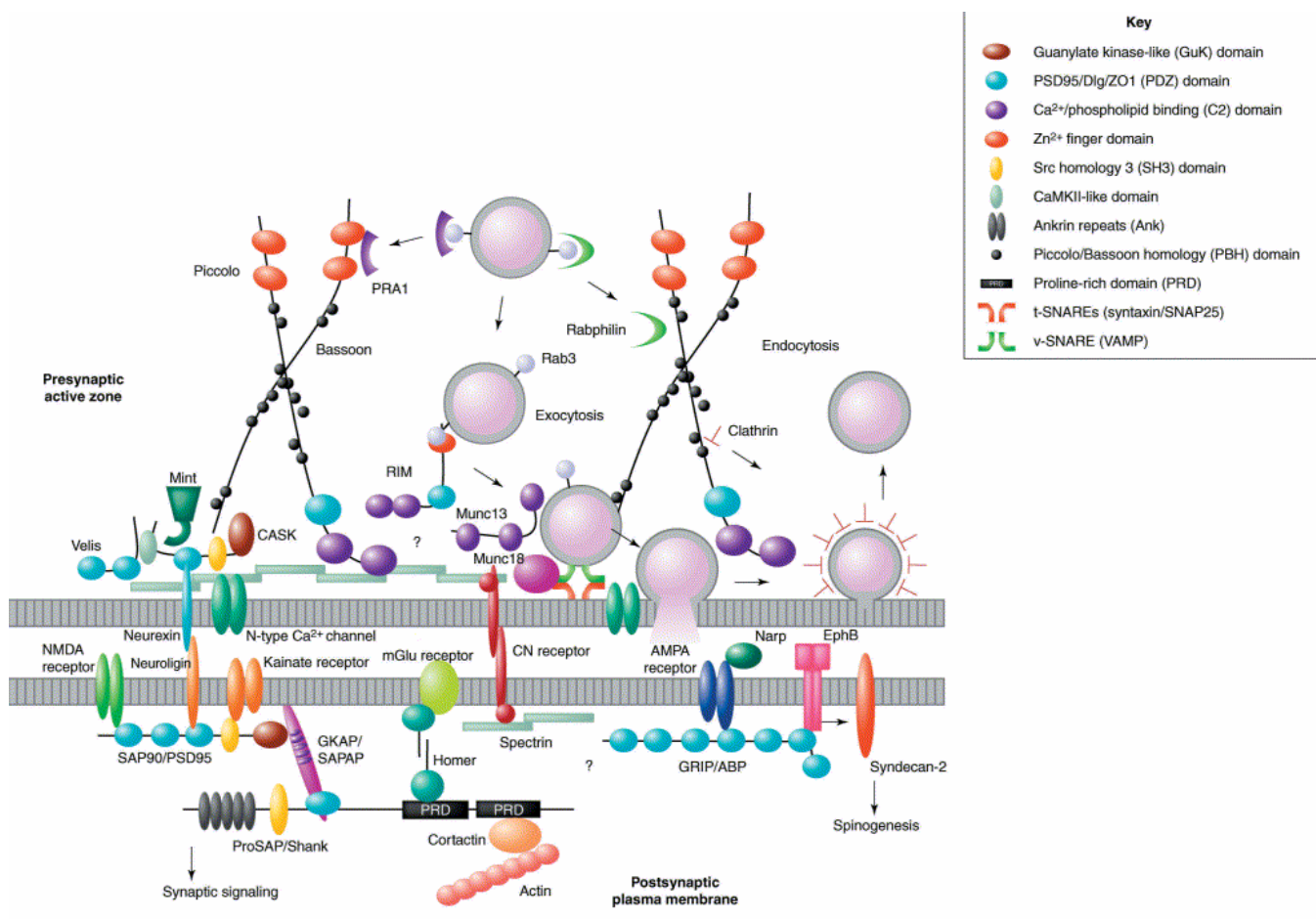

TRENOS in Neurosesiences

Fig. 1-2 Representative molecular composition of CNS glutamatergic synapse (Adapted from Garner et al., 2002).

Historically, much of what we know about the development of chemical synapses is based on studies of the vertebrate neuromuscular junction (NMJ) - a large peripheral synapse formed between a motor neuron and a skeletal muscle fibre. However, in recent years, with the incremental technical 
advances a picture of the synaptogenesis in the mammalian central nervous system has also begun to emerge.

\subsubsection{Vertebrate NMJ}

The vertebrate NMJ (Fig. 1-3) is ideal for studying chemical synaptogenesis because it is large, relatively simple and unparalleled accessible to experimentation. During development (starting at about E11 in mice) multinucleated skeletal muscle fibres form by fusion of precursor myoblasts, shortly after myotubes begin to form (at E12-13 in mice), motor neurons innervate the muscle at a specialized region of the muscle membrane called the end-plate. At the region where the motor axon approaches the muscle fibre, the axon loses its myelin sheath and splits into several fine branches. The ends of the fine branches form multiple expansions or varicosities, called synaptic boutons, from which the neurotransmitter (here is Ach) is released from the motor neuron. Each bouton is positioned over a postjunctional fold, a deep and narrow depression of muscle cell membrane that contains high densities of transmitter receptors (here is acetylcholine receptors, AchRs), and this precise apposition of postsynaptic specializations to the motor nerve terminal insures that transmitter comes into contact with its receptor within microseconds after release, thereby facilitating efficient synaptic transmission.

\subsubsection{Initial AchR clustering}

Once the myotube forms, the AchR is starting to be expressed and inserted into the muscle surface, but with low density (usually about 1000 per $\left.\mu \mathrm{m}^{2}\right)$; after synaptic maturation, by contrast, the AchRs are highly concentrated at the synaptic sites (to a density up to 10,000 per $\mu \mathrm{m}^{2}$ ) and depleted at the extrasynaptic membrane (10 per $\mu \mathrm{m}^{2}$ or less). One of the central questions is the contribution of muscle versus motor nerve in initiating 

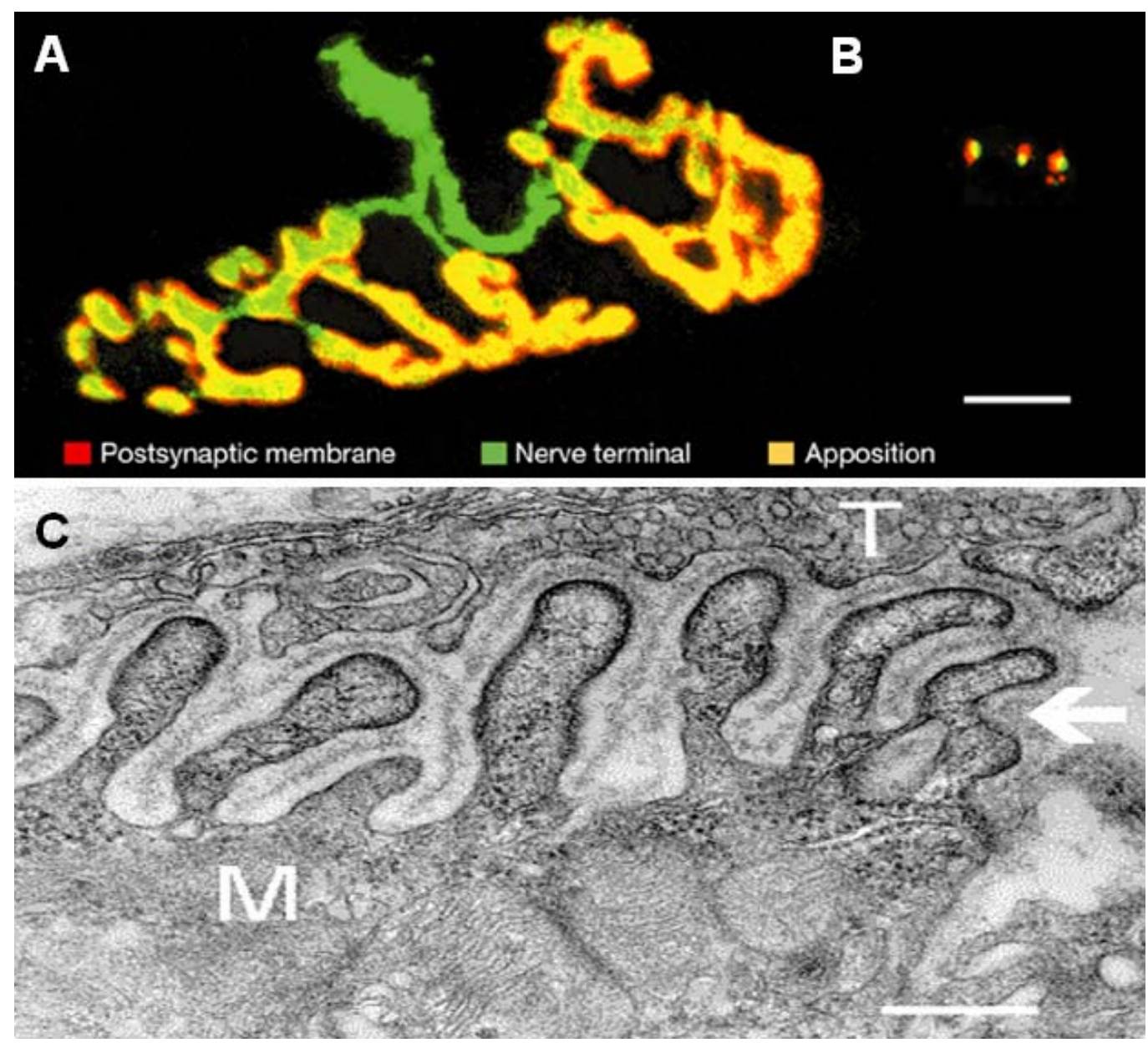

Fig.1-3 Vertebrate neuromuscular junction. A, The neuromuscular junction of an adult mouse. Scale $=5 \mu \mathrm{m}$. B, Three synapses on cultured mouse hippocampal neurons shown at the same scale(Adapted from Sanes J et al. 2001). C, Electron micrograph of motor end plate of abdominal muscle of frog. $\mathrm{T}$ - axon terminal, $\mathrm{M}$ - muscle fiber, arrow - foldings covered with basal lamina. Scale $=0.3 \mu \mathrm{m}$ (Adapted from www.synapses.mcg.edu/)

and maintaining postsynaptic differentiation. Recently, more and more evidences suggest that the muscle itself has the capacity to be prepatterned. Particularly pertinent observations are, during the brief period when synaptogenesis begins in mouse muscle, some AchR clusters are not apposed by nerves and some nerve endings are not opposed to AchR clusters; moreover, the AchR clusters are present in muscles even without innervation, most of these 
small clusters aggregate in the central end-plate band of the muscle, although this central band appears to be wider and more poorly defined than the one in innervated muscle (Lin et al., 2001; Yang et al., 2001).

\subsubsection{Arin-MuSK Signaling Pathway}

Upon innervation, AchR clusters are restricted to the central band of muscle. In the past three decades, numerous studies have established that motor nerve plays a major role in organizing this type of postsynaptic differentiation via two intramuscular mechanisms: clustering of AchRs at the synaptic contact sites; and selective transcription of AchR genes by myonuclei associated with synaptic sites (Burden, 1998; Sanes and Lichtman, 1999; Lin et al., 2001). Several nerve derived molecules have been identified and agrin is proved to be crucial in vivo. Agrin, isolated by McMahan and colleagues, is a large heparan sulphate proteoglycan that is synthesized by motor neurons, transported down motor axons, and released from nerve terminals, where it stably associates with the basal lamina of the synaptic cleft (Wallace, 1989; McMahan, 1990; Bowe and Fallon, 1995; Sanes and Lichtman, 2001). Agrin was identified from its ability to induce the aggregation AchRs on cultured myotubes, and was soon found to also aggregate many other components of the postsynaptic apparatus (Wallace, 1989). In Agrin-deficient mice, the number and density of AchR clusters, as well as synapse-specific transcription, are reduced markedly, which convincingly demonstrates that Agrin is crucial for AchR clustering in the muscle membrane opposite the presynaptic terminals (Gautam et al., 1996; Ruegg and Bixby, 1998).

MuSK, a transmembrane receptor tyrosine kinase, was discovered due to its selective abundance in synapse-rich Torpedo electric organ, whose principal cells are modified muscle cells that are innervated by cholinergic synapses (Jennings et al., 1993). Studies in mice reveal that MuSK is specifically expressed in skeletal muscles and is colocalized with AchRs in the postsynaptic membrane at the NMJ (Valenzuela et al., 1995). 
Unexpectedly, muscles in MuSK-null mutant mice show totally no detectable signs of postsynaptic differentiation, although the AchR genes are expressed at normal level (DeChiara et al., 1996; Lin et al., 2001; Yang et al., 2001). Several studies have established the linkage between Agrin and Musk signaling: application of Agrin to myotubes leads to rapid activation of MuSK; expression of a dominant negative form of MuSK inhibits Agrin-induced cluster formation in cultured myotubes (Glass et al., 1996); $\mathrm{MuSK}^{-/-}$ myotubes are completely unresponsive to agrin, but agrin sensitivity is restored by introducing wild-type MuSK (Zhou et al., 1999). Taken together, these results demonstrate that MuSK is essential for agrin-induced postsynaptic differentiation.

A crucial component downstream of MuSK that affects the postsynaptic differentiation is rapsyn, a $43-\mathrm{kDa}$ membrane associated cytoplasmic protein. Rapsyn is identified from its tight connection with AchR. It appears as soon as AchR starts clustering, perfectly colocalizes with AchR clusters, and in the electric organ it follows 1:1 stoichiometry (LaRochelle and Froehner, 1986; Noakes et al., 1993). Co-expression of AchR and rapsyn in all of the heterologous cells tested so far results into the formation of AchR- Rapsyn co-clusters, whereas AchRs are diffusely distributed when expressed along (Froehner et al., 1990; Phillips et al., 1991). Consistently, in rapsyn mutant mice, no AchR clusters form on the muscle surface (Gautam et al., 1995). Thus, rapsyn is necessary for all forms of AchR clusting. Intriguingly, rapsyn can cluster AchRs when they are co-expressed in heterologous non-muscle cells that lack agrin and MuSK (Apel et al., 1997). By contrast, not even spontaneous (agrin-independent) AchR clusters form in MuSK deficient myotubes (Zhou et al., 1999), and overexpression of rapsyn in wild-type myotubes induces virtually no further cluster formation (Yoshihara and Hall, 1993; Han et al., 1999). This raises the possibility that muscles contains intrinsic activity to prevent the MuSK signaling independent clustering of rapsyn, which might be an efficient way to avoid the AchR clustering at the 
wrong sites.

How does activation of Agrin-MuSK signaling lead to the clustering of AchRs? Previously it has been shown that cytoskeletal components are closely associated with AchR clusters, and disruption of cytoskeleton prevents the AchR cluster formation (Bloch and Pumplin, 1988). Thus, it is plausible that the activation of MuSK signaling upon agrin binding provide intrinsic cytoskeletal regulatory capacity required for remodelling and postsynaptic assembly. Consistent with this view, it has been found that agrin induces the polymerization of actin at the AchR clustering sites (Dai et al., 2000), moreover, it has been demonstrated that agrin-mediated clustering of AchRs depends on activation of $\mathrm{Rac} / \mathrm{Cdc} 42$, leading to the formation of AchR microclusters, followed by Rho activation, resulting in the consolidation of these microclusters into larger AcgR clusters (Weston et al., 2000; Weston et al., 2003).

Although there is little doubt that Agrin/ MuSK signaling is the major pathway that is crucial for NMJ synaptogenesis, the intracellular signaling mechanisms downstream of MuSK have only begun to be elucidated recently. In response to agrin binding, MuSK undergoes tyrosine phosphorylation, this might create docking sites for signaling molecules or promote proper folding of the kinase. As an example, the juxtamembrane tyrosine (Y553) is required for agrin-induced AchR clustering (Zhou et al., 1999; Herbst and Burden, 2000). Very recently, the Abl kinases were shown to be required for the agrin/ MuSK dependant synaptic assembly, Abl kinases and MuSK can effect reciprocal tyrosine phosphorylation and form a complex after agrin engagement (Finn et al., 2003). Dishevelled (Dvl), a signaling molecule important for planar cell polarity, has also been found to interact directly with MuSK and PAK, forming a signaling scaffold which is important for agrin/ MuSK mediated AchR clusting (Luo et al., 2002). Additionally, evidences have been provided that geranylgeranyltransferase I (GGT), a zinc 
metalloenzyme that tethers proteins to plasma membrane by prenylation (Zhang and Casey, 1996), serves as one important component in the agrin/ MuSK pathway, which reveals an important role of prenylation in regulating synapse formation and/ or maintenance (Luo et al., 2003).

\subsubsection{Developmental role of AchR per se}

During postsynaptic differentiation, do AchRs only occupy the slots within assembled scaffold and contribute nothing for the assembly? In tested non-muscle cells, this seems true, but in muscle cells it is obviously not the case: Rapsyn fails to cluster at synaptic sites in zebrafish mutants deficient in the AchRs, suggesting the requirement of AchRs for proper synaptic localization of postsynaptic components (Ono et al., 2001); consistently, in the variants of C2 myotubes virtually lacking AchRs, agrin also fails to aggregate rapsyn (Marangi et al., 2001); moreover, in mutant mice that lack an adult AchR subunit, the postsynaptic membrane undergoes a profound reorganization in which levels of several membrane and cytoskeletal components decline in parallel with AchR loss, and residual material forms abnormal small islands at the NMJ (Missias et al., 1997). Therefore, the AchR per se is not merely a passive ligand, but plays an active role in organizing synaptic assembly. Interestingly, this is reminiscent of the assembly of inhibitory CNS synapses, in which $\mathrm{GABA}_{\mathrm{A}}$ receptor subtypes are localized at synapses through their interaction with gephyrin (Kneussel et al., 1999). Gephyrin is not clustered at postsynaptic sites of mice lacking GABA $_{\mathrm{A}}$ receptor $\gamma$ subunits, suggesting that gephyrin requires the presence of $\mathrm{GABA}_{\mathrm{A}}$ receptors to efficiently form synaptic aggregates (Essrich et al., 1998). Thus, neurotransmitter receptors of both vertebrate NMJ and inhibitory CNS synapses are actively involved in postsynaptic assembly during synaptogenesis.

\subsubsection{Mammalian CNS excitatory synapses}

In the central nervous system, most excitatory synapses utilize glutamate 
as the neurotransmitter. Glutamatergic synapses contain excitatory, glutamate responsive ionotropic and metabotropic receptors (Hollmann and Heinemann, 1994; McGee and Bredt, 2003). Ionotropic glutamate receptors are supposed to be tetrameric complexes, and are categorized into three major classes, on the basis of pharmacology, electrophysiology and sequence homology: AMPA ( $\alpha$-amino-3-hydroxy-5-methyl-4-isoxazoleproprionic acid) receptors, NMDA (N-methyl-D-aspartic acid) receptors and kainate receptors.

Morphologically, most mature excitatory synapses are constructed on the characteristic tiny actin-rich structures---dendritic spines---the small lateral dendritic protrutions. Spines have variable size (up to a few $\mu \mathrm{m}$ in length and $0.8 \mu^{3}$ in volume) and shape (such as thin, stubby or mushroom-like) (Harris and Kater, 1994; Hering and Sheng, 2001; Li and Sheng, 2003) (Fig. 1-4). During brain development, there is morphological transition in spines. During the first two postnatal weeks, synapses are frequently observed on the dendritic shaft, on stubby spines and on filopodia (Fiala et al., 1998), with maturation of the brain, such synapses are gradually substituted by, or converted to, synapses on mushroom-like spines (Harris and Kater, 1994).

As described before, the pre- and postsynaptic membrane of vertebrate NMJ are separated by an extracellular basal membrane, the extracellular-matrix molecule agrin is secreted from the nerve terminal and induces the postsynaptic differentiation. However, in central synapses, the extracellular matrix is not well characterized and, so far, no secreted molecules has been convincingly shown to be crucial for the development of mammalian central synapses. Narp (neuronal activity-regulated pentraxin), a secreted molecule that accumulates at synaptic sites, can interact with and cluster AMPA receptors (O'Brien et al., 1999; O'Brien et al., 2002). However, just as its name implied, Narp is a neuronal activity-regulated molecule, it recruits AMPA receptors only when a synapse is undergoing activity-dependant changes. Narp is less likely to play a major role during synaptogenesis, since AMPA receptor clustering is not essential for initial 
synapse formation (see below for details).
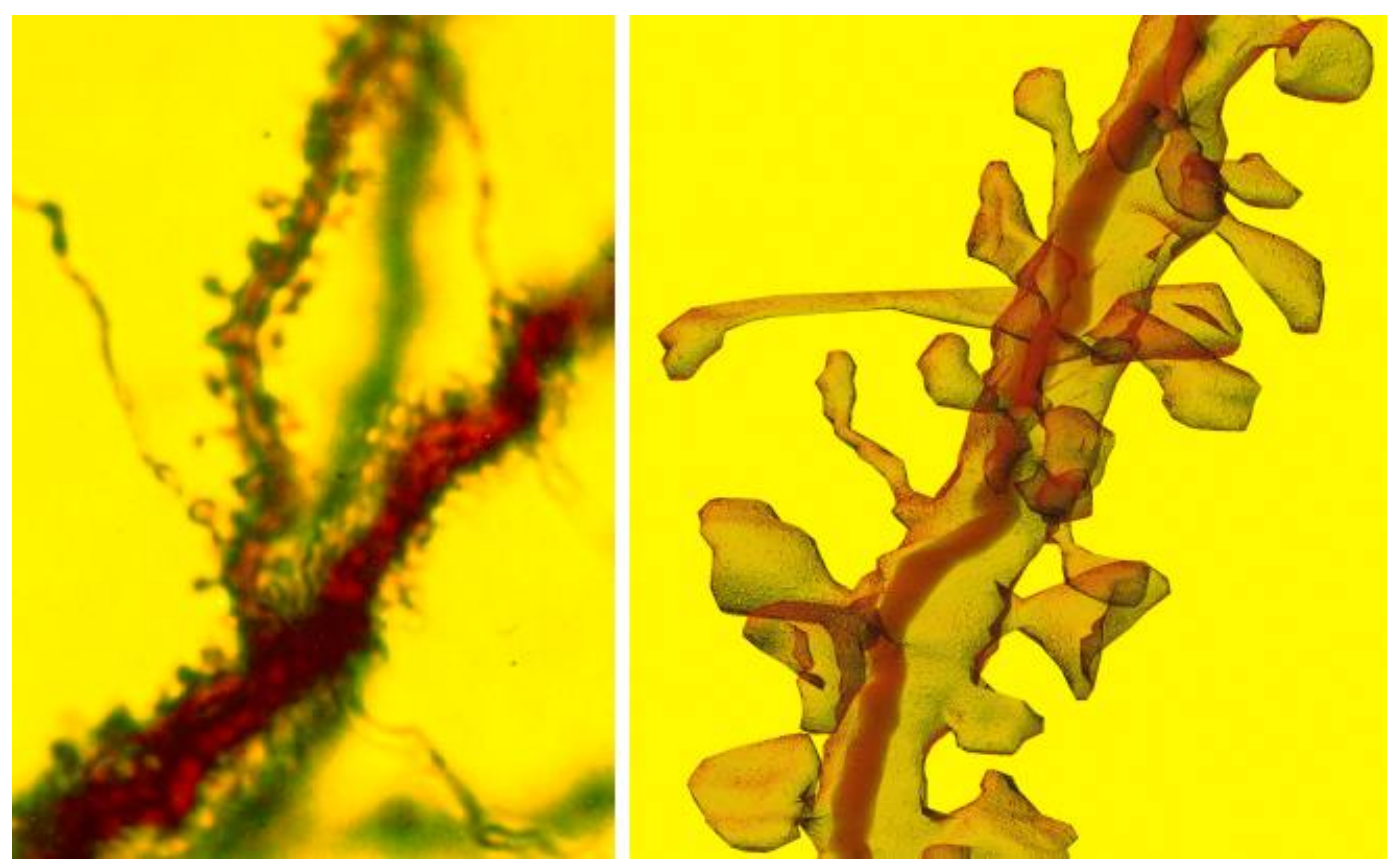

Fig. 1-4 Spiny dendrites from hippocampal pyramidal neuron.

Left: Light microscope image. Right: Reconstruction from serial electron microscopy (Adapted from www.synapses.mcg.edu/).

\subsubsection{Cell adhesion molecules in the axon-dendrite contact}

Synaptic assembly appears to be a multi-step process that is initiated from the axon-dendrite contact. Initial contact is followed by a stabilization step at the contact site and, subsequently, by further pre- and postsynaptic differentiation. Each process seems to require the interaction of various classes of cell adhesion molecules (CAMs). The Cadherin family of homophilic cell adhesion molecues is essential for the formation and stability of epithelian cell junctions. Cadherins are found to localize at both pre- and postsynaptic side, dominant-negative studies of the neuronal cadherins indicate that they are important for synapse integrity and morphological maturation of dendritic spines (Togashi et al., 2002). However, due to the large number of various Cadherin and Cadherin-related genes that are expressed in the central nervous system, it is still not possible to conclude 
whether cadherin plays an initiating role in synapse formation or a promoting role in subsequent synaptic growth.

Various types of integrins are expressed in the brain with different distribution patterns (Pinkstaff et al., 1999). Integrins function as cell-matrix or cell-cell adhesion molecules by binding, respectively, to extracellular matrix proteins or to immunoglobulin superfamily proteins (Aplin et al., 1998). Based on our current understanding, integrins have diverse functions in early stage of CNS development, including neuronal migration (Milner and Campbell, 2002), axon pathfinding and outgrowth (Hoang and Chiba, 1998; Pasterkamp and Kolodkin, 2003), they also appear to have a role in synapse maturation (Chavis and Westbrook, 2001).

Immunoglobulin superfamily molecules contain certain numbers of extracellular cyctein-looped domains first found in immunoglobulins. Synaptic cell adhesion molecule (SynCAM) belongs to such a superfamily and is found to be localized on the synaptic membranes. Importantly, expression of SynCAM in nonneuronal cells both induces neighboring neurons to form functional presynaptic terminals and, if gluamate receptors are coexpressed, endows the postsynaptic membranes with the capacity of responding to glutamate (Biederer et al., 2002). Thus, SynCAM seems an ideal candidate initiating central synapse formation, although its in vivo role has yet to be examined. Very recently, another two immunoglobulin superfamily members, SYG-1 and SYG-2 were isolated in genetic screen for C. elegans mutants defective in targeting specificity during synaptogenesis (Shen and Bargmann, 2003; Shen et al., 2004). SYG-1 is present at the presynaptic neuron HSNL and localizes to synaptic sites at the early stages of synaptic formation, while SYG-2 is expressed transiently in the guidepost epithelial cells during synapse formation. SYG-2 can interact with SYG-1 and thus instruct the accumulation of synaptic vesicles and the subsequent synaptic assembly at proper site. 
Presynaptic neurexins and postsynaptic neuroligins represent another group of synaptic CAMs proposed to function in synapse differentiation. The extracellular domain of presynaptic beta-neurexin interacts directly with the extracellular AchE-like domain of postsynaptic neuroligin (Nguyen and Sudhof, 1997). In a co-culture system, the overexpression of neuroligin in heterologous cells can induce the structural and functional presynaptic specialization in contacting axons (Scheiffele et al., 2000). More convincingly, in the context of intracellular interactions, the cytoplasmic tail of $\beta$-neurexin binds with PDZ protein CASK, a presynaptic scaffold protein; comparably, the intracellular part of neuroligin binds with PSD-95, which is an important PDZ domain scaffold protein of the postsynaptic density. Thus the direct 'talk' between $\beta$-neurexin and neuroligin across the synaptic cleft might bridge the adhesive and signaling components of both presynaptic transmitter release machinery and postsynaptic reception apparatus, allowing for a stepwise assembly of trans-synaptic complexes (Dean et al., 2003). Recent studies in knock-out mice have revealed that, in the absence of $\alpha$-neurexins the central synapse morphology is largely normal, but the presynaptic calcium channel activity and neurotransmitter release are severely impaired (Missler et al., 2003). Therefore, $\alpha$-neurexins probably have a role in organizing the molecular machinery at presynaptic side. Up to now, the in vivo role of $\beta$-neurexin in synaptic assembly remains to be answered.

Finally, Ephrin-Bs (EphB) are small transmembrane proteins identified as ligands for the EphB receptor tyrosine kinase. Through binding to EphB receptor, EphB can induce the clustering of NMDA receptors on the surface of fibroblasts and immature cultured hippocampal neurons (Dalva et al., 2000). The NMDA receptor clustering is mediated by a ligand-induced association of the extracellular domain of EphB receptor with the large extracellular domain of NR1 subunit, while the tyrosine kinase activity of EphB receptor are not necessary for this process. EphBs are unlikely to be the general inducers of postsynaptic differentiation, since in immature neurons it can only facilitate the 
clustering of NMDA receptors and its partner $\mathrm{Ca}^{2+}$-calmodulin-dependant protein kinase II (CaMKII) but not the other known PSD components (such as SAP90/ PSD95), moreover, although EpgB2 knock-out mice show deficits in activity-dependant synaptic plasticity, they have normal synapse density and structure, as well as largely intact synaptic function (Grunwald et al., 2001; Henderson et al., 2001). Syndecan2, a cell-surface heparan sulfate proteoglycan, is able to enhance the maturation of dendritic spines: when syndecan 2 is overexpressed in cultured immature hippocampal neurons, dendritic spines display morphology typical of mature spines (Ethell and Yamaguchi, 1999). Interestingly, EphB receptor is shown to be the upstream activators of syndecan2 (Ethell et al., 2001), thus EphB receptor pathway might not only function during the initial formation of synapses but also participate in their maturation process.

\subsubsection{Assembly of synapse}

The development of the time-lapse imaging techniques has allowed one to obtain information on the dynamic behavior of essential biological processes in living cells. Synaptic transmission can be detected within an hour of initial axon-dendrite contact, so the accumulation of certain pre- and postsynaptic proteins must occur even earlier. The time-lapse studies have shown that many synaptic components are assembled within tens of minutes after the initial contact of axons and dendrites, much faster than previous estimation (Friedman et al., 2000; Okabe et al., 2001; Washbourne et al., 2002).

\subsection{Presynaptic assembly}

Recently, evidence is growing to support the opinion that the presynaptic specialization is constructed from essentially two types of preassembled 'packets' of vesicles and/ or proteins (Hannah et al., 1999; Jahn and Sudhof, 1999; Sudhof, 2000). The biogenesis of these two precursor complexes occurs in the soma, apparently through the Golgi body. 
Using time-lapse imaging of green fluorescent protein (GFP) tagged VAMP (vesicle associated membrane protein, a synaptic vesicle protein), it has been found that even before the period of peak synaptogenesis in culture, VAMP-GFP is present in axons as mobile clusters (about 0.5-1.5 $\mu \mathrm{m}$ ) larger than single synaptic vesicles. These VAMP labelled puncta have varied vesicular and tubular membrane structures, as shown by EM, and they colocalize with presynaptic membrane proteins (such as the voltage-gated calcium channels) and other synaptic vesicle associated proteins (such as synaptic vesicle protein 2 and synapsin I). It is estimated that the amount of VAMP required in a single presynaptic bouton corresponds to 1-4 such precursor packets (Ahmari et al., 2000).

A second type of precursor packet, which is associated with dense-core vesicles that have a diameter of about $80 \mathrm{~nm}$, has been visualized and purified (Zhai et al., 2001). In contrast to synaptic vesicles, these dense-core vesicles contain many active zone components, including the presynaptic scaffold proteins Piccolo, Bassoon and RIM (Rab3-interacting molecule), the SNARE (soluble N-ethylmaleimide-sensitive-factor attachment protein receptor) proteins syntaxin and SNAP-25 (synaptosomal-associated protein 25), and the CAM N-Cadherin, suggesting that they constitute 'active zone presursor vesicles' that, upon fusing with the presynaptic plasma membrane, lead to rapid formation of active zones (Zhai et al., 2001). It was proposed that incorporation of only 2-3 of these transport vesicles supplies enough material for an active zone of average size (Shapira et al., 2003).

A common feature of these two types of precursor packets is that they are mobile along axons prior to synaptogenesis, but promptly immobilized at nascent synapses. During neuronal development, the Piccolo/ Bassoon dense-core vesicles are present earlier than the VAMP containing synaptic vesicle precursor packet, which might suggest that the formation of new neurotransmitter release sites is preceded by the recruitment and assembly of 
active zone components. The molecular mechanisms that target these precursor complexes to the nascent synaptic site still remain elusive, however, studies from immunoglobulin superfamily proteins (SYG-1 and SYG-2, see above) have already provided possible clue.

\subsection{Postsynaptic assembly}

Compared to the assembly of presynaptic specialization, the process of postsynaptic assembly is not well characterized yet, which is at least partially due to the fact that postsynaptic specialization is more complex and heterogeneous than presynaptic active zone. One of the best-studied complexes is associated with NMDA receptors. This complex comprises molecules such as SAP90/ PSD95, alfa-actinin, and CaMKII, neuroligin, the microtubule-binding protein Cript, the guanylate kinase domain-binding proteins GKAP/ SAPAPs, and other proteins such as ProSAP/ Shank, Homer and cortactin (which provide additional links to the actin cytoskeleton and other glutamate receptor complexes (Garner et al., 2002). The rapid recruitment of PSD-95 and the presence of non-synaptic clusters of PSD-95 (Rao et al., 1998; Marrs et al., 2001) are consistent with the idea that 'prefabricated' complexes are used to assemble the PSD---which is analogous to presynaptic differentiation. However, it is not known whether the non-synaptic clusters of PSD-95 represent real packets of PSD proteins in delivery. In sharp contrast to this view, however, independent studies suggest that although some synaptic PSD-95 clusters might be derived from the transport of pre-existing non-synaptic precursor packets, the de novo accumulation of PSD-95 clusters at nascent synapses seems to usually occur from diffuse 'cytoplasmic pools' (Bresler et al., 2001; Marrs et al., 2001). Notably, this model is supported by an updated report in which more PSD components have been examined, all the tagged PSD components tested are recruited to new synaptic sites gradually, with no discernable discrete transport packets involved (Bresler et al., 2004). Interestingly, in this new study, the non-synaptic PSD clusters are also observed, but they appear no more mobile than synaptic clusters, hence unlikely representing the transport intermediates. 
Ionotropic glutamate receptors are essential components of postsynaptic apparatus, in addition to converting the chemical signal released from presynaptic terminal to an electrical response in the postsynaptic neuron, they are critically involved in activity-dependant, long-term changes in synaptic strength, which may represent a central physiological correlate to learning and memory. Time-lapse imaging of GFP-tagged NR1 (one subunit of NMDA receptors) and GluR1 (one subunit of AMPA receptors) indicates that NMDA receptors and AMPA receptors are present in largely non-overlapping mobile clusters before synaptogenesis starts, NMDA receptors are recruited earlier than AMPA receptors to the axon-dendrite contact sites (Washbourne et al., 2002). It is still uncertain whether these glutamate receptor clusters represent prefabricated packets of synaptic components, or just reflect distinct classes of transport vesicles containing certain types of glutamate receptors. The fact that, the NMDA receptor scaffolding protein PSD-95 is transported to synaptic sites largely independently of NMDA receptors (Rao et al., 1998; Friedman et al., 2000; Rao et al., 2000; Washbourne et al., 2002), supports the latter interpretation. The non-overlapping of mobile AMPA and NMDA receptor clusters and the different dynamics of their recruitment to synaptic sites reflect the presence of distinct regulatory mechanisms controlling their synaptic traffic and localization. Consistently, the cytoplasmic parts of AMPA and NMDA receptor subunits bind to very different sets of structural and regulatory proteins (Scannevin and Huganir, 2000; Sheng, 2001; Sheng and Lee, 2001). The AMPA receptor binding protein, Stargazin, is crucial for the synaptic targeting of AMPA receptors, but not for the morphological development of synapses (Chen et al., 2000). Also combining with the discovery of 'silent synapses' present in rat hippocampus and somatosensory cortex, which only contain NMDA receptors but lack AMPA receptors (Liao et al., 1995; Isaac et al., 1997), it is clear that the AMPA receptor incorporation at the synaptic sites is not required for initial synaptic assembly. 


\subsubsection{Time sequence of pre- and postsynaptic assembly}

Although more and more pre- and postsynaptic proteins have been tagged with fluorescent probes and recorded in living cells, there is incomplete agreement on the time course of pre- and postsynaptic assembly. For example, DsRed tagged NMDA receptors have been reported to cluster at nascent synapses within a few minutes of axon-dendrite contact in young cortical culture (3-4 days in vitro) (Washbourne et al., 2002). However, another study in more mature hippocampal neurons (about 14 days in vitro) showed that the accumulation of NMDA receptors at nascent synapses takes 1-2 hours, and therefore lags behind the accumulation of presynaptic markers (Okabe et al., 2001). Thus, it is still not absolutely sure whether one synaptic side differentiates before the other, or whether the synaptic assembly undergoes simultaneously on both sides. Because the discrepancy raised here is at least partially due to the difference of experimental materials, it is also possible that divergent mechanisms for synaptic assembly are used by different neuron types, or even different stages of the same type. Further studies are apparently required to determine this basic issue in synaptogenesis, and it will be much more benificial to analyze, if possible, this process in vivo. Despite the uncertainty of the time course of pre- and postsynaptic assembly, as soon as a synapse is established, presynaptic active zone and PSD are exquisitely overlapped, indicating a close coordination of presynaptic and postsynaptic maintainence.

\subsubsection{Drosophila NMJ as a model for studying synaptogenesis}

\subsubsection{Structural properties of Drosophila NMJ}

The Drosophila neuromuscular junction (NMJ) is an excellent model system for addressing fundamental mechanisms governing synapse formation and growth. The advantageous features of Drosophila NMJ include its structural accessibility and amenability to powerful genetic, electrophysiological and microscopic techniques. 
In Drosophila late stage embryo and larva, body wall musculature shows a segmentally repeated pattern of 30 muscle cells (in each symmetric hemisegment) innervated by about 45 motor axons. Each of these muscles is easily identified by its distinct position and size, and is innervated by specific motorneurons. The NMJ morphology is highly sterotyped, the axons of motorneurons spread onto the muscle surface, forming a synaptic arbor composed of series of varicosities connected by thin axonal processes (Fig. 1-5 A). These varicosities, or called synaptic boutons, are categorized by three classes (Gramates and Budnik, 1999; Rheuben et al., 1999). The major class of boutons (type I) contain clear vesicles and are glutamatergic, and at larval stage, they are surrounded by the characteristic subsynaptic reticulum (SSR), an elaborately layered structure formed from the muscle membrane at the junctional region (Atwood et al., 1993; Jia et al., 1993)(Fig. 1-5 B). All 30 body wall muscles are innervated by type I glutamatergic boutons, some muscles are also innervated by type II and type III boutons, which contain octopamine or peptide neurotransmitters (Koh et al., 2000). The neuromuscular contact differentiates into distinct boutons containing functional synapses in late stage embryo, and the boutons continuously grow and sprout throughout the whole larval life. Extensive studies have been made on the third instar larval type I glutamatergic synapses, detailed analysis revealed that type I boutons can be further divided into type I small (Is) and type I big (Ib) by size, the extent of the folding of SSR, and electrophysiological properties. Due to the tiny size of embryo, it is not used as frequently as larva, however, it has been proved to be invaluable in understanding molecular events relevant to initial synapse formation. Unlike the vertebrate NMJ, the NMJ of Drosophila is not covered by glial cells, leaving $10-20 \mu \mathrm{m}$ of neuronal surface without insulation from the hemolymph. (Hall and Sanes, 1993; Auld et al., 1995).

Notably, Drosophila NMJ shares several important features with the excitatory central synapses in vertebrate brain: it is glutamatergic (unlike the cholinergic NMJ in vertebrate) and contains homologous ionotropic glutamate 

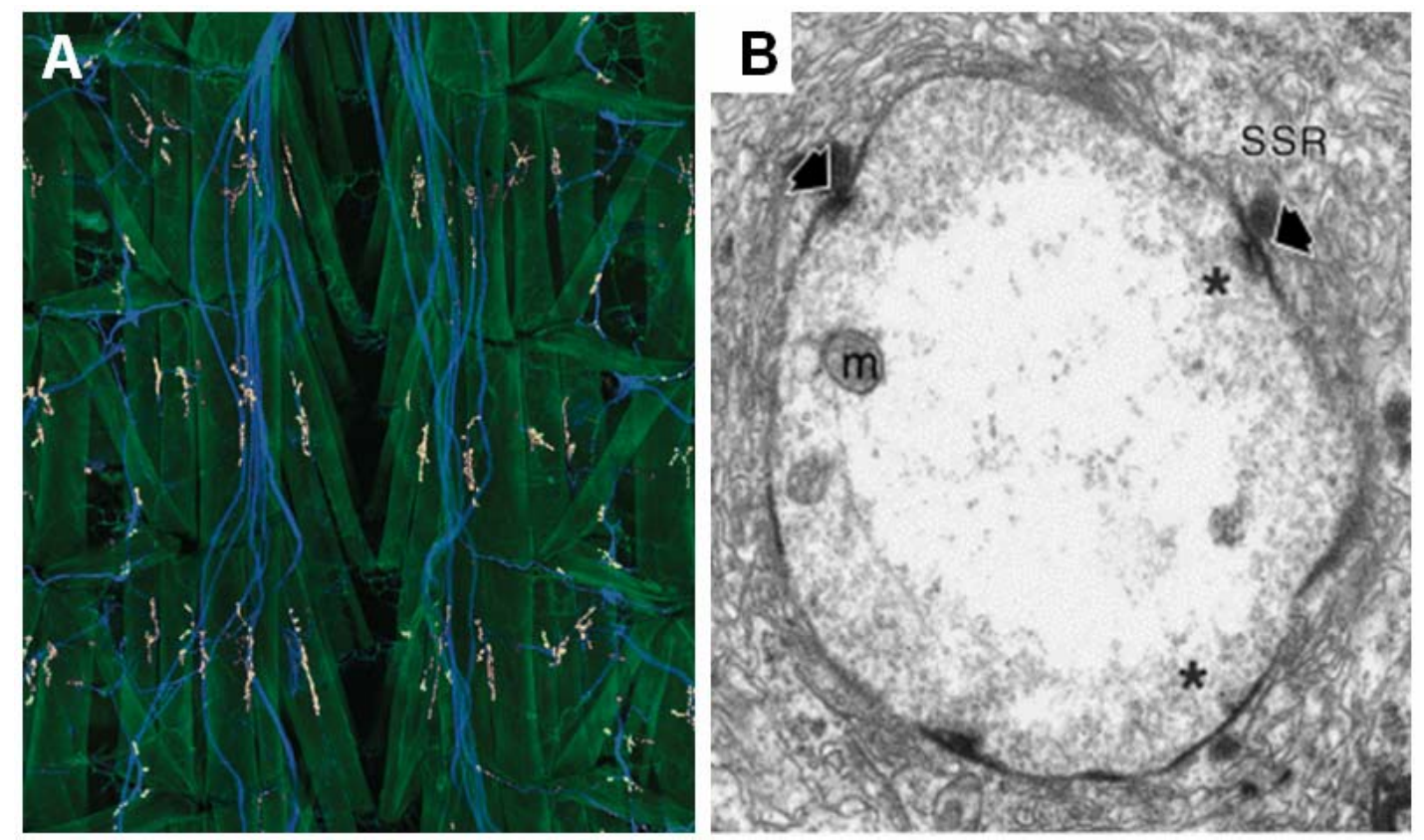

\section{Fig.1-5 Structure of Drosophila NMJ.}

A, Three segments of a dissected third instar Drosophila larva. Muscles are stained as green, neuromuscular junctions stained as red and nerves stained as blue (Adapted from (Daniels et al., 2004). B, Electron micrographs depicting cross-sections through a type-I bouton on muscle 6 in wild-type larvae. The subsynaptic reticulum (SSR), active zones (asterisks), T-bars (thick arrows), and mitochondria (m) are marked (Adapted from (Aberle et al., 2002).

receptors; there is no obvious basal lamina layer that separates pre- and postsynaptic membrane, so the synapse appears close membrane-membrane apposition ultrastructurally (Fig. 1-5 B); it is organized into a series of boutons that can be added or eliminated during development and plasticity (Petersen et al., 1997). During the period that larva grows from first instar to third instar, which normally takes several days, the muscles undergo a dramatic increase in size (more than 100 folds), therefore requiring a concomitant addition of more synaptic boutons and further elaboration of NMJ branches to efficiently depolarize the muscles.

Although the NMJ architecture changes dramatically during postembryonic 
development, the characteristics of individual neuromuscular synapses are rather similar between embryo and larva at ultrastructural level (Prokop, 1999): the pre- and postsynaptic membranes appear smoother and more electron dense compared to the extrasynaptic areas, indicating that the molecular composition of synaptic fields differs from extrasynaptic membranes. The intracellular face of presynaptic membrane harbors active zones, which contain characteristic T-shaped electron-dense structures (T-bars) surrounded by clusters of synaptic vesicles (Fig. 1-5 B). T-bars can still be seen in neuronal terminals that are depleted of synaptic vesicles and therefore appear to be independent molecular structures (Poodry and Edgar, 1979). The exact molecular composition and function of T-bars still remain unclear.

\subsubsection{Initiation and maintenance of neuromuscular connection 1.1.4.2.1 Initiation of neuromuscular contact---target recognition (pathfinding)}

So far, most studies on NMJ formation have been carried out on the ventral longitudinal muscles 6 and 7, which are innervated exclusively by glutamatergic type I boutons. It is believed that most NMJs, if not all, develop in a similar way and share the common architecture. At embryonic stage 15/ 16, muscles start sending out myopodia preferentially from the future innervation site (Ritzenthaler et al., 2000), and they are contacted by filopodia sent out from the motoneuronal growth cones in a random fashion. Those motoneuronal processes which are in contact with inappropriate muscles are soon withdrawn (Broadie and Bate, 1993; Yoshihara et al., 1997). This specific targeting recognition process is suggested to be mediated by both repulsion from inappropriate target muscle and contact-mediated attraction to the right target muscle (Bate and Broadie, 1995; Keshishian et al., 1996). Example of repellent molecule is the Toll, which is present on the surfaces of certain muscles and can prevent innervating by irrelevant motorneurons, removal of Toll leads to the ectopic 
innervation. Fasciclin III (FasIII), one homophilic cell adhesion molecule, appears to be mediate attractive cell-cell recognition, since misexpression of FasIII introduces innervation at the non-target cell. Up to now, many targeting cues have been identified, such as caspricious, semaphorins, connectin, neuromusculin and netrins (Bate and Broadie, 1995; Chiba and Rose, 1998), acting as either repellent or attractive molecules.

\subsection{Stabilization and maintenance of neuromuscular connection by cell adhesion molecules}

As soon as the initial neuromuscular contact is established, it has to be stabilized for further differentiation. This process, which should be also required for the new bouton growth during postembryonic period, is believed to be largely mediated by multiple cell adhesion molecules. Upon initial neuromuscular contact at embryonic stage 13, usually the junction consists of only short stretches of apposed membranes, often interrupted by stretches of non-connected cell surfaces (Schuster et al., 1996; Yoshihara et al., 1997; Prokop A et al., 1998). While at the mature NMJ of late stage 17 embryo such contact develops into an extended cell-cell junction, indicating that adhesive properties change during NMJ maturation. Consistent with this notion, FasIII, which contributes to target recognition and potentially also early phase of contact stabilization, disappears from NMJs of later stages (Broadie and Bate, 1993). In contrast, FasII, another homophilic CAM belonging to the immunoglobulin superfamily, is initially expressed strongly on the surfaces of all motor axons and at low levels in muscles during NMJ formation (Schuster et al., 1996). As synaptic connections are established, FasII is progressively

clustered at pre- and postsynaptic membranes. The late junctional maintenance of FasII is largely, but not all, mediated by the scaffold protein Discs large (Dlg), the Drosophila Membrane-Associated Guanylate Kinase (MAGUK) homologous to mammalian PSD-95/ SAP90 family proteins (Budnik et al., 1996; Thomas et al., 1997; Zito et al., 1997), which itself is not detectable at the NMJ until late stage 17 (Guan et al., 1996). Deleting FasII does not interfere with 
NMJ formation, but strongly affects postembryonic stabilization and maintenance of NMJs, suggesting its crucial role in controlling synaptic stability and growth (Schuster et al., 1996). Furthermore, it seems to be of developmental importance that FasII function is restricted to the late phase of NMJ differentiation: overexpression of FasII in muscles during initial NMJ formation seems to render muscle surfaces too 'sticky' and interferes with precise neuromuscular target recognition, as a result, ectopic motoneuronal branches (which normally occur only transiently) become trapped and form NMJs on inappropriate muscles (Davis et al., 1997). Apparently, there must exist other yet unidentified adhesion molecule(s) required for initial evens of synapse formation.

Another example relevant to switch in adhesive properties at NMJ comes from analysis of mef2 mutant embryos, where the initial neuromuscular contact can be genetically separated from the late phase of neuromuscular adhesion. In mef2 mutant embryos, muscle founder cells form, and motoneurons establish initial contact with proper target cells (Prokop et al., 1996). However, the muscle founder cells remain immature and fail to acquire properties of general muscle differentiation. In late-stage 17 mef2 mutant embryos, proper cell junctions between motoneurons and improperly differentiated muscle fibers are never observed, but instead the abnormal muscle surfaces are covered by basement membrane. This phenotype could be explained by lack of mef2-dependent late synaptic CAMs, which directly maintain the established neuromuscular connection. Alternatively, mef2 might be required to exclude basement membrane receptors from the NMJ, thus preventing dissociation of the pre- and postsynaptic membranes from competitive invasion of basement membrane material. No matter which explanation is true, it is clear that appropriate adhesive strength is the prerequisite for proper NMJ maturation and further maintenance. 


\subsubsection{Synapse formation}

Synapse formation involves the coordinated assembly of pre- and postsynaptic apparatus. Positioning cues are required to direct the juxtaposition of synaptic components at the pre- and postsynaptic membranes. In Drosophila NMJ, candidate that might serve as positioning cue is the special type of cleft-spanning adhesion molecules, as supposed by the function of Neuroligin/ Neuexin at vertebrate central synapses and immunoglobulin superfamily members SYG-1/ SYG-2 in C. elegans (see above), but this still remains to be proved.

In contrast to the essential role that nerve-secreted factor agrin plays in vertebrate NMJ differentiation, no agrin orthologue seems to be present in the Drosophila genome. However, recent evidence indicates that other sets of secreted molecules, Wnts and members of the TGF-beta family might take similar responsiblility in the development of glutamatergic synapses at Drosophila NMJ. Wingless (Wg), a member of the Wnt family of ligands, is secreted from motorneuron, loss of $\mathrm{Wg}$ leads to dramatic impairment of synapse formation, with boutons lacking active zones and postsynaptic structures (Packard et al., 2002). In consistence with this, a mammalian Wg homologue Wnt7a has been implicated in presynaptic differentiation in the cerebellum (Hall et al., 2000). Wishful thinking (wit) encodes a BMP (bone-morphogenetic-protein) type II receptor, which is present in a subsets of neurons, including motorneurons; its ligand, the BMP ortholog Glass Bottom Boat (Gbb), mainly fuctions in muscles. Mutations in both wit and Gbb result in much smaller NMJs, defective synaptic transmission and presynaptic abmormalities (such as synaptic membrane detatchment at active zone region, presence of floating T-bodies) (Aberle et al., 2002; McCabe et al., 2003). Taken together, these studies indicate that $\mathrm{Wg}$ and $\mathrm{Gbb}$ function in opposite directions at Drosophila NMJ to assure proper differentiation of pre- and postsynaptic structures. 
Before motorneuron innervation, low amounts of glutamate receptors are already expressed in muscles and diffusely distributed over the muscle surfaces (Broadie and Bate, 1993; Currie et al., 1995; Saitoe et al., 1997). Upon the initial neuromuscular contact, glutamate receptors begin clustering rapidly at the innervation sites, and within a few hours, new glutamate receptors are synthesized and inserted into newly formed synapses. Compared to the innervation-independent spontaneous AchR clustering at vertebrate NMJ, the clustering of glutamate receptors is innervation-dependent, the aneural muscles neither cluster existing receptors nor synthesize additional receptors. Intriguingly, disruption or complete elimination of neurotransmission does not seem to inhibit glutamate receptor field formation (Broadie et al., 1995; Featherstone and Broadie, 2000), however, the glutamate receptors will not cluster if the action potential of the neuron is blocked genetically or with tetrodotoxin (Broadie and Bate, 1993). Thus, glutamate receptor clustering appears to require an electrical activity dependant signal derived from motorneuron, and this signal is not related to neurotransmission. The latter notion is completely consistent with findings in mammalian central synapses: in Munc-13 or Munc-18 null mutant mice, in which neurotransmitter release is completely absent, both brain anatomy and synapses appear normal (Verhage et al., 2000; Varoqueaux et al., 2002). Thus, evidences from both Drosophila and vertebrates highly suggest that, at least in glutamatergic synapses, synaptic activity is not necessary for initiating synaptogenesis.

Analysis on mutants deficient in muscle development indicates that the presynaptic apparatus can be assembled independently of the postsynaptic cell (Prokop et al., 1996), which again contrasts with vertebrate NMJ differentiation. In the latter case, muscular secretion of s-laminin into synaptic basement membrane is essential for the assembly of morphologically normal active zones (Noakes et al., 1995). Anyhow, although the assembly of active zones at Drosophila NMJ can be independent of muscle cells, the precise apposition of 
pre- and postsynaptic apparatus must rely on the close communication between motorneuron and target muscle.

\subsection{Structure and functional relevance of ionotropic glutamate receptors}

The ionotropic glutamate receptors (iGluRs), including AMPA, NMDA and Kainate types, mediate excitatory synaptic transmission at most mammalian central synapses. In addition to converting transmitter released from presynaptic terminal to electrical response in the postsynaptic neuron, these receptors are thought to contribute to the activity-dependant, long-term changes in synaptic strength which is proposed to underlie learning and memory (Contractor and Heinemann, 2002). Besides the physiological importance of iGluRs, their dysfunction is implicated in various neuropathologies, including epilepsy, stroke damage and the perception of pain (Dingledine et al., 1999).

\subsubsection{Modular design of ionotropic glutamate receptors during evolution}

Ligand-gated ion channels are generally formed as homo- or hetero-oligomeric assemblies of integral membrane protein subunits. One important feature of iGluR structure is its modular construction. As illustrated in Fig.1-6 A, one typical iGluR subunit consists of an amino-terminal domain (NTD), a ligand-binding domain (S1S2), three transmembrane domains, a re-entrant pore loop, and a carboxy-terminal domain (Hollmann and Heinemann, 1994; Madden, 2002). The NTD shows homology to the leucine/ isoleucine/ valine-binding protein LIVBP, one of the bacterial periplasmic binding proteins (PBPs). In NR2 subunits, it forms or contributes to the binding site of many NMDA receptor modulators and affects receptor desensitization. For the non-NMDA receptors, it is supposed to be involved in receptor assembly. The $\mathrm{S} 1 \mathrm{~S} 2$ ligand-binding domain is formed by two sequences ( the S1 region which is the N-terminal of transmembrane domain 1, and the S2 region which is C-terminal of transmembrane domain 2) sharing sequence and structural 
homology with the glutamine-binding protein QBP. The re-entrant pore loop is homologous to the P-loop sequences of other channels, the P-loop sequence lines the channel and determines many of its electrophysiological properties. In addition, the cytoplasmic carboxy-terminal contains various interaction domains and phosphorylation sites, which likely controls the transport and localization of these receptors. The modular feature of iGluR indicates that it might have been assembled from discrete components during evolution, consistent with the 'genes-in-pieces' hypothesis (Gilbert, 1978). Supporting this notion, a prokaryotic glutamate receptor ion channel (GluR0) was found, which lacks NTD and has only two transmembrane domains but is indeed glutamate-gated and selective for potassium ions (Chen et al., 1999).
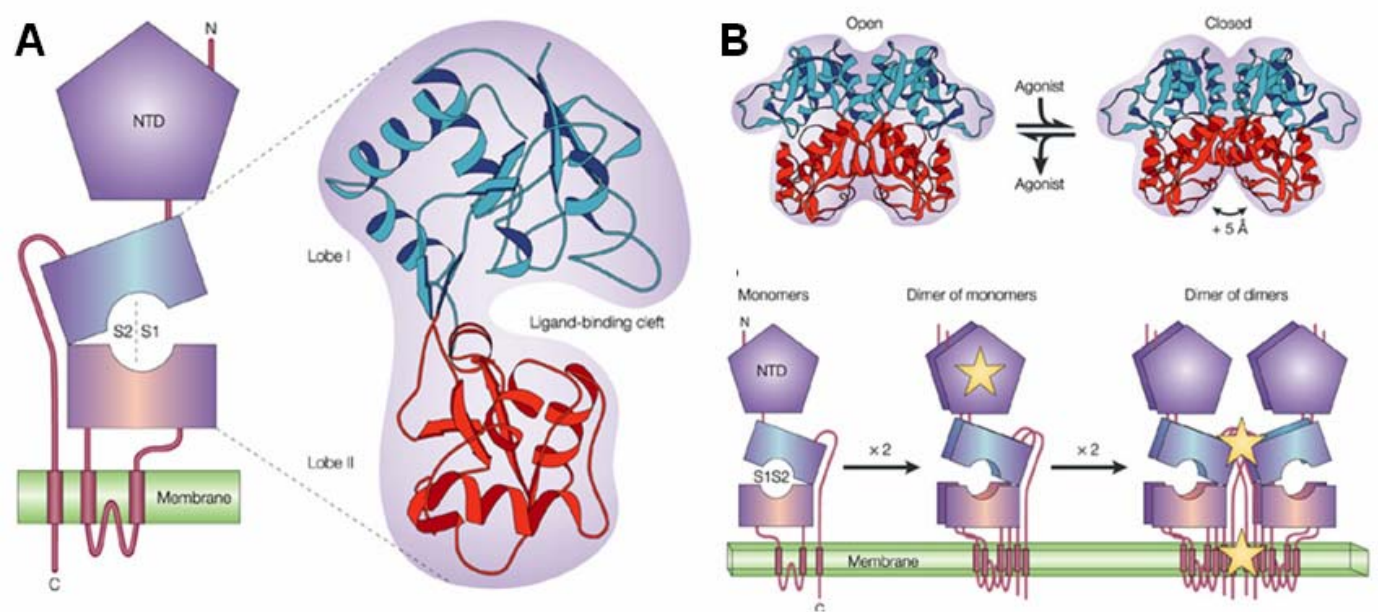

Fig.1-6 Structure of iGluR subunit and model of iGluR assembly. A, The modular structure of iGluR subunit. B, 'Dimer of dimers' model of iGluR assembly (Adapted from Madden, 2002).

\subsubsection{Molecular mechanisms underlying selective assembly and transport of ionotropic glutamate receptors}

Despite the schematic understanding of the sequence of building blocks that constitutes glutamate receptor subunits, still little is known about the spatial disposition of these domains within individual subunit and assembled functional 
ion channel. Recently, dissecting the molecular details underlying the functional assembly and cell surface trafficking of various types of iGluRs has become one of the central focuses in molecular neuroscience.

On the basis of electrophysiological, biochemical and hydrodynamic analysis, iGluRs are proposed to be tetramers (Laube et al., 1998; Mano and Teichberg, 1998; Rosenmund et al., 1998; Kuusinen et al., 1999; Safferling et al., 2001). AMPA receptors are homo- or hetero-tetramers composed of GluR1-4 (Hollmann and Heinemann, 1994; Dingledine et al., 1999). Kainate receptors are homo- or hetero-tetramers of the subunits GluR5-7, KA1 and KA2. NMDA receptors require both NR1 and at least one type of the four NR2 subunits (A-D) to form functional channels, in some cases NR3 subunits are also included (Meguro et al., 1992; Monyer et al., 1992; Perez-Otano et al., 2001; Matsuda et al., 2003). Recently, using a series of epitope-tagged receptor chimeras (Ayalon and Stern-Bach, 2001), Ayalon and Stern-Bach provided evidence of sequential assembly of tetrameric iGluRs as dimmer of dimmers (Fig.1-7 B). In this model, initial subunit dimerization is mediated primarily by interactions between compatible NTDs. Then, assembly of functional receptors requires that these dimmers undergo a second dimerization, this time requiring compatibility between the S2 and transmembrane domains of the subunits. This secondary dimerization is not observed in the absence of NTD compatibility, indicating that it might be weaker than the primary dimerization. This dimmer-of-dimmers model of iGluR assembly is consistent with images of GluR2 homomers determined by electron microscopy (Safferling et al., 2001).

In non-neuronal cells, the step of exit from the endoplasmic reticulum (ER) is often under the most stringent control during transport of membrane proteins to the cell surface (Ellgaard and Helenius, 2003). Like other multimeric cell membrane proteins, glutamate receptors are synthesized, folded, assembled in the ER; similarly, traffic of glutamate receptors through ER is also tightly regulated, which decides the type and number of glutamate receptor at synapse 
(Vandenberghe and Bredt, 2004). ER contains rigorous quality control system to ensure that only correctly folded and assembled proteins exit to the Golgi body, which in the case of glutamate receptors is evidenced by the presence of various ER retention and export signals in NMDA, AMPA and Kainate receptor subunits. For NMDA and Kainate receptors, both ER retention and export signals localize within the intracellular tails (Standley and Baudry, 2000; Scott et al., 2001; Xia et al., 2001; Ren et al., 2003; Hawkins et al., 2004; Jaskolski et al., 2004). Intriguingly, AMPA receptor subunit GluR2 contains a unique ER retention motif which just localizes in the re-entrant membrane loop (Greger et al., 2002), this single arginine (R607) is generated by mRNA editing and critically controls the ion permeability (Burnashev et al., 1992). Unlike other known ER export signal, the ER export signal of AMPA receptor subunit Glu1 appears within the extra-cellular NTD (Xia et al., 2002).

\subsubsection{Properties of ionotropic glutamate receptors expressed at Drosophila neuromuscular synapses}

In contrast to mammalian acetylcholinergic NMJ, the Drosophila type I junctional bouton is glutamatergic. So far, three ionotropic glutamate receptor subunits have been identified to be specifically expressed at the postsynaptic muscle cell (Schuster et al., 1991; Marrus et al., 2004). These glutamate receptor subunits are structurally and functionally similar to mammalian AMPA-/ Kainate-type receptors. GluR-IIA and GluR-IIB are genomic neighbors and their encoding proteins share $44 \%$ amino acid identity, animals with double knock-out of both genes die at late embryonic stage, while the lethality can be fully rescued by transgenic expression of either gene; moreover, modulation of subunit composition via genetically changing the copy number of GluR-IIA and GluR-IIB gene results into distinct single channel kinetic properties and is able to trigger long term changes of synaptic strength (Petersen et al., 1997; DiAntonio et al., 1999; Sigrist et al., 2002). GluRIII (also called GluR-IIC) is crucial for animal vitality and required for synaptic localization of GluR-IIA and GluR-IIB; while GluR-IIA and GluR-IIB do not precisely colocalize at 
many synapses, they both perfectly colocalize with GluR-III, implicating that GluR-III acts as a obligate subunit for functional receptor assembly (Marrus et al., 2004).

\subsection{The aim and strategy of this work}

The purpose of this study is to improve our understanding of the molecular mechanisms relevant to the synaptogenesis and growth control/ regulation of Drosophila NMJ. To this end, newly developed DNA microarray technique was applied to sort potential genes relevant to the NMJ formation and functioning through the whole genome. Via RNA profiling of postsynaptic cell (here is the somatic body wall muscle), a pool of genes with specific or enriched expression pattern were obtained, which would greatly facilitate systematic characterization of certain interesting functional groups. Data mining from these obtained RNA profiles and online genomic resources resulted into the identification of several muscle expressed but uncharacterized ionotropic glutamate receptor subunit genes. The expression pattern of these subunits were further verified at both RNA and protein level, via quantitative RT-PCR, in situ hybridization and immunohistochemistry. Ionotropic glutamate receptors play the major roles in mediating the signal transmission at excitatory glutamatergic synapses in the central nervous system, moreover, these receptors are also thought to contribute to the activity-dependant, long-term plasticity of neuronal circuits which is proposed to underlie learning and memory (Contractor A et al., 2002). The Drosophila neuromuscular synapse provides an excellent platform for investigating the behavior of glutamate receptors, due to its structural and functional similarity to excitatory central synapse and its rather simple organization. Reasonably, characterizing the functional significance of these newly identified glutamate receptor subunits at Drosophila NMJ in principle would help better understand their orthologs in the central synapses and thus became the main body of this thesis work. 
To study the in vivo roles of these novel glutamate receptor subunits, knock-out animals were generated via standard transposon-based mutagenesis screening. The null mutant animals of either subunit die at late embryonic stage, indicating that they are all essential genes. Further genetic, cell biological and electrophysiological studies were performed and uncovered a tight interdependence of all essential glutamate receptor subunits for synaptic localization and functioning, implying a novel "strictly hetero-tetrameric stoichiometry" model which is so far not reported in mammalian ionotropic glutamate receptors.

Closer inspection on glutamate receptor deprived synapses was also performed, with the combination of immunohistochemistry and electron microscopy based ultrastructural analysis. Surprisingly, it was found that with deprivation of glutamate receptors, the typical postsynaptic differentiation and synaptic compartmentation displayed severe defects, further analysis of a series of neurotransmission deficient mutant backgrounds excluded the involvement of neurotransmission activity in instructing differentiation of synaptic specializations. Thus, the glutamate receptor per se, but not its ligand-gated ion channel activity, is essential for normal synaptic construction. This important 'structural' role of glutamate receptor in organizing synaptic differentiation is firstly reported, raising highly interesting questions such as, which part of glutamate receptor is responsible for facilitating synaptic assembly? Do the ionotropic glutamate receptors in mammalian central synapses play similar roles during synaptogenesis? 


\section{Materials and methods}

\subsection{Materials (Chemicals, enzymes and molecular biology kits)}

All of the chemicals were purchased from Roth and Sigma unless stated elsewhere. For molecular cloning, all of the enzymes, including various restriction endonuclease enzymes, T4 DNA ligases, Alkaline Phosphotase were purchased from Roche (Mannheim, Germany). The Vent polymerase used for PCR cloning was from NEB (USA). All of the oligonucleotides were synthesized from MWG (Germany). The E. Coli strains used for transformation were either self-made DH5 $\alpha$ chemical competent cell or SURE electroporation competent cell from Stratagene (USA). Unless described elsewhere all of the molecular biology kits were from Qiagen (Germany).

\subsection{Methods}

\subsubsection{Genechip analysis and real-time RT-PCR}

\subsubsection{Genechip analysis}

Part I Protocol for the Affymatrix GeneChip Target Preparation

Material and Reagents:

Qiagen RNeasy RNA purification kit

3M Sodium Acetate, pH 5.2 Sigma

$80 \%$ ethanol kept cold

Absolute ethanol kept cold

Glycogen 5mg/ml, Roche

Ethedium bromide

7.5M Ammonium Acetate, Sigma

1). Synthesis of Double-Stranded cDNA from Total RNA

Materials and Reagents:

T7- (dT)24 Primer, (MWG Corp) HPLC purified DNA

Phase lock Gel, Eppendorf 
Phenol/ Chloroform/isoamyl alcohol, Roth

Superscript Choice System, Invitrogen

Ice bath

Heat/cool block for $70^{\circ} \mathrm{C}, 42^{\circ} \mathrm{C}, 16^{\circ} \mathrm{C}$

PE 9700 PCR machine

\subsection{First strand cDNA synthesis}

Prepare an Rnase- free $0.5 \mathrm{ml}$ reaction tube containing the following reagents.

They are to be added in the order listed. The total reaction volume is $20 \mu l$.

1. In a $0.5 \mathrm{ml}$ Rnase-free tube add total volume of $10 \mu \mathrm{l}$ of total RNA sample $(2.5 \mu \mathrm{g})$.

2. Add $1 \mu 1$ of T7- (dT) 24 primer (100 pmol/ $\mu \mathrm{l})$.

3. Incubate at $70^{\circ} \mathrm{C}$ for $10 \mathrm{Min}$, quick spin and place on ice.

4. Add $4 \mu 1$ of $5 X$ First strand cDNA buffer.

5. Add $2 \mu 1$ of 0.1 M DTT.

6. Then add $1 \mu 1$ of $10 \mathrm{mM}$ dNTP mix.

7. Add this mixture to the RNA sample then Incubate at $42^{\circ} \mathrm{C}$ for $2 \mathrm{~min}$.

8. While in the bath Add $2 \mu 1 \mathrm{SSII}$

9. Incubate for 1 hour at $42^{\circ} \mathrm{C}$.

10. Place reaction on ice and proceed onto second strand synthesis.

11. Equilibrate a $16^{\circ} \mathrm{C}$ bath.

\subsection{Second Strand Synthesis}

12. Briefly centrifuge first strand reaction and return to ice.

13. Add the following reagents in the order listed to the first strand reaction.

14. Make sure to add the reagents while the reaction is on ice.

$91 \mu \mathrm{l}$ of DEPC-treated $\mathrm{dH}_{2} \mathrm{O}$

$30 \mu \mathrm{l}$ of $5 \mathrm{X}$ Second Strand Reaction Buffer

$3 \mu \mathrm{l}$ of $10 \mathrm{mM}$ dATP, dCTP, dGTP, DTTP

$1 \mu \mathrm{l}$ of DNA ligase $(10 \mathrm{U} / \mu \mathrm{l})$

$4 \mu 1$ of DNA polymerase I $(10 \mathrm{U} / \mu \mathrm{l})$ 
$1 \mu \mathrm{l}$ of Rnase $\mathrm{H}(2 \mathrm{U} / \mu \mathrm{l})$

To yield a final reaction volume of $150 \mu 1$.

15. Gently tap tube to mix. Briefly spin to remove condensation.

16. Incubate the reaction at $16^{\circ} \mathrm{C}$ for 2 hours in a cooling water bath

17. While in the bath add $2 \mu 1$ of T4 DNA Polymerase.

18. Incubate for an additional 5 minutes at $16^{\circ} \mathrm{C}$.

19. Add $10 \mu \mathrm{l}$ of $0.5 \mathrm{M} \mathrm{Na}_{2}$ EDTA

The reaction product can be stored at $-20^{\circ} \mathrm{C}$ for later use.

\subsection{Clean Up of Double-Stranded cDNA}

STEP 1-Phase Lock Gels Phenol/Chloroform Extraction

1. Pellet the Phase lock gel in a micro-centrifuge tube by spinning it at full speed (1,3200rpm) for 20 to 30 seconds.

2. Add $162 \mu 1$ of Phenol: Chloroform: isoamyl alcohol (saturated with $10 \mathrm{mM}$ Tris- $\mathrm{HCl} \mathrm{pH} 8.0 / 1 \mathrm{mM}$ EDTA) to the cDNA reaction sample, mix by vortexing for one minute.

3. Transfer the entire cDNA Phenol Chloroform mixture into the PLG tube. Spin the PLG tube at full speed for 2 minutes

4. Avoiding contact with the gel transfer the aqueous upper phase to a fresh 1.5 $\mathrm{ml}$ tube (Tilt the tube for better recovery).

Proceed to step 2.

STEP 2 - Ethanol precipitation

1. Add $1 \mu 1$ of glycogen to the sample.

2. Add 0.5 volumes of $7.5 \mathrm{M}$ NH4Ac plus 2.5 volumes of absolute ethanol (stored at $-20^{\circ} \mathrm{C}$ ) to the sample and vortex.

3. Immediately spin at full speed in a micro-centrifuge at room temp for $20 \mathrm{~min}$, discard supernatant.

4. Wash pellet with $500 \mu \mathrm{l}$ of $80 \%$ ethanol (stored at $-20^{\circ} \mathrm{C}$ )

5. Spin at full speed for 5minutes at room temp.

6. Remove the ethanol very carefully so as not to disturb the pellet and repeat the wash. (This is an optional stopping point. The pellet may be left in the wash 
at $-20^{\circ} \mathrm{C}$ overnight.)

7. Air dry the pellet at room temp completely (this may require over an hour).

8 . Resuspend the pellet in $12 \mu 1$ of Rnase-free water.

9. Proceed onto Synthesis of biotin-labeled cRNA.

To reduce overdrying leave the pellet in a very small volume of the last wash.

2). Synthesis of biotin-labeled cRNA

Materials and Reagents:

Enzo RNA transcript labeling Kit 9 (BioArray High Yeald RNA Transcript

Labeling Kit)

Rneasy Mini Kit, Qiagen

DEPC-treated water

10X TBE, BioWhittaker

Reagent preparation

5X Fragmentation Buffer:

In an Rnase-free vessel combine

$4.0 \mathrm{ml}$ of $1 \mathrm{M}$ Tris-acetate $\mathrm{pH} 8.1$ (adjusted with glacial acetic acid).

$0.64 \mathrm{~g} \mathrm{MgOAc}$.

$0.98 \mathrm{~g} \mathrm{KOAc.}$

DEPC-treated water to make a final volume of $20 \mathrm{~mL}$.

Mix thoroughly then filter through a $0.2 \mu \mathrm{m}$ vacuum filter unit. This reagent should be aliquoted and stored.

2.1 protocol for the IVT reaction

1. Equilibrate a $37^{\circ} \mathrm{C}$ bath / block

2. Thaw the Enzo reagents on ice.

3. When thawed check for precipitate, vortex if necessary.

4. The volume of cDNA to be used in the IVT reaction should corresponding to the total RNA range of 8.0 and $16.0 \mu \mathrm{g}$.

5. From the Enzo BioArray RNA labeling kit add the following reagents in the 
order listed.

Reagent Volume

Template DNA Variable to give $1 \mu \mathrm{g}$ of cDNA $(10 \mu \mathrm{l})$

Deionized water To make final volume equal to $40 \mu \mathrm{l}(\sim 12 \mu \mathrm{l})$

10X HY Buffer $4 \mu 1$

10X Biotin-labeled nucleotides $4 \mu 1$

10X DTT $4 \mu \mathrm{l}$

10X RNase inhibitor $4 \mu 1$

20X T7 RNA polymerase $2 \mu \mathrm{l}$

Total volume $40 \mu 1$

6. Carefully mix the reagents in the tube then spin the sample briefly

7. Immediatly place the tube in a water bath at $37^{\circ} \mathrm{C}$ incubate for 5.5 hours gently mixing every 30 to 45 minutes

8. Remove $1 \mu 1$ aliquot for gel analysis

9. At the end of the incubation the reaction may be stored at $-20.0^{\circ} \mathrm{C}$ for later use.

2.2 Purification and Quantification of in vitro Transcription STEP 1 - Column purification of RNA

1. Pre-heat a small working stock of Rnase-free water at $60-65^{\circ} \mathrm{C}$.

2. Pre- -heat elution buffer at $60-65^{\circ} \mathrm{C}$.

3. Aliquot one half $(\sim 20 \mu \mathrm{l})$ of the cRNA reaction into a $1.5 \mathrm{ml}$ tube.

4. Adjust volume to $100 \mu \mathrm{l}$ with Rnase-free water.

5. Add $350 \mu 1$ of buffer RLT (lysis buffer) to the sample. Mix thoroughly.

6. Add $250 \mu 1$ of ethanol (96-100\%) to the lysate and mix well by pipetting up and down.

7. Apply sample $(700 \mu 1)$ to Rneasy mini-spin column sitting in a 2 ml-collection tube.

8. Centrifuge for $30 \mathrm{sec}$ at 10,000 rpm.

9. Reapply the flow-through onto the column and spin again.

10. Transfer the column into a new $2 \mathrm{ml}$-collection tube.

11. Add $500 \mu 1$ of buffer RPE (ensure ethanol has been added to the buffer) and 
let stand for $1 \mathrm{~min}$.

12. Spin at max for 30 s and discard flow-through.

13. Add another $500 \mu \mathrm{l}$ of buffer RPE onto the column and let it stand for $1 \mathrm{~min}$.

14. Centrifuge and discard flow-through.

15. Spin at full speed for an additional $3 \mathrm{~min}$.

16. Transfer the column to a new $1.5 \mathrm{ml}$ tube and pipet $33 \mu \mathrm{l}$ of the pre-heated Rnase free water directly onto the membrane. Let stand for $1 \mathrm{~min}$.

17. Spin at full speed for $1 \mathrm{~min}$.

18. Repeat elution using the flow-through from the first elution.

STEP 2 - Ethanol precipitation: if the cRNA yield is poor

19. Add 0.5 volume of $7.5 \mathrm{M}$ Ammonium Acetate and 2.5 times volume of absolute ethanol

20. Incubate at $-20^{\circ} \mathrm{C}$ for 60 min then spin at full speed for 30 minutes.

21 . Decant the supernatant then wash the pellet by adding $500 \mu 1$ of $80 \%$ Ethanol and spin at full speed for 5 minutes.

22. Decant the supernatant and repeat the wash step

23. Decant the final wash and dry the pellet at room temperature.

STEP 3 - Electrophoresis

24. Prepare sample in a RNA gel-loading buffer.

25. Heat sample $65^{\circ} \mathrm{C}, 5 \mathrm{~min}$.

26. Run one lane of unpurified labeled cRNA $(0.5 \mu 1)$ along side a lane of the purified labeled probe $(1.5 \mu \mathrm{l})$ on standard 1\% agarose gel.

Fragmentation of cRNA for target preparation

27. Add $2 \mu 1$ of $5 \mathrm{X}$ Fragmentation buffer to every $8 \mu 1$ of RNA plus water. The final RNA concentration in the reaction can range between $0.8 \mu \mathrm{g} / \mu \mathrm{l}$ and 2 $\mu \mathrm{g} / \mu 1$.

28. The following are guidelines that should be followed:

$20 \mu \mathrm{g}$ cRNA 1 to $16 \mu \mathrm{l}$

$5 \mathrm{X}$ Fragmentation buffer $4 \mu 1$

Rnase-free water to $20 \mu \mathrm{L}$ 
Component Volume

$15 \mu \mathrm{g}$ cRNA (extreme case) 1 to $12 \mu \mathrm{l}$

$5 X$ Fragmentation buffer $3 \mu 1$

Rnase-free water $15 \mu 1$

29. Incubate the reaction at $94^{\circ} \mathrm{C}$ for $35 \mathrm{~min}$, quick spin and place on ice immediately following the incubation.

30. Run $1 \mu \mathrm{g}$ of the fragmented RNA on a $1 \%$ agarose gel to check the completion of fragmentation.

31. The fragmented RNA is ready for hybridization.

Hybridization was performed in Affymetrix hybridization oven at $50^{\circ} \mathrm{C}$ for 16 hours. The hybridized Gene chips were then stained and washed in Affymetrix fluidics station following the standard program. Data analysis was performed via Microarray Suite 4.0 and exported via Excel.

\subsubsection{Real-time RT-PCR}

1. Extract total RNAs using QIAGEN's RNeasy kit (as described in 2.2.1.1), check the concentration of each sample.

2. Perform reverse transcription using QIAGEN's Omniscript Reverse Transcriptase:

Master mix:

10X Buffer RT

$2.0 \mu 1$

dNTP Mix (5mM each dNTP) $\quad 2.0 \mu \mathrm{l}$

Random hexamer $\quad 2.0 \mu \mathrm{l}$

Rnase inhibitor (40 units $/ \mu 1$ ) $\quad 0.25 \mu 1$

Ominiscript Reverse Transcriptase $\quad 1.0 \mu l$

Rnase-free $\mathrm{H}_{2} \mathrm{O} \quad \mathrm{X} \mu \mathrm{l}$ 
Template RNA

Incubate the mixture for 1 hour at $37^{\circ} \mathrm{C}$, then inactivate the Reverse Transcriptase by heating the reaction mixture to $93^{\circ} \mathrm{C}$ for $5 \mathrm{~min}$ followed by rapid cooling on ice.

3. Perform the real-time PCR in 96 well plate :

Master mix $(50 \mu 1)$ :

2X PCR mixture

$25 \mu 1$

$\mathrm{H}_{2} \mathrm{O}$

$22 \mu 1$

Primer (forward)

$1.0 \mu 1$

Primer (reverse)

$1.0 \mu 1$

cDNA template

$1.0 \mu 1$

4. Quantitate the relative transcript level by analyzing $\mathrm{Ct}$ value of each sample.

5. Check the amplified products by agrose gel electrophoresis.

\subsubsection{Fly stocks and genetics}

\subsubsection{Fly stocks, crosses and rearing conditions}

Fly stocks used are: All of the wild type control strain used is $w^{1}(B)$ except stated elsewhere. $\mathrm{w}^{-} ; \mathrm{Df}(2 \mathrm{~L})^{\mathrm{SP22}}\left(\right.$ Gglur-IIA $^{-/}$, Gglur-IIB $\left.^{-/}\right) /$Cyo, Kr::GFP (Petersen et al., 1997). w; $\mathrm{wf}^{-2}(2 \mathrm{~L}) \mathrm{cl}^{\mathrm{h}}{ }^{4} /$ Cyo, Kr::GFP. w;OK319-Gal4. $\mathrm{w}^{-}$;UAS-TNTe (Sweeney et al., 1995). Syx ${ }^{229}$ is a null mutation allele of syx-1A that completely eliminates mRNA and protein expression (Schulze et al., 1995). $S h i^{\text {ts } 1}$ mutants were shifted from a permissive $\left(25^{\circ} \mathrm{C}\right)$ to a non-permissive temperature $\left(32^{\circ} \mathrm{C}\right)$ after $13 \mathrm{~h}$ of embryogenesis (Featherstone D et al., 2002). $\mathrm{III}^{1} / \mathrm{Gla}$ Bc (Marrus et al., 2004).

Double mutants in GluR-IIA and GluR-IIB were described previously (Petersen et al., 1997; DiAntonio et al., 1999). In short, GluR-IIA and GluR-IIB 
double mutant embryos were recovered by crossing $D f(2 L) G l u R-I I A \& B^{S P 22}$ to $D f(2 L) C^{h 4}$, mutant embryos were selected using GFP marked balancer chromosomes.

To generate GluRIIA hypo, GluR-IIB ${ }^{\text {null }}$ condition having an extremely reduced amount of GluR-IIA and no GluR-IIB expression, genomic fragment of GluR-IIA encompassing promotor region and the whole open reading frame while missing most part of the 3'-UTR was used. This transgene still produces full length GluR-IIA while in dramatically reduced amount due a loss of message stability (see 3.4.2 for details). This construct was expressed from pUAST vector (using the GluR-IIA endogenous promotor). A single transgene copy rescues embryos null for both GluR-IIA and GluR-IIB (Df(2L)GluRIIA\& $B^{S P 22} / D f(2 L) C l^{\text {h4 }}$ ) giving GluRIIA $A^{\text {hypo }}$, GluR-IIB ${ }^{\text {null }}$ larvae (see results).

Unless stated elsewhere, all of the crosses were established at $25^{\circ} \mathrm{C}$ with $60 \%$ humidity.

\subsubsection{Mutagenesis screening}

The GluR-IID and GluR-IIE loci are situated at 92F4. In a recently released pBac transposon insertion collection (Thibault et al., 2004), a piggyBac transposon ( $\mathrm{pBac}\{\mathrm{RB}\} \mathrm{e} 01443, \# 17952$,) was found to be inserted into intron 6 at amino acid position 427 of the GluR-IID open reading frame. This allele GluR-IIDe $e^{01443}$ is embryonic lethal both homozygous or over $D f(3 R) H-B 79$ (Bloomington Drosophila stock center) which deletes a large genomic region including the GluR-IID and GluR-IIE locus, and over deficiency GluRIID\&-IIE $E^{E 3}$ (see below). For GluR-IIE, imprecise excision screening was performed using the $\mathrm{P}$ element line GE28753 (commercially available with Genexcel), which is inserted $\sim 150 \mathrm{bp}$ downstream of the end of the GluR-IIE transcript. In brief, P-element GE28753 was remobilized by crossing to the $\Delta 2-3$ transposase source, white eye progenies were selected and mated individually, 
then single fly genomic PCR reactions were performed to map deletions flanking the P-element insertion site. Nearly 1,000 eye color revertants were checked, one line (GluR-IIE $E^{E 1}$ ) was found to delete $1.2 \mathrm{~kb}$ flanking region in direction of the GluR-IIE gene, removing the C-term and transmembrane domain 4 of GluR-IIE. GluR-IIE ${ }^{E 1}$ is embryonic lethal homozygously, over $d f(3 R) H-B 79$ and over GluR-IID\&-IIE $E^{E 3}$. GluR-IID\&-IIE $E^{E 3}$ is a larger deletion also recovered from exision mutagenesis of GE28753, which removes both GluR-IIE and GluRIID and thus was used in combination with either GluR-IID ${ }^{e 01443}$ or GluR-IIE $E^{E 1}$ as deficiency.

\subsubsection{Molecular Constructs and Transgenes}

A genomic fragment covering the DGluR-IIA gene (containg $1.3 \mathrm{~kb}$ sequence 5' of the ATG) was firstly subcloned into pSL1180 as EcoRI/ XhoI fragment $(5.6 \mathrm{~Kb})$ from a BAC clone RPCI-98-35L07. The wild type rescue construct was made by directly inserting the EcoRI/ XhoI fragment into pUAST (Brand and Perrimon, 1993). For the 3' UTR deleted version, the subclone was cut with NcoI and XhoI, end-blunted and religated, then the EcoRI/ Asp718 fragment $(4.7 \mathrm{~Kb})$ was inserted into pUAST. All constructs were confirmed by double-strand sequencing, transgenic flies were produced in w1 background via standard procedures and crossed into the $\operatorname{Df}(2 \mathrm{~L}) \mathrm{cl}^{\mathrm{h} 4} / \mathrm{Gla}$, Bc background.

Genomic fragments covering the GluR-IID gene and GluR-IIE gene were generated by PCR using for IID

5'GGTCTAGAGCGGCCGCGGCCACGAACTGACCCACGGTTTC3' and 5'GCGGCCCTCGAGCGACGTCAAGGATGTGCCCAC3'

and for IIE

5'GGTCTAGAGCGGCCGCACCTCCCCAAGCTGTCAACTTC3' and 5'GCGGCCCTCGAGACTGCTCAAAGCTGCTGCCCTG3'. The products were double strand sequenced and cloned into $p U A S T$. Several independent lines of transgenic animals were generated. For overexpression studies, 
UAS-GluRIID and UAS-GluRIIE were generated by introducing the full length cDNA into the transformation vector $p U A S T$. Full-length cDNAs of GluR-IID (RE24732) and GluR-IIE (RE07945) were obtained from Berkeley Drosophila Genome Project cDNA libraries.

The construct for inducible RNA interference (RNAi-GluR-IIE) was made based on the pUASTi plasmid (contains an intron between insertion sites for sense and antisense frgaments; generous gift by Amin Ghabria, Krasnow lab). Selected cDNA fragments coving part of 5'-UTR and coding region were PCR-amplified by using the following primer pairs:

5'GCGCGCCTCGAGCTGTTCGGGAAACTCAAGAAT3' and 5'GGTCTAGAGCGGCCGCCGTGGTTAGCTCGTTCAAAATG3' and 5'GCTGGTACCTGTTCGGGAAACTCAAGAAT3' and 5'GCGTCTAGATCGTGGTTAGCTCGTTCAAAATG3'

The two fragments were inserted into pUASTi plasmid sequentially and verified by sequencing. Several independent lines of transgenic animals carrying UAS-GluR-IIE were generated.

To generate GluR-IIE::EGFP fusion. The EGFP ORF was inserted into the C-terminal of GluR-IIE coding sequence (the insertion site and the linker sequences are as described in (Sheridan et al., 2002) via 'blant-end ligation' strategy. Briefly, a HindIII fragment covering the C-terminal region of GluRIIE was cut from full-length cDNA (RE07945) and subcloned into pSL1180; PCR reaction was performed to generate the whole length linear fragment using the following primers which are standing at the EGFP insertion site but opposite in direction:

GGCAGATGTGTATAAGAGACAGTCGCCAGTCCTCGATGTCAGTAGCTT and

AGATGTGTATAAGAGACAGGACTGGCGACTGCGCGAGTAGATGG, at the same time EGFP coding sequence was amplified from EGFP vector (Clontech) using the following primers with 5' phosphate modification: 
pGGCGCGCCGAGCAAGGGCGAGGAGCTGTTCACCGG

and

pCGGGCGCGCCGCCCTTGTACAGCTCGTCCATGCCGAGA, the two PCR products were put together for blant-end ligation and the positive clones with right orientation of EGFP were verified by PCR and sequencing; then the HindIII fragment (now containing EGFP insert) was put back into the full-length cDNA backbone and the reading frame was verified by PCR and sequencing; finally the full-length DGluRIIE cDNA tagged with EGFP was PCR cloned into the NotI/ XbaI site of pUAST and pFasBac vector and verified by sequencing. Several independent lines of transgenic animals were generated from injecting the pUAST construct. The pFastBac construct was used for driving expression in Sf9 cells (for details see 2.2.5)

\subsubsection{In Situ Hybridization}

I. Preparation of embryos

1. Collect embryos every 3 hours for 24 hours and wash $3 \mathrm{X}$ with $0.02 \%$ Triton X (Tx)

( $15 \mathrm{ml}$ for up to $2 \mathrm{ml}$ embryos in a $50 \mathrm{ml}$ falcon tube).

2. Dechorionate $3 \mathrm{~min}$. in $50 \%$ bleach.

3. Wash $3 \mathrm{X}$ with $0.02 \% \mathrm{Tx}$.

4. Fill tube with equal parts heptane and $4 \%$ formaldehyde/PBS (filtered).

5. Incubate $25 \mathrm{~min}$. with frequent shaking.

6. Remove LOWER aqueous phase and replace with equal volume methanol.

7. Shake for $1 \mathrm{~min}$. allowing embryos to settle. Do not vortex.

8. Remove UPPER phase along with embryos and vitelline membranes and remaining at the interphase.

9. Rinse settled embryos $3 \mathrm{X}$ with methanol (Embryos can be stored at -20 degrees $\mathrm{C}$ at this point).

10. Rehydrate in 3:1 (MeOH: 4\% formaldehyde/PBS) for 2 min., then 1:3 (MeOH: 4\% formaldehyde/PBS) for $5 \mathrm{~min}$. 
11. Fix $10 \mathrm{~min}$. in $4 \%$ formaldehyde/PBS.

12. Rinse $6 \mathrm{X}$ with $\mathrm{PBS}+0.1 \%$ Tween 20 (PBT).

II. Preparation of Probe

1. Perform restriction digestion of the vectors containing the sequences of interest. For preparing antisense RNA probes of, GluR-IID and GluR-IIE, RE24732 (GluR-IID) and RE07945 (GluR-IIE) plasmids were cut with NotI, respectively.

2. Run the digest on agrose gel and extract the DNA (elute the DNA in $10 \mu 1$ of $\mathrm{H} 2 \mathrm{O}$ )

3. Run $1 \mu 1$ of DNA on a test gel.

4. In-vitro transcription:

Set the following reaction mixture:

DNA $\quad 9 \mu 1$

RNAse free water $\quad 3 \mu \mathrm{l}$

10xTrancription Buffer $2 \mu 1$

RNAse inhibitor $\quad 2 \mu 1$

DIG-labelling mix $\quad 2 \mu 1$

T3 RNA polymerase $\quad 2 \mu \mathrm{l}$

Incubate at $37^{\circ} \mathrm{C}$ for 2 hours.

5. Then add $2 \mu \mathrm{l}$ RNAse free DNAse and incubate at $37^{\circ} \mathrm{C}$ for 15 minutes.

6. Extract the RNAs by using RNAeasy Kit.

7. Fragmentation of RNA

RNA sample in Rnase free water $25 \mu 1$

2xcarbonate buffer $\quad 25 \mu 1$

8. Divide the above mixture into two parts. Incubate $25 \mu 1$ at $60^{\circ} \mathrm{C}$ for $5 \mathrm{~min}$ and incubate other $25 \mu \mathrm{l}$ at $60^{\circ} \mathrm{C}$ for $15 \mathrm{~min}$.

9. Add Rnase free water to the fragmented RNA sample to get a final volume of $100 \mu 1$, add $10 \mu 1$ of NaAc buffer (PH 5.2) and $1 \mu 1$ of LPA, and $250 \mu l$ of 
absolute ethanol. Mix and centrifuge for $5 \mathrm{~min}$ at full speed, remove supernatant and wash the pellet with $75 \%$ ethanol.

10. Dissolve the RNAs sample with $25 \mu$ of probe resuspension buffer.

III. Hybridisation of embryos (fillets)

1. Wash the prepared embryos (fillets) in 1ml Pre-hybridisation Buffer(-DS)

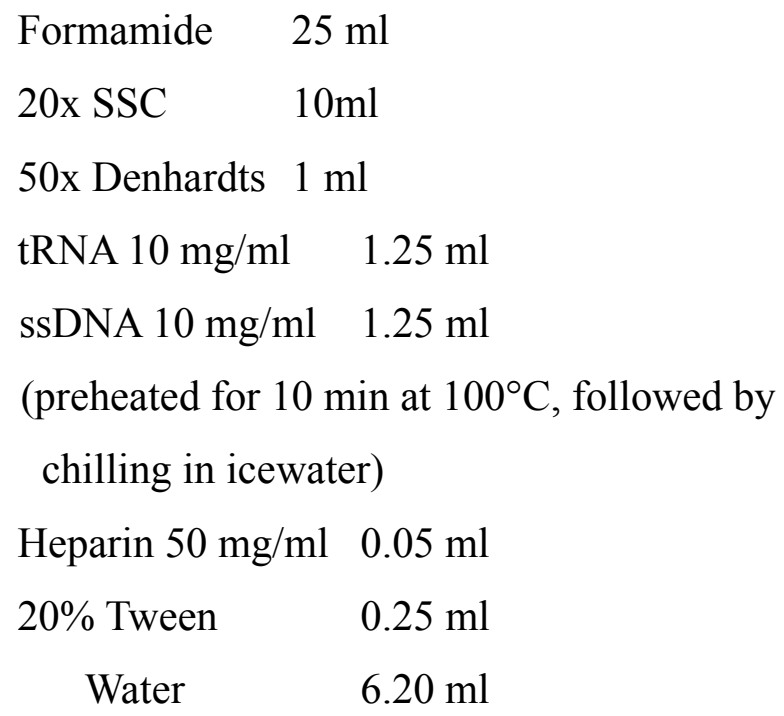

Combine $9 \mathrm{ml}$ with $1 \mathrm{ml}$ water to get the Pre-hybridisation Buffer (-DS) 2. Incubate the samples in $1 \mathrm{ml}$ of Pre-hybridisation Buffer(-DS) for $1 \mathrm{hr}$ at room temperature.

3. Add $1 \mu \mathrm{l}$ of probe to $0.5 \mathrm{ml}$ hybridisation Buffer (+DS) (9:1). Incubate overnight at $55^{\circ} \mathrm{C}$.

4. 2x wash with $0.5 \mathrm{ml}$ Washbuffer prewarmed at $55^{\circ} \mathrm{C}$.

Wash buffer:

Formamide $250 \mathrm{ml}$

20x SSC $\quad 50 \mathrm{ml}$

Water $\quad 200 \mathrm{ml}$

$20 \%$ Tween $202.5 \mathrm{ml}$

$5.7 \times 0.5 \mathrm{ml}$ warmed wash buffer $1 \mathrm{hr}$ at $55^{\circ} \mathrm{C}$.

6. Incubate in $0.5 \mathrm{ml}$ warmed wash buffer overnight at $55^{\circ} \mathrm{C}$.

7. 2x wash with PBTw. 
8. Incubate for $30 \mathrm{~min}$ in PBTw at RT.

9. Incubate in $0.5 \mathrm{ml} \mathrm{PBTw}+5 \% \mathrm{NGS}+$ Antikörper (1:2000) (anti-DIG-AP

FAB-Frag.) for $2 \mathrm{hrs}$. at RT.

10. 2x wash with PBTw.

11. 9x 10 min. PBTw at RT.

12. $2 \mathrm{x}$ wash with Alkaline Phosphatase Buffer $+0.1 \%$ Tween-20

$$
\begin{aligned}
& 100 \mathrm{mM} \mathrm{NaCl}^{2} \quad(=20 \mathrm{ml} 5 \mathrm{M} \mathrm{NaCl}) \\
& 5 \mathrm{mM} \mathrm{MgCl}_{2} \quad\left(=5 \mathrm{ml} \mathrm{MgCl}_{2}\right) \\
& 100 \mathrm{mM} \text { Tris/HCl pH } 9.5 \quad(=100 \mathrm{ml} 1 \mathrm{M} \text { Tris }+875 \\
& \text { ml water }) \\
& 1 / 100 \mathrm{~V} 10 \% \text { Tween-20 }
\end{aligned}
$$

13. 5 min of incubation in AP buffer at RT.

14. Transfer the samples into 24 well plates, wash one time with $0.2 \mathrm{ml}$ of BM Purple AP substrate, then add $0.2 \mathrm{ml}$ of BM Purple AP substrate.

15. At proper stages, stop staining by 3 times of wash with PBTw and add $80 \%$ Glycerin/ H2O. Store the plate at 4 degrees.

IIII. Mounting and light microscopy

\subsubsection{Biochemistry (collaborated with Tobias Schwarz)}

GluR-IID and GluR-IIE-EGFP were inserted into pFastbac1 vector (Invitrogen) and transformed into DH10Bac cells. Then the recombinant bacmid DNA was used for transfecting Sf9 cells with the aid of the Bac-to-Bac Baculovirus expression system (Invitrogen). For immunoblot experiments Sf9 cells were infected with the recombinant GluR-IID and GluR-IIE-EGFP baculovirus at a MOI (multiplicity of infection) of 1. Sf9 cells were harvested 40 hours post-infection washed twice with PBS, denatured in Laemmli buffer and heated at $100^{\circ} \mathrm{C}$ for 10 minutes.

Drosophila wild-type embryos were collected and dechorionated 18h AEL, froze in liquid nitrogen and stored at $-80^{\circ} \mathrm{C}$. Embryo lysates were prepared as described (Gillespie and Wasserman, 1994). The embryonic debris was removed 
by centrifugation at $13000 \mathrm{rpm}$ for 15 minutes and the supernatant was transferred to a new tube and centrifuged again for further purification. A portion of both pellet and supernatant fraction were mixed with an equal volume of $2 \mathrm{x}$ Laemmli buffer and heated at $100^{\circ} \mathrm{C}$ for 10 minutes.

Standard western blot was performed as described before. Anti-IID (1:1000), anti-IIC (1:1000) and rabbit anti-GFP antibodies (1:2000, Abcam) were incubated for 2 hours with the blot membranes at $4^{\circ} \mathrm{C}$. For the visualization of the bands horseradish peroxidase conjugated secondary antibodies were used with the ECL Western blot system (Amersham).

\subsubsection{Immunohistochemistry}

\subsubsection{Embryonic and Larval preparation}

Solutions used for dissection:

Haemolymph-like (HL-3) saline without Ca

$14 \mathrm{ml} 5 \mathrm{M} \mathrm{NaCl}$

$5 \mathrm{ml} 1 \mathrm{M} \mathrm{KCl}$

$20 \mathrm{ml} 1 \mathrm{M} \mathrm{MgCl}_{2}$

$5 \mathrm{ml}$ Trehalose

$115 \mathrm{ml} 1 \mathrm{M}$ Sucrose

Add distilled and deionized water to $1000 \mathrm{ml}$, adjust $\mathrm{PH}$ to 7.2

10X PBS (Phosphate-buffered saline)

$80 \mathrm{~g} \mathrm{NaCl}$

$2 \mathrm{~g} \mathrm{KCl}$

$2 \mathrm{~g} \mathrm{KH}_{2} \mathrm{PO}_{4}$

11.5g Na $2 \mathrm{HPO}_{4} \cdot 2 \mathrm{H}_{2} \mathrm{O}$

Add distilled and deionized water to $1000 \mathrm{ml}$, adjust $\mathrm{PH}$ to 7.4 
PBT

$1 \mathrm{X}$ PBS

$0.05 \%$ Tween-20

4\% PFA (Fixation solution)

$4 \%$ paraformaldehyde

$1 \mathrm{X}$ PBS

Bouwin Fixation solution

Antibodies

Rabbit anti-GluR-IIC/III antibodies were generated against a c-terminal peptide (PRRSLDKSLDRTPKS). Rabbit anti-GluR-IID antibodies were generated against a c-terminal peptide of GluR-IID (ESLKTDSEENMPVED). Both sera were affinity purified and used at 1:500 dilution. Other primary antibodies were used at the following concentrations: mouse monoclonal anti-GluR-IIA antibody (8B4D2, DSHB), 1:100; Goat anti-HRP-Cy5, 1:250; mouse anti-FasII (1D4, DSHB), 1:40; mouse anti-Dlg (DSHB), 1:500; Nc82 (generous gift of Erich Buchner, Würzburg), 1:100. Except for samples stained with 8B4D2, which were fixed for 5 min with cold methanol, all of the other stainings were fixed for 10 min with $4 \%$ paraformaldehyd.

Dissection and staining

Mid stage 3rd instar larvae were put on a dissection plate, both ends were fixed by fine pins and the specimen was covered by a drop of ice cold HL-3 solution (see below). Dissection scissors were used to make a small hole at the dorsal midline of the larva (near to the posterior end) which was then completely opened along the dorsal midline from the hole to the anterior end. The epidermis was stretched flat and pinned down, then the internal organs and central nervous system were removed carefully with forceps. Late stage embryos (20- $22 \mathrm{hrs}$ after egg laying) were dissected on sylgard plates, fixed 
with fine clips and opened using a pair of sharp tungsten needles. The dissected samples were fixed and then incubated with primary antibodies overnight, followed by fluorescence-labelled secondary antibodies (Dianova) and mounted in VectaShield mounting media (Vector Laboratories).

\subsubsection{Confocal and epifluorescent microscopy}

Imaging of embryonic and larval body wall preparations was performed on a Leica DM IRE2 microscope equipped with a Leica TCS SP2 AOBS scan head, using a Leica HCX PL Apo CS 63x 1.32 NA OIL UV objective. Epifluoresence images were taken on a Zeiss Axioscope with Axiocam camera, using a 100x oil objective of NA 1.4. Image processing was performed using ImageJ and Photoshop software.

\subsubsection{Ultrastructural analysis (collaborated with Carolin Wichmann)}

Dissected preparations were primary fixed in a mixture of $4 \%$ paraformaldehyde and $0.5 \%$ glutaraldehyde in $0.1 \mathrm{M}$ PBS (pH7.2) for $10 \mathrm{~min}$ and fixed additionally $60 \mathrm{~min}$ with secondary fixative comprising of $2 \%$ glutaraldehyde in $0.1 \mathrm{M}$ sodium cacodylate buffer ( $\mathrm{pH} 7.2$ ), washed three times for $5 \mathrm{~min}$ in sodium cacodylate buffer, and postfixed on ice for $1 \mathrm{hr}$ with $1 \%$ osmium tetroxide, followed by an $1 \mathrm{hr}$ washing step in sodium cacodylate buffer and three brief washing steps in destilled water. The samples were stained en bloc with $1 \%$ uranyl acetate in destilled water for $1 \mathrm{hr}$ on ice. After a brief wash with destilled water, the samples were dehydrated in increasing ethanol concentrations $(30 \%, 50 \%, 70 \%, 95 \%, 100 \%)$, infiltrated in Epon resin $(100 \%$ EtOH/Epon 1:1, 30 min and $90 \mathrm{~min} ; 100 \%$ Epon, over night) and embedded for $24 \mathrm{hr}$ at $85^{\circ} \mathrm{C}$.

The samples were trimmed, and series of $80 \mathrm{~nm}$ ultrathin sections were cut from muscle 6/7, abdominal segment 2 and 3 with a diamond knife on a Reichert Ultracut Ultramicrotome and mounted on Formvar-coated grids. 
The sections were stained in uranyl acetate (4\%) and photographed with a Philips (EM 301) transmission electron microscope.

\subsubsection{Eletrophysiology (collaborated with Robert Kittel)}

Intracellular recordings were made at $22^{\circ} \mathrm{C}$ from muscle fiber 6 of abdominal segments 2 and 3, of late third instar larvae. The larvae were dissected in ice-cold, calcium-free haemolymph-like saline (HL-3) according to Stewart et al. (1994). Larval filets were rinsed with $2 \mathrm{ml}$ of HL-3 saline containing $1 \mathrm{mM} \mathrm{Ca}^{2+}$, before being transferred to the recording chamber where two-electrode voltage clamp (TEVC) recordings were performed in $1 \mathrm{mM}$ extra cellular $\mathrm{Ca}^{2+}$. The larval NMJ was visualized with a fixed-stage upright microscope (Olympus, 40x water immersion lens). Whole muscle recordings of both miniature and evoked postsynaptic currents were recorded in TEVC mode (AxoClamp 2B, Axon Instruments) using sharp microelectrodes (borosilicate glass with filament, $1,5 \mathrm{~mm}$ outer diameter) with resistances of 15-35 $\mathrm{M} \Omega$ and filled with $3 \mathrm{M} \mathrm{KCL}$. All cells selected for analysis had resting potentials between -55 and $-70 \mathrm{mV}$. For stimulation, the cut end of the segmental nerve was pulled into a fire-polished suction electrode and brief $(300 \mu \mathrm{s})$ depolarizing pulses were passed at $0.2 \mathrm{~Hz}$ (npi stimulus generator and isolation unit). To ensure the stable recruitment of both innervating motoneurons, the amplitude of the pulse was determined by increasing the stimulation strength to 1.5 times the amplitude needed to reach the threshold of double motoneuron recruitment. The clamp was tuned such that it responded to a voltage step from -60 to $-70 \mathrm{mV}$ with settling times of $1 \mathrm{~ms}$ for mEJCs and $500-750 \mu$ s for eEJCs, this gave voltage errors of maximally $4 \mathrm{mV}$ for eEJCs of approx. 100nA. Both eEJCs (voltage clamp at $-60 \mathrm{mV}$ ) and $\mathrm{mEJCs}$ (voltage clamp at $-80 \mathrm{mV}$ ) were low-pass filtered at $1 \mathrm{kHz}$. For each cell, 20 eEJCs and 90s of mEJCs recordings were used for subsequent analysis (pClamp9, Axon Instruments). For the paired-pulse stimulation, responses to 20 consecutive paired stimuli (separated by $4 \mathrm{~s}$ rest) with $20 \mathrm{~ms}$ interpulse interval were averaged. 


\section{Results}

\subsection{Establishing the tissue specific transcriptome of Drosophila larval body wall muscles}

The total number of genes in Drosophila genome is predicted to be approximately 14,000 . The high-density DNA microarray technique provides a fast and high throughput way to identify genes expressed at specific tissues or involved in certain processes (Stathopoulos and Levine, 2002; Butler et al., 2003; Li and White, 2003). Here, initial goal was to obtain a whole genome scaled map of molecular components involved in the construction and regulation of the postsynaptic muscle cells. Thus, using a Drosophila wild type strain (CS10), the transcripts of $3^{\text {rd }}$ instar larval epidermis preparations, mainly consisting of body wall somatic muscles (with cuticles) but not central nervous systems, were prepared, amplified, labeled and hybridized to the Affymetrix Drosophila Genome arrays, generating the RNA expression profiles. RNA expression profiles of total larvae of Drosophila CS10 strain were also produced and set as baseline to compare with those from epidermis samples. Since equal amount of RNAs were utilized for different samples, transcripts displaying higher expression level in epidermis samples might represent the muscle specific or enriched genes and were selected.

The transcripts were ranked by average fold increase. Transcripts showing 2 fold or greater enrichment in epidermis samples were collected and shown in the appendix. One direct way to check the specificity of the data obtained is to check those transcripts with known tissue distribution pattern. As expected, among the pool of nearly 700 transcripts showing increasing abundance in epidermis samples, about one tenth are already known to be specifically (or abundantly) present in muscles or cuticles, thus directly proving the reliability of the data. These genes are categorized into various groups, including RNA-binding proteins, transcription factors, cell adhesion molecules, enzymes, 
motor proteins, receptors and ion channels, and structural proteins, etc.(representatively shown in Table 3-1).

Table 3-1 Described muscle / cuticle-specific transcripts displaying enrichment in gene chip analysis of body-wall-preparations

\begin{tabular}{|c|c|c|}
\hline Gene & Fold change & Proposed Function \\
\hline \multicolumn{3}{|l|}{ RNA binding proteins } \\
\hline How (held out wings) & 4.5 & RNA binding \\
\hline \multicolumn{3}{|l|}{ Transcription factors } \\
\hline Mef2 & 4.4 & RNA polymerase II transcription factor \\
\hline Muscle LIM protein at $84 \mathrm{~B}$ & 4.4 & transcription factor \\
\hline \multicolumn{2}{|l|}{ Cell adhesion molecules } & cell adhesion \\
\hline \multicolumn{3}{|l|}{ Enzymes } \\
\hline Mlc-k & 12.2 & myosin light chain kinse \\
\hline myosin light chain Kinase & 5 & myosin light chain kinase \\
\hline CamKII & 3.8 & Calcium/ Calmodulin-dependent protein kinase II \\
\hline dlg1 (discs large 1) & 2.9 & Synaptic and muscular scaffolding \\
\hline \multicolumn{3}{|l|}{ Motor proteins } \\
\hline Tropomysin 1 & 2.6 & Molecular motor \\
\hline Tropomysin 2 & 3.6 & Molecular motor \\
\hline upheld (troponin T) & 3.6 & tropomyosin binding \\
\hline sanpodo (tropomodulin) & 5.8 & tropomyosin binding \\
\hline Mhc (myosin II heavy chain) & 2.5 & muscle motor protein \\
\hline Mlc2 (myosin light chain 2) & 2.1 & muscle motor protein \\
\hline \multicolumn{3}{|l|}{ Receptors and ion channels } \\
\hline GluR-IIA & 4.3 & glutamate receptor \\
\hline GluR-IIB & 2.6 & glutamate receptor \\
\hline GluR-III/ IIC & 3.5 & glutamate receptor \\
\hline \multicolumn{3}{|l|}{ Structural proteins } \\
\hline Lcp65Ag1 & 3.3 & structural constituent of larval cuticle \\
\hline Lcp1 & 2.9 & structural constituent of larval cuticle \\
\hline Lcp2 & 2.8 & structural constituent of larval cuticle \\
\hline Lcp65Ag2 & 2.1 & structural constituent of larval cuticle \\
\hline Lcp4 & 2.1 & structural constituent of larval cuticle \\
\hline Lcp3 & 2 & structural constituent of larval cuticle \\
\hline \multicolumn{3}{|l|}{ Others } \\
\hline Mp20 (muscle protein 20) & 2.9 & calcium-binding \\
\hline TpnC73F (Troponin C) & 4.3 & calcium-binding \\
\hline TpnC47D & 2.7 & calcium-binding \\
\hline
\end{tabular}




\subsection{Identification of novel ionotropic glutamate receptor subunits expressed at the Drosophila neuromuscular synapse}

\subsubsection{Enrichment of various transcripts encoding novel ionotropic glutamate receptor subunits in larval body wall muscles}

The Drosophila genome encodes about 30 potential ionotropic glutamate receptor subunits (Littleton, 2000; Littleton and Ganetzky, 2000; Sprengel et al., 2001). Using the Affymetrix Drosophila gene chip result, the expression level of each subunit gene was compared between larval body wall and whole larvae RNA pools. As expected, GluR-IIA and GluR-IIB mRNA (Schuster et al., 1991; Petersen et al., 1997) were found to be enriched in body wall preparations. Another subunit annotated as CG4226, first referred to as GluR-IIC (Saitoe et al., 2001) and later as GluR-III (Marrus et al., 2004) was found to be enriched in body walls as well. A recent publication has shown that GluR-III null mutants die at late embryonic stages most likely due to a defect of glutamatergic transmission. In addition to these already described subunits with muscle expression, another glutamate receptor (annotated as CG18039) was found to be also enriched in body wall preparations.

Since the probe design of the Drosophila gene chip used here is based on an earlier version of the Drosophila genome annotation database, some genes might be omitted. To check if there are any newly anotated glutamate receptor genes which were not represented by the chip used, each of these selected glutamate receptor genes was blasted against the updated Drosophila genome annotation database (www.Flybase.com). Indeed, when blasting CG18039 coding sequence one gene annotated as CG31201 was found, which is strikingly similar to CG18039 (see below, Fig3-2). Interestingly, these two genes are located as direct neighbors within the genome, which is reminiscent of the organization of GluR-IIA and GluR-IIB (Petersen et al., 1997). Recently, it has been found that genes with similar expression pattern often cluster at certain regions of genome (Boutanaev et al., 2002; Roy et al., 2002), likely facilitating 
transcriptional control of to be co-expressed genes. Intuitively, CG31201 might thus be expressed in a similar pattern with its neighbor CG18039.

Next, real time quantitative RT-PCR was applied to independently quantify the expression level of these candidate glutamate receptor genes. As shown in table3-2, real time quantitative PCR data were consistent with the observation from gene chip analysis. Moreover, CG31201 was shown to be enriched within body wall mRNA pool as well. From now on we will refer to the locus encoding subunit CG18039 as GluR-IID and the locus encoding CG31201 as GluR-IIE. These names are meant to reflect their muscle expression (see below) along with Glu-IIA, -IIB and -IIC/III.

\begin{tabular}{lccccc}
\hline & $\begin{array}{c}\mathrm{C}_{\mathrm{t}} \\
\text { (body wall) }\end{array}$ & \% control & $\begin{array}{c}\mathrm{C}_{\mathrm{t}} \\
\text { (total larva) }\end{array}$ & \% control & $\begin{array}{c}\text { Body wall } \\
\text { /total larva }\end{array}$ \\
\hline $\begin{array}{l}\text { GluR-IIA } \\
\text { (CG6992) }\end{array}$ & 26,7 & 15,4 & 28,3 & 4,4 & 3,5 \\
$\begin{array}{l}\text { GluR-IIB } \\
\text { (CG7234) }\end{array}$ & 28,2 & 5,6 & 29,5 & 1,9 & 3,0 \\
$\begin{array}{l}\text { GluR-IIC/III } \\
\text { (CG4226) }\end{array}$ & 26,4 & 18,7 & 28,9 & 3,1 & 6,3 \\
$\begin{array}{l}\text { GluR-IID } \\
\text { (CG18039) }\end{array}$ & 25,5 & 35,5 & 27,0 & 10,5 & 3,4 \\
$\begin{array}{l}\text { GluR-IIE } \\
\text { (CG31201) }\end{array}$ & 24,5 & 73,7 & 26,3 & 17,5 & 4,2 \\
$\begin{array}{l}\text { tbp-1 } \\
\text { (internal } \\
\text { control) }\end{array}$ & 23,8 & 100,0 & 23,8 & 100,0 & 1,0 \\
\hline
\end{tabular}

Table 3-2 GluR-IID and GluR-IIE are enriched in larval body wall mRNA.

Abundances of glutamate receptor subunit transcripts estimated using real time PCR. The control transcript (proteasome subunit tbp-1) had the same abundance in body wall preps and whole larvae. The glutamate receptor subunits shown were enriched in body wall RNA when compared to whole larva RNA. The abundances of mRNAs are expressed as $C_{t}$ values indicating the cycle number with which amplification exceeds detection threshold ( $\mathrm{Ct}$ difference of one indicates a two-fold difference in abundance). All data are averages of three independent experiments where each sample was run two times in parallel.

\subsubsection{Expression pattern of new ionotropic glutamate receptor genes}


To further confirm the muscle expression of these new subunit genes, in situ hybridization on Drosophila embryos and larvae was performed. In fact, the mRNAs of GluR-IID (Fig.3-1A-D) and GluR-IIE (Fig.3-1E, F) are specifically expressed in somatic muscles of both Drosophila embryo (Fig.3-1A-C, E, F) and larva (Fig.3-1D). Expression of GluR-IID (Fig.3-1C-D) and GluR-IIE (Fig.3-1E) starts in somatic muscles of late stage 12 embryos (Fig.3-1A, E) and extends throughout embryonic and larval development (Fig.3-1D, F), a pattern very similar to that of GluR-IIA, -IIB and -IIC/III (data not shown; Petersen et al., 1997; Marrus et al., 2004). Intriguingly, in addition to the somatic muscle expression GluR-IID is also abundantly present in cardiac precursor cells (Fig.3-1A, C), which was not found for the other muscle subunit genes.

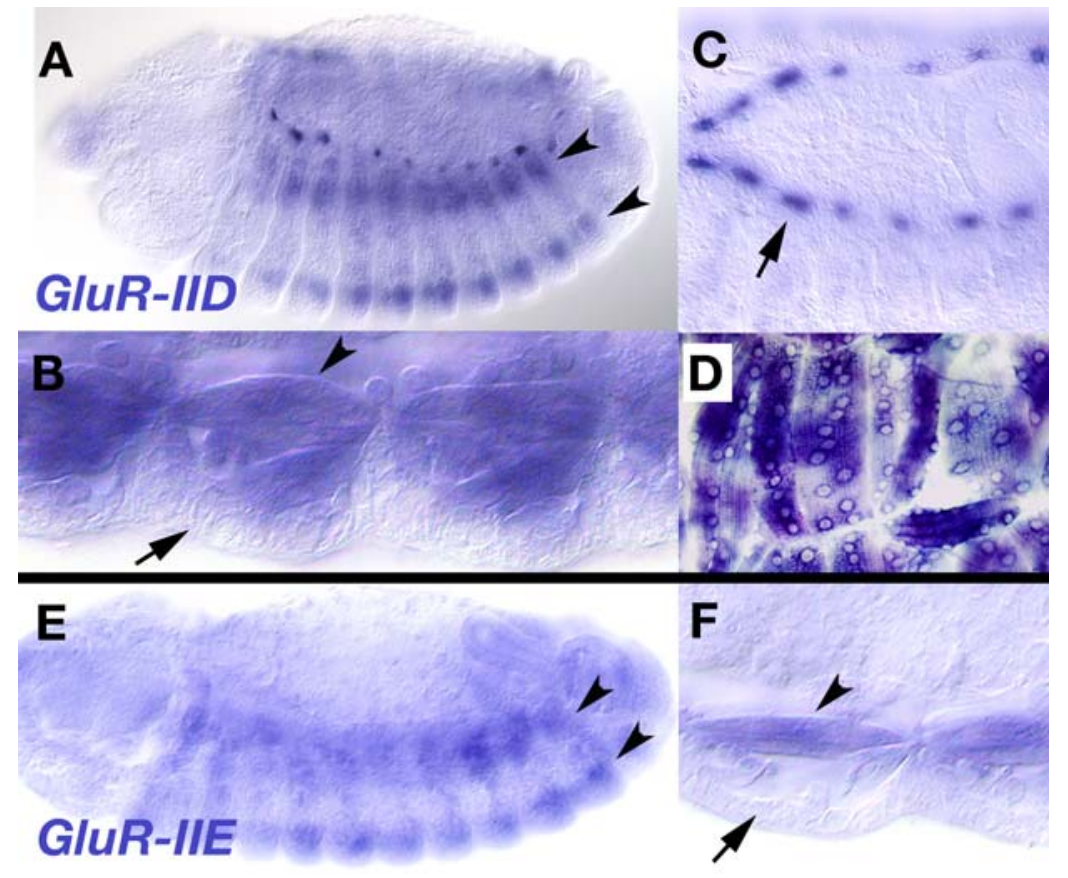

Figure 3-1. GluR-IID and GluR-IIE: novel glutamate receptor subunits with muscle specific expression

In situ hybridizations on Drosophila embryos (A-C, E,F) and larvae (D) for GluRIID (A-D) and GluRIIE mRNA (E,F). Both subunits are expressed specifically in presumptive somatic muscle cells (A, B, E, F, arrowheads) but are for example not found in the adjacent epidermis (A, B, F, arrows). Expression of GluR-IID and GluR-IIE transcript in the presumptive somatic muscles starts in late stage 12 and peaks at around stage 14 (A, E), to then persist during later embryogenesis (B: stage 16; E: stage 17) and larval development (D). GluR-IID is also expressed within heart precursor cells (A and B, arrows). 


\subsubsection{GluR-IID and GluR-IIE represent a new type of muscle-expressed glutamate receptor subunit}

In a 'simple' synaptic model system like the Drosophila NMJ the coexistence of so many distinct glutamate receptor subunits is somewhat unexpected. To get a closer view of the relationship among them, full-length cDNA clones of GluR-IIC, GluR-IID and GluR-IIE were obtained, the reading frames were verified by double strand sequencingand the deduced amino acid sequences compared. Clearly, these proteins encode all structural features typical in glutamate receptor subunits. Importantly, all putative transmembrane domains were found at similar positions (see Figure 3-2A). As previously noted (Marrus et al., 2004), the GluR-IIC/III sequence is closely related to GluR-IIA and IIB. GluR-IID and GluR-IIE are also highly related with each other (Fig. 3-2A, B). However, GluR-IID and GluR-IIE are distant from GluR-IIA, IIB and IIC/III group (Fig.3-2B). The fact is, GluR-IID and GluR-IIE are even slightly closer to human kainate receptor GluR6 than they are to the GluR-IIA, -IIB and -IIC/III group (see dendrogram in Fig.3-2B). Thus, in contrast to the GluR-IIA, -IIB, -IIC/III type, GluR-IID and GluR-IIE represent a new type of glutamate receptor subunits which is expressed in somatic Drosophila muscle. In a previous alignment entailing all identified ionotropic glutamate receptor subunits of the Drosophila genome, several other non-NMDA type subunits group in between GluRIID, -IIE and GluR-IIA, -IIB, -IIC/III groups (Littleton and Ganetzky, 2000). Notably, all of the muscle glutamate receptor subunits (from GluR-IIA to -IIE) have direct orthologs in Drosophila Pseudoobscura (data not shown), suggesting that the differentiation of the insect muscle-expressed glutamate receptor subunits into two structurally different groups might be conserved. 


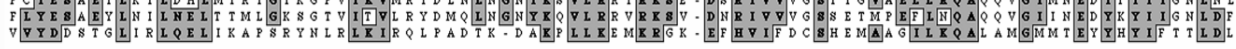

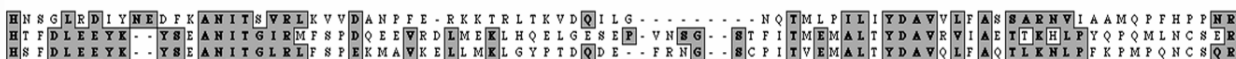

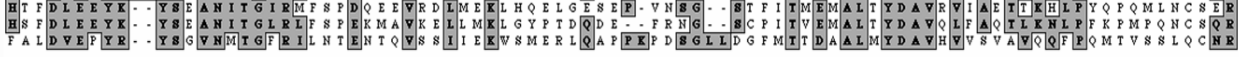

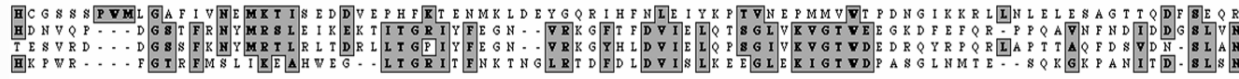

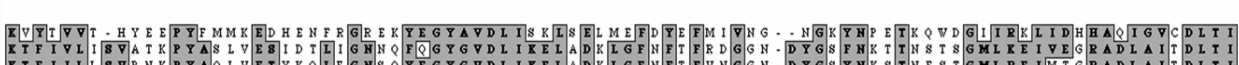

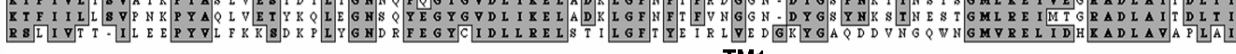

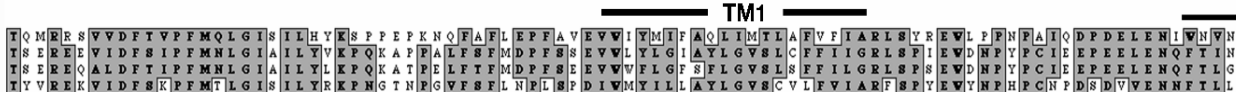

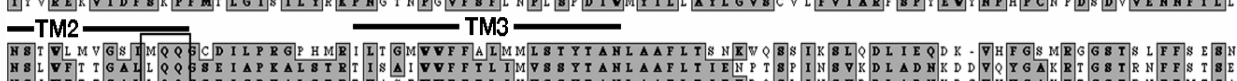

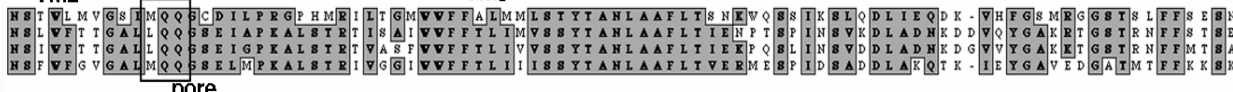

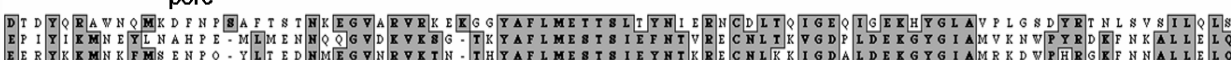

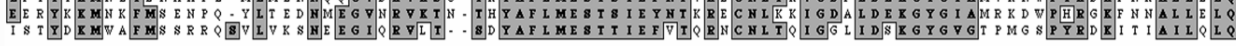

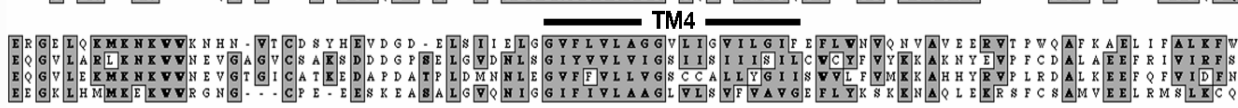

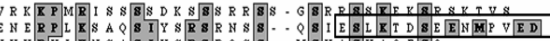

B

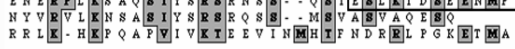

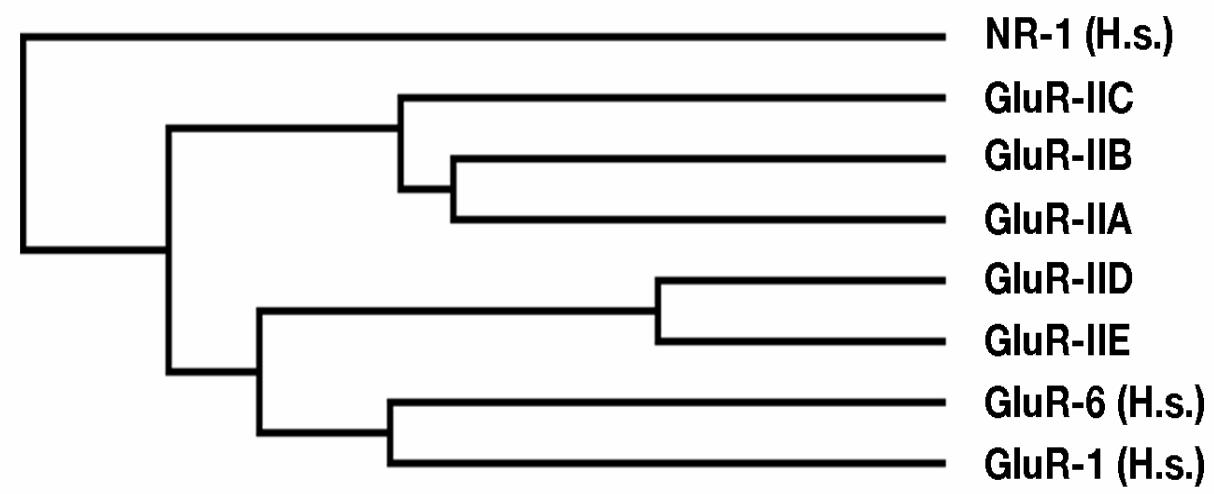

Figure 3-2 Sequence analysis of GluR-IID and GluR-IIE

A, sequence alignment of predicted amino acid sequences of (from top to bottom) GluRIIC/III, GluR-IID, GluR-IIE and human kainate receptor subunit GluR-6, similar amino acids are indicated by shaded boxes. Putative trans-membrane domains (TM1-4), the channel pore region and the c-terminal peptide of GluR-IID which was used for immunization are indicated as well.

$\mathrm{B}$, dendrogram analysis comparing muscle expressed glutamate receptor subunits of Drosophila, together with AMPA receptor subunit GluR1, kainate receptor subunit GluR-6 and NMDA receptor subunit NR-1 (all Homo sapiens). Dendrogram was generated using MacVector software. 


\subsubsection{GluR-IIC, GluR-IID and GluR-IIE are specifically localized at postsynaptic densities of neuromuscular synapses}

To directly test the subcellular distribution of these novel glutamate receptor subunits, polyclonal antibodies were produced. In brief, rabbits were immunized with GluR-IIC/III C-terminus peptide (PRRSLDKSLDRTPKS) and GluR-IID C-terminus peptide (ESLKTDSEENMPVED) respectively. Collected sera were affinity-purified via their corresponding peptides. In western blotting assay, the antibodies against GluR-IIC/III and GluR-IID recognize a single band of about predicted $109 \mathrm{kD}$ and $102 \mathrm{KD}$ in wild type Drosophila embryo extracts respectively (Fig.3-3A, right lane). Cross reactivity of our sera between receptor subunits is not observed, since the antiserum against DGluR-IID can specifically recognize SF9-cells infected with recombinant virus containing GluR-IID cDNA but not the SF9-cells with expressing GluR-IIE (Fig.3-3A, left lanes).

For immuno-fluorescence microscopy studies, previously a monoclonal antibody (8B4D2) against GluR-IIA has been characterized. In both late stage embryos and larvae 8B4D2 stains the postsynaptic membrane along the NMJ with a discrete dotty manner (Fethetherston et al., 2001; Packard et al., 2002). This antibody thus allows direct visualization of PSDs opposite the corresponding active zones. In the wild type larvae and embryos stained with our antibodies against GluR-IIC and GluR-IID, similar findings were obtained: localization of both GluR-IIC and GluR-IID is confined to typical punctae (Fig.3-3B, C, arrow heads) corresponding to individual postsynaptic densities (PSDs), which are surrounded by the HRP labels known to have perisynaptic expression (Sone et al., 2000). Moreover, these punctae are found directly opposite to the presynaptic Nc82 label. The Nc82 monoclonal antibody specifically recognizes the presynaptic active zone (Heimbeck et al., 1999; Wucherpfennig et al., 2003). Thus, it can be concluded that both GluR-IIC and GluR-IID are expressed within the PSD region of individual neuromuscular 
synapses.
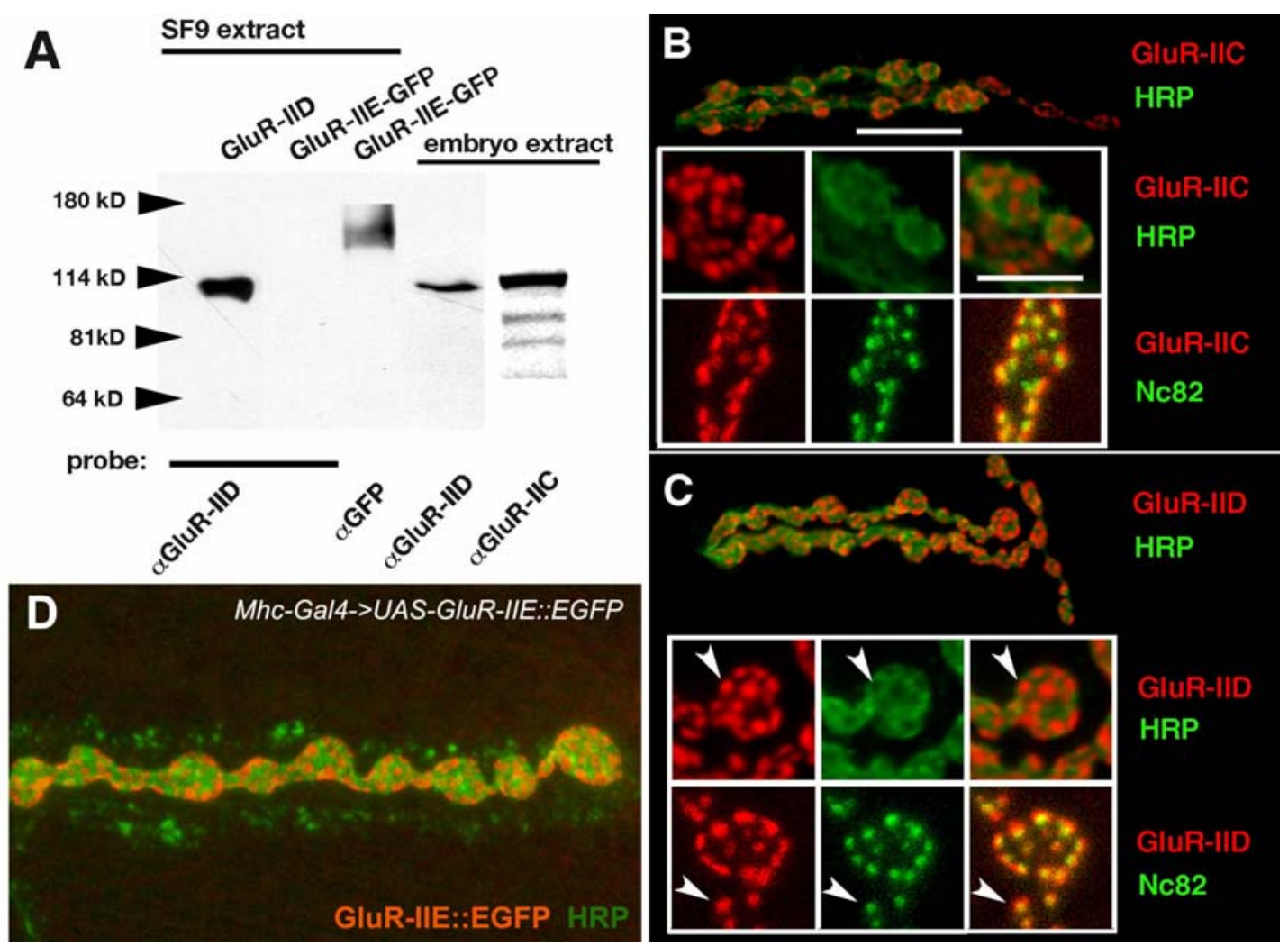

Fig.3-3 GluR-IID and GluR-IIE are expressed within postsynaptic densities A: western blot analysis: the anti-IID peptide antibody recognizes SF9-cell expressed GluR-IID and endogenous GluR-IID from Drosophila embryo extract. It does not cross react with the related GluR-IIE protein, which is SF9-cell expressed as a GFP fusion and is recognized using anti-GFP antibody with the predicted size of about 145 $\mathrm{kD}$. GluR-IIC/III is recognized with our peptide antibody in Drosophila embryo extract with predicted size as well.

B-C, shown are epifluorescence pictures (upper two panels in B and C) and confocal pictures (lower panels in $\mathrm{B}$ and $\mathrm{C}$ ) of receptor subunits GluR-IIC/III (B, red) and GluR-IID (C, red) together with the perisynaptic marker HRP (upper panels in B and C, green) or active zone marker Nc82 (lower panels in B and C, green), scale bars: 8 and $4 \mu \mathrm{m}$.

D, confocal picture of GluR-IIE::EGFP (red) together with perisynaptic marker HRP (green).

Several GluR-IIE-specific peptides were used to immunize rabbits as well. Unfortunately, specific antibodies could not be obtained, mainly due to the fact that the GluR-IIE amino acid sequence is closely related to GluR-IID. In fact, it is hard to find a GluR-IIE specific peptide sequence which would be proper for 
immunization. To overcome this difficulty, the enhanced green fluoerscent protein (EGFP) reading frame was inserted into the near C-terminus GluR-IIE to generate an autofluorescent fusion protein. Fusion of EGFP into the same position of mammalian GluR2 has been previously shown not to interfere with glutamate receptor assembly and function (Sheridan et al., 2002). Indeed, upon overexpressing this GluR-IIE::EGFP in larval muscle (driven by MHC-Gal4), an antibody directed against EGFP strongly labeled the postsynaptic density region (Fig3-3D). This result makes it very likely that the endogenous GluR-IIE protein localizes to the PSD region as well.

\subsection{GluR-IID and GluR-IIE are essential for viability}

To genetically investigate what roles GluR-IID and GluR-IIE play in NMJ glutamatergic transmission, null mutants for each of the two genes were required. For Drosophila molecular genetics, transposon mediated mutagenesis often is an efficient way of knocking out a candidate gene function. In the presence of transposase activity, integrated transposons can be remobilized and randomly jump into other chromosmal locations. Upon remobilization, apart from the transposon seqeunce as well often genomic sequences flanking the insertion site of the mobilized transposon will be eliminated as well. Thus, transposon mapping in the near of candidate loci can be used to produce gene-specific deletions. Here, this strategy was used to obtain mutants of GluR-IID and GluR-IIE.

From a collection of piggyBac transposon lines which covers $53 \%$ of all loci annotated for Drosophila (Thibault et al., 2004), one line (pBac $\{\mathrm{RB}\} \mathrm{e} 01443)$ was described to have an insertion in the GluR-IID locus. This line was obtained and genomic PCR was performed using one primer corresponding to the flanking region the proposed insertion site and another primer corresponding to the end of the piggyBac transposon. The resulting PCR 


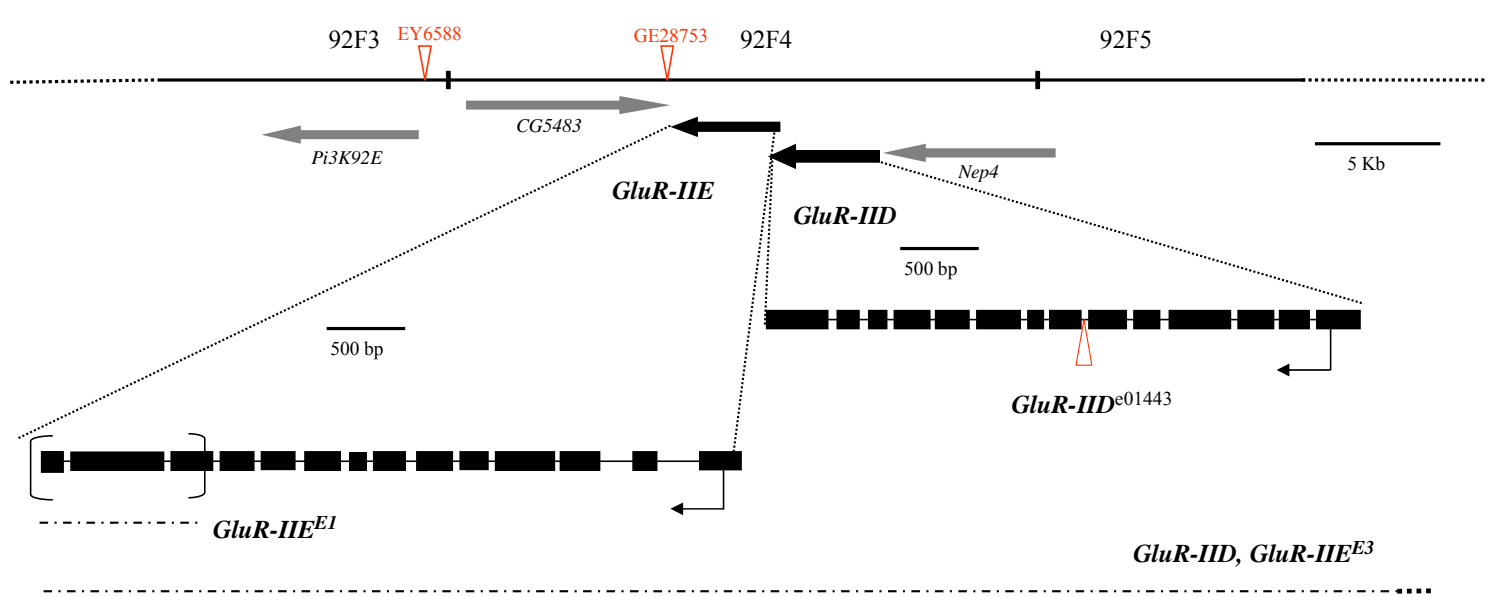

GluR-IIE genomic rescue

Figure 3-4 Genetic analysis of GluR-IID and GluR-IIE

GluR-IID and GluR-IIE map to position 92F on chromosome III. Exon-intron structure of both loci is shown, exons are boxed. The null allele GluR-IIDe01443 is based on a piggyBac transposon insertion within the open reading frame of the GluR-IID locus. Null allele GluR-IIEE1 lacks c-terminal sequence of the protein including the last transmembrane domain of the receptor subunit. GluR-IIEE3 deletes at least GluR-IIE and GluR-IID alleles and thus is used as deficiency allele. The genomic stretches used for genomic rescue constructs are shown below. 
product was purified and subjected to sequencing. It was found that in fact in this line the transposon has integrated directly into intron 6 of DGluR-IID (Fig.3-4). This could well interfere with normal transcript splicing and hence the generation of GluRIID protein. Interestingly, this line is embryonic lethal in homozygous condition. We from now on refer to this allele as GluR-IID ${ }^{\mathrm{e} 01443}$.

For GluR-IIE, imprecise excision mutagenesis screening was performed using transposon line EY6588 (http://flypush.imgen.bcm.tmc.edu/pscreen/) and later GE28753 (http://www.genexel.com) whose insertion sites are located 14Kb and $150 \mathrm{bp}$ downstream of the 3' end of GluR-IIE transcript, respectively (Fig.3-4). From the screening, about 30 imprecise excision events (Fig.3-4) were recovered, genomic PCR with a series of primer pairs corresponding to the flanking region of GE28753 insertion position were utilized to recover the deleted region in each line. Among them, a line called GluR-IIE ${ }^{E 1}$ was found to have the genomic sequence encoding the C-terminal part of GluR-IIE including the last transmembrane domain deleted. Importantly however, the GluR-IIE $E^{E 1}$ deletion does not extend into neighboring loci. Similar to GluR-IID ${ }^{\mathrm{e} 01443}$, GluR-IIE $E^{E 1}$ is also embryonic lethal in homozygous condition. In addition, no GluR-IID protein could be observed at the neuromuscular synapses of either GluR-IID ${ }^{\mathrm{e} 01443}$ or GluR-IIE ${ }^{E 1}$ homozygous embryos (see 3.4.1 for details). GluR-IID $D^{\mathrm{e} 01443}$ and GluR-IIE $E^{E 1}$ are also embryonic lethal over another independent excision allele GluR-IID\&IIE E3 (Fig.3-4), a larger deficiency which deletes both GluR-IID and GluR-IIE genomic sequences. However, GluR-IID $D^{\mathrm{e} 01443}$ is fully viable when crossed over GluR-IIE ${ }^{E 1}$. Both GluR-IID $D^{\mathrm{e} 01443}$ and GluR-IIE $E^{E 1}$ are fully rescued by transgenic addition of one copy of genomic GluR-IID or GluR-IIE, respectively; however, neither the GluR-IID mutant nor the GluR-IIE mutant can be rescued by the respective other transgene. Thus, several lines of evidence (here and 3.4.1) suggest that GluR-IID $D^{\mathrm{e} 01443}$ and GluR-IIE E1 represent specific null mutant alleles for GluR-IID and GluR-IIE, respectively. While the neighboring loci GluR-IID and GluR-IIE encode very similar proteins with largely overlapping expression patern, both of them are essential for embryonic viability. 


\subsection{Reciprocal dependence of all essential glutamate receptor subunits for synaptic expression and function}

3.4.1 Complete synaptic absence of all other glutamate receptor subunits after knockout of any essential subunit genes

Homozygous GluR-IID ${ }^{\mathrm{e} 01443}$ and GluR-IIE ${ }^{E 1}$ embryos were subjected to further inspection. These embryos, while apparently developing normally, show no coordinated movements and do not hatch. The same phenotype is also observed previously within GluR-IIC/III single or GluR-IIA\&IIB double null mutant embryos. This phenotype was interpreted as failure of neuromuscular neurotransmission due to the absence of functional postsynaptic glutamate receptors (Petersen et al., 1997; Marrus et al., 2004). Consistent with a role in synaptic transmission, GluR-IID and GluR-IIE mRNA expression starts within embryonic muscles well before the onset of neurotransmission (Fig.3-1), just like the cases of GluR-IIA, -IIB and -IIC (Petersen et al., 1997; Marrus et al., 2004).

In mammals, ionotropic glutamate receptors appear to be composed of rather closely related subunits (Wenthold et al., 1996; Mulle et al., 2000). Hence, whether the rather similar GluR-IID and GluR-IIE subunits directly form glutamate receptors was investigated via immuno-fluorescence microscopy. In rpinciple, if the formation of a certain type of glutamate receptor fails, the synaptic localization of its consituent receptor subunits must be affected. In wild type late stage embryos, GluR-IID proteins stably localize at the PSD region of all NMJ synapses (Fig.3-5B, wild type). In GluR-IIE E1 embryos, neuromuscular contacts still form, as shown by staining against anti-HRP and Nc82 antibodies (Fig.3-5A, blue and green channel). However, GluR-IID is completely absent from GluR-IIE ${ }^{E 1}$ embryonic NMJs (Fig.3-5B). This finding suggests that GluR-IIE and GluR-IID might join into a common glutamate receptor which in turn is essential for synaptic neurotransmission at the embryonic NMJ. 


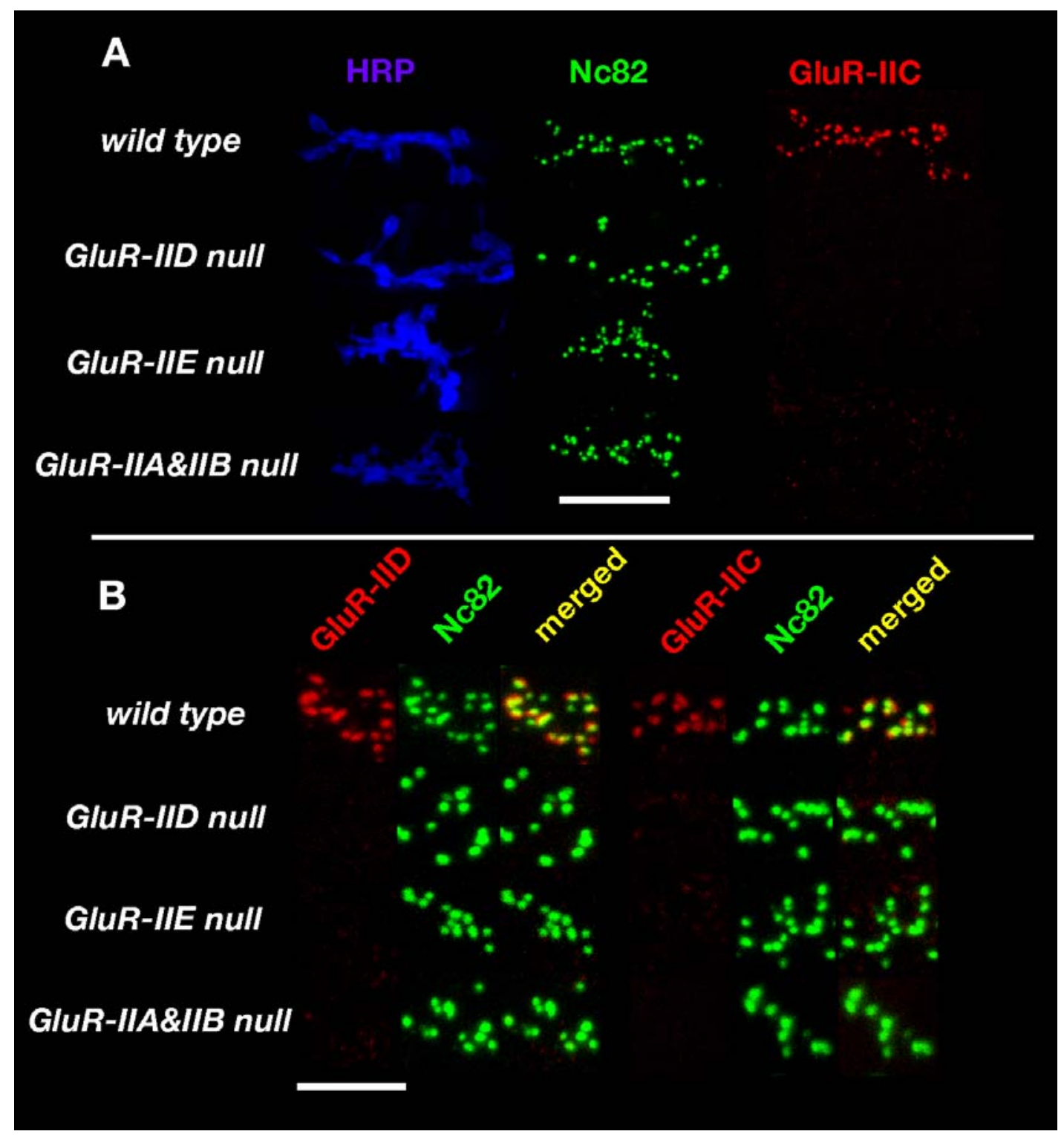

Figure 3-5 Interdependence between glutamate receptor subunits for NMJ expression

A, Confocal images of NMJs in wild-type, GluR-IID null, GluR-IIE null or GluR-IIA\&IIB double null mutant embryos (20-22 hrs old) stained with antibodies against GluR-IIC/III (red), Nc82 (green) and HRP (blue). Nc82 staining indicates differentiation of presynaptic release sites at the mutant NMJs. Synaptic expression of all glutamate receptor subunits fails at the mutant NMJs. Scale bar: $10 \mu \mathrm{m}$ $\mathrm{B}$, Higher magnifications of confocal stainings similar as in A. Stainings of either GluR-IID (left panels) or GluR-IIC/III (right panels) are shown together with Nc82. Scale bar: $5 \mu \mathrm{m}$.

Based on the analysis of GluR-IIC/III and GluRII-A hypomorphic larvae, it 
has been suggested that GluR-IIC/III is an obligatory subunit, which associates with either GluR-IIA or GluR-IIB to form functionally distinct glutamate receptors (Marrus et al., 2004). This idea was tested again but directly by immunostaining GluRII-A\&IIB double mutant embryos. Consistent with Marrus et al., GluR-IIC/III label is completely absent from NMJs of Glur-IIA\&IIB null mutant embryos (Fig.3-5A and B). Thus GluR-IIA together with GluR-IIB can be considered a "synthetic essential subunit". In principle, the two "groups" of subunits (GluR-IID, -IIE versus -IIA, -IIB, -IIC/III) could be independently essential for embryonic neurotransmission. Alternatively, synaptic localization and thus receptor function could be interdependent between both groups. To distinguish these two possibilities, it was first checked whether the synaptic localization of the GluRIIC/III glutamate receptor subunit is dependent on the presence of GluR-IID and GluR-IIE. Clearly, no synaptic localization of GluR-IIC/III can be found in either GluR-IID or GluR-IIE null mutant embryos (Fig.3-5A and B). Vice versa, the synaptic expression of GluR-IID was absent from GluR-IIA\&-IIB double null mutant embryos (Fig. 5B). Thus, at the embryonic NMJ, the synaptic expression of all essential glutamate receptor subunits is absolutely interdependent. The easiest explanation for these data is that at the Drosophila NMJ the glutamate receptor is formed by four different essential glutamate receptor subunits: GluR-IIC, GluR-IID, and GluR-IIE together with either GluR-IIA or GluR-IIB.

\subsubsection{Genetically depriving the expression level of any single essential glutamate receptor subunit results into corresponding reduction of all other subunits}

If the glutamate receptor is indeed assembled from four different subunits, partially suppressing the level of any single such subunit should interfere with the synaptic expression of the other essential subunits and the overall glutamate receptor formation. To test this, several independent experiments have been performed. 
First, the expression of GluR-IIE was knocked down in vivo via transgenic RNA interference technique (Kennerdell and Carthew, 2000). When RNA interference against GluR-IIE was conducted in a muscle-specific manner, the GluR-IIE transcript level was knocked down to about $20 \%$ of wild type level while the transcription level of all other muscle subunit genes remained unchanged (not shown), indicating the highly selective RNA degradation mediated by RNA interference. In contrast to the GluR-IIE null mutants, these animals do not die at embryonic stage but instead develop into mature larvae and adult flys. So the effects on the distribution of other subunits in $3^{\text {rd }}$ larvae were examined. Significantly, all of the other known subunits (GluR-IIA, GluR-IIB, GluR-IIC/III and GluR-IID) were greatly reduced at NMJ when the RNA interference was driven in muscles (using G14-Gal4 or MHC-Gal4), while

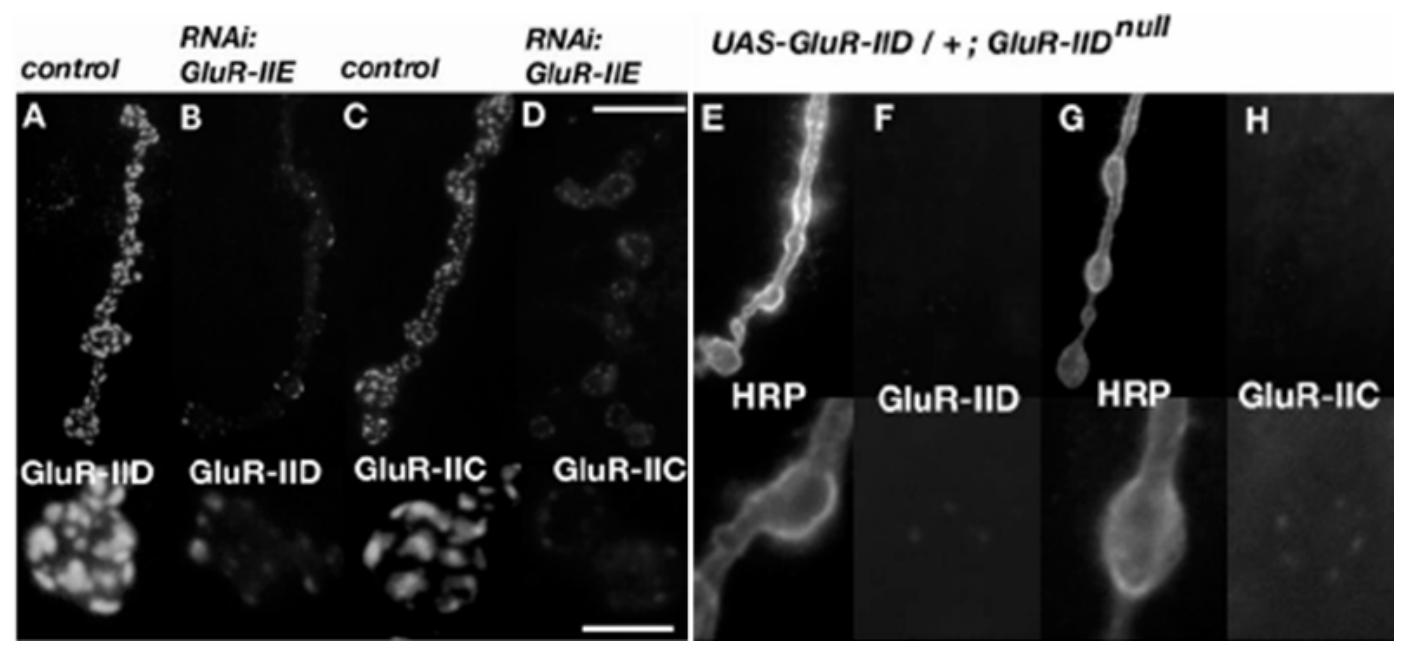

Figure 3-6 A partial reduction of either GluR-IIE or GluR-IID provokes a significant reduction of all glutamate receptor subunits at the NMJ

(A-D) Larval NMJs (muscle 4, abdominal segment 2) stained for GluR-IID (A, B) or GluR-IIC/III (C, D) in control larvae (G14-gal4/ +, A and C) or larvae experiencing muscle specific RNA interference against GluR-IIE (G14-gal/+; UAS-GluR-IIE-RNAi, $\mathrm{B}$ and D). Suppression of GluR-IIE leads also to a reduction in the NMJ expression of GluR-IIC/III and GluR-IID. Lower pictures represent higher magnifications.

(E-H) Shown are NMJs (muscle 4, abdominal segment 2) of GluR-IID null mutant larvae rescued with a single copy of UAS-GluR-IID (UAS-GluR-IID/+; GluR-IID ${ }^{e 01443} /$ GluR-IID\&IIE E3. Only trace amounts of GluR-IID (F) and GluR-IIC (H) are expressed at the NMJ, HRP stainings $(E, G)$ are added to visualize the NMJ. Non-linear contrasting had to be used to make the trace amounts of GluR-IID (F) and GluR-IIC (H) visible. Scale bar in upper panel 7,5 $\mu \mathrm{m}$, in lower panel showing higher magnification $2,5 \mu \mathrm{m}$. 
there is no obvious effects when driving the expression of RNA interference construct in the central nervous system (using ELAV-Gal4) (Fig.3-6B, D; data not shown), which further verify that GluR-IIE functions specifically in somatic muscles and intrinsically associates with other subunits.

For a second experiment, it was found that minimal amounts of GluR-IID (produced by the "leaky" expression from pure UAS-cDNA transgenes) can already rescue GluR-IID null mutant embryos into mature larvae (Fig.3-6E-H). In these rescued larvae, both GluR-IID and all other glutamate receptor subunits were strongly reduced at the NMJs (Fig.3-6H). Re-expressing GluR-IID to normal level by using the muscle specific Gal4-driver line Mhc-Gal4 at $18^{\circ} \mathrm{C}$ restored the synaptic localization of GluR-IID and all other glutamate receptor subunits (not shown; overexpression of GluR-IID cDNA in both wild type and GluR-IID null background at $25^{\circ} \mathrm{C}$ resulted into significant reduction of synaptic glutamate receptor level with abnormal accumulation of GluR-IID proteins within the ER). Thus, the levels of GluR-IID and GluR-IIE within the muscles directly control the overall amount of glutamate receptors that localize at the NMJ.

The next question addressed is, whether vice versa the amounts of muscle GluR-IIA, -IIB and -IIC/III can control synaptic level of GluR-IID and -IIE. To this end, a genetic situation in which GluRIIB was fully absent and simultaneously GluR-IIA was suppressed to dramatically low level (GluRIIA ${ }^{\text {hypo, }}$, $G l u R-I I B^{\text {null }}$, see 2.2.2.1) was used. Previously it has been proposed that local postsynaptical translation plays important roles in controlling the growth and transmission efficacy of synapses at Drosophila NMJ. GluR-IIA appears might be a substrate under subsynaptic translational control (Sigrist et al., 2000). Since translational control elements often localize within the 3'UTR region of a gene, transgenic flys expressing GluR-IIA lacking most 3'UTR were produced and tested for their ability to rescue the GluR-IIA\&IIB double null mutants, which is 
embryonic lethal (Diantonio et al., 1999). Among several independent transgenic lines tested, only one of them could rescue the embryonic lethality with low rate and the rescued larvae were clearly paralyzed. Real-time quantitative PCR assay showed that the GluR-IIA message level was below 5\% of endogenous level. In accordance with the low message level, the GluR-IIA protein was hardly detectable at the larval NMJ (not shown). Notably, the level of both GluR-IIC/III and GluR-IID were drastically low (Fig.3-7B, D). Collectively, all the above data suggest all essential glutamate receptor subunits together controls receptor formation and proper synaptic localization. Furthermore, this rule seems true for both initial formation and further development of NMJs, where many new synapses get added continuously (Schuster et al., 1996; Gramates and Budnik, 1999).

Fig.3-7 Minimal mounts of GluR-IIA and no- IIB: expression of all glutamate receptor subunits and postsynaptic sensitivity are strongly reduced

(A-D) Shown are 3rd instar larval NMJs (muscle 4, abdominal segment 2) stained for GluR-IID (A, B) or GluR-IIC/III (C, D) in wild type controls (A, C) or in animals having only about 5\% GluR-IIA and no GluR-IIB (GluR-IIAhypo, GluR-IIBnull, see Material and Methods). NMJ morphology in HRP labeling is shown in insets to allow NMJ visualization independent of receptor label. In GluR-IIAhypo, GluR-IIBnull larvae, the synaptic localization of both GluR-IID and GluR-IIC/III is strongly reduced. Apparent differences in between residual GluR-IID and GluR-IIC/III are likely due to slightly different sensitivity of our antibodies.

E-G, Postsynaptic sensitivity is dramatically reduced at larval NMJs of GluRIIAhypo,GluRIIBnull larvae. E, F, Two-electrode voltage-clamp recordings of eEJCs and mEJCs from muscle 6 of abdominal segment A2 and A3. Representative traces of mEJC recordings (E) and average traces of 10 consecutively recorded eEJCs (F) of the indicated genotypes are shown. The mean amplitudes of mEJCs (white bars) are indistinguishable between wild type and GluRIIA\&IIB double mutant larvae rescued with a complete genomic GluRIIA transgene (exact genotype: $\operatorname{df}(2 \mathrm{~L}) \mathrm{clh} 4$, $\mathrm{P}$ [GluRIIA 43 'UTR] / GluRIIA\&BSP22). In all muscle cells of GluRIIAhypo,GluRIIBnull larvae, no signs of mEJCs could be recorded despite extensive and sensitive recording. Evoked responses (F) are also dramatically reduced in GluRIIAhypo,GluRIIBnull larvae compared to both control groups. The increase in evoked response in animals rescued with wild type GluRIIA (and thus without expressing of GluRIIB) when compared to wild type has been described before (Petersen et al., 1997; DiAntonio et al., 1999). G, Paired pulses (stimulation interval ms) provoke slight facilitation in the controls but depression in GluRIIAhypo,GluRIIB-/larvae. Data are derived from the indicated number of cells (number in the column) and represent means \pm SEM. 

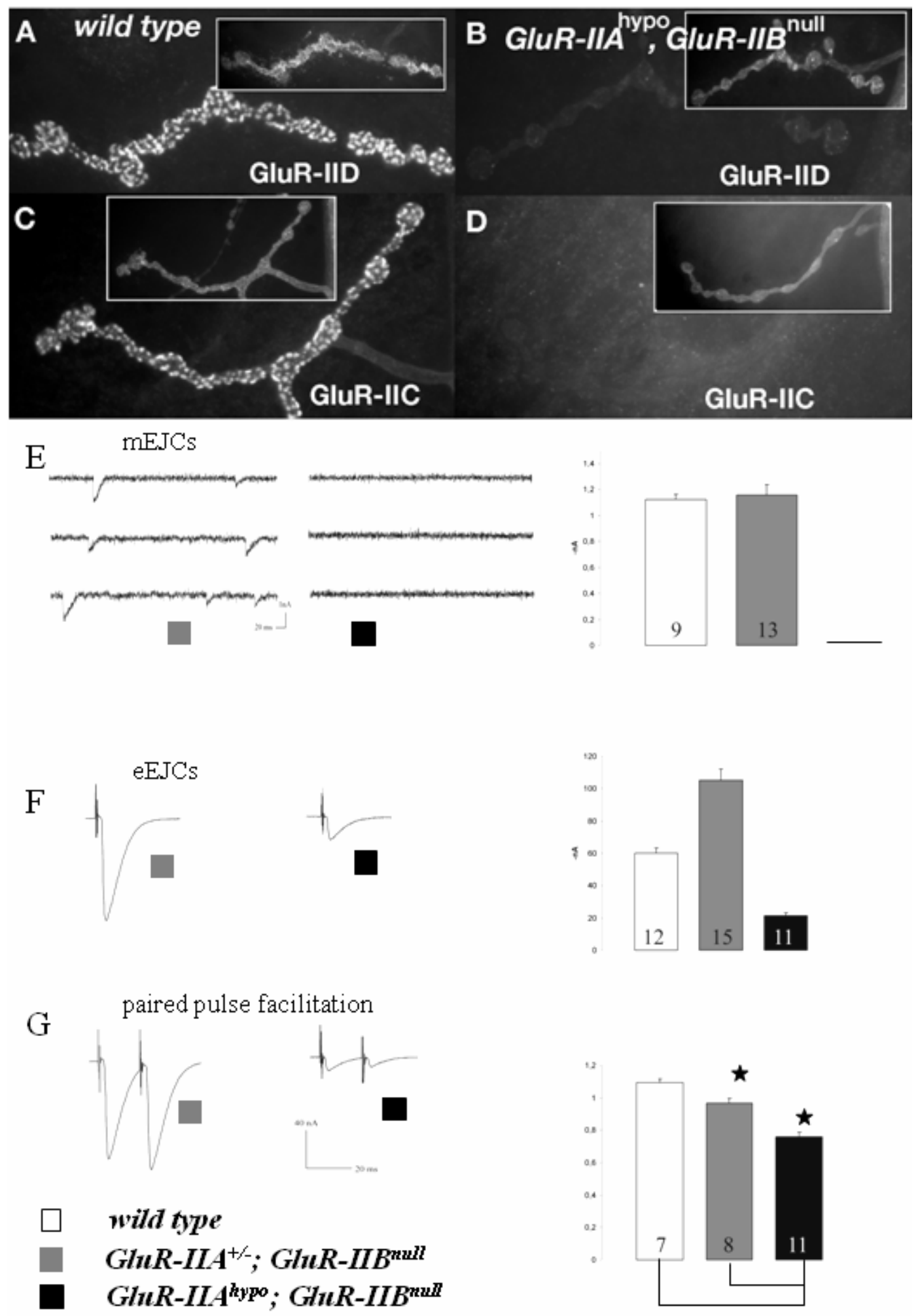


\subsubsection{Depriving synaptic glutamate receptor subunits results into dramatic weakness of postsynaptic activity}

To test whether the physiological function of glutamate receptor correlates well with its presence at synapse reflected from the above morphological data, electrophysiological analysis is highly necessary. Among the different genetic situations which partially deprive certain glutamate receptor subunits in embryos and larvae, the GluRIIA ${ }^{\text {hypo }}$, GluR-IIB ${ }^{\text {null }}$ larvae maintain the lowest overall level of glutamate receptors (Fig.3-7 B, D) and display clear signs of paralysis. Thus GluRIIA hypo, GluR-IIB ${ }^{\text {null }}$ larvae were subjected to two-electrode-voltage-clamp recordings in collaboration with Robert Kittle (Fig.3-7 E, F). As expected, compared to wild type conditions, spontaneous miniature junctional currents (mEJCs) in these animals were below the detection limit indicating an extreme drop of postsynaptic glutamate receptor function. Accordingly, evoked currents (eEJCs) were also significantly low (wild type: 61 $\mathrm{nA}, \mathrm{n}=12$, GluRIIAhypo, GluR-IIBnull: $21 \mathrm{nA}, \mathrm{n}=11 ; \mathrm{p}<0.00005$, Mann-Whitney, two-sided non-parametric test).

In conclusion, strongly reducing the expression of a single 'essential' glutamate receptor subunit is sufficient in severely downregulating the overall level of functional glutamate receptors at the NMJ, which in turn results into weakening of postsynaptic activity in response to neurotransmitter release.

\subsection{Structural role of ionotropic glutamate receptor during pre- and postsynaptic differentiation of neuromuscular synapse}

\subsubsection{Normal differentiation of presynaptic transmitter release machinery at glutamate receptor deprived synapse}

As shown in chapter 3.4, GluRIIA ${ }^{\text {hypo }}$, GluR-IIB ${ }^{\text {null }}$ larval junctions produce evoked currents of about one third of that of wild type junctions. Even more drastically, the spontaneous currents are hardly detectable. We considered the fact that evoked responses appeared less affected than spontaneous responses in 
GluRIIA $^{\text {hypo }}$, GluR-IIB ${ }^{\text {null }}$ larvae as indication of normal or even increased presynaptic transmitter vesicle release as compensation of severe postsynaptic defects. Consistent with the concomitant increase in presynaptic vesicle release, paired pulse stimulation of GluRIIA ${ }^{\text {hypo }}$, GluR-IIB ${ }^{\text {null }}$ NMJ leads to depression of evoked currents while as for wild type controls the currents remain slightly increased in the same condition. The organization of presynaptic release machinery was further characterized morphologically. The active zone is the region of the presynaptic plasma membrane where synaptic vesicles dock, fuse, and release the neurotransmitters (Shapira et al., 2003). Monoclonal antibody NC82 was shown to specifically label the Active zones of synapses of Drosophila NMJ (Wucherpfennig et al., 2003) and central synapses (Heimbeck et al., 1999). In GluRIIA ${ }^{\text {hypo }}$, GluR-IIB ${ }^{\text {null }}$ larvae, nc82 labels are clearly present at NMJ with both density and individual size comparable to wild type controls (Fig.3-8 C, D; Fig.3-9 D-E). $\alpha$-Adaptin is essential for presynaptic endocytosis/vesicle retrieval (Gonzalez-Gaitan and Jackle, 1997) and is restricted to the presynaptic membrane, forming a network-like structure that surrounds the active zones (Gonzalez-Gaitan and Jackle, 1997) (Fig. 3-8 A-C). In GluRIIA $^{\text {hypo }}$, GluR-IIB ${ }^{\text {null }}$ larvae, subcellular distribution of $\alpha$-Adaptin is not apparently distinct with that of wild type controls (Fig.3-8 A, B), suggesting that the differentiation of presynaptic release apparatus is not affected by depriving postsynaptic glutamate receptor.

Fig. 3-8 Normal organization of presynaptic neurotransmitter release machinery components at glutamate receptor deprived synapses

NMJs double-labeled with the antibodies against presynaptic component $\alpha$-Adaptin (Gonzalez-Gaintan M et al., 1997; A-B) and Nc82 antibody recognizing an active zone epitope (Wucherpfennig et al., 2003, Heimbeck et al., 1999; C-D). In wild type animals, $\alpha$-Adaptin is restricted to the presynaptic membrane, forming a network-like structure that surrounds the active zones represented by $\mathrm{Nc} 82$ spots (A, C, E). In GluRIIA $^{\text {hypo }}$, GluRIIB $^{-/-}$larvae, both Nc82 and $\alpha$-Adaptin spots appear no different from wild type control (B, D, F). 

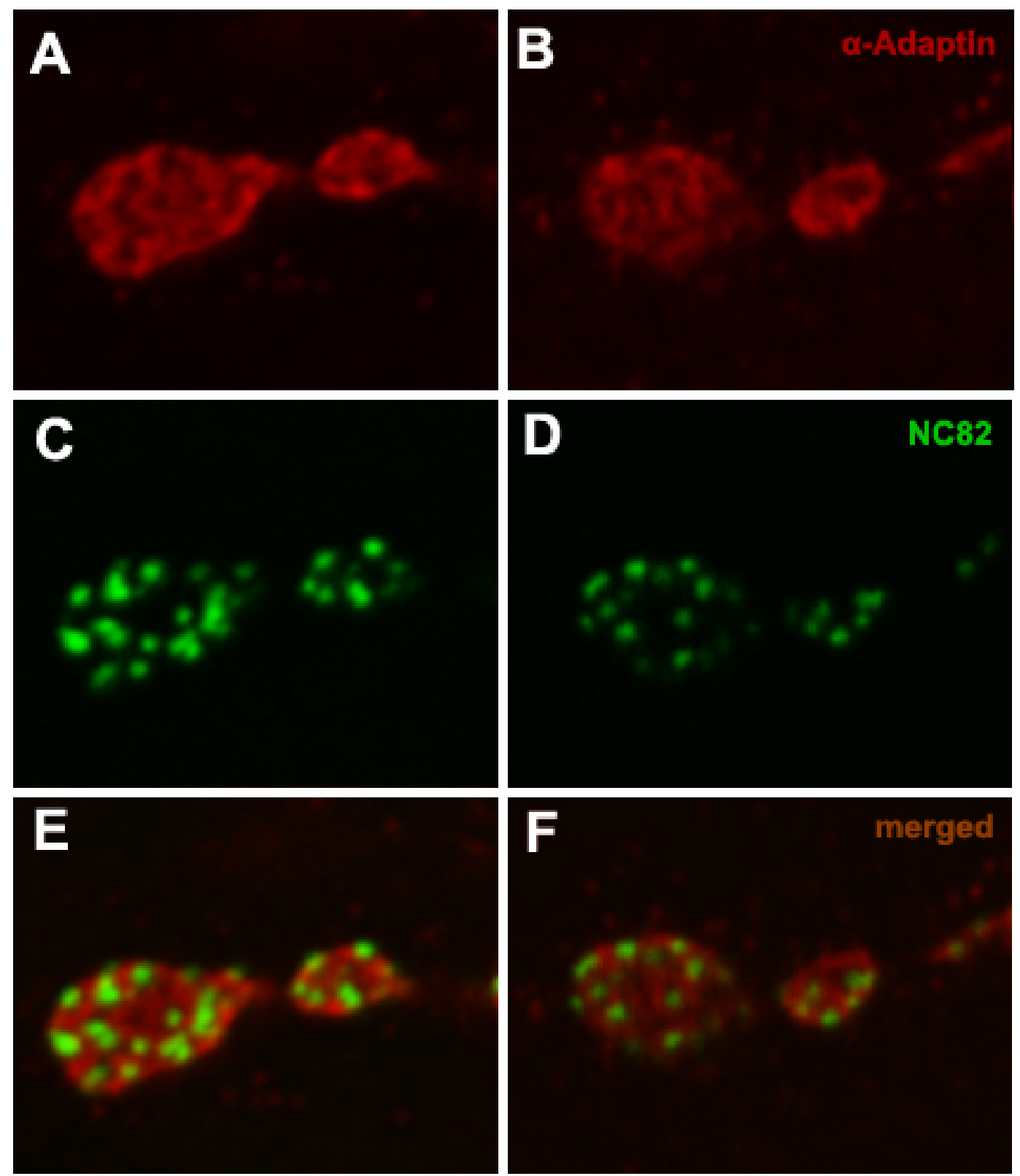

Wild type

GluR-IIAhypo, GluR-IIBnuII

3.5.2 Defective assembly of PSD specialization at glutamate receptor deprived but not presynaptic neurotransmission activity deprived synapse

The differentiation of postsynaptic apparatus was also analyzed in GluRIIA $^{\text {hypo }}$, GluR-IIB ${ }^{\text {null }}$ larvae. So far, only a few PSD components have been identified and characterized at Drosophila NMJ (Sone et al., 2000; Wan et al., 
2000; Parnas et al., 2001). PAKs are a family of serine/threonine kinases that serve as targets for the small GTP-binding proteins Cdc42 and Rac, they are implicated in modulating the cytoskeleton organization (Bagrodia and Cerione, 1999). In Drosophila, dPak has been shown to specifically localize at the PSD of neuromuscular synapse at both immunofluorescence microscopic and immuno-EM level (Sone et al., 2000; Wan et al., 2000). In dpak mutants, the levels of DLG and GluRIIA at the synapse are reduced and the SSR folding is also disrupted (Parnas et al., 2001; Albin and Davis, 2004) In wild type larvae, dPAK antibodies labeled the well defined patches with approximately even distribution over the surface of bouton, (Fig. 3-9), and as expected NC82 perfectly colocalized with dPAK staining (Fig.3-9 ). In GluRIIA ${ }^{\text {hypo }}$, GluR-IIB ${ }^{\text {null }}$ bouton, however, the dPAK staining displayed apparently lower density, with the size of individual spots much more diverse---from huge irregular aggregates to tiny dots, and often, at some bouton areas the dPAK even did not aggregate at all but just 'wrap' the bouton like cloud, (Fig.3-9, arrow).

The above data suggest that, when glutamate receptors are deprived from synapse other constitutive components of postsynaptic specialization can not be assembled efficiently. In principle, these defects could be either due to the absence of glutamate receptor per se or lack of glutamate mediated transmission activity. To distinguish these possibilities, several genetic situations in which the NMJ transmission was either strongly reduced (survival to larval stage) or

Fig.3-9 Defective PSD assembly at glutamate receptor deprived but not transmission deprived synapses

NMJs triple-labeled with the Nc82 antibody recognizing an active zone epitope (Wucherpfennig et al., 2003, Heimbeck et al., 1999; D-F), antibodies against PSD marker DPAK (Sone et al., 2000; G-I)) and HRP (A-C). In wild type animals, Nc82 spots localize at presynaptically juxtaposed to postsynaptic DPAK spots (J). In GluRIIA $^{\text {hypo }}$,GluRIIB ${ }^{-/-}$larvae, Nc82 spots localize normally (E), however, DPAK spots display significantly lower density and more variable individual size $(\mathrm{H})$. In OK319-Gal4->UAS-TNT larvae, both Nc82 and DPAK spots appear no different from wild type control (F, I, L). 

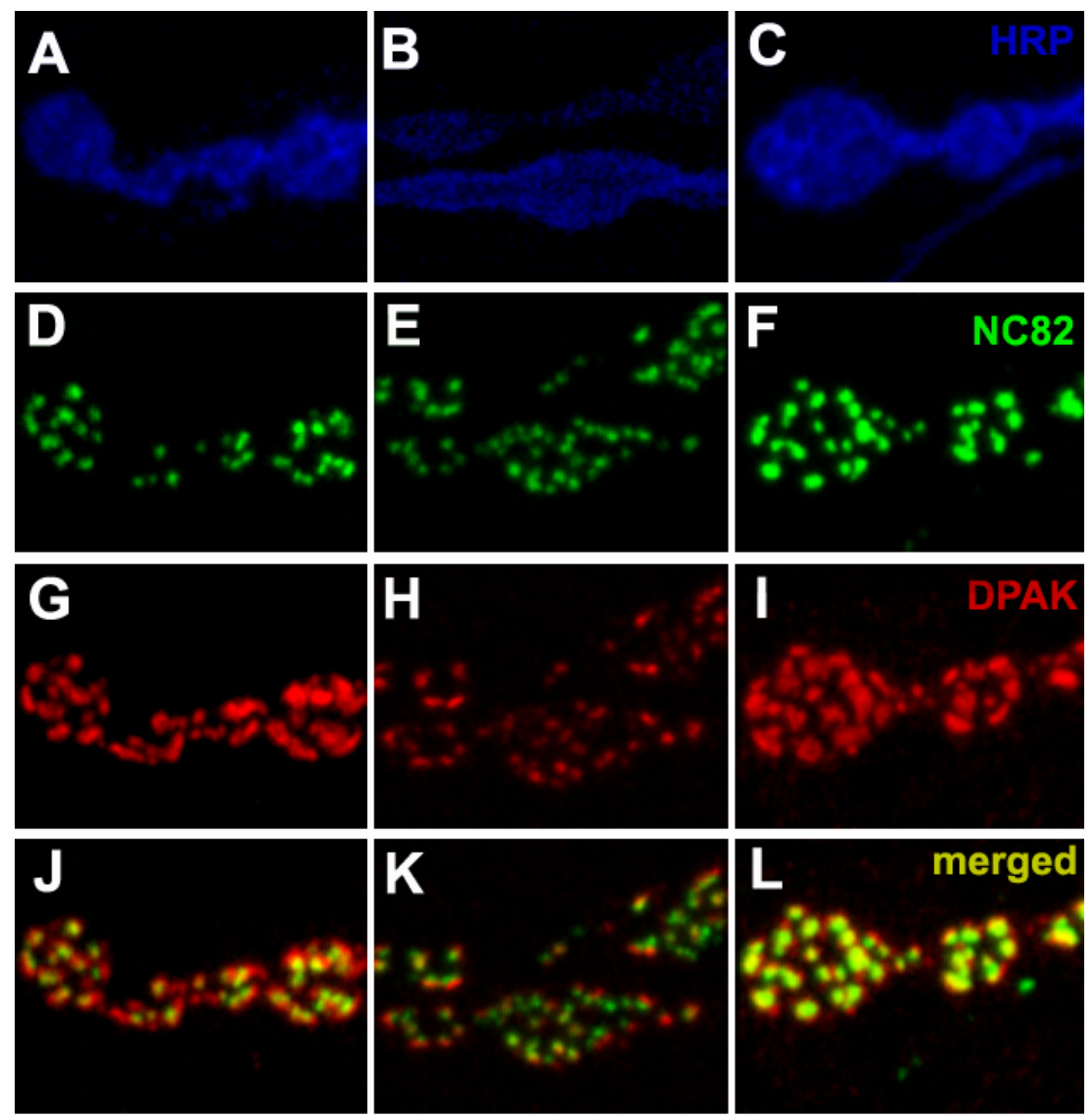

Wild type

GluR-IIAhypo, GluR-IIBnull Ok319-Gal4->UAS-TNT

completely suppressed (lethal at late embryonic stage) were analyzed for postsynaptic assembly. Targeted expression of tetanus toxin light chain (TNT) in Drosophila neurons completely eliminates evoked, but not spontaneous synaptic vesicle release (Sweeney et al., 1995). When using the motor neuron driver ok319-Gal4 to drive TNT expression, low number of animals can survive to $3^{\text {rd }}$ instar larval stage (this survival is likely due to the mosaic property of the driver, Cahir O'Kane, personal communication). Surviving larvae were very strongly paralyzed, much stronger than GluRIIA ${ }^{\text {hypo }}$, GluR-IIB ${ }^{\text {null }}$ larvae. However, the distribution of neither postsynaptic components $\mathrm{dPAK}$ nor glutamate receptor 
show any discernible defects in all the NMJs checked, in contrast they both colocalize well with active zone shown by NC82 staining (Fig.3-9 F, I).

Since the expression of tetanus toxin does not fully eliminate spontaneous synaptic vesicle release (Sweeney et al., 1995), it might be argued that in the above ok319-Gal4 driven situation remaining activity (especially spontaneous activity) could sufficiently induce the assembly of postsynaptic specializations at normal level. Indeed, recently a tight link between spontaneous vesicle exocytosis and glutamate receptor clustering during Drosophila embryonic development has been reported (Saitoe et al., 2001). It should be noted, however, that this report is clearly controversial to some other reports (Broadie et al., 1995; Featherstone et al., 2001). It has been proved previously that, in either null mutants of syntaxin or temperature sensitive allele of dynamin $\left(\mathrm{shi}^{\mathrm{ts} 1}\right)$ reared at nonpermissive temperature, both spontaneous and evoked vesicle exocytosis and hence synaptic transmission are completely eliminated (Broadie et al., 1995). Using these 'cleaner' conditions, the immuno-fluoresence microscopic analysis was performed with our antibodies against GluR-IIC and GluR-IID. In contrast to the findings of Saitoe $M$ et al but consistent with those of Featherstone et al, the glutamate receptor localization within individual PSDs is essentially unaffected as seen in GluR-IIC and GluR-IID staining (Fig.3-10). Moreover, in these animals DPAK proteins also cluster at normal size at the PSD region as evidenced by colocalization with NC82 labels (Fig3-10). Therefore, it is the glutamate receptor per se but not synaptic transmission activity essential for the postsynaptic specialization (including glutamate receptor field) assembly.

Besides the main finding described above, another interesting phenomena found is found. At the NMJs of glutamate receptor deprived larvae (GluRIIA ${ }^{\text {hypo }}$, GluR-IIB ${ }^{\text {null }}$ ), often postsynaptic dPAK label was not opposed by presynptic active zone marker (identified via NC82 staining) (Fig.3-9). One explanation is that these free NC82 labels might represent new synapse growth sites. However, 
this is quite unlikely since in wild type NMJs stained with same antibodies, the free NC82 labels without aligned dPAK patches were very rarely found (Fig.3-9). Intriguingly, free dPAK labels could be stably detected in wide type NMJs, and in most cases such free dPAK patches are small and localize at the tips of junctions or the linkage regions between boutons, which likely represent the potential growth sites of new synapses. Thus, these free dPAK labels might reflect the precursors of new synapses, which implicates that, in contrast to the finding in synapses of cultured hippocampal neurons, at Drosophila NMJ the postsynaptic assembly might precede presynptic assembly. In GluRIIA ${ }^{\text {hypo, }}$ GluR-IIB $^{\text {null }}$ NMJs, the precise coordination of pre- and postsynaptic differentiation is strongly affected. However, it is unlikely that this coordination defect is merely due to absence of glutamate receptor per se, since in embryos with complete loss of presynptic transmitter release, although individual PSDs are assembled rather normally, there also exist free active zones (Fig.3-10 F). Because the glutamate receptor null mutants lack both glutamate receptor and the concomitant transmission activity, it remains to be further addressed whether the precise coordination of pre- and postsynaptic differentiation is activity-dependant.

Fig. 3-10 Glutamate receptor per se but not presynaptic vesicle release are important for proper synapse formation at embryo NMJ

Confocal microscopy on embryonic NMJs (muscle 6 and 7), stained with the synaptic markers in wild type (A-D), in GluR-IIA\&IIB ${ }^{-/-}$double mutant embryos (B, E) and in shibire temperature sensitive mutant $\left(s h i^{\text {ts1 }}\right)$ embryos reared at nonpermissive temperature $(\mathrm{C}, \mathrm{F})$.

A-C, Embryonic NMJs double labeled with active zone recognizing antibody Nc82, glutamate receptor subunits GluR-IIC/ III. In situation with defective presynaptic release (C), postsynaptic glutamate receptor fields appear identical as in wild type control. As expected, staining for GluR-IIC, which depends on the presence of either GluR-IIA or GluR-IIB expression, is absent from synapses of dglurIIA\&IIB-/-(B).

D-F, Embryonic NMJs double labeled with the active zone recognizing antibody Nc82 and antibodies against PSD marker DPAK. . Wild type embryos show typical DPAK patches juxtaposed to presynaptic Nc82 labels (D). In GluR-IIA\&IIB ${ }^{-/-}$and shibire shits embryos reared at nonpermissive temperature, $\mathrm{Nc} 82$ often is not associated with postsynaptic DPAK spots (E, F), DPAK spots are irregular in size and shape and show lower density in GluR-IIA\&IIB ${ }^{-/}$embryos (E). 

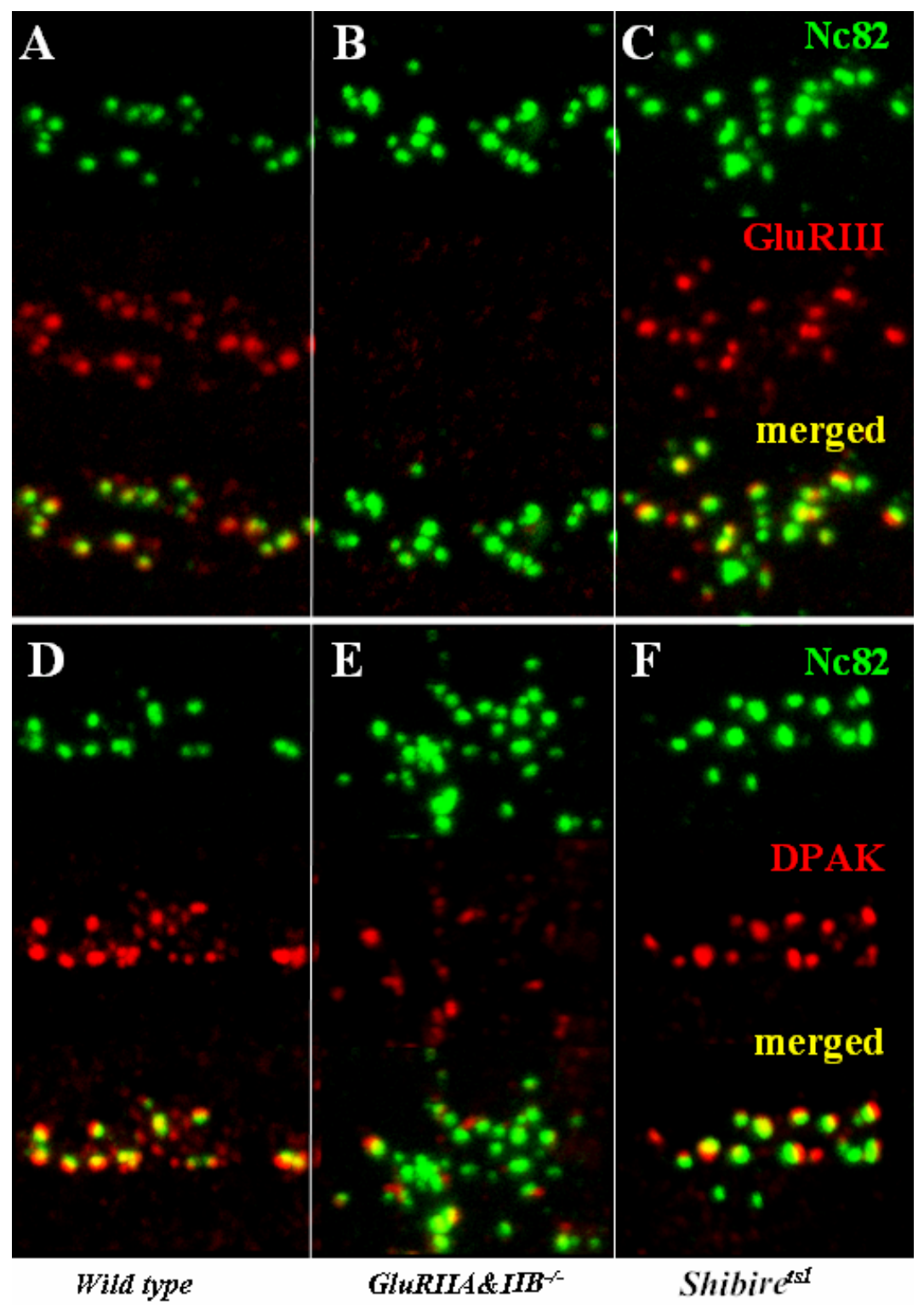

\subsubsection{Ultrastructural evidence of abnormal synaptic differentiation at glutamate receptor deprived synapse}

Next, to check whether such assembly defects of postsynaptic components at glutamate receptor deprived synapse could be directly visualized at the ultrastructural level, neuromuscular junctions 6 and 7 of $3^{\text {rd }}$ instar GluR-IIA ${ }^{\text {hypo }}$, GluR-IIB ${ }^{\text {null }}$ larvae were subjected to transmission electron microscopy (collaborated with Carolin Wichman). At normal Drosophila neuromuscular synapses, active zones are characterized by the presence of 

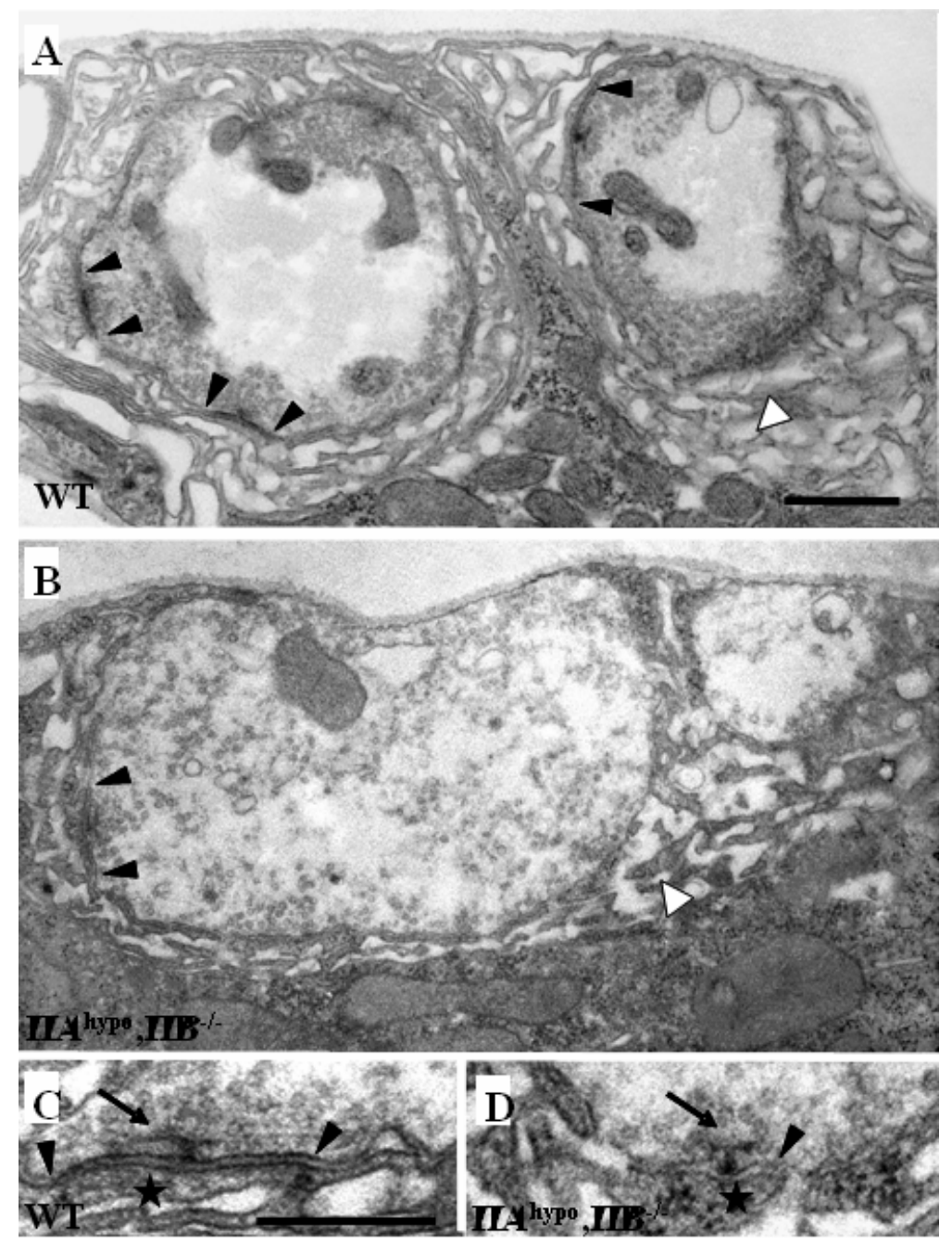

Fig.3-11 Electron microscopy: defective postsynaptic assembly at glutamate receptor deprived synapses

(A) Electron micrograph of type I nerve terminals of NMJ 6/7 in a wild type situation showing several clearly visible electron dense synapses (black arrowheads) and the SSR (white arrowhead). The synaptic vesicles are arranged along the presynaptic membrane. (B) GluRIIA ${ }^{\text {hypo }}$, GluRIIB ${ }^{-/ 2}$ type 1 b bouton of NMJ 6/7, respectively. The black arrowheads indicate a mutant, clearly electron translucend synapses decorated with a T-bar. The SSR (white arrowhead) is significantly reduced. (C, D) Synaptic sites in wild type (C) and GluRIIA ${ }^{\text {hypo }}$, GluRIIB ${ }^{-/}$(D) larvae. The electron dense T-bars are indicated by arrows. The PSD, in wild type detectable as electron dense material $(C$, asterisk) is in GluRIIA ${ }^{\text {hypo }}$, GluRIIB $^{-/-}$synapses absent (D, asterisk) or in less frequent weaker cases dramatically reduced (not shown). In GluRIIA ${ }^{\text {hypo }}$, GluRIIB $^{-/-}$synapses the lack of PSD organization also affects the close alignment of the pre- and postsynaptic membranes, which usually extends over several $100 \mathrm{~nm}$ (C, arrowheads). In GluRIIA $^{\text {hypo }}$, GluRIIB $^{-/-}$larvae this alignment is severely defective and the SSR formation already begins in direct vicinity to the T-bar (D, arrowhead) or extends asymmetrically. 
electron-dense T-shaped structures called 'T-bars' at the presynaptic side (Wojtowicz et al., 1994; Cooper et al., 1995; Cooper et al., 1996). PSDs are opposite to the T-bars and easily recognized by electron dense linear membrane-membrane appositions (Fig.3-11), a characteristic structure also found in mammalian CNS glutamatergic synapses. Within the PSD region, preand postsynaptic membrane associate closely with each other and are visualized as linear apposition in cross section (Fig.3-11). At most synaptic sites, the PSD diameter clearly exceeds the diameter of the presynaptic T-bar. In region between individual PSDs (so called "perisynaptic region"), pre- and postsynaptic membrane (motorneuron and muscle membrane) are organized into various in-foldings and associate only at restricted contact points. This structure is continuous with the subsynaptic reticulum (SSR).

In GluR-IIA ${ }^{\text {hypo }}$,GluR-IIB ${ }^{\text {null }}$ larvae, apparently normal presynaptic T-bars were observed in boutons. However, PSD organization is severely defective (Fig.3-11) in these animals: the muscle membrane domains juxtaposed to the presynaptic T-bars, where typically PSDs should be formed, show very low amount or even complete absence of electron dense material (Fig. 3-11 D). Moreover, the typical extended and close alignment between pre- and postsynaptic membranes could not be found from GluRIIA $^{\text {hypo }}$, GluRIIB ${ }^{\text {null }}$ larvae, in which with only significantly narrower and "wrinkled" apposition of membranes observed (Fig3-11 D). Thus, the ultrastructural analysis directly shows that in the absence of glutamate receptors, the PSD can not be efficiently assembled. In contrast, in the dpak mutants, despite the dramatic reduction of SSR foldings, the ultrastructure of individual synapse appeared rather normal, with smooth electron dense thickening at PSD region (Parnas et al., 2001). Rather normal ultrastructural active zone and PSD assembly was also found in embryos with neuronal TNT expression or lack of Syntaxin (Broadie et al., 1995). In combination of all these data, it is the glutamate receptor per se, obviously independent of transmission activity, controls the normal PSD assembly. 


\subsubsection{Defective compartmentation of the synaptic and perisynaptic zones at glutamate receptor deprived but not neurotransmission deprived synapse}

The ultrastructural analysis showed that the close apposition in between pre- and postsynaptic membrane is largely abolished after reducing glutamate receptor level. It is thus interesting to know, whether the strong defects in ultrastructural PSD organization at glutamate receptor deprived synapses might also reflect defects in the molecular organization of synaptic compartments. Therefore, to address this issue, the GluRIIA ${ }^{\text {hypo }}$, GluRIIB $^{\text {null }}$ larval NMJs were further investigated by antibody staining against compartment specific marker molecules. At normal Drosophila NMJ boutons, individual synapses are separated and surrounded by so called "perisynaptic" or "periactive zone" compartment (Sone et al., 2000; Sigrist et al., 2002), characterized by the presence of various molecular markers such as Fasciclin II (FasII) and Disc Large (Dlg). FasII is one NCAM-related cell adhesion molecule involved in synaptic growth, stabilization and structural plasticity (Shuster et al., 1996). DLG is the Drosophila PSD95 homologue, it belongs to the membrane-associated guanylate kinase homologs (MAGUKs) family and is generally involved in synaptic clustering of various molecules such as potassium channel Shaker and FasII through the PDZ domains mediated interactions (Tejedor et al., 1997; Thomas et al., 1997; Zito et al., 1997). As reported previously, in wild type controls both FasII and DLG label the boutons in a characterized network pattern, which is clearly complementary with dPAK staining (Fig.3-12 A, D). Since normally presynaptic active zone is always precisely aligned with DPAK as evidenced by NC82 and DPAK double staining (see Fig.3-9), the perisynaptic components such as FasII and DLG clearly decorate the synaptic region.

At GluRIIA ${ }^{\text {hypo }}$, GluRIIB $^{\text {null }}$ boutons, both FasII and DLG staining appear reduced and lose the typical network pattern, in contrast, they are distributed almost evenly throughout boutons (Fig.3-12 B, E). Notably, this homogeneous distribution pattern of Fas II and DLG indicates the intrusion of perisynaptic 

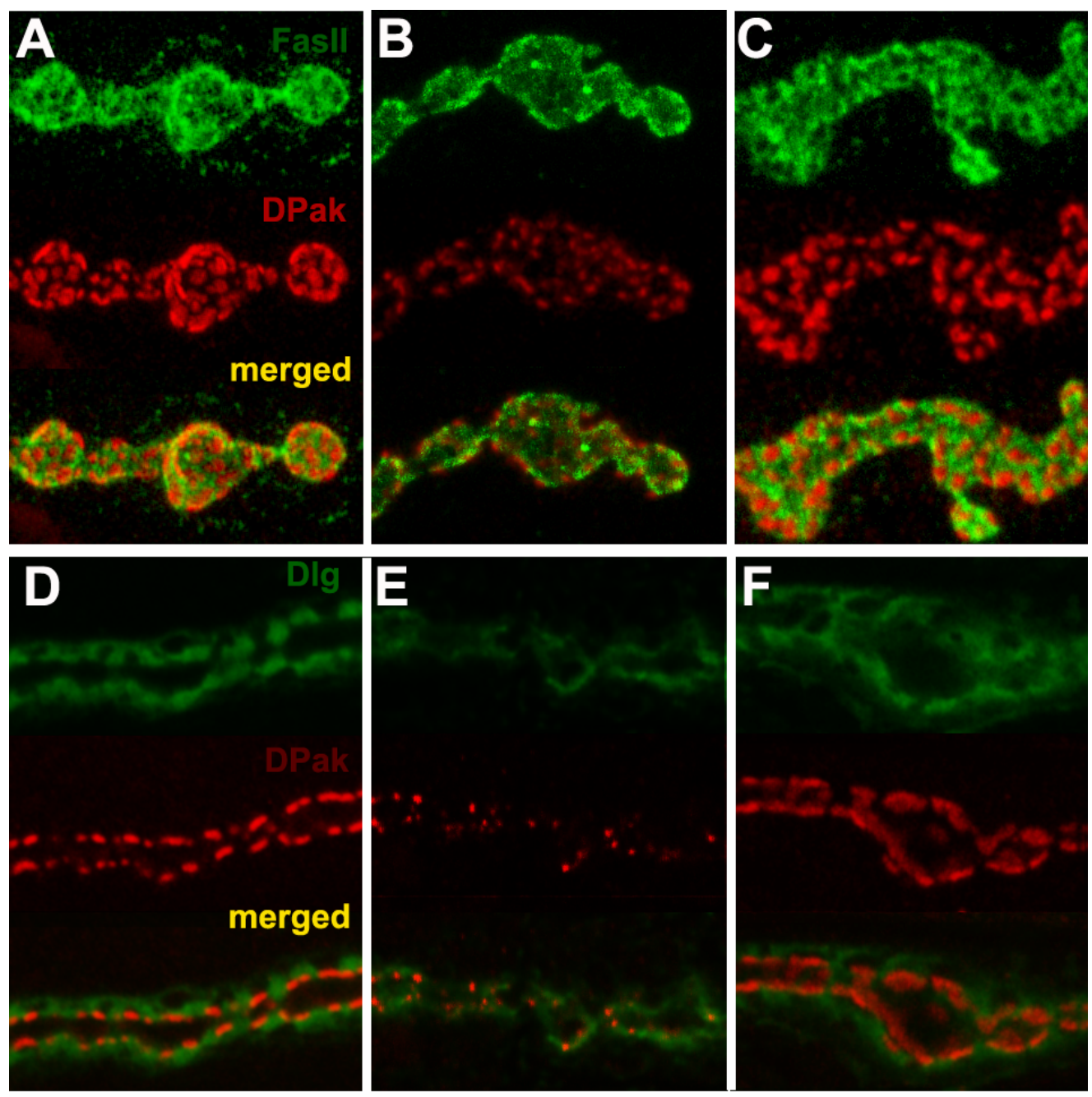

Wild type

GluR-IIAhypo, GluR-IIBnull

OK319-Gal4->UAS-TNT

Fig.3-12 Defective synaptic compartments at glutamate receptor deprived but not neurotransmission deprived synapses

Confocal microscopy on NMJs of mid 3rd instar larvae at muscle 4, stained with compartment specific antibodies in wild type (A, D), GluRIIA ${ }^{\text {hypo }}$, GluRIIB $^{-/}$larvae (B, E) and animals with presynaptic expression of Tetanus-toxin light chain (OK319-Gal4->UAS-TNT, Sweeney, et al., 1995) (C, F).

A-C, NMJs double labeled with FASII together with DPAK. In wild type animals, FasII shows typical perisynaptic distribution surrounding individual PSDs which are marked by DPAK expression (A). In GluRIIA ${ }^{\text {hypo }}$, GluRIIB ${ }^{-/-}$larvae, FasII show rather even distribution over the whole bouton (B), DPAK spots display significantly lower density and more variable individual size (B). In OK319-Gal4->UAS-TNT larvae, both FasII and DPAK show normal distribution (C).

D-F, single cross section image of larval NMJs double labeled with DLG and DPAK. In wild type animals, DLG shows typical perisynaptic distribution surrounding individual PSDs which are marked by DPAK expression (D). In GluRIIA ${ }^{\text {hypo }}$, GluRIIB $^{-1-}$ larvae, DLG show rather even distribution over the whole bouton surface thus invade the synaptic area (E), DPAK spots display significantly lower density and more variable individual size and overlay with DLG labels (E). In OK319-Gal4->UAS-TNT larvae, both DLG and DPAK show normal distribution $(\mathrm{F})$. 
components into the synaptic/ active zone region, thus destroying the compartmentation of synaptic and perisynaptic area in boutons. Concerning the still rather normal localization of active zones within these boutons, clearly the major driving force to prevent perisynaptic components entering synaptic area comes from the efficiently assembled PSDs, glutamate receptors play either direct or indirect roles in this process.

\subsubsection{Similar defects in synaptic differentiation in the complete absence of glutamate receptors}

The above studies using glutamate receptor largely deprived larvae strongly support the structural role of glutamate receptor in organizing synaptic differentiation. To further confirm this finding, analysis on synapses with complete loss of all glutamate receptors is needed. To this end, the GluR-IIA\&IIB double null mutant background was used. At late stage embryos of this mutant, none of any known muscle glutamate receptor subunits can be detected at NMJs (Fig.3-5 B; Fig.3-10). In consistent with the result obtained

from GluRIIA ${ }^{\text {hypo }}$, GluRIIB $^{\text {null }}$ larvae, although the active zone localize normally at the presynaptic terminal, much fewer amount of assemble dPAK aggregates are present at junctions compared to control conditions (Fig.3-10 E). 


\section{Discussion}

\subsection{Genomic tools could speed up identifying essential genes involved in synapse formation and modulation}

Drosophila has long been an excellent model organism due to the availability of well established, classical genetic tools. With the recent release of the whole Drosophila genome sequence information and development of systematic functional genomic research tools, reverse genetics has further strengthened the power of this model organism. Recently genomic scale microarray screening has been stably applied to a wide range of studies to obtain molecular insights or identify novel relevant components of complicated regulatory processes (Furlong et al., 2001; Stathopoulos and Levine, 2002; Butler et al., 2003; Li and White, 2003).

Here, to screen genes relevant to synapse formation and growth control at whole genome scale, the microarray technique was applied to the Drosophila model synapse system, the NMJ. Thus, the Drosophila body wall filets which mainly consist of somatic muscles were sampled and the RNAs were extracted. Tissue specific RNA profiling was performed and pools of genes (about 700) with specific or enriched expression in postsynaptic muscles were obtained. These genes represent wide variety of functional groups, including enzymes, signal transduction components, receptors and ion channels, cell adhesion molecules, transcriptional factors, RNA-binding proteins, structural proteins and so on. Among them, some are previously already shown to be specifically or abundantly expressed within muscle cells, indicating that our strategy is absolutely feasible and the gene chip methodology is highly reliable. Notably, a large body of genes within the pools are not characterized yet, thus the challenge will be to study their functional significance in high-throughput way. To this end, the application of RNA interference (RNAi) will be the best choice since recently Drosophila whole genomic RNAi has been set up by various labs and proved to be very efficient assay (Kiger et al., 2003; Kim et al., 2004). 
Undoubtedly, the microarray oriented reverse genetics platform can well complement the classical genetic screening based strategy and their application into neuronal system will greatly enhance our precise understanding of molecular details related to synapse construction and modulation. The main part of this thesis work is studying the molecular behaviors of glutamate receptor subunits, which is originated from the microarray screening and thus provide direct proof of its value.

\subsection{GluR-IID and GluR-IIE are novel glutamate receptor subunits crucial for glutamate receptor assembly and thus neurotransmission at neuromuscular synapse}

The glutamatergic neuromuscular synapses of Drosophila, rather similar to the excitatory CNS synapses of vertebrate brain in terms of ultrastructure and molecular composition, have been intensely utilized to investigate synaptic function in vivo capitalizing on the efficient genetics and superb experimental accessibility of this model system. At neuromuscular synapses three non-NMDA type glutamate receptor subunits (GluR-IIA, -IIB, -IIC/III) have been described previously. While only GluR-IIA\&IIB double null mutants but not either single null mutants completely lack neurotransmission (Petersen et al., 1997; DiAntonio et al., 1999), lack of GluRIIC/ III alone is fatal with a phenotype consistent with the absence of neurotransmission at neuromuscular synapses (Marrus et al., 2004). The full picture of subunit composition of synaptic glutamate receptors at the NMJ still remains elusive.

Here via the genomic scale microarray screening, I report the identification of two additional non-NMDA glutamate receptor subunits, GluR-IID and GluR-IIE, which are proved to be specifically localized at the postsynaptic site of all neuromuscular synapses. Interestingly, while GluR-IID and GluR-IIE are genomic neighbors and very similar to each other, they are not particularly close to GluR-IIA, -IIB and -IIC/III. Specific null mutants for both GluR-IID and GluR-IIE have been obtained to further functional characterization. While both 
GluR-IID and GluR-IIE null embryos still fully develop, they do not hatch and are incapable of performing any coordinated movement. Consistent with my finding, via embryonic electrophysiological recording, Featherstone et al. have recently demonstrated that no postsynaptic sensitivity to glutamate was detected at the embryonic muscles of one independent GluR-IID null mutant allele (personal communication). Thus, GluR-IID and GluR-IIE appear critically important for mediating glutamate-gated ionic currents at the postsynaptic site of neuromuscular synapses. Since in the case of GluR-IIA\&IIB double as well as the GluR-IIC/III single mutant background the same paralysis phenotype was found, it is therefore most likely, that they also lack any glutamate gated ionic current.

Taken together, lack of either GluR-IIC/III, -IID, -IIE or both -IIA and -IIB leads to total loss of glutamate receptor function at the embryonic NMJ. The following evidences support that this is directly due to the loss of the respective glutamate receptor subunits hence all the functional glutamate receptors within the embryonic muscles. Firstly, despite the fact that GluR-IID is also present in cardio-precursor cells, the expression of GluR-IIA, -IIB, -IIC/III and -IIE is most likely restricted to the somatic muscles (Petersen et al., 1997; DiAntonio et al., 1999; Fig.3-1). Secondly, the paralysis phenotype of all these null mutants could always be fully rescued by a muscle-specific re-expression of the corresponding cDNAs (DiAntonio et al., 1999; Marrus et al., 2004; 3.4). Thirdly, muscle-specific down-regulation of GluR-IIE via RNA interference could effectively down-regulate synaptic expression of GluR-IID, -IIA, -IIB and -IIC/III as well. Last, in each of these null mutants, all of the other subunits are also completely absent at NMJ. Thus clearly, lack of any essential glutamate receptor subunits within the postsynaptic muscle cells will result into a complete loss of all glutamate receptors, which is the direct cause of embryonic paralysis. 


\subsection{Implications in the in vivo stoichiometry of Drosophila glutamate receptor based on genetic analysis}

Several recent studies have suggested that the mammalian ionotropic glutamate receptors form as a tetramer of subunits (Kuusinen A et al., 1999; Rosenmund C et al., 1998; Laube B et al., 1998; Mano I et al., 1998; Safferling $\mathrm{M}$ et al., 2001). At the Drosophila Neuromuscular synapses, the synaptic expression of all glutamate receptor subunits is completely abolished after eliminating either GluR-IID or IIE alone or GluR-IIA and IIB together (Fig. 3-5), clearly indicating that essential glutamate receptor subunits (GluR-IIC/III, -IID and -IIE) are obligatory part of all postsynaptic glutamate receptors. In addition, the presence of either GluRIIA or GluR-IIB seems obligate for receptor formation as well. One likely scenario therefore is that the glutamate receptor population at the Drosophila NMJ is a mix of (IIA)(IIC/III)(IID)(IIE) receptors with (IIB)(IIC/III)(IID)(IIE) receptors. However, since the current tetrameric structure model of ionotropic glutamate receptors was suggested solely on the basis of either electrophysiological and biochemical studies in heterologous system or crystal structure analysis of ligand-binding domains, an unequivocal determination of the stoichiometry of functional glutamate receptor thus still awaits efficient method that reliably resolute the structure of the endogeneous receptor itself. In theory, as was proposed in the past (Premkumar et al., 1997), pentameric (or higher) stoichiometry still cannot be totally excluded in the moment. Therefore, stoichiometry as (A)2(C)(D)(E), (B)2(C)(D)(E) or $(A)(B)(C)(D)(E)$ still need to be considered.

Previous studies have established that GluR-IIA- and GluR-IIB-containing receptors differ strongly in their biophysical properties, with GluR-IIA-containing receptors showing slow desensitization kinetics and GluR-IIB-containing receptors showing fast desensitization kinetics (DiAntonio et al., 1999). Thus, GluR-IIA and GluR-IIB containing complexes are likely to play different roles in synaptic function and development. Acordingly, it has 
been recently found that, increased postsynaptic GluR-IIA level or reduced GluR-IIB gene dosage results into an increased strength of NMJ transmission and an addition of boutons harboring increased numbers of synapses; this phenotypes were suppressed by overexpression of GluR-IIB (Sigrist et al., 2002). Thus, GluR-IIC/III, IID and IIE might establish a "receptor assembly platform" where the incorporation of either the GluR-IIA or the GluR-IIB subunit could determine the specific functions of the respective glutamate receptor.

As discussed above, the interdependency between all essential subunits observed for synaptic localization is easiest explained by assuming the existence of common glutamate receptor. Another possibility might be that eliminating a certain glutamate receptor subunit could provoke an early defect during the developmental set up of neuromuscular synapses, which in turn might interfere with the localization of other glutamate receptor complexes. This way, the genetic elimination of a certain subunit could also cause the loss of synaptic expression (and thus also synaptic function) of another subunit even if these two subunits would not be coassembled in the same glutamate receptor complex. At this juncture, such a scenario appears unlikely because of the following reasons. First, partial suppression of any essential glutamate receptor subunit always provokes a corresponding down-regulation of other essential glutamate receptor subunits at larval neuromuscular synapses (Fig.3-6 and3-7; Marrus et al., 2004). This suggests that all essential subunits obey a tight stoichiometric relationship, where the availability of the least abundant subunit defines the overall amount of glutamate receptors, and that this relation exists throughout development. Secondly, Featherstone et al. have convincingly demonstrated a complete loss of glutamate mediated currents on embryonic muscle surfaces of one independent GluR-IID null mutant allele (Featherstone D et al., personal communication). In contrast, extrasynaptic glutamate receptor mediated currents have been stably detected on wild type embryonic muscle membranes using the same experimental settings (Broadie and Bate, 1993; Featherstone et al., personal 
communication). Thus, elimination of GluR-IID abolishes not only synaptic but also extrasynaptic populations of glutamate receptors on embryonic muscles. Therefore, if interaction between independently forming glutamate receptors is involved in their proper targeting, it would have to be absolutely essential already during the intracellular transport and insertion of glutamate receptors. Since all known muscle receptor subunits from GluR-IIA to GluR-IID (GluR-IIE also quite likely the case as evidenced by EGFP fusion protein distribution) are clearly expressed in substantial amounts within postsynaptic densities, the existence of subunits evolved only to mediate the intracellular transport or assembly of glutamate receptor complexes appears rather unlikely unless it was omitted from the Gene chip analysis.

Collectively, the in vivo data presented here clearly favor the notion that, at Drosophila neuromuscular synapses the functional glutamate receptors can be composed of four different subunits. To our knowledge, a "strictly hetero-tetrameric stoichiometry" has so far not been described for other types of ionotropic glutamate receptors in all model synapses. This finding thus potentially reveal that, apart from our current understanding on glutamate receptor behavior mainly based on heterologous studies, the in vivo glutamate receptors might have a rather fixed stochiometry, which is reminiscent of that of other types of ligand-gated ion channels such as the nicotinic acetylcholine receptor (Colquhoun and Sivilotti, 2004).

\subsection{Structural role of ionotropic glutamate receptor per se in organizing synaptic assembly}

In the brain, huge amount of neurons are precisely connected via synapses, which display respectively characteristic structural properties at both pre and postsynaptic sides. Most excitatory synapses are glutamatergic, and their initial formation, stabilization, and elimination are under strict control all the time. Moreover, the local changes in synapse strength are proposed to be relevant to 
the process of learning and memory. Apparently, it is of great importance to understand how the original synaptic contact is initiated at the proper site and how exquisite coordination of presynaptic and postsynaptic development is accomplished. Although up to now great efforts have been devoted in these directions with more and more synaptic components identified and characterized, still the molecular details driving synaptic assembly remain elusive.

At central excitatory synapses, synaptic transmission is mediated by various ionotropic glutamate receptors including AMPA, kainite and NMDA subfamilies. Recent work has revealed that certain classes of AMPA receptors recycle rapidly at the synaptic sites, suggesting that placeholders or 'slots' might be brought to, or assembled at the postsynaptic membrane with de novo insertion of AMPA receptors. The molecular identity of the slots is unknown, but is likely to involve one or more receptor-binding or scaffolding proteins (Barry and Ziff, 2002).

Taking the advantages of the powerful Drosophila genetic tools and the rather simpler architecture of $\mathrm{NMJ}$, the consequences of losing synaptic glutamate receptor partially or completely in vivo were investigated. Despite the rather normal differentiation of transmitter release machinery at the presynaptic membrane, which is consistent with previous findings that the presynaptic differentiation can undergo at the nerve terminal even without contact with the postsynaptic cell (Prokop et al., 1996), the postsynaptic assembly is apparently deficient, as evidenced by not only inefficient clustering of other PSD component ( $\mathrm{dPAK})$ but also the intrusion of perisynaptic components (FASII and DLG) into synaptic (active zone) territory. PAK is known to regulate cytoskeleton dynamics, the cytoskeleton organization is crucial for the clustering of synaptic components and /or maintaining the synaptic structure at diverse types of synapses (Kirsch and Betz, 1995; Allison et al., 1998; Dai et al., 2000; Hirai, 2000). Recently it has been proposed that dPAK is important in 
clustering glutamate receptors at Drosophila NMJ since in dpak hypomorphic mutant animals, glutamate receptor subunit GluR-IIA is significantly reduced but still localize normally at synapses (Parnas et al., 2001). Interestingly, the data presented here indicate that proper aggregation and synaptic distribution of dPAK is definitely dependant on the availability of glutamate receptors; with glutamate receptors depleted the $\mathrm{APAK}$ is no longer able to cluster and localize properly. At mammalian neuromuscular synapse it has been shown that agrin-mediated clustering of AchR is mediated by the corporation of various signaling pathways: first the activation of Rac/ $\mathrm{Cdc} 42$, leading to formation of AchR microclusters, followed by Rho activation, resulting into combining these microclusters into larger clusters (Weston C et al., 2003). DPAK does not necessarily need to interact directly with glutamate receptors, but via various direct and indirect interactions glutamate receptors might be able to recruit various PSD components (including dPAK, other signaling molecules and scaffold proteins) during synaptogenesis, as soon as these components are recruited together, the relevant signaling cascades are efficiently activated and thus trigger the membrane and cytoskeleton remodeling which finally results into the stable development of mature synaptic structure; in depletion of glutamate receptors, either the dPAK signaling cascade is not efficiently activated to reorganize actin cytoskeleton essential for proper clustering, or activating dPAK cascade along is not sufficient for effective PSD components clustering and maintaining. Currently, it remains elusive whether other signaling pathways are involved in PSD formation, but based on the clear defects in synaptic compartmentation that perisynaptic components intrude into synaptic region, the cytoskeleton organization must have been improperly regulated. This notion is also supported from ultrastructural analysis, the electron dense material typical present at the PSD region is essentially absent from glutamate receptor deprived synapses, implicating that the components of postsynaptic scaffold are either not recruited together at all or no longer stable without the integration of glutamate receptors. Interestingly, at the neuromuscular synapses of dapk hypomorphic mutant, despite much fewer SSR, the ultrastructure of 
both PSD and active zone appears quite normal, further supporting the relatively minor role of $\mathrm{dPAK}$ in postsynaptic differentiation. Notably, the role of glutamate receptor in synaptic development was shown not to be relevant to its ion channel activity, since in all the backgrounds tested which deprive presynaptic neurotransmitter release (partially or completely), the glutamate receptor can be clustered as effectively as wild type control. Correspondingly, the postsynaptic reception apparatus in these transmission depleted conditions appear functional since responses can be stably detected when applying glutamate at junctions (Broadie, 1996).

Another interesting phenomena at glutamate receptor deprived synapse is that the number of presynaptic specializations exceeds postsynaptic ones, thus resulting into free active zones without postsynaptic partners, which is hardly found in normal conditions. In principle, this can be due to either lack of glutamate receptor per se, or that of the transmission activity. The latter idea appears more likely since in complete transmitter release deficient situations, free active zones were also observed although certain levels of PSDs (glutamate receptor fields) were assembled normally.

In conclusion, apart from functioning as ligand-gated ion channel, the glutamate receptor at Drosophila NMJ also plays crucial role in organizing and /or maintaining the synaptic architecture, which is reminiscent of AchR at vertebrate NMJ (Ono F et al., 2001; Marangi PA et al., 2001; Missias AC et al., 1997) and glycin and GABA receptor at mammalian central synapses (Essrich et al., 1998). In mammalian CNS synapses similar roles of ionotropic glutamate receptors have not been reported, mainly due to the fact that there exist highly divergent classes of glutamate receptors at central synapses and each type of receptors play distinct roles, therefore it is not easy to obtain a condition with all types of glutamate receptors removed together. 


\section{Reference}

Aberle H, Haghighi AP, Fetter RD, McCabe BD, Magalhaes TR, Goodman CS (2002) wishful thinking encodes a BMP type II receptor that regulates synaptic growth in Drosophila. Neuron 33:545-558.

Ahmari SE, Buchanan J, Smith SJ (2000) Assembly of presynaptic active zones from cytoplasmic transport packets. Nat Neurosci 3:445-451.

Albin SD, Davis GW (2004) Coordinating structural and functional synapse development: postsynaptic p21-activated kinase independently specifies glutamate receptor abundance and postsynaptic morphology. J Neurosci 24:6871-6879.

Allison DW, Gelfand VI, Spector I, Craig AM (1998) Role of actin in anchoring postsynaptic receptors in cultured hippocampal neurons: differential attachment of NMDA versus AMPA receptors. $J$ Neurosci 18:2423-2436.

Apel ED, Glass DJ, Moscoso LM, Yancopoulos GD, Sanes JR (1997) Rapsyn is required for MuSK signaling and recruits synaptic components to a MuSK-containing scaffold. Neuron 18:623-635.

Aplin AE, Howe A, Alahari SK, Juliano RL (1998) Signal transduction and signal modulation by cell adhesion receptors: the role of integrins, cadherins, immunoglobulin-cell adhesion molecules, and selectins. Pharmacol Rev 50:197-263.

Atwood HL, Govind CK, Wu CF (1993) Differential ultrastructure of synaptic terminals on ventral longitudinal abdominal muscles in Drosophila larvae. J Neurobiol 24:1008-1024.

Auld VJ, Fetter RD, Broadie K, Goodman CS (1995) Gliotactin, a novel transmembrane protein on peripheral glia, is required to form the blood-nerve barrier in Drosophila. Cell 81:757-767.

Ayalon G, Stern-Bach Y (2001) Functional assembly of AMPA and kainate receptors is mediated by several discrete protein-protein interactions. Neuron 31:103-113.

Bagrodia S, Cerione RA (1999) Pak to the future. Trends Cell Biol 9:350-355.

Barry MF, Ziff EB (2002) Receptor trafficking and the plasticity of excitatory synapses. Curr Opin Neurobiol 12:279-286.

Bate M, Broadie K (1995) Wiring by fly: the neuromuscular system of the Drosophila embryo. Neuron 15:513-525.

Benson DL, Colman DR, Huntley GW (2001) Molecules, maps and synapse specificity. Nat Rev Neurosci 2:899-909.

Biederer T, Sara Y, Mozhayeva M, Atasoy D, Liu X, Kavalali ET, Sudhof TC (2002) SynCAM, a synaptic adhesion molecule that drives synapse assembly. Science 297:1525-1531.

Bloch RJ, Pumplin DW (1988) Molecular events in synaptogenesis: nerve-muscle adhesion and postsynaptic differentiation. Am J Physiol 254:C345-364.

Boutanaev AM, Kalmykova AI, Shevelyov YY, Nurminsky DI (2002) Large clusters of co-expressed genes in the Drosophila genome. Nature 420:666-669.

Bowe MA, Fallon JR (1995) The role of agrin in synapse formation. Annu Rev Neurosci 18:443-462.

Brand AH, Perrimon N (1993) Targeted gene expression as a means of altering cell fates and generating dominant phenotypes. Development 118:401-415.

Bresler T, Ramati Y, Zamorano PL, Zhai R, Garner CC, Ziv NE (2001) The dynamics of SAP90/PSD-95 recruitment to new synaptic junctions. Mol Cell Neurosci 18:149-167.

Bresler T, Shapira M, Boeckers T, Dresbach T, Futter M, Garner CC, Rosenblum K, Gundelfinger ED, Ziv NE (2004) Postsynaptic density assembly is fundamentally different from presynaptic active zone assembly. J Neurosci 24:1507-1520.

Broadie K, Bate M (1993) Activity-dependent development of the neuromuscular synapse during Drosophila embryogenesis. Neuron 11:607-619.

Broadie K, Bate M (1993) Innervation directs receptor synthesis and localization in Drosophila embryo synaptogenesis. Nature 361:350-353.

Broadie K, Prokop A, Bellen HJ, O'Kane CJ, Schulze KL, Sweeney ST (1995) Syntaxin and synaptobrevin function downstream of vesicle docking in Drosophila. Neuron 15:663-673.

Broadie KS (1996) Regulation of the synaptic vesicle cycle in Drosophila. Biochem Soc Trans 24:639-645.

Budnik V, Koh YH, Guan B, Hartmann B, Hough C, Woods D, Gorczyca M (1996) Regulation of 
synapse structure and function by the Drosophila tumor suppressor gene dlg. Neuron 17:627-640.

Burden SJ (1998) The formation of neuromuscular synapses. Genes Dev 12:133-148.

Burnashev N, Khodorova A, Jonas P, Helm PJ, Wisden W, Monyer H, Seeburg PH, Sakmann B (1992) Calcium-permeable AMPA-kainate receptors in fusiform cerebellar glial cells. Science 256:1566-1570.

Butler MJ, Jacobsen TL, Cain DM, Jarman MG, Hubank M, Whittle JR, Phillips R, Simcox A (2003) Discovery of genes with highly restricted expression patterns in the Drosophila wing disc using DNA oligonucleotide microarrays. Development 130:659-670.

Chavis P, Westbrook G (2001) Integrins mediate functional pre- and postsynaptic maturation at a hippocampal synapse. Nature 411:317-321.

Chen GQ, Cui C, Mayer ML, Gouaux E (1999) Functional characterization of a potassium-selective prokaryotic glutamate receptor. Nature 402:817-821.

Chen L, Chetkovich DM, Petralia RS, Sweeney NT, Kawasaki Y, Wenthold RJ, Bredt DS, Nicoll RA (2000) Stargazin regulates synaptic targeting of AMPA receptors by two distinct mechanisms. Nature 408:936-943.

Chiba A, Rose D (1998) "Painting" the target: how local molecular cues define synaptic relationships. Bioessays 20:941-948.

Colquhoun D, Sivilotti LG (2004) Function and structure in glycine receptors and some of their relatives. Trends Neurosci 27:337-344.

Contractor A, Heinemann SF (2002) Glutamate receptor trafficking in synaptic plasticity. Sci STKE 2002:RE14.

Cooper RL, Marin L, Atwood HL (1995) Synaptic differentiation of a single motor neuron: conjoint definition of transmitter release, presynaptic calcium signals, and ultrastructure. J Neurosci 15:4209-4222.

Cooper RL, Harrington CC, Marin L, Atwood HL (1996) Quantal release at visualized terminals of a crayfish motor axon: intraterminal and regional differences. J Comp Neurol 375:583-600.

Currie DA, Truman JW, Burden SJ (1995) Drosophila glutamate receptor RNA expression in embryonic and larval muscle fibers. Dev Dyn 203:311-316.

Dai Z, Luo X, Xie H, Peng HB (2000) The actin-driven movement and formation of acetylcholine receptor clusters. J Cell Biol 150:1321-1334.

Dalva MB, Takasu MA, Lin MZ, Shamah SM, Hu L, Gale NW, Greenberg ME (2000) EphB receptors interact with NMDA receptors and regulate excitatory synapse formation. Cell 103:945-956.

Daniels RW, Collins CA, Gelfand MV, Dant J, Brooks ES, Krantz DE, Diantonio A (2004) Increased Expression of the Drosophila Vesicular Glutamate Transporter Leads to Excess Glutamate Release and a Compensatory Decrease in Quantal Content. J Neurosci 24:10466-10474.

Davis GW, Schuster CM, Goodman CS (1997) Genetic analysis of the mechanisms controlling target selection: target-derived Fasciclin II regulates the pattern of synapse formation. Neuron 19:561-573.

Dean C, Scholl FG, Choih J, DeMaria S, Berger J, Isacoff E, Scheiffele P (2003) Neurexin mediates the assembly of presynaptic terminals. Nat Neurosci 6:708-716.

DeChiara TM, Bowen DC, Valenzuela DM, Simmons MV, Poueymirou WT, Thomas S, Kinetz E, Compton DL, Rojas E, Park JS, Smith C, DiStefano PS, Glass DJ, Burden SJ, Yancopoulos GD (1996) The receptor tyrosine kinase MuSK is required for neuromuscular junction formation in vivo. Cell 85:501-512.

DiAntonio A, Petersen SA, Heckmann M, Goodman CS (1999) Glutamate receptor expression regulates quantal size and quantal content at the Drosophila neuromuscular junction. J Neurosci 19:3023-3032.

Dingledine R, Borges K, Bowie D, Traynelis SF (1999) The glutamate receptor ion channels. Pharmacol Rev 51:7-61.

Dingledine R, Borges K, Bowie D, Traynelis SF (1999) The glutamate receptor ion channels. Pharmacol Rev 51:7-61.

Ellgaard L, Helenius A (2003) Quality control in the endoplasmic reticulum. Nat Rev Mol Cell Biol 4:181-191.

Essrich C, Lorez M, Benson JA, Fritschy JM, Luscher B (1998) Postsynaptic clustering of major GABAA receptor subtypes requires the gamma 2 subunit and gephyrin. Nat Neurosci 1:563-571.

Ethell IM, Yamaguchi Y (1999) Cell surface heparan sulfate proteoglycan syndecan-2 induces the maturation of dendritic spines in rat hippocampal neurons. J Cell Biol 144:575-586.

Ethell IM, Irie F, Kalo MS, Couchman JR, Pasquale EB, Yamaguchi Y (2001) EphB/syndecan-2 
signaling in dendritic spine morphogenesis. Neuron 31:1001-1013.

Featherstone DE, Broadie K (2000) Surprises from Drosophila: genetic mechanisms of synaptic development and plasticity. Brain Res Bull 53:501-511.

Fernandez-Chacon R, Sudhof TC (1999) Genetics of synaptic vesicle function: toward the complete functional anatomy of an organelle. Annu Rev Physiol 61:753-776.

Fiala JC, Feinberg M, Popov V, Harris KM (1998) Synaptogenesis via dendritic filopodia in developing hippocampal area CA1. J Neurosci 18:8900-8911.

Finn AJ, Feng G, Pendergast AM (2003) Postsynaptic requirement for Abl kinases in assembly of the neuromuscular junction. Nat Neurosci 6:717-723.

Friedman HV, Bresler T, Garner CC, Ziv NE (2000) Assembly of new individual excitatory synapses: time course and temporal order of synaptic molecule recruitment. Neuron 27:57-69.

Froehner SC, Luetje CW, Scotland PB, Patrick J (1990) The postsynaptic 43K protein clusters muscle nicotinic acetylcholine receptors in Xenopus oocytes. Neuron 5:403-410.

Furlong EE, Andersen EC, Null B, White KP, Scott MP (2001) Patterns of gene expression during Drosophila mesoderm development. Science 293:1629-1633.

Garner CC, Zhai RG, Gundelfinger ED, Ziv NE (2002) Molecular mechanisms of CNS synaptogenesis. Trends Neurosci 25:243-251.

Gautam M, Noakes PG, Mudd J, Nichol M, Chu GC, Sanes JR, Merlie JP (1995) Failure of postsynaptic specialization to develop at neuromuscular junctions of rapsyn-deficient mice. Nature 377:232-236.

Gautam M, Noakes PG, Moscoso L, Rupp F, Scheller RH, Merlie JP, Sanes JR (1996) Defective neuromuscular synaptogenesis in agrin-deficient mutant mice. Cell 85:525-535.

Gilbert W (1978) Why genes in pieces? Nature 271:501.

Gillespie SK, Wasserman SA (1994) Dorsal, a Drosophila Rel-like protein, is phosphorylated upon activation of the transmembrane protein Toll. Mol Cell Biol 14:3559-3568.

Glass DJ, Bowen DC, Stitt TN, Radziejewski C, Bruno J, Ryan TE, Gies DR, Shah S, Mattsson K, Burden SJ, DiStefano PS, Valenzuela DM, DeChiara TM, Yancopoulos GD (1996) Agrin acts via a MuSK receptor complex. Cell 85:513-523.

Gonzalez-Gaitan M, Jackle H (1997) Role of Drosophila alpha-adaptin in presynaptic vesicle recycling. Cell 88:767-776.

Gramates LS, Budnik V (1999) Assembly and maturation of the Drosophila larval neuromuscular junction. Int Rev Neurobiol 43:93-117.

Greger IH, Khatri L, Ziff EB (2002) RNA editing at arg607 controls AMPA receptor exit from the endoplasmic reticulum. Neuron 34:759-772.

Grunwald IC, Korte M, Wolfer D, Wilkinson GA, Unsicker K, Lipp HP, Bonhoeffer T, Klein R (2001) Kinase-independent requirement of EphB2 receptors in hippocampal synaptic plasticity. Neuron 32:1027-1040.

Guan B, Hartmann B, Kho YH, Gorczyca M, Budnik V (1996) The Drosophila tumor suppressor gene, $\mathrm{dlg}$, is involved in structural plasticity at a glutamatergic synapse. Curr Biol 6:695-706.

Hall AC, Lucas FR, Salinas PC (2000) Axonal remodeling and synaptic differentiation in the cerebellum is regulated by WNT-7a signaling. Cell 100:525-535.

Hall ZW, Sanes JR (1993) Synaptic structure and development: the neuromuscular junction. Cell 72 Suppl:99-121.

Han H, Noakes PG, Phillips WD (1999) Overexpression of rapsyn inhibits agrin-induced acetylcholine receptor clustering in muscle cells. J Neurocytol 28:763-775.

Hannah MJ, Schmidt AA, Huttner WB (1999) Synaptic vesicle biogenesis. Annu Rev Cell Dev Biol 15:733-798

Harris KM, Kater SB (1994) Dendritic spines: cellular specializations imparting both stability and flexibility to synaptic function. Annu Rev Neurosci 17:341-371.

Hawkins LM, Prybylowski K, Chang K, Moussan C, Stephenson FA, Wenthold RJ (2004) Export from the endoplasmic reticulum of assembled N-methyl-d-aspartic acid receptors is controlled by a motif in the $\mathrm{c}$ terminus of the NR2 subunit. J Biol Chem 279:28903-28910.

Heimbeck G, Bugnon V, Gendre N, Haberlin C, Stocker RF (1999) Smell and taste perception in Drosophila melanogaster larva: toxin expression studies in chemosensory neurons. J Neurosci 19:6599-6609.

Henderson JT, Georgiou J, Jia Z, Robertson J, Elowe S, Roder JC, Pawson T (2001) The receptor tyrosine kinase EphB2 regulates NMDA-dependent synaptic function. Neuron 32:1041-1056.

Herbst R, Burden SJ (2000) The juxtamembrane region of MuSK has a critical role in agrin-mediated signaling. Embo J 19:67-77.

Hering H, Sheng M (2001) Dendritic spines: structure, dynamics and regulation. Nat Rev Neurosci 
2:880-888.

Hirai H (2000) Clustering of delta glutamate receptors is regulated by the actin cytoskeleton in the dendritic spines of cultured rat Purkinje cells. Eur J Neurosci 12:563-570.

Hoang B, Chiba A (1998) Genetic analysis on the role of integrin during axon guidance in Drosophila. J Neurosci 18:7847-7855.

Hollmann M, Heinemann S (1994) Cloned glutamate receptors. Annu Rev Neurosci 17:31-108.

Isaac JT, Crair MC, Nicoll RA, Malenka RC (1997) Silent synapses during development of thalamocortical inputs. Neuron 18:269-280.

Jahn R, Sudhof TC (1999) Membrane fusion and exocytosis. Annu Rev Biochem 68:863-911.

Jaskolski F, Coussen F, Nagarajan N, Normand E, Rosenmund C, Mulle C (2004) Subunit composition and alternative splicing regulate membrane delivery of kainate receptors. J Neurosci 24:2506-2515.

Jennings CG, Dyer SM, Burden SJ (1993) Muscle-specific trk-related receptor with a kringle domain defines a distinct class of receptor tyrosine kinases. Proc Natl Acad Sci U S A 90:2895-2899.

Jia XX, Gorczyca M, Budnik V (1993) Ultrastructure of neuromuscular junctions in Drosophila: comparison of wild type and mutants with increased excitability. J Neurobiol 24:1025-1044.

Kennerdell JR, Carthew RW (2000) Heritable gene silencing in Drosophila using double-stranded RNA. Nat Biotechnol 18:896-898.

Keshishian H, Broadie K, Chiba A, Bate M (1996) The Drosophila neuromuscular junction: a model system for studying synaptic development and function. Annu Rev Neurosci 19:545-575.

Kiger AA, Baum B, Jones S, Jones MR, Coulson A, Echeverri C, Perrimon N (2003) A functional genomic analysis of cell morphology using RNA interference. J Biol 2:27.

Kim YO, Park SJ, Balaban RS, Nirenberg M, Kim Y (2004) A functional genomic screen for cardiogenic genes using RNA interference in developing Drosophila embryos. Proc Natl Acad Sci U S A 101:159-164.

Kirsch J, Betz H (1995) The postsynaptic localization of the glycine receptor-associated protein gephyrin is regulated by the cytoskeleton. J Neurosci 15:4148-4156.

Kneussel M, Brandstatter JH, Laube B, Stahl S, Muller U, Betz H (1999) Loss of postsynaptic GABA(A) receptor clustering in gephyrin-deficient mice. J Neurosci 19:9289-9297.

Koh YH, Gramates LS, Budnik V (2000) Drosophila larval neuromuscular junction: molecular components and mechanisms underlying synaptic plasticity. Microsc Res Tech 49:14-25.

Kuusinen A, Abele R, Madden DR, Keinanen K (1999) Oligomerization and ligand-binding properties of the ectodomain of the alpha-amino-3-hydroxy-5-methyl-4-isoxazole propionic acid receptor subunit GluRD. J Biol Chem 274:28937-28943.

LaRochelle WJ, Froehner SC (1986) Determination of the tissue distributions and relative concentrations of the postsynaptic $43-\mathrm{kDa}$ protein and the acetylcholine receptor in Torpedo. $\mathrm{J}$ Biol Chem 261:5270-5274.

Laube B, Kuhse J, Betz H (1998) Evidence for a tetrameric structure of recombinant NMDA receptors. J Neurosci 18:2954-2961.

Li TR, White KP (2003) Tissue-specific gene expression and ecdysone-regulated genomic networks in Drosophila. Dev Cell 5:59-72.

Li Z, Sheng M (2003) Some assembly required: the development of neuronal synapses. Nat Rev Mol Cell Biol 4:833-841.

Liao D, Hessler NA, Malinow R (1995) Activation of postsynaptically silent synapses during pairing-induced LTP in CA1 region of hippocampal slice. Nature 375:400-404.

Lin W, Burgess RW, Dominguez B, Pfaff SL, Sanes JR, Lee KF (2001) Distinct roles of nerve and muscle in postsynaptic differentiation of the neuromuscular synapse. Nature 410:1057-1064.

Littleton JT (2000) A genomic analysis of membrane trafficking and neurotransmitter release in Drosophila. J Cell Biol 150:F77-82.

Littleton JT, Ganetzky B (2000) Ion channels and synaptic organization: analysis of the Drosophila genome. Neuron 26:35-43.

Luo ZG, Je HS, Wang Q, Yang F, Dobbins GC, Yang ZH, Xiong WC, Lu B, Mei L (2003) Implication of geranylgeranyltransferase I in synapse formation. Neuron 40:703-717.

Luo ZG, Wang Q, Zhou JZ, Wang J, Luo Z, Liu M, He X, Wynshaw-Boris A, Xiong WC, Lu B, Mei L (2002) Regulation of AChR clustering by Dishevelled interacting with MuSK and PAK1. Neuron 35:489-505.

Madden DR (2002) The structure and function of glutamate receptor ion channels. Nat Rev Neurosci 3:91-101.

Mano I, Teichberg VI (1998) A tetrameric subunit stoichiometry for a glutamate receptor-channel complex. Neuroreport 9:327-331. 
Marangi PA, Forsayeth JR, Mittaud P, Erb-Vogtli S, Blake DJ, Moransard M, Sander A, Fuhrer C (2001) Acetylcholine receptors are required for agrin-induced clustering of postsynaptic proteins. Embo J 20:7060-7073.

Marrs GS, Green SH, Dailey ME (2001) Rapid formation and remodeling of postsynaptic densities in developing dendrites. Nat Neurosci 4:1006-1013.

Marrus SB, Portman SL, Allen MJ, Moffat KG, DiAntonio A (2004) Differential localization of glutamate receptor subunits at the Drosophila neuromuscular junction. J Neurosci 24:1406-1415.

Matsuda K, Fletcher M, Kamiya Y, Yuzaki M (2003) Specific assembly with the NMDA receptor 3B subunit controls surface expression and calcium permeability of NMDA receptors. J Neurosci 23:10064-10073.

McCabe BD, Marques G, Haghighi AP, Fetter RD, Crotty ML, Haerry TE, Goodman CS, O'Connor MB (2003) The BMP homolog Gbb provides a retrograde signal that regulates synaptic growth at the Drosophila neuromuscular junction. Neuron 39:241-254.

McGee AW, Bredt DS (2003) Assembly and plasticity of the glutamatergic postsynaptic specialization. Curr Opin Neurobiol 13:111-118.

McMahan UJ (1990) The agrin hypothesis. Cold Spring Harb Symp Quant Biol 55:407-418.

Meguro H, Mori H, Araki K, Kushiya E, Kutsuwada T, Yamazaki M, Kumanishi T, Arakawa M, Sakimura K, Mishina M (1992) Functional characterization of a heteromeric NMDA receptor channel expressed from cloned cDNAs. Nature 357:70-74

Milner R, Campbell IL (2002) The integrin family of cell adhesion molecules has multiple functions within the CNS. J Neurosci Res 69:286-291.

Missias AC, Mudd J, Cunningham JM, Steinbach JH, Merlie JP, Sanes JR (1997) Deficient development and maintenance of postsynaptic specializations in mutant mice lacking an 'adult' acetylcholine receptor subunit. Development 124:5075-5086.

Missler M, Zhang W, Rohlmann A, Kattenstroth G, Hammer RE, Gottmann K, Sudhof TC (2003) Alpha-neurexins couple Ca2+ channels to synaptic vesicle exocytosis. Nature 423:939-948.

Monyer H, Sprengel R, Schoepfer R, Herb A, Higuchi M, Lomeli H, Burnashev N, Sakmann B, Seeburg PH (1992) Heteromeric NMDA receptors: molecular and functional distinction of subtypes. Science 256:1217-1221.

Mulle C, Sailer A, Swanson GT, Brana C, O'Gorman S, Bettler B, Heinemann SF (2000) Subunit composition of kainate receptors in hippocampal interneurons. Neuron 28:475-484.

Nguyen T, Sudhof TC (1997) Binding properties of neuroligin 1 and neurexin 1beta reveal function as heterophilic cell adhesion molecules. J Biol Chem 272:26032-26039.

Noakes PG, Phillips WD, Hanley TA, Sanes JR, Merlie JP (1993) 43K protein and acetylcholine receptors colocalize during the initial stages of neuromuscular synapse formation in vivo. Dev Biol 155:275-280.

Noakes PG, Gautam M, Mudd J, Sanes JR, Merlie JP (1995) Aberrant differentiation of neuromuscular junctions in mice lacking s-laminin/laminin beta 2. Nature 374:258-262.

O'Brien R, Xu D, Mi R, Tang X, Hopf C, Worley P (2002) Synaptically targeted narp plays an essential role in the aggregation of AMPA receptors at excitatory synapses in cultured spinal neurons. $\mathrm{J}$ Neurosci 22:4487-4498.

O'Brien RJ, Xu D, Petralia RS, Steward O, Huganir RL, Worley P (1999) Synaptic clustering of AMPA receptors by the extracellular immediate-early gene product Narp. Neuron 23:309-323.

Okabe S, Miwa A, Okado H (2001) Spine formation and correlated assembly of presynaptic and postsynaptic molecules. J Neurosci 21:6105-6114.

Okabe S, Urushido T, Konno D, Okado H, Sobue K (2001) Rapid redistribution of the postsynaptic density protein PSD-Zip45 (Homer 1c) and its differential regulation by NMDA receptors and calcium channels. J Neurosci 21:9561-9571.

Ono F, Higashijima S, Shcherbatko A, Fetcho JR, Brehm P (2001) Paralytic zebrafish lacking acetylcholine receptors fail to localize rapsyn clusters to the synapse. J Neurosci 21:5439-5448.

Packard M, Koo ES, Gorczyca M, Sharpe J, Cumberledge S, Budnik V (2002) The Drosophila Wnt, wingless, provides an essential signal for pre- and postsynaptic differentiation. Cell 111:319-330.

Parnas D, Haghighi AP, Fetter RD, Kim SW, Goodman CS (2001) Regulation of postsynaptic structure and protein localization by the Rho-type guanine nucleotide exchange factor dPix. Neuron 32:415-424.

Pasterkamp RJ, Kolodkin AL (2003) Semaphorin junction: making tracks toward neural connectivity. Curr Opin Neurobiol 13:79-89.

Perez-Otano I, Schulteis CT, Contractor A, Lipton SA, Trimmer JS, Sucher NJ, Heinemann SF (2001) 
Assembly with the NR1 subunit is required for surface expression of NR3A-containing NMDA receptors. J Neurosci 21:1228-1237.

Petersen SA, Fetter RD, Noordermeer JN, Goodman CS, DiAntonio A (1997) Genetic analysis of glutamate receptors in Drosophila reveals a retrograde signal regulating presynaptic transmitter release. Neuron 19:1237-1248.

Phillips GR, Huang JK, Wang Y, Tanaka H, Shapiro L, Zhang W, Shan WS, Arndt K, Frank M, Gordon RE, Gawinowicz MA, Zhao Y, Colman DR (2001) The presynaptic particle web: ultrastructure, composition, dissolution, and reconstitution. Neuron 32:63-77.

Phillips WD, Maimone MM, Merlie JP (1991) Mutagenesis of the 43-kD postsynaptic protein defines domains involved in plasma membrane targeting and AChR clustering. J Cell Biol 115:1713-1723.

Pinkstaff JK, Detterich J, Lynch G, Gall C (1999) Integrin subunit gene expression is regionally differentiated in adult brain. J Neurosci 19:1541-1556.

Poodry CA, Edgar L (1979) Reversible alteration in the neuromuscular junctions of Drosophila melanogaster bearing a temperature-sensitive mutation, shibire. J Cell Biol 81:520-527.

Prange O, Murphy TH (2001) Modular transport of postsynaptic density-95 clusters and association with stable spine precursors during early development of cortical neurons. J Neurosci 21:9325-9333.

Prokop A (1999) Integrating bits and pieces: synapse structure and formation in Drosophila embryos. Cell Tissue Res 297:169-186.

Prokop A, Landgraf M, Rushton E, Broadie K, Bate M (1996) Presynaptic development at the Drosophila neuromuscular junction: assembly and localization of presynaptic active zones. Neuron 17:617-626.

Prokop A, Martin-Bermudo MD, Bate M, Brown N (1998) Absence of PS integrins or laminin A affects extracellular adhesion, but not intracellular assembly, of hemiadherens and neuromuscular junctions in Drosophila embryos. Dev Biol 196:58-76.

Rao A, Cha EM, Craig AM (2000) Mismatched appositions of presynaptic and postsynaptic components in isolated hippocampal neurons. J Neurosci 20:8344-8353.

Rao A, Kim E, Sheng M, Craig AM (1998) Heterogeneity in the molecular composition of excitatory postsynaptic sites during development of hippocampal neurons in culture. J Neurosci 18:1217-1229.

Ren Z, Riley NJ, Needleman LA, Sanders JM, Swanson GT, Marshall J (2003) Cell surface expression of GluR5 kainate receptors is regulated by an endoplasmic reticulum retention signal. J Biol Chem 278:52700-52709.

Rheuben MB, Yoshihara M, Kidokoro Y (1999) Ultrastructural correlates of neuromuscular junction development. Int Rev Neurobiol 43:69-92.

Ritzenthaler S, Suzuki E, Chiba A (2000) Postsynaptic filopodia in muscle cells interact with innervating motoneuron axons. Nat Neurosci 3:1012-1017.

Rosenberg M, Meier J, Triller A, Vannier C (2001) Dynamics of glycine receptor insertion in the neuronal plasma membrane. J Neurosci 21:5036-5044.

Rosenmund C, Stern-Bach Y, Stevens CF (1998) The tetrameric structure of a glutamate receptor channel. Science 280:1596-1599.

Roy PJ, Stuart JM, Lund J, Kim SK (2002) Chromosomal clustering of muscle-expressed genes in Caenorhabditis elegans. Nature 418:975-979.

Ruegg MA, Bixby JL (1998) Agrin orchestrates synaptic differentiation at the vertebrate neuromuscular junction. Trends Neurosci 21:22-27.

Safferling M, Tichelaar W, Kummerle G, Jouppila A, Kuusinen A, Keinanen K, Madden DR (2001) First images of a glutamate receptor ion channel: oligomeric state and molecular dimensions of GluRB homomers. Biochemistry 40:13948-13953.

Saitoe M, Tanaka S, Takata K, Kidokoro Y (1997) Neural activity affects distribution of glutamate receptors during neuromuscular junction formation in Drosophila embryos. Dev Biol 184:48-60.

Saitoe M, Schwarz TL, Umbach JA, Gundersen CB, Kidokoro Y (2001) Absence of junctional glutamate receptor clusters in Drosophila mutants lacking spontaneous transmitter release. Science 293:514-517.

Sanes JR, Lichtman JW (1999) Development of the vertebrate neuromuscular junction. Annu Rev Neurosci 22:389-442.

Sanes JR, Lichtman JW (2001) Induction, assembly, maturation and maintenance of a postsynaptic apparatus. Nat Rev Neurosci 2:791-805.

Scannevin RH, Huganir RL (2000) Postsynaptic organization and regulation of excitatory synapses. Nat 
Rev Neurosci 1:133-141.

Scheiffele P, Fan J, Choih J, Fetter R, Serafini T (2000) Neuroligin expressed in nonneuronal cells triggers presynaptic development in contacting axons. Cell 101:657-669.

Schulze KL, Broadie K, Perin MS, Bellen HJ (1995) Genetic and electrophysiological studies of Drosophila syntaxin-1A demonstrate its role in nonneuronal secretion and neurotransmission. Cell 80:311-320.

Schuster CM, Davis GW, Fetter RD, Goodman CS (1996) Genetic dissection of structural and functional components of synaptic plasticity. I. Fasciclin II controls synaptic stabilization and growth. Neuron 17:641-654.

Schuster CM, Ultsch A, Schloss P, Cox JA, Schmitt B, Betz H (1991) Molecular cloning of an invertebrate glutamate receptor subunit expressed in Drosophila muscle. Science 254:112-114.

Scott DB, Blanpied TA, Swanson GT, Zhang C, Ehlers MD (2001) An NMDA receptor ER retention signal regulated by phosphorylation and alternative splicing. J Neurosci 21:3063-3072.

Shapira M, Zhai RG, Dresbach T, Bresler T, Torres VI, Gundelfinger ED, Ziv NE, Garner CC (2003) Unitary assembly of presynaptic active zones from Piccolo-Bassoon transport vesicles. Neuron 38:237-252.

Shen K, Bargmann CI (2003) The immunoglobulin superfamily protein SYG-1 determines the location of specific synapses in C. elegans. Cell 112:619-630.

Shen K, Fetter RD, Bargmann CI (2004) Synaptic specificity is generated by the synaptic guidepost protein SYG-2 and its receptor, SYG-1. Cell 116:869-881.

Sheng M (2001) Molecular organization of the postsynaptic specialization. Proc Natl Acad Sci U S A 98:7058-7061.

Sheng M, Lee SH (2001) AMPA receptor trafficking and the control of synaptic transmission. Cell 105:825-828.

Sheridan DL, Berlot CH, Robert A, Inglis FM, Jakobsdottir KB, Howe JR, Hughes TE (2002) A new way to rapidly create functional, fluorescent fusion proteins: random insertion of GFP with an in vitro transposition reaction. BMC Neurosci 3:7.

Sigrist SJ, Thiel PR, Reiff DF, Schuster CM (2002) The postsynaptic glutamate receptor subunit DGluR-IIA mediates long-term plasticity in Drosophila. J Neurosci 22:7362-7372.

Sigrist SJ, Thiel PR, Reiff DF, Lachance PE, Lasko P, Schuster CM (2000) Postsynaptic translation affects the efficacy and morphology of neuromuscular junctions. Nature 405:1062-1065.

Sone M, Suzuki E, Hoshino M, Hou D, Kuromi H, Fukata M, Kuroda S, Kaibuchi K, Nabeshima Y, Hama C (2000) Synaptic development is controlled in the periactive zones of Drosophila synapses. Development 127:4157-4168.

Sprengel R, Aronoff R, Volkner M, Schmitt B, Mosbach R, Kuner T (2001) Glutamate receptor channel signatures. Trends Pharmacol Sci 22:7-10.

Standley S, Baudry M (2000) The role of glycosylation in ionotropic glutamate receptor ligand binding, function, and trafficking. Cell Mol Life Sci 57:1508-1516.

Stathopoulos A, Levine M (2002) Whole-genome expression profiles identify gene batteries in Drosophila. Dev Cell 3:464-465.

Sudhof TC (2000) The synaptic vesicle cycle revisited. Neuron 28:317-320.

Sweeney ST, Broadie K, Keane J, Niemann H, O'Kane CJ (1995) Targeted expression of tetanus toxin light chain in Drosophila specifically eliminates synaptic transmission and causes behavioral defects. Neuron 14:341-351.

Tejedor FJ, Bokhari A, Rogero O, Gorczyca M, Zhang J, Kim E, Sheng M, Budnik V (1997) Essential role for dlg in synaptic clustering of Shaker K+ channels in vivo. J Neurosci 17:152-159.

Thibault ST, Singer MA, Miyazaki WY, Milash B, Dompe NA, Singh CM, Buchholz R, Demsky M, Fawcett R, Francis-Lang HL, Ryner L, Cheung LM, Chong A, Erickson C, Fisher WW, Greer K, Hartouni SR, Howie E, Jakkula L, Joo D, Killpack K, Laufer A, Mazzotta J, Smith RD, Stevens LM, Stuber C, Tan LR, Ventura R, Woo A, Zakrajsek I, Zhao L, Chen F, Swimmer C, Kopczynski C, Duyk G, Winberg ML, Margolis J (2004) A complementary transposon tool kit for Drosophila melanogaster using P and piggyBac. Nat Genet 36:283-287.

Thomas U, Kim E, Kuhlendahl S, Koh YH, Gundelfinger ED, Sheng M, Garner CC, Budnik V (1997) Synaptic clustering of the cell adhesion molecule fasciclin II by discs- large and its role in the regulation of presynaptic structure. Neuron 19:787-799.

Togashi H, Abe K, Mizoguchi A, Takaoka K, Chisaka O, Takeichi M (2002) Cadherin regulates dendritic spine morphogenesis. Neuron 35:77-89.

Valenzuela DM, Stitt TN, DiStefano PS, Rojas E, Mattsson K, Compton DL, Nunez L, Park JS, Stark JL, Gies DR, et al. (1995) Receptor tyrosine kinase specific for the skeletal muscle lineage: expression in embryonic muscle, at the neuromuscular junction, and after injury. Neuron 
15:573-584

Vandenberghe W, Bredt DS (2004) Early events in glutamate receptor trafficking. Curr Opin Cell Biol 16:134-139.

Varoqueaux F, Sigler A, Rhee JS, Brose N, Enk C, Reim K, Rosenmund C (2002) Total arrest of spontaneous and evoked synaptic transmission but normal synaptogenesis in the absence of Munc13-mediated vesicle priming. Proc Natl Acad Sci U S A 99:9037-9042.

Verhage M, Maia AS, Plomp JJ, Brussaard AB, Heeroma JH, Vermeer H, Toonen RF, Hammer RE, van den Berg TK, Missler M, Geuze HJ, Sudhof TC (2000) Synaptic assembly of the brain in the absence of neurotransmitter secretion. Science 287:864-869.

Wallace BG (1989) Agrin-induced specializations contain cytoplasmic, membrane, and extracellular matrix-associated components of the postsynaptic apparatus. J Neurosci 9:1294-1302.

Wan HI, DiAntonio A, Fetter RD, Bergstrom K, Strauss R, Goodman CS (2000) Highwire regulates synaptic growth in Drosophila. Neuron 26:313-329.

Washbourne P, Bennett JE, McAllister AK (2002) Rapid recruitment of NMDA receptor transport packets to nascent synapses. Nat Neurosci 5:751-759.

Wenthold RJ, Petralia RS, Blahos J, II, Niedzielski AS (1996) Evidence for multiple AMPA receptor complexes in hippocampal CA1/CA2 neurons. J Neurosci 16:1982-1989.

Weston C, Yee B, Hod E, Prives J (2000) Agrin-induced acetylcholine receptor clustering is mediated by the small guanosine triphosphatases Rac and Cdc42. J Cell Biol 150:205-212.

Weston C, Gordon C, Teressa G, Hod E, Ren XD, Prives J (2003) Cooperative regulation by Rac and Rho of agrin-induced acetylcholine receptor clustering in muscle cells. J Biol Chem 278:6450-6455.

White JG, Southgate E, Thomson JN, Brenner S (1976) The structure of the ventral nerve cord of Caenorhabditis elegans. Philos Trans R Soc Lond B Biol Sci 275:327-348.

Wojtowicz JM, Marin L, Atwood HL (1994) Activity-induced changes in synaptic release sites at the crayfish neuromuscular junction. J Neurosci 14:3688-3703.

Wucherpfennig T, Wilsch-Brauninger M, Gonzalez-Gaitan M (2003) Role of Drosophila Rab5 during endosomal trafficking at the synapse and evoked neurotransmitter release. J Cell Biol 161:609-624.

Xia H, Hornby ZD, Malenka RC (2001) An ER retention signal explains differences in surface expression of NMDA and AMPA receptor subunits. Neuropharmacology 41:714-723.

Xia H, von Zastrow M, Malenka RC (2002) A novel anterograde trafficking signal present in the $\mathrm{N}$-terminal extracellular domain of ionotropic glutamate receptors. J Biol Chem 277:47765-47769.

Yamagata M, Sanes JR, Weiner JA (2003) Synaptic adhesion molecules. Curr Opin Cell Biol 15:621-632.

Yang X, Arber S, William C, Li L, Tanabe Y, Jessell TM, Birchmeier C, Burden SJ (2001) Patterning of muscle acetylcholine receptor gene expression in the absence of motor innervation. Neuron 30:399-410.

Yoshihara CM, Hall ZW (1993) Increased expression of the 43-kD protein disrupts acetylcholine receptor clustering in myotubes. J Cell Biol 122:169-179.

Yoshihara M, Rheuben MB, Kidokoro Y (1997) Transition from growth cone to functional motor nerve terminal in Drosophila embryos. J Neurosci 17:8408-8426.

Zhai RG, Vardinon-Friedman H, Cases-Langhoff C, Becker B, Gundelfinger ED, Ziv NE, Garner CC (2001) Assembling the presynaptic active zone: a characterization of an active one precursor vesicle. Neuron 29:131-143.

Zhang FL, Casey PJ (1996) Protein prenylation: molecular mechanisms and functional consequences. Annu Rev Biochem 65:241-269.

Zhou H, Glass DJ, Yancopoulos GD, Sanes JR (1999) Distinct domains of MuSK mediate its abilities to induce and to associate with postsynaptic specializations. J Cell Biol 146:1133-1146.

Zito K, Fetter RD, Goodman CS, Isacoff EY (1997) Synaptic clustering of Fascilin II and Shaker: essential targeting sequences and role of Dlg. Neuron 19:1007-1016. 


\section{Appendix}

\section{Genes enriched in larval body wall preparations}

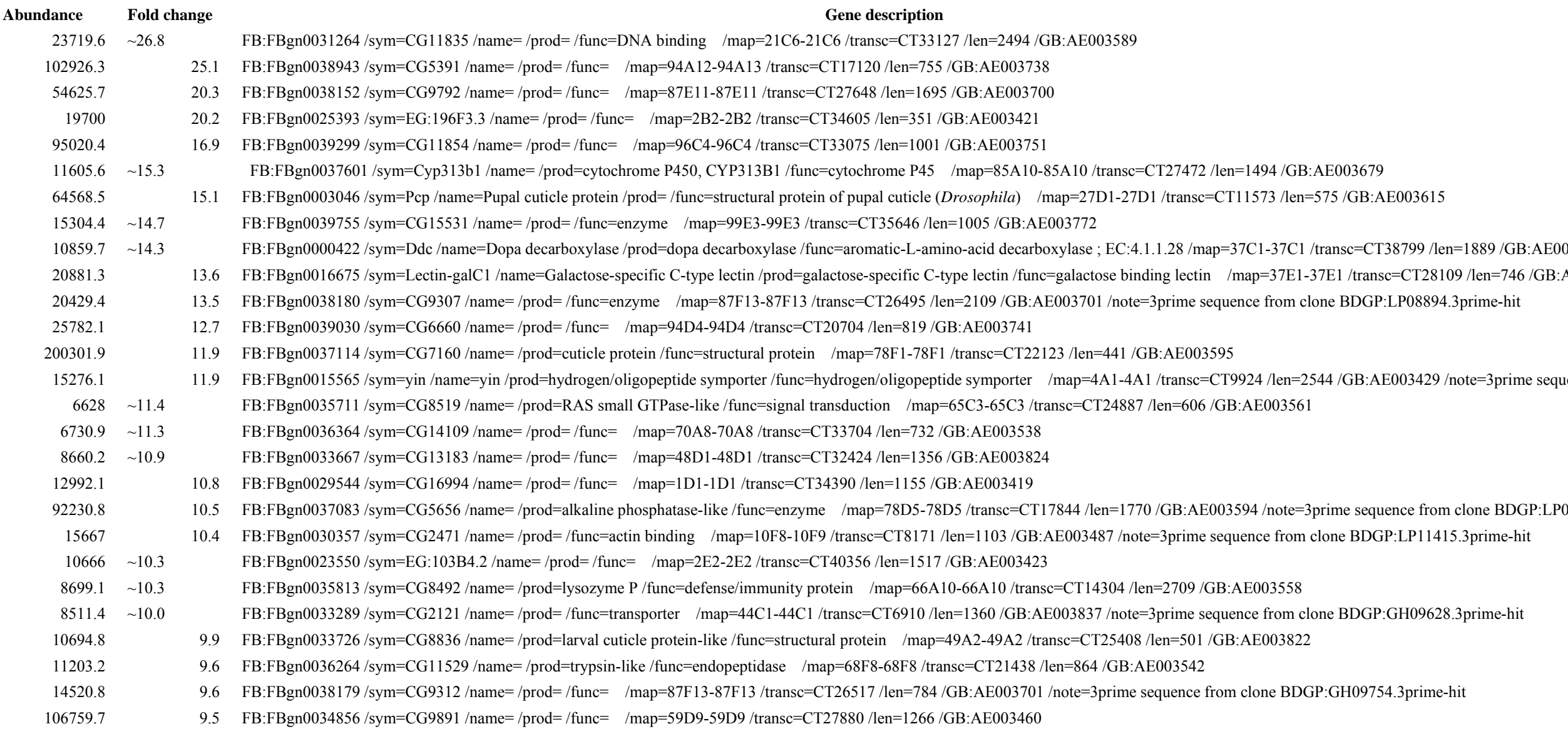

Gene description

FB:FBgn0031264 $/$ sym $=$ CG11835 $/$ name $=/$ prod $=/$ func=DNA binding $/$ map=21C6-21C6 $/$ transc $=$ CT33127 /len=2494 /GB:AE003589

25.1 FB:FBgn0038943 $/$ sym $=$ CG5391 $/$ name $=/$ prod $=/$ func $=/$ map $=94$ A12-94A13 $/$ transc $=\mathrm{CT} 17120 /$ len $=755 /$ GB:AE003738

20.3 FB:FBgn0038152/sym $=$ CG9792 $/$ name $=/$ prod $=/$ func $=/$ map $=87$ E $11-87$ E $11 /$ transc $=$ CT27648 $/$ len $=1695 /$ GB:AE003700

20.2 FB:FBgn0025393 /sym=EG:196F3.3 /name $=/$ prod= $/$ func $=/$ map=2B2-2B2 $/$ transc $=$ CT34605 /len=351 /GB:AE003421

16.9 FB:FBgn0039299/sym=CG1 $1854 /$ name $=/$ prod $=/$ func $=/$ map=96C4-96C4 $/$ transc=CT33075/len=1001 /GB:AE003751

FB:FBgn0037601 /sym=Cyp313b1 /name $=/$ prod=cytochrome P450, CYP313B1 /func=cytochrome P45 /map=85A10-85A10/transc=CT27472 /len=1494 /GB:AE003679

15.1 FB:FBgn0003046/sym=Pcp /name=Pupal cuticle protein /prod=/func=structural protein of pupal cuticle (Drosophila) $/$ map=27D1-27D1/transc=CT11573/len=575/GB:AE003615 FB:FBgn0039755 /sym=CG15531 /name=/prod= /func=enzyme $/$ map=99E3-99E3 $/$ transc=CT35646/len=1005 /GB:AE003772

4.3

$13.6 \mathrm{FB} \cdot \mathrm{FBgn} 0016675 / \mathrm{sym}=$ Lectin-galC1/name $=$ Galactose-specific $\mathrm{C}$-type lectin $/ \mathrm{prod}=$ galactose-specific C-type lectin $/ \mathrm{func}=\mathrm{galactose}$ binding lectin $/ \mathrm{map}=37 \mathrm{E} 1-37 \mathrm{E} 1 /$ transc $=\mathrm{CT} 28109 / \mathrm{len}=746 / \mathrm{GB}$ -

13.5 FB:FBgn0038180/sym=CG9307 /name=/prod= /func=enzyme /map=87F13-87F13/transc=CT26495/len=2109/GB:AE003701 /note=3prime sequence from clone BDGP:LP08894.3prime-hit

12.7 FB:FBgn0039030 $/$ sym $=$ CG6660 $/$ name $=/$ prod $=/$ func $=/$ map=94D4-94D4 $/$ transc $=$ CT20704 $/$ len $=819 /$ GB:AE003741

11.9 FB:FBgn0037114 $/$ sym=CG7160 $/$ name $=/$ prod=cuticle protein $/$ func=structural protein $/$ map=78F1-78F1 $/$ transc=CT22123/len=441 /GB:AE003595

11.9 FB:FBgn0015565/sym=yin /name=yin /prod=hydrogen/oligopeptide symporter /func=hydrogen/oligopeptide symporter $/$ map=4A1-4A1/transc=CT9924/len=2544 /GB:AE003429/note=3prime sequ FB:FBgn0035711 /sym=CG8519/name=/prod=RAS small GTPase-like /func=signal transduction /map=65C3-65C3/transc=CT24887/len=606/GB:AE003561

FB:FBgn0036364 $/$ sym $=$ CG14109 $/$ name $=/$ prod $=/$ func $=/$ map=70A8-70A $8 /$ transc $=$ CT33704 $/$ len $=732 /$ GB:AE003538

FB:FBgn0033667/sym $=$ CG13183 $/$ name $=/$ prod $=/$ func $=/$ map $=48 D 1-48 D 1 /$ transc $=$ CT32424 $/$ len $=1356 /$ GB:AE003824

10.8 FB:FBgn0029544 $/$ sym $=$ CG16994 $/$ name $=/$ prod $=/$ func $=/$ map=1D1-1D1 $/$ transc $=$ CT34390 $/$ len=1155 $/$ GB:AE003419

10.5 FB:FBgn0037083/sym=CG5656/name $=/$ prod=alkaline phosphatase-like $/$ func=enzyme $/$ map=78D5-78D5 /transc=CT17844 /len=1770 /GB:AE003594 /note=3prime sequence from clone BDGP:LP0

10.4 FB:FBgn0030357/sym=CG2471/name=/prod=/func=actin binding $/$ map=10F8-10F9/transc=CT8171/len=1103 /GB:AE003487/note=3prime sequence from clone BDGP:LP11415.3prime-hit FB:FBgn0023550 $/$ sym=EG:103B4.2 $/$ name $=/$ prod $=/$ func $=/$ map $=2 \mathrm{E} 2-2 \mathrm{E} 2 /$ transc $=\mathrm{CT} 40356 / \mathrm{len}=1517 / \mathrm{GB}: \mathrm{AE} 003423$

FB:FBgn0035813 /sym=CG8492/name $=/$ prod=lysozyme $\mathrm{P} /$ func=defense/immunity protein $/ \mathrm{map}=66 \mathrm{~A} 10-66 \mathrm{~A} 10 /$ transc $=\mathrm{CT} 14304 / \mathrm{len}=2709 / \mathrm{GB}: \mathrm{AE} 003558$

FB:FBgn0033289/sym=CG2121 /name=/prod=/func=transporter $/$ map=44C1-44C1 /transc=CT6910/len=1360 /GB:AE003837/note=3prime sequence from clone BDGP:GH09628.3prime-hit

9.9 FB:FBgn0033726/sym=CG8836/name=/prod=larval cuticle protein-like /func=structural protein $/$ map=49A2-49A2/transc=CT25408/len=501 /GB:AE003822

9.6 FB:FBgn0036264/sym=CG11529/name $=/$ prod=trypsin-like $/$ func $=$ endopeptidase $/ \mathrm{map}=68 \mathrm{~F} 8-68 \mathrm{~F} 8 /$ transc $=\mathrm{CT} 21438 /$ len $=864 / \mathrm{GB}: \mathrm{AE} 003542$

9.6 FB:FBgn0038179/sym $=$ CG9312 $/$ name $=/$ prod $=/$ func $=/$ map $=87 F 13-87 F 13 /$ transc $=$ CT26517 /len=784 /GB:AE003701 /note=3prime sequence from clone BDGP:GH09754.3prime-hit

9.5 FB:FBgn0034856 $/$ sym $=$ CG9891 $/$ name $=/$ prod $=/$ func $=/$ map $=59 \mathrm{D} 9-59 D 9 /$ transc $=\mathrm{CT} 27880 /$ len $=1266 / \mathrm{GB}: \mathrm{AE} 003460$ 


$\begin{aligned} 8081 & \sim 9.1 \\ 7297.3 & \sim 9.1 \\ 6026.4 & \sim 8.9 \\ 10038.8 & \\ 17201.8 & \\ 30755.5 & \\ 22531.1 & \\ 10634.5 & \\ 11276.3 & \\ 19312.7 & \\ 7383.3 & \sim 7.7 \\ 6329 & \sim 7.4 \\ 19301.8 & \\ 7576.3 & \sim 7.3 \\ 22097.3 & \\ 63005.9 & \\ 5453.9 & \sim 7.2 \\ 101119.9 & \\ 6255.2 & \sim 7.1 \\ 5606.4 & \sim 7.0 \\ 7498.3 & \\ 5278.4 & \sim 7.0 \\ 5163.4 & \sim 6.8 \\ 69802.4 & \\ 11072.1 & \\ 19916.4 & \\ 7115.8 & \\ 2204.1 & \sim 6.6 \\ 4567.9 & \sim 6.6 \\ 5343.8 & \sim 6.6 \\ 78514.5 & \\ 80923 & \\ & \\ & \\ & \\ & \\ & \\ & \\ & \\ & \\ & \\ & \\ & \\ & \\ & \\ & \\ & \end{aligned}$

80923
FB:FBgn0032349 $/$ sym $=$ CG4779 $/$ name $=/$ prod $=/$ func $=/$ map $=32 \mathrm{D} 4-32 \mathrm{D} 4 /$ transc $=\mathrm{CT} 15337 /$ len $=1272 / \mathrm{GB}: \mathrm{AE} 003631$ FB:FBgn0033858 $/$ sym $=$ CG13335 $/$ name $=/$ prod $=/$ func $=/$ map $=50 \mathrm{~B} 7-50 \mathrm{~B} 7 /$ transc $=\mathrm{CT} 32654 / \mathrm{len}=408 / \mathrm{GB}: \mathrm{AE} 003818$ FB:FBgn0040868 $/ \mathrm{sym}=\mathrm{CG} 15762 /$ name $=/$ prod $=/$ func $=/ \mathrm{map}=12 \mathrm{~B} 10-12 \mathrm{~B} 10 /$ transc $=\mathrm{CT} 36016 / \mathrm{len}=240 / \mathrm{GB}: \mathrm{AE} 003493$

8.9 FB:FBgn0037319/sym=CG2666/name $=/$ prod=chitin synthase-like $/$ func=enzyme $/$ map=83A5-83A5 /transc=CT9021 /len=3897/GB:AE003603

8.6 FB:FBgn0034443/sym=CG10460 /name $=/$ prod $=/$ func=endopeptidase $/ \mathrm{map}=56 \mathrm{D} 4-56 \mathrm{D} 4 /$ transc=CT29364 /len=485 /GB:AE003796

8.5 FB:FBgn0035964/sym=CG4665/name=dihydropteridine reductase $/$ prod=dihydropteridine reductase-like $/$ func $=$ dihydropteridine reductase $/ \mathrm{map}=66 \mathrm{~F} 6-66 \mathrm{~F} 6 /$ transc $=\mathrm{CT} 15055 /$ len $=708 / \mathrm{GB}: \mathrm{AE} 0035$

8.3 FB:FBgn0035427 /sym $=\mathrm{CG} 14959 /$ name $=/$ prod $=/$ func $=/ \mathrm{map}=63 \mathrm{C} 2-63 \mathrm{C} 2 /$ transc $=\mathrm{CT} 34804 / \mathrm{len}=450 / \mathrm{GB}: \mathrm{AE} 003477$

8.2 FB:FBgn0029821/sym=CG4020/name $=/$ prod $=/$ func $=/ \mathrm{map}=5 \mathrm{C} 6-5 \mathrm{C} 6 /$ transc $=\mathrm{CT} 13322 /$ len $=1485 / \mathrm{GB}: \mathrm{AE} 003435$

7.8 FB:FBgn0035300 $/$ sym $=\mathrm{CG} 1139 /$ name $=/$ prod $=$ amino-acid permease-like $/$ func=transporter $/ \mathrm{map}=62 \mathrm{~B} 11-62 \mathrm{~B} 11 / \mathrm{transc}=\mathrm{CT} 1131 / \mathrm{len}=1511 / \mathrm{GB}: \mathrm{AE} 003473$

7.7 FB:FBgn0038348 $/$ sym $=$ CG18519 $/$ name $=/$ prod $=/$ func $=/$ map $=88 \mathrm{~F} 8-88 \mathrm{~F} 8 /$ transc $=\mathrm{CT} 42266 / \mathrm{len}=3671 / \mathrm{GB}: \mathrm{AE} 003709$

FB:FBgn0034253 /sym=CG10936/name $=/$ prod $=/$ func $=$ endopeptidase $/$ map=54D3-54D4 /transc=CT30629 /len=4407 /GB:AE003802

FB:FBgn0038629 $/$ sym $=$ CG14304 $/$ name $=/$ prod $=/$ func $=/$ map=91B4-91B4 $/$ transc $=$ CT33934 /len=3585 $/$ GB:AE003722

7.3 FB:FBgn0031791 $/$ sym $=\mathrm{CG} 9486 /$ name $=/$ prod $=/$ func $=$ enzyme $/ \mathrm{map}=26 \mathrm{C} 1-26 \mathrm{C} 1 /$ transc $=\mathrm{CT} 26862 / \mathrm{len}=651 / \mathrm{GB}: \mathrm{AE} 003613$

FB:FBgn0037503 $/$ sym $=$ CG14598 $/$ name $=/$ prod $=/$ func $=/$ map $=84 \mathrm{D} 3-84 \mathrm{D} 3 /$ transc $=\mathrm{CT} 34346 /$ len $=690 /$ GB:AE003671

7.3 FB:FBgn0037761 $/$ sym $=$ CG8534 $/$ name $=/$ prod $=/$ func $=/$ map $=85 \mathrm{E} 10-85 \mathrm{E} 10 /$ transc $=\mathrm{CT} 24919 / \mathrm{len}=818 / \mathrm{GB}: \mathrm{AE} 003684$

7.2 FB:FBgn0030326 $/ \mathrm{sym}=\mathrm{CG} 2444 / \mathrm{name}=/ \mathrm{prod}=/$ func $=/ \mathrm{map}=10 \mathrm{D} 4-10 \mathrm{D} 4 /$ transc $=\mathrm{CT} 8084 / \mathrm{len}=635 / \mathrm{GB}: \mathrm{AE} 003487$

FB:FBgn0000594 /sym=Est-P /name=Esterase P $/$ prod=carboxylesterase $/$ func=carboxyesterase $;$ EC:3.1.1.1 /map=68F8-68F8 /transc=CT38084 /len=1635 /GB:AE003542

7.1 FB:FBgn0034162 $/ \mathrm{sym}=\mathrm{CG} 6426 /$ name $=/$ prod=calcium binding protein-like $/$ func=ligand binding or carrier $/ \mathrm{map}=53 \mathrm{D} 15-53 \mathrm{D} 15 /$ transc $=\mathrm{CT} 19886 / \mathrm{len}=426 / \mathrm{GB}: \mathrm{AE} 003805$ FB:FBgn0033136 $/$ sym $=\mathrm{CG} 12838 /$ name $=/$ prod $=/$ func $=/$ map $=42 \mathrm{E} 2-42 \mathrm{E} 2 /$ transc $=\mathrm{CT} 31970 / \mathrm{len}=633 / \mathrm{GB}: \mathrm{AE} 003842$

FB:FBgn0026190/sym=prolyl-4-hydroxylase-alpha /name=prolyl 4-hydroxylase alpha /prod=procollagen-proline,2-oxoglutarate-4-dioxygenase, alpha subunit /func=procollagen-proline,2-oxoglutarate

7 FB:FBgn0037905 $/$ sym $=$ CG14703 $/$ name $=/$ prod $=/$ func $=/$ map=86E6-86E $6 /$ transc $=$ CT34493 $/$ len $=1397 /$ GB:AE003691

FB:FBgn0033099 $/$ sym $=$ CG $9435 /$ name $=/$ prod $=/$ func $=/$ map $=42 \mathrm{C} 3-42 \mathrm{C} 3 /$ transc $=$ CT26754 $/$ len $=594 /$ GB:AE003789

FB:FBgn0031564 /sym=CG2816/name =/prod=chymotrypsin inhibitor-like /func=enzyme inhibitor $/$ map=24A5-24A5/transc=CT9603/len=292/GB:AE003579

6.7 FB:FBgn0033595 $/$ sym $=\mathrm{CG} 18337 /$ name $=/$ prod $=/$ func $=/$ map $=47 \mathrm{E} 1-47 \mathrm{E} 1 /$ transc $=\mathrm{CT} 41641 / \mathrm{len}=540 / \mathrm{GB}: \mathrm{AE} 003826$

6.7 FB:FBgn0022702 /sym=Cht2 /name=Chitinase $2 /$ prod=chitinase /func=chitinase ; EC:3.2.1.14/map=62B1-62B1/transc=CT6562/len=1597/GB:AE003472 /note=3prime sequence from clone BDGP:L

6.6 FB:FBgn0022770 /sym=Peritrophin-A /name=Peritrophin A /prod=peritrophin A /func=chitin binding /map=19C1-19C1 /transc=CT35567/len=1232/GB:AE003572 /note=3prime sequence from clon FB:FBgn0010222/sym=Nmdmc /name=NAD-dependent methylenetetrahydrofolate dehydrogenase $/$ prod=NAD-dependent methylenetetrahydrofolate dehydrogenase $/$ func $=$ methenyltetrahydrofolate cy 6.6 /GB:AE003

FB:FBgn0039098 $/$ sym $=$ CG13822 $/$ name $=/$ prod $=/$ func $=/$ map=95B1-95B1 $/$ transc $=$ CT33322 $/$ len $=651 /$ GB:AE003744

FB:FBgn0035553/sym=CG13722/name=/prod=prolin-rich protein-like /func=structural protein $/$ map=64B12-64B12/transc=CT33188/len=2124/GB:AE003481

FB:FBgn0027570/sym=BcDNA:GH07643 /name=/prod=membrane-anchored zinc metalloendopeptidase-like $(\mathrm{M} 13$ peptidase $) /$ func $=$ endopeptidase $/$ map $=82 \mathrm{D} 6-82 \mathrm{D} 7 /$ transc $=\mathrm{CT} 27583 /$ len $=2739 / \mathrm{C}$ 6.5 FB:FBgn0039482 $/$ sym $=$ CG14258 $/$ name $=/$ prod $=/$ func $=/$ map $=97$ E6-97E6 $/$ transc $=$ CT33878 $/$ len $=777 /$ GB $:$ AE003759

6.5 FB:FBgn0034517/sym=CG18066/name $=/$ prod $=/$ func $=/$ map=57A8-57A8 $/$ transc=CT40491/len=625 /GB:AE003791 /note $=3$ prime sequence from clone BDGP:GH09112.3prime-hit 
6.5 FB:FBgn0030976 $/$ sym $=\mathrm{CG} 7378 /$ name $=/$ prod $=$ protein phosphatase-like $/$ func $=$ protein phosphatase $/$ map $=17 \mathrm{E} 8-17 \mathrm{E} 8 /$ transc $=\mathrm{CT} 22699 / \mathrm{len}=738 / \mathrm{GB}: \mathrm{AE} 003510$

6.5 FB:FBgn0033022 $/ \mathrm{sym}=\mathrm{CG} 10398 /$ name $=/$ prod $=/$ func $=/ \mathrm{map}=41 \mathrm{C} 1-41 \mathrm{C} 1 /$ transc $=\mathrm{CT} 29180 / \mathrm{len}=501 / \mathrm{GB}: \mathrm{AE} 003786$

6.4 FB:FBgn0038053 $/$ sym $=C$ CG18549 $/$ name $=/$ prod $=/$ func $=/$ map $=87 \mathrm{C} 1-87 \mathrm{C} 1 /$ transc $=\mathrm{CT} 17408 / \mathrm{len}=1634 / \mathrm{GB}: \mathrm{AE} 003696$

6.4 FB:FBgn0031542 $/$ sym $=\mathrm{CG} 15414 /$ name $=/$ prod $=/$ func $=/$ map $=23 F 3-23 F 3 /$ transc $=\mathrm{CT} 35473 /$ len $=963 /$ GB:AE003579

FB:FBgn0035755 $/ \mathrm{sym}=\mathrm{CG} 14830 /$ name $=/$ prod $=/$ func $=/ \mathrm{map}=65 \mathrm{E} 10-65 \mathrm{E} 10 /$ transc $=\mathrm{CT} 34646 / \mathrm{len}=972 / \mathrm{GB}: \mathrm{AE} 003559$

6.3 FB:FBgn0035684/sym=CG10461 /name $=/$ prod=cuticle protein-like $/$ func=structural protein $/$ map=65A5-65A5 /transc=CT29366/len=321 /GB:AE003563

6.3 FB:FBgn0034860 $/$ sym $=$ CG9812 $/$ name $=/$ prod $=/$ func $=/$ map=59D10-59D $11 /$ transc $=C T 42639 /$ len=611 $/$ GB:AE003460

6.3 FB:FBgn0030816/sym $=$ CG16700/name $=/$ prod=amino-acid permease-like $/$ func $=\mathrm{G}$ protein linked receptor $/ \mathrm{map}=15 \mathrm{D} 2-15 \mathrm{D} 3 /$ transc $=\mathrm{CT} 37167 / \mathrm{len}=1673 / \mathrm{GB}: \mathrm{AE} 003504$ FB:FBgn0038449 $/$ sym $=$ CG17562 $/$ name $=/$ prod $=/$ func=enzyme $/$ map=89D5-89D5 /transc=CT34716 /len=1416 /GB:AE003714

6.2 FB:FBgn0030558 /sym=CG1461 /name $=/$ prod=tyrosine aminotransferase $/$ func=enzyme $/$ map=12E1-12E1 /transc=CT3554/len=1844 /GB:AE003495

FB:FBgn0039111/sym=CG10371/name=/prod $=/$ func $=$ protein phosphatase $/$ map=95B7-95B7/transc=CT29124/len=585/GB:AE003744

6.1 FB:FBgn0039895/sym=CG11288 /name $=/$ prod=fibrillin-like $/$ func $=$ cell adhesion $/ \mathrm{map}=102 \mathrm{~A} 3-102 \mathrm{~A} 3 /$ transc $=\mathrm{CT} 31491 / \mathrm{len}=914 / \mathrm{GB}: \mathrm{AE003844}$

6.1 FB:FBgn0031737/sym=CG11142/name=/prod=peritrophin-like $/$ func=structural protein $/ \mathrm{map}=26 \mathrm{~A} 1-26 \mathrm{~A} 1 /$ transc $=\mathrm{CT} 31147 / \mathrm{len}=1457 / \mathrm{GB}: \mathrm{AE} 003611$

6 FB:FBgn0038366 $/$ sym $=$ CG4576 $/$ name $=/$ prod $=/$ func $=/$ map $=89$ A $8-89$ A $8 /$ transc $=$ CT14818 $/$ len $=2581 /$ GB:AE003710

FB:FBgn0023540 $/$ sym=EG:152A3.3 $/$ name $=/$ prod $=/$ func $=/$ map=2E1-2E1 $/$ transc $=$ CT12189/len=1918 /GB:AE003423 /note=3prime sequence from clone BDGP:HL01173.3prime-hit

5.9 FB:FBgn0035936 $/$ sym $=$ CG4999 $/$ name $=/$ prod $=/$ func $=/$ map $=66 \mathrm{E} 3-66 \mathrm{E} 3 /$ transc $=\mathrm{CT} 16000 /$ len $=1131 /$ GB:AE003553

5.9 FB:FBgn0020439 $/$ sym=fau $/$ name $=/$ prod=anoxia upregulated protein $/$ func $=/$ map $=86 C 4-86 C 4 /$ transc $=$ CT41978 $/$ len=2121 /GB:AE003688

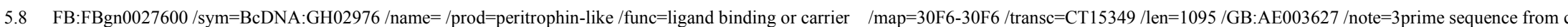
FB:FBgn0037186/sym=CG11241 /name $=/$ prod $=$ pyridoxal-phosphate-dependent aminotransferase class-III $/$ func $=$ enzyme $/$ map $=79 F 2-79 F 2 /$ transc $=\mathrm{CT} 31379 /$ len $=1856 / \mathrm{GB}: \mathrm{AE} 003598 /$ note $=3 \mathrm{prime}$ FB:FBgn0030595 $/$ sym $=$ CG14406 $/$ name $=/$ prod $=/$ func $=/$ map $=13$ A1-13A $1 /$ transc $=$ CT34057 $/$ len $=312 /$ GB:AE003497

BDGP:GH188.3prime-hit $/$ ESTpos=maps in FB:FBgn0036365 $/$ sym $=$ CG10732 $/$ name $=/$ prod $=/$ func $=$ signal transduction $/$ map $=7$ A8-7A8 $/$ transc $=\mathrm{CT} 369 /$ len $=535$

5.8 FB:FBgn0010019/sym=Cyp4g1/name=Cytochrome P45-4g1/prod=cytochrome P450, CYP4G1/func=cytochrome P45 ; EC:1.14.14.1/map=1B3-1B3 /transc=CT13187/len=2267 /GB:AE003417/nc 5.7 FB:FBgn0033725/sym=CG8502/name $=/$ prod $=$ cuticle protein /func=structural protein $/$ map=49A2-49A2/transc=CT24855/len=1413 /GB:AE003822 /note=3prime sequence from clone BDGP:LP07

5.7 FB:FBgn0029838 $/$ sym $=$ CG4666 $/$ name $=/$ prod $=/$ func $=/$ map $=5 \mathrm{D} 2-5 \mathrm{D} 2 /$ transc $=\mathrm{CT} 15013 / \mathrm{len}=729 / \mathrm{GB}: \mathrm{AE} 003436$

FB:FBgn0035304/sym=CG1282 $/$ name $=/$ prod=titin/twitchin-like $/$ func=cell adhesion $/ \mathrm{map}=62 \mathrm{C} 3-62 \mathrm{C} 3 /$ transc $=\mathrm{CT} 2687 /$ len=2392 $/ \mathrm{GB}: \mathrm{AE} 003473$

FB:FBgn0028922 /sym=BG:DS00929.8/name $=/$ prod $=/$ func $=/$ map=35C1-35C1 $/$ transc $=$ CT12287 /len $=1508 /$ GB:AE003646

5.6 FB:FBgn0030394 /sym=CG2560/name $=/$ prod $=$ cuticle protein-like $/$ func $=$ structural protein $/ \mathrm{map}=11 \mathrm{~B} 7-11 \mathrm{~B} 7 /$ transc $=\mathrm{CT} 8657 / \mathrm{len}=893 / \mathrm{GB}: \mathrm{AE} 003489$

FB:FBgn0036185 $/$ sym $=$ CG7346 $/$ name $=/$ prod=ATP-binding cassette transporter $/$ func=enzyme $/$ map=68C10-68C11 /transc=CT22647/len=1794 $/$ GB:AE003544

FB:FBgn0033290 $/$ sym $=$ CG8698 $/$ name $=/$ prod $=/$ func $=/ \mathrm{map}=44 \mathrm{C} 1-44 \mathrm{C} 1 /$ transc $=\mathrm{CT} 8775 / \mathrm{len}=504 / \mathrm{GB}: \mathrm{AE} 003837$

FB:FBgn0035289 $/ \mathrm{sym}=\mathrm{CG} 12026 /$ name $=/$ prod $=/$ func $=/$ map $=62 \mathrm{~B} 9-62 \mathrm{~B} 9 /$ transc $=\mathrm{CT} 1797 / \mathrm{len}=1455 / \mathrm{GB}: \mathrm{AE} 003473 /$ note $=3$ prime sequence from clone BDGP $:$ LP10272.3prime-hit

5.5 FB:FBgn0031872/sym=CG9211/name=/prod=/func=cell adhesion $/$ map=27C5-27C6 /transc=CT26314 /len=3247 /GB:AE003615 /note=3prime sequence from clone BDGP:GH03927.3prime-hit

5.5 FB:FBgn0032497 $/$ sym $=$ CG6043 $/$ name $=/$ prod $=/$ func $=/$ map $=34$ A11 $-34 \mathrm{~B} 1 /$ transc $=\mathrm{CT} 18935 /$ len $=3263 /$ GB:AE003639 
FB:FBgn0035457 $/$ sym $=\mathrm{CG} 12604 /$ name $=/$ prod $=/$ func $=/$ map $=63 \mathrm{E} 8-63 \mathrm{E} 8 / \mathrm{transc}=\mathrm{CT} 34822 / \mathrm{len}=936 / \mathrm{GB}: \mathrm{AE} 003479$

5.4 FB:FBgn0037534/sym=CG2781/name $=/$ prod $=/$ func=1,3-beta-glucan synthase $/$ map=84E5-84E5 /transc $=\mathrm{CT} 9465 /$ len $=831 / \mathrm{GB}: \mathrm{AE} 003677$

5.3 FB:FBgn0034920 $/ \mathrm{sym}=\mathrm{CG} 5597 /$ name $=/$ prod $=/$ func $=/ \mathrm{map}=60 \mathrm{~A} 8-60 \mathrm{~A} 8 /$ transc $=\mathrm{CT} 17698 / \mathrm{len}=897 /$ GB:AE003462 $/$ note $=3$ prime sequence from clone BDGP $:$ GH04238.3prime-hit

5.3 FB:FBgn0035985/sym=CG3672/name=/prod=/func=structural protein $/$ map=67B2-67B2/transc=CT12317/len=997 /GB:AE003552/note=3prime sequence from clone BDGP:GH12638.3prime-hit FB:FBgn0030065/sym=CG12075/name=/prod=/func=transcription factor $/ \mathrm{map}=8 \mathrm{~B} 4-8 \mathrm{~B} 4 /$ transc=CT5152/len=3406 /GB:AE003445 /note=3prime sequence from clone BDGP:LD23217.3prime-hit

5.3 FB:FBgn0032230 $/$ sym $=$ CG13139 $/$ name $=/$ prod $=/$ func $=/$ map $=31 \mathrm{D} 10-31 \mathrm{D} 10 /$ transc $=\mathrm{CT} 32379 / \mathrm{len}=807 / \mathrm{GB}: \mathrm{AE} 003628$

FB:FBgn0037805 $/$ sym $=$ CG1 $1871 /$ name $=/$ prod $=/$ func $=/$ map $=86$ A1-86A1 $/$ transc $=$ CT37022 $/$ len $=3575 /$ GB:AE003686

5.3 FB:FBgn0035705 /sym=CG8352/name $=/$ prod $=/$ func $=/$ map=65B4-65B5 /transc=CT24625/len=1728 /GB:AE003562 /note=3prime sequence from clone BDGP:LD26402.3prime-hit

5.3 FB:FBgn0031971 /sym $=$ CG7224 $/$ name $=/$ prod $=/$ func $=/$ map $=28 \mathrm{D} 11-28 \mathrm{D} 11 /$ transc $=\mathrm{CT} 22279 / \mathrm{len}=755 /$ GB:AE003619 $/$ note $=3$ prime sequence from clone BDGP $:$ GH18422.3prime-hit

5.2 FB:FBgn0033509/sym=CG12908/name=/prod=nidogen-like $/$ func=cell adhesion $/$ map=47A1-47A1 /transc=CT32053 /len=4591 /GB:AE003830 /note=3prime sequence from clone BDGP:LD35637

5.2 FB:FBgn0036985/sym=CG5847/name $=/$ prod $=/$ func $=$ structural protein $/$ map=77B9-77B9 $/$ transc=CT18331/len=6855/GB:AE003591

5.2 FB:FBgn0022701 /sym=Cht3 /name=Chitinase $3 /$ prod=chitinase $/$ func=chitinase ; EC:3.2.1.14 / map=40D5-40D5/transc=CT40797/len=3899/GB:AE002743

5.2 FB:FBgn0039690 /sym=CG1969 /name =/prod $=/$ func=glucosamine-phosphate N-acetyltransferase $;$ EC:2.3.1.4 /map=99C1-99C1/transc=CT4002 /len=1206 /GB:AE003771 /note=3prime sequence fro

5.2 FB:FBgn0029898/sym=CG14439/name=/prod=permease-like $/$ func=transporter $/ \mathrm{map}=6 \mathrm{C} 11-6 \mathrm{C} 11 /$ transc $=\mathrm{CT} 34101 / \mathrm{len}=1608 / \mathrm{GB}: \mathrm{AE} 003438$

5.2 FB:FBgn0032993 /sym=CG18117/name=/prod=chitinase-like /func=enzyme $/ \mathrm{map}=40 \mathrm{E} 1-40 \mathrm{E} 1 /$ transc=CT40795 /len=1393 /GB:AE002743 /note=3prime sequence from clone BDGP:LP05745.3prin

5.2 FB:FBgn0032601 /sym=yellow-b $/$ name=yellow-b $/$ prod $=/$ func $=/$ map=36A8-36A8 $/$ transc=CT39906 /len=1625 /GB:AE003652 /note=3prime sequence from clone BDGP:LD43175.3prime-hit

5.1 FB:FBgn0035398 /sym=CG1869 /name $=/$ prod=chitinase $/$ func=enzyme $/$ map=63B8-63B $/$ transc=CT5720/len=2346 /GB:AE003477

FB:FBgn0039658 /sym=CG11956/name=/prod=/func=/map=99A5-99A5/transc=CT39158/len=2937/GB:AE003768 /note=3prime sequence from clone BDGP:LD35296.3prime-hit

5.1 FB:FBgn0010388/sym=Dro $/$ name=Drosocin $/$ prod=drosocin $/$ func=antibacterial response protein $/ \mathrm{map}=51 \mathrm{C} 2-51 \mathrm{C} 2 /$ transc=CT30318/len=195/GB:AE003813

5 FB:FBgn0031908/sym=CG5177/name =/prod=/func=enzyme /map=27F6-27F6/transc=CT16575/len=980 /GB:AE003617 /note=3prime sequence from clone BDGP:LD18740.3prime-hit FB:FBgn0000152/sym=Axs /name=Abnormal X segregation $/$ prod $=/$ func $=/$ map=15A1-15A1/transc=CT27428/len=2396 /GB:AE003503

5 FB:FBgn0032281 $/$ sym $=$ CG17107 $/$ name $=/$ prod $=/$ func $=/$ map $=32 \mathrm{~A} 1-32 \mathrm{~A} 1 /$ transc $=$ CT33651 $/$ len $=291 /$ GB:AE003629

5 FB:FBgn0002440 /sym=1(3)mbn /name=lethal (3) malignant blood neoplasm $/$ prod $=/$ func $=/$ map $=65$ A4-65A4 $/$ transc $=\mathrm{CT} 29378 /$ len=1957 $/$ GB:AE003563

5 FB:FBgn0035103 $/$ sym $=$ CG7047 $/$ name $=/$ prod $=$ arrestin-like $/$ func $=/$ map $=61$ A5-61A5 $/$ transc $=$ CT42521 $/$ len $=1187 /$ GB:AE003467

5 FB:FBgn0039894/sym=CG10323/name=/prod=fibulin/fibrillin-like /func=cell adhesion $/ \mathrm{map}=102 \mathrm{~A} 1-102 \mathrm{~A} 3 /$ transc $=\mathrm{CT} 7856 / \mathrm{len}=1684 / \mathrm{GB}: \mathrm{AE} 003844$

5 FB:FBgn0036271 /sym=CG10335/name $=/$ prod=porphobilinogen synthase-like $/$ func $=$ enzyme $/$ map $=69$ A3 $-69 A 3 /$ transc $=$ CT29022 $/$ len $=984 / \mathrm{GB}: \mathrm{AE} 003542$

5 FB:FBgn0034420/sym=CG10737/name =/prod=protein kinase-like /func=protein kinase /map=56B6-56C1/transc=CT21097/len=3105/GB:AE003797 /note=3prime sequence from clone BDGP:GN FB:FBgn0013718 $/ \mathrm{sym}=$ nuf $/$ name $=$ nuclear fallout $/$ prod $=/$ func $=$ cytoskeletal structural protein $/$ map=70D2-70D2 $/$ transc $=$ CT23449 $/$ len $=2261 / \mathrm{GB}:$ AE003535

4.9 FB:FBgn0033130/sym=CG12843/name=Tetraspanin 42Ei /prod=tetraspanin /func $=/ \mathrm{map}=42 \mathrm{E} 1-42 \mathrm{E} 1 /$ transc $=\mathrm{CT} 31975 / \mathrm{len}=967 / \mathrm{GB}: \mathrm{AE} 003842$

4.9 FB:FBgn0040631/sym=CG13249 $/$ name $=/$ prod $=/$ func $=/$ map=77C2-77C2 $/$ transc=CT32502 /len=432 /GB:AE003591 /note=3prime sequence from clone BDGP $:$ LD43096.3prime-hit FB:FBgn0032382/sym=CG14935/name=/prod=maltase 2-like /func=enzyme $/$ map=33B2-33B2/transc=CT34763/len=1790/GB:AE003634

4.9 FB:FBgn0038774 /sym=CG5023/name $=/$ prod=calponin-like /func=ligand binding or carrier $/$ map=92D2-92D2/transc=CT16114 /len=697 /GB:AE003729 $/$ note $=3$ prime sequence from clone BDGP: 
4.9 FB:FBgn0032774 /sym=CG17549 /name $=/$ prod $=/$ func $=/$ map=37E1-37E1 /transc=CT38763/len=1051 /GB:AE003662 /note=3prime sequence from clone BDGP:GH19142.3prime-hit FB:FBgn0035155/sym=CG12015/name $=/$ prod $=/$ func $=$ transporter $/$ map=61C $9-61 \mathrm{C} 9 /$ transc $=\mathrm{CT} 1655 / \mathrm{len}=728 / \mathrm{GB}: \mathrm{AE} 003469$

FB:FBgn0036561 /sym=CG5891/name $=/$ prod=ankyrin-like $/$ func $=$ cytoskeletal structural protein $/$ map=72D1-72D3 $/$ transc=CT18415 /len=3517 /GB:AE003528

4.8 FB:FBgn0038250/sym=CG3505/name $=/$ prod $=/$ func $=$ endopeptidase $/ \mathrm{map}=88 \mathrm{C} 11-88 \mathrm{C} 11 /$ transc=CT11805/len=1161 /GB:AE003705 /note=3prime sequence from clone BDGP:LP10895.3prime-hit 4.7 FB:FBgn0033724/sym $=$ CG8501 $/$ name $=/$ prod $=/$ func $=/$ map=49A2-49A2 /transc=CT24861 /len=902 /GB:AE003822 /note=3prime sequence from clone BDGP:HL07915.3prime-hit

4.7 FB:FBgn0027617/sym=Rbp9/name=/prod $=/$ func=RNA binding $/$ map=23C1-23C2 /transc=CT38165/len=3320/GB:AE003581 /note $=3$ prime sequence from clone BDGP:GH07919.3prime-hit

4.7 FB:FBgn0036454/sym=CG17839 $/$ name $=/$ prod $=/$ func $=$ cell adhesion $/$ map=71A1-71A1 /transc=CT39634/len=3570 /GB:AE003533

4.7 FB:FBgn0034603/sym=CG9480/name=/prod=glycogenin glucosyltransferase-like /func=enzyme $/$ map=57C7-57C7/transc=CT26834/len=950/GB:AE003453

FB:FBgn0003888 /sym=betaTub60D /name=betaTubulin6D /prod=beta-tubulin /func=cytoskeletal structural protein $/ \mathrm{map}=60 \mathrm{C} 5-60 \mathrm{C} 5 /$ transc $=\mathrm{CT} 11425 /$ len=1542 $/ \mathrm{GB}: \mathrm{AE} 003463$

4.7 FB:FBgn0038151/sym=CG17044 $/$ name $=/$ prod $=/$ func $=/$ map $=87$ E11-87E $11 /$ transc $=$ CT37841 /len $=1281 /$ GB:AE003700

4.6 FB:FBgn0029662 $/ \mathrm{sym}=\mathrm{CG} 12206 /$ name $=/$ prod $=/$ func $=/ \mathrm{map}=3 \mathrm{D} 6-3 \mathrm{D} 6 /$ transc $=\mathrm{CT} 10991 / \mathrm{len}=1446 / \mathrm{GB}: \mathrm{AE} 003427$

FB:FBgn0034112 $/$ sym $=$ CG3896 $/$ name $=/$ prod $=/$ func=ligand binding or carrier $/$ map=53A3-53A4/transc=CT12657/len=3264 $/$ GB:AE003807

4.6 FB:FBgn0037067/sym $=\mathrm{CG} 11310 /$ name $=/$ prod $=/$ func $=$ structural protein $/ \mathrm{map}=78 \mathrm{C} 8-78 \mathrm{C} 8 /$ transc $=\mathrm{CT} 23453 /$ len=384 $/ \mathrm{GB}: \mathrm{AE} 003594$

4.6 FB:FBgn0033679/sym=CG8888 /name=/prod=/func=enzyme /map=48E4-48E6 /transc=CT25512 /len=1694 /GB:AE003823 /note=3prime sequence from clone BDGP:GH26015.3prime-hit

4.6 FB:FBgn0033630 $/ \mathrm{sym}=\mathrm{CG} 13200 /$ name $=/$ prod $=/$ func $=/ \mathrm{map}=47 \mathrm{~F} 6-47 \mathrm{~F} 6 /$ transc $=\mathrm{CT} 32444 / \mathrm{len}=309 / \mathrm{GB}: \mathrm{AE} 003826$

4.6 FB:FBgn0034522 $/$ sym $=$ CG13432 $/$ name $=/$ prod $=/$ func $=/$ map $=57$ A9-57A10 $/$ transc $=$ CT32789 $/$ len $=1355 /$ GB:AE003791

4.6 FB:FBgn0040992 $/ \mathrm{sym}=\mathrm{CG} 10570 /$ name $=/$ prod $=/$ func $=/$ map $=37 \mathrm{~B} 1-37 \mathrm{~B} 1 /$ transc $=\mathrm{CT} 29654 / \mathrm{len}=802 / \mathrm{GB}: \mathrm{AE} 003660 /$ note $=3$ prime sequence from clone BDGP:GH23934.3prime-hit

4.6 FB:FBgn0030798 $/ \mathrm{sym}=\mathrm{CG} 13003 / \mathrm{name}=/ \mathrm{prod}=/$ func $=/ \mathrm{map}=15 \mathrm{~A} 10-15 \mathrm{~A} 10 /$ transc $=\mathrm{CT} 32210 / \mathrm{len}=3477 / \mathrm{GB}: \mathrm{AE} 003503$

4.6 FB:FBgn0029843 /sym=CG5894/name=/prod=endothelin-converting enzyme-like zinc metalloendopeptidase $(\mathrm{M} 13$ peptidase $)$-like /func=endopeptidase $/ \mathrm{map}=5 \mathrm{D} 2-5 \mathrm{D} 2 /$ transc $=\mathrm{CT} 18487 /$ len=2314 FB:FBgn0038896 $/$ sym $=$ CG6328 $/$ name $=/$ prod $=/$ func $=/$ map $=93 F 4-93 F 4 /$ transc $=$ CT19790 $/$ len $=1317 /$ GB:AE003736

FB:FBgn0038977 $/$ sym $=$ CG5376 $/$ name $=/$ prod $=/$ func $=$ motor $/$ map=94B4-94B $/$ transc $=$ CT17018 $/$ len $=763 /$ GB:AE003739

FB:FBgn0039433/sym=CG5467/name=/prod=/func= /map=97B9-97B9 /transc=CT17334/len=2934 /GB:AE003757 /note=3prime sequence from clone BDGP:GH07007.3prime-hit

4.5 FB:FBgn0037697/sym=CG9363/name $=/$ prod $=/$ func=enzyme $/$ map=85D18-85D18 /transc=CT26609/len=1037 /GB:AE003682 /note=3prime sequence from clone BDGP:GH17960.3prime-hit

4.5 FB:FBgn0037721 /sym=CG9427 /name $=/$ prod $=/$ func $=/$ map=85E1-85E2 $/$ transc=CT26734/len=836/GB:AE003683 /note=3prime sequence from clone BDGP:GH19763.3prime-hit

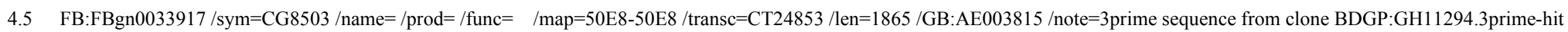
FB:FBgn0031193 $/$ sym $=$ CG17602 $/$ name $=/$ prod $=/$ func $=/$ map $=20 A 1-20 A 1 /$ transc $=$ CT34368 $/$ len $=1152 /$ GB:AE003574

4.4 FB:FBgn0038088 $/$ sym $=$ CG10126/name $=/$ prod $=$ calcium binding protein-like $/$ func $=$ ligand binding or carrier $/$ map $=87 \mathrm{D} 3-87 \mathrm{D} 3 /$ transc $=\mathrm{CT} 28495 /$ len $=879 / \mathrm{GB}: \mathrm{AE} 003697 /$ note $=3$ prime sequence fro

4.4 FB:FBgn0015801 /sym=Reg-5/name=Rhythmically expressed gene $5 /$ prod $=/$ func $=/ \mathrm{map}=60 \mathrm{D} 15-60 \mathrm{D} 16 /$ transc $=\mathrm{CT} 9939 /$ len $=1650 /$ GB:AE003465 $/$ note $=3$ prime sequence from clone BDGP:HL047

4.4 FB:FBgn0036547/sym $=$ CG17032 $/$ name $=/$ prod $=/$ func $=/$ map $=72 \mathrm{C} 1-72 \mathrm{C} 1 /$ transc $=\mathrm{CT} 32296 / \mathrm{len}=1007 / \mathrm{GB}: \mathrm{AE} 003529$

4.4 FB:FBgn0033109/sym $=\mathrm{CG} 9446 /$ name $=/$ prod $=/$ func $=$ chaperone $/ \mathrm{map}=42 \mathrm{C} 8-42 \mathrm{C} 8 /$ transc $=\mathrm{CT} 11617 / \mathrm{len}=1880 / \mathrm{GB}: \mathrm{AE} 003790$

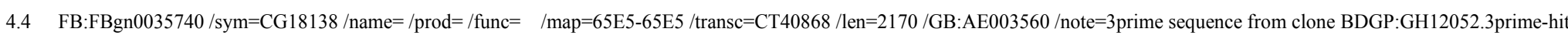

4.4 FB:FBgn0035917/sym=CG6416/name $=/$ prod $=/$ func $=$ enzyme $/$ map=66D9-66D10/transc=CT20013 /len=1175 /GB:AE003554/note=3prime sequence from clone BDGP:GH19182.3prime-hit 
$3588.9 \sim 4.4$

$2988.3 \sim 4.4$

110661.9

34249.9

25547.3

11289.1

82296.8

37898.4

11320.1

3702.5

4432.4

6566.3

1895.4

16413.7

26327.7

$3681.9 \sim 4.2$

20932.2

$2247.7 \sim 4.2$

$3098.4 \sim 4.2$

$3047.3 \sim 4.2$

11736.9

23343.7

175868.2

99849.6

180907.8

58068.8

10733.4
FB:FBgn0036101 /sym=CG6449 /name $=/$ prod=ninjurin $/$ func $=$ cell adhesion $\quad /$ map=67E5-67E $5 /$ transc $=$ CT20102 $/$ len $=591 /$ GB:AE003547

BDGP:SD1276.3prime-hit /ESTpos=maps 3prime of FB:FBgn0003638/sym=su(w[a]) /name=suppressor of white-apricot $/$ prod=/func=RNA binding $/$ map=1E1-1E1/transc=CT1162 /len=584

4.3 FB:FBgn0034044 /sym=CG12969 /name $=/$ prod $=/$ func $=$ enzyme $/$ map=52C7-52C $/$ transc=CT32161 /len=1203 /GB:AE003809

4.3 FB:FBgn0032685/sym=CG10211/name=/prod=peroxidase-like /func=enzyme $/$ map=37A1-37A1/transc=CT28633 /len=3691/GB:AE003659 /note=3prime sequence from clone BDGP:LD42267.3p

4.3 FB:FBgn0033033 $/ \mathrm{sym}=\mathrm{CG} 11066 /$ name $=/$ prod $=/$ func $=$ endopeptidase $/ \mathrm{map}=41 \mathrm{E} 3-41 \mathrm{E} 4 /$ transc $=\mathrm{CT} 30959 / \mathrm{len}=1377 / \mathrm{GB}: \mathrm{AE} 003785$

4.3 FB:FBgn0030355/sym $=\mathrm{CG} 2467 /$ name $=/$ prod $=/$ func $=/$ map=10F7-10F $/$ transc $=$ CT8145 $/$ len $=3418 /$ GB:AE003487 $/$ note $=3$ prime sequence from clone BDGP:GH09980.3prime-hit

4.3 FB:FBgn0031037/sym=CG14207/name $=/$ prod=heat shock protein HSP20-like /func=chaperone $/$ map=18D8-18D8 /transc $=\mathrm{CT} 33820 /$ len=1175 /GB:AE003512 /note $=3$ prime sequence from clone B FB:FBgn0000275/sym=Pka-R1 /name $=\mathrm{cAMP}-$ dependent protein kinase R1 /prod $=\mathrm{cAMP}-$ dependent protein kinase, regulatory subunit /func $=\mathrm{cAMP}-$ dependent protein kinase regulator $/$ map $=77 \mathrm{~F} 1-7$ 4.3 clon

4.3 BDGP:GH4896.3prime-hit /ESTpos=maps in FB:FBgn0033462/sym=CG12138/name=/prod=/func=DNA binding $/ \mathrm{map}=46 \mathrm{C} 4-46 \mathrm{C} 4 /$ transc $=\mathrm{CT} 7814 / \mathrm{len}=700$

FB:FBgn0011656/sym=Mef2 /name=Myocyte enhancing factor $2 /$ prod= /func=RNA polymerase II transcription factor $/$ map=46C6-46C7 /transc=CT3413/len=3211 /GB:AE003831 /note=3prime sec

4.3 FB:FBgn0031938 $/$ sym $=$ CG18590 $/$ name $=/$ prod $=/$ func $=/$ map $=28 \mathrm{C} 2-28 \mathrm{C} 2 /$ transc $=\mathrm{CT} 42499 / \mathrm{len}=176 / \mathrm{GB}: \mathrm{AE} 003618$

4.3 FB:FBgn0036673 /sym=CG11915/name=/prod=/func=ligand binding or carrier $/$ map=73D3-73D3 /transc=CT37088 /len=2999 /GB:AE003525 /note=3prime sequence from clone BDGP:GH17145.3 FB:FBgn0033936 $/$ sym $=$ CG17386 $/$ name $=/$ prod= $/$ func=RNA binding $/$ map=51A1-51A1/transc=CT33484/len=2609 $/$ GB:AE003814

4.3 FB:FBgn0032456/sym=CG6214 /name=/prod=ATP-binding cassette transporter; multidrug resistance protein-like $/$ func $=$ ion channel $/ \mathrm{map}=33 \mathrm{~F} 2-33 \mathrm{~F} 3 /$ transc $=\mathrm{CT} 19412 /$ len $=6461 / \mathrm{GB}: \mathrm{AE} 003637 / \mathrm{n}$

4.2 FB:FBgn0033133 $/$ sym $=$ CG12841 $/$ name $=/$ prod $=/$ func $=/$ map $=42 \mathrm{E} 1-42 \mathrm{E} 1 /$ transc $=\mathrm{CT} 31973 / \mathrm{len}=648 / \mathrm{GB}: \mathrm{AE} 003842$

FB:FBgn0004620/sym=Glu-RIIA /name=Glutamate receptor IIA /prod=glutamate receptor/func=glutamate receptor /map=25F1-25F1/transc=CT21587/len=3022 /GB:AE003610

4.2 FB:FBgn0029517 $/$ sym $=$ CG13377 $/$ name $=/$ prod $=/$ func $=/$ map $=1$ A5-1 $55 /$ transc $=$ CT32709 $/$ len $=993 /$ GB:AE003417

FB:FBgn0038987 $/$ sym $=$ CG6926 $/$ name $=/$ prod $=/$ func $=/$ map $=94 B 8-94 B 8 /$ transc $=C T 21454 /$ len $=789 /$ GB:AE003739

FB:FBgn0032616 $/$ sym $=$ CG15131 $/$ name $=/$ prod $=/$ func $=/$ map $=36$ A10-36A10 $/$ transc $=$ CT35026 $/$ len $=862 /$ GB:AE003652

FB:FBgn0022703 /sym=Cht1 /name=Chitinase $1 /$ prod=chitinase $/$ func=chitinase ; EC:3.2.1.14/map=40D5-40E1/transc=CT39094/len=1527/GB:AE002743

4.2 FB:FBgn0038720 $/$ sym $=$ CG6231 $/$ name $=/$ prod $=$ organic cation transporter $/$ func=transporter $/$ map $=92 \mathrm{~A} 13-92 \mathrm{~A} 13 /$ transc $=\mathrm{CT} 19536 / \mathrm{len}=1743 / \mathrm{GB}: \mathrm{AE} 003727$

4.2 FB:FBgn0030876 $/$ sym $=\mathrm{CG} 6762 /$ name $=/$ prod $=/$ func $=/ \mathrm{map}=16 \mathrm{D} 7-16 \mathrm{D} 7 /$ transc $=\mathrm{CT} 20293 / \mathrm{len}=580 / \mathrm{GB}: \mathrm{AE} 003507$

4.2 FB:FBgn0033729 $/ \mathrm{sym}=\mathrm{CG} 8510 /$ name $=/$ prod=cuticle protein-like $/$ func $=$ structural protein $/ \mathrm{map}=49 \mathrm{~A} 3-49 \mathrm{~A} 3 / \mathrm{transc}=\mathrm{CT} 24877 / \mathrm{len}=381 / \mathrm{GB}: \mathrm{AE} 003822$

4.2 FB:FBgn0001258 /sym=ImpL3 /name=Ecdysone-inducible gene L3 /prod=L-lactate dehydrogenase $/$ func=L-lactate dehydrogenase $; \mathrm{EC}: 1.1 .1 .27 / \mathrm{map}=65 \mathrm{~A} 8-65 \mathrm{~A} 8 /$ transc $=\mathrm{CT} 28577 /$ len=999 $/ \mathrm{GB}: \mathrm{AE} 0$

4.1 FB:FBgn0032777 $/$ sym $=$ CG18576 $/$ name $=/$ prod $=/$ func $=/$ map $=37 \mathrm{E} 1-37 \mathrm{E} 1 /$ transc $=\mathrm{CT} 42454 / \mathrm{len}=1593 / \mathrm{GB}: \mathrm{AE} 003662$

4.1 FB:FBgn0037069/sym=CG7658 /name $=/$ prod=cuticle protein-like $/$ func $=$ structural protein $/ \mathrm{map}=78 \mathrm{C} 8-78 \mathrm{C} 8 /$ transc $=\mathrm{CT} 23381 / \mathrm{len}=511 / \mathrm{GB}: \mathrm{AE} 003594$

4.1 FB:FBgn0017397/sym=how $/$ name=held out wings $/$ prod= $/$ func=RNA binding $/$ map=94A1-94A2/transc=CT28865 /len=1922 /GB:AE003737

FB:FBgn0032851 $/$ sym $=$ CG13970 $/$ name $=/$ prod $=/$ func $=/$ map $=38 \mathrm{C} 1-38 \mathrm{C} 1 /$ transc $=\mathrm{CT} 33523 / \mathrm{len}=618 / \mathrm{GB}: \mathrm{AE} 003665$

FB:FBgn0004852 /sym=Ac76E /name=Adenylyl cyclase 76E /prod=adenylate cyclase $/$ func=adenylate cyclase $;$ EC:4.6.1.1 /map=76D8-76E1/transc=CT23940 $/$ len=5189 $/$ GB:AE003515 /note=3prime

4.1 FB:FBgn0030759 $/ \mathrm{sym}=\mathrm{CG} 13014 /$ name $=/$ prod $=/$ func $=/ \mathrm{map}=14 \mathrm{~F} 2-14 \mathrm{~F} 2 /$ transc $=\mathrm{CT} 32227 / \mathrm{len}=699 / \mathrm{GB}: \mathrm{AE} 003502$

4.1 FB:FBgn0032025/sym $=\mathrm{CG} 7778 /$ name $=/$ prod $=/$ func $=/ \mathrm{map}=29 \mathrm{~B} 1-29 \mathrm{~B} 2 /$ transc $=\mathrm{CT} 23662 / \mathrm{len}=1222 / \mathrm{GB}: \mathrm{AE} 003620 /$ note $=3$ prime sequence from clone BDGP:GH17475.3prime-hit

4.1 FB:FBgn0014863 /sym=Mlp84B /name=Muscle LIM protein at 84B /prod=muscle LIM protein at 84B /func=transcription factor $/ \mathrm{map}=84 \mathrm{C} 3-84 \mathrm{C} 3 /$ transc $=\mathrm{CT} 1058 /$ len=1863 $/ \mathrm{GB}: \mathrm{AE} 003672$ 
4.1 FB:FBgn0038842 $/$ sym $=$ CG5630 $/$ name $=/$ prod $=/$ func $=/$ map $=93$ A4-93A4 $/$ transc $=C T 17794 /$ len $=1360 /$ GB:AE003732

4.1 FB:FBgn0037213 $/$ sym=CG12581 $/$ name $=/$ prod $=/$ func $=/$ map=82A1-82A1 /transc=CT34405/len=2382 /GB:AE003607 /note=3prime sequence from clone BDGP:GM02933.3prime-hit FB:FBgn0031700 $/$ sym $=$ CG14022 $/$ name $=/$ prod $=/$ func $=$ enzyme $/$ map=25D5-25D $/$ transc $=$ CT33581 /len=334 /GB:AE003609

4.1 FB:FBgn0034067/sym=CG8399 $/$ name $=/$ prod $=/$ func $=/$ map=52E1-52E3 $/$ transc=CT18617/len=2268 /GB:AE003808 /note=3prime sequence from clone BDGP:LD47639.3prime-hit FB:FBgn0037275/sym=CG14655/name=/prod=/func=nucleic acid binding $/$ map=82E1-82E1/transc=CT34432/len=2167/GB:AE003605/note=3prime sequence from clone BDGP:GH23506.3prim

4 FB:FBgn0034575/sym=CG15652/name $=/$ prod $=/$ func $=/$ map $=57$ B16-57B16 $/$ transc $=$ CT35836/len=1311 /GB:AE003452 /note=3prime sequence from clone BDGP:GH14953.3prime-hit

4 FB:FBgn0033532/sym $=$ CG18380 $/$ name $=/$ prod $=/$ func $=/$ map $=47$ A11-47A11 $/$ transc $=$ CT41783 /len $=657 /$ GB:AE003829

FB:FBgn0008649 /sym=dei /name $=$ delilah /prod $=/$ func $=$ specific RNA polymerase II transcription factor $/$ map=97B2-97B2 /transc=CT17256/len=1862 /GB:AE003756 /note $=3$ prime sequence from c

4 FB:FBgn0022800/sym=HD-14 /name $=/$ prod=fibroblast growth factor receptor-like /func=protein tyrosine kinase ; EC:2.7.1.11 inferred from sequence similarity $/ \mathrm{map}=96 \mathrm{C} 2-96 \mathrm{C} 2 /$ transc $=\mathrm{CT} 28777 / 1$

4 FB:FBgn0039667/sym=CG2010 /name=/prod= /func= /map=99B3-99B3 /transc=CT6431/len=1752 /GB:AE003769 /note=3prime sequence from clone BDGP:GH26184.3prime-hit

4 FB:FBgn0004028/sym=wupA /name=wings up A /prod=troponin I /func=cytoskeletal structural protein /map=16F7-17A1/transc=CT40012/len=389/GB:AE003507 FB:FBgn0033345 / $/$ ym $=$ CG13750 $/$ name $=/$ prod $=/$ func $=/$ map $=44 F 9-44 F 9 /$ transc $=$ CT33227 $/$ len $=228 /$ GB:AE003835

FB:FBgn0030758 /sym=CG9819/name=/prod=/func=protein phosphatase $/$ map=14F1-14F1/transc=CT27722/len=1724 /GB:AE003502

4 FB:FBgn0032284 $/$ sym $=$ CG7294 $/$ name $=/$ prod $=/$ func $=/$ map $=32 \mathrm{~A} 1-32 \mathrm{~A} 1 /$ transc $=\mathrm{CT} 22525 / \mathrm{len}=527 / \mathrm{GB}: \mathrm{AE} 003629$

4 FB:FBgn0031097/sym=CG17052/name=/prod=peritrophin-like /func=structural protein $/ \mathrm{map}=19 \mathrm{C} 1-19 \mathrm{C} 1 /$ transc $=\mathrm{CT} 37860 / \mathrm{len}=1383 / \mathrm{GB}: \mathrm{AE} 003572$

4 FB:FBgn0032279 $/ \mathrm{sym}=\mathrm{CG17104} /$ name $=/$ prod $=/$ func $=/ \mathrm{map}=32 \mathrm{~A} 1-32 \mathrm{~A} 1 /$ transc=CT38006 $/$ len=1746 $/$ GB:AE003629 $/$ note $=3$ prime sequence from clone BDGP:LD16579.3prime-hit

4 FB:FBgn0039742/sym=CG15528/name=/prod=protein phosphatase-like /func=protein phosphatase /map=99D5-99D5/transc=CT35642/len=639/GB:AE003772

4 FB:FBgn0036674 /sym=CG11916/name $=/$ prod= $/$ func=transcription factor $/$ map=73D4-73D4/transc=CT37092/len=707 /GB:AE003525

4 FB:FBgn0022341 /sym $=\mathrm{CG} 17467 /$ name $=/$ prod $=/$ func $=/$ map $=102 \mathrm{E} 3-102 \mathrm{E} 3 /$ transc $=\mathrm{CT} 38621 / \mathrm{len}=1002 / \mathrm{GB}: \mathrm{AE} 003846$

4 FB:FBgn0034701 $/$ sym $=$ CG13505 $/$ name $=/$ prod $=/$ func $=/$ map $=58 \mathrm{C} 5-58 \mathrm{C} 5 /$ transc $=\mathrm{CT} 32873 / \mathrm{len}=1011 / \mathrm{GB}: \mathrm{AE} 003456$

3.9 FB:FBgn0036675/sym=CG9959/name $=/$ prod $=/$ func=transcription factor $/$ map=73D4-73D5 /transc=CT28055/len=883 /GB:AE003525

FB:FBgn0032178 $/$ sym=CG4804 /name $=/$ prod=serpin $/$ func=serpin $/$ map=31A1-31A1 /transc $=C$ T15443 /len=1259 /GB:AE003627

3.9 FB:FBgn0028855/sym=BG:DS07721.3 $/$ name $=/$ prod $=/$ func $=/$ map=35B4-35B4 $/$ transc $=$ CT35230 $/$ len=465 $/$ GB:AE003644

3.9 FB:FBgn0038674 /sym=CG14285/name $=/$ prod $=/$ func=transcription factor binding $/$ map=91E4-91E4 $/$ transc $=\mathrm{CT} 33914 /$ len $=894 / \mathrm{GB}: \mathrm{AE} 003724$

3.9 FB:FBgn0036572/sym=CG5165/name=/prod=phosphoglucomutase-like /func=enzyme /map=72D7-72D7/transc=CT16539/len=1766 /GB:AE003528 /note=3prime sequence from clone BDGP:LD

3.9 FB:FBgn0035710/sym $=$ SP1173 $/$ name $=/$ prod $=/$ func $=/$ map $=65 \mathrm{C} 1-65 \mathrm{C} 1 /$ transc $=\mathrm{CT} 28481 / \mathrm{len}=2494 / \mathrm{GB}: \mathrm{AE} 003561 /$ note $=3$ prime sequence from clone BDGP:GH14073.3prime-hit

3.9 FB:FBgn0004507 /sym=GlyP /name=Glycogen phosphorylase /prod=glycogen phosphorylase /func=phosphorylase ; EC:2.4.1.1 inferred from direct assay $/ \mathrm{map}=22 \mathrm{C} 3-22 \mathrm{C} 3 /$ transc=CT22383 /len=287

3.9 FB:FBgn0032474 /sym $=\mathrm{CG} 9828 /$ name $=/$ prod $=/$ func $=$ chaperone $/ \mathrm{map}=34 \mathrm{~A} 8-34 \mathrm{~A} 8 /$ transc $=\mathrm{CT} 27734 / \mathrm{len}=1253 / \mathrm{GB}: \mathrm{AE} 003639$

FB:FBgn0037972 $/$ sym $=$ CG10005 $/$ name $=/$ prod $=/$ func $=/$ map $=87$ A8 -87 A $8 /$ transc $=$ CT28191 $/$ len $=684 /$ GB:AE003693

3.9 FB:FBgn0004577/sym=Pxd /name=Peroxidase $/$ prod=peroxidase $/$ func=peroxidase $; \mathrm{EC}: 1.11 .1 .7 / \mathrm{map}=89 \mathrm{E} 11-89 \mathrm{E} 11 /$ transc $=\mathrm{CT} 11695 / \mathrm{len}=2071 / \mathrm{GB}: \mathrm{AE} 003716$

3.8 FB:FBgn0035494/sym=CG14993 /name=/prod=fumarylacetoacetase-like /func=enzyme $/$ map=64A5-64A5 /transc=CT34846/len=1050/GB:AE003480 FB:FBgn0040587 $/$ sym $=$ CG17618 $/$ name $=/$ prod $=/$ func $=/$ map $=94$ C4 $-94 \mathrm{C} 4 /$ transc $=$ CT38876 $/$ len $=576 /$ GB:AE003740 
3.8 FB:FBgn0032332 $/ \mathrm{sym}=\mathrm{CG} 14917 / \mathrm{name}=/ \mathrm{prod}=/ \mathrm{func}=/ \mathrm{map}=32 \mathrm{D} 1-32 \mathrm{D} 1 / \operatorname{transc}=\mathrm{CT} 34744 / \mathrm{len}=1863 / \mathrm{GB}: \mathrm{AE} 003630$ FB:FBgn0035385/sym=CG2114/name $=/$ prod=G-protein coupled receptor-like $/$ func $=\mathrm{G}$ protein linked receptor $/ \mathrm{map}=63 \mathrm{~B} 2-63 \mathrm{~B} 2 /$ transc $=\mathrm{CT} 2366 /$ len $=1650 / \mathrm{GB}: \mathrm{AE} 003476$

3.8 BDGP:GH1742.3prime-hit /ESTpos=maps in FB:FBgn0033441 / $/ \mathrm{sym}=\mathrm{CG} 1776 /$ name $=/$ prod=myosin light chain kinase $/$ func $=$ protein kinase $/ \mathrm{map}=46 \mathrm{~A} 3-46 \mathrm{~A} 4 / \mathrm{transc}=\mathrm{CT} 5322 / \mathrm{len}=488$

3.8 FB:FBgn0037167/sym=CG11425/name=/prod=phosphatidate phosphohydrolase type 2-like /func=enzyme /map=79E4-79E4/transc=CT21215/len=956 /GB:AE003597

3.8 FB:FBgn0032785 /sym=CG10026/name = /prod=alpha-tocopherol transfer relative protein /func=transcription factor $/ \mathrm{map}=37 \mathrm{E} 3-37 \mathrm{E} 3 / \mathrm{transc}=\mathrm{CT} 28189 / \mathrm{len}=1143 / \mathrm{GB}: \mathrm{AE} 003663 /$ note $=3 \mathrm{prime}$ sequ

3.8 FB:FBgn0014141/sym=cher /name=cheerio /prod=filamin /func=actin binding /map=89F1-89F $1 /$ transc=CT12961 /len=7617 /GB:AE003716 /note=3prime sequence from clone BDGP:SD05640.3pri FB:FBgn0010015/sym=CanA1 /name=Calcineurin A1 /prod=protein serine/threonine phosphatase /func=protein serine/threonine phosphatase ; EC:3.1.3.16 | inferred from sequence similarity $/$ map=10

3.8 FB:FBgn0032280 $/$ sym $=\mathrm{CG} 17105 /$ name $=/$ prod $=/$ func $=/$ map $=32 \mathrm{~A} 1-32 \mathrm{~A} 1 /$ transc $=\mathrm{CT} 33652 / \mathrm{len}=312 / \mathrm{GB}: \mathrm{AE} 003629$

FB:FBgn0030586/sym=CG12539/name=/prod=glucose dehydrogenase (acceptor) /func=enzyme $/$ map=13A1-13A1 /transc=CT34058/len=1503/GB:AE003497

3.8 FB:FBgn0032283 $/$ sym $=$ CG7296 $/$ name $=/$ prod $=/$ func $=/$ map $=32 \mathrm{~A} 1-32 \mathrm{~A} 1 /$ transc $=\mathrm{CT} 22519 / \mathrm{len}=575 / \mathrm{GB}: \mathrm{AE} 003629$

3.8 FB:FBgn0036550/sym $=\mathrm{CG} 17026 /$ name $=/$ prod $=/$ func $=/ \mathrm{map}=72 \mathrm{C} 1-72 \mathrm{C} 1 /$ transc $=\mathrm{CT} 37805 / \mathrm{len}=855 / \mathrm{GB}: \mathrm{AE} 003529$ FB:FBgn0033092 $/$ sym $=$ CG9422 $/$ name $=/$ prod $=/$ func $=/$ map $=42 \mathrm{C} 1-42 \mathrm{C} 1 /$ transc $=\mathrm{CT} 26724 / \mathrm{len}=621 / \mathrm{GB}: \mathrm{AE} 003789$ FB:FBgn0038451/sym=CG14893 /name=/prod=/func=enzyme $/$ map=89D6-89D6 /transc=CT34717/len=1491 /GB:AE003714

3.8 FB:FBgn0031198 $/$ sym $=\mathrm{CG} 13238 /$ name $=/$ prod $=/$ func $=/$ map $=20 \mathrm{~A} 1-20 \mathrm{~A} 1 /$ transc $=\mathrm{CT} 32487 / \mathrm{len}=405 / \mathrm{GB}: \mathrm{AE} 003574$

3.8 FB:FBgn0011296/sym=1(2)efl /name=lethal (2) essential for life /prod=/func=chaperone /map=59F4-59F4/transc=CT14702/len=717/GB:AE003461 /note=3prime sequence from clone BDGP:GH0

3.8 FB:FBgn0035303/sym=CG5699/name=/prod=/func=cell adhesion $/ \mathrm{map}=62 \mathrm{C} 3-62 \mathrm{C} 3 /$ transc=CT2689 /len=1990 /GB:AE003473 /note=3prime sequence from clone BDGP:GH09541.3prime-hit

3.8 FB:FBgn0031534/sym=CG2774 /name=/prod=sorting nexin 1-like /func=transporter $/$ map=23F2-23F2/transc=CT9125/len=1450/GB:AE003579

3.8 FB:FBgn0031421 /sym $=$ CG9867 $/$ name $=/$ prod $=/$ func $=/$ map=22E1-22E2 $/$ transc $=$ CT27848 $/$ len=1826 $/$ GB:AE003583 $/$ note $=3$ prime sequence from clone BDGP:GH05422.3prime-hit FB:FBgn0039927/sym=CG11155/name=/prod=glutamate receptor-like /func=ion channel $/$ map=102E7-102F1/transc=CT30863/len=2062/GB:AE003846

FB:FBgn0031080 $/$ sym $=$ CG12655 $/$ name $=/$ prod $=/$ func $=/$ map $=19 A 3-19$ A3 $/$ transc $=$ CT35310 $/$ len $=496 /$ GB:AE00261 1

3.7 FB:FBgn0033034 $/ \mathrm{sym}=\mathrm{CG} 15900 /$ name $=/$ prod $=/$ func $=/ \mathrm{map}=41 \mathrm{E} 4-41 \mathrm{E} 4 /$ transc $=\mathrm{CT} 34160 / \mathrm{len}=519 / \mathrm{GB}: \mathrm{AE} 003785$

3.7 FB:FBgn0032422 $/ \mathrm{sym}=\mathrm{CG} 6579 /$ name $=/$ prod $=/$ func $=/ \mathrm{map}=33 \mathrm{C} 3-33 \mathrm{C} 4 /$ transc $=\mathrm{CT} 20347 / \mathrm{len}=834 / \mathrm{GB}: \mathrm{AE} 003635$

3.7 FB:FBgn0023023 /sym=CRMP $/$ name $=$ Collapsin Response Mediator Protein $/$ prod $=/$ func $=$ enzyme $/$ map $=83 B 3-83 B 4 /$ transc $=\mathrm{CT} 3290 /$ len $=2554 / \mathrm{GB}: \mathrm{AE} 003602 /$ note $=3$ prime sequence from clone B

3.7 FB:FBgn0038096 $/$ sym $=$ CG7340 $/$ name $=/$ prod $=/$ func $=/$ map=87D7-87D7 $/$ transc $=$ CT22347 /len=1953 /GB:AE003698 $/$ note $=3$ prime sequence from clone BDGP $:$ LD13869.3prime-hit

3.7 FB:FBgn0038009/sym $=$ CG17738 $/$ name $=/$ prod $=/$ func $=/$ map $=87 \mathrm{~B} 8-87 \mathrm{~B} 8 /$ transc $=$ CT34537 $/$ len $=333 /$ GB:AE003695 FB:FBgn0037813 $/$ sym $=\mathrm{CG} 17100 /$ name $=/$ prod $=/$ func $=/ \mathrm{map}=86 \mathrm{~A} 5-86 \mathrm{~A} 5 /$ transc $=\mathrm{CT} 34464 / \mathrm{len}=2030 / \mathrm{GB}: \mathrm{AE} 003687$

3.7 FB:FBgn0034797/sym=CG12781/name=/prod=/func=protein kinase $/$ map=59B4-59B4/transc=CT36793/len=4470/GB:AE003459/note=3prime sequence from clone BDGP:GH04942.3prime-hit

3.7 BDGP:LD43683.3prime-hit /ESTpos=maps in FB:FBgn0031097/sym=CG17052/name=/prod=peritrophin-like /func=structural protein $/ \mathrm{map}=19 \mathrm{C} 1-19 \mathrm{C} 1 /$ transc $=\mathrm{CT} 3786 /$ len $=595$

3.7 FB:FBgn0035550/sym=CG11349 $/$ name $=/$ prod $=/$ func $=$ structural protein $/ \mathrm{map}=64 \mathrm{~B} 12-64 \mathrm{~B} 12 / \operatorname{transc}=\mathrm{CT} 31656 /$ len $=1880 / \mathrm{GB}: \mathrm{AE} 003481$

3.7 FB:FBgn0015276/sym=Pcmt/name=Protein-L-isoaspartate (D-aspartate) O-methyltransferase /prod=protein-L-isoaspartate (D-aspartate) O-methyltransferase /func=protein-L-isoaspartate (D-aspartate)

3.7 FB:FBgn0033309/sym=CG8735/name $=/$ prod $=/$ func=electron transfer $/$ map=44D1-44D2/transc=CT8725/len=1421 /GB:AE003836/note=3prime sequence from clone BDGP:GH24644.3prime-hit FB:FBgn0040874 $/$ sym $=\mathrm{CG} 15600 /$ name $=/$ prod $=/$ func $=/$ map $=13 \mathrm{E} 12-13 \mathrm{E} 12 /$ transc $=\mathrm{CT} 35723 / \mathrm{len}=153 / \mathrm{GB}: \mathrm{AE} 003499$ 
3.7 FB:FBgn0036902 $/$ sym $=$ CG8748 $/$ name $=/$ prod $=/$ func $=/$ map $=76 \mathrm{C} 4-76 \mathrm{C} 4 /$ transc $=\mathrm{CT} 25246 /$ len $=1263 / \mathrm{GB}: \mathrm{AE} 003516$

3.7 FB:FBgn0036901 /sym=CG8756/name $=/$ prod=low-density lipoprotein-receptor-like $/$ func=receptor $/$ map=76C3-76C3 /transc $=\mathrm{CT} 25252 /$ len=2157 /GB:AE003516 $/$ note $=3$ prime sequence from clone FB:FBgn0032469 $/$ sym $=$ CG9932 $/$ name $=/$ prod $=/$ func $=$ nucleic acid binding $/$ map=34A5-34A5 $/$ transc=CT27960/len=6516 $/$ GB:AE003638 FB:FBgn0040981 /sym=CG15268 $/$ name $=/$ prod $=/$ func $=/$ map $=35$ B9-35B $/$ transc $=$ CT35215 $/$ len $=195 /$ GB:AE003645

3.7 FB:FBgn0034042 /sym $=$ CG8242 $/$ name $=/$ prod $=/$ func $=$ transcription factor $/ \mathrm{map}=52 \mathrm{C} 7-52 \mathrm{C} 7 /$ transc $=\mathrm{CT} 24455 /$ len $=4875 / \mathrm{GB}: \mathrm{AE} 003809$

3.6 FB:FBgn0033502 $/ \mathrm{sym}=\mathrm{CG} 12910 /$ name $=/$ prod $=/$ func $=/ \mathrm{map}=46 \mathrm{~F} 7-46 \mathrm{~F} 7 /$ transc $=\mathrm{CT} 32055 /$ len $=1690 / \mathrm{GB}: \mathrm{AE} 003830 /$ note $=3$ prime sequence from clone BDGP $:$ GH17442.3prime-hit

3.6 FB:FBgn0031064 /sym=CG12531/name $=/$ prod=amino-acid permease $/$ func=transporter $/ \mathrm{map}=18 \mathrm{E} 3-18 \mathrm{E} 5 /$ transc $=\mathrm{CT} 33850 / \mathrm{len}=2415 / \mathrm{GB}: \mathrm{AE} 003513$

3.6 FB:FBgn0035612/sym=CG10625/name=/prod=/func= /map=64E2-64E2 /transc=CT29764/len=3883 /GB:AE003565 /note=3prime sequence from clone BDGP:LD39545.3prime-hit FB:FBgn0034194 /sym=CG15611/name $=/$ prod=guanyl-nucleotide exchange factor $/$ func=signal transduction $/ \mathrm{map}=53 \mathrm{~F} 12-53 \mathrm{~F} 13 /$ transc $=\mathrm{CT} 35747 /$ len=1742 $/ \mathrm{GB}: \mathrm{AE} 003804 /$ note $=3$ prime sequence

3.6 FB:FBgn0000565 /sym=Eip71CD /name=Ecdysone-induced protein 28/29kD /prod=protein-methionine-S-oxide reductase-like /func=protein-methionine-S-oxide reductase ; EC:1.8.4.6 | inferred from s FB:FBgn0004879/sym=plx /name=pollux $/$ prod $=/$ func $=/$ map $=83$ B8-83B9 $/$ transc $=$ CT1567 /len $=5472 /$ GB:AE003602

3.6 FB:FBgn0034075/sym=CG18658/name=/prod=peptide-aspartate beta-dioxygenase-like /func=enzyme $/ \mathrm{map}=52 \mathrm{~F} 1-52 \mathrm{~F} 1 /$ transc $=\mathrm{CT} 18815 /$ len=1662 $/$ GB:AE003808 FB:FBgn0033942 $/$ sym $=\mathrm{CG} 10112 /$ name $=/$ prod $=/$ func=structural protein $/ \mathrm{map}=51 \mathrm{~A} 6-51 \mathrm{~A} 6 /$ transc $=\mathrm{CT} 28465 / \mathrm{len}=435 / \mathrm{GB}: \mathrm{AE} 003814$

3.6 FB:FBgn0037911 /sym=CG10898 /name $=/$ prod=7,8-dihydro-8-oxoguanine-triphosphatase $/$ func=DNA repair protein $/$ map $=86 \mathrm{E} 13-86 \mathrm{E} 13 /$ transc $=\mathrm{CT} 30521 / \mathrm{len}=1936 / \mathrm{GB}: \mathrm{AE} 003692 /$ note $=3$ prime 3.6 FB:FBgn0040993 $/$ sym $=$ CG17325 $/$ name $=/$ prod $=/$ func $=/$ map $=37$ B1 -37 B $2 /$ transc $=$ CT35069 $/$ len $=765 /$ GB:AE003660

3.6 FB:FBgn0028915/sym=BG:DS01068.5/name=/prod=serine endopeptidase $/$ func=endopeptidase $/ \mathrm{map}=35 \mathrm{~A} 1-35 \mathrm{~A} 1 /$ transc=CT23067 /len=530/GB:AE003643

3.6 FB:FBgn0036659/sym=CG9701/name=/prod=beta-glucosidase-like /func=ion channel $/$ map=73B5-73B5 /transc=CT27420/len=1936/GB:AE003526 /note=3prime sequence from clone BDGP:LP0

3.6 FB:FBgn0004117/sym=Tm2 $/$ name=Tropomyosin $2 /$ prod=tropomyosin $/$ func=motor $/$ map=88E11-88E $12 /$ transc=CT15467 /len=914 /GB:AE003708

3.6 FB:FBgn0036875/sym=CG9449/name=/prod=acid phosphatase-like /func=enzyme /map=76B6-76B6/transc=CT26772/len=1179/GB:AE003516

3.6 FB:FBgn0033968 $/ \mathrm{sym}=\mathrm{CG} 10200 /$ name $=/$ prod $=/$ func $=/ \mathrm{map}=51 \mathrm{C} 5-51 \mathrm{C} 5 /$ transc $=\mathrm{CT} 28697 /$ len $=659 /$ GB:AE003813 $/$ note $=3$ prime sequence from clone BDGP $:$ LP02570.3prime-hit

3.5 FB:FBgn0033919/sym $=\mathrm{CG} 8547 /$ name $=/$ prod $=/$ func $=$ DNA binding $/ \mathrm{map}=50 \mathrm{~F} 1-50 \mathrm{~F} 1 /$ transc $=\mathrm{CT} 24943 / \mathrm{len}=1313 / \mathrm{GB}: \mathrm{AE} 003815$ FB:FBgn0035590 $/$ sym $=\mathrm{CG} 10673 /$ name $=/$ prod $=/$ func=protein kinase $/$ map $=64 \mathrm{C} 12-64 \mathrm{C} 12 /$ transc $=\mathrm{CT} 29894 /$ len $=675 / \mathrm{GB}: \mathrm{AE} 003567$

3.5 FB:FBgn0030745/sym=CG4239/name $=/$ prod $=/$ func $=/ \mathrm{map}=14 \mathrm{C} 3-14 \mathrm{C} 4 /$ transc=CT13936/len=1639 /GB:AE003502 /note=3prime sequence from clone BDGP:GH25683.3prime-hit FB:FBgn0000719 $/$ sym=fog $/$ name $=$ folded gastrulation $/$ prod $=/$ func $=/$ map=20A4-20A5 $/$ transc $=$ CT16970 $/$ len=3059 $/$ GB:AE003573 $/$ note $=3$ prime sequence from clone BDGP:SD02223.3prime-hit

3.5 FB:FBgn0031293/sym=CG4226/name=/prod=AMPA/kainate-selective ionotrophic glutamate receptor-like /func=ion channel $/ \mathrm{map}=21 \mathrm{D} 4-21 \mathrm{D} 4 /$ transc $=\mathrm{CT} 13021 / \mathrm{len}=2979 / \mathrm{GB}: \mathrm{AE} 003588 / \mathrm{note}=3$

3.5 FB:FBgn0026077/sym=Gasp /name=/prod=peritrophin-like /func=chitin binding $/$ map=83D4-83D4/transc=CT28895/len=1460/GB:AE003600/note=3prime sequence from clone BDGP:LD05259.

3.5 FB:FBgn0036051/sym $=$ CG8154 $/$ name $=/$ prod $=/$ func $=/$ map $=67 \mathrm{C} 2-67 \mathrm{C} 2 /$ transc $=\mathrm{CT} 24352 / \mathrm{len}=1726 / \mathrm{GB}: \mathrm{AE} 003550$

3.5 BDGP:LD31422.3prime-hit /ESTpos=maps in FB:FBgn0004624/sym=CaMKII /name=Calcium/calmodulin-dependent protein kinase II /prod=calcium/calmodulin-dependent protein kinase II /func=cal

3.5 FB:FBgn0038181/sym=CG9297/name $=/$ prod $=/$ func $=$ enzyme $/$ map $=87 F 13-87 \mathrm{~F} 13 /$ transc $=$ CT26475 /len=2985 $/$ GB:AE003701

3.5 FB:FBgn0031199 $/ \mathrm{sym}=\mathrm{CG} 9557 /$ name $=/$ prod $=/$ func $=/ \mathrm{map}=20 \mathrm{~A} 1-20 \mathrm{~A} 1 /$ transc $=\mathrm{CT} 17180 /$ len $=420 / \mathrm{GB}: \mathrm{AE} 003574$

3.5 FB:FBgn0038294 $/ \mathrm{sym}=\mathrm{CG} 6803 /$ name $=/$ prod $=/$ func $=/ \mathrm{map}=88 \mathrm{E} 6-88 \mathrm{E} 6 /$ transc $=\mathrm{CT} 21060 / \mathrm{len}=1488 / \mathrm{GB}: \mathrm{AE} 003707 /$ note $=3$ prime sequence from clone BDGP $:$ GH14252.3prime-hit FB:FBgn0033996 /sym=CG11807/name=/prod=/func=receptor $/$ map=51E7-51E7/transc=CT14564/len=1924 /GB:AE003811 /note=3prime sequence from clone BDGP:SD03973.3prime-hit 
3.4 FB:FBgn0032897 /sym $=$ CG9336 $/$ name $=/$ prod $=/$ func $=/$ map $=38 \mathrm{~F} 1-38 \mathrm{~F} 1 /$ transc $=\mathrm{CT} 5238 / \mathrm{len}=660 / \mathrm{GB}: \mathrm{AE} 003668$

3.4 FB:FBgn0034632 $/$ sym $=$ CG15668 $/$ name $=/$ prod $=/$ func $=/$ map $=57$ E8 -57 E9 $/$ transc $=$ CT35852 $/$ len=1976 $/$ GB:AE003454 $/$ note $=3$ prime sequence from clone BDGP $:$ GH02495.3prime-hit

3.4 FB:FBgn0035844 $/ \mathrm{sym}=\mathrm{CG} 13676 /$ name $=/ \mathrm{prod}=/$ func $=/ \mathrm{map}=66 \mathrm{~B} 10-66 \mathrm{~B} 10 /$ transc $=\mathrm{CT} 33112 / \mathrm{len}=2625 / \mathrm{GB}: \mathrm{AE} 003556$

3.4 FB:FBgn0036903/sym=CG8747/name=/prod=low-density lipoprotein-like /func=receptor $/ \mathrm{map}=76 \mathrm{C} 4-76 \mathrm{C} 4 /$ transc=CT25242 /len=651 /GB:AE003516

3.4 FB:FBgn0034819 $/$ sym $=\mathrm{CG} 9877 /$ name $=/$ prod $=/$ func $=/$ map $=59$ C4 $-59 \mathrm{C} 4 /$ transc $=\mathrm{CT} 27864 /$ len $=267 / \mathrm{GB}: \mathrm{AE} 003459$

FB:FBgn0003995/sym=vvl/name=ventral veins lacking /prod=/func=RNA polymerase II transcription factor /map=65C5-65D1/transc=CT28091/len=2556/GB:AE003561

3.4 FB:FBgn0001123/sym=G-salpha60A /name $=\mathrm{G}$ protein salpha $6 \mathrm{~A} /$ prod $=\mathrm{G}$ protein alphas-subunit $/$ func=heterotrimeric G protein $/ \mathrm{map}=60 \mathrm{~A} 12-60 \mathrm{~A} 12 / \mathrm{transc}=\mathrm{CT} 40310 /$ len $=1626 / \mathrm{GB}: \mathrm{AE} 003462 / \mathrm{no}$

3.4 FB:FBgn0033872/sym=CG6329/name $=/$ prod $=/$ func $=/$ map=50C12-50C12 /transc=CT19784 /len=1312 /GB:AE003817 /note $=3$ prime sequence from clone BDGP:HL02087.3prime-hit

3.4 FB:FBgn0003162 /sym=Pu /name=Punch /prod=GTP cyclohydrolase I /func=GTP cyclohydrolase I ; EC:3.5.4.16/map=57C5-57C6/transc=CT26766 /len=1623 /GB:AE003453 /note=3prime sequence

3.4 FB:FBgn0038610/sym=CG7675/name=/prod=/func=enzyme /map=91A2-91A2/transc=CT30419/len=1100/GB:AE003721 /note=3prime sequence from clone BDGP:GH26851.3prime-hit

3.4 FB:FBgn0028694/sym $=$ Rpn $11 /$ name $=/$ prod $=19 \mathrm{~S}$ proteasome regulatory particle, non-ATPase protein, subunit S13 $/$ func $=/ \mathrm{map}=25 \mathrm{C} 3-25 \mathrm{C} 3 /$ transc $=\mathrm{CT} 41032 / \mathrm{len}=1165 / \mathrm{GB}: \mathrm{AE} 003608$ FB:FBgn0036028 $/$ sym $=$ CG16717 $/$ name $=/$ prod $=/$ func $=/$ map $=67$ B11-67B $11 /$ transc $=$ CT37199 $/$ len $=903 /$ GB:AE00355

3.4 FB:FBgn0031200 $/ \mathrm{sym}=\mathrm{CG} 9558 /$ name $=/$ prod $=/$ func $=/ \mathrm{map}=20 \mathrm{~A} 1-20 \mathrm{~A} 1 /$ transc $=\mathrm{CT} 17174 / \mathrm{len}=261 / \mathrm{GB}: \mathrm{AE} 003574$

3.4 FB:FBgn0039897/sym=CG1674 $/$ name $=/$ prod $=/$ func $=$ motor $/$ map=102A3-102A $4 /$ transc $=$ CT4686 /len=2388 $/$ GB:AE003844

FB:FBgn0011327/sym=Uch-L3 /name=Ubiquitin C-terminal hydrolase /prod=ubiquitinyl hydrolase L3 /func=ubiquitinyl hydrolase 1 /map=67B5-67B5/transc=CT11565 /len=1137/GB:AE003552 /1

3.3 FB:FBgn0030309/sym $=\mathrm{CG} 1572 / \mathrm{name}=/ \mathrm{prod}=/$ func $=/ \mathrm{map}=10 \mathrm{C} 4-10 \mathrm{C} 4 /$ transc $=\mathrm{CT} 4080 / \mathrm{len}=955 /$ GB:AE003486 $/$ note $=3$ prime sequence from clone BDGP $:$ LD04844.3prime-hit

3.3 FB:FBgn0016756/sym=Ubp64E /name=Ubiquitin-specific protease 64E /prod=ubiquitin-specific protease /func=ubiquitin-specific protease $/ \mathrm{map}=64 \mathrm{E} 13-64 \mathrm{~F} 1 /$ transc $=\mathrm{CT} 17382 / \mathrm{len}=4202 / \mathrm{GB}: \mathrm{AE} 00$

3.3 FB:FBgn0033446/sym $=\mathrm{CG} 1648 /$ name $=/$ prod $=/$ func $=/ \mathrm{map}=46 \mathrm{~B} 10-46 \mathrm{~B} 10 /$ transc $=\mathrm{CT} 4516 / \mathrm{len}=960 / \mathrm{GB}: \mathrm{AE} 003832 /$ note $=3$ prime sequence from clone BDGP $:$ GH20817.3prime-hit

FB:FBgn0039335/sym=CG5127/name=/prod=vacuolar protein sorting-like /func=transporter $/$ map=96E1-96E1 /transc=CT16445/len=2021 /GB:AE003752/note=3prime sequence from clone BDG

3.3 FB:FBgn0033596/sym $=\mathrm{CG} 7738 /$ name $=/$ prod $=/$ func $=/$ map $=47 \mathrm{E} 1-47 \mathrm{E} 1 /$ transc $=\mathrm{CT} 23557 / \mathrm{len}=405 / \mathrm{GB}: \mathrm{AE} 003826$

3.3 FB:FBgn0032002/sym=CG8353 /name=/prod=cytidine deaminase-like $/$ func=enzyme $/$ map=28F4-28F4 /transc=CT24599 /len=513/GB:AE003620

FB:FBgn0037542/sym=CG2723 /name = /prod= /func= /map=84E6-84E6/transc=CT9257/len=1314 /GB:AE003677 /note=3prime sequence from clone BDGP:LD11958.3prime-hit

3.3 FB:FBgn0019972/sym=Ice $/$ name=Ice $/$ prod=caspase /func=caspase ; EC:3.4.22.- $/ \mathrm{map}=99 \mathrm{C} 4-99 \mathrm{C} 4 /$ transc=CT4706 /len=1932/GB:AE003771 /note=3prime sequence from clone BDGP:GH24292.3pr

3.3 FB:FBgn0023517/sym=EG:63B12.4 /name $=/$ prod= $/$ func $=/$ map=2B13-2B13 /transc=CT34629/len=1066 /GB:AE003422 /note=3prime sequence from clone BDGP:GH02880.3prime-hit

FB:FBgn0036843 $/$ sym $=$ CG6812 $/$ name $=/$ prod $=/$ func $=/$ map $=75 F 4-75 F 5 /$ transc $=$ CT2 $1117 /$ len $=1585 /$ GB:AE003518

3.3 FB:FBgn0039719/sym=CG15515/name $=/$ prod=cuticle protein-like $/$ func $=$ structural protein $/$ map=99D1-99D1 $/$ transc=CT35628 /len=327 /GB:AE003771

3.3 FB:FBgn0031821 /sym $=$ CG9542 $/$ name $=/$ prod $=/$ func $=/$ map=26D7-26D7 $/$ transc $=$ CT26992 $/$ len $=903 /$ GB:AE003613

3.3 FB:FBgn0039208 $/$ sym $=$ CG6643 $/$ name $=/$ prod $=/$ func $=$ enzyme $/ \mathrm{map}=96$ A7-96A7 $/$ transc $=$ CT20638 $/$ len=2769 $/$ GB:AE003748 $/$ note $=3$ prime sequence from clone BDGP:GH21511.3prime-hit FB:FBgn0031502/sym=CG3524/name=/prod=/func=enzyme /map=23C5-23D1/transc=CT11835/len=7590/GB:AE003581/note=3prime sequence from clone BDGP:GH02912.3prime-hit FB:FBgn0035054 $/$ sym $=$ CG9189 $/$ name $=/$ prod $=/$ func $=/$ map $=60 \mathrm{D} 14-60 \mathrm{D} 14 /$ transc $=\mathrm{CT} 26264 / \mathrm{len}=1121 / \mathrm{GB}: \mathrm{AE} 003465$

3.3 FB:FBgn0039529 $/$ sym $=$ CG5612 $/$ name $=/$ prod $=/$ func $=/$ map=98A4-98A4 $/$ transc $=$ CT17752 $/$ len $=1352 /$ GB:AE003761

FB:FBgn0029542 $/$ sym $=\mathrm{CG} 3708 /$ name $=/$ prod $=/$ func $=/$ map $=1 \mathrm{C} 5-1 \mathrm{C} 5 /$ transc $=\mathrm{CT} 12445 /$ len $=1188 / \mathrm{GB}: \mathrm{AE} 003419 /$ note $=3$ prime sequence from clone BDGP $: \mathrm{GH} 17085.3$ prime-hit 
FB:FBgn0040542 $/$ sym $=C G 12815 /$ name $=/$ prod $=/$ func $=/$ map $=85$ F15-85F15 $/$ transc $=$ CT31943 $/$ len $=213 /$ GB:AE003686

3.3 FB:FBgn0031695/sym=Cyp4ac3 /name=/prod=cytochrome P450, CYP4AC3 /func=cytochrome P45 /map=25D2-25D2/transc=CT33590/len=939/GB:AE003609 /note=3prime sequence from clon FB:FBgn0031153/sym=CG15448/name=/prod=/func=transmembrane receptor $/$ map=19E7-19E7/transc=CT35512/len=669 /GB:AE003569 FB:FBgn0031211/sym=CG2776/name=/prod=/func=ligand binding or carrier $/$ map=21A4-21A4/transc=CT9453/len=615 /GB:AE003590

3.2 FB:FBgn0027540 /sym=BcDNA:GH12504/name=/prod=/func=transmembrane receptor $/$ map=85B3-85B4/transc=CT27712/len=3771 /GB:AE003680/note=3prime sequence from clone BDGP:GH

3.2 BDGP:GH1453.3prime-hit /ESTpos=maps in FB:FBgn0031737/sym=CG11142/name=/prod=peritrophin-like /func=structural protein $/ \mathrm{map}=26 \mathrm{~A} 1-26 \mathrm{~A} 1 /$ transc $=\mathrm{CT} 31147 /$ len=899 FB:FBgn0031251 /sym $=$ CG4213 $/$ name $=/$ prod $=/$ func $=$ motor $/$ map $=21 \mathrm{C} 2-21 \mathrm{C} 2 /$ transc $=\mathrm{CT} 13886 / \mathrm{len}=3696 / \mathrm{GB}: \mathrm{AE} 003589$

3.2 FB:FBgn0038407/sym=CG6126/name=/prod=sugar transporter /func=transporter $/$ map=89B13-89B13/transc=CT19169/len=2108 /GB:AE003712 /note=3prime sequence from clone BDGP:GH092

3.2 FB:FBgn0034525/sym=CG13435/name=/prod=/func=ligand binding or carrier $/ \mathrm{map}=57 \mathrm{~B} 1-57 \mathrm{~B} 1 /$ transc=CT32792 /len=1983 /GB:AE003791

3.2 FB:FBgn0003499 /sym=sr /name=stripe /prod=/func=RNA polymerase II transcription factor $/$ map=90E1-90E2 $/$ transc=CT23724 /len=4829/GB:AE003720 FB:FBgn0035772 $/$ sym $=$ CG8582 $/$ name $=/$ prod $=/$ func $=/$ map $=65 F 4-65 F 4 /$ transc $=$ CT14420 $/$ len $=1739 /$ GB:AE003559

FB:FBgn0036562 $/$ sym $=$ CG5600 $/$ name $=/$ prod $=/$ func $=/$ map=72D3-72D4 /transc $=C$ T17536 $/$ len $=1858 /$ GB:AE003528 $/$ note $=3$ prime sequence from clone BDGP:GH16214.3prime-hit

3.2 FB:FBgn0039606 /sym $=$ CG1448 /name $=/$ prod $=/$ func $=/$ map=98E6-98E6 $/$ transc $=C$ T3509 /len=1544 /GB:AE003767 /note=3prime sequence from clone BDGP:LD34202.3prime-hit

$3.2 \quad \mathrm{FB}$

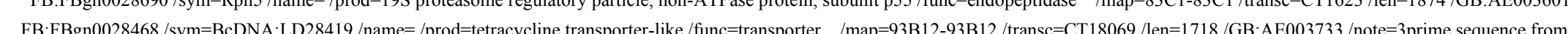
FB:FBgn0039870 $/$ sym=CG1896/name $=/$ prod $=/$ func $=/$ map=100E2-100E2 $/$ transc=CT5870 /len=806 /GB:AE003779 /note=3prime sequence from clone BDGP:LD39576.3prime-hit

3.2 FB:FBgn0010424 /sym=TpnC73F /name=Troponin $\mathrm{C}$ at $73 \mathrm{~F} /$ prod=troponin $\mathrm{C} /$ func=calcium binding $/ \mathrm{map}=73 \mathrm{E} 4-73 \mathrm{E} 5 /$ transc $=\mathrm{CT} 23822 /$ len=935 $/ \mathrm{GB}: \mathrm{AE} 003525$

FB:FBgn0034029 $/$ sym $=$ CG8190 $/$ name $=/$ prod=translation initiation factor 2B-gamma-like $/$ func=translation factor $/$ map $=52 \mathrm{~A} 10-52 \mathrm{~A} 10 /$ transc $=\mathrm{CT} 20341 /$ len $=1503 / \mathrm{GB}: \mathrm{AE} 003810 /$ note $=3$ prime sec 3.2 FB:FBgn0030028 $/ \mathrm{sym}=\mathrm{CG} 10965 / \mathrm{name}=/ \mathrm{prod}=/ \mathrm{func}=/ \mathrm{map}=7 \mathrm{D} 21-7 \mathrm{D} 21 /$ transc $=\mathrm{CT} 7616 / \mathrm{len}=1052 / \mathrm{GB}: \mathrm{AE} 003443 /$ note $=3$ prime sequence from clone BDGP $:$ GH26991.3prime-hit

3.2 FB:FBgn0038149 $/$ sym $=$ CG9796 $/$ name $=/$ prod $=/$ func $=/$ map=87E10-87E10 /transc=CT27692/len=1109 /GB:AE003700 /note $=3$ prime sequence from clone BDGP $:$ LD47508.3prime-hit FB:FBgn0034045/sym=CG8249/name=/prod=glucose transporter-like /func=transporter $/$ map=52D2-52D2 /transc=CT21797/len=1807/GB:AE003809 FB:FBgn0034579 $/$ sym $=$ CG9353 $/$ name $=/$ prod $=/$ func $=/$ map $=57$ B20-57B20 $/$ transc $=$ CT26571 $/$ len=647 $/$ GB:AE003452 $/$ note $=3$ prime sequence from clone BDGP $:$ SD09147.3prime-hit

3.2 FB:FBgn0011716/sym=spdo /name=sanpodo /prod=tropomodulin /func=tropomyosin binding /map=100A1-100A1/transc=CT3919/len=5374/GB:AE003774 /note=3prime sequence from clone BD 3.2 FB:FBgn0032899 $/$ sym $=$ CG9338 $/$ name $=/$ prod $=/$ func $=/$ map $=38 \mathrm{~F} 1-38 \mathrm{~F} 1 /$ transc $=$ CT5240 $/$ len $=660 /$ GB:AE003668 $/$ note $=3$ prime sequence from clone BDGP $:$ GH07967.3prime-hit

3.2 FB:FBgn0029958/sym=CG12151/name=/prod=pyruvate dehydrogenase phosphatase /func=protein phosphatase $/ \mathrm{map}=7 \mathrm{~B} 8-7 \mathrm{~B} 8 /$ transc $=\mathrm{CT} 8439 / \mathrm{len}=1910 / \mathrm{GB}: \mathrm{AE} 003441 /$ note $=3$ prime sequence fr

3.2 FB:FBgn0032596/sym $=\mathrm{CG} 17331 /$ name $=/$ prod=20S proteasome, beta2 subunit $/$ func $=$ endopeptidase $/ \mathrm{map}=36 \mathrm{~A} 7-36 \mathrm{~A} 7 /$ transc $=\mathrm{CT} 32562 / \mathrm{len}=760 / \mathrm{GB}: \mathrm{AE} 003652$

3.2 FB:FBgn0038817 /sym $=$ CG18039 $/$ name $=/$ prod $=/$ func $=/$ map $=92 F 1-92 F 1 /$ transc $=C T 13538 /$ len $=961 /$ GB:AE003731

3.2 FB:FBgn0027501/sym=BcDNA:LD24639/name=/prod=UDP-N-acetylglucosamine pyrophosphorylase-like $/$ func=enzyme $/ \mathrm{map}=26 \mathrm{D} 5-26 \mathrm{D} 5 /$ transc $=\mathrm{CT} 26974 / \mathrm{len}=2033 / \mathrm{GB}: \mathrm{AE} 003613 / \mathrm{note}=3 \mathrm{pri}$

3.2 FB:FBgn0039898/sym=CG1748 /name=/prod=RHO GTPase activator-like /func=signal transduction /map=102A4-102A4/transc=CT4742/len=717/GB:AE003844

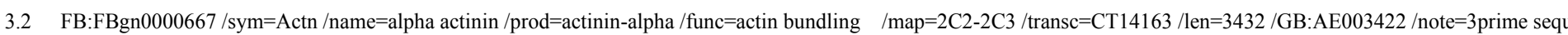
FB:FBgn0028428 $/$ sym $=\mathrm{Ih} /$ name $=/$ prod=voltage-gated ion channel protein $/$ func=voltage-gated ion channel $/ \mathrm{map}=50 \mathrm{~F} 1-50 \mathrm{~F} 1 /$ transc $=\mathrm{CT} 24973 /$ len $=3924 / \mathrm{GB}: \mathrm{AE} 003815$ FB:FBgn0038866 $/$ sym $=$ CG5810 $/$ name $=/$ prod $=/$ func $=$ cell adhesion $/$ map=93C3-93C $3 /$ transc $=\mathrm{CT} 18214 /$ len=1287 $/$ GB:AE003733 
FB:FBgn0037762 $/$ sym $=$ CG16905 $/$ name $=/$ prod $=/$ func $=/$ map $=85 \mathrm{E} 10-85 \mathrm{E} 10 /$ transc $=\mathrm{CT} 37510 / \mathrm{len}=781 / \mathrm{GB}: \mathrm{AE} 003684$

3.1 FB:FBgn0004586 /sym=grh /name=grainy head /prod=/func=specific RNA polymerase II transcription factor $/ \mathrm{map}=54 \mathrm{~F} 1-54 \mathrm{~F} 4 /$ transc $=\mathrm{CT} 42182 / \mathrm{len}=4748 / \mathrm{GB}: \mathrm{AE} 003801 /$ note $=3$ prime sequence fre FB:FBgn0034366 /sym=CG5489 /name=/prod=/func=enzyme $/$ map=55E4-55E $4 /$ transc $=$ CT42577 /len=1577 /GB:AE003799

FB:FBgn0028895/sym=BG:DS02740.8/name=/prod=/func=nucleic acid binding /map=35F8-35F8/transc=CT32539/len=1158 /GB:AE003650

FB:FBgn0039807/sym=CG15546/name $=/$ prod $=/$ func $=/$ map=100B1-100B1 $/$ transc $=$ CT35662 $/$ len $=1233 /$ GB:AE003775

FB:FBgn0037817/sym=Cyp12e1 /name=/prod=cytochrome P450, CYP12E1/func=cytochrome P45 /map=86A7-86A7/transc=CT34465/len=1518/GB:AE003687

3.1 FB:FBgn0023548 $/$ sym $=$ msta $/$ name $=/$ prod $=/$ func $=/$ map $=2 \mathrm{E} 2-2 \mathrm{E} 2 /$ transc $=\mathrm{CT} 40358 / \mathrm{len}=3150 / \mathrm{GB}: \mathrm{AE} 003423$

FB:FBgn0032490 $/$ sym $=$ CG16813 $/$ name $=/$ prod $=/$ func $=/$ map $=34$ A10-34A10 $/$ transc $=$ CT35578 $/$ len $=555 /$ GB:AE003639

FB:FBgn0031457/sym=CG3077/name=/prod=/func=ligand binding or carrier /map=23B1-23B1/transc=CT10334/len=1317 /GB:AE003582/note=3prime sequence from clone BDGP:LD12265.3p

3.1 FB:FBgn0024989/sym=EG:125H10.1/name=/prod=/func=motor $/$ map=1A8-1A8/transc=CT12604/len=2479/GB:AE003417

FB:FBgn0031933 $/ \mathrm{sym}=\mathrm{CG} 7068 /$ name $=/$ prod $=/$ func $=/ \mathrm{map}=28 \mathrm{C} 1-28 \mathrm{C} 1 /$ transc $=\mathrm{CT} 21827 /$ len=4745 $/$ GB:AE003618 $/$ note $=3$ prime sequence from clone BDGP:GH05679.3prime-hit

3.1 FB:FBgn0033807/sym=CG12251/name=aquaporin /prod=aquaporin /func=water transporter $/$ map=49F11-49F11/transc=CT14932/len=1180/GB:AE003819

3.1 FB:FBgn0023175 /sym=Prosalpha7 /name=Proteasome alpha7 subunit $/$ prod=20S proteasome, alpha7 subunit /func=multicatalytic endopeptidase ; EC:3.4.99.46 /map=46B13-46B13 /transc $=\mathrm{CT} 3927 / /$

3.1 FB:FBgn0030917 $/$ sym $=$ CG6267 $/$ name $=/$ prod $=/$ func $=/$ map=17A11 -17 A $11 /$ transc $=$ CT19600 $/$ len $=678 /$ GB:AE003508

FB:FBgn0031027/sym=CG14201/name=/prod=/func=transcription factor $/$ map=18D3-18D3/transc=CT33814/len=702 /GB:AE003512

FB:FBgn0032330 $/$ sym $=$ CG12291 $/$ name $=/$ prod $=/$ func $=/$ map $=32$ D1 $-32 \mathrm{D} 1 /$ transc $=$ CT19324 $/$ len $=382 /$ GB:AE003630

3.1 FB:FBgn0034142 /sym=CG8306 /name $=/$ prod $=/$ func $=$ enzyme $/$ map $=53 \mathrm{C} 7-53 \mathrm{C} 8 /$ transc $=\mathrm{CT} 24503 / \mathrm{len}=1937 / \mathrm{GB}: \mathrm{AE} 003806$

3.1 FB:FBgn0001250/sym=if /name=inflated /prod=integrin, alpha-subunit $/$ func $=$ cell adhesion receptor $/ \mathrm{map}=15 \mathrm{~A} 3-15 \mathrm{~A} 5 /$ transc $=\mathrm{CT} 27194 /$ len $=5726 / \mathrm{GB}: \mathrm{AE} 003503$

3.1 FB:FBgn0036370 $/$ sym $=$ CG14108 $/$ name $=/$ prod $=/$ func $=/$ map $=70 B 1-70 B 1 /$ transc $=\mathrm{CT} 33703 /$ len $=435 /$ GB:AE003537

3.1 FB:FBgn0003060 $/$ sym $=$ CG9757 $/$ name $=/$ prod $=/$ func $=/ \mathrm{map}=87 \mathrm{~F} 5-87 \mathrm{~F} 5 /$ transc $=\mathrm{CT} 27573 / \mathrm{len}=566 / \mathrm{GB}: \mathrm{AE} 003701$

3.1 FB:FBgn0000014/sym=abd-A /name=abdominal A /prod=/func=specific RNA polymerase II transcription factor $/$ map=89E4-89E4/transc=CT29034 /len=1931/GB:AE003715

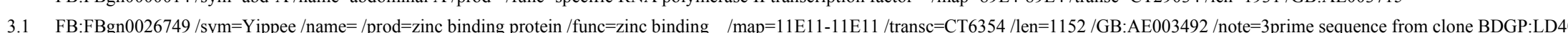

3.1 FB:FBgn0038293/sym=CG6904/name=/prod=/func=enzyme /map=88E5-88E5/transc=CT21366/len=2705/GB:AE003707/note=3prime sequence from clone BDGP:LD46952.3prime-hit

3.1 FB:FBgn0001112/sym=Gld /name=Glucose dehydrogenase $/$ prod=glucose dehydrogenase (acceptor) $==\mathrm{EC} 1.1 .99 .10 /$ func=glucose dehydrogenase $($ acceptor $)$; EC:1.1.99.10 $/$ map $=84 \mathrm{C} 7-84 \mathrm{C} 8 /$ transc=

3 FB:FBgn0003206 $/$ sym=Ras64B $/$ name $=$ Ras oncogene at 64B $/$ prod $=/$ func $=$ RAS small GTPase $/ \mathrm{map}=64 \mathrm{~A} 10-64 \mathrm{~A} 10 /$ transc $=\mathrm{CT} 1405 / \mathrm{len}=588 / \mathrm{GB}: \mathrm{AE} 003480$

3 FB:FBgn0033600/sym=CG9077 /name $=/$ prod $=$ cuticle protein $/$ func $=$ structural protein $/$ map=47E1-47E1 /transc $=\mathrm{CT} 26058 /$ len=396 /GB:AE003826 FB:FBgn0029585/sym=CG11511/name=/prod=/func=transcription factor $/ \mathrm{map}=2 \mathrm{~B} 6-2 \mathrm{~B} 6 /$ transc=CT34608 /len=1000/GB:AE003421

FB:FBgn0003511/sym=Sry-beta $/$ name=Serendipity beta $/$ rod $=/$ func=RNA polymerase II transcription factor $/$ map=99D5-99D5/transc=CT5812/len=1247/GB:AE003772/note=3prime sequence fi

3 FB:FBgn0003071 /sym=Pfk /name=Phosphofructokinase $/$ prod=6-phosphofructokinase $/$ func $=6$-phosphofructokinase $; \mathrm{EC}: 2.7 .1 .11 / \mathrm{map}=46 \mathrm{E} 4-46 \mathrm{E} 4 /$ transc $=\mathrm{CT} 13302 / \mathrm{len}=3176 / \mathrm{GB}: \mathrm{AE} 003830 /$ note $=$

3 FB:FBgn0040559 $/ \mathrm{sym}=\mathrm{CG} 14359 / \mathrm{name}=/ \mathrm{prod}=/$ func $=/ \mathrm{map}=88 \mathrm{~B} 1-88 \mathrm{~B} 1 / \mathrm{transc}=\mathrm{CT} 33994 / \mathrm{len}=237 / \mathrm{GB}: \mathrm{AE} 003703$

3 FB:FBgn0028693/sym=Rpn12/name=/prod=19S proteasome regulatory particle, non-ATPase protein, subunit S14/func=endopeptidase $/ \mathrm{map}=73 \mathrm{~A} 8-73 \mathrm{~A} 8 /$ transc $=\mathrm{CT} 13736 /$ len $=989 / \mathrm{GB}: \mathrm{AE} 00352$ FB:FBgn0038536 $/$ sym $=\mathrm{CG} 7655 /$ name $=/$ prod $=$ multipass nuclear envelope protein-like $/$ func $=$ transmembrane receptor $/$ map $=90 \mathrm{C} 1-90 \mathrm{C} 1 /$ transc $=\mathrm{CT} 23407 /$ len $=965 / \mathrm{GB}: \mathrm{AE} 003718 / \mathrm{note}=3 \mathrm{prime}$ seq 
44484.6

3552.4

38368.3

41645.2

14310.2

104072.4

30341.6

4080.2

4064.4

16524

86366.2

32359.3

2061.3

29445.5

38242.4

29174.8

6818

10857

67758.7

13006.7

1649.5

34712.3

25527.5

1435.5

32146.3

2417.1

6095.4

22931.3

$1923 \sim 2.9$

$2417.5 \sim 2.9$

143693.8
3 FB:FBgn0029533 $/$ sym $=$ CG5254 $/$ name $=/$ prod $=/$ func $=/$ map $=1 \mathrm{C} 1-1 \mathrm{C} 1 /$ transc $=\mathrm{CT} 16777 /$ len $=993 / \mathrm{GB}: \mathrm{AE} 003418$

3 FB:FBgn0032128 $/$ sym $=$ CG13115 $/$ name $=/$ prod $=/$ func $=/$ map=30B11-30B $11 /$ transc $=$ CT32352 $/$ len=956 $/$ GB:AE003625 $/$ note $=3$ prime sequence from clone BDGP:GH05993.3prime-hit

3 FB:FBgn0035030/sym $=\mathrm{CG} 3541 / \mathrm{name}=/ \mathrm{prod}=/ \mathrm{func}=/ \mathrm{map}=60 \mathrm{D} 7-60 \mathrm{D} 8 /$ transc $=\mathrm{CT} 11882 / \mathrm{len}=2417 /$ GB:AE003464 $/$ note $=3$ prime sequence from clone BDGP:GH12163.3prime-hit

3 FB:FBgn0022359/sym=Sodh-2 /name=Sorbitol dehydrogenase-2 /prod=L-iditol 2-dehydrogenase /func=L-iditol 2-dehydrogenase ; EC:1.1.1.14 /map=86C7-86C7 /transc=CT14906 /len=1345/GB:AEC

3 FB:FBgn0028685 $/$ sym=Rpt4 /name=/prod=19S proteasome regulatory particle, triple-A protein, subunit S10b $/$ func=proteasome ATPase ; EC:3.6.4.8 $/$ map $=5 \mathrm{E} 1-5 \mathrm{E} 1 /$ transc $=\mathrm{CT} 11623 / \mathrm{len}=1453 / \mathrm{GB}$

3 BDGP:HL664.3prime-hit /ESTpos=maps 3prime of FB:FBgn0038181/sym=CG9297/name $=/$ prod $=/$ func $=$ enzyme $/$ map $=87 \mathrm{~F} 13-87 \mathrm{~F} 13 /$ transc $=\mathrm{CT} 26475 /$ len $=608$

3 FB:FBgn0028691 /sym=Rpn4 /name=/prod=19S proteasome regulatory particle, non-ATPase protein, subunit S13/func=endopeptidase /map=95B5-95B5/transc=CT28751/len=1330/GB:AE00374

3 FB:FBgn0005771/sym=noc /name=no ocelli /prod=/func=RNA polymerase II transcription factor $/$ map=35A4-35A4 /transc=CT14619/len=2668/GB:AE003644 /note=3prime sequence from clone $\mathrm{B}$

3. FB:FBgn0031637/sym=CG2950/name =/prod=/func=RNA binding $/ \mathrm{map}=25 \mathrm{~B} 3-25 \mathrm{~B} 3 /$ transc=CT40278 /len=2649 /GB:AE003575/note=3prime sequence from clone BDGP:GH06479.3prime-hit

3 FB:FBgn0040520 $/$ sym $=$ CG12447 $/$ name $=/$ prod $=/$ func $=/$ map=20A1-20A $1 /$ transc $=$ CT32486 $/$ len $=132 /$ GB:AE003574

FB:FBgn0003944/sym=Ubx /name=Ultrabithorax /prod=/func=specific RNA polymerase II transcription factor /map=89D8-89E2/transc=CT29154/len=2204 /GB:AE003714

3 FB:FBgn0034497/sym=CG9090/name = /prod=phosphate transporter /func=carrier type transporter $/$ map=56F16-56F16/transc=CT25968 /len=1371 /GB:AE003792

2.9 FB:FBgn0029876 /sym=CG3960/name=/prod=/func=actin binding /map=6B3-6C1 /transc=CT13158/len=2129 /GB:AE003438 /note=3prime sequence from clone BDGP:GH02414.3prime-hi

FB:FBgn0027550/sym=BcDNA:GH10711/name=/prod=/func=receptor $/$ map=32A4-32A5/transc=CT20185 /len=2550/GB:AE003629/note=3prime sequence from clone BDGP:GH10711.3prime-

2.9 FB:FBgn0034399 $/ \mathrm{sym}=\mathrm{CG} 15083 / \mathrm{name}=/ \mathrm{prod}=/ \mathrm{func}=/ \mathrm{map}=55 \mathrm{~F} 3-55 \mathrm{~F} 3 /$ transc $=\mathrm{CT} 34958 / \mathrm{len}=453 / \mathrm{GB}: \mathrm{AE} 003798$

2.9 FB:FBgn0033553/sym $=$ CG12323 $/$ name $=/$ prod $=/$ func $=/$ map $=47 \mathrm{C} 1-47 \mathrm{C} 1 /$ transc $=\mathrm{CT} 22275 /$ len=1114 $/$ GB:AE003828 $/$ note $=3$ prime sequence from clone BDGP:LD08717.3prime-hit

2.9 FB:FBgn0031307/sym=CG4726/name $=/$ prod=sodium/phosphate cotransporter /func=transporter $/$ map=21E4-21E4 /transc=CT15243/len=2003 /GB:AE003587

2.9 FB:FBgn0032129 $/$ sym $=$ CG4405 $/$ name $=/$ prod $=/$ func $=$ RNA binding $/ \mathrm{map}=30 \mathrm{~B} 11-30 \mathrm{~B} 12 /$ transc $=\mathrm{CT} 14344 / \mathrm{len}=3801 / \mathrm{GB}: \mathrm{AE} 003625$

2.9 FB:FBgn0035927/sym=CG5775 /name $=/$ prod $=/$ func $=/$ map=66E1-66E1 $/$ transc $=$ CT18132 $/$ len=837 /GB:AE003554 /note=3prime sequence from clone BDGP:SD08909.3prime-hit

2.9 FB:FBgn0034470/sym $=\mathrm{CG} 11218 / \mathrm{name}=/$ prod $=$ antennal binding protein X-like $/$ func $=$ ligand binding or carrier $/ \mathrm{map}=56 \mathrm{E} 4-56 \mathrm{E} 4 / \mathrm{transc}=\mathrm{CT} 31326 / \mathrm{len}=574 / \mathrm{GB}: \mathrm{AE} 003795$

2.9 FB:FBgn0036891/sym=CG9372/name=/prod=serine protease-like /func=endopeptidase $/$ map=76B11-76B11/transc=CT26619/len=1569/GB:AE003516

FB:FBgn0039706 $/$ sym $=$ CG18040 $/$ name $=/$ prod $=/$ func $=/$ map $=99$ C5-99C $6 /$ transc $=$ CT40392 $/$ len $=786 /$ GB:AE003771

2.9 FB:FBgn0032427 /sym=CG5453 /name $=/$ prod $=/$ func $=/$ map=33D4-33D4/transc=CT17294 /len=819 /GB:AE003636/note=3prime sequence from clone BDGP:GH02216.3prime-hit

2.9 FB:FBgn0010397/sym=L

FB:FBgn0001624/sym=dlg1 /name=discs large $1 /$ prod=guanylate kinase $/$ func=guanylate kinase $; \mathrm{EC}: 2.7 .4 .8 / \mathrm{map}=10 \mathrm{~B} 11-10 \mathrm{~B} 12 / \mathrm{transc}=\mathrm{CT} 41310 / \mathrm{len}=631 / \mathrm{GB}: \mathrm{AE} 003486 / \mathrm{note}=3 \mathrm{prime}$ sequence fr

2.9 FB:FBgn0035517 $/ \mathrm{sym}=\mathrm{CG} 1265 / \mathrm{name}=/ \mathrm{prod}=/ \mathrm{func}=/ \mathrm{map}=64 \mathrm{~B} 4-64 \mathrm{~B} 4 /$ transc $=\mathrm{CT} 2591 / \mathrm{len}=549 / \mathrm{GB}: \mathrm{AE} 003480$

FB:FBgn0032013 $/$ sym $=$ CG7851 $/$ name $=/$ prod $=/$ func $=/$ map $=29 A 4-29 \mathrm{~A} 4 /$ transc $=C T 23800 /$ len $=1391 /$ GB:AE003620

2.9 FB:FBgn0030494 /sym=CG15757 /name $=/$ prod $=$ cuticle protein-like $/$ func $=$ structural protein $/ \mathrm{map}=12 \mathrm{~A} 1-12 \mathrm{~A} 1 /$ transc=CT36009 $/$ len=522 $/ \mathrm{GB}: \mathrm{AE} 003492$

2.9 FB:FBgn0040575 $/$ sym $=$ CG15922 $/$ name $=/$ prod $=/$ func $=/$ map=92E10-92E $10 /$ transc $=$ CT35885 $/$ len $=159 /$ GB:AE003731

FB:FBgn0035199/sym=CG9134/name=/prod=C-type lectin-like /func=ligand binding or carrier $/$ map=61F4-61F4 /transc=CT10115/len=1161 /GB:AE003471 /note=3prime sequence from clone BD FB:FBgn0004957 /sym=por $/$ name=porcupine $/$ prod $=/$ func $=/$ map=17A10-17A10 $/$ transc $=$ CT19254 $/$ len=2331 $/$ GB:AE003508

2.9 FB:FBgn0003075/sym=Pgk /name=Phosphoglycerate kinase $/$ prod=phosphoglycerate kinase $/$ func=phosphoglycerate kinase $;$ EC:2.7.2.3 /map=23A7-23A7/transc=CT10484 /len=1361 /GB:AE003582 
2.9 FB:FBgn0035542 $/$ sym $=$ CG1 $1347 /$ name $=/$ prod $=/$ func $=\quad /$ map $=64 \mathrm{~B} 11-64 \mathrm{~B} 11 /$ transc $=\mathrm{CT} 31652 /$ len=2045 $/$ GB:AE003481 $/$ note $=3$ prime sequence from clone BDGP:GH28550.3prime-hit

2.8 FB:FBgn0031914 /sym=CG5973 /name=/prod=/func=ligand binding or carrier $/$ map=27F7-28A1/transc=CT18751 /len=1519/GB:AE003617 /note=3prime sequence from clone BDGP:HL01515.3p 2.8 FB:FBgn0028688 /sym=Rpn7 /name $=/$ prod=19S proteasome regulatory particle, non-ATPase protein, subunit S10a /func=endopeptidase $/ \mathrm{map}=94 \mathrm{~B} 3-94 \mathrm{~B} 3 / \mathrm{transc}=\mathrm{CT} 17076 / \mathrm{len}=1374 / \mathrm{GB}: \mathrm{AE} 0037$ 2.8 FB:FBgn0035062 $/ \mathrm{sym}=\mathrm{CG} 16914 / \mathrm{name}=/$ prod=larval cuticle protein-like $/$ func $=$ structural protein $/ \mathrm{map}=60 \mathrm{D} 15-60 \mathrm{D} 15 /$ transc $=\mathrm{CT} 37520 / \mathrm{len}=279 / \mathrm{GB}: \mathrm{AE} 003465$

2.8 FB:FBgn0034064 /sym=CG8392 /name $=/$ prod $=20$ S proteasome, beta1 subunit $/$ func $=$ endopeptidase $/ \mathrm{map}=52 \mathrm{E} 1-52 \mathrm{E} 1 /$ transc $=\mathrm{CT} 18263 /$ len $=829 / \mathrm{GB}: \mathrm{AE} 003808 /$ note $=3$ prime sequence from clone 2.8 FB:FBgn0040941 $/$ sym $=$ CG15308 $/$ name $=/$ prod $=/$ func $=/$ map=9B6-9B6 $/$ transc $=$ CT35285 $/$ len $=249 /$ GB:AE003449

2.8 FB:FBgn0031629/sym=CG3244 /name=/prod=selectin-like /func=ligand binding or carrier $/$ map=25B1-25B1/transc=CT10874 /len=1267/GB:AE003575

2.8 FB:FBgn0032727 $/$ sym $=$ CG10623 $/$ name $=/$ prod $=/$ func $=/$ map=37B8-37B8 $/$ transc $=$ CT29758 $/$ len $=1114 /$ GB:AE00366

2.8 FB:FBgn0020369/sym=Pros $45 /$ name=Saccharomyces cerevisiae UAS construct a of Cheng /prod=19S proteasome regulatory particle, triple-A protein, subunit S8 /func=proteasome ATPase ; EC 3.6 .

2.8 FB:FBgn0034975 /sym=CG11290/name $=/$ prod=histone acetyltransferase-like $/$ func=enzyme $/$ map=60B5-60B5 /transc=CT31509/len=6956 /GB:AE003463 /note=3prime sequence from clone BDGP FB:FBgn0024887 /sym=kin $17 /$ name $=/$ prod= $/$ func $=$ DNA binding $/$ map=77B4-77B4 $/$ transc $=$ CT17834 /len=1241 /GB:AE003591

2.8 BDGP:GH13437.3prime-hit /ESTpos=maps 3prime of FB:FBgn0039493 $/$ sym=CG5889 $/$ name $=/$ prod $=/$ func $=/$ map=97E11-97F1 $/$ transc=CT18483 $/$ len=374

2.8 FB:FBgn0028544/sym=BG:DS00180.3/name=/prod=/func= /map=34E1-34E1/transc=CT25718/len=1397 /GB:AE003641/note=3prime sequence from clone BDGP:HL02234.3prime-hit

2.8 FB:FBgn0031067/sym $=$ CG12533 $/$ name $=/$ prod $=/$ func $=$ actin binding $/ \mathrm{map}=18 \mathrm{~F} 1-18 \mathrm{~F} 1 /$ transc $=\mathrm{CT} 33853 / \mathrm{len}=2079 / \mathrm{GB}: \mathrm{AE} 003513$

2.8 FB:FBgn0034140 $/ \mathrm{sym}=\mathrm{CG} 8317 /$ name $=/$ prod $=/$ func $=/ \mathrm{map}=53 \mathrm{C} 7-53 \mathrm{C} 7 /$ transc $=\mathrm{CT} 24573 / \mathrm{len}=693 / \mathrm{GB}: \mathrm{AE} 003806$

2.8 FB:FBgn0014869/sym=Pglym78 /name=Phosphoglyceromutase /prod=phosphoglycerate mutase /func=phosphoglycerate mutase ; EC:5.4.2.1 /map=99A1-99A1 /transc=CT4904 /len=1125 /GB:AE003

2.8 FB:FBgn0004066 /sym=Pros28.1 /name=Proteasome $28 \mathrm{kD}$ subunit $1 /$ prod=20S proteasome, alpha4 subunit /func=multicatalytic endopeptidase $; \mathrm{EC}: 3.4 .99 .46 / \mathrm{map}=14 \mathrm{~B} 11-14 \mathrm{~B} 11 /$ transc $=\mathrm{CT} 11501 / 1$

2.8 FB:FBgn0000084 /sym=AnnX /name=Annexin X/prod=annexin X /func=calcium-dependent phospholipid binding /map=19C1-19C1/transc=CT17636/len=1216/GB:AE002611 /note=3prime seque

2.8 FB:FBgn0035334 /sym=CG8993 /name=/prod=mitochondrial thioredoxin-like /func=chaperone $/ \mathrm{map}=62 \mathrm{E} 1-62 \mathrm{E} 1 / \mathrm{transc}=\mathrm{CT} 25846 / \mathrm{len}=484 / \mathrm{GB}: \mathrm{AE} 003474$

2.8 FB:FBgn0032538 $/$ sym $=$ CG16885 $/$ name $=/$ prod $=/$ func $=/$ map $=34 \mathrm{E} 1-34 \mathrm{E} 1 /$ transc $=\mathrm{CT} 35249 / \mathrm{len}=780 / \mathrm{GB}: \mathrm{AE} 003641$

2.8 FB:FBgn0033959 $/$ sym $=\mathrm{CG} 18372 /$ name $=/$ prod $=/$ func $=/$ map $=51 \mathrm{C} 2-51 \mathrm{C} 2 /$ transc $=\mathrm{CT} 41763 / \mathrm{len}=674 / \mathrm{GB}: \mathrm{AE} 003813$

2.8 FB:FBgn0031322 $/ \mathrm{sym}=\mathrm{CG} 5001 / \mathrm{name}=/ \mathrm{prod}=/$ func $=$ chaperone $/ \mathrm{map}=21 \mathrm{~F} 1-21 \mathrm{~F} 1 /$ transc $=\mathrm{CT} 15884 / \mathrm{len}=1356 / \mathrm{GB}: \mathrm{AE} 003587$

2.8 FB:FBgn0026380 $/$ sym $=$ Prosbeta3 $/$ name $=/$ prod $=20 \mathrm{~S}$ proteasome, beta3 subunit $/$ func $=$ multicatalytic endopeptidase $; \mathrm{EC}: 3.4 .99 .46 / \mathrm{map}=85 \mathrm{C} 3-85 \mathrm{C} 3 / \mathrm{transc}=\mathrm{CT} 32122 / \mathrm{len}=915 / \mathrm{GB}: \mathrm{AE} 003681 / \mathrm{note}=$ 2.8 BDGP:LD8622.3prime-hit /ESTpos=maps 3prime of FB:FBgn0013718 $/$ sym=nuf $/$ name=nuclear fallout $/$ prod $=/$ func=cytoskeletal structural protein $/ \mathrm{map}=7 \mathrm{D} 2-7 \mathrm{D} 2 /$ transc $=\mathrm{CT} 23449 /$ len=291

2.8 FB:FBgn0035788 $/ \mathrm{sym}=\mathrm{CG} 8541 /$ name $=/$ prod $=$ cuticle protein $/$ func=ligand binding or carrier $/ \mathrm{map}=66 \mathrm{~A} 1-66 \mathrm{~A} 1 / \mathrm{transc}=\mathrm{CT} 15726 / \mathrm{len}=828 / \mathrm{GB}: \mathrm{AE} 003559$

FB:FBgn0038063 $/$ sym $=\mathrm{CG} 6989 /$ name $=/$ prod=beta adrenergic receptor-like $/$ func $=\mathrm{G}$ protein linked receptor $/ \mathrm{map}=87 \mathrm{C} 2-87 \mathrm{C} 2 /$ transc $=\mathrm{CT} 21650 / \mathrm{len}=1224 / \mathrm{GB}: \mathrm{AE} 003696$

FB:FBgn0000462 /sym=dl /name=dorsal /prod=/func=specific RNA polymerase II transcription factor $/$ map=36C2-36C2 /transc=CT42418/len=4565 /GB:AE003655

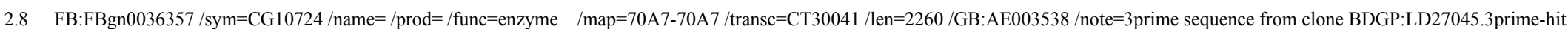
FB:FBgn0039484/sym=CG6124 /name $=/$ prod $=/$ func=cell adhesion $/$ map=97E6-97E6 $/$ transc $=$ CT19227/len=2673 /GB:AE003759

2.8 FB:FBgn0037744/sym=CG8417/name=/prod=mannose-6-phosphate isomerase $/$ func=enzyme $/$ map=85E8-85E8 /transc=CT24707 /len=1398 $/$ GB:AE003684 $/$ note $=3$ prime sequence from clone BDC 2.8 FB:FBgn0020304/sym=drongo $/$ name=drongo $/$ prod= /func=defense/immunity protein $/$ map=21D2-21D2/transc=CT42210 /len=2383/GB:AE003588 $/$ note=3prime sequence from clone BDGP:GH1 2.8 FB:FBgn0035390 $/$ sym $=\mathrm{CG} 1893 /$ name $=/$ prod $=/$ func $=/$ map $=63$ B5 $-63 \mathrm{~B} 5 /$ transc $=\mathrm{CT} 5862 / \mathrm{len}=834 / \mathrm{GB}: \mathrm{AE} 003476$ 
2.8 FB:FBgn0005666 /sym=bt /name=bent /prod=projectin /func=cell adhesion $/$ map=102D6-102E1 /transc=CT3598 /len=23764 /GB:AE003843 /note=3prime sequence from clone BDGP:GH07636.3pri FB:FBgn0037651/sym $=$ CG1 $1978 /$ name $=/$ prod $=/$ func $=/$ map $=85 \mathrm{C} 3-85 \mathrm{C} 3 /$ transc $=\mathrm{CT} 32124 / \mathrm{len}=456 / \mathrm{GB}: \mathrm{AE} 003681$

FB:FBgn0033526 $/$ sym=CG12892 $/$ name $=/$ prod $=/$ func $=$ enzyme $/$ map=47A9-47A9 $/$ transc $=$ CT32037 /len=2244 $/$ GB:AE003829

2.8 FB:FBgn0016762 $/$ sym=angel $/$ name $=$ angel $/$ prod $=/$ func $=$ enzyme $/$ map=59F4-59F4 $/$ transc $=$ CT17360 $/$ len=1184 $/$ GB:AE003461 /note=3prime sequence from clone BDGP:GH06351.3prime-hit

2.8 FB:FBgn0030526 $/ \mathrm{sym}=\mathrm{CG} 11102 / \mathrm{name}=/$ prod $=/$ func $=/ \mathrm{map}=12 \mathrm{~B} 9-12 \mathrm{~B} 9 /$ transc $=\mathrm{CT} 31063 / \mathrm{len}=1671 / \mathrm{GB}: \mathrm{AE} 003493$

2.8 FB:FBgn0003738/sym=Tpi /name=Triose phosphate isomerase /prod=triosephosphate isomerase /func=triosephosphate isomerase ; EC:5.3.1.1/map=99E1-99E1/transc=CT6334/len=1186/GB:AE003 2.8 BDGP:LD1876.3prime-hit $/$ ESTpos=maps 3prime of FB:FBgn0038501 $/ \mathrm{sym}=\mathrm{CG} 5319 / \mathrm{name}=/ \mathrm{prod}=/$ func $=/ \mathrm{map}=9 \mathrm{~A} 6-9 \mathrm{~A} 6 / \mathrm{transc}=\mathrm{CT} 1693 / \mathrm{len}=567$

2.8 FB:FBgn0002789 /sym=Mp20/name=Muscle protein $2 /$ prod=calcium-binding protein /func=calcium binding $/$ map=49F15-49F15/transc=CT15161 /len=852 /GB:AE003819

2.7 FB:FBgn0030753/sym=CG4420 /name=/prod=/func=DNA binding /map=14D1-14D1/transc=CT14402/len=1880 /GB:AE003502/note=3prime sequence from clone BDGP:GM04721.3prime-hit

2.7 FB:FBgn0039914/sym=CG1901/name=/prod=transforming growth factor beta-like $/$ func=signal transduction $/$ map=102D1-102D1/transc=CT5854/len=2528 $/$ GB:AE003843

2.7 FB:FBgn0032282 $/$ sym $=$ CG7299 $/$ name $=/$ prod $=/$ func $=/$ map $=32 \mathrm{~A} 1-32 \mathrm{~A} 1 /$ transc $=\mathrm{CT} 22515 / \mathrm{len}=534 / \mathrm{GB}: \mathrm{AE} 003629$

2.7 FB:FBgn0037138/sym=CG7145/name=/prod=1-pyrroline-5-carboxylate dehydrogenase-like /func=enzyme /map=79A5-79A5/transc=CT22083/len=1890/GB:AE003595 FB:FBgn0036656/sym $=$ CG13026 $/$ name $=/$ prod $=/$ func $=/$ map $=73$ B5-73B $5 /$ transc $=$ CT32244 $/$ len $=405 /$ GB:AE003526

2.7 FB:FBgn0033593 $/$ sym $=$ CG9080 $/$ name $=/$ prod $=/$ func $=/$ map $=47 \mathrm{E} 1-47 \mathrm{E} 1 /$ transc $=\mathrm{CT} 26066 / \mathrm{len}=435 / \mathrm{GB}: \mathrm{AE} 003826$

2.7 FB:FBgn0000486 /sym=Dox-A2 /name=Diphenol oxidase A2 /prod=proteasome, regulatory subunit S3 /func=multicatalytic endopeptidase ; EC:3.4.99.46 | inferred from sequence similarity /map=37B 2.7 FB:FBgn0035232 $/$ sym $=\mathrm{CG} 12099 /$ name $=/$ prod $=/$ func $=/$ map $=62 \mathrm{~A} 7-62 \mathrm{~A} 7 /$ transc $=\mathrm{CT} 6023 / \mathrm{len}=2445 / \mathrm{GB}: \mathrm{AE} 003472$

2.7 FB:FBgn0010423 /sym=TpnC47D /name=Troponin C at 47D /prod=troponin C /func=calcium binding /map=47E1-47E1 /transc=CT26048/len=586/GB:AE003826

2.7 FB:FBgn0004169/sym=up /name=upheld /prod=troponin T /func=tropomyosin binding /map=12A2-12A4/transc=CT41714/len=1424/GB:AE003493

BDGP:GH27479.3prime-hit /ESTpos=maps in FB:FBgn0031850/sym=CG11326/name=/prod=thrombospondin-3 like $/$ func=cell adhesion $/ \mathrm{map}=26 \mathrm{~F} 6-27 \mathrm{~A} 1 /$ transc $=\mathrm{CT} 31613 /$ len $=523$

2.7 FB:FBgn0000568 /sym=Eip75B /name=Ecdysone-induced protein 75B /prod=nuclear receptor NR1D3 /func=ligand-dependent nuclear receptor $/ \mathrm{map}=75 \mathrm{~A} 10-75 \mathrm{~B} 6 /$ transc=CT24290 /len=4527 /GB:A FB:FBgn0035345 $/$ sym $=C$ CG16764 $/$ name $=/$ prod $=/$ func $=/$ map $=62$ E $5-62$ E $6 /$ transc $=$ CT37287 $/$ len $=577 /$ GB:AE003475

2.7 FB:FBgn0037821 /sym=CG14682 $/$ name $=/$ prod $=/$ func=nucleic acid binding $/ \mathrm{map}=86 \mathrm{C} 2-86 \mathrm{C} 2 /$ transc=CT34468 $/$ len=3926 $/$ GB:AE003688 $/$ note $=3$ prime sequence from clone BDGP:GH12580.3prin

2.7 FB:FBgn0039136/sym $=$ CG5902 $/$ name $=/$ prod $=/$ func $=/$ map=95D1-95D $1 /$ transc $=$ CT18529 $/$ len=1603 $/$ GB:AE003745 $/$ note $=3$ prime sequence from clone BDGP:SD10002.3prime-hit

2.7 FB:FBgn0037747/sym=CG8481/name=/prod=N-acetyltransferase /func=enzyme /map=85E8-85E8/transc=CT24815/len=1390/GB:AE003684 /note=3prime sequence from clone BDGP:GH04732

2.7 FB:FBgn0034412 /sym $=$ CG15105 /name $=/$ prod $=/$ func $=$ transcription factor $/ \mathrm{map}=56 \mathrm{~A} 1-56 \mathrm{~A} 2 /$ transc $=\mathrm{CT} 34980 /$ len $=3668 /$ GB:AE003797 $/$ note $=3$ prime sequence from clone BDGP:GH06739.3prim

2.7 FB:FBgn0038130 $/$ sym $=$ CG8630 $/$ name $=/$ prod $=/$ func $=$ enzyme $/$ map $=87$ E5-87E5 $/$ transc $=$ CT25031 /len $=1227 /$ GB:AE003699

2.7 FB:FBgn0002772/sym=Mlc1 /name=Myosin alkali light chain $1 /$ prod=myosin muscle class II essential light chain /func=muscle motor protein $/$ map=98A6-98A6/transc=CT17694 /len=722 /GB:AEC 2.7 FB:FBgn0039268 $/$ sym=CG11819 $/$ name $=/$ prod $=/$ func=protein kinase $/ \mathrm{map}=96 \mathrm{~B} 15-96 \mathrm{~B} 16 /$ transc $=\mathrm{CT} 36931 / \mathrm{len}=2933 / \mathrm{GB}: \mathrm{AE} 003750$

FB:FBgn0023180/sym=Orc6 $/$ name=Origin recognition complex subunit $6 /$ prod=origin recognition complex, subunit $6 /$ func $=$ DNA replication factor $/ \mathrm{map}=46 \mathrm{~B} 13-46 \mathrm{~B} 13 /$ transc $=\mathrm{CT} 4175 /$ len $=774$ 2.7 FB:FBgn0032218 $/ \mathrm{sym}=\mathrm{CG} 5381 / \mathrm{name}=/ \mathrm{prod}=/$ func $=$ transcription factor $/ \mathrm{map}=31 \mathrm{D} 8-31 \mathrm{D} 8 /$ transc $=\mathrm{CT} 17078 / \mathrm{len}=1884 / \mathrm{GB}: \mathrm{AE} 003628$

2.7 FB:FBgn0033631/sym=CG9027/name=/prod=superoxide dismutase-like /func=enzyme /map=47F6-47F7/transc=CT25938/len=477/GB:AE003826

2.7 FB:FBgn0004646 /sym=ogre /name =optic ganglion reduced /prod=innexin /func=ion channel $/$ map=6E4-6E4 /transc=CT9674/len=2219/GB:AE003439 /note=3prime sequence from clone BDGP:HL 
FB:FBgn0038572 $/$ sym $=$ CG7901 $/$ name $=/$ prod $=/$ func $=/$ map $=90 \mathrm{E} 4-90 \mathrm{E} 4 /$ transc $=\mathrm{CT} 42545 /$ len $=937 /$ GB:AE003721

2.7 FB:FBgn0028695/sym=Rpn $/$ name $=/$ prod=19S proteasome regulatory particle, non-ATPase protein, subunit S2 $/$ func=endopeptidase $/$ map=76D7-76D7/transc=CT23606 /len=2967 /GB:AE003515 FB:FBgn0037806 $/$ sym $=$ CG1 $1872 /$ name $=/$ prod $=/$ func $=/$ map $=86$ A $1-86$ A $2 /$ transc $=$ CT37024 $/$ len $=4311 /$ GB:AE003686

2.7 FB:FBgn0034969/sym=CG10485/name $=/$ prod=ribosomal protein L12-like $/$ func=structural protein of ribosome $/ \mathrm{map}=60 \mathrm{~B} 2-60 \mathrm{~B} 2 / \mathrm{transc}=\mathrm{CT} 29426 / \mathrm{len}=675 / \mathrm{GB}: \mathrm{AE} 003462$

2.7 FB:FBgn0039909 /sym=CG1970/name $=/$ prod=NADH-ubiquinone oxidoreductase $/$ func $=$ enzyme $/ \mathrm{map}=102 \mathrm{C} 5-102 \mathrm{C} 5 /$ transc $=\mathrm{CT} 6146 /$ len $=1482 / \mathrm{GB}: \mathrm{AE} 003843$

2.7 FB:FBgn0003149/sym=Prm /name=Paramyosin /prod=paramyosin /func=structural protein of muscle /map=66D14-66D14/transc=CT18619/len=2721 /GB:AE003554/note=3prime sequence from c

2.6 FB:FBgn0003178 /sym=PyK /name=Pyruvate kinase /prod=pyruvate kinase $/$ func=pyruvate kinase $;$ EC:2.7.1.40 /map=94A15-94A15/transc=CT21861/len=2091 /GB:AE003738/note=3prime sequenc FB:FBgn0016697/sym=ProsMA5 $/$ name=Proteasome alpha subunit $/$ prod=20S proteasome, alpha5 subunit $/$ func $=$ multicatalytic endopeptidase $; \mathrm{EC}: 3.4 .99 .46 / \mathrm{map}=54 \mathrm{C} 1-54 \mathrm{C} 1 /$ transc $=\mathrm{CT} 30641 /$ len= 2.6 BDGP:LD33318.3prime-hi

FB:FBgn0038591/sym=CG7150/name=/prod=transcriptional adaptor-like /func=transcription factor binding /map=90F4-90F4/transc=CT22097/len=1876 /GB:AE003721

2.6 FB:FBgn0032148 /sym=CG13122 /name $=/$ prod= /func= /map=30D1-30D1/transc=CT32359/len=1567 /GB:AE003626/note=3prime sequence from clone BDGP:HL02309.3prime-hit

2.6 FB:FBgn0003074 /sym=Pgi /name=Phosphoglucose isomerase /prod=phosphogluconate dehydrogenase (decarboxylating)/func=phosphogluconate dehydrogenase (decarboxylating); EC:1.1.1.44/map=

2.6 FB:FBgn0031187/sym=CG14619/name =/prod=ubiquitin-specific protease $/$ func=ubiquitin-specific protease $/ \mathrm{map}=19 \mathrm{~F} 5-19 \mathrm{~F} 6 /$ transc $=\mathrm{CT} 34376 /$ len=2865 $/ \mathrm{GB}: \mathrm{AE} 003574 /$ note $=3$ prime sequence fro

2.6 FB:FBgn0033126 $/$ sym $=C$ CG10106 $/$ name $=/$ prod $=/$ func $=/$ map $=42$ E $1-42 \mathrm{E} 1 /$ transc $=$ CT7946 $/$ len $=1113 /$ GB:AE003842

FB:FBgn0037543 $/$ sym $=$ CG10903 $/$ name $=/$ prod $=/$ func $=/$ map $=84$ E7-84E7 $/$ transc $=C T 10979 /$ len $=831 /$ GB:AE003677

FB:FBgn0040733 $/$ sym $=$ CG15068 $/$ name $=/$ prod $=/$ func $=/$ map $=55$ C $9-55$ C $9 /$ transc $=$ CT34939 $/$ len $=189 /$ GB:AE003799

2.6 FB:FBgn0034952 $/ \mathrm{sym}=\mathrm{CG} 18021 /$ name $=/$ prod $=/$ func $=/ \mathrm{map}=60 \mathrm{~A} 15-60 \mathrm{~A} 15 /$ transc $=\mathrm{CT} 40326 / \mathrm{len}=2017 /$ GB:AE003462 $/$ note $=3$ prime sequence from clone BDGP $:$ GH20492.3prime-hit

2.6 FB:FBgn0036108 $/ \mathrm{sym}=\mathrm{CG} 7941 / \mathrm{name}=/$ prod $=$ cuticle protein-like $/$ func $=$ structural protein $/ \mathrm{map}=67 \mathrm{~F} 4-67 \mathrm{~F} 4 /$ transc $=\mathrm{CT} 23954 / \mathrm{len}=405 / \mathrm{GB}: \mathrm{AE} 003546$

2.6 FB:FBgn0035464 $/ \mathrm{sym}=\mathrm{CG} 12006 /$ name $=/$ prod $=/$ func $=/ \mathrm{map}=63 \mathrm{~F} 1-63 \mathrm{~F} 1 /$ transc $=\mathrm{CT} 1387 / \mathrm{len}=1873 / \mathrm{GB}: \mathrm{AE} 003479 /$ note $=3$ prime sequence from clone BDGP:LD47795.3prime-hit

2.6 BDGP:LD23884.3prime-hit $/$ ESTpos=maps in FB:FBgn0030430/sym=CG4410/name=/prod=chaperone-like protein /func=chaperone $/ \mathrm{map}=11 \mathrm{C} 4-11 \mathrm{C} 4 /$ transc $=\mathrm{CT} 4259 / \mathrm{len}=500$

2.6 FB:FBgn0023215 /sym=EG:114E2.2/name=/prod=/func=transcription factor binding /map=3F2-3F2 /transc=CT9776/len=1830/GB:AE003428 /note=3prime sequence from clone BDGP:GH28809.

2.6 FB:FBgn0037252/sym $=\mathrm{CG} 14650 /$ name $=/$ prod $=/$ func $=$ chaperone $/ \mathrm{map}=82 \mathrm{C} 1-82 \mathrm{C} 1 /$ transc $=\mathrm{CT} 34422 / \mathrm{len}=3606 /$ GB:AE003606 $/$ note $=3$ prime sequence from clone BDGP:GH27269.3prime-hit

2.6 FB:FBgn0033179/sym $=$ CG11139 $/$ name $=/$ prod $=/$ func $=/ \mathrm{map}=43 \mathrm{C} 4-43 \mathrm{C} 5 /$ transc $=\mathrm{CT} 31143 /$ len=1419 $/$ GB:AE003841 /note $=3$ prime sequence from clone BDGP:GH01724.3prime-hit

2.6 FB:FBgn0037314 $/$ sym $=$ CG12000 $/$ name $=/$ prod $=20 \mathrm{~S}$ proteasome, beta4 subunit-like $/$ func $=$ endopeptidase $/ \mathrm{map}=83 \mathrm{~A} 4-83 \mathrm{~A} 4 /$ transc $=\mathrm{CT} 1070 /$ len $=1216 / \mathrm{GB}: \mathrm{AE} 003603 / \mathrm{note}=3$ prime sequence from

2.6 FB:FBgn0028689/sym=Rpn6 /name=Proteasome p44.5 subunit/prod=19S proteasome regulatory particle, non-ATPase protein, subunit S9 /func=multicatalytic endopeptidase ; EC:3.4.99.46 inferred

2.6 FB:FBgn0012042/sym=AttA /name=Attacin-A /prod=attacin $/$ func=antibacterial response protein $/$ map $=51 \mathrm{C} 2-51 \mathrm{C} 2 /$ transc $=\mathrm{CT} 28545 / \mathrm{len}=895 / \mathrm{GB}: \mathrm{AE} 003813 / \mathrm{note}=3$ prime sequence from clone $\mathrm{BD}$

2.6 FB:FBgn0022986 /sym=qkr58E-1 /name=quaking related 58E-1 /prod=/func=RNA binding /map=58D8-58D8 /transc=CT12115/len=1618/GB:AE003457 /note=3prime sequence from clone BDGP:

2.6 FB:FBgn0032538 $/$ sym $=$ CG16885 $/$ name $=/$ prod $=/$ func $=/$ map $=34 \mathrm{E} 1-34 \mathrm{E} 1 /$ transc $=\mathrm{CT} 35249 / \mathrm{len}=780 / \mathrm{GB}: \mathrm{AE} 003641$

2.6 FB:FBgn0039110/sym=CG10225/name=/prod=/func=structural protein $/ \mathrm{map}=95 \mathrm{~B} 7-95 \mathrm{~B} 7 /$ transc=CT28739/len=1726 /GB:AE003744 /note=3prime sequence from clone BDGP:LD02979.3prime-1

2.6 FB:FBgn0002787/sym=Mov34/name=Mov34/prod=19S proteasome regulatory particle, non-ATPase protein,subunit S12 /func=multicatalytic endopeptidase ; EC:3.4.99.46/map=60D1-60D1/transc

2.6 FB:FBgn0030479/sym=CG1987/name=/prod=RNA binding protein-like /func=RNA binding /map=11E11-11E11 /transc=CT6330/len=393/GB:AE003492

2.6 FB:FBgn0000480/sym=Doa $/$ name=Darkener of apricot $/$ prod=protein serine/threonine kinase $/$ func=protein kinase $/$ map $=98 \mathrm{~F} 1-98 \mathrm{~F} 2 /$ transc $=\mathrm{CT} 4592 / \mathrm{len}=2232 / \mathrm{GB}: \mathrm{AE} 003767 /$ note $=3 \mathrm{prime}$ sequen FB:FBgn0039688/sym=CG1964 /name=/prod=ADAM10 family metalloendopeptidase/disintegrin-like /func=endopeptidase $/$ map=99C1-99C1/transc=CT3146/len=4614/GB:AE003770 
2.6 FB:FBgn0002641 /sym=mal /name=maroon-like $/$ prod=molybdopterin cofactor sulfurase-like $/$ func=molybdopterin cofactor sulfurase $/ \mathrm{map}=19 \mathrm{D} 2-19 \mathrm{D} 3 / \mathrm{transc}=\mathrm{CT} 4746 /$ len=2717 $/ \mathrm{GB}: \mathrm{AE} 003571 / \mathrm{nc}$

2.6 FB:FBgn0039423 $/ \mathrm{sym}=\mathrm{CG} 6166 /$ name $=/$ prod $=/$ func $=/ \mathrm{map}=97 \mathrm{~A} 9-97 \mathrm{~A} 9 /$ transc $=\mathrm{CT} 19364 / \mathrm{len}=2031 / \mathrm{GB}: \mathrm{AE} 003756 /$ note $=3$ prime sequence from clone BDGP:GH14066.3prime-hit FB:FBgn0038041 /sym $=\mathrm{CG} 6525 /$ name $=/$ prod $=/$ func $=$ ligand binding or carrier $/$ map $=87 \mathrm{~B} 15-87 \mathrm{~B} 15 /$ transc $=\mathrm{CT} 20279 / \mathrm{len}=6051 / \mathrm{GB}: \mathrm{AE} 003695$

2.5 FB:FBgn0034688 $/ \mathrm{sym}=\mathrm{CG} 11474 /$ name $=/$ prod $=/$ func $=/ \mathrm{map}=58 \mathrm{C} 1-58 \mathrm{C} 1 /$ transc=CT36283/len=1521 /GB:AE003456 /note=3prime sequence from clone BDGP:LD03212.3prime-hit

2.5 FB:FBgn0029851/sym $=\mathrm{CG} 14445 /$ name $=/$ prod $=/$ func $=/ \mathrm{map}=5 \mathrm{D} 6-5 \mathrm{D} 6 / \mathrm{transc}=\mathrm{CT} 34116 / \mathrm{len}=1143 / \mathrm{GB}: \mathrm{AE} 003437$

FB:FBgn0034242 $/$ sym $=$ CG14480 $/$ name $=/$ prod $=/$ func $=/$ map $=54 \mathrm{D} 1-54 \mathrm{D} 1 /$ transc $=\mathrm{CT} 34191 / \mathrm{len}=750 /$ GB:AE003802

2.5 FB:FBgn0028687/sym=Rpt1 /name=/prod=19S proteasome regulatory particle, triple-A protein, subunit S7 /func=proteasome ATPase ; EC:3.6.4.8 /map=43E6-43E6 /transc=CT3016/len=1397 /GB: FB:FBgn0035081 /sym=CG2858 /name $=/$ prod $=/$ func $=$ enzyme $/$ map=60E5-60E5 $/$ transc $=$ CT $9732 /$ len $=1449 /$ GB:AE003465

FB:FBgn0038688/sym=CG3768/name $=/$ prod=calcium binding protein-like /func=ligand binding or carrier $/$ map=91F8-91F8 /transc=CT12576/len=1030 /GB:AE003725 /note=3prime sequence fron 2.5 FB:FBgn0029715/sym=CG11444 /name $=/$ prod $=/$ func $=/$ map $=4$ C4 $-4 \mathrm{C} 4 /$ transc $=$ CT9463 /len=1230 /GB:AE003431 /note=3prime sequence from clone BDGP:GM14292.3prime-hit

2.5 FB:FBgn0024988 /sym=EG:131F2.2 /name $=/$ prod= /func=enzyme $/ \mathrm{map}=2 \mathrm{~B} 12-2 \mathrm{~B} 12 /$ transc=CT34614 /len=2176 /GB:AE003422 /note=3prime sequence from clone BDGP:SD04906.3prime-hit

2.5 FB:FBgn0037346/sym=CG2922 $/$ name $=/$ prod $=/$ func $=/$ map=83B4-83B4 $/$ transc=CT7240/len=2082 $/$ GB:AE003602 $/$ note $=3$ prime sequence from clone BDGP $:$ LD21309.3prime-hit

2.5 FB:FBgn0036109 $/$ sym $=$ CG18349 $/$ name $=/$ prod $=/$ func $=/$ map $=67 F 4-67 F 4 /$ transc $=$ CT41690 $/$ len $=536 /$ GB:AE003546

2.5 Drosophila gene for Gapdh2 (_5,_M,_3 represent transcript regions 5 prime, Middle, and 3 prime respectively)

2.5 FB:FBgn0001092 /sym=Gapdh2 /name=Glyceraldehyde 3 phosphate dehydrogenase $2 /$ prod=glyceraldehyde 3-phosphate dehydrogenase (phosphorylating) 2 /func=glyceraldehyde 3-phosphate dehydro 2.5 FB:FBgn0038420 $/$ sym $=$ CG10311 $/$ name $=/$ prod $=/$ func $=/$ map $=89 \mathrm{~B} 22-89 \mathrm{~B} 22 /$ transc $=\mathrm{CT} 28967 / \mathrm{len}=719 / \mathrm{GB}: \mathrm{AE} 003713$

FB:FBgn0033745/sym=CG8824/name $=/$ prod=beta-N-acetylhexosaminidase-like $/$ func $=$ enzyme $/$ map $=49 A 9-49 \mathrm{~A} 9 /$ transc $=\mathrm{CT} 25388 /$ len $=2555 / \mathrm{GB}: \mathrm{AE} 003822 /$ note $=3$ prime sequence from clone BI 2.5 FB:FBgn0038878 $/$ sym=CG3301 $/$ name $=/$ prod $=/$ func $=$ enzyme $/$ map=93D4-93D4 /transc=CT11093 /len=913 /GB:AE003734 /note=3prime sequence from clone BDGP:GH01837.3prime-hit FB:FBgn0031206 /sym $=$ CG12466 $/$ name $=/$ prod $=/$ func=enzyme $/ \mathrm{map}=20 \mathrm{~B} 1-20 \mathrm{~B} 1 /$ transc=CT32678 /len=1204 /GB:AE003573 /note=3prime sequence from clone BDGP:GH12380.3prime-hit

2.5 FB:FBgn0030672/sym=CG9281/name=/prod=ATP-binding cassette transporter /func=enzyme $/$ map=13E14-13E14/transc $=\mathrm{CT} 26402 /$ len $=2568 / \mathrm{GB}: \mathrm{AE} 003500 /$ note $=3$ prime sequence from clone B

2.5 FB:FBgn0026781/sym=Prosalpha1 $/$ name=Proteasome alpha1 subunit $/$ prod $=20 \mathrm{~S}$ proteasome alpha1 subunit $/$ func $=$ multicatalytic endopeptidase $; \mathrm{EC}: 3.4 .99 .46 / \mathrm{map}=43 \mathrm{~F} 1-43 \mathrm{~F} 1 / \mathrm{transc}=\mathrm{CT} 42048 / \mathrm{len}=$

2.5 FB:FBgn0036811 $/$ sym $=$ CG6884 $/$ name $=/$ prod $=/$ func $=/$ map $=75 \mathrm{D} 4-75 \mathrm{D} 4 /$ transc $=\mathrm{CT} 21320 /$ len $=610 /$ GB:AE003519

$2.5 \quad$ FB:FBgn0032574/sym $=\mathrm{CG} 18629 / \mathrm{name}=/ \mathrm{prod}=/$ func $=/ \mathrm{map}=35 \mathrm{E} 1-35 \mathrm{E} 1 / \mathrm{transc}=\mathrm{CT} 41822 / \mathrm{len}=588 / \mathrm{GB}: \mathrm{AE} 003649$

2.5 FB:FBgn0034094/sym=CG3666/name=/prod=transferrin-like /func=ligand binding or carrier $/$ map=52F10-52F10/transc=CT12185/len=2352/GB:AE003807/note=3prime sequence from clone BD 2.5 FB:FBgn0038154/sym $=$ CG18290 $/$ name $=/$ prod $=/$ func $=/$ map $=87$ E $11-87$ E $11 /$ transc $=$ CT41497 /len $=1195 /$ GB:AE003700

2.5 FB:FBgn0000250/sym=cact $/$ name $=$ cactus $/$ prod $=/$ func=transcription factor, cytoplasmic sequestering $/ \mathrm{map}=35 \mathrm{~F} 8-35 \mathrm{~F} 9 /$ transc=CT18347 /len=1918 $/ \mathrm{GB}: \mathrm{AE} 003650 /$ note $=3$ prime sequence from clo 2.5 FB:FBgn0040754 $/$ sym $=$ CG17059 $/$ name $=/$ prod $=/$ func $=/$ map $=49 \mathrm{~F} 13-49 \mathrm{~F} 13 /$ transc $=\mathrm{CT} 37898 / \mathrm{len}=358 / \mathrm{GB}: \mathrm{AE} 003819$

2.5 FB:FBgn0000045/sym=Act79B /name=Actin 79B /prod=actin /func=muscle motor protein $/ \mathrm{map}=79 \mathrm{~B} 3-79 \mathrm{~B} 3 / \mathrm{transc}=\mathrm{CT} 22987 / \mathrm{len}=1172 / \mathrm{GB}: \mathrm{AE} 003596$

2.5 FB:FBgn0023174 /sym=Prosbeta2 $/$ name=Proteasome beta2 subunit $/$ prod=20S proteasome, beta2 subunit $/$ func=multicatalytic endopeptidase $; \mathrm{EC}: 3.4 .99 .46 / \mathrm{map}=71 \mathrm{~A} 3-71 \mathrm{~A} 3 / \mathrm{transc}=\mathrm{CT} 10524 / \mathrm{len}=1$ 2.5 FB:FBgn0001301 /sym=kel /name=kelch /prod=/func=transcription factor $/ \mathrm{map}=36 \mathrm{E} 3-36 \mathrm{E} 5 /$ transc=CT22235/len=5604 /GB:AE003657 /note=3prime sequence from clone BDGP:LD29455.3prime-

$2.4 \mathrm{FB}: \mathrm{FBg} 0032202 / \mathrm{syn}=\mathrm{CG} 18619 / \mathrm{name}=/ \mathrm{p}$ 
396588.5

2.4 FB:FBgn0002531/sym=Lcp $1 /$ name=Larval cuticle protein $1 /$ prod=larval cuticle protein $1 /$ func=structural protein of larval cuticle $($ Drosophila $) \quad / \mathrm{map}=44 \mathrm{C} 1-44 \mathrm{C} 1 /$ transc $=\mathrm{CT} 36649 /$ len=543 $/ \mathrm{GB}: \mathrm{AE}$

2.4 FB:FBgn0033129 $/$ sym $=$ CG12844 $/$ name $=/$ prod $=/$ func $=/$ map $=42 \mathrm{E} 1-42 \mathrm{E} 1 /$ transc $=\mathrm{CT} 31976 / \mathrm{len}=673 / \mathrm{GB}: \mathrm{AE} 003842$

FB:FBgn0035649 $/$ sym $=$ CG10483 $/$ name $=/$ prod $=/$ func $=/$ map $=64 F 5-64 F 5 /$ transc $=$ CT29432 /len=2221 /GB:AE003564 /note=3prime sequence from clone BDGP:GH26628.3prime-hit BDGP:LD4689.3prime-hit $/$ ESTpos=maps in FB:FBgn0037937/sym=CG6913 $/$ name $=/$ prod $=/$ func $=$ DNA binding $/ \mathrm{map}=86 \mathrm{~F} 1-86 \mathrm{~F} 1 /$ transc $=\mathrm{CT} 21412 / \mathrm{len}=536$

2.4 BDGP:LD1981.3prime-hit /ESTpos=maps 3prime of FB:FBgn0027356/sym=amphiphysin /name=amphiphysin /prod=amphiphysin /func=protein kinase $/ \mathrm{map}=49 \mathrm{~B} 3-49 \mathrm{~B} 3 / \mathrm{transc}=\mathrm{CT} 259 / \mathrm{len}=527$

2.4 FB:FBgn0015282 /sym=Pros26.4 /name=Proteasome $26 \mathrm{~S}$ subunit subunit 4 ATPase /prod=19S proteasome regulatory particle, triple-A protein, subunit S4 /func=proteasome ATPase ; EC:3.6.4.8 /map FB:FBgn0030941 $/$ sym $=$ CG6531 $/$ name $=/$ prod $=/$ func $=/$ map $=17$ C3-17C3 $/$ transc $=C T 20295 /$ len $=1260 /$ GB:AE003509

2.4 FB:FBgn0029534 $/$ sym $=$ CG5273 $/$ name $=/$ prod $=/$ func $=/$ map $=1 \mathrm{C} 1-1 \mathrm{C} 1 /$ transc $=\mathrm{CT} 16821 /$ len $=2072 /$ GB:AE003418

2.4 FB:FBgn0015283 /sym=Pros54 /name=Proteasome 54kD subunit /prod=19S proteasome regulatory particle, non-ATPase protein, subunit S5a /func=multicatalytic endopeptidase ; EC:3.4.99.46 /map= FB:FBgn0033026 /sym=CG12183/name $=/$ prod $=/$ func=actin binding $/$ map=41C4-41C4 /transc=CT9399/len=1982 /GB:AE003786

2.4 FB:FBgn0039238 $/$ sym $=$ CG7016 $/$ name $=/$ prod $=/$ func $=/$ map $=96 \mathrm{~B} 4-96 \mathrm{~B} 4 /$ transc $=\mathrm{CT} 21718 / \mathrm{len}=1089 / \mathrm{GB}: \mathrm{AE} 003749$

FB:FBgn0036359/sym $=$ CG14105 $/$ name $=/$ prod $=/$ func $=/$ map $=70$ A $8-70 A 8 /$ transc $=$ CT33698 $/$ len $=558 /$ GB:AE003538

2.4 FB:FBgn0035089/sym=CG9358 $/$ name $=/$ prod $=/$ func=ligand binding or carrier $/ \mathrm{map}=60 \mathrm{E} 7-60 \mathrm{E} 7 /$ transc $=\mathrm{CT} 26583 / \mathrm{len}=366 / \mathrm{GB}: \mathrm{AE} 003465$

2.4 FB:FBgn0038260/sym=CG14855/name=/prod=organic cation transporter-like /func=transporter $/$ map=88D5-88D5/transc=CT34672 /len=1671 /GB:AE003706

2.4 FB:FBgn0029639 $/ \mathrm{sym}=\mathrm{CG} 14419 /$ name $=/ \mathrm{prod}=/ \mathrm{func}=/ \mathrm{map}=3 \mathrm{C} 3-3 \mathrm{C} 3 /$ transc $=\mathrm{CT} 34076 / \mathrm{len}=588 / \mathrm{GB}: \mathrm{AE} 003425$

2.4 FB:FBgn0001091 /sym=Gapdh1 /name=Glyceraldehyde 3 phosphate dehydrogenase 1 /prod=glyceraldehyde 3-phosphate dehydrogenase (phosphorylating) 1 /func=glyceraldehyde 3-phosphate dehydro FB:FBgn0030991 $/$ sym $=C$ CG7453 $/$ name $=/$ prod $=/$ func $=/$ map $=18$ B4 -18 B $4 /$ transc $=C T 22925 /$ len $=1285 /$ GB:AE00351

2.4 FB:FBgn0035978 /sym=CG4347 /name=/prod=/func=UTP--glucose-1-phosphate uridylyltransferase $/$ map=67A9-67B1/transc=CT14147/len=2009/GB:AE003552 /note=3prime sequence from clon FB:FBgn0024993 /sym=EG:100G10.6 /name = /prod=/func=transcription factor $/ \mathrm{map}=3 \mathrm{~B} 4-3 \mathrm{~B} 5 /$ transc=CT9011 /len=1402 /GB:AE003425

2.4 FB:FBgn0036111/sym=CG6391/name=/prod=diphosphoinositol polyphosphate phosphohydrolase $/$ func=enzyme $/$ map=67F4-67F4/transc=CT19950/len=1704 /GB:AE003546

2.4 FB:FBgn0032392 $/$ sym $=$ CG17749 $/$ name $=/$ prod $=/$ func $=/$ map $=33$ B9-33B $/$ transc $=$ CT39339 $/$ len $=476 /$ GB:AE003634

2.4 FB:FBgn0036580 $/$ sym $=$ CG13072 $/$ name $=/$ prod $=/$ func $=/$ map $=72 \mathrm{D} 12-72 \mathrm{D} 12 /$ transc $=\mathrm{CT} 32291 / \mathrm{len}=402 / \mathrm{GB}: \mathrm{AE} 003528$

2.4 FB:FBgn0033683 $/ \mathrm{sym}=\mathrm{CG} 18343 / \mathrm{name}=/ \mathrm{prod}=/ \mathrm{func}=/ \mathrm{map}=48 \mathrm{E} 10-48 \mathrm{E} 10 /$ transc $=\mathrm{CT} 41671 / \mathrm{len}=463 / \mathrm{GB}: \mathrm{AE} 003823$

2.4 FB:FBgn0039358 $/ \mathrm{sym}=\mathrm{CG} 5028 / \mathrm{name}=/ \mathrm{prod}=/$ func $=$ enzyme $/ \mathrm{map}=96 \mathrm{E} 10-96 \mathrm{E} 10 /$ transc $=\mathrm{CT} 16155 / \mathrm{len}=1312 / \mathrm{GB}: \mathrm{AE} 003753$

FB:FBgn0015569/sym=alpha-Est10 /name=alpha-Esterase-1 /prod=esterase, unknown substrate /func=esterase, unknown substrate ; EC:3.1.1.- /map=84D5-84D5 /transc=CT1811 /len=1650 /GB:AE0 2.4 FB:FBgn0024754 /sym=Flo /name=flotillin /prod=flotillin /func=ligand binding or carrier $/$ map=52A13-52A13/transc=CT37018 /len=1779/GB:AE003810 /note=3prime sequence from clone BDGP: 2.4 FB:FBgn0030151/sym=CG1354/name=/prod=GTP-binding protein /func=ligand binding or carrier $/ \mathrm{map}=8 \mathrm{~F} 10-8 \mathrm{~F} 10 /$ transc $=\mathrm{CT} 3048 /$ len $=1200 / \mathrm{GB}: \mathrm{AE} 003448$

2.4 FB:FBgn0028686 $/ \mathrm{sym}=\mathrm{Rpt} 3 / \mathrm{name}=/$ prod=19S proteasome regulatory particle, triple-A protein, subunit S6b $/$ func=proteasome ATPase $; \mathrm{EC}: 3.6 .4 .8 / \mathrm{map}=10 \mathrm{~A} 6-10 \mathrm{~A} 6 /$ transc $=\mathrm{CT} 35131 / \mathrm{len}=1242 / \mathrm{G}$ 2.4 FB:FBgn0000064 /sym=Ald /name=Aldolase /prod=fructose-bisphosphate aldolase /func=fructose-bisphosphate aldolase ; EC:4.1.2.13 inferred from direct assay /map=97A6-97A6/transc=CT41919/1 2.4 FB:FBgn0037007 $/ \mathrm{sym}=\mathrm{CG} 5059 / \mathrm{name}=/ \mathrm{prod}=/ \mathrm{func}=/ \mathrm{map}=77 \mathrm{C} 4-77 \mathrm{C} 4 /$ transc $=\mathrm{CT} 16233 / \mathrm{len}=939 / \mathrm{GB}: \mathrm{AE} 003591$

2.4 FB:FBgn0004551/sym=Ca-P60A /name=Calcium ATPase at 6A /prod=calcium-transporting ATPase, sarco/endoplasmic reticulum type /func=calcium-transporting ATPase ; EC:3.6.1.38 /map=60A11 2.4 FB:FBgn0027591/sym=BcDNA:GH04245/name=/prod=/func=/map=41A1-41A2 /transc=CT9123 /len=3519/GB:AE003787 /note=3prime sequence from clone BDGP:LD16758.3prime-hit 
FB:FBgn0030563 $/$ sym $=\mathrm{CG} 18157 /$ name $=/$ prod $=/$ func $=/$ map $=12 \mathrm{E} 2-12 \mathrm{E} 2 /$ transc $=\mathrm{CT} 40954 / \mathrm{len}=594 / \mathrm{GB}: \mathrm{AE} 003495$

2.4 FB:FBgn0040954 $/$ sym $=\mathrm{CG} 13779 /$ name $=/$ prod $=/$ func $=/$ map $=27 \mathrm{D} 5-27 \mathrm{D} 5 /$ transc $=\mathrm{CT} 33267 / \mathrm{len}=240 / \mathrm{GB}: \mathrm{AE} 003616$

2.4 FB:FBgn0035981/sym=CG4452/name=/prod=/func=endopeptidase /map=67B1-67B1/transc=CT14468 /len=1633/GB:AE003552/note=3prime sequence from clone BDGP:LD21662.3prime-hit

2.4 FB:FBgn0040764 $/$ sym $=$ CG13230 $/$ name $=/$ prod $=/$ func $=/$ map=47D4-47D4 $/$ transc $=$ CT32474 /len=219 $/$ GB:AE003827

2.4 Drosophila gene for Gapdh2 (_5,_M, 3 represent transcript regions 5 prime, Middle, and 3 prime respectively)

2.4 FB:FBgn0026777 /sym=Rad23 /name=/prod=/func=DNA repair protein $/$ map=102A8-102A8 /transc=CT5572/len=1477 /GB:AE003844 /note=3prime sequence from clone BDGP:LD10153.complet FB:FBgn0036074 $/$ sym $=$ CG1 $1965 /$ name $=/$ prod $=/$ func $=/$ map $=67 D 2-67 D 2 /$ transc $=$ CT37129 $/$ len $=1464 /$ GB:AE003549

2.4 FB:FBgn0015614 /sym=CanB2 /name=Calcineurin B2 /prod=calcineurin, calcium-binding, regulatory (B)-subunit $2 /$ func=calcium binding $/$ map=43E16-43E16 /transc=CT31322 /len=718 /GB:AE0

2.3 FB:FBgn0024753/sym=Flo-2 /name=flotillin $2 /$ prod=flotillin $2 /$ func= /map=13A3-13A4/transc=CT34056/len=1791 /GB:AE003497/note=3prime sequence from clone BDGP:LP11503.3prime-hit

2.3 FB:FBgn0027493/sym=BcDNA:LD32788/name=/prod=adenylosuccinate synthase /func=adenylosuccinate synthase ; EC:6.3.4.4/map=92F13-92F13/transc=CT35906/len=1986/GB:AE003732 /not

2.3 FB:FBgn0039213 /sym=CG6668 /name $=/$ prod $=/$ func $=/$ map=96A14-96A15/transc=CT20689/len=2852 /GB:AE003748 /note=3prime sequence from clone BDGP:GH09383.3prime-hit

FB:FBgn0034946/sym=CG3065/name $=/$ prod $=/$ func $=$ nucleic acid binding $/$ map=60A14-60A14 /transc $=$ CT10306 /len=1916 /GB:AE003462 /note $=3$ prime sequence from clone BDGP:GM01315.3pr 2.3 FB:FBgn0033886 $/ \mathrm{sym}=\mathrm{CG} 13349 /$ name $=/ \mathrm{prod}=/$ func $=/ \mathrm{map}=50 \mathrm{C} 20-50 \mathrm{C} 20 /$ transc $=\mathrm{CT} 32670 / \mathrm{len}=1170 / \mathrm{GB}: \mathrm{AE} 003816$

2.3 FB:FBgn0002284 $/ \mathrm{sym}=$ Pros $26 /$ name $=$ Proteasome $26 \mathrm{kD}$ subunit $/ \mathrm{prod}=20 \mathrm{~S}$ proteasome, beta6 subunit $/$ func $=$ multicatalytic endopeptidase $; \mathrm{EC}: 3.4 .99 .46 / \mathrm{map}=73 \mathrm{~A} 10-73 \mathrm{~A} 10 / \mathrm{transc}=\mathrm{CT} 13566 /$ len $=9$

2.3 FB:FBgn0017565 $/ \mathrm{sym}=$ Nacalpha $/$ name $=$ Nascent polypeptide associated complex protein alpha subunit $/$ prod=nascent polypeptide associated complex protein alpha subunit $/ \mathrm{func}=/ \mathrm{map}=49 \mathrm{C} 2-49 \mathrm{C} 2$

2.3 FB:FBgn0030310/sym $=$ CG11709 $/$ name $=/$ prod=peptidoglycan recognition protein-like $/$ func $=$ defense/immunity protein $/$ map $=10 \mathrm{C} 4-10 \mathrm{C} 4 /$ transc $=\mathrm{CT} 35112 /$ len $=612 / \mathrm{GB}: \mathrm{AE} 003486$

2.3 FB:FBgn0030724 /sym=CG9212/name=/prod=/func=4-nitrophenylphosphatase $/ \mathrm{map}=14 \mathrm{A6}-14 \mathrm{~A} 6 /$ transc=CT26318 /len=1359/GB:AE003501 /note=3prime sequence from clone BDGP:LD01807.3 FB:FBgn0029970 $/$ sym $=$ CG17256 $/$ name $=/$ prod $=$ protein serine/threonine kinase-like $/$ func $=$ protein kinase $/$ map=7C6-7C6 $/$ transc $=\mathrm{CT} 38234 /$ len $=2435 / \mathrm{GB}: \mathrm{AE} 003442 /$ note $=3$ prime sequence from cle

2.3 FB:FBgn0036630/sym $=\mathrm{CG} 4561 /$ name $=/$ prod $=/$ func $=$ tyrosine--tRNA ligase $/ \mathrm{map}=73 \mathrm{~A} 1-73 \mathrm{~A} 1 /$ transc $=\mathrm{CT} 14730 /$ len $=1717 / \mathrm{GB}: \mathrm{AE} 003527$

BDGP:GH06247.3prime-hit /maps to FB:FBgn0036239 (/sym=CG5684/name=/prod=/func=transcription factor $)$ and FB:FBgn0036238 (/sym=CG5688 $/$ name $=/$ prod=/func=motor $)$

2.3 FB:FBgn0003151/sym=Pros35/name=Proteasome 35kD subunit /prod=20S proteasome, alpha6 subunit /func=multicatalytic endopeptidase $; \mathrm{EC}: 3.4 .99 .46 / \mathrm{map}=31 \mathrm{~B} 3-31 \mathrm{~B} 4 / \mathrm{transc}=\mathrm{CT} 15762 /$ len=94

2.3 FB:FBgn0035206 $/$ sym=CG9186 $/$ name $=/$ prod $=/$ func $=/$ map=61F6-61F6 $/$ transc $=$ CT8283 /len=1400 $/$ GB:AE003471 /note $=3$ prime sequence from clone BDGP:LP01162.3prime-hit

2.3 FB:FBgn0010406/sym=RNaseX25 /name=Ribonuclease X25/prod=ribonuclease-like /func=ribonuclease /map=66A22-66A22/transc=CT24383/len=1658 /GB:AE003557/note=3prime sequence fro FB:FBgn0029798 $/$ sym=CG4078 $/$ name $=/$ prod= $/$ func=DNA repair protein $/ \mathrm{map}=5 \mathrm{~B} 3-5 \mathrm{~B} 3 /$ transc=CT13546 $/$ len=3246 $/$ GB:AE003435

2.3 FB:FBgn0005649/sym=Rox8 /name=Rox8 /prod=nucleolysin-like /func=poly(A) binding /map=95D5-95D5/transc=CT17178 /len=3165/GB:AE003746

FB:FBgn0039901 $/$ sym $=$ CG10322 $/$ name $=/$ prod $=/$ func $=/$ map $=102 \mathrm{~A} 7-102 \mathrm{~A} 8 /$ transc $=\mathrm{CT} 7685 /$ len $=630 /$ GB:AE003844

2.3 FB:FBgn0033352/sym=CG8232/name=/prod=PAB-dependent poly(A)-specific ribonuclease subunit /func=enzyme $/ \mathrm{map}=44 \mathrm{~F} 9-44 \mathrm{~F} 11 /$ transc=CT8229 $/$ len=3925 $/ \mathrm{GB}: \mathrm{AE} 003835 /$ note=3prime sequ

2.3 FB:FBgn0039265 $/ \mathrm{sym}=\mathrm{CG1} 1790 / \mathrm{name}=/ \mathrm{prod}=/ \mathrm{func}=$ chaperone $/ \mathrm{map}=96 \mathrm{~B} 15-96 \mathrm{~B} 15 / \mathrm{transc}=\mathrm{CT} 33049 / \mathrm{len}=794 / \mathrm{GB}: \mathrm{AE} 003750$

2.3 FB:FBgn0034618 /sym=CG9485 /name $=/$ prod=glycogen debranching enzyme $/$ func $=$ enzyme $/$ map $=57$ D4-57D5 /transc $=$ CT26790 $/$ len=4780 $/$ GB:AE003453 $/$ note $=3$ prime sequence from clone BDGI

2.3 FB:FBgn0038922 /sym=CG6439/name $=/$ prod=isocitrate dehydrogenase $[\mathrm{NAD}]$ subunit $/$ func=enzyme $/ \mathrm{map}=93 \mathrm{~F} 14-93 \mathrm{~F} 14 /$ transc $=\mathrm{CT} 20062 /$ len=1598 $/ \mathrm{GB}: \mathrm{AE} 003737 /$ note $=3 \mathrm{prime}$ sequence from FB:FBgn0016122/sym=Acer /name=Angiotensin-converting enzyme-related $/$ prod=angiotensin I-converting enzyme /func=peptidyl-dipeptidase A ; EC:3.4.15.1/map=29D1-29D2/transc=CT29700 /le 2.2 BDGP:LD28328.3prim

2.2 Drosophila gene for Gapdh2 (_5,_M,_3 represent transcript regions 5 prime, Middle, and 3 prime respectively) 
FB:FBgn0025186/sym=ari2 $/$ name $=$ ariadne $2 /$ prod $=/$ func $=/$ map=58C7-58D $1 /$ transc $=$ CT17832 $/$ len $=3002 /$ GB:AE003456

FB:FBgn0004237 /sym $=$ Hrb87F $/$ name $=$ Heterogeneous nuclear ribonucleoprotein at $87 \mathrm{~F} /$ prod=heterogeneous nuclear ribonucleoprotein A1 /func $=$ ribonucleoprotein $\quad / \mathrm{map}=87 \mathrm{~F} 7-87 \mathrm{~F} 7 /$ transc $=\mathrm{CT} 2725$ 2.2 BDGP:GH05625.3

2.2 FB:FBgn0003150/sym=Pros $29 /$ name=Proteasome $29 \mathrm{kD}$ subunit $/$ prod=20S proteasome, alpha3 subunit $/$ func=multicatalytic endopeptidase $; \mathrm{EC}: 3.4 .99 .46 / \mathrm{map}=57 \mathrm{~B} 15-57 \mathrm{~B} 15 /$ transc $=\mathrm{CT} 26525 /$ len= 2.2 FB:FBgn0032537/sym=CG18634 $/$ name $=/$ prod $=/$ func $=/$ map $=34 \mathrm{E} 1-34 \mathrm{E} 1 /$ transc $=\mathrm{CT} 42158 / \mathrm{len}=552 / \mathrm{GB}: \mathrm{AE} 003641$

2.2 FB:FBgn0035631/sym=CG5495/name=Thioredoxin-like /prod=thioredoxin-like /func=thioredoxin $/ \mathrm{map}=64 \mathrm{~F} 1-64 \mathrm{~F} 1 /$ transc $=\mathrm{CT} 17420 /$ len=957 $/ \mathrm{GB}: \mathrm{AE} 003565 /$ note $=3$ prime sequence from clone B

2.2 FB:FBgn0027910/sym=BcDNA:GM14618 $/$ name $=/$ prod $=/$ func $=/$ map $=25 \mathrm{C} 2-25 \mathrm{C} 3 /$ transc $=\mathrm{CT} 26154 / \mathrm{len}=1161 / \mathrm{GB}:$ AE003608

2.2 FB:FBgn0033356/sym $=$ CG8229 $/$ name $=/$ prod $=/$ func $=/$ map=44F12-45A1 / transc=CT8591 /len=1918 /GB:AE003835 /note=3prime sequence from clone BDGP:LD27667.3prime-hit

2.2 FB:FBgn0000579/sym=Eno $/$ name=Enolase $/$ prod=phosphopyruvate hydratase $/$ func=phosphopyruvate hydratase $;$ EC:4.2.1.11 /map=22B1-22B1/transc=CT32526 /len=1931/GB:AE003585

2.2 FB:FBgn0040985 $/ \mathrm{sym}=\mathrm{CG} 6115 / \mathrm{name}=/ \mathrm{prod}=/$ func $=/ \mathrm{map}=36 \mathrm{~A} 11-36 \mathrm{~A} 11 /$ transc $=\mathrm{CT} 19203 / \mathrm{len}=566 / \mathrm{GB}: \mathrm{AE} 003653$

2.2 FB:FBgn0022959/sym=yps /name=ypsilon schachtel $/$ prod $=/$ func $=/ \mathrm{map}=68 \mathrm{~F} 4-68 \mathrm{~F} 4 /$ transc $=\mathrm{CT} 17850 /$ len=2254 $/$ GB:AE003542 $/$ note $=3$ prime sequence from clone BDGP:LD37574.3prime-hit

2.2 FB:FBgn0032776 $/$ sym $=$ CG18061 $/$ name $=/$ prod $=/$ func $=/$ map=37E1-37E1 $/$ transc $=$ CT40481 /len=2027 /GB:AE003662 $/$ note $=3$ prime sequence from clone BDGP:SD04793.3prime-hit

FB:FBgn0011754/sym=PhKgamma /name=Phosphorylase kinase gamma /prod=phosphorylase kinase, catalytic gamma subunit /func=phosphorylase kinase catalyst $/$ map=10D2-10D4 /transc=CT55 2.2 BDGP:GH28523.3pri

2.2 FB:FBgn0032203 $/ \mathrm{sym}=\mathrm{CG} 4946 / \mathrm{name}=/ \mathrm{prod}=/ \mathrm{func}=/ \mathrm{map}=31 \mathrm{C} 6-31 \mathrm{C} 6 /$ transc $=\mathrm{CT} 15842 / \mathrm{len}=834 / \mathrm{GB}: \mathrm{AE} 003628$

2.2 FB:FBgn0032941 /sym=CG8669 /name $=/$ prod $=/$ func $=/$ map=39D2-39D2 /transc $=$ CT5302 /len=1793 /GB:AE003670 /note=3prime sequence from clone BDGP:GH1 1210.3 prime-hit FB:FBgn0029913/sym=CG3044 /name=/prod=chitinase $/$ func=enzyme $/$ map=6D3-6D3/transc=CT10053 /len=1299 /GB:AE003439

FB:FBgn0004170/sym=sc /name=scute /prod=/func=specific RNA polymerase II transcription factor $/$ map=1B2-1B2/transc=CT12777/len=1038/GB:AE003417

2.2 FB:FBgn0037377 $/$ sym $=\mathrm{CG} 1218 /$ name $=/$ prod $=/$ func $=/ \mathrm{map}=83 \mathrm{C} 1-83 \mathrm{C} 1 /$ transc $=\mathrm{CT} 2292 / \mathrm{len}=1479 / \mathrm{GB}: \mathrm{AE} 003601 /$ note $=3$ prime sequence from clone BDGP $:$ LD28626.3prime-hit FB:FBgn0040737/sym $=$ CG14503 $/$ name $=/$ prod $=/$ func $=/$ map $=55$ C5-55C $5 /$ transc $=$ CT34218 $/$ len $=177 /$ GB:AE003800

2.2 FB:FBgn0028490/sym=BcDNA:GH07269/name=/prod=/func=DNA binding /map=33A2-33A2/transc=CT20317 /len=3403 /GB:AE003632 /note=3prime sequence from clone BDGP:GH07269.3p FB:FBgn0028854/sym=BG:DS07721.6 $/$ name $=/$ prod $=/$ func $=/$ map=35B5-35B $/$ transc $=$ CT35229 $/$ len $=3129 /$ GB:AE003644

2.2 FB:FBgn0031024 $/ \mathrm{sym}=\mathrm{CG} 12233 / \mathrm{name}=/ \mathrm{prod}=/$ func $=$ enzyme $/ \mathrm{map}=18 \mathrm{D} 3-18 \mathrm{D} 3 /$ transc $=\mathrm{CT} 13154 / \mathrm{len}=1375 / \mathrm{GB}: \mathrm{AE} 003512$

2.2 FB:FBgn0034110/sym $=\mathrm{CG} 3615 /$ name $=/$ prod $=/$ func $=/$ map=53A2-53A2 $/$ transc $=$ CT12045/len=2831 /GB:AE003807 /note=3prime sequence from clone BDGP:SD01812.3prime-hit

2.2 FB:FBgn0037537/sym=CG2767/name=/prod=alcohol dehydrogenase /func=enzyme /map=84E5-84E6/transc=CT9417/len=1293/GB:AE003677 /note=3prime sequence from clone BDGP:LD2467

2.2 FB:FBgn0015239/sym=Hr78 /name=Hormone-receptor-like in $78 /$ prod=nuclear receptor NR2D1 /func=ligand-dependent nuclear receptor $/ \mathrm{map}=78 \mathrm{D} 7-78 \mathrm{D} 7 /$ transc=CT22217 /len=2246 $/ \mathrm{GB}: \mathrm{AE} 003$

2.2 FB:FBgn0033128 /sym=CG12142 /name=Tetraspanin 42Eg /prod=tetraspanin /func $=/$ map=42E1-42E1 /transc $=$ CT7934 /len=1170 /GB:AE003842 /note $=3$ prime sequence from clone BDGP:GM0696

2.2 FB:FBgn0036661 $/ \mathrm{sym}=\mathrm{CG} 9705 /$ name $=/$ prod $=$ calcium-regulated heat stable protein-like $/$ func $=/$ map $=73 \mathrm{C} 1-73 \mathrm{C} 1 /$ transc $=\mathrm{CT} 27440 /$ len $=1386 / \mathrm{GB}: \mathrm{AE} 003526 /$ note $=3$ prime sequence from clone $\mathrm{BD}$

2.2 FB:FBgn0027108/sym=inx2 /name=/prod=innexin $2 /$ func=neurotransmitter transporter $/$ map=6E4-6E5 /transc=CT14874 /len=1819/GB:AE003439

2.2 FB:FBgn0035904 $/ \mathrm{sym}=\mathrm{CG} 6776 /$ name $=/$ prod $=$ glutathione transferase $/$ func $=$ glutathione transferase $/ \mathrm{map}=66 \mathrm{D} 4-66 \mathrm{D} 4 /$ transc $=\mathrm{CT} 21027 / \mathrm{len}=776 / \mathrm{GB}: \mathrm{AE} 003555$

2.2 FB:FBgn0004432 /sym=Cyp1 /name=Cyclophilin $1 /$ prod=cyclophilin /func=cyclophilin $/$ map=14B15-14B15/transc=CT27926/len=910/GB:AE003501 /note=3prime sequence from clone BDGP:SD FB:FBgn0037190/sym=CG7651 /name=/prod=P-type ATPase /func=transporter $/$ map=79F3-79F3 /transc=CT22775/len=3566 /GB:AE003598

2.2 FB:FBgn0036299 $/ \mathrm{sym}=\mathrm{CG} 10620 /$ name $=/$ prod=transferrin-like $/$ func=transporter $/ \mathrm{map}=69 \mathrm{C} 4-69 \mathrm{C} 4 /$ transc $=\mathrm{CT} 29752 /$ len=2860 $/$ GB:AE003541 /note=3prime sequence from clone BDGP:LD22449. 2.2 FB:FBgn0037245 $/$ sym $=$ CG14648 $/$ name $=/$ prod $=/$ func $=$ enzyme $/$ map=82B3-82B3 $/$ transc $=$ CT34420 $/$ len $=1773 /$ GB:AE003606 
2.2 FB:FBgn0033385 $/$ sym $=$ CG8055 $/$ name $=/$ prod $=/$ func $=\quad /$ map=45B1-45B1/transc=CT8052/len=1251 /GB:AE003834 /note=3prime sequence from clone BDGP:GH13992.3prime-hit FB:FBgn0034578 $/$ sym $=$ CG15653 $/$ name $=/$ prod $=/$ func $=/$ map $=57 \mathrm{~B} 20-57 \mathrm{~B} 20 /$ transc $=\mathrm{CT} 35837 / \mathrm{len}=513 / \mathrm{GB}: \mathrm{AE} 003452$

FB:FBgn0025802/sym=Sbf $/$ name $=$ SET domain binding factor $/$ prod $=/$ func=ligand binding or carrier $/$ map=86F9-86F9 $/$ transc $=\mathrm{CT} 21495 /$ len $=6619 / \mathrm{GB}: \mathrm{AE} 003693 /$ note $=3$ prime sequence from clon 2.2 FB:FBgn0031454/sym=CG9960 $/$ name $=/$ prod $=/$ func $=/$ map=23B1-23B1 $/$ transc=CT28077 /len=799 /GB:AE003582 /note=3prime sequence from clone BDGP:GH13185.3prime-hit FB:FBgn0039443 $/$ sym $=$ CG14242 $/$ name $=/$ prod $=/$ func $=/$ map $=97 \mathrm{C} 1-97 \mathrm{C} 1 /$ transc $=\mathrm{CT} 33862 /$ len $=687 / \mathrm{GB}: \mathrm{AE} 003757$

2.1 FB:FBgn0037465 $/$ sym $=$ CG1 $105 /$ name $=/$ prod $=/$ func $=/$ map $=84 \mathrm{C} 1-84 \mathrm{C} 1 /$ transc $=\mathrm{CT} 1653 /$ len $=1336 / \mathrm{GB}: \mathrm{AE} 003673$

FB:FBgn0034494 /sym=CG10444 /name=/prod=sodium-dependent multivitamin transporter-like $/$ func=transporter $/ \mathrm{map}=56 \mathrm{~F} 15-56 \mathrm{~F} 16 /$ transc $=\mathrm{CT} 29322 / \mathrm{len}=2772 / \mathrm{GB}: \mathrm{AE} 003792 /$ note $=3$ prime seq

2.1 FB:FBgn0028684 /sym=Rpt5 /name=Tat-binding protein-1 /prod=19S proteasome regulatory particle, triple-A protein, subunit S6a /func=proteasome ATPase ; EC:3.6.4.8 /map=95B7-95B7/transc=C

2.1 FB:FBgn0034583/sym=CG10527 /name $=/$ prod $=/$ func $=/$ map=57B20-57B20 /transc=CT29543/len=1133 /GB:AE003452/note=3prime sequence from clone BDGP:LD46156.3prime-hit

2.1 FB:FBgn0016700/sym=Rab1 /name=Rab-protein $1 /$ prod=/func=RHO small GTPase $/$ map=93D4-93D4 /transc=CT11153/len=1791/GB:AE003734

2.1 FB:FBgn0038298/sym=CG18525/name $=/$ prod $=/$ func $=/$ map=88E7-88E7 /transc=CT42292/len=1494 /GB:AE003708 /note=3prime sequence from clone BDGP:GH14439.3prime-hit

2.1 FB:FBgn0037686/sym=CG9354/name=/prod=ribosomal protein L34-like/func=structural protein of ribosome /map=85D15-85D15/transc=CT25494/len=771 /GB:AE003682 FB:FBgn0036722 $/$ sym $=$ CG13729 $/$ name $=/$ prod $=/$ func $=/$ map $=74$ C1-74C1 $/$ transc $=$ CT33196 $/$ len $=360 /$ GB:AE003524

2.1 FB:FBgn0002626/sym=RpL32 /name=Ribosomal protein L32 /prod=ribosomal protein L32/func=large-subunit cytosol ribosomal protein /map=99D5-99D5 /transc=CT6405/len=505 /GB:AE003772

2.1 FB:FBgn0030087/sym=CG7766 $/$ name $=/$ prod $=/$ func=protein kinase $/ \mathrm{map}=8 \mathrm{C} 13-8 \mathrm{C} 14 /$ transc $=\mathrm{CT} 23171 / \mathrm{len}=3994 / \mathrm{GB}: \mathrm{AE} 003446$

2.1 FB:FBgn0023211 /sym=Elongin-C $/$ name=Elongin $\mathrm{C} /$ prod=elongin $\mathrm{C} /$ func=transcription factor $/ \mathrm{map}=56 \mathrm{D} 7-56 \mathrm{D} 7 / \operatorname{transc}=\mathrm{CT} 26431 / \mathrm{len}=557 / \mathrm{GB}: \mathrm{AE} 003796$

FB:FBgn0040974 $/$ sym $=$ CG9260 $/$ name $=/$ prod $=/$ func $=/$ map $=34 \mathrm{C} 1-34 \mathrm{C} 1 /$ transc $=\mathrm{CT} 26204 / \mathrm{len}=460 /$ GB:AE003640

2.1 FB:FBgn0002533/sym=Lcp2 /name=Larval cuticle protein $2 /$ prod=larval cuticle protein $2 /$ func $=$ structural protein of larval cuticle $($ Drosophila $) \quad /$ map $=44 \mathrm{C} 1-44 \mathrm{C} 1 /$ transc $=\mathrm{CT} 3631 /$ len $=526 / \mathrm{GB}: \mathrm{AEO}$

2.1 FB:FBgn0026088 $/$ sym=EG:63B12.12 $/$ name $=/$ prod $=/$ func $=/$ map=2B14-2B $14 /$ transc $=$ CT34631 /len $=460 /$ GB:AE003422

FB:FBgn0039353/sym=CG5046/name=/prod= /func=cell adhesion $/$ map=96E10-96E10/transc=CT16193/len=840 /GB:AE003753

2.1 FB:FBgn0011768 /sym=Fdh /name=Formaldehyde dehydrogenase /prod=formaldehyde dehydrogenase (glutathione) /func=formaldehyde dehydrogenase (glutathione) ; EC:1.2.1.1 | inferred from direct

2.1 FB:FBgn0032725/sym=CG10679/name $=/$ prod= $/$ func=structural protein of ribosome $/$ map=37B8-37B8 /transc=CT29904 /len=255/GB:AE003661

2.1 FB:FBgn0032134 /sym=CG3864 /name $=/$ prod $=/$ func $=/$ map=30C1-30C2 $/$ transc=CT12877/len=966 /GB:AE003625 /note=3prime sequence from clone BDGP:SD03042.3prime-hit

2.1 FB:FBgn0033635/sym $=\mathrm{CG} 7777 /$ name $=/$ prod=water transporter $/$ func $=$ transporter $/$ map $=47 F 13-47 F 13 /$ transc $=\mathrm{CT} 23658 /$ len $=1371 / \mathrm{GB}: \mathrm{AE} 003826 /$ note $=3$ prime sequence from clone

2.1 FB:FBgn0002535/sym=Lcp $4 /$ name=Larval cuticle protein $4 /$ prod=larval cuticle protein $4 /$ func $=$ structural protein of larval cuticle $($ Drosophila $) \quad / \mathrm{map}=44 \mathrm{C} 1-44 \mathrm{C} 1 /$ transc $=\mathrm{CT} 6607 /$ len $=682 / \mathrm{GB}: \mathrm{AEO}$

2.1 FB:FBgn0002741 /sym=Mhc /name=Myosin heavy chain $/$ prod=myosin II heavy chain $/$ func=muscle motor protein $/$ map=36A8-36A9/transc=CT39920/len=5794 /GB:AE003652

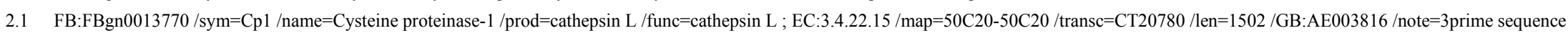
FB:FBgn0033024/sym=CG10416/name=/prod=/func=RNA-directed DNA polymerase, group II intron encoded $/$ map=41C2-41C2/transc=CT29248 /len=1195/GB:AE003786

2.1 FB:FBgn0030847/sym $=$ CG12991 $/$ name $=/$ prod $=/$ func $=/$ map=16B8-16B8 $/$ transc $=$ CT32195 $/$ len $=1380 /$ GB:AE003506

FB:FBgn0035026/sym=CG12252/name $=/$ prod=RNA polymerase CTD phosphatase $/$ func=protein phosphatase $/$ map=60D5-60D5/transc $=\mathrm{CT} 15027 /$ len=3150 $/$ GB:AE003464 $/$ note $=3$ prime sequenc 2.1 FB:FBgn0037752/sym=CG8495/name=/prod=ribosomal protein S29-like /func=structural protein of ribosome $/$ map=85E9-85E9/transc=CT24837/len=979/GB:AE003684

2.1 FB:FBgn0030350 $/$ sym $=\mathrm{CG} 1844 /$ name $=/$ prod $=/$ func $=/$ map=10F4-10F4 $/$ transc $=$ CT5645 /len=828 /GB:AE003487 /note=3prime sequence from clone BDGP $:$ GH03581.3prime-hit 
2.1 FB:FBgn0035549 $/$ sym $=$ CG1 $1346 /$ name $=/$ prod $=/$ func $=$ structural protein $/ \mathrm{map}=64 \mathrm{~B} 12-64 \mathrm{~B} 12 / \operatorname{transc}=\mathrm{CT} 31648 /$ len $=564 / \mathrm{GB}: \mathrm{AE} 003481$ BDGP:LD32469.3prime-hit /maps to FB:FBgn0032432 (/sym=CG5442 /name=/prod=/func=) and FB:FBgn0032431 (/sym=CG5435/name $=/$ prod=/func= $)$

2.1 FB:FBgn0038570 $/ \mathrm{sym}=\mathrm{CG} 7217 /$ name $=/$ prod $=/$ func $=/ \mathrm{map}=90 \mathrm{E} 4-90 \mathrm{E} 4 /$ transc $=\mathrm{CT} 22257 / \mathrm{len}=728 / \mathrm{GB}: \mathrm{AE} 003721 /$ note $=3$ prime sequence from clone BDGP $:$ LD45324.3prime-hit

2.1 FB:FBgn0023081/sym=gek /name=genghis khan /prod=protein serine/threonine kinase $/$ func=protein serine/threonine kinase $;$ EC:2.7.1.37 /map=60B5-60B5/transc=CT13314 /len=5090 /GB:AE00346 2.1 FB:FBgn0037874 $/$ sym $=\mathrm{CG} 4800 /$ name $=/$ prod $=/$ func $=/$ map $=86 \mathrm{D} 7-86 \mathrm{D} 7 /$ transc $=\mathrm{CT} 15437 / \mathrm{len}=782 / \mathrm{GB}: \mathrm{AE} 003690$

2.1 FB:FBgn0020249/sym=stck /name=steamer duck /prod= /func=transcription factor $/$ map=84F15-84F16/transc=CT23960 /len=1335 /GB:AE003678 /note=3prime sequence from clone BDGP:LD393 FB:FBgn0039333 $/$ sym $=$ CG1 $1917 /$ name $=/$ prod $=/$ func $=/$ map $=96$ D2-96D $2 /$ transc $=C T 37090 /$ len $=718 /$ GB:AE003751

2.1 FB:FBgn0024923 /sym=TER94/name=Saccharomyces cerevisiae UAS construct a of McKearin /prod=transitional endoplasmic reticulum adenosinetriphosphatase /func=adenosinetriphosphatase ; EC:3

2.1 FB:FBgn0027844 /sym=CAH1 /name=Carbonic anhydrase 1 /prod=carbonate dehydratase /func=carbonate dehydratase ; EC:4.2.1.1 /map=34D1-34D1 /transc=CT23642/len=1439 /GB:AE003641 /note FB:FBgn0033900/sym=CG8257/name=/prod=cysteine--tRNA ligase-like /func=enzyme /map=50E2-50E2 /transc=CT24479/len=1770 /GB:AE003815 /note=3prime sequence from clone BDGP:GH

2.1 FB:FBgn0000116/sym=Argk /name=Arginine kinase $/$ prod=arginine kinase $/$ func=arginine kinase $; \mathrm{EC}: 2.7 .3 .3 / \mathrm{map}=66 \mathrm{~F} 2-66 \mathrm{~F} 2 /$ transc $=\mathrm{CT} 16565 /$ len=2106 $/ \mathrm{GB}: \mathrm{AE} 003553 / \mathrm{note}=3 \mathrm{prime}$ sequence fro

2 FB:FBgn0037253 $/$ sym $=$ CG9798 $/$ name $=/$ prod $=/$ func $=/$ map $=82 \mathrm{C} 1-82 \mathrm{C} 2 /$ transc $=\mathrm{CT} 27682 / \mathrm{len}=1914 / \mathrm{GB}: \mathrm{AE} 003606$

2 FB:FBgn0027066/sym=BcDNA:LD08743/name $=/$ prod $=/$ func $=$ cytoskeletal structural protein $/$ map $=42 \mathrm{C} 3-42 \mathrm{C} 4 /$ transc $=\mathrm{CT} 10989 / \mathrm{len}=1860 / \mathrm{GB}: \mathrm{AE} 003789$

2 FB:FBgn0030541/sym=CG1 1584/name=/prod=/func=ligand binding or carrier $/$ map=12D3-12D3/transc=CT36540/len=3048 /GB:AE003495

2 BDGP:GH18222.3prime-hit /ESTpos=maps in FB:FBgn0033295/sym=CG8689/name=/prod=maltase L-like /func=enzyme /map=44C3-44C3/transc=CT6492/len=521

2 FB:FBgn0011726/sym=tsr /name=twinstar $/$ prod=cofilin $/$ func=actin binding $/$ map=60B2-60B2 /transc=CT13858 /len=733 /GB:AE003462

2 FB:FBgn0033134 $/$ sym $=$ CG12840 $/$ name $=$ Tetraspanin 42El $/$ prod=tetraspanin $/$ func $=/$ map=42E2-42E2 $/$ transc $=$ CT31972 $/$ len=1034 $/$ GB:AE003842 $/$ note $=3$ prime sequence from clone BDGP:GH1495

2 FB:FBgn0026630 $/$ sym $=$ nes $/$ name $=$ nessy $/$ prod $=/$ func $=/$ map $=76$ A3-76A3 $/$ transc $=$ CT27290 $/$ len $=2247 /$ GB:AE003517

2 FB:FBgn0039595/sym=CG10001/name=/prod=allatostatin receptor-like /func=allatostatin receptor $/$ map=98E2-98E2/transc=CT28187/len=990/GB:AE003766

2 FB:FBgn0032518/sym=CG9282/name=/prod=ribosomal protein L24/func=structural protein of ribosome /map=34B6-34B6/transc=CT26439/len=774 /GB:AE003640

2 J04423 E coli bioD gene dethiobiotin synthetase ( -5 and -3 represent transcript regions 5 prime and 3 prime respectively)

2 FB:FBgn0010808 $/$ sym=l(3)03670 $/$ name $=/$ prod $=/$ func $=/$ map=100B4-100B4 $/$ transc $=$ CT4906 /len=906 $/$ GB:AE003776

2 FB:FBgn0035272/sym $=\mathrm{CG} 13922 /$ name $=/$ prod $=/$ func $=$ structural protein of ribosome $/$ map $=62 \mathrm{~B} 4-62 \mathrm{~B} 4 /$ transc $=\mathrm{CT} 33461 /$ len $=830 / \mathrm{GB}: \mathrm{AE} 003472$

2 FB:FBgn0035201 $/$ sym $=$ CG9146 $/$ name $=/$ prod $=/$ func $=/$ map $=61 \mathrm{~F} 5-61 \mathrm{~F} 5 /$ transc $=\mathrm{CT} 26188 / \mathrm{len}=4092 / \mathrm{GB}: \mathrm{AE} 003471$

2 FB:FBgn0027525/sym=BcDNA:LD21529/name $=/$ prod $=/$ func $=/$ map=47C3-47C3 /transc=CT23459/len=1643 /GB:AE003828 /note=3prime sequence from clone BDGP:LD21529.3prime-hit

2 FB:FBgn0025366 /sym=Ip259/name=Intronic Protein $259 /$ prod= /func= /map=31E1-31E1/transc=CT16819/len=838 /GB:AE003628 /note=3prime sequence from clone BDGP:GM13959.3prime-hit

2 FB:FBgn0033188 /sym=CG1600 /name = /prod=/func=enzyme /map=43D3-43D3 /transc=CT37723/len=1747 /GB:AE003840/note=3prime sequence from clone BDGP:GH18014.3prime-hit

2 BDGP:LP1 1629.3prime-hit /ESTpos=maps 3prime of FB:FBgn0033505/sym=CG3451 /name $=/$ prod $=/$ func $=$ cell cycle regulator $/ \mathrm{map}=46 \mathrm{F9}-46 \mathrm{F9} / \mathrm{transc}=\mathrm{CT} 7898 / \mathrm{len}=250$

2 FB:FBgn0000261 /sym=Cat /name=Catalase /prod=catalase $/$ func=catalase $;$ EC:1.11.1.6 $/$ inferred from direct assay $/$ map=75D7-75D8 /transc=CT21282 /len=1977 /GB:AE003519

2 FB:FBgn0031213/sym=CG11372/name $=/$ prod $=/$ func=ligand binding or carrier $/ \mathrm{map}=21 \mathrm{~A} 5-21 \mathrm{~A} 5 /$ transc $=\mathrm{CT} 31742 / \mathrm{len}=1517 / \mathrm{GB}: \mathrm{AE} 003590$

2 FB:FBgn0025828 /sym=EG:EG0003.7/name=/prod=cytosolic translation release factor-like /func=cytosolic translation release factor /map=53D14-53D15/transc=CT19804/len=790/GB:AE003805 2 FB:FBgn0025582/sym=Int6 $/$ name $=$ Int6 homologue $/$ prod=translation initiation factor 3 , subunit $6 /$ func $=$ cytosolic translation initiation factor $/ \mathrm{map}=73 \mathrm{C} 1-73 \mathrm{C} 1 /$ transc $=\mathrm{CT} 27364 / \mathrm{len}=1560 / \mathrm{GB}: \mathrm{AE}$ 
2 FB:FBgn0030531/sym=CG11058/name=/prod=/func=enzyme /map=12B9-12B10/transc=CT30929/len=2254 /GB:AE003493 /note=3prime sequence from clone BDGP:GH10492.3prime-hit

2 FB:FBgn0015268 /sym=Nap1 /name=Nucleosome assembly protein $1 /$ prod=nucleosome assembly protein $1 /$ func=nucleosome assembly chaperone $/ \mathrm{map}=60 \mathrm{~A} 9-60 \mathrm{~A} 9 /$ transc $=\mathrm{CT} 16956 /$ len $=1381 / \mathrm{G}$

2 FB:FBgn0025637/sym=skpA /name=/prod=/func=cell cycle regulator /map=1B10-1B11/transc=CT32694/len=1059/GB:AE003418/note=3prime sequence from clone BDGP:HL01263.3prime-hit

2 FB:FBgn0020236/sym=ATPCL /name=ATP citrate lyase /prod=ATP-citrate (pro-S)-lyase /func=ATP-citrate (pro-S)-lyase ; EC:4.1.3.8 /map=52E1-52E1 /transc=CT18257 /len=3745/GB:AE003808

2 FB:FBgn0038446 $/$ sym $=$ CG14903 $/$ name $=/$ prod $=/$ func $=/$ map $=89 \mathrm{D} 3-89 \mathrm{D} 3 /$ transc $=\mathrm{CT} 34727 / \mathrm{len}=360 / \mathrm{GB}: \mathrm{AE} 003714$

2 FB:FBgn0035471 $/$ sym $=$ CG10849 $/$ name $=/$ prod $=/$ func $=/$ map=63F6-63F6 $/$ transc $=$ CT30379 $/$ len=1056 $/$ GB:AE003479 $/$ note $=3$ prime sequence from clone BDGP:SD09294.3prime-hit

2 FB:FBgn0038826/sym=CG17838/name=/prod=RNA binding protein /func=RNA binding /map=92F10-92F10/transc=CT39628 /len=1802 /GB:AE003732 /note=3prime sequence from clone BDGP 2 FB:FBgn0003498/sym=sqd /name=squid /prod=/func=ribonucleoprotein $/ \mathrm{map}=87 \mathrm{~F} 7-87 \mathrm{~F} 7 /$ transc=CT39414/len=1137/GB:AE003701

2 FB:FBgn0001297/sym=kay /name=kayak/prod=/func=DNA binding /map=99C2-99C4/transc=CT35622/len=2642 /GB:AE003771 /note=3prime sequence from clone BDGP:SD04477.3prime-hit 


\section{Lebenslauf}

Ich bin am 13.12.1975 in Tianjin, Volkrepublik China, geboren und habe die chinesische Staatsangehörigkeit. 1994 schloss ich die No.1 High School in Tianjin ab und begann im selben Jahr mein Studium an der Nankai Universität in Tianjin. 1998 erhielt ich den Bachelor of Science im Hauptfach Molekularbiologie. 2001erhielt ich den Master of Science (First class honours degree) der Nankai Universität in Tianjin.

Am 15. August 2001 begann ich in der Abteilung von Dr. Stephan J. Sigrist am European Neuroscience Institute des Bereichs Humanmedizin der Georg-August-Universität Göttingen unter der Anleitung von Prof. Dr. Friedrich-Wilhelm Schürmann die Arbeit an der Dissertation mit dem Thema „Identification and functional characterization of novel ionotropic glutamate receptor subunits at Drosophila neuromuscular synapse“. 